\title{
PROCEEDINGS OF THE
}

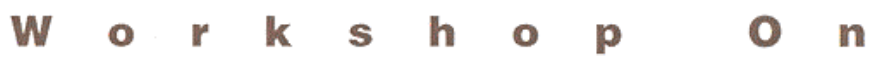

Measurement

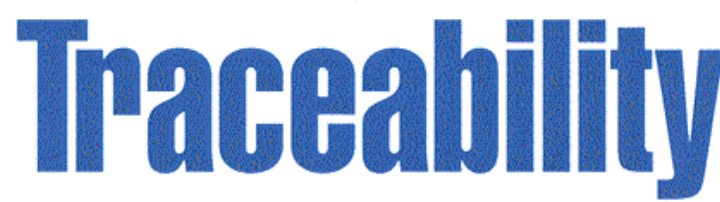

For Clinical Laboratory Testing and In Vitro Diagnostic Test Systems

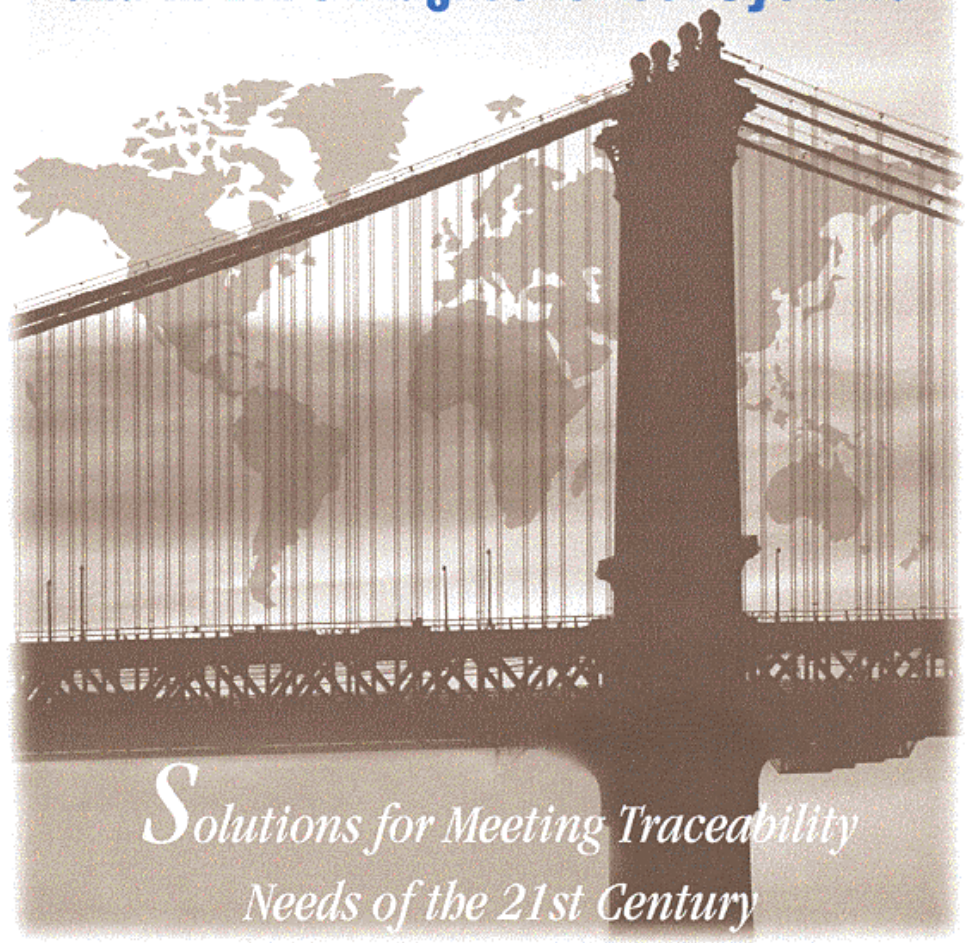

November 2-3,2000 - Gaithersburg, MD

NLT

National Institute of Standards and Technology

Technology Administration, U.S. Department of Commerce

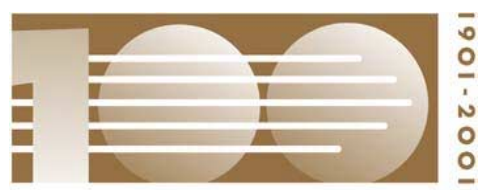

NISTIR 6742

NIST CENTEN N I AL 


\section{NISTIR 6742}

Proceedings of the Workshop on Measurement Traceability for Clinical Laboratory Testing and In Vitro Diagnostic Test Systems

Ellyn S. Beary, Editor

May 2001

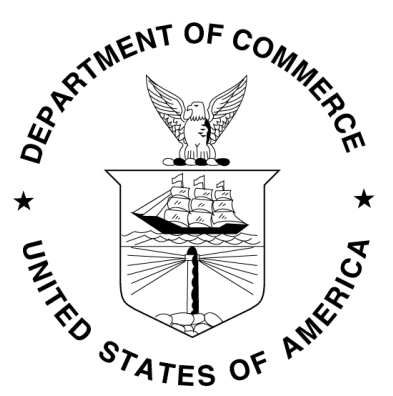

U. S. DEPARTMENT OF COMMERCE

Donald L. Evans, Secretary

TECHNOLOGY ADMINISTRATION

Karen H. Brown, Acting Under Secretary for Technology

NATIONAL INSTITUTE OF STANDARDS AND TECHNOLOGY Karen H. Brown, Acting Director 
Compiled by Gretchen Kennedy

For information contact NIST/CSTL

Telephone:301-975-8300

Facsimile:301-975-3845

E-Mail: cstl@nist.gov

Web Sites:www.nist.gov

www.cstl.nist.gov

Any mention of commercial products is for information only. It does not imply recommendation or endorsement by the National Institute of Standards and Technology, nor does it imply that the materials or equipment mentioned are necessarily the best available for this purpose.

Version of February 2001 


\section{TABLE OF CONTENTS}

(Note: The Agenda Items are linked, and Bookmarks are available to facilitate navigation through the document)

I. INTRODUCTION AND WELCOME ............................................... 1 - 7

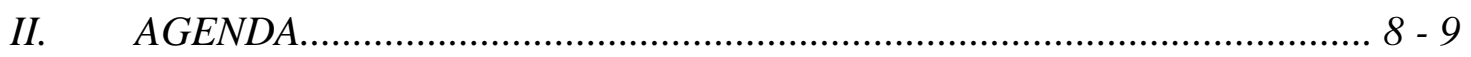

III. MANUSCRIPTS AND VIEW GRAPHS …………............................ 11 - 266

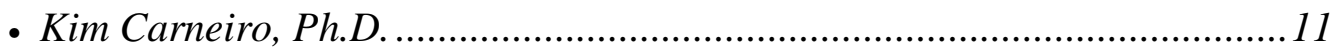

- Emil Voelkert, Ph.D. ...........................................................................

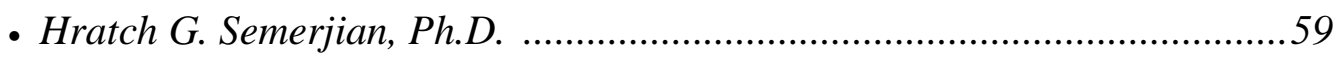

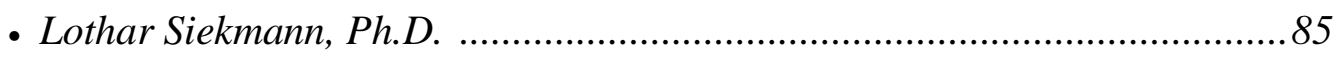

- Heinz Schimmel, Ph.D................................................................115

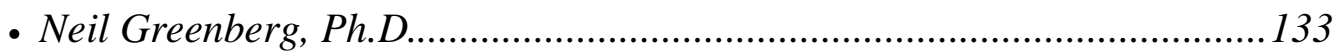

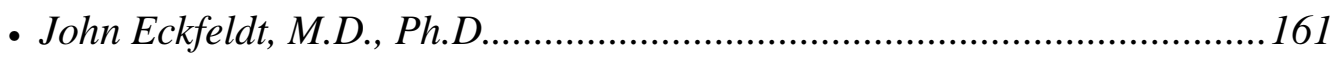

- Willie E. May, Ph.D. ...............................................................................189

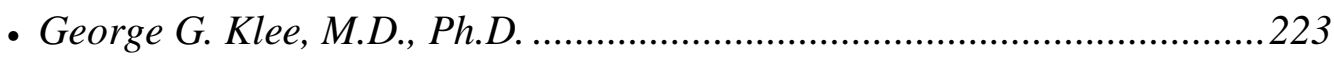

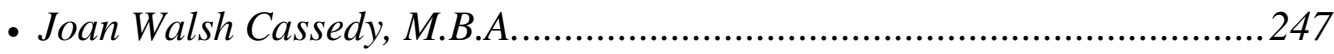

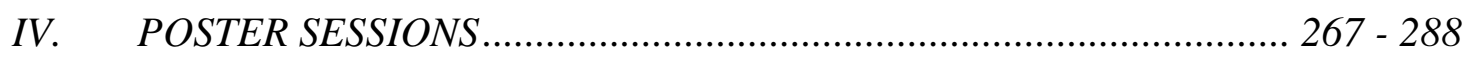

V. BREAKOUT SESSION REPORTS AND DISCUSSION …………....... 289 - 339

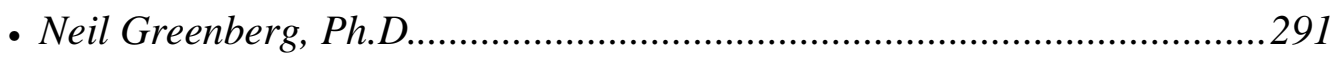

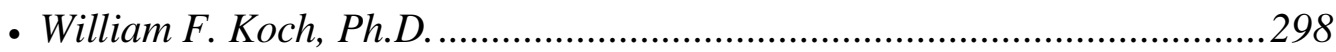

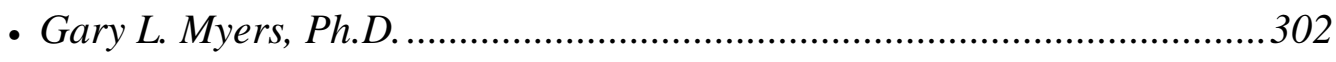

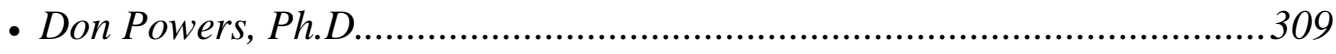

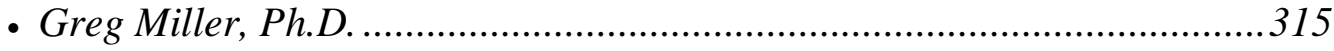

VI. SUMMARIES AND ACTION PLAN …………………………........ 341 - 346

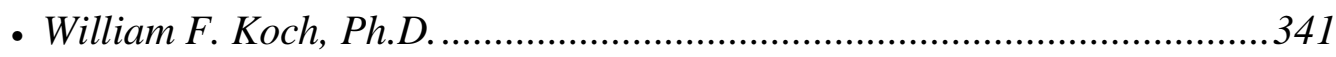

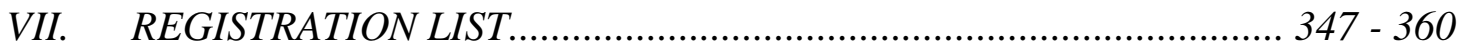




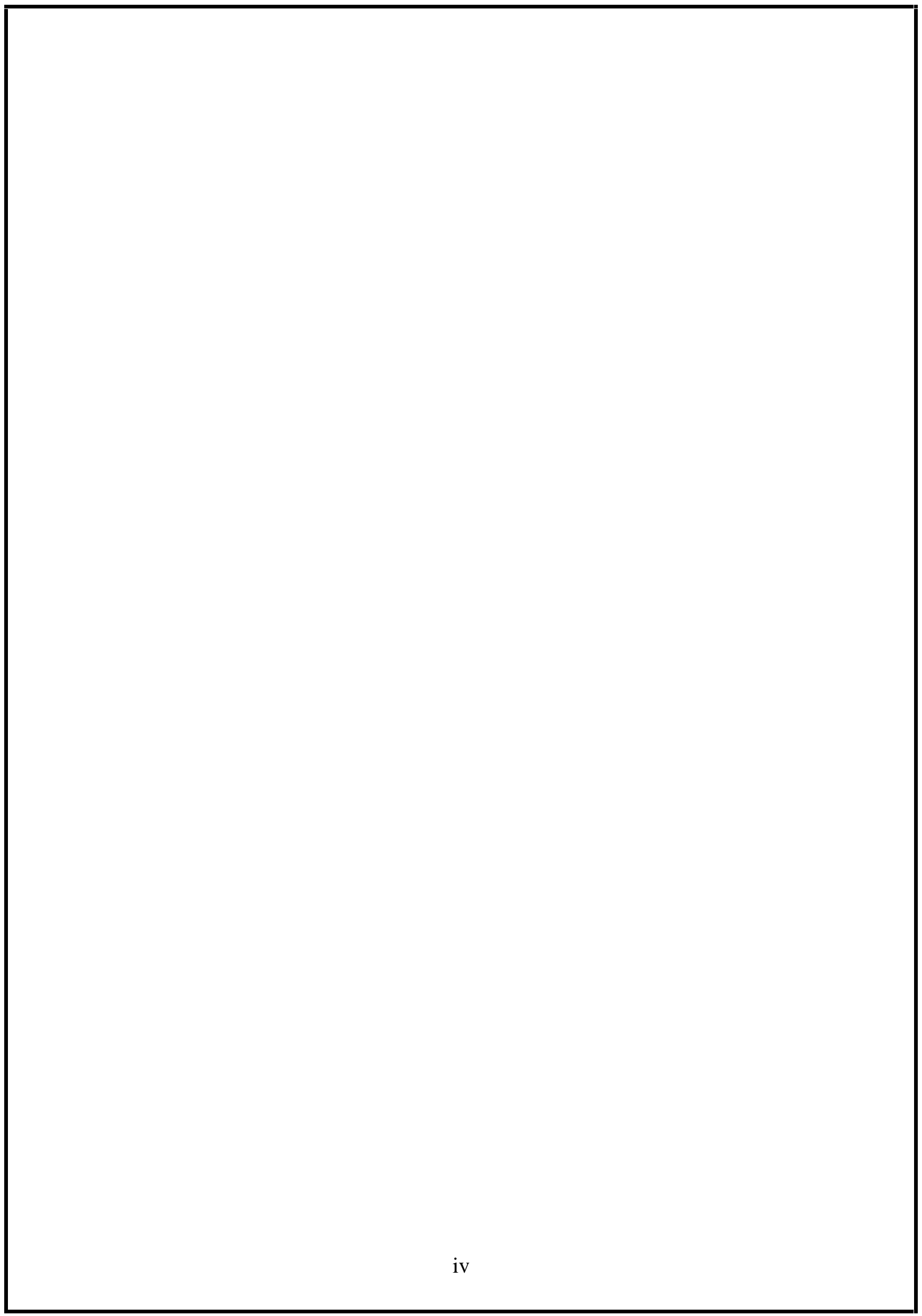




\section{WELCOME}

Dear Colleague,

A workshop on Measurement and Traceability for Clinical Laboratory Testing and In Vitro Diagnostic Test Systems will be held at the National Institute of Standards and Technology (NIST) on November 2 and 3, 2000. It is becoming increasingly evident that a formal national traceability structure will be essential for U.S. clinical device manufacturers to compete in the global marketplace. One of the driving forces for this workshop is the proposed ISO standard for Calibration traceability of In Vitro Diagnostic Medical Devices, which is being drafted in response to the European Union Directive. This Directive requires that all IVD assays be traceable to the highest available reference material or method. NIST is committed to provide the nation's measurement infrastructure, and indeed, has many primary standard reference materials that provide Traceability in the clinical arena. But more is required to properly position the U.S. clinical community to compete in this changing environment.

This workshop will provide a forum for all stakeholders from manufacturers of clinical devices and secondary reference material producers, to government agencies and professional associations. The goal is to assess the measurement needs of the clinical community, and collaboratively produce a plan that addresses international standards issues and assures U.S. manufacturers of an accessible national traceability system.

This letter serves as a call to action. Your participation is important to assess the current and future needs of the integrated clinical community and to ensure a vital U.S. role in the world market.

Sincerely,

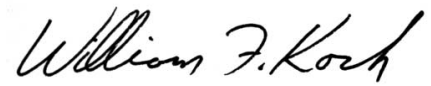

Deputy Director

Chemical Science and Technology Laboratory, NIST

Immediate Past Present, NCCLS 


\section{OVERVIEW}

The National Institute of Standards and Technology (NIST), in cooperation with the NCCLS (National Committee for Clinical Laboratory Standards) and with the sponsorship of the Centers for Disease Control and Prevention (CDC), the Advanced Medical Technology Association (AdvaMed), the College of American Pathologists (CAP), and the American Association for Clinical Chemistry (AACC), is planning a workshop on measurement traceability for clinical laboratory testing and in vitro diagnostic test systems for the IVD industry, professional organizations, government, and national and international standards developing organizations. The workshop will be held on the NIST-site in Gaithersburg, Maryland, November 2-3, 2000. The purpose of the workshop is to actively involve all interested parties in the solution to establishing the means and processes for assuring measurement traceability and reference systems for the healthcare community.

\section{Topics to be Discussed:}

The workshop will include scientific experts who participated in development of the standards for measurement traceability in ISO Technical Committee 212 (ISO/TC 212). Speakers will discuss the technical requirements for establishing measurement traceability, and present practical approaches that manufacturers may follow to satisfy the requirements of these ISO standards. Additional presentations will focus on the state of the art in the calibration of IVD test systems, review global resources already available to manufacturers, and identify areas for which improvements are needed. Breakout sessions will be scheduled for participants to discuss reference measurement methods, reference materials, and laboratories that perform reference measurement procedures

Recommendations will be developed to address needs in the area of clinical measurement traceability and how progress can be accelerated through cooperation among the stakeholders. The emphasis will be on solutions that are in compliance with international standards and industry's needs.

\section{Stakeholders:}

- IVD manufacturer's representatives involved in product development, quality assurance and regulatory affairs

- Representatives of regulatory agencies and notified bodies

- Providers of proficiency testing programs, laboratory accreditation, and external quality assessment materials

- Providers of reference methods and materials for clinical laboratory analysis, supporting measurement traceability of commercial IVD systems

- Laboratory professionals involved in standardization of laboratory methods 


\section{Background:}

Traceability to internationally recognized and accepted standards is an important component in assuring the accuracy and comparability of clinical laboratory measurements. In addition, the global marketplace is presenting new demands for measurement traceability. NIST has a long history of providing such measurement standards in several fields including the clinical laboratory community and is continuing its efforts to develop new reference methods and materials for important health-status markers to meet on-going and future needs for traceability. NCCLS is a globally recognized, voluntary consensus standards-developing organization that enhances the value of medical testing within the healthcare community through the development and dissemination of standards, guidelines, and best practices. NCCLS has the Secretariat responsibility for the ISO Technical Committee 212 (ISO/TC 212) on Clinical Laboratory Testing and In Vitro Diagnostic Test Systems (TC 212), and is the home of the National Reference System for the Clinical Laboratory (NRSCL), a collection of broadly understood reference systems intended to improve the comparability of test results, consistent with the needs of medical practice. Recently, a new opportunity has emerged that applies new pressure on the quest for traceability and the demand for reference systems. Prompted by the European Union's In Vitro Diagnostics Directive (IVDD), the European Committee for Standardization's Technical Committee 140 (CEN/TC 140), in vitro diagnostic systems, began drafting a standard on metrological traceability. By working closely together, CEN/TC 140 and ISO/TC 212 will develop identical European and ISO standards on this topic. Full implementation of the IVD Directive is expected by December 2003 and will require that calibration of all IVD assays be traceable to the highest available reference material or method. 


\section{SPONSORS}

\section{NLT}

National Institute of Standards and Technology Technology Administration, U.S. Department of Commerce
The National Institute of Standards and Technology (NIST), located in Gaithersburg, Maryland, is an agency of the U.S. Department of Commerce's Technology Administration.

NIST's mission is to promote U.S. economic growth by working with industry to develop and apply technology, measurements, and standards. NIST's Chemical Science and Technology Laboratory (CSTL) works to provide the chemical measurement infrastructure to enhance U.S. industry's productivity and competitiveness, assure equity in trade, and improve public health, safety, and environmental decision-making. This is accomplished through the development and critical evaluation of analytical methods, and provision of certified reference materials and reference data that support measurement accuracy and traceability for diverse communities such as those performing health-related measurements.

\section{http://www.nist.gov/}

The College of American Pathologists (CAP) is headquartered in Northfield, Illinois with its Division of Government and Professional Affairs located in Washington, DC. The College is a medical society serving more than 15,000 physician members and the laboratory community throughout the world. It is the world's largest association composed exclusively of pathologists and is widely considered the leader in providing laboratory quality improvement programs. The mission of the College, the principal

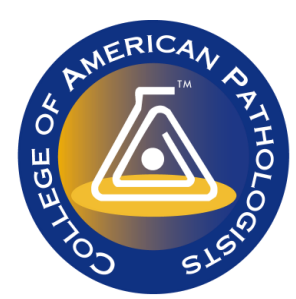

Advancing Excellence organization of board-certified pathologists, is to represent the interests of patients, the public, and pathologists by fostering excellence in the practice of pathology and laboratory medicine worldwide.

\section{http://www.cap.org/}

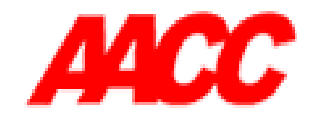

Advancing Clinical Laboratory Science Worldwide

The American Association for Clinical Chemistry (AACC), headquartered in Washington $\mathrm{DC}$, is an international scientific/medical society of clinical laboratory professionals, physicians, research scientists and other individuals involved with clinical chemistry and other clinical laboratory science-related disciplines. Founded in 1948, the society currently has 11,000 members in 93 countries. The AACC was formed to further the public interest by encouraging the study, advancing the science, and improving the practice of clinical chemistry. To this end, they establish standards for education, training and certification of clinical chemists and sponsor activities that promote scientific knowledge in health-related fields.

\section{http://www.aacc.org/}




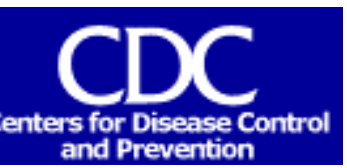

The Centers for Disease Control and Prevention (CDC), located in Atlanta, Georgia, is an agency of the U.S. Department of Health and Human Services. In fulfillment of its mission "to promote health and quality of life by preventing and controlling disease, injury, and disability", the CDC monitors public health, maintains national health statistics, supports immunization services, guards against international disease transmission, fosters health environments, and supports research into disease and injury prevention including occupational injuries.

\section{http://www.cdc.gov/}

NCCLS, headquartered in Wayne, PA, is a globally recognized, voluntary consensus standards-developing organization that enhances the value of medical testing within the healthcare community through the development and dissemination of standards, guidelines, and best practices. NCCLS is

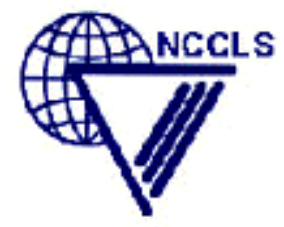
fundamentally and strategically committed to facilitating development of medical-testing standards worldwide and to global harmonization through the NCCLS and ISO standardization processes. NCCLS has the Secretariat responsibility for the ISO Technical Committee 212 (ISO/TC 212) on Clinical Laboratory Testing and In Vitro Diagnostic Test Systems (TC 212), and is the home of the National Reference System for the Clinical Laboratory (NRSCL), a collection of broadly understood reference systems intended to improve the comparability of test results, consistent with the needs of medical practice.

\section{http://www.nccls.org/}

AdvaMed (formerly HIMA) is headquartered in Washington, DC and is the largest medical technology trade association in the world, supported by more than 800 medical device, diagnostic products and health information systems manufacturers of all sizes. AdvaMed is the only medical device association that operates globally to promote a legal, regulatory and economic climate that advances health care by assuring worldwide patient access to the benefits of medical technology. Together, AdvaMed companies form a strong and effective partnership to support policies and practices that provide timely delivery of new medical technologies throughout the global health-care market.

http://www.himanet.com/

\section{Industry Sponsors}

The following corporation sponsors provided funding support.

BD (Becton, Dickinson and Co.)

Beckman Coulter, Inc.

Ortho-Clinical Diagnostics, Inc.

Diagnostics Products Corporation Roche Diagnostics 


\section{Workshop on Measurement Traceability For Clinical Laboratory Testing And In-Vitro Diagnostic Test Systems \\ November 2-3, 2000}

\section{Steering Committee}

Basil Doumas, Co-Chair

Medical College of Wisconsin

Neil Greenberg, Co-Chair

Ortho-Clinical Diagnostics

Carolyn Jones, Co-Chair

Advanced Medical Technology Association (formerly HIMA)

William Koch, Co-Chair

National Institute of Standards and Technology

Ellyn Beary, National Institute of Standards and Technology

W. Gregory Cooper, Bio-Rad Laboratories

John Eckfeldt, University of Minnesota

Chandra Jain, Beckman Coulter, Inc.

David Kelly, NCCLS

Arthur Kessner, Beckman Coulter, Inc.

George Klee, Mayo Clinic

Willie May, National Institute of Standards and Technology

Peter Maxim, Food and Drug Administration

Kenneth McNeil, Dade Behring, Inc.

W. Gregory Miller, Virginia Commonwealth University

Gary Myers, Centers for Disease Control

Debbie Nadel, American Association for Clinical Chemistry

Donald Powers, Powers Consulting Services

Terry Pry, Abbott Laboratories

Jean Rhame, American Association for Clinical Chemistry

John Thompson, Dade Behring, Inc.

Michael Welch, National Institute of Standards and Technology

Beth Ann Wise, NCCLS

The steering committee was supported by many others who contributed to the success of the workshop, and we are most grateful for all their efforts. 


\section{NIST Organizing Committee}

Ellyn Beary

Jeanice Brown Thomas

Jennifer Colbert

Michael Epstein

Amy Grafmuller

Kathleen Kilmer

William Koch

Willie May

Donna Sirk

Kimberly Snouffer

Teresa Vicente

Michael Welch
Chemical Science and Technology Laboratory, NIST

Analytical Chemistry Division, NIST

Standard Reference Materials Program, NIST

Analytical Chemistry Division, NIST

Analytical Chemistry Division, NIST

Conference Program, NIST

Chemical Science and Technology Laboratory, NIST

Analytical Chemistry Division, NIST

Analytical Chemistry Division, NIST

Conference Program, NIST

Conference Program, NIST

Analytical Chemistry Division, NIST 


\section{$A G E N D A$}

Traceability Workshop: November 2-3, 2000

Invited Talks, November 2,

Chair: Basil Doumas, Ph.D., Medical College of Wisconsin, USA

Introduction

9:00-9:15

Invited Talk 1

9:15-9:45

Invited Talk 2

9:45 - 10:15

Invited Talk 3

10:15 - 10:45

Invited Talk 4

$11: 15-11: 45$

Invited Talk 5

$11: 45-12: 15$

Invited Talk 6

12:15 - 12:45

Invited Talk 7

2:00 - 2:30

Invited Talk 8

2:30 - 3:00

Invited Talk 9

$3: 30-4: 00$

Invited Talk 10

4:00 - 4:30
Welcome from NIST Deputy Director

EC IVD Directive - Expectations and

Implications

Standardization Activities to Support the Implementation of the IVD-Directive:

Harmonized Standards and the European Free Market

Status of MRAs and International

Standardization

Traceability of Calibration for IVD

Industry: Process for Credentialing

Materials, Reference Methods, and

Reference Laboratories in Europe

European Reference Materials Activities in the Clinical Field

IVD Directive Requirement for Calibration Traceability from the U.S. Industry

Perspective

History of Reference Systems for Clinical Measurements

Development of Reference Methods and Reference Materials in the U.S.

Importance of Commutable Human

Specimens and Test Value

Distributions for Assay Calibration

Reference Systems Models from Other

Industries
Karen Brown, Ph.D. NIST, USA

Kim Carneiro, Ph.D. Danish Institute of Fundamental Metrology Denmark

Emil Voelkert, Ph.D. Roche Diagnostics Germany CEN/TC 140, Chair

Hratch G. Semerjian, Ph.D. NIST, USA

Lothar Siekmann, Ph.D. University of Bonn, Germany

Heinz Schimmel, Ph.D. Institute for Reference Materials \& Measurements (IRMM), Belgium

Neil Greenberg, Ph.D., Ortho Diagnostics, USA

John Eckfeldt, M.D., Ph.D., CAP

Fairview-University Medical Center, USA

Willie E. May, Ph.D.

NIST, USA

George G. Klee, M.D, Ph.D. Mayo Clinic, USA

Joan Walsh Cassedy, MBA ACIL, USA

Poster Session all day, November 2

Chair: Michael J. Welch, Ph.D., NIST, USA 


\section{Breakout Sessions, November 3, 2000}

\section{Breakout Session Convener}

Session 1

Neil Greenberg, Ph.D., D.A.B.C.C. Ortho Clinical Diagnostics

\section{Topic of Breakout}

Title: Priorities for National and International Investments in New or Improved Reference Systems in Support of Clinical Laboratory Measurement

Objective: To identify and rank the factors that are necessary for determining priorities for development of new reference systems.
Session 2

Don Powers, Ph.D.

Private Consultant
Title: Reference Materials and Reference Measurement Procedures to Support Traceability Requirements of the IVD Directive.

Objective: To identify practical ways to close important gaps in available reference measurement procedures and materials, and to explore ways to maximize cooperation among existing organizations developing and providing reference systems.

\section{Session 3}

Greg Miller, Ph.D.

Medical College of Virginia Hospitals at Virginia Commonwealth University
Title: Impact of Method-Material Matrix Interactions on Calibration Traceability Protocols for Successful Harmonization of Patient Results.

Objective: To develop a statement of limitations in the commutability of reference materials and the appropriate use of such reference materials for traceability and harmonization of results.
Session 4

William F. Koch, Ph.D., F.A.C.B. NIST/NCCLS
Title: Development of International Consensus on the Credentialing Process for Reference Systems

Objective: To develop an international consensus on the credentialing process for reference systems.

\section{Session 5}

Gary L. Myers, Ph.D., F.A.C.B.

Centers for Disease Control and Prevention
Title: Creating and Sustaining Reference Method Laboratory Networks

Objective: To develop criteria necessary for the implementation and maintenance of reference laboratory networks

\section{Afternoon Session: Reports from Breakout Session Chair: Willie E. May, Ph.D., NIST, USA}




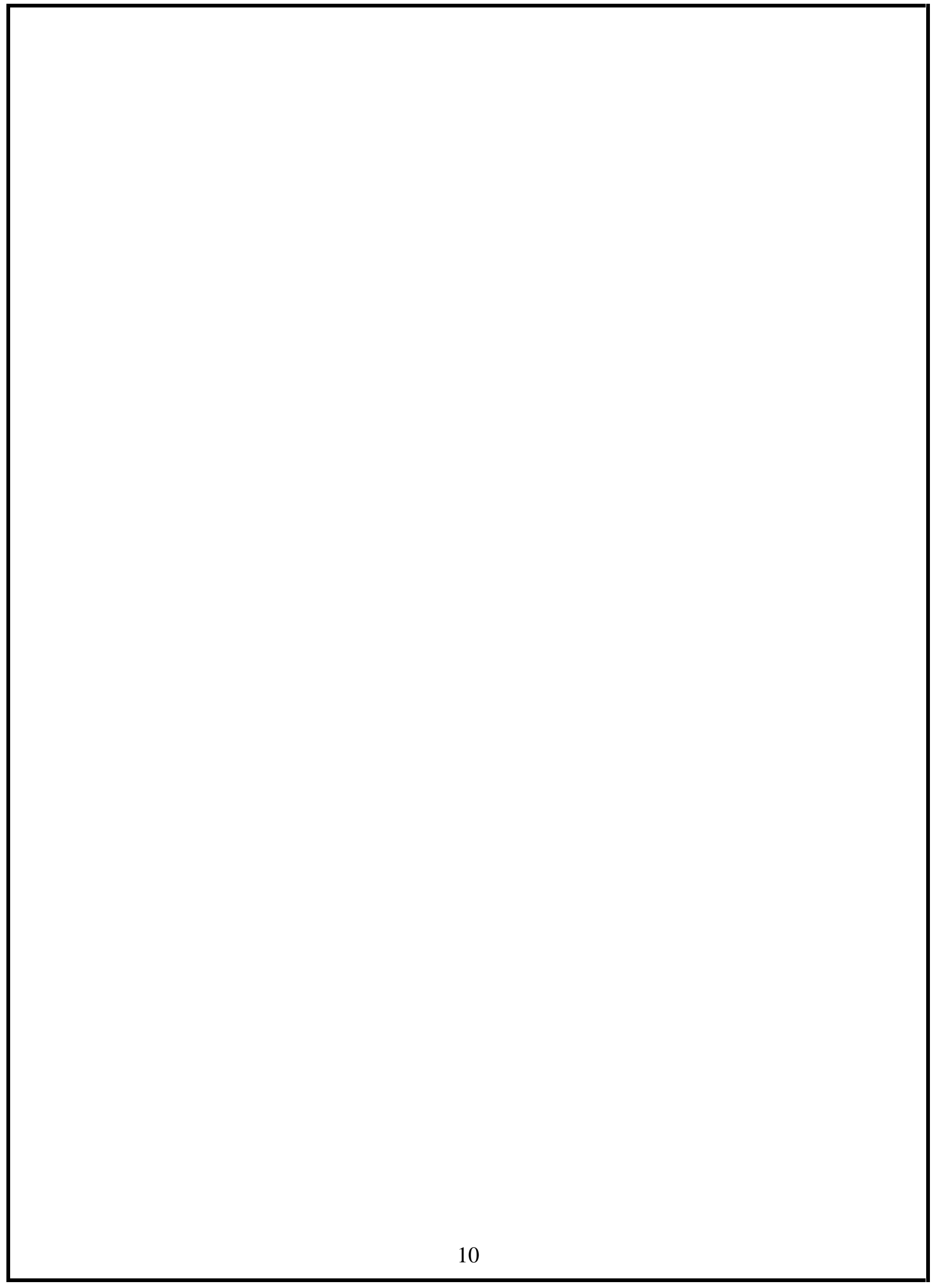




\section{IVD Directive - Expectations and Implications Kim Carneiro \\ Danish Institute of Fundamental Metrology}

\section{THE IVD DIRECTIVE}

\subsection{What is a European Directive?}

Within the framework of common European legislation, the "new approach" Directives play an important role. A directive is a legislative document issued by the European Council of Ministers and the European Parliament, to be implemented by the member states. Implementation is means that the member states must issue appropriate national legislation to make the directive function in the particular member state. There is a time limit for the implementation, which for the IVD directive is December 2003.

The purpose of a directive is to ensure that "devices", such as products and materials that are sold in Europe are functional and safe. However, the directive itself provides no details. Instead it is states the essential requirements for devices in question for the well being of European citizens. It is then up to the manufacturer or importer to demonstrate that the products meet these requirements.

Associated with any directive are one or more voluntary EN/ISO standards that detail the essential requirements and tell the producer how to test the device in order to demonstrate conformity with the directive. These standards prescribe a way to qualify compliance with the directive; but if the producer wishes to demonstrate compliance in another way, he is free to do so as long as complete compliance is demonstrated. One reason why the standards are voluntary is to avoid impediments of technological progress, for instance in testing methods. For the same reason details of test methods are also seldom given. At any point in time, a manufacturer may use test methods other than those mentioned in the standard to demonstrate conformity with the directive.

If a product is subject to more than one directive, careful considerations must be given to resolve any discrepancies in compliance requirements. Sometimes compliance must be with both directives; sometimes one has priority over the other.

Although the tests required may be done by any competent laboratory (and competence is increasingly demonstrated through accreditation), the conformity assessment itself and the associated type approval are done by a notified body. Hence, each member state will inform the European Commission about which body is authorized to issue the pattern approval.

Once the product has been type approved, each product is marked with a CE-mark by the producer/importer. The CE-mark serves as a manufacturer's declaration that "this product complies with all relevant EU-directives." Manufacturers that perform CEmarking must register, and are subjected to inspection. 
Although the above describes a typical case, there are variations on the theme. Certain products must be initially verified for proper functioning, while others must be re-verified. In the case of potential severe danger in the case of malfunctioning of the product, each product must be inspected by an independent competent body. In short, the complexity of the device and the potential hazard due to improper functioning are reflected in the tests.

\subsection{An Example: The Toy directive}

Before going to the IVD directive, let us take a look at another example, namely the toy directive. This states requirements for toys that are essential for their harmlessness in the hands of children. It distinguishes between children below and above 3 years, because the behavior in playing with toys changes at that age.

The voluntary standard EN 71 specifies how to test toys. For instance it gives the dimensions of a standard throat of a 3-year-old child that the toys (or parts thereof) may not easily pass through. If the toy does not pass this test, the CE-marking must be accompanied by a clear statement such as "this toy is not intended for the use of children below 3 years of age."

In Denmark the notified body for the toy directive is the Agency for Consumer Protection. Any producer or importer may CE-mark (i.e. declaring compliance with the directive).

If the toy in question must be connected to an electrical supply, the low voltage directive comes into play. However, in the case of a children's lamp (for instance with the shape of a doll) the lamp must be considered safe as a toy and not as an electrical appliance. This means that the lamp must be equipped with a $12 \mathrm{~V}$ transformer and cannot be connected directly to the 220 volt supply.

\subsection{The IVD Directive ${ }^{\mathrm{i}}$}

This directive, issued on 27 October 1998 for implementation in the member states by December 2003, applies to in vitro diagnostics medical devices and their accessories. The details of its scope and definitions are stated in Article 1.

Article 3 relates to the essential requirements which are detailed ANNEX 1. It consists of two parts A and B:

\section{A: GENERAL REQUIREMENTS (1/2 page)}

Clause 1 of part A states the following:

"The devices must be designed and manufactured in such a way that, when used under the conditions and to the purposes intended, they will not compromise, directly or indirectly, the clinical conditions or the safety of the patients, the safety or health of users or, where applicable, other persons, or the safety of property. Any risk which may be associated with their use must be acceptable and be compatible with a high level of protection of health and safety."

Clause 3 makes reference to metrology in the following way: 
"The devices...must achieve performances in terms of ...accuracy, repeatability, reproducibility... as stated by the manufacturer.

The traceability of values assigned to calibrtors and/or control materials must be assured through available reference measurements and/or available reference materials of higher order."

Hence, clinical measurements must possess the metrology quality of traceability. This has so far only been sporadically the case. headings:

B: SPECIFIC REQUIREMENTS (4 pages) detailed under the following

- Chemical and physical properties

- Infection and microbial contamination

- Manufacturing and environmental properties

- Devices which are instruments or apparatus with measuring function

Here is stated that accuracy limits must be stated by the manufacturer, and that units must be legal units of the EU.

- Protection against radiation

- Requirements for medical devices connected to or equipped with an energy source

- $\quad$ Requirements for devices for self-testing

- Information supplied by the manufacturer

The rest of the directive details the implementation of the directive including the setting up of a Committee on Medical Devices (Article 7). This committee may examine any question connected with the implementation of the directive.

\subsection{The "Voluntary" Standard"}

The standard associated with the IVD directive will be discussed in a subsequent lecture.

\section{EXPECTATIONS}

Within the general spirit of European Directives, the expectations of the IVD directive may be discussed under the following three headlines:

\subsection{Better European Healthcare by Protecting the Patient}

By stating the general and specific requirement for clinical instrumentation, one expects (of course) that the patient, the end-user of the clinical investigation, will get a better treatment and be protected from the hazards of the clinical devices. Each of the 8 points in the specific requirements has to be read in that light.

Of particular interest is the new focus on measurement traceability as a means of improving laboratory chemistry. 


\subsection{Less Costs Due to Less Double Analysis}

No less important is the fact that one expects significant reductions in the costs of healthcare, costs that are in Europe predominantly included in the public budget. Although the directive at least initially imposes costs on the manufacturer of medical devices, these costs have to be weighed against the significant reductions in erroneous and superfluous analytical activities. Let us look at two examples:

Saving Money From Repetitive Measurements

According the "German Health Report 1998" (available on the internet at www.gbe-bund.de) the costs of repeat measurements amount to 1.5 billion US \$ per year in Germany. This is a number that speaks for itself.

\section{Evolution of Danish Public Chemical Laboratories}

An example that illustrates that the raising of quality standards may lead to more efficient laboratories in the public sector, let us consider the evolution of public chemical laboratories in Denmark during the last ten years. Although not clinical laboratories, their fate may well be indicative of what will happen to the clinical laboratories in the next few years.

Up to 1990 , some 70 of the 228 local administrations in Denmark operated general-purpose laboratories for the surveillance of the environment and foodstuff (MLKlaboratories). In order to offer its services to other clients, one laboratory applied for accreditation; but just before being granted the accreditation from Department of Commerce, the Department of the Interior ruled that this would be illegal, with respect to the operating laws of the laboratories.

During 1992-93, the Department of the Interior came to appreciate the value of accreditation as a means of controlling the quality of the laboratories, and cost reductions were expected. Suddenly, many laboratories saw accreditation as a necessary evil for survival, and this opened a "race for accreditation", so that some 30 laboratories applied for accreditation. ized.

In 2000 , there are 11 accredited public laboratories, which are all highly special-

Hence, by setting strict quality standards for the operation of its laboratories, the Department of the Interior caused considerable structural changes, increased laboratory competence, and significantly reduced the public costs.

\subsection{Openness and Fairness}

Although the system of new approach directives has sometimes been accused of being complicated and unfair to producers outside the European Union, the idea is to create an open and fair market, subjected to regulations that are essential. The system has the following virtues.

- The directives state the essential requirements in an unambiguous way, without prescribing specific tests that could constitute technical barriers to trade. 
- The voluntary standards give practical solutions to the requirements. Other solutions are not excluded.

\section{IMPLICATIONS}

A European directive puts demands on the manufacturer, who is held responsible for the compliance of his products. This of course is also the case for the IVD directive. Manufacturers must ensure that their instruments can be calibrated against reference standards and procedures (implicit from the traceability requirement), that repeatability and reproducibility are quantified and documented, and that accuracy is substantiated by uncertainty calculations. These points conveniently may be categorized as establishing a metrology infrastructure appropriate for in vitro diagnostics.

The IVD directive will probably also lead to a global coordination, as well as to a common approach to quality assurance. These three topics are discussed below.

\subsection{The Establishment of a Metrology Infrastructure for Laboratory Medicine}

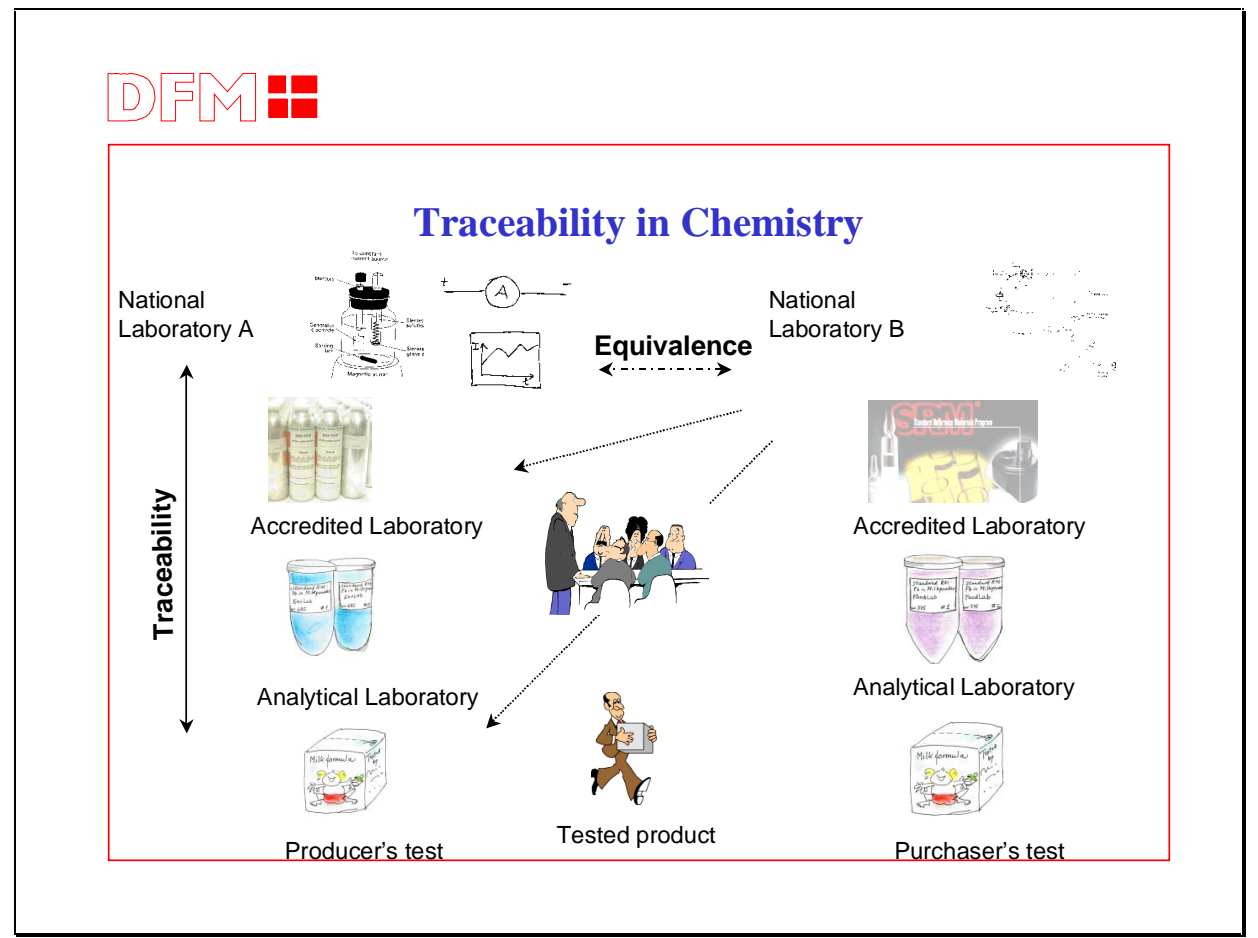

FIGURE 1.

The establishment of a metrology infrastructure for the IVD directive is conveniently discussed in using the general scheme for chemical traceability above. This scheme is itself an adaptation of the scheme that has been developed for most physical quantities under the auspices of the meter convention during the last 125 years. A producer (lower left) of a clinical test-device performs measurements that are traceable to some analytical laboratory, which uses stable control materials and validated procedures in its analysis. The measurements of that analytical laboratory is itself traceable to the certified reference materials and methods of an accredited laboratory, which in turn uses reference materials 
that have been certified at a national laboratory (NMI). Seen from the upper corner of the scheme, the national laboratories have disseminated traceable measurements to the producer. Hence, the producer is traceable to the international system of units, the SI, or some other reference system.

The NMI has another important task, namely to ensure equivalence with other NMIs that do similar dissemination in their countries. When the scheme works properly, all users are sure to arrive at the same measurement results, so that repetitive measurements can be avoided, and one has arrived at the situation where a product is "once tested - globally accepted". In IVD terms this phrase would be "once analyzed, accepted by all doctors".

However, that "everything works properly" requires quality assurance and includes comparisons between laboratories.

The ideal situation of traceability to the SI is only the rare example in the IVD analytical reality. However, it is useful to discuss the opposite situation, namely no traceability at all in terms of the scheme below:

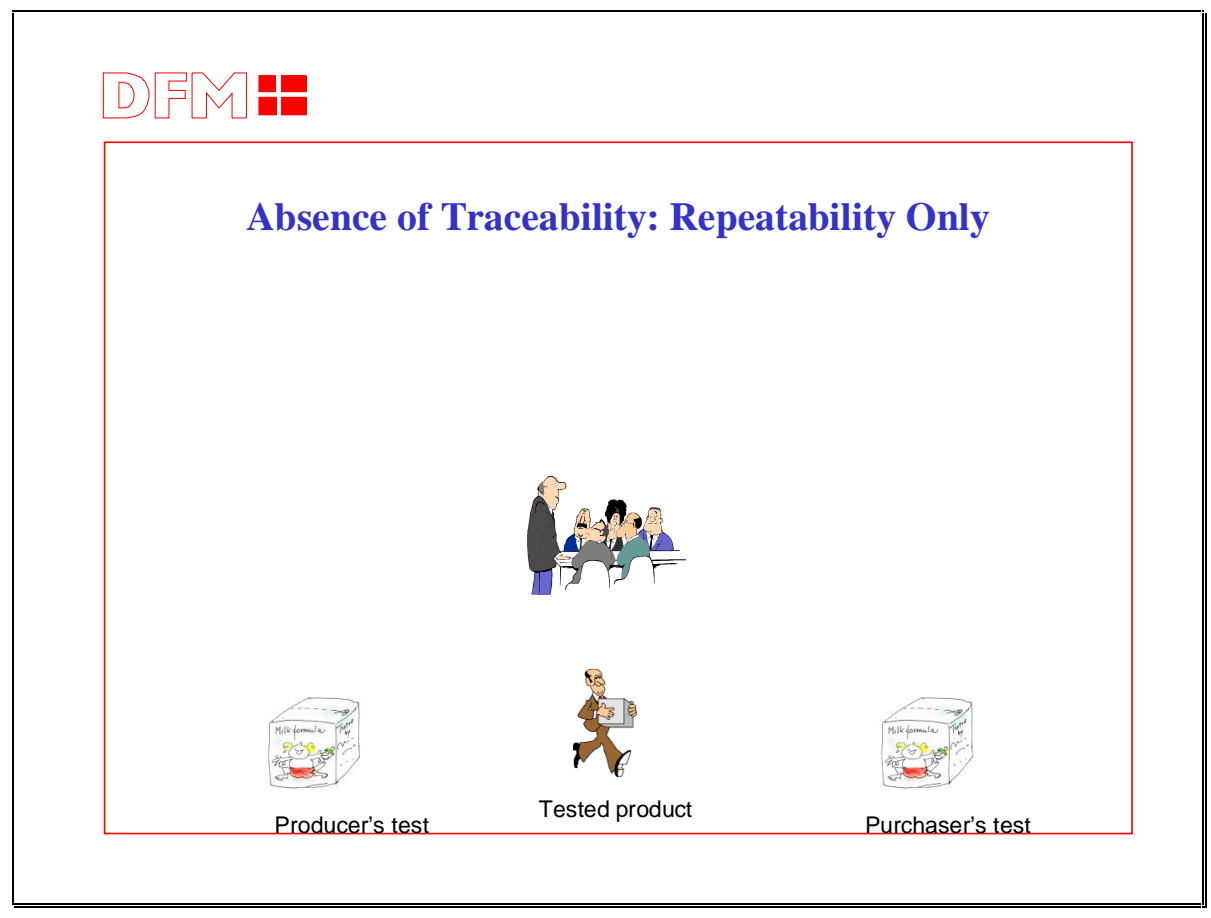

FIGURE 2.

The second figure illustrates that in the absence of traceability, one can only study repeatability, i.e. the stability of the measurements without changes to the circumstances under which the measurements are performed. Since the repeatability of a chemical measurement typically contributes with only a small fraction to the total uncertainty of the measurement, the lack of traceability implies lack of compliance with the IVD directive. 
What the IVD directive requires is traceability to some reference (material and procedure) of higher order, possibly the SI. This is demonstrated in the figure below:

This figure shows the requirement of the IVD directive, namely that traceability to some stated reference is achieved and documented, which ensures that both reproducibility and accuracy can be stated by the manufacturer. In some cases, the stated reference is the SI.

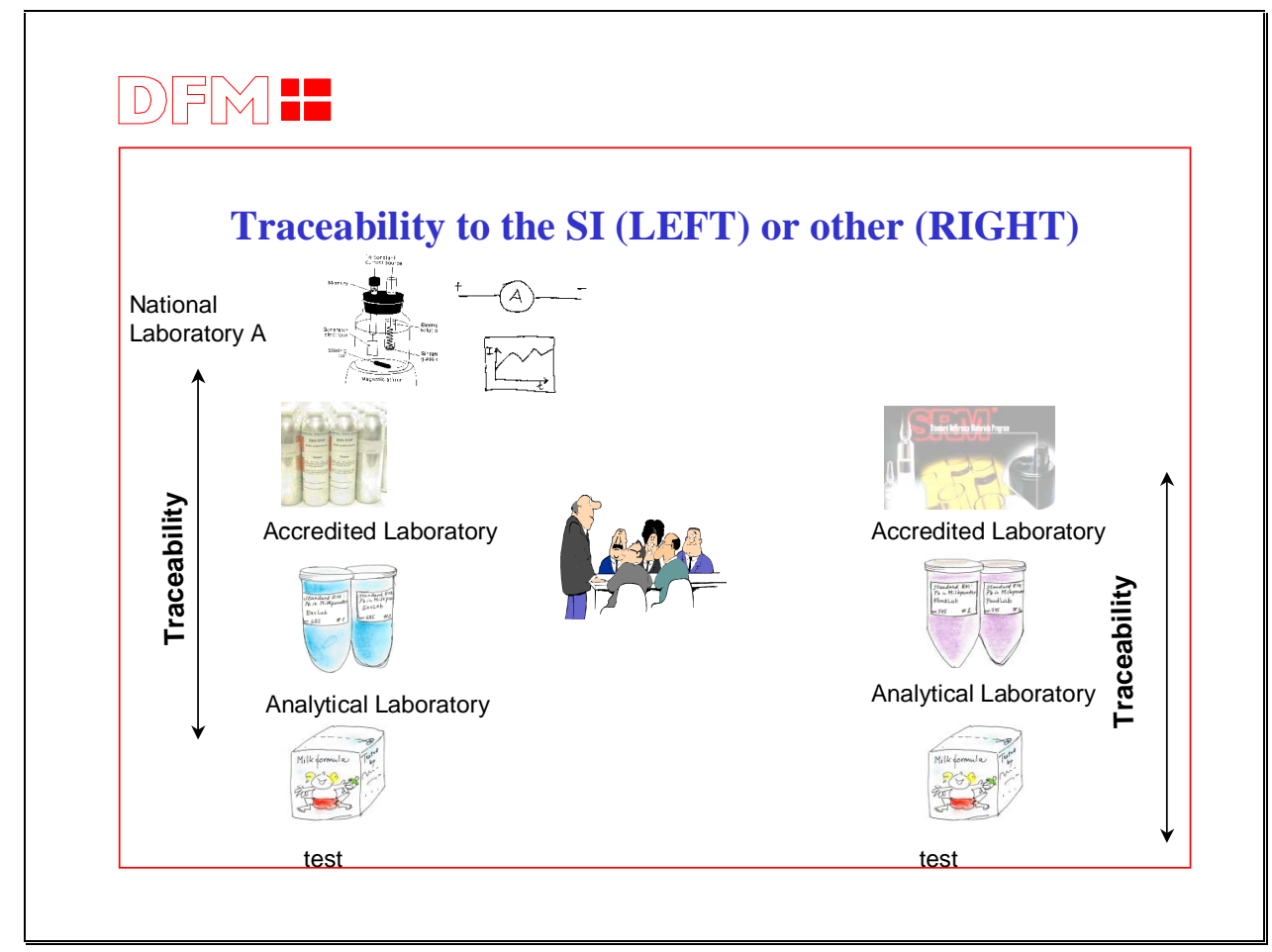

FIGURE 3.

\subsection{Global Coordination}

There are three reasons why the IVD directive will lead to global coordination:

- The IVD industry is a global one. Hence, whichever region sets essential requirements the industry will ensure proliferation to other regions.

- The task of setting up the metrology infrastructure exceeds the capabilities of any region. Therefore, regions must unite forces to deliver the necessary knowledge for its practical implementation.

- Finally, patients travel globally, acquire global diseases and will eventually request global healthcare. 


\subsection{A Common Approach to Quality Assurance}

Although a common approach to traceability has been achieved globally amongst NMIs, the way in which traceability is disseminated within each country has not been fully agreed upon; and different approaches to quality assurance are still prevailing. As quality in measurement is of paramount importance in the IVD field, a global approach to quality should emerge, so that the above global coordination can become effective.

\section{CONCLUSIONS}

The European IVD directive is designed for the well being of Europeans. Since it has global implications it will be followed up by global research.

In order to make it work, a coordinated approach has to be adopted for the basic concepts of quality in measurements. An infrastructure must be developed for the IVD sector.

\section{ACKNOWLEDGMENTS}

I would like to thank my colleagues in Metrology in the Healthcare initiative ${ }^{\mathrm{iii}}$ for introducing me to the field of IVD and to share with me their knowledge of clinical chemistry. I would also like to thank my colleagues in the various groups within the framework programs for research and technological development of the European Union $^{\text {iv } v}$ who have taught me that metrology in chemistry has facets other than metrology in physics.

\section{References}

${ }^{\text {i }}$ Directive 98/79EC of the European Parliament and of the Council of 27 October 1998 on in vitro diagnostic medical devices.

ii prEN ISO 17511 (CEN/TC 140) In vitro diagnostic medical devices- Measurement of quantities in samples of biological origin- Metrological traceability of values assigned to calibrators and control materials.

iii Consultative meeting on measurements in healthcare (minutes June 23, 2000).

iv Metrology in Chemistry. Current Activities and Future Requirements in Europe (EUR 19074 EN, ISBN 92-828-7465-6).

$\checkmark$ Metrology in Chemistry. Position paper of the High Level Expert Group on Measurement and Testing under the $5^{\text {th }}$ Frame Work Program. 


\section{KIM CARNEIRO}

\section{Danish Institute of Fundamental Metrology}

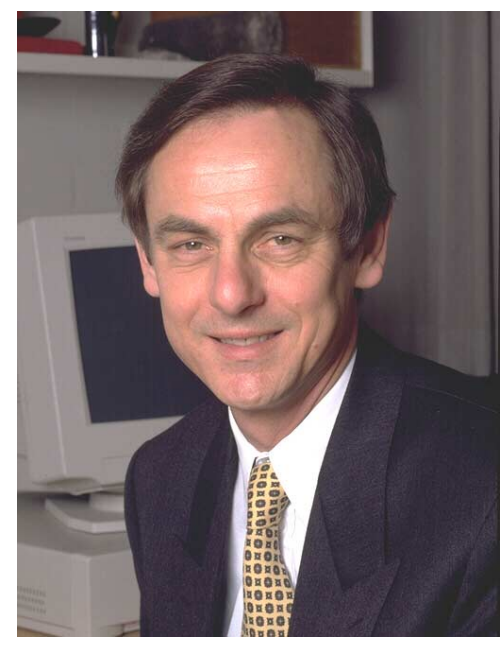

Kim Carneiro, M.Sc., Ph.D., is the current director of the Danish Institute of Fundamental Metrology (DFM), a Member of the Board of the SYMBION Foundation, and Chairman of the Board of Image Metrology ApS. Dr. Carneiro received his Ph.D. in physics from the University of Copenhagen in 1974, followed by a postdoctoral research position at Brookhaven National Laboratory that lasted until 1976. He then took a professorship at the University of Copenhagen from 1976 to 1986 with a sabbatical at IBM Research in San Jose, CA from 1982-1983. In 1986, Dr. Carneiro accepted his current position at DFM where he worked on the construction of quality assurance at the DFM, helped build the metrological organization in Denmark, served as Scientific Advisor to IPQ-Portugal and served as Strategic Advisor to INMETRO Brazil. Dr. Carneiro is a member of the Danish Physical Society, Solid Section, holding the position of Chairman from 19761979, the European Physical Society, the Danish Academy for Natural Sciences, and the Academy of Technical Sciences, ATV. He is currently a member of numerous standardisation committees, evaluation committees, and technical working groups, including the EUROMET Committee, serving as chairman from 1994-1998, the UN-ECE working party on technical harmonisation and standardisation policies as Co-rapporteur for Metrology, the Management group of the Centre for Fundamental Metrology Manager, the Steering Group for European Metrology in Chemistry of the European Commission, and the Supporting group for $5^{\text {th }}$ Framework programme "Competitive and Sustainable Growth" of the Danish Research Ministry. 


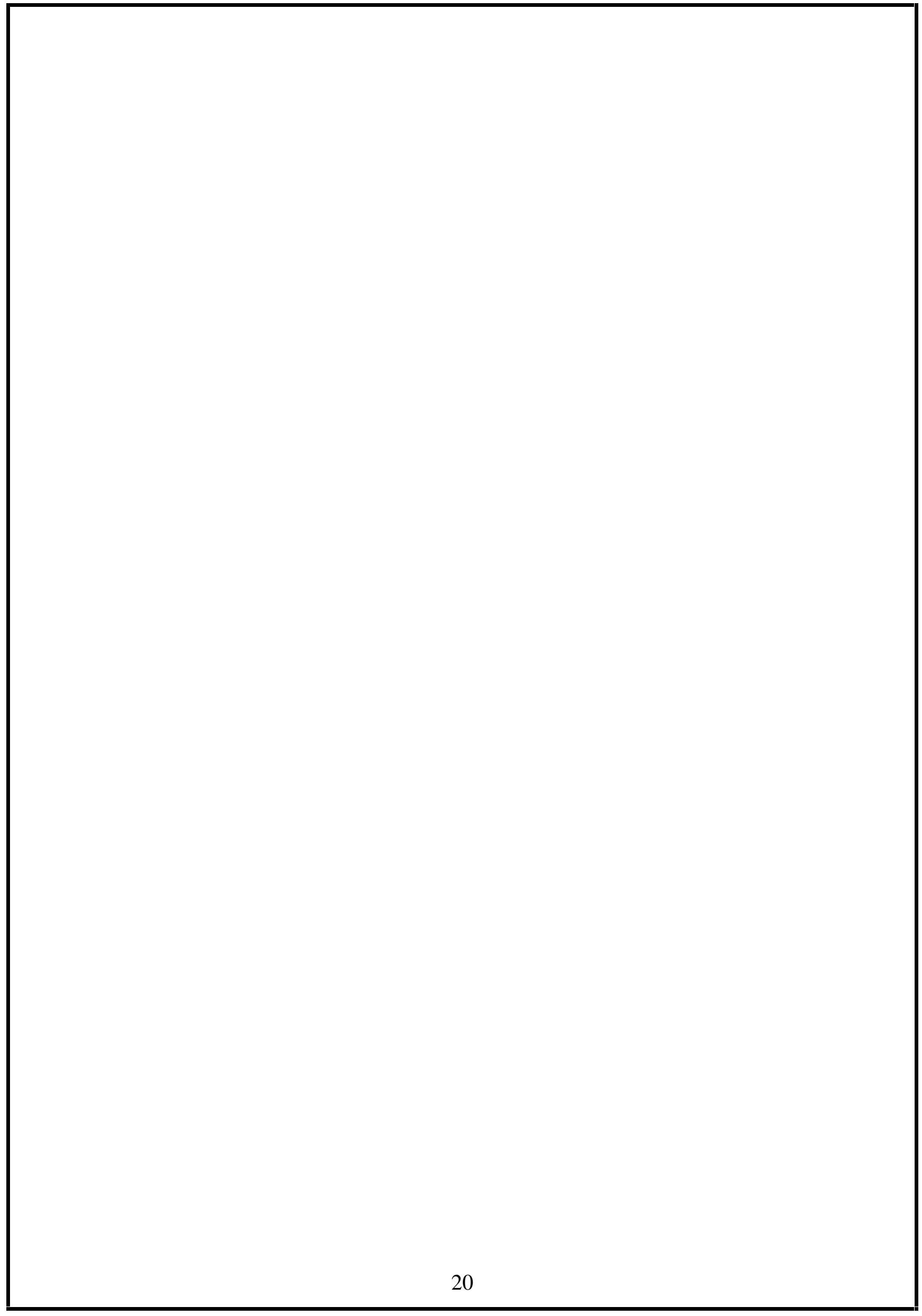




\title{
IVD Directive - Expectations and Implications
}

\author{
Visuals Presented By
}

Kim Carneiro

Danish Institute of Fundamental Metrology 

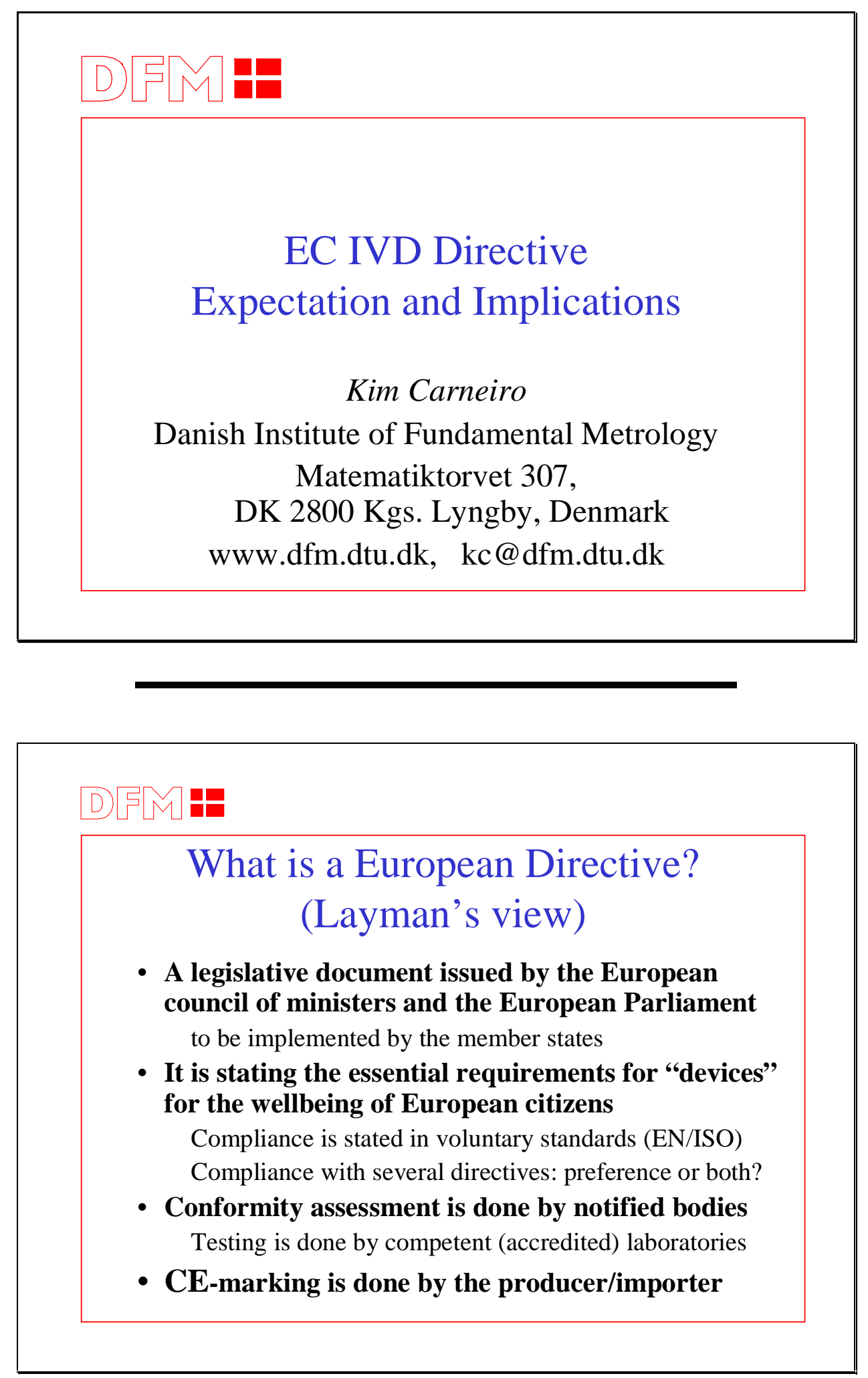


\section{Example: The toy directive}

- The toy directive states requirements for toys that are essential for their safety

- The standard EN 71 tells you how to test toys

Distinguish between children below and above 3 years

- In Denmark the notified body is the Agency for Consumer protection

- Any producer or importer may CE-mark (i.e. declaring compliance with the directive).

Registration required

- Preference over low-voltage directive:

Children's lamps must be supplied by $12 \mathrm{~V}$

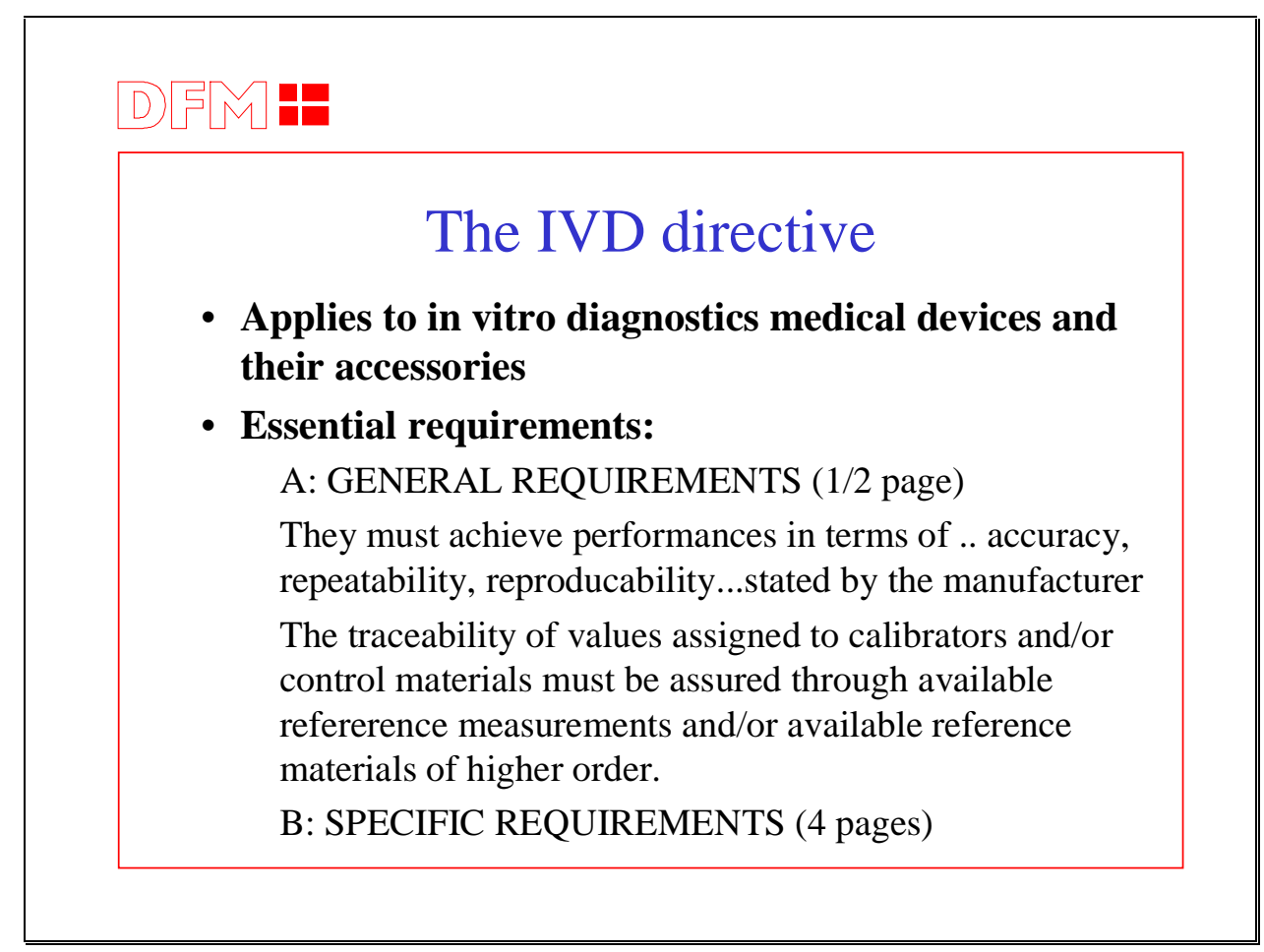




\section{The IVD directive: \\ Expectations}

- Better European healthcare by protecting the patient

- Less costs due to less double analysis

- Openness and Fairness

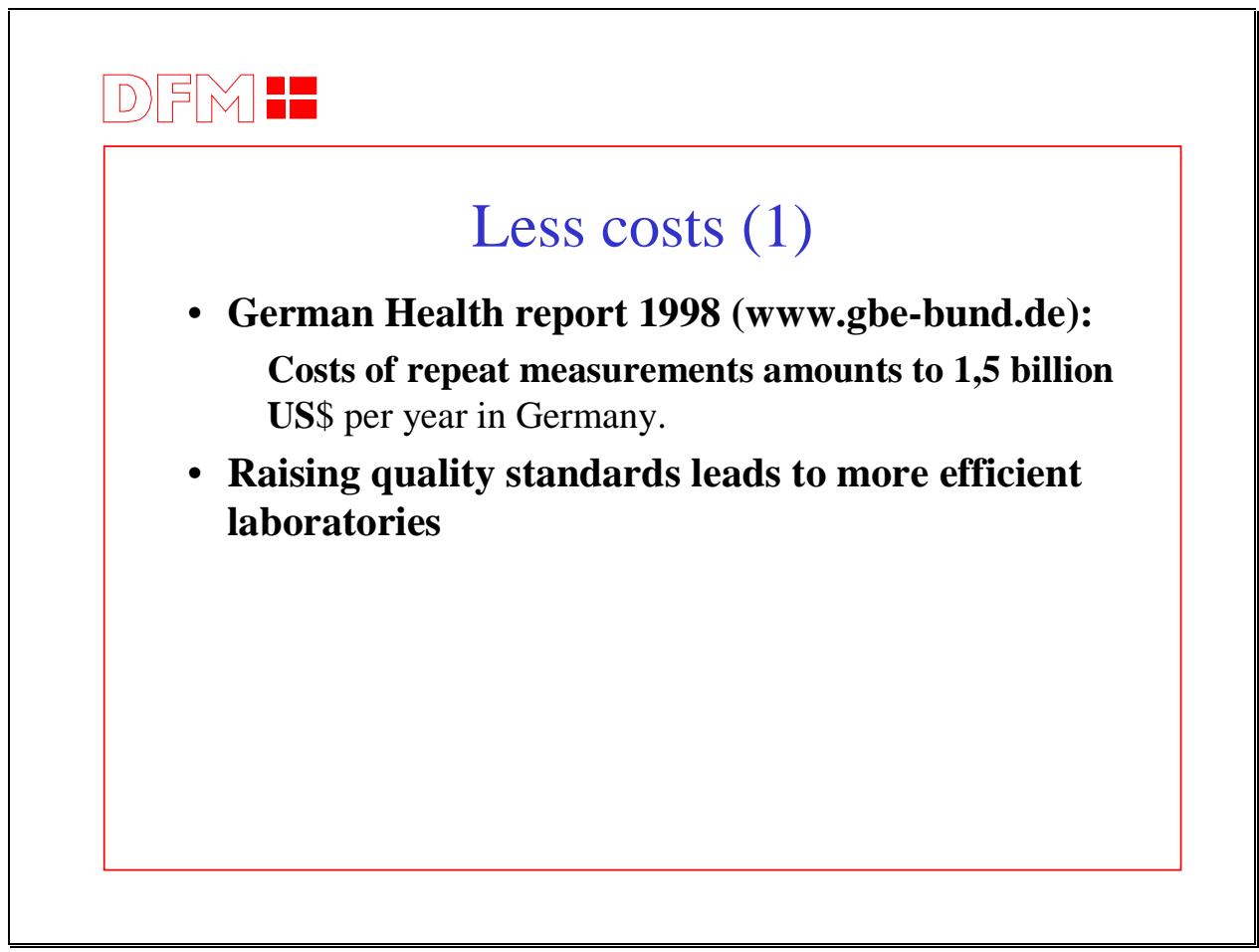




\section{Less costs (2)}

Evolution of Danish Public Laboratories

- Up to 1990

Some 70 of the 228 Local administrations in Denmark operate general purpose laboratories for the surveillance of environmental and foodstuff ("MLK-laboratories")

One applies for accreditation; but that is illegal

- During 1992-93

About 30 laboratories applies for accreditation

- In 2000

There are 11 accredited public laboratories (specialised)

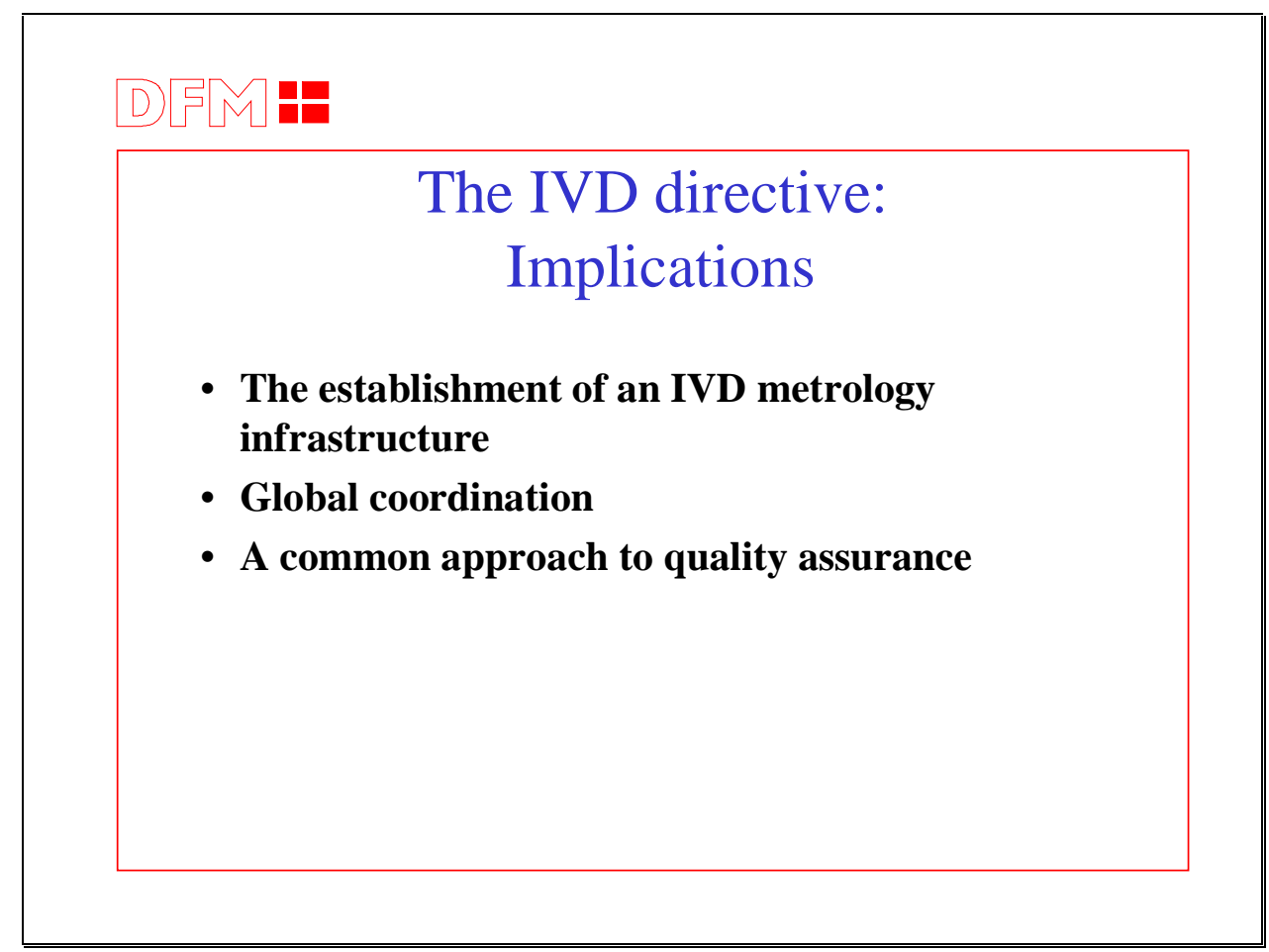




\section{D) $5 \mathrm{M}=$}

\section{What is Metrology?}

Repeatability

Traceability to SI or other

Uncertainties (Accuracy)

Comparisons (Reproducibility)

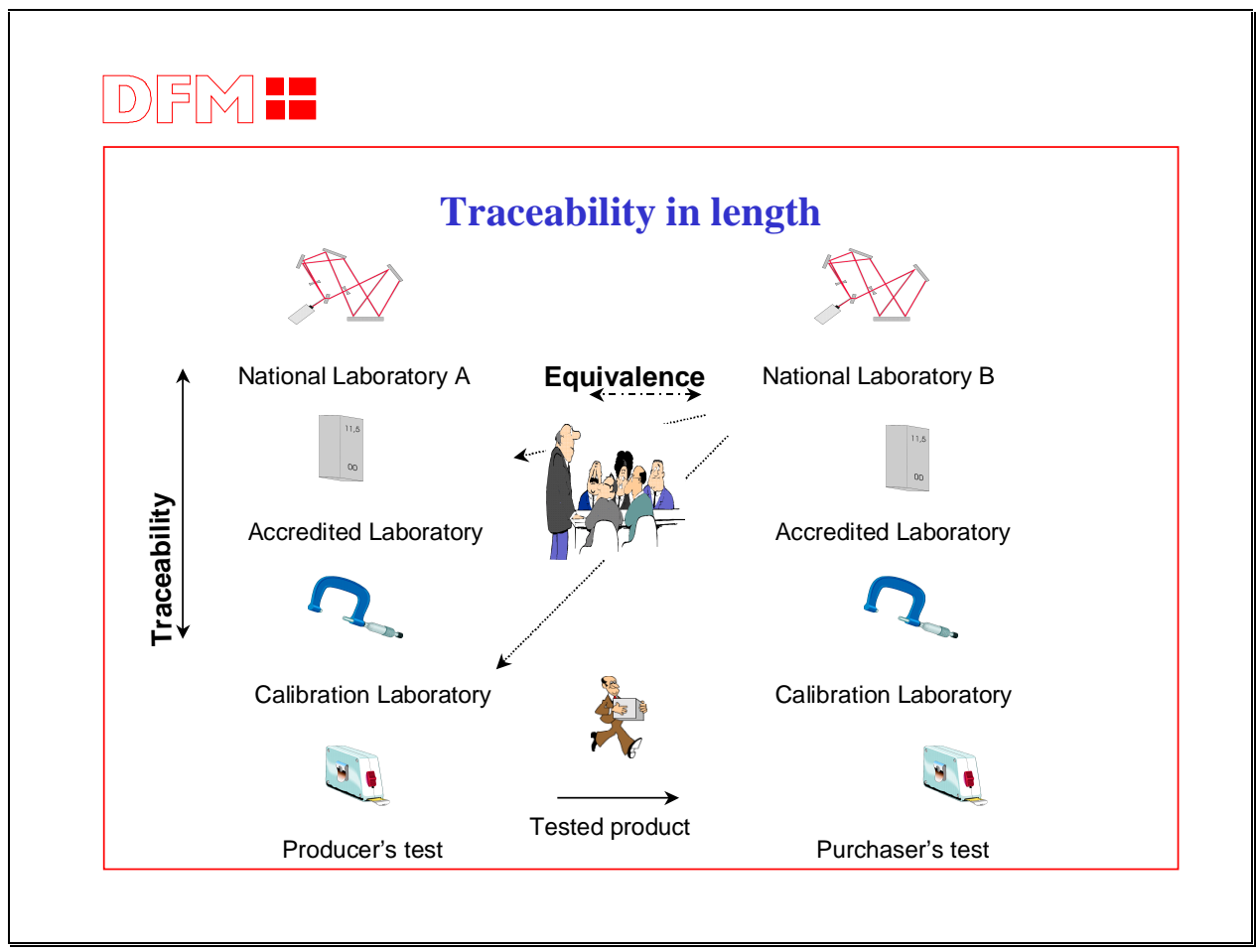



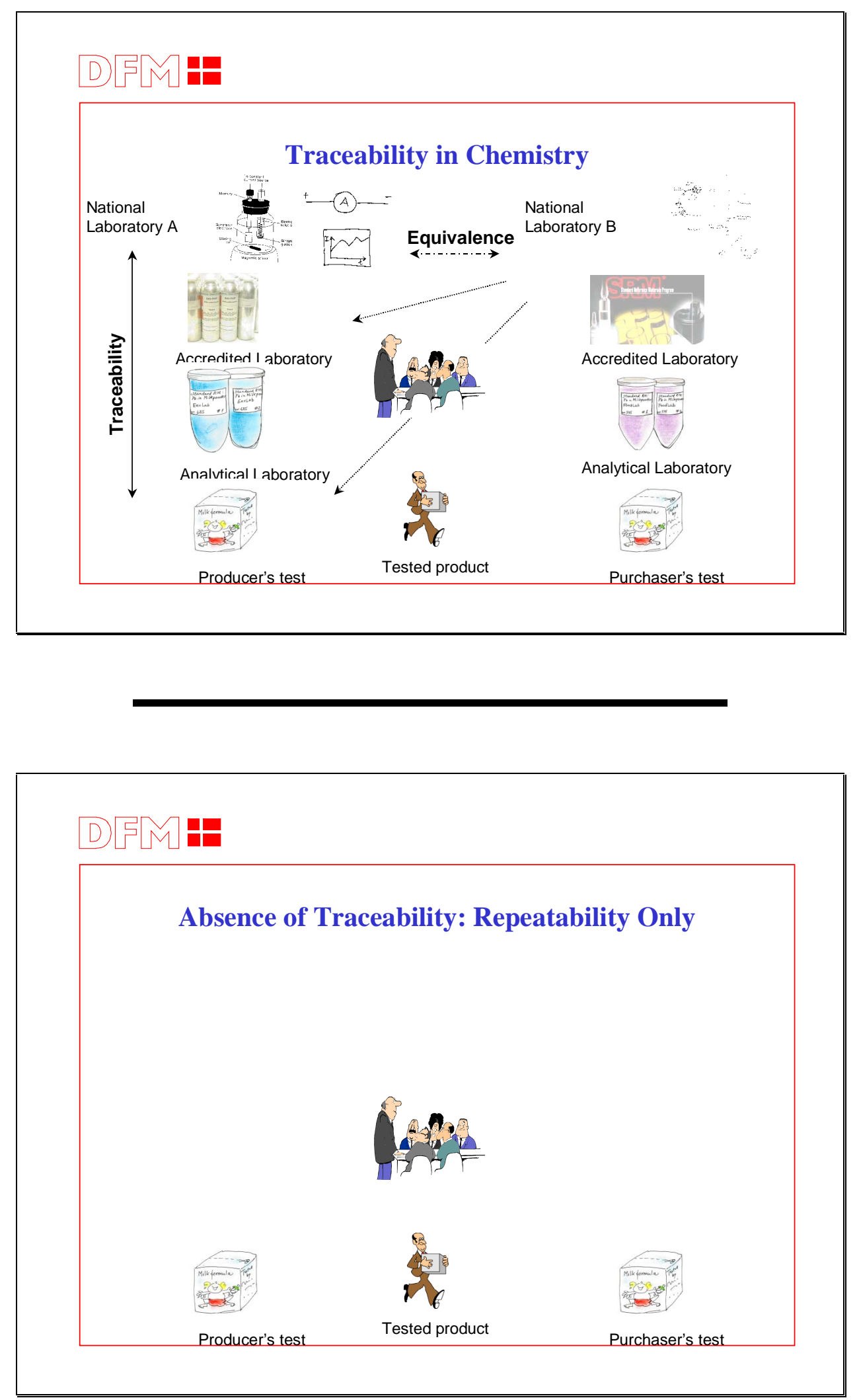

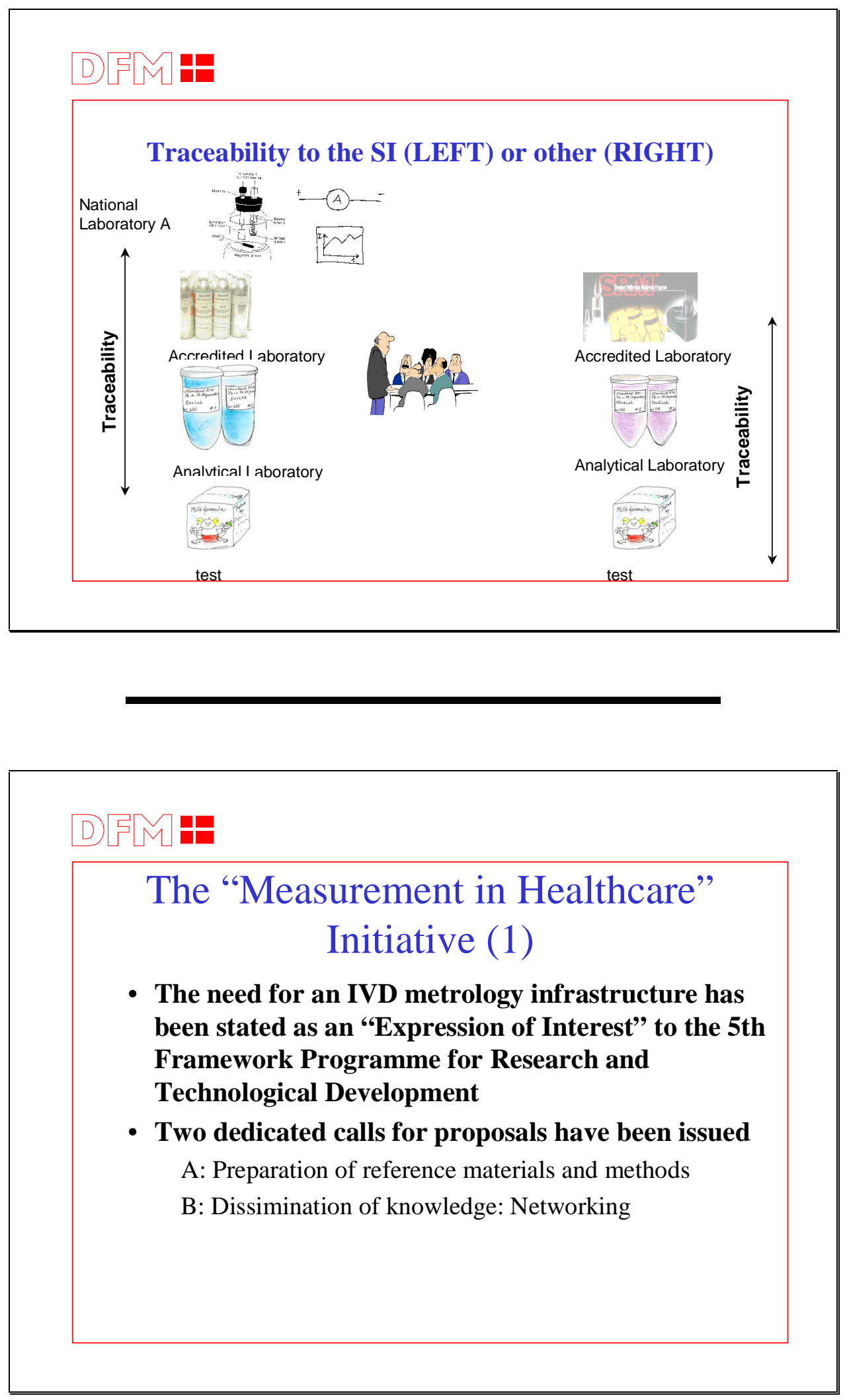

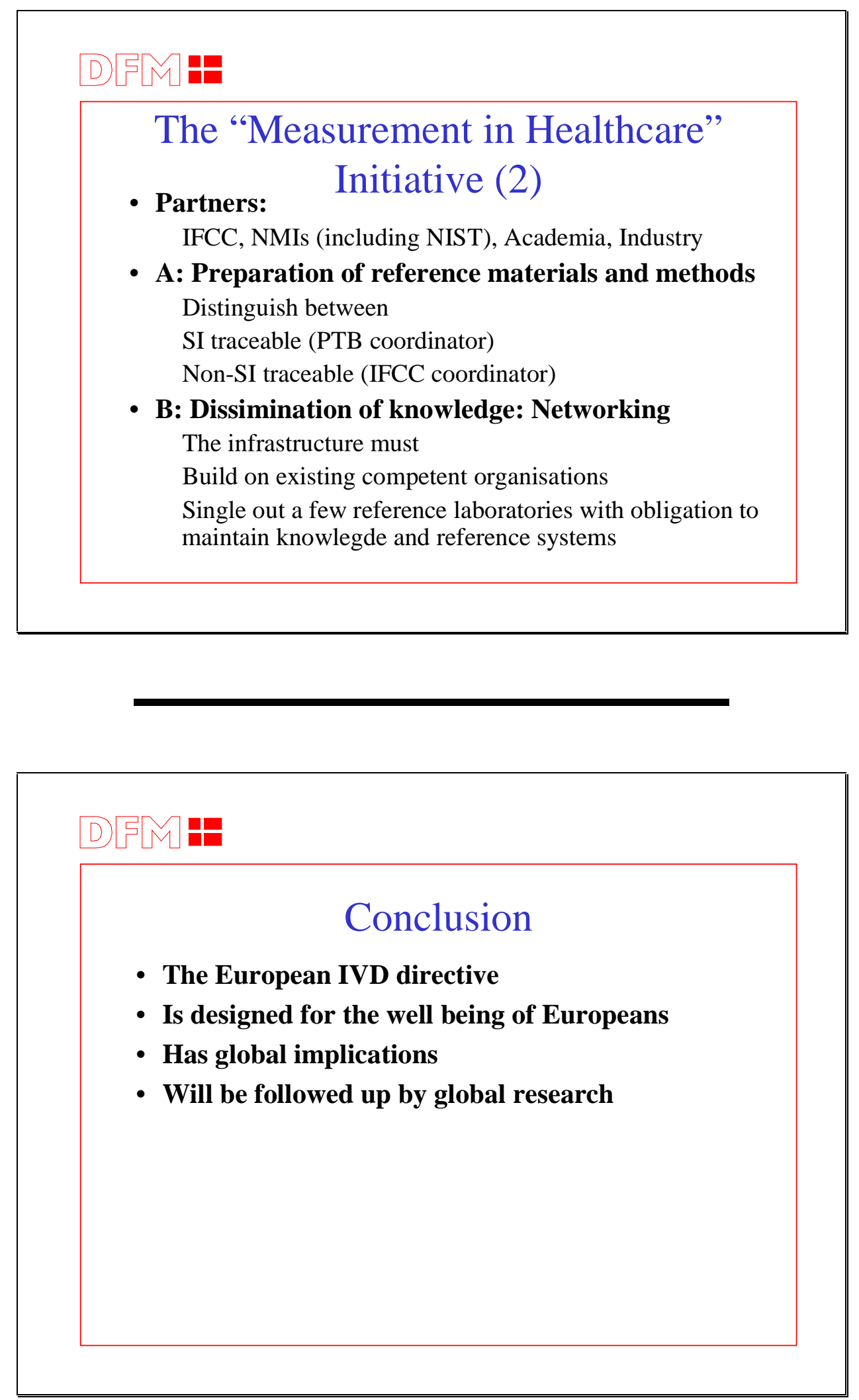


\section{References (1)}

- DIRECTIVE 98/79/EC OF THE EUROPEAN PARLIAMENT AND OF THE COUNCIL of 27 October 1998

on in vitro diagnostic medical devices

\section{- prEN ISO 17511 (CEN/TC 140)}

In vitro diagnostic medical devices- Measurement of quantities in samples of biological origin- Metrological traceability of values assigned to calibrators and control materials

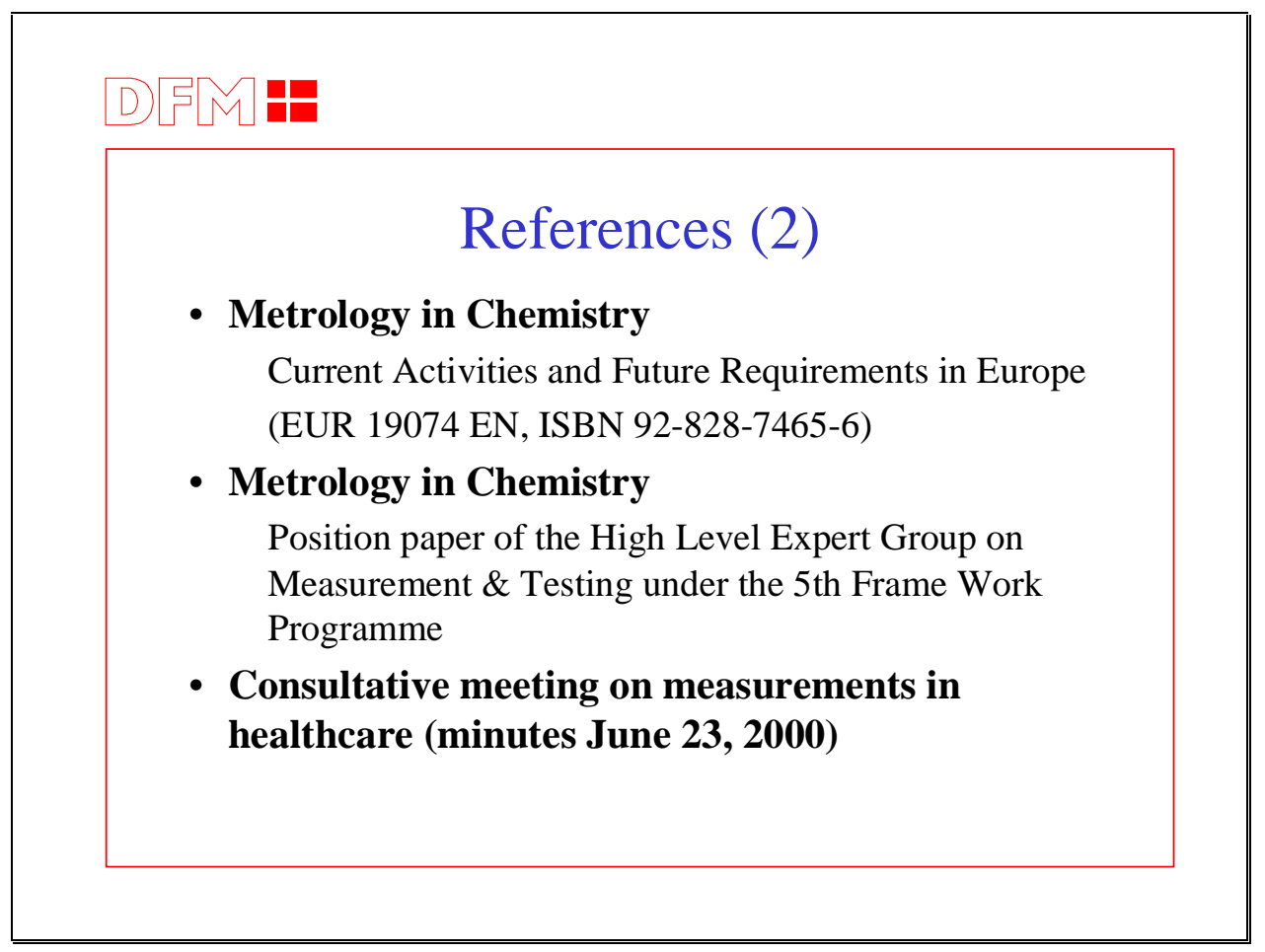




\section{Standardization Activities to Support the Implementation of the IVD Directive Emil Voelkert Roche Diagnostics GmbH Germany}

\section{HARMONIZED STANDARDS AND THE FREE EUROPEAN MARKET}

Europe has seen rather important changes in the past twenty years. The countries in Western Europe are growing together economically and, to some extent, also politically. One of the major ongoing trends is the disappearance of trade barriers between the countries.

This implies not only the removal of trade barriers, like excise taxes or other import restrictions between the European countries, but goes much further on to harmonization and mutual recognition of regulations, standards and technical specifications.

Presently, all products that are legally manufactured and marketed in one European country can move freely throughout the community. There are possible exceptions, but they are acceptable only if a legitimate purpose is pursued, e.g. environmental protection, health issues.

The harmonization process was initially rather slow, because elaborate technical specifications had to be generated and unanimously agreed upon by the European Council and the European Parliament.

Since 1993, a different philosophy, the "New Approach" was implemented. It involves a regulatory approach, defining only "Essential Requirements" in rather general terms that the products must meet. The Essential Requirements specify the elements necessary to protect public interests. They are mandatory and only products complying with the Essential Requirements may be placed on the market and put into service.

The actual technical specifications that the products must meet to fulfil the Essential Requirements, are defined in Harmonized Standards. As before, the application of standards is voluntarily and manufacturers can always choose to demonstrate fulfilling the Essential Requirements by other means. However, adherence to the Harmonized Standards leads to presumption of conformity with the Essential Requirements. This approach provides a high level of protection. Safeguard clauses allow contesting the conformity of a product or are against failure or shortcomings of Harmonized Standards.

This development occurred and is occurring in an environment, where quality assurance concepts and accreditation systems are continuously developed and applied. At the same time mutual recognition of testing and certification is supported by the European member states in order to minimize differences in the quality infrastructures that exist between the states and the industrial sector, involving metrology systems, testing laboratories, etc. These activities extend beyond the European Union in order to develop this approach to a global scale. 
Although the Harmonized Standards do not constitute a specific category amongst the European standards, they have nevertheless a special meaning in the context of the European directives. They are very relevant to the presumption of conformity regarding the fulfillment of the Essential Requirements of products.

The European Commission mandates the standards after consultation with the member states, and the mandate is transmitted to one of the European standard organizations (CEN, CENELEC, ETSI). The European standard organization accepts the mandate and develops a work program. The standards will then be prepared according to General Guidelines that are agreed upon by the European Commission and the European standard organizations. The procedure involves the participation of all interested parties, i.e. users, consumers, manufacturers and public authorities.

After the standards have been developed and agreed upon, they are adopted by all European standard organizations. They are being transposed into national standards in all member states and any contradicting national standards have to be withdrawn.

The European standard organization transmits reference to the corresponding directive of the European Commission that will publish this in the Official Journal of the European Commission. The approach does not foresee a process under which public authorities verify or approve the contents of the standards.

Common Technical Specifications (CTS) fulfill a similar function as Harmonized Standards, and adherence to the CTS also leads to the presumption of compliance with the Essential Requirements to which the specifications refer. They are developed by experts from all interested constituencies for selected products, which are mainly used for the evaluation of safety of blood products. Presently only CTS are drafted for only Annex II, list A products. Also in the case of the CTS, the application is voluntarily. In principle the manufacturer can show by other means that this product maintains at least the same level of performance and safety as demanded by the CTS. In practice, the CTS will probably always be applied. In addition, references of the CTS to the Essential Requirements are published in the Official Journal of the European Communities.

Increasingly, standardization work is shifted from a national to a European or international global level. Between the two standardization organizations, CEN and ISO, there exists an agreement to avoid duplication of work by these two organizations, and sets out procedures for cooperation and mutual recognition of developed standards. The two Technical Committees (TC) charged with the generation of standards for laboratory medicine and in vitro diagnostics are ISO/TC 212 - "Clinical laboratory testing and in vitro diagnostics test systems" on the international level and CEN/TC 140 - "In vitro diagnostics medical devices" on the European side. These two technical committees cooperate closely and in many instances experts participate in the working groups of both TCs.

There are, however, some differences in focus of the two corresponding committees, ISO TC 212 and CEN/TC 140. They are as follows:. CEN/TC 140 is occupied with the development of "Harmonized Standards" mandated by the Commission. In view of the fact that the IVD Directive regulates IVD products, the emphasis is on products and processes relevant to the manufacturers. ISO/TC 212 has a wider scope that includes laboratory medicine, as well as and user aspects. However, there are several agenda items 
that are jointly developed under the Vienna Agreement, that are either ISO lead or CEN lead.

The working groups and the national standardization organizations in the CEN area have to develop the standards with a view of the technical coherence at national and European level. This is of importance, because of the implied compulsory national implementation and the obligation to withdraw any conflicting national standard. Another goal is to coordinate with other international activities, especially ISO.

CEN and ISO Technical Committees involve national standardization organizations that assume the role of the secretariat. These are: NCCLS in the case ISO/TC 212; and, DIN in the case of CEN/TC 140.

National "mirror committees" play an important role at the different stages of development of the standards. They provide the necessary input from experts of the interested parties on a national level and provide the platform for commenting and voting on the standards.

In the IVD Directive (98/79/EC) there are three major references to standards and CTS:

In Article 5 -Reference to standards, the publication of references in the Official Journal of the European Communities is described. Member states shall presume compliance with the Essential Requirements when manufacturers adhere to harmonized standards and CTS.

In Article 6 -Committee on Standards and Technical Regulation describes the role of this committee assisting the European Commission.

In Article 8 -Safeguard clause. The right of member states is described to take appropriate interim measures when - amongst other things - standards are applied incorrectly or show shortcomings themselves.

With this philosophy and in this environment, the European standard organizations are charged with the development of standards for this purpose and in this context.

The general principles that are applied to these tasks involve the participation of all interested parties in a consensus process to develop documents that fit the purpose laid down in the principles of the new approach for the directives. Formal adoption is done by a vote of all CEN national members. The votes are weighted in order to account for differences in the population of the European countries.

There are several mechanisms that can be used to generate standards. In most cases, a Technical Committee is charged to generate a suitable document. Delegates of national delegations are invited for working groups in order to represent their expertise and their national points of view. During this period they have to consider relevant work and national regulations falling within the scope of their tasks. Another route is to transfer the execution of the standard work to ISO, as formalized by the Vienna Agreement. This will lead to a global standard, which will become a "harmonized" standard in the European countries. In some areas another approach might be taken, the "questionnaire procedure", whereby an appropriate existing reference document (often as ISO document) is adopted. 
The development of standards goes usually through four stages. At first, a draft is generated by experts from all interested constituencies, manufacturers, health authorities and users, based on the scope and justification of the mandate. The document drawn up by these experts goes on to the enquiry stage where comments are invited via the national standards institutes. After considering and accepting or rejecting the comments the national standard institutes will vote on the acceptance or rejection of the proposed standard using their weighted votes. When the majority of votes are in favor, the standard will be accepted and published.

There are also interfaces to other committees dealing with topics related to medical devices - IVDs are medical devices - on more general terms. This is necessary to achieve a consolidated perspective in the whole field.

The subsequent tables give an overview of the present status of published standards and projects of both CEN/TC 140 and ISO TC 212.

Work in CEN/TC 140 is spread over several working groups focussing on different aspects. Some of the projects are developed in parallel, either under CEN or under ISO lead. A few of the projects listed are revisions of earlier versions.

\section{TABLE 1}

\begin{tabular}{|c|c|c|c|}
\hline \multicolumn{2}{|c|}{ CEN/TC 140} & \multicolumn{2}{|l|}{ In vitro diagnostic medical devices } \\
\hline WG & Topic & Title & Document \\
\hline WG 1 & \multicolumn{3}{|c|}{ Labelling and performance evaluation } \\
\hline \multirow{6}{*}{+2} & & $\begin{array}{l}\text { Information supplied by the manufacturer with in vitro diagnostic reagents for } \\
\text { professional use }\end{array}$ & EN $375 \mathrm{rev}$ \\
\hline & & $\begin{array}{l}\text { Information supplied by the manufacturer with in vitro diagnostic reagents for } \\
\text { self-testing }\end{array}$ & EN $376 \mathrm{rev}$ \\
\hline & & Instructions for use for in vitro diagnostic instruments for professional use & EN $591 \mathrm{rev}$ \\
\hline & & $\begin{array}{l}\text { Instructions for use for } \\
\text { in vitro diagnostic instruments for self-testing }\end{array}$ & EN $592 \mathrm{rev}$ \\
\hline & & Performance evaluation of in vitro diagnostic medical devices & prEN 13612 \\
\hline & $\mathscr{H}$ & Requirements for marking of in vitro diagnostic instruments & EN 1658 \\
\hline \multicolumn{2}{|c|}{ CEN/TC 257} & Graphical Symbols for use in the labelling of medical devices & EN 980 \\
\hline \multirow[t]{5}{*}{ WG 2} & \multicolumn{3}{|c|}{ GMP for IVDs } \\
\hline & & Stability testing of in vitro diagnostic medical devices & PrEN 13640 \\
\hline & & $\begin{array}{l}\text { Sampling procedures used for acceptance testing of in vitro medical devices - } \\
\text { Statistical aspects }\end{array}$ & $\begin{array}{l}\text { PrEN xxx } \\
\text { (in print) }\end{array}$ \\
\hline & & $\begin{array}{l}\text { Elimination or reduction of risk of infection related to in vitro diagnostic } \\
\text { medical devices }\end{array}$ & prEN 13641 \\
\hline & H & $\begin{array}{l}\text { In vitro diagnostic systems - Guidance on the application of EN } 29001 \text { and EN } \\
46001 \text { and of EN } 29002 \text { and EN } 46002 \text { for in vitro diagnostic medical devices }\end{array}$ & EN 928 \\
\hline \multirow[t]{2}{*}{ WG 3} & \multicolumn{3}{|c|}{ Quality management in the medical laboratory } \\
\hline & & Quality management in the medical laboratory & $\begin{array}{l}\text { ISO/DIS } \\
\text { 15189:1999 }\end{array}$ \\
\hline
\end{tabular}




\section{TABLE 1 (cont'd)}

\begin{tabular}{|c|c|c|}
\hline \multirow{2}{*}{$\begin{array}{l}\text { WG } \\
4\end{array}$} & \multicolumn{2}{|l|}{ Reference Systems } \\
\hline & $\mathscr{H} \begin{array}{l}\text { In vitro diagnostic medical devices - Measurement of quantities in samples of biological } \\
\text { origin - Presentation of reference measurement procedures }\end{array}$ & EN 12286 \\
\hline & $\begin{array}{l}\text { In vitro diagnostic medical devices - Measurement of quantities in samples of biological } \\
\text { origin - Description of reference materials }\end{array}$ & EN 12287 \\
\hline & $\begin{array}{l}\text { In vitro diagnostic medical devices - Measurement of quantities in samples of biological } \\
\text { origin -Metrological traceability of values assigned to calibrators and control materials }\end{array}$ & $\begin{array}{l}\text { prEN ISO } \\
17511 \\
\text { (in print) }\end{array}$ \\
\hline & $\begin{array}{l}\text { In vitro diagnostic medical devices - Measurement of quantities in samples of biological } \\
\text { origin -Metrological traceability of values for catalytic concentration of enzymes assigned to } \\
\text { calibrators and control materials }\end{array}$ & $\begin{array}{l}\text { prEN ISO } \\
18153 \\
\text { (in print) }\end{array}$ \\
\hline & Requirements for reference measurement laboratories in laboratory medicine & $\begin{array}{l}\text { prEN ISO } \\
15195 \\
\text { (in print) }\end{array}$ \\
\hline \multirow{4}{*}{\begin{tabular}{l|l|l} 
WG \\
\end{tabular}} & \multicolumn{2}{|l|}{ Specimen containers } \\
\hline & $\begin{array}{l}\text { In vitro diagnostic systems - Transport packages for medical and biological specimens - } \\
\text { Requirements, tests }\end{array}$ & EN 829 \\
\hline & Single-use receptacles for human venous blood specimen collection & \begin{tabular}{|l|} 
prEN ISO 6710 \\
(in preparation)
\end{tabular} \\
\hline & $\begin{array}{l}\text { In vitro diagnostic medical devices - Single-use receptacles for the collection of specimens, } \\
\text { other than blood, from humans }\end{array}$ & $\begin{array}{l}\text { prEN xxx } \\
\text { (in preparation) }\end{array}$ \\
\hline \multirow{2}{*}{$\begin{array}{l}\text { WG } \\
6 \\
\end{array}$} & \multicolumn{2}{|l|}{ Staining in Biology } \\
\hline & $\begin{array}{l}\text { In vitro diagnostic medical devices - Information supplied by the manufacturer with in vitro } \\
\text { diagnostic reagents for staining in biology }\end{array}$ & EN 12376 \\
\hline \multirow[t]{3}{*}{$\begin{array}{l}\text { WG } \\
7\end{array}$} & \multicolumn{2}{|l|}{ Culture media } \\
\hline & H $\begin{array}{l}\text { In vitro diagnostic medical devices - Culture media for microbiology - Performance criteria } \\
\text { for culture media }\end{array}$ & EN 12322 \\
\hline & In vitro diagnostic systems - Culture media for microbiology - Terms and definitions & EN 1659 \\
\hline \multirow[t]{3}{*}{$\begin{array}{l}\text { WG } \\
8\end{array}$} & \multicolumn{2}{|l|}{ IVDs for self-testing } \\
\hline & $\begin{array}{l}\text { Determination of performance criteria for in vitro blood glucose monitoring systems for } \\
\text { management of human diabetes mellitus }\end{array}$ & $\begin{array}{l}\text { ISO/DIS } \\
15197: 2000 \\
\text { prEN } 15197 \\
\end{array}$ \\
\hline & General requirements for in vitro diagnostic medical devices for self-testing & prEN 13532 \\
\hline \multirow[t]{2}{*}{$\begin{array}{l}\text { WG } \\
9\end{array}$} & \multicolumn{2}{|l|}{ Use of external quality assessment schemes } \\
\hline & $\begin{array}{l}\text { Use of external quality assessment schemes in the assessment of the performance of in vitro } \\
\text { diagnostic procedures }\end{array}$ & $\begin{array}{l}\text { prEN xxx } \\
\text { (in print) }\end{array}$ \\
\hline
\end{tabular}

There are also several standards that have been harmonized, i.e. references to the Essential Requirements have been published in the Official Journal of the European Commission; they are marked by "Ho". 
In ISO/TC 212 there are three Working Groups dealing with the areas "Quality Management in the Clinical Laboratory", "Reference Systems" (parallel work with CEN/TC 140) and "IVD Products".

TABLE 2

\begin{tabular}{|c|c|c|c|}
\hline \multirow{2}{*}{\begin{tabular}{|l|} 
ISO/TC \\
212 \\
WG \\
\end{tabular}} & \multicolumn{3}{|c|}{ Clinical laboratory testing and in vitro diagnostic test systems } \\
\hline & Topic & Title & Document \\
\hline \multirow[t]{3}{*}{ WG 1} & \multicolumn{3}{|c|}{ Quality management in the Clinical Laboratory } \\
\hline & & Quality management in the medical laboratory & $\begin{array}{l}\text { ISO/DIS } \\
15189 * *\end{array}$ \\
\hline & & Safety management for medical laboratories & $\begin{array}{l}\mathrm{ISO} / \mathrm{CD} \\
15190 *\end{array}$ \\
\hline \multirow[t]{6}{*}{ WG 2} & \multicolumn{3}{|c|}{ Reference Systems } \\
\hline & & $\begin{array}{l}\text { Measurement of quantities in samples of biological origin - Contents and description } \\
\text { of reference measurement procedures utilizing nominal and ordinal scales }\end{array}$ & $\begin{array}{l}\text { ISO/DIS } \\
15193\end{array}$ \\
\hline & & $\begin{array}{l}\text { Measurement of quantities in samples of biological origin - Contents and description } \\
\text { of reference materials }\end{array}$ & $\begin{array}{l}\text { ISO/DIS } \\
15194\end{array}$ \\
\hline & & $\begin{array}{l}\text { Clinical Laboratory Medicine - Requirements for laboratories performing reference } \\
\text { procedures }\end{array}$ & $\begin{array}{l}\mathrm{ISO} / \mathrm{CD} \\
15195 *\end{array}$ \\
\hline & & Metrological traceability of values assigned to calibrators and control materials & $\begin{array}{l}\mathrm{ISO} / \mathrm{CD} 17 \\
511 *\end{array}$ \\
\hline & & $\begin{array}{l}\text { Traceability of assigned values for catalytic concentration of enzymes in calibrators } \\
\text { and control materials }\end{array}$ & $\begin{array}{l}\mathrm{ISO} / \mathrm{CD} \\
18153 *\end{array}$ \\
\hline \multirow[t]{6}{*}{ WG 3} & \multicolumn{3}{|c|}{ IVD Products } \\
\hline & & $\begin{array}{l}\text { Determination of analytical performance goals for laboratory procedures based on } \\
\text { medical needs }\end{array}$ & \begin{tabular}{|l|l|l|l} 
ISO/CD \\
15196
\end{tabular} \\
\hline & & $\begin{array}{l}\text { Determination of performance criteria for in vitro blood glucose monitoring systems } \\
\text { for management of human diabetes mellitus }\end{array}$ & $\begin{array}{l}\text { ISO/DIS } \\
15197\end{array}$ \\
\hline & & $\begin{array}{l}\text { Clinical Laboratory Medicine } \\
\text { Recommendation for validation of user quality control }\end{array}$ & $\begin{array}{l}\mathrm{ISO} / \mathrm{CD} \\
15198 *\end{array}$ \\
\hline & & Performance of measurement systems for self-testing of oral anticoagulant therapy & $\begin{array}{l}\text { ISO/AWI } \\
17593\end{array}$ \\
\hline & & Information supplied by the manufacturer with IVD reagents for staining in biology & \begin{tabular}{|l} 
ISO/DIS \\
19001
\end{tabular} \\
\hline Status: & & * DIS pending & parallel CEN \\
\hline $\begin{array}{l}\text { August } \\
2000\end{array}$ & & ** FDIS pending & \\
\hline
\end{tabular}


Most of the standards from both committees are either in the stage of voting or enquiry.

By their mandate, the Harmonized Standards are developed primarily with the view of the IVD-Directive as well as the fulfillment of the Essential Requirements in mind. However, in most cases attempts were also made to include perspectives from areas outside Europe. This is most obvious in the case of standards that are developed in parallel both on ISO and CEN levels. It is definitely in the interest of the manufacturers, and to a very large extent also of the users, to have a framework of identical standards on a global scale. But there are situations where, due to differences in the national regulations, some problems exist that have to be considered. Many of these complications arise from discrepancies in nomenclature and definitions of terms in their legal context. There are several cases where the meanings of terms differ, due to the regulatory environment, mainly between USA and Europe. In the long run, harmonization of definition is very desirable also in this area.

There are a few areas that merit special consideration. Due to the linguistic heterogeneity of the European countries the question of languages has a much higher importance than in the USA, and poses some problems for manufacturers in the first place. Therefore, special approaches have been developed in the context of labeling. Another basic principle that underlies the European approach is the requirement for metrological "traceability", which is in fact the general theme of this workshop.

There have been some delays in the final preparation of the standards, which put some time pressure on the work of the standardization: the CE mark can already be applied as of 7 June 2000. So far, only four standards have been formally harmonized for IVD products. Most of the mandated standards are, however, in a late stage of development, either at the voting stage or in enquiry. On the assumption that the standard will be accepted, perhaps with some minor modification, they will provide the required framework even at this stage.

On the verge of transposing the IVD Directive into national law, the requirement for labeling in the national language came up in several countries of the EU, often because of political motives. While this demand is without any doubt justified for products for self-testing and lay use, the situation is different for products used by professionals in central laboratories. In those cases, medical technologists are usually highly trained and have, in many countries, a command of at least one major European language besides their native language. There are now 11 different major languages in the European Economic Area and assuming that the European Union expands eastwards, there might be another dozen of languages to consider. Thinking in extremes, this would then either require separate products for almost two dozens of countries, or products that have labels and contain instructions for use in all the required national languages. Compromise solutions will have to be chosen.

In addition, the meaning of "labeling" is in fact not identical in the US and Europe, and moreover it is open to (national) interpretation. This includes labels on bottles and packs, it may include instructions for use, handbooks, software and even promotional material. Considering the fact that only 4-5 million people speak some of the European languages, this demand for national languages constitutes an additional burden for 
manufacturers and may in fact inadvertently create a trade barrier. It becomes rather obvious that, for example, the translation of software for an analyzer system is not economically feasible when only a few units are placed onto the national market. Therefore, compromises have to be reached for products that are intended for use by professionals. Approaches in this direction are foreseen in some of the standards. They include the use of symbols, which will be very helpful for labels on bottles and packs, as well as the possibility of providing detailed instructions for use by alternate means other than the insert in the pack, e.g. system manuals, CD ROMs, Internet, Fax polling etc...

The main topic of this workshop is, of course, traceability. In the description of the Essential Requirements for IVDs, traceability of values assigned to calibrators and/or control material must be assured to available reference measurement procedures and/or available reference materials of higher order. This has to be implemented by the manufacturers and the information pertaining to the traceability of the calibration has to be supplied to the user in the instructions for use. This requirement by the IVD Directive demands the application of reference measurement procedures and materials, and supports further development. For this purpose, five standards have been elaborated: ISO/DIS 15193 "Contents and description of reference measurement procedures utilizing nominal and ordinal scales", ISO/DIS 15194 "Contents and description of reference materials", ISO/CD 15195 "Requirements for laboratories performing reference procedures", ISO/CD 17511 "Metrological traceability of values assigned to calibrators and control materials" and ISO/CD 18153 "Metrological traceability of assigned values for catalytic concentration of enzymes in calibrators and control materials". The last two of these are of particular relevance to manufacturers

The approach for metrological traceability described in the Standard ISO/CD 17511 describes a spectrum of different situations, ranging from cases where the complete traceability chain can be established, to situations in which the chain ends at a manufacturer's master calibrator. The essential idea is shown here in a somewhat simplified scheme:

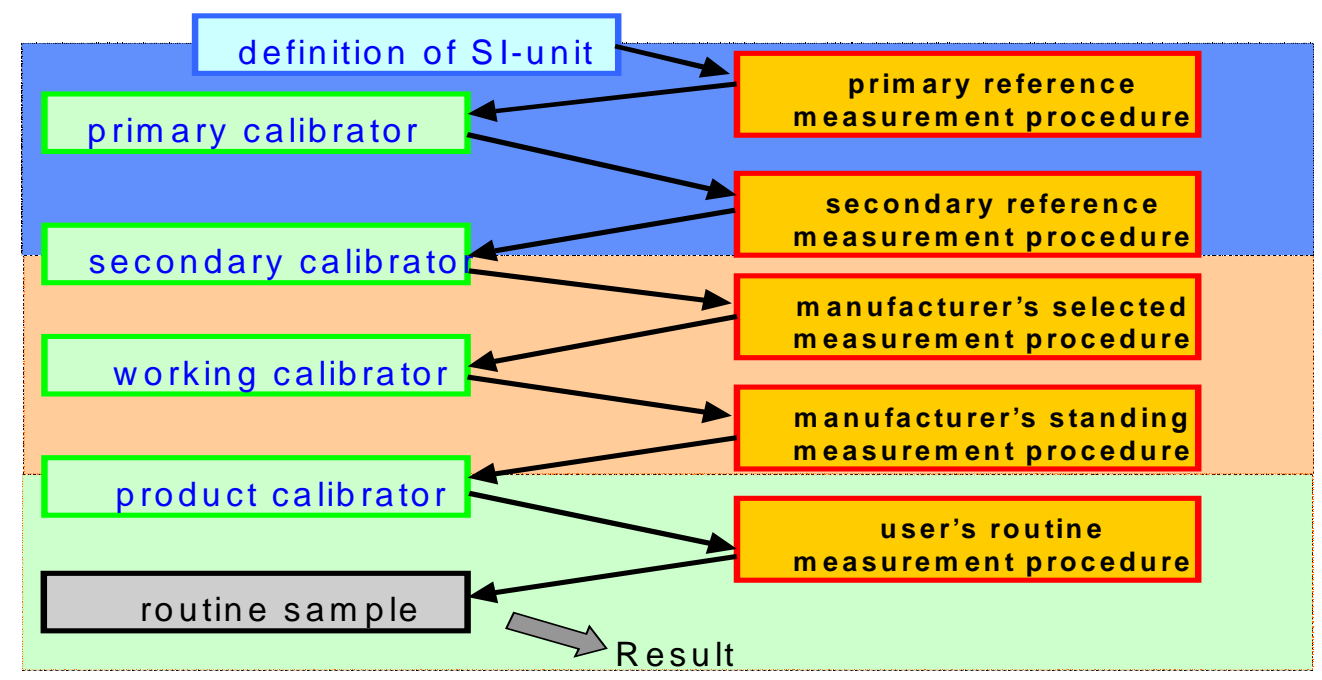


To establish and describe the traceability chain, first of all, it is necessary to define the intended use of the product (with respect to the medical decision it will support, the system to which it will be applied, blood, plasma, urine etc. and the kind of quantity that is measured). The primary or secondary calibrator (e.g. NIST material, WHO) or the reference measurement procedure of highest order must be decided upon and a transfer protocol has to be established.

Both reference measurement procedures and materials should be internationally agreed upon and accepted, preferably with the support of a recognized scientific organization. The reference laboratories should be accredited or at least a quality management system should be established.

There are some hurdles to take with regard to the actual application of this approach to a wide range of analytes. In cases where the entity is a clearly defined molecule, traceability to the highest possible metrological level is possible and can be successfully applied. It has led in the past to an improvement of quality and comparability of measurements of patient results. This applies at present to some 30 analytes of the approximately 400 to 600 types of quantities routinely determined in the medical laboratory.

The traceability for the majority of these parameters will have to end at a lower hierarchical level. However, it is also the aim to develop materials and procedures for these analytes thus leading to a higher metrological level. On the way toward that goal, problems of different nature have to be solved. Very often analytes are mixtures of chemical entities or are heterogeneous by nature. Protein binding plays a role and immunological methods inherently show variability, because of the possibility (or probability) of being directed towards different epitopes. Last but not least, matrix effects may play a role and must be taken into account.

The standard, due to the metrological approach, also requires the determination of the uncertainty along the traceability chain for calibrators and controls. Uncertainty is, of course, increasing as one moves down the traceability chain. The determination of uncertainty is, however, not trivial, particularly in situations where several calibrators are used to establish a calibration curve.

Information on the uncertainty of calibrator set values must be made available to users on request.

Reference materials and reference measurement procedures will provide "Golden Standards" for the calibration of methods. How to proceed further and how to address some of the issues in this field, is the topic of this workshop. It is encouraging to see that these efforts are taken up on a global scale with initiatives arising both in the USA and Europe. Improvement of quality of laboratory data is to be expected from these approaches, and will provide further steps in comparability of patient results, hopefully on a global scale. However, it should be kept in mind that measurements of clinically useful parameters are not an aim per se, but rather the fulfillment of medical needs.

To summarize now the importance and the impact on standards, in particular, with perspective to the European situation: 
The harmonized standards are still voluntary in their application but they are (almost) legally binding. The same is true for the Common Technical Specifications. Both play a very important role at the presumption of conformity with the Essential Requirements for IVDs. They are designed to guarantee a high level of quality of performance and safety. To achieve this, the documents describe the necessary framework of conditions that have to be met. Their global application is in the interest of manufacturers and users, as several of the major standards for this purpose have been developed in parallel, and with close cooperation between ISO/TC 212 and CEN/TC 140.

Additional information on the Internet:

HTTP://www.Newapproach.org/NewApproach/Concepts.htm

http://www.iso.ch/wtotbt/wtotbt.htm

http://www.cenorm.be/standardization/standardization.htm

http://www.wssn.net/WSSN/ 


\section{EMIL VOELKERT}

\section{Roche Diagnostics GmbH}

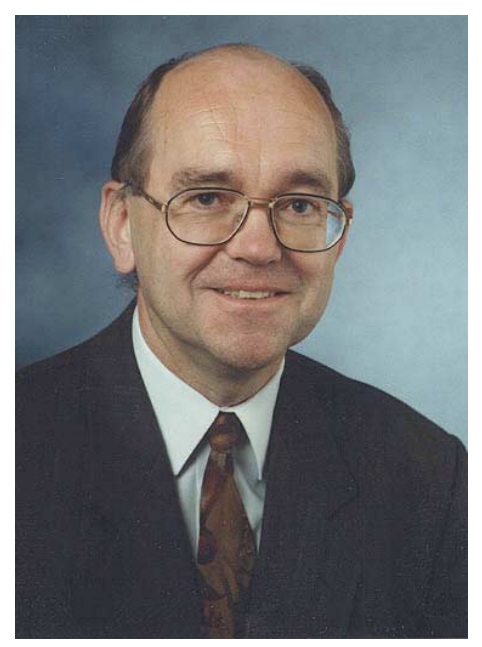

Emil Voelkert, Ph.D., is the Senior Vice President of Integrated Health Care Diagnostics at Roche Diagnostics GmbH Mannheim. Dr. Voelkert received his Ph.D. from the University of Saskatchewan, Canada and in 1970 joined Boeringer Mannheim in Mannheim, Germany, serving as Head of the Applications Laboratory Diagnostics. In 1979, he became the Head of Evaluation Clinical Chemistry and then Head of Product Management German Sales Organization Diagnostics in 1985. He was Head of Marketing German Sales Organization, Laboratory Systems from 1991 to 1996. He then took the position of General Manager Boeringer Mannheim Switzerland from 1996 until 1998, when he assumed his current position at Roche. Dr. Voelkert is a member of a number of standards organizations, including Chairman of the CEN/TC1 140, the EDMA Chairman of WP Standardisation, KAM, a member of the NCCLS Board of Directors, and both a board member and chairman of WP science and technology at VDGH. 


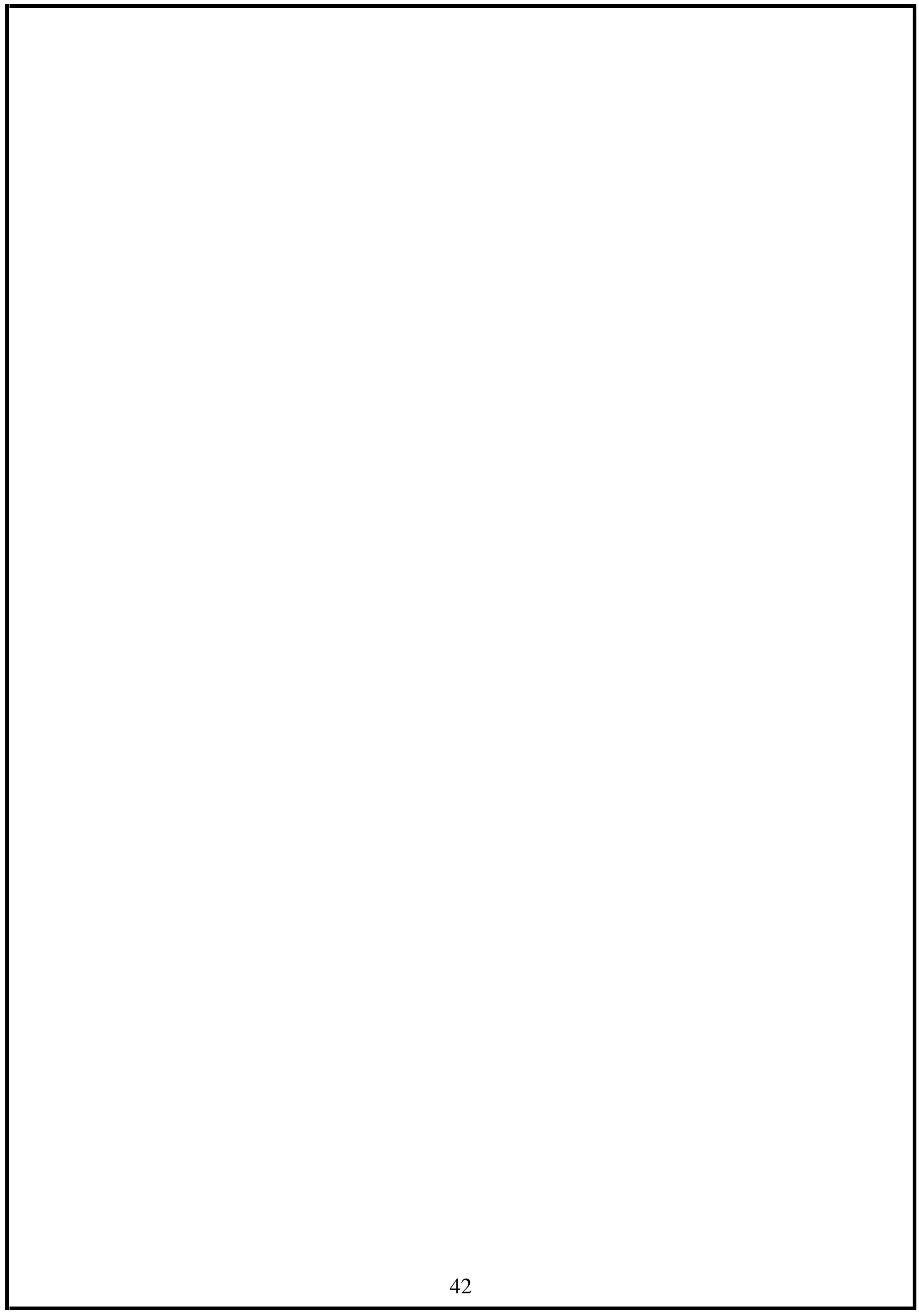


Standardization Activities to Support the Implementation of the IVD Directive

Visuals Presented By

Emil Voelkert

Roche Diagnostics GmbH

Germany 
Standardization activities to support the implementation of the IVD-Directive Harmonized standards and the European free market

NIST Workshop

Gaithersburg, Nov. 2-3, 2000

E. Voelkert, Roche Diagnostics GmbH

\section{Underlying idea:}

Free trade in a single market

Any product lawfully produced and marketed in one Member State must be admitted to the market of any other member state

this implies the prevention of barriers, mutual recognition and technical harmonization 


\section{New Approach: Basic philosophy}

- Legislative harmonization limited to Essential Requirement, which products must meet

- Technical specifications laid down in harmonized standards or in Common Technical Specifications

- Application of standards is voluntary, however

- adherence to these standards leads to the presumption of conformity with the corresponding essential requirements

- Safeguard clause to protect against failure or shortcomings of standards

\section{Harmonized Standards}

- mandated by the European Commission

- prepared according General Guidelines agreed between the European Commission and the European Standards Organizations

- participation of all interested parties (manufacturers, users, consumer associations trade unions)

- participation of public authorities only in certain areas (health, safety, etc ...)

- uniform application throughout the community

- adopted by all European standards organizations

- contradicting national standards have to be withdrawn

- references are published in the Official Journal of the European Communities

NIST Workshop EV 


\section{Common Technical Specifications}

- For selected devices, mainly used for the evaluation of safety of blood supply and organ donations (Annex II products)

- developed by experts, replacing national specifications

- similar role as harmonized standards - manufacturers are required to comply with CTS -

exceptions are possible, however, alternate solutions have to maintain a level at least equivalent thereto

- references published in the Official Journal of the European Communities

adherence to these specifications leads to the

presumption of compliance with the essential requirements to which the specifications refer to

\section{Aspects of standardization work}

- Increasingly Standardization is shifted from a national to a European and to a global level

- Duplicate work by CEN and ISO is avoided ("Vienna Agreement" foresees "fast track procedures" for mutual recognition)

- Underlying philosophy:

CEN: Standards mandated by European Commission related to IVD-Directive - product focus

ISO: Global acceptance - laboratory focus

- In CEN and ISO national standardization institutes take the role of the secretariat (DIN, NCCLS)

- national "mirror committees" are established to find a consensus and provide input via the national standardization institutes 


\section{Standardization Organizations Focus on In vitro diagnostics}

\section{CEN Comité Européen de Normalisation}

European Federation of national standards bodies

CEN/TC 140 In vitro diagnostic medical devices

secretariate: DIN

ISO International Organisation for Standardization World-wide federation of national standards bodies ISO/TC 212 Clinical laboratory testing and in vitro diagnostic test systems

secretariate: NCCLS

\section{Basis in the IVD-Directive}

- Article 5 - Reference to Standards Presumed compliance with the Essential Requirements when adhering to standards and CTS

- Article 6 - Committee on Standards and Technical Regulations assisting the Commission in matters related to standards

- Article 8 - Safeguard clause

Right of the Member States to take appropriate interim measures ... when standards are applied incorrectly or there are shortcomings of the standards themselves 


\section{CEN Principles}

- Integration of all interested parties

- Consensus - voluntary agreement by interested parties

- formal adoption by weighted majority vote of all CEN National Members

- technical coherence at national and European level

- compulsory national implementation

- withdrawal of conflicting standards

- Co-ordination with other international activities, especially ISO

\section{CEN Routes}

- ISO

transfer of execution of standard work to ISO (and vice versa), formalized by "Vienna Agreement"

- Questionnaire procedure

use of an appropriate "reference" document, often an ISO document

- Technical Committee delegates from national delegations to represent expertise and national points of view. Relevant work falling within its scope has to be considered

This path can also be used to modify ISO work 


\section{The Stages at the Development}

- Draft

Experts from manufacturers, health authorities and users develop a draft, based on scope purpose and justification of the standard

- Enquiry Comments are invited via the national standards institutes

- Voting

National standards institutes vote on acceptance or rejection of proposed standard (weighted votes)

- Publication

Actually, the situation is more complex...

There are several other TCs at CEN and ISO that also have an influence:

- ISO/TC 176 Quality management and assurance

- ISO/TC 210 Quality management and general aspects for medical devices

- CEN/TC 204 Sterilisation of medical devices

- CEN/TC 257 Symbols and information provided with MD; Nomenclature for regulatory data exchange

- ISO/TC 210/ Application of risk management to IEC/SC 62A medical devices 


\section{CEN/TC 140 Projects (1)}

\begin{tabular}{|c|c|c|c|}
\hline \multicolumn{2}{|c|}{ CEN/TC 140} & \multicolumn{2}{|l|}{ In vitro diagnostic medical devices } \\
\hline WG & Topic & Title & Document \\
\hline \multirow[t]{7}{*}{ WG 1} & \multicolumn{3}{|c|}{ Labelling and performance evaluation } \\
\hline & & $\begin{array}{l}\text { Information supplied by the manufacturer with in vitro diagnostic reagents } \\
\text { for professional use }\end{array}$ & EN $375 \mathrm{rev}$ \\
\hline & & $\begin{array}{l}\text { Information supplied by the manufacturer with in vitro diagnostic reagents } \\
\text { for self-testing }\end{array}$ & EN $376 \mathrm{rev}$ \\
\hline & & Instructions for use for in vitro diagnostic instruments for professional use & EN $591 \mathrm{rev}$ \\
\hline & & Instructions for use for in vitro diagnostic instruments for self-testing & EN $592 \mathrm{rev}$ \\
\hline & & Performance evaluation of in vitro diagnostic medical devices & prEN 13612 \\
\hline & & Requirements for marking of in vitro diagnositc instruments & EN 1658 \\
\hline \multicolumn{2}{|c|}{ CENTC 257} & Graphical Symbols for use in the labelling of medical devices & EN 980 \\
\hline \multirow[t]{5}{*}{ WG 2} & \multicolumn{3}{|c|}{ GMP for IVDs } \\
\hline & & Stability testing of in vitro diagnostic medical devices & PrEN 13640 \\
\hline & & $\begin{array}{l}\text { Sampling procedures used for acceptance testing of in vitro medical } \\
\text { devices - Statistical aspects }\end{array}$ & \begin{tabular}{|l|l} 
PrEN $x x x$ \\
(in print)
\end{tabular} \\
\hline & & $\begin{array}{l}\text { Elimination or reduction of risk of infection related to in vitro diagnostic } \\
\text { medical devices }\end{array}$ & prEN 13641 \\
\hline & $\hat{W}$ & $\begin{array}{l}\text { In vitro diagnostic systems - Guidance on the application of EN } 29001 \text { and } \\
\text { EN } 46001 \text { and of EN } 29002 \text { and EN } 46002 \text { for in vitro diagnostic medical } \\
\text { devices }\end{array}$ & EN 928 \\
\hline
\end{tabular}

NIST Workshop EV

认 Harmonized Standard

\section{CEN/TC 140 Projects (2)}

\begin{tabular}{|c|c|c|c|}
\hline WG 3 & Quality & management in the medical laboratory & \\
\hline & & Quality management in the medical laboratory & \begin{tabular}{|l} 
ISO/DIS \\
$15189: 1999$
\end{tabular} \\
\hline \multirow[t]{6}{*}{ WG 4} & \multicolumn{3}{|c|}{ Reference Systems } \\
\hline & $\hat{W}$ & $\begin{array}{l}\text { In vitro diagnostic medical devices - Measurement of quantities in samples } \\
\text { of biological origin - Presentation of reference measurement procedures }\end{array}$ & EN 12286 \\
\hline & & $\begin{array}{l}\text { In vitro diagnostic medical devices - Measurement of quantities in samples } \\
\text { of biological origin - Description of reference materials }\end{array}$ & EN 12287 \\
\hline & & $\begin{array}{l}\text { In vitro diagnostic medical devices - Measurement of quantities in samples } \\
\text { of biological origin -Metrological traceability of values assigned to } \\
\text { calibrators and control materials }\end{array}$ & $\begin{array}{l}\text { prEN ISO } 17511 \\
\text { (in print) }\end{array}$ \\
\hline & & $\begin{array}{l}\text { In vitro diagnostic medical devices - Measurement of quantities in samples } \\
\text { of biological origin -Metrological traceability of values for catalytic } \\
\text { concentration of enzymes assigned to calibrators and control materials }\end{array}$ & $\begin{array}{l}\text { prEN ISO } 18153 \\
\text { (in print) }\end{array}$ \\
\hline & & $\begin{array}{l}\text { Requirements for reference measurement laboratories in laboratory } \\
\text { medicine }\end{array}$ & $\begin{array}{l}\text { prEN ISO } 15195 \\
\text { (in print) }\end{array}$ \\
\hline \multirow[t]{4}{*}{ WG 5} & \multicolumn{3}{|c|}{ Specimen containers } \\
\hline & & $\begin{array}{l}\text { In vitro diagnostic systems - Transport packages for medical and biological } \\
\text { specimens - Requirements, tests }\end{array}$ & EN 829 \\
\hline & & Single-use receptacles for human venous blood specimen collection & $\begin{array}{l}\text { prEN ISO } 6710 \\
\text { (in preparation) }\end{array}$ \\
\hline & & $\begin{array}{l}\text { In vitro diagnostic medical devices - Single-use receptacles for the } \\
\text { collection of specimens, other than blood, from humans }\end{array}$ & $\begin{array}{l}\text { prEN } x \times x \\
\text { (in preparation) }\end{array}$ \\
\hline
\end{tabular}

NIST Workshop EV 


\section{CEN/TC 140 Projects (3)}

\begin{tabular}{|c|c|c|c|}
\hline WG 5 & Spe & en containers & \\
\hline & & $\begin{array}{l}\text { In vitro diagnostic systems - Transport packages for medical and biological } \\
\text { specimens - Requirements, tests }\end{array}$ & EN 829 \\
\hline ISO/TC & & Single-use receptacles for human venous blood specimen collection & $\begin{array}{l}\text { prEN ISO } 6710 \\
\text { (in preparation) }\end{array}$ \\
\hline & & $\begin{array}{l}\text { In vitro diagnostic medical devices - Single-use receptacles for the } \\
\text { collection of specimens, other than blood, from humans }\end{array}$ & $\begin{array}{l}\text { prEN xxx } \\
\text { (in preparation) }\end{array}$ \\
\hline WG 6 & Stai & g in Biology & \\
\hline & & $\begin{array}{l}\text { In vitro diagnostic medical devices - Information supplied by the } \\
\text { manufacturer with in vitro diagnostic reagents for staining in biology }\end{array}$ & EN 12376 \\
\hline WG 7 & Cult & media & \\
\hline & & $\begin{array}{l}\text { In vitro diagnostic medical devices - Culture media for microbiology - } \\
\text { Performance criteria for culture media }\end{array}$ & EN 12322 \\
\hline & & $\begin{array}{l}\text { In vitro diagnostic systems - Culture media for microbiology - Terms and } \\
\text { definitions }\end{array}$ & EN 1659 \\
\hline WG 8 & IVD & r self-testing & \\
\hline & & $\begin{array}{l}\text { Determination of performance criteria for in vitro blood glucose monitoring } \\
\text { systems for management of human diabetes mellitus }\end{array}$ & $\begin{array}{l}\text { ISO/DIS 15197:2000 } \\
\text { prEN 15197 }\end{array}$ \\
\hline & & General requirements for in vitro diagnostic medical devices for self-testing & prEN 13532 \\
\hline WG 9 & Use & external quality assessment schemes & \\
\hline & & $\begin{array}{l}\text { Use of external quality assessment schemes in the assessment of the } \\
\text { performance of in vitro diagnostic procedures }\end{array}$ & \begin{tabular}{|l|} 
prEN xxx \\
(in print)
\end{tabular} \\
\hline & & & parallel ISO/TC 212 \\
\hline
\end{tabular}

NIST Workshop EV

\section{ISO/TC 212 Projects (1)}

\begin{tabular}{|c|c|c|}
\hline WG 1 & Quality management in the Clinical Laboratory & \\
\hline & Quality management in the medical laboratory & ISO/DIS $15189^{* *}$ \\
\hline & \begin{tabular}{|l|l} 
& Safety management for medical laboratories \\
\end{tabular} & ISO/CD 15190 * \\
\hline WG 2 & Reference Systems & \\
\hline & $\begin{array}{l}\text { Measurement of quantities in samples of } \\
\text { biological origin - Contents and description of } \\
\text { reference measurement proce dures utilizing } \\
\text { nominal and ordinal scales }\end{array}$ & ISO/DIS 15193 \\
\hline & $\begin{array}{l}\text { Measurement of quantities in samples of } \\
\text { biological origin - Contents and description of } \\
\text { reference materials }\end{array}$ & ISO/DIS 15194 \\
\hline & $\begin{array}{l}\text { Clinical Laboratory Medicine - Requirements for } \\
\text { laboratories performing reference procedures }\end{array}$ & ISO/CD 15195 * \\
\hline & $\begin{array}{l}\text { Metrological traceability of values assigned to } \\
\text { calibrators and control materials }\end{array}$ & ISO/CD 17511 * \\
\hline & $\begin{array}{l}\text { Traceability of assigned values for catalytic } \\
\text { concentration of enzymes in calibrators and } \\
\text { control materials }\end{array}$ & ISO/CD 18153 * \\
\hline & & * DIS pending \\
\hline & & ${ }^{\star \star}$ FDIS pending \\
\hline
\end{tabular}

NIST Workshop EV 
ISO/TC 212 Projects (2)

\begin{tabular}{|l|l|l|}
\hline WG 3 & IVD Products \\
\hline & $\begin{array}{l}\text { Determination of analytical performance goals } \\
\text { for laboratory procedures based on medical } \\
\text { needs }\end{array}$ & ISO/CD 15196 \\
\hline & $\begin{array}{l}\text { Determination of performance criteria for in } \\
\text { vitro blood glucose monitoring systems for } \\
\text { management of human diabetes mellitus }\end{array}$ & ISO/DIS 15197 \\
\hline & $\begin{array}{l}\text { Clinical Laboratory Medicine } \\
\text { Recommendation for validation of user quality } \\
\text { control }\end{array}$ & ISO/CD 15198 * \\
\hline $\begin{array}{l}\text { Performance of measurement systems for self- } \\
\text { testing of oral anticoagulant therapy }\end{array}$ & ISO/AWI 17593 \\
\hline & $\begin{array}{l}\text { Information supplied by the manufacturer with } \\
\text { IVD reagents for staining in biology }\end{array}$ & ISO/DIS 19001 \\
\hline & & * DIS pending \\
\hline
\end{tabular}

\section{Special aspects}

- Globalisation and global recognition

- Harmonization of Nomenclature and Definitions

- Labeling

- Languages

- Use of symbols

- Traceability

- Reference methods / -material

- Uncertainty

- Timeline (IVD-Directive is effective as of 7.6.2000) 


\section{Labeling}

- What is labeling?

- Labels on bottles and pack

- instructions for use

- software

- The European languages

- there are 11 different (major) languages in the EU

- there are at least another 11 in the Eastern parts

- at the transposition of the IVD-Directive more countries demand their national language

- Choices

- one version for all countries

- 11 country specific versions of products

\section{Labeling}

- Use of Symbols

e.g.

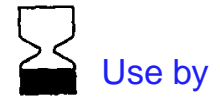

1

Store between

(Temperature)

- Use of alternate means of providing detailed information (instructions for use)

- System manual

- CD-ROM

- FAX on demand

- Internet 


\section{Traceability - Basis}

- The IVD-Directive:

Essential Requirements (Annex 1)

General requirements ...

The traceability of values assigned to calibrators and/or control materials must

be assured through available reference measurement procedures and/or available

reference materials of a higher order.

8. Information supplied by the manufacturer ...

(k) information appropriate to users on:

- internal quality control including specific validation procedures,

the traceability of the calibration of the device;

- The Standards ISO/CD 17511 and ISO/CD 18153

Metrological traceability of values assigned to calibrators and control materials

Metrological traceability of assigned values for catalytic

concentration of enzymes in calibrators and control materials

NIST Workshop EV

\section{The standard prEN ISO 17511 describes several situations}

- Quantities traceable to SI-units

- International conventional reference measurement procedure and international calibration material available

- International conventional reference measurement procedure available

- International conventional calibration material available

- Neither international conventional reference measurement procedure and international calibration material available 


\section{Traceability - the approach}

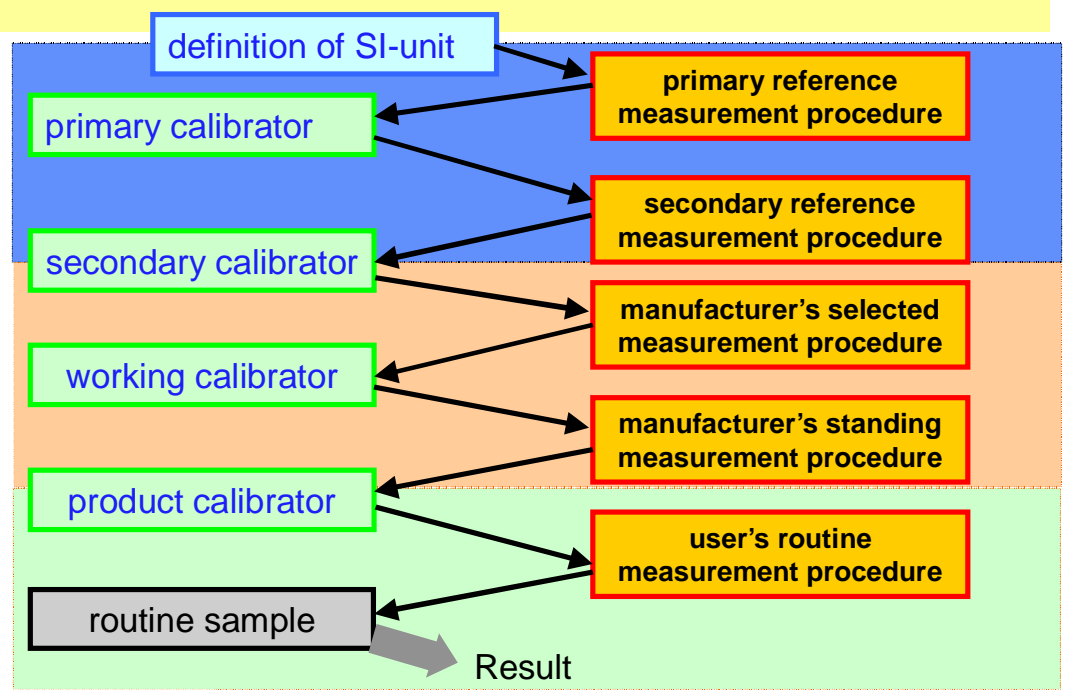

NIST Workshop EV

\section{Traceability chain}

- Define intended use of IVDMD

- medical decision

- system (serum, plasma, urine, ...)

- kind of quantity

- Describe traceability chain to methods of higher metrological order (including uncertainty)

- Characterization of calibrators used (e.g. NIST, WHO ...)

- Responsibility of manufacturer starts with product calibrator and ends at highest reference used by manufacturer

- Establish transfer protocol

NIST Workshop EV 


\section{Traceability - considerations}

- Reference method internationally agreed

- Reference material suitable for intended purpose

- analyte clearly defined

- matrix

- International recognition of methods and materials, supported by scientific organizations

- Reference laboratories accredited

\section{Traceability - problems}

- Analytes - of the approx. $\mathbf{4 0 0}$ to $\mathbf{6 0 0}$ analytes routinely investigated in the clinical laboratory there are only some 30 , which are clearly described chemical entities

- Analytes are often mixtures

- Heterogeneity of many analytes (e.g. ferritin)

- Analytes bound to proteins and in free state

- Immunological procedures show inherent variability (different epitopes)

- Matrix influences

- Uncertainty - has to be indicated (on request)

- a priori calculation of uncertainty is often rather complex

- useful for routine purposes ? 


\section{Conclusions}

- Harmonized standards and Common Technical Specifications are (almost) legally binding

- they are an important element at the presumption of conformity

- they guarantee a high level of performance and safety

- they tend to be "horizontal"

- their global application is in the interest of manufacturers and users

- there is a close co-operation between ISO/TC 212 and CEN/TC 140 


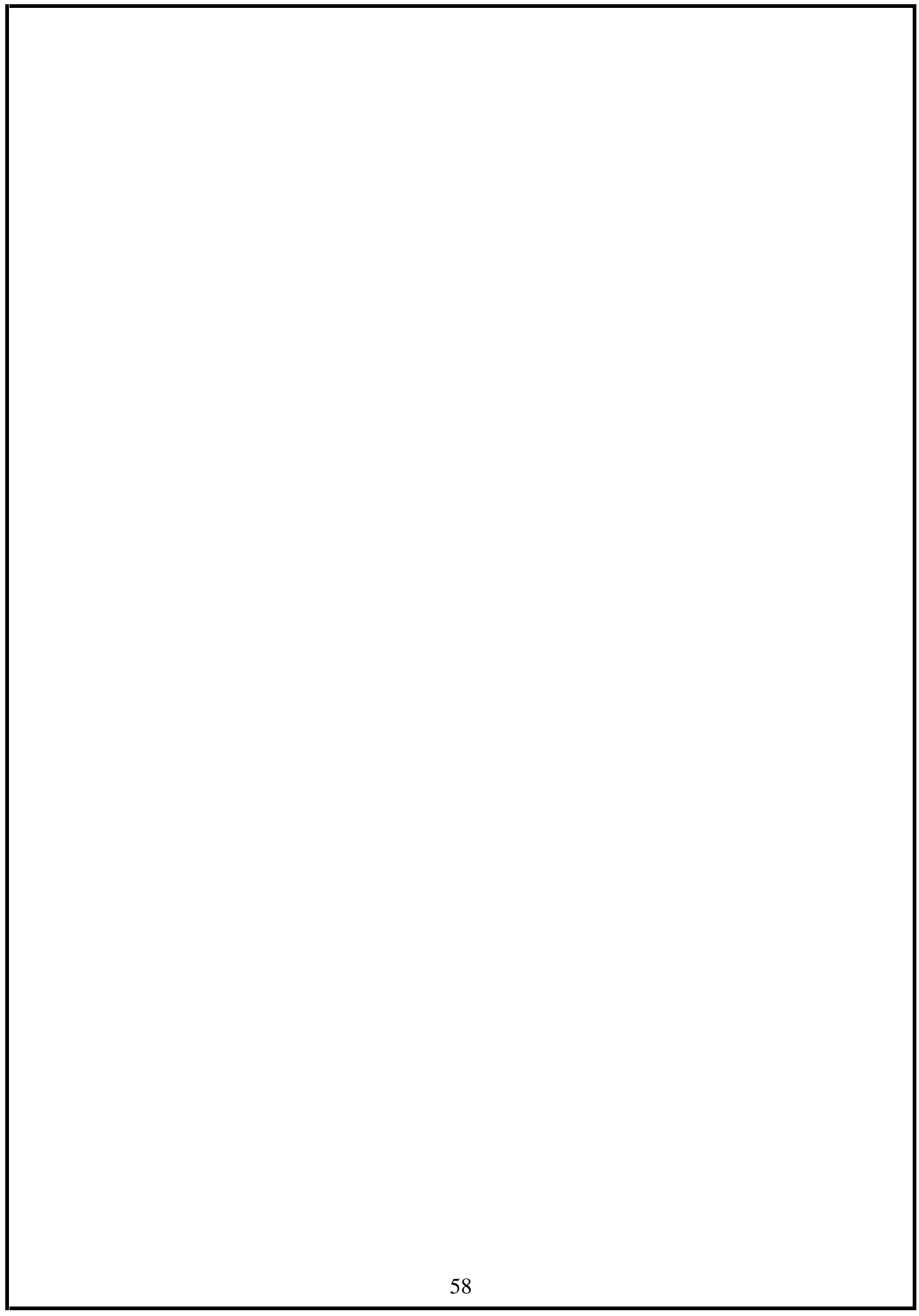




\section{Mutual Recognition of Measurements: How Do They Impact International Trade? \\ Hratch G. Semerjian and Ellyn S. Beary \\ National Institute of Standards and Technology}

\section{Introduction}

Advances in technology, globalization of trade, and accelerated growth of international trade are the primary factors driving economic growth. These changes, in turn, have created an increasing need for strengthening the national metrology infrastructure and ensuring mutual recognition of measurements performed in each country. International trade is growing at a rate of $15 \%$ per year, and tariff-based barriers are being replaced with technical barriers to trade. To support this rate of growth and to overcome the technical barriers to trade, measurements performed by industry to characterize their products and for conformity assessment must be globally recognized. Each nation must therefore ensure the quality and accuracy of their measurement system to promote mutual recognition in the global marketplace.

Technology is one of the main driving forces for economic growth; according to a recent study, in the United States alone, more than $50 \%$ of the economic growth is attributed to technological advances [1]. Advanced technologies such as microelectronics, biotechnology, and nanotechnology, require measurements of higher spatial resolution, sensitivity, and selectivity. In addition, the deregulation of many mature industries, such as natural gas and electricity, requires more frequent and accurate measurements to help ensure equity in trade.

The federal role in metrology is becoming ever more important as the health of domestic industries becomes increasingly dependent on global trade. In the U.S., the federal government has the constitutional responsibility to provide the weights and measures for the nation, and this responsibility is vested in NIST (Figure 1). NIST maintains both basic and derived SI units (International System of Units) and also provided standards and calibrations that are traceable to NIST. NIST invests ap-

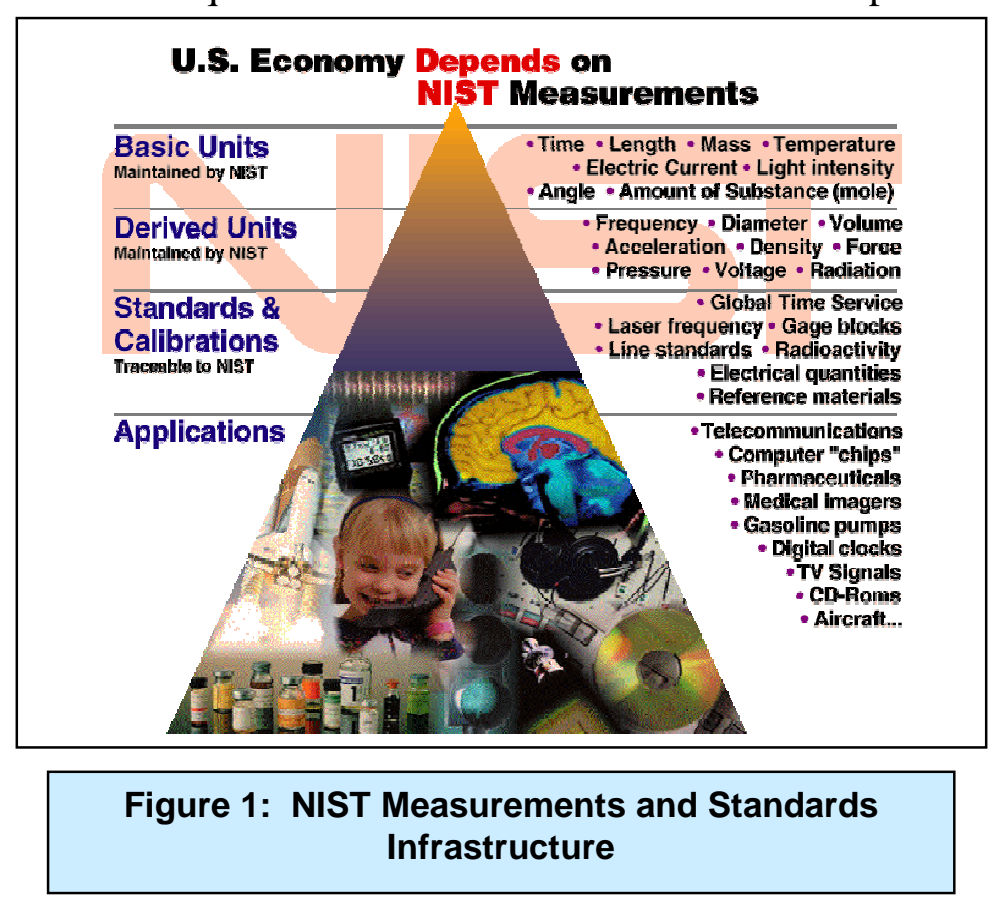


proximately $\$ 500$ million per year $(0.7 \%$ of the federal R\&D funds) in metrology programs, and supports a $\$ 10$ billion private sector investment in measurements and standards. More than half of the $\$ 7.6$ trillion per year U.S. GDP in sales is supported by this measurement infrastructure.

The measurement and standards infrastructure developed by NIST is designed to address needs of rapidly growing, high technology industries, as well as mature industries. NIST provides measurements and standards for these industries not only to provide better tools to characterize products, but also tools to improve processes, enhance process yields, and promote competitiveness in the global market. This paper provides an overview of the international agreements that support global trade, and describes the role NIST plays in facilitating mutual recognition of measurements and standards.

\section{Measurements and Standards for International Trade}

Traceability and Comparability: The metrology infrastructure to ensure global recognition of measurements and standards must have two major elements: a) vertical traceability system to ensure the quality and accuracy of measurements; and b) comparability of the standards realized and maintained by the National Metrology Institutes (NMIs) of different countries. Accreditation of measurement capabilities and conformity assessment for certification of products require demonstration of measurement traceability to national standards and comparability among the national standards of the trading partners (Figure 2). The VIM (International Vocabulary of Basic and General Terms in Metrology) definition of traceability is:

"... the property of the result of a measurement or the value of a standard whereby it can be related to stated reference, usually national or international standards, through an unbroken chain of comparisons all having stated uncertainties."

Indeed, traceability carries an additional requirement in that it can only exist when scientifically rigorous evidence is collected on a continuing basis showing that the measurement is producing documented results for which the total measurement uncertainty is quantified. A national traceability system links field measurements to the SI by providing tools such as reference materials, reference data, and calibrations.

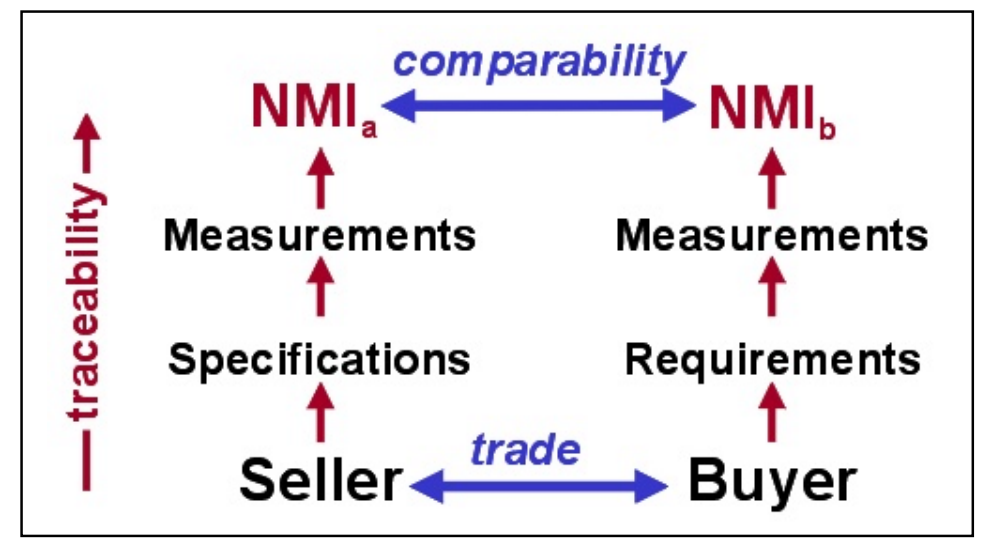

Figure 2: NMIs are responsible for national traceability and international comparability. The function of comparability is becoming more critical in global trade. 
NMIs have a unique and pivotal role to play in ensuring the comparability of physical and chemical measurements. NMIs must be active in advancing the state of measurement science to support the needs of their own domestic industry. NIST, the NMI of the United States, was founded on March 3, 1901 as the National Bureau of Standards, and was the U.S. federal government's first physical science research laboratory. NIST provides traceability to the SI and derived units, ensuring that products and goods sold meet the claimed specifications.

In addition to providing vertical traceability, the NMI also serves to link the national infrastructure to the international measurement system. NMIs have been collaborating and carrying out international comparisons of their national measurement standards for more than one hundred years. However, the ad hoc recognition that resulted from these long-standing interactions among the NMIs is no longer considered sufficient to promote international trade and to ensure equity in trade. The NMI function of establishing comparability is more critical in today's global market, and must be established on a more formal and systematic basis. The International Committee of Weights and Measures (CIPM) has played a critical role in the negotiation and implementation of a Mutual Recognition Arrangement (MRA), as a mechanism to demonstrate comparability of measurements and standards between NMIs. Details of this new MRA will be described later in this paper.

All NMIs interact on a global basis with other NMIs and strive to have their measurement and standards capabilities recognized by their counterparts. These efforts can facilitate the harmonization of systems of measurement and standards and eventually lead to mutual recognition among trading partners. Today's more formalized system reflects a major change in philosophy, where measurements and data from each NMI are openly shared and accessible to all. In formal comparisons, degrees or levels of comparability may be demonstrated among participants, and is defined in terms of a "degree of equivalence" that is needed for "agreement for the purpose at hand" to be decided by the end user. This formal international measurement infrastructure spear-headed by the metrological community is in harmony with the EU/US Mutual Recognition Agreement designed to facilitate international trade.

The EU/US Mutual Recognition Agreement: This international trade agreement was negotiated at the highest level of governments in recognition of EU/US economic interdependence. This agreement, signed in October 1998, provides for mutual recognition of specified conformity assessment activities undertaken in the exporting country to the regulatory requirements of the importing country. It covers testing activities, product certification, and quality system registration, where relevant [2]. Since NMIs provide the traceability to the SI required for these services, mutual recognition of the capabilities of the NMIs is a prerequisite for the mutual recognition of metrology services in general. This treaty was designed to address regulatory issues in the following areas: Avionics, Environmental Protection, Pharmaceutical and Medical Devices, Electromagnetic Compatibility and Interference, and Occupational Health and Safety. It is estimated that this treaty will eliminate duplicative product testing on approximately $\$ 60$ billion worth of traded goods. [3] 
EU IVD Directive: The European Community expressed the need for legislative directives that would create a single European market as far back as the 1980s. In fact, it was the medical products industry throughout Europe that identified the need for a set of directives to regulate its products. This industry group sought the assistance of the European Commission to develop directives with the goal of setting specific rules to regulate the distribution of medical devices and diagnostic products throughout the European market, and requiring only a single approval for each product [4].

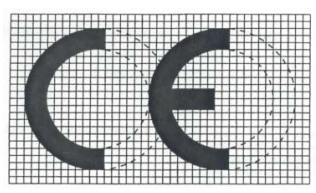
It is not surprising that the EU/US Mutual Recognition Agreement of 1998 targeted "Pharmaceutical and Medical Devices" as a focus area. While this agreement engaged the NMIs, the EU Directive 98/79/EC on In Vitro Diagnostic (IVD) Medical Devices that was issued around the same time, was the impetus for the industry to take notice. This IVD Directive was issued in October 1998 and required that all IVD devices sold in Europe after December 2003 bear the CE mark. This mark would indicate traceability of its

The CE Marking of in vitro diagnostic devices applies to reagents and reagent products, calibrator materials, or instruments including specimen receptacles intended by the manufacturer for the in vitro examination of human tissue, blood, or fluid samples for the purpose of proving information about a patient's state of health. measurement systems to "standards of a higher order" [5]. The implementation timetable provides the following options: CE marking of IVD Devices could begin as early as June 2000, and devices already on the market prior to December 7, 2003 could be sold without CE marking until December 7, 2005. Health status markers that are impacted by the IVD Directive are classified into two categories: "List A" includes approximately 100 welldefined chemical species, potentially traceable to SI units; and "List B" consists of more than 500 less well-defined chemical species, potentially not traceable to SI units. Since it is generally agreed that the phrase "standards of a higher order" refers to nationally and internationally recognized Certified Reference Materials (CRMs), it is clear that international collaboration and cooperation is required to meet the industry need for new standards. NIST's CRMs sold under the trademark Standard Reference Material (SRM $\left.{ }^{\circledR}\right)$ are widely recognized and used worldwide.

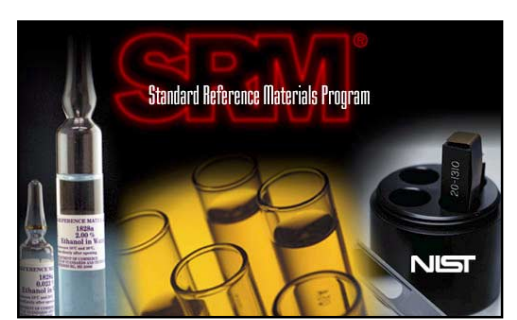

NIST's Standard Reference Materials Program: The SRM Program at NIST is of long standing, and has produced chemical and physical standards for almost 100 years. These standards continue to promote the development of new technologies, and are as relevant at the turn of this century as they were at the turn of the last. NIST standards have ushered in many eras of technological advances in the U.S. from the industrial age to the new age of DNA based technologies. Approximately 1300 SRMs are currently available for use in: (1) industrial materials production and analysis; (2) environmental analysis; (3) healthcare measurements; and (4) basic measurements in science and metrology. SRMs are crucial reference points in the establishment of a comprehensive measurement system for the entire nation [6]. This system has met the needs of U.S. industry and commerce for a century and contin- 
ues to evolve to satisfy more demanding measurement requirements. SRMs are used both in the U.S. and abroad to provide traceability, i.e., an unbroken chain that connects field measurements to SI units. The process of traceability is ongoing, not stagnant, and NIST continually strives to provide its customers with the robust, flexible, and globally recognized measurement and standards infrastructure that is necessary to compete in the $21^{\text {st }}$ century market place. Healthcare related standards, of particular interest to the IVD manufacturing community, are discussed in the following section.

\section{Measurements and Standards for Healthcare}

Chemical metrology is at the heart of accurate medical diagnosis and the development of measures to improve our health and ensure long life. In the U.S., about $\$ 1.5$ trillion are spent each year on health care, which corresponds to about $14 \%$ of our GDP [7]. About $13 \%$ of these expenditures are for measurements. It is estimated that over one third of these measurements are performed for non-diagnostic purposes, such as quality assurance and quality control (QA/QC) measurements and retests at a cost of about $\$ 50$ to $\$ 60$ billion annually. Clearly, improvements in the reliability of chemical measurements in this area would have a significant economic impact for the U.S. Indeed, improvements in the accuracy of just one analyte (cholesterol) is estimated to have resulted in cost savings of about $\$ 100$ million per year.

NIST works closely with the American Association for Clinical Chemistry (AACC), the Centers for Disease Control and Prevention (CDC), the College of American Pathologists (CAP), the National Conference of Clinical Laboratory Standards (NCCLS), the Food and Drug Administration (FDA), and other organizations interested in health-related standards to help prioritize our standards activities and the development of SRMs.

For more than 20 years NIST has developed, maintained, and refined "Definitive Methods" for 12 health status markers to support the national reference system for clinical measurements (Figure 3). "Definitive Methods" are robust analytical methods for which all sources of uncertainty are known and quantified. Such methods have been instrumental in improving the accuracy and reliability of clinical laboratory measurements. For example, in the area of cholesterol measurements, it has been estimated that

\begin{tabular}{|c|c|}
\hline $\begin{array}{l}\text { - Calcium } \\
\text { - Chloride } \\
\text { - Cholesterol } \\
\text { - Creatinine } \\
\text { - Glucose } \\
\text { - Lithium } \\
\text { - Magnesium } \\
\text { - Potassium } \\
\text { - Sodium } \\
\text { - Triglycerides } \\
\text { - Urea } \\
\text { - Uric Acid }\end{array}$ & $\begin{array}{l}\text { Cancer, Blood Clotting } \\
\text { Kidney Function } \\
\text { Heart Disease } \\
\text { Kidney Function } \\
\text { Diabetes } \\
\text { Antipsychotic Treatment } \\
\text { Heart Disease } \\
\text { Electrolyte Balance } \\
\text { Electrolyte Balance } \\
\text { Heart Disease } \\
\text { Kidney Function } \\
\text { Gout }\end{array}$ \\
\hline
\end{tabular}
the measurement uncertainty was reduced from $\pm 18 \%$ relative in 1969 to between \pm 5.5 to $7.5 \%$ relative by 1994 [8]. The improvement in accuracy has been attributed to the release a pure cholesterol SRM in 1969, and the serum cholesterol SRMs released in 1981 and 1988. In addition to using NIST definitive methods for the certification of 
SRMs, they are also used to value-assign high-priority serum pools that serve as the anchor point for CDC developed reference methods. These standards are also used by the College of American Pathologist (CAP) for proficiency testing of more than 20,000 U.S. clinical laboratories. Improved accuracy of measurements, facilitated by this program, has led to better diagnosis, treatment, and reduced healthcare costs. Maintaining these anchor points for the clinical measurements reference system also facilitates the development and use of new technologies that are better, faster, and less expensive.

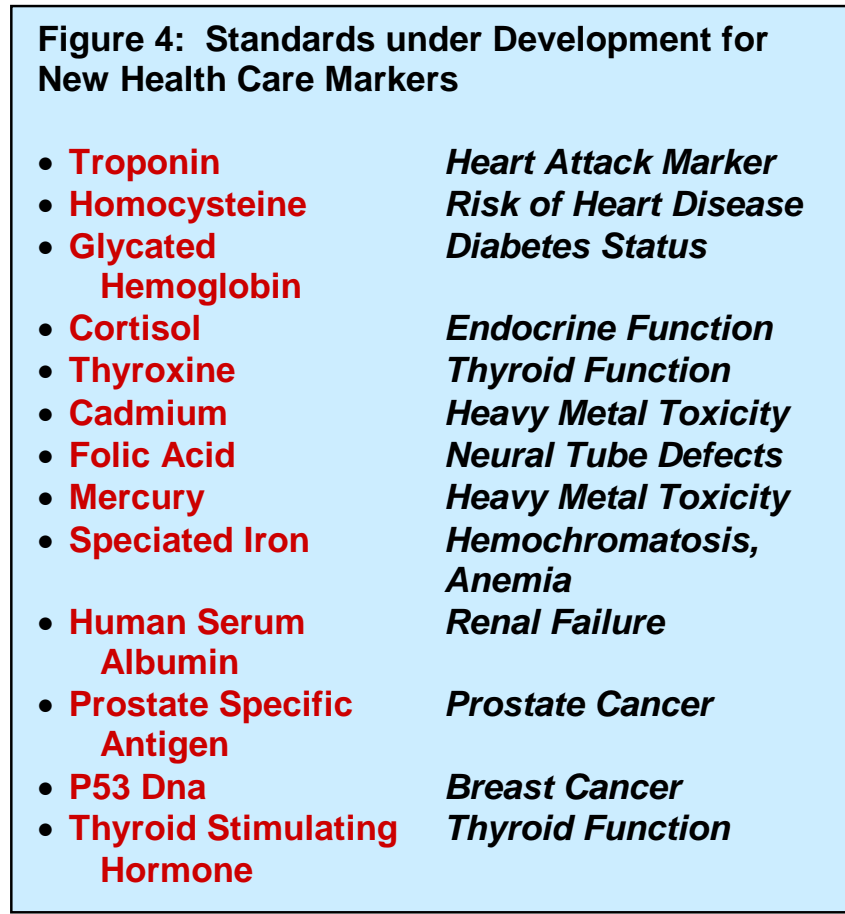

A new generation of health status markers, emerging now, shows great promise from the clinical diagnostic perspective, but offers new and more difficult challenges for standardization. Many of the new markers are proteins, peptides, or other large biomolecules, usually present at very low concentrations. Because of the large market for tests for these new markers, many different approaches have been developed commercially, resulting in vast disagreements among results obtained with different manufacturer test kits. NIST has focused on basic research to establish reference systems for several new biomarkers shown in Figure 4.

NIST views its SRMs as a cornerstone to traceability. NIST also works to ensure recognition of its standards worldwide, and to promote fair trade practices. One mechanism in which NIST ensures mutual recognition of its traceability structure worldwide is through proactive participation in the CIPM) Mutual Recognition MRA. This MRA provides the mechanism for implementation of the EU/US Mutual Recognition Agreement.

\section{CIPM Mutual Recognition Arrangement}

The CIPM MRA was signed in October 1999 by the directors of the NMIs of thirty-eight member states of the Metre Convention, and representatives of two international organizations. Since then, additional members have joined the MRA. The MRA provides the framework for "mutual recognition of national measurement standards, and of calibration and measurement certificates issued by $\mathrm{Na}$ tional Metrology Institutes" [9].

\section{Convention du Metré}

As far back as 1875 when the Convention du Metré was signed in Paris, the necessity for international collaboration and agreement on metrological issues was recognized. The United States, while not a world power at the time, was one of the seventeen original signatory nations. The Convention remains the basis of all international agreement on units of measurement. Currently, there are 49 Member States, including all the major industrialized countries. 
The CIPM MRA has as its objectives: a) to establish the degree of equivalence of measurement standards maintained by NMIs; b) to provide for the mutual recognition of calibration and measurement certificates issued by NMIs; and thereby c) to provide governments and other parties with a secure technical foundation for wider agreements related to international trade, commerce, and regulatory affairs. Implementation of the MRA involves international key and supplementary comparisons and quality systems and demonstration of competence by NMIs. The CIPM Consultative Committees are responsible for identifying key comparisons that are needed to demonstrate comparability for a broad range of measurements. Consultative Committees are organized to focus on the following metrology areas: amount of substance (mole) (CCQM), electricity and magnetism (CCEM), ionizing radiation (CCRI), length (CCL), mass and related quantities (CCM), photometry and radiometry (CCPR), thermometry (CCT), and acoustics, ultrasound, and vibration (CCAUV). Results of these comparisons and demonstrations are maintained in a database on the World Wide Web.

The BIPM Key Comparisons Database was originally developed by NIST for the BIPM and is now operated by the BIPM (http://kcdb.bipm.org/BIPM-KCDB/). This database is defined in the text of the CIPM MRA as "the database maintained by the BIPM which contains Appendices A, B, C, and D of the Mutual Recognition Arrangement" and are as follows:

Appendix $A$ is the list of the NMIs that are signatories to the arrangement, Appendix $B$ contains the results of key and supplementary comparisons, Appendix $C$ contains calibration and measurement capabilities (CMCs),

Appendix $D$ is the list of key and supplementary comparisons.

The principal components of the CIPM MRA are contained in Appendices B and C. Appendix B lists the metrology areas, protocol specifications, participating laboratories, participating countries, measuring conditions, results, and associated uncertainties for Key and Supplementary Comparisons. CIPM Key Comparisons (KCs) are carried out by the CIPM Consultative Committees or the BIPM; these are carried out at the highest metrological level. Participation in the CIPM KCs is open to all signatories of the CIPM

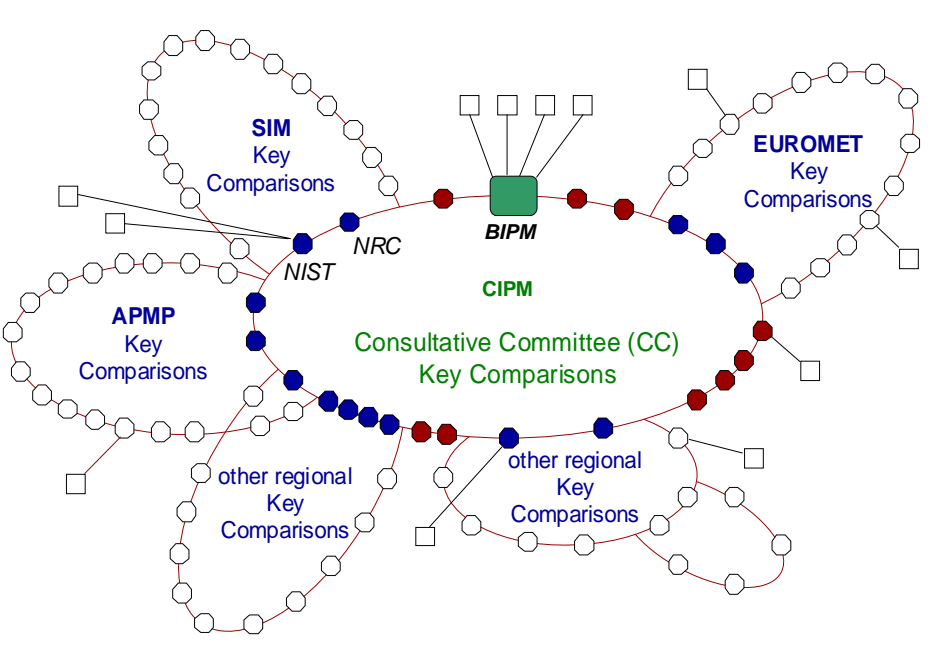

Figure 5: The figure shows the interconnectivity among the RMOs, the BIPM, and the CIPM Consultative Committees. The RMOs serve to link their respective regions to the international measurement infrastructure. 
MRA. Key Comparisons are also carried out by regional metrology organizations (RMOs) such as SIM (Systema Interamericano de Metrologia), EUROMET (European Collaboration on Measurement Standards), and APMP (Asia-Pacific Metrology Programme). The RMO KCs must be linked to corresponding CIPM Key Comparisons by means of joint participants (Figure 5). RMOs may also carry out Supplementary Comparisons that address regional needs, not covered by Key Comparisons, to provide confidence in calibration and measurement certificates.

The CIPM CCQM (Consultative Committee on Amount of Substance) is the committee responsible for the realization of the SI unit "mole", and demonstrating the comparability of all chemical composition related measurements. Currently, the CCQM Key Comparisons are focused on measurements related to health care, food and nutrition (including drinking water), environment, advanced materials, commodities, forensics, and general analytical applications (e.g., calibration solutions, high purity materials, $\mathrm{pH}$, conductivity, etc).

NIST is the pilot (lead) laboratory for several of the CIPM Key Comparisons, including the CCQM-K6, cholesterol in human serum. This comparison has been completed and its report is in a final draft stage. Planned Key Comparisons that address clinical needs are glucose in serum and creatinine in serum. One of the Key Comparisons that has been completed is CCQM-K2 comparison, lead and cadmium in water. The data have been reviewed and approved for posting in the database; therefore they can be discussed here as an example. Along with the analytical results, a statement of the degree of equiva-

\section{MATRIX OF EQUIVALENCE}

Key comparison CCQM-K2

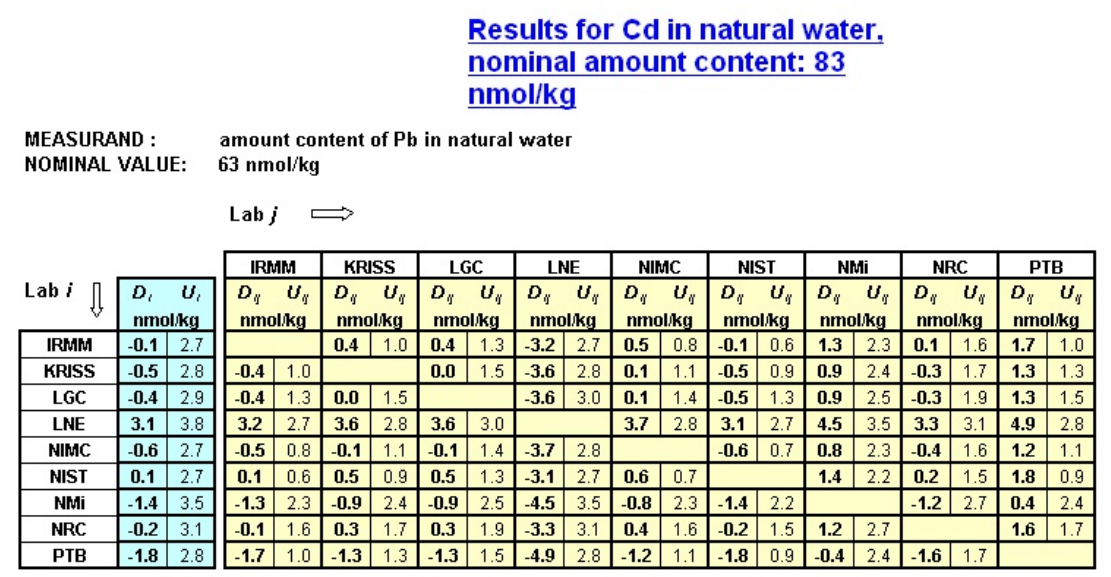

$A$ look at the matrix for Cd? ok

Figure 6: A Matrix of Equivalence is shown for CIPM Key Comparison (CCQM - K2) in the results section of the BIPM Key Comparisons Database. Each cell in the matrix shows the degree of equivalence between two NMls. lence is included, which is defined as the magnitude $\left(\mathrm{D}_{\mathrm{i}}\right)$ and uncertainty in the deviation $\left(\mathrm{U}_{\mathrm{i}}\right)$ from the key comparison reference value. An example of a "Matrix of Equivalence" from the BIPM Key Comparisons Database, along with the graphic representation of the same data, are shown in Figures 6 and 7, respectively. A given authority can use the results in the BIPM Key Comparisons Database to determine if the 
degree of equivalence demonstrated between two NMIs ( $\mathrm{D}_{\mathrm{ij}}$ and $\mathrm{U}_{\mathrm{ij}}$, corresponding to each cell in the matrix) is sufficient for the purpose at hand, such as regulatory compliance or international trade.

Appendix C of the BIPM Database provides detailed information on the existing metrological capabilities of each NMI. These calibration and measurement capabilities statements (CMCs) are developed and submitted by the participating NMI and provide the following information: the partici-

GRAPH OF EQUIVALENCE

Key comparison CCQM-K2

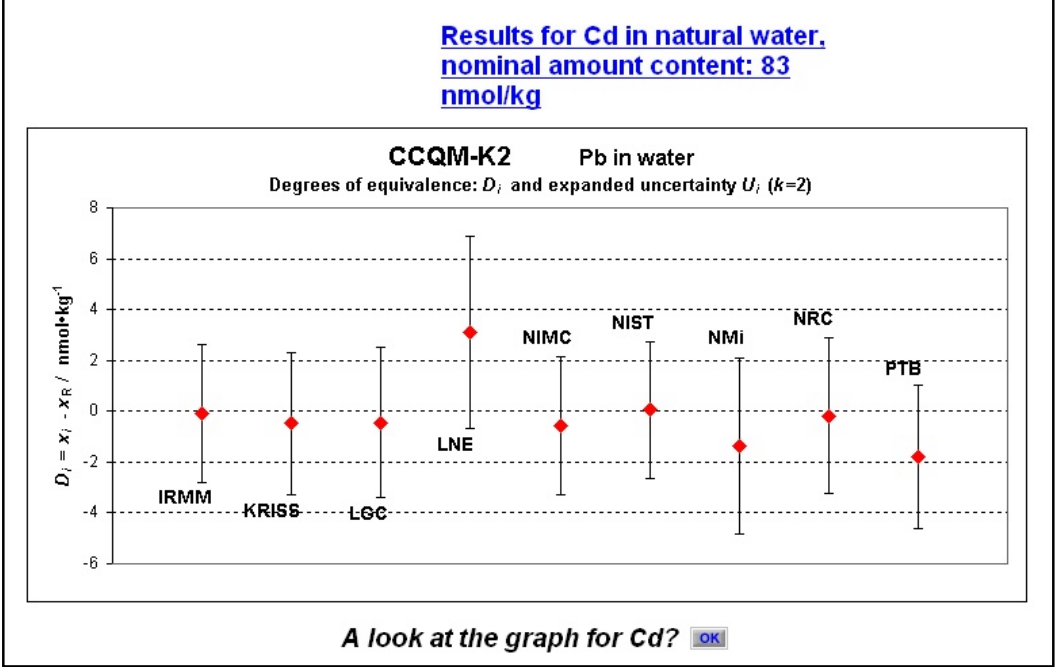

Figure 7: The graphic depiction of the "degree of equivalence" for CCQM - K2 indicating how each NMI's value compares with a reference value, and more importantly, how they compare to each other.

pating organization and country, metrology or calibration area, applicable range of measurements, uncertainties obtained by the institution, and the means of traceability to the SI. Currently, Appendix $\mathrm{C}$ is populated only in the areas of electricity and magnetism, and length. A draft template suggested for the amount of substance capability declaration has been prepared and includes the following categories: matrix, measurand, dissemination range of measurement capability, associated uncertainties, range in certified values of reference materials, and expanded uncertainties of certified values, source of traceabililty, measurement technique, and link to Appendix B if applicable.

A Joint Committee of Regional Metrology Organizations and the BIPM, known as the JCRB, provides oversight for results included in Appendix B, and is responsible for determining the degree(s) of equivalence of results from individual NMIs as discussed above. The statement of the degree of equivalence is a very important component of the CIPM MRA, as it demonstrates comparability at the NMI level.

The CIPM Key Comparisons that are carried out by its members are at the highest metrological level with traceability to the SI units. In the Americas, NIST, the National Research Council (NRC) of Canada, and the Centro Nacional de Metrologia (CENAM) of Mexico are members of the CIPM Consultative Committee on Amount of Substance, and as such take part in the CIPM Key Comparisons. However, in order to expand trade throughout each region, comparability of measurements is extended to non-members through Regional Metrology Organization (RMO) activities. 
Systema Interamericano de Metrologia (SIM) is the regional metrology organization (RMO) of the Americas and thus includes the United States. The metrology organizations that make up SIM are known as: NORAMET (North America), CAMET (Central America), CARIMET (Carribean), ANDIMET (Andes Region), and SURAMET (Southern America). Regional cooperation leads to a wider harmonization of measurements and standards, facilitates free flow of trade, and is a necessary first step to globalization. NIST, CENAM, and NRC Canada link SIM countries to the BIPM, and as such, provide greater opportunities for international trade and commerce.

Figure 8 shows the traceability and comparability link between two specific RMOs, SIM and EUROMET, through the NMIs that are members of the CIPM Consultative Committees. In addition to the KCs, RMOs can carry out Supplementary Comparisons (SCs) among its members to provide linkage to the BIPM/CIPM membership. Sec-

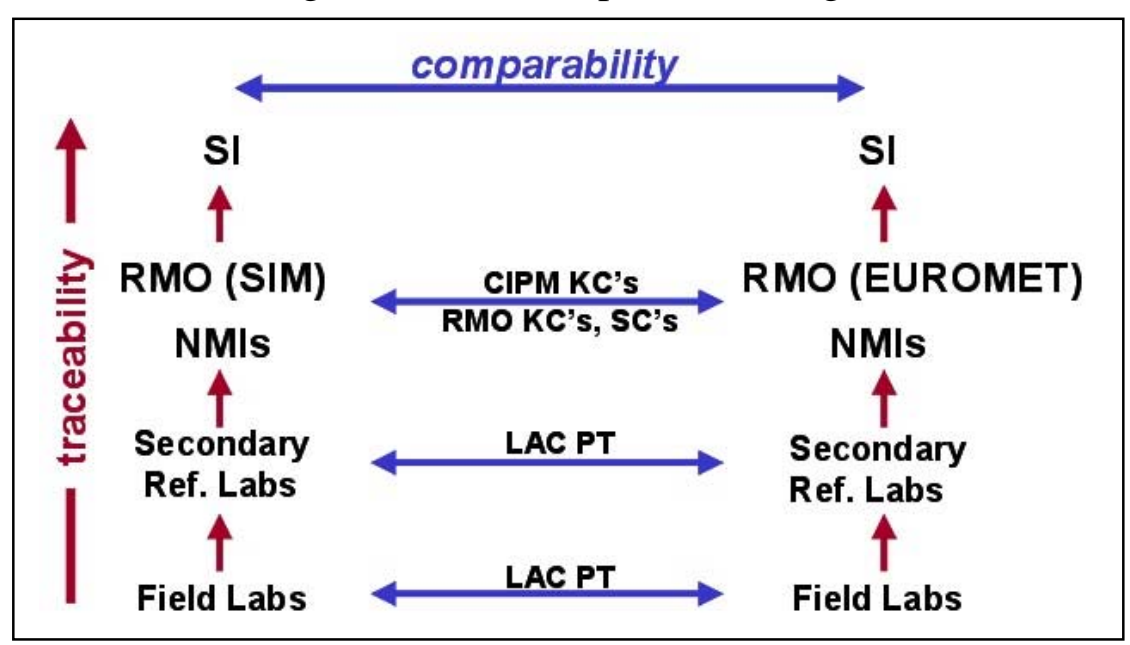

Figure 8: Comparability at the highest level is established among NMIs. However, for specific purposes and when mutually accepted among all parties, comparability between laboratories lower in the traceability structure may also be established through accreditation processes and proficiency testing. ondary Reference Laboratories may participate in SCs organized by their own RMO, SCs of other RMOs, as well as in Laboratory Accreditation (LAC) and Proficiency Testing (PT) Programs. The fundamental concept here is to establish confidence in the capabilities and the quality of measurements provided by a particular laboratory. Therefore,

Secondary Reference Laboratories and Field Laboratories can demonstrate comparability via LAC and PT programs, as well as via traceability to the NMIs participating in CIPM Key Comparisons.

NIST's Chemical Science and Technology Laboratory staff provide leadership for SIM by chairing the Chemical Metrology Working Group of SIM, and by serving as the U.S. representative to the JCRB, in order to assure the effective, fair, and metrologically sound implementation of the MRA throughout the world. 


\section{Summary}

The EU/US Mutual Recognition Agreement is a treaty that was negotiated to promote equity in trade by removing technical barriers. This treaty provides for mutual recognition of standards and conformance infrastructure. While the "Pharmaceutical and Medical Devices" area explicitly addresses health (and safety) issues, all of the areas specified in the treaty deal with health and safety regulations. This crosscutting focus indicates the value placed on health-related issues at the highest level of governments, and an understanding that economic, environmental and human health issues are inter-

"Global economic integration, in itself is neither a panacea nor a curse. It is, however, a powerful phenomenon that incorporates many aspects of technological, economic, political and cultural change. We cannot, nor should we, wish to stop it. We can and must, however, shape it so that it advances our prosperity and values."

Alan P. Larson, U. S. Under Secretary of State for Economic, Business and Agricultural Affairs, February, 2000 twined.

The EU IVD Directive, to become fully effective in December 2003, reminds us of the importance of traceability for international trade, as well as for improving accuracy of measurements. It is estimated that approximately $60 \%$ of the IVD devices sold in Europe are imported from the U.S. U.S. manufacturers recognize the urgent need to be active participants in building the measurement and standards infrastructure that ensures continued and open commerce for their products.

The CIPM MRA recently developed by the metrological community is an overarching framework that facilitates the implementation of the EU/US Mutual Recognition Agreement and provides comparability of measurements and standards worldwide. An important part of the CIPM MRA is the BIPM Key Comparisons Database that demonstrates equivalence of measurements among signatory NMIs. While the Key Comparisons are conducted at the NMI level, comparability is expanded on a regional basis through each RMO. The comparability of measurement capabilities demonstrated through the Key Comparisons is designed to facilitate trade by eliminating the need for retesting a product at every port of entry. The linkage through the national traceability structure helps to ensure that the measurements performed by industry to characterize their products are recognized in the global marketplace. It is clear that such a system is required to sustain the growth of global trade, and to remove technical barriers to trade. Over the past few years, the international metrological community has been building the framework for fair and efficient trading practices by demonstrating comparability of selected measurements through the implementation of the CIPM MRA. The IVD manufacturers and the medical community along with the NMIs must now work in partnership to identify the highest priority measurement standards for the timely implementation of the regulations imposed by the IVD Directive thus meeting its requirement of traceability to "standards of a higher order." 


\section{References}

1. M. Boskin, L. Lau, "Generalized Solow-Neutral Technical Progress and Postwar Economic Growth,” NBER Working Paper, W8023, December 2000.

2. "Agreement on Mutual Recognition between the European Community and the United States of America," December 1, 1998. (Text available on the internet at http;//www.useu.be).

3. "The US/EU Partnership for the $21^{\text {st }}$ Century," remarks given by U.S. Under Secretary of State Eizenstat at the Nixon Center, October 16, 1998. (Text available on the internet at http://www.useu.be/DOCS/Index.html).

4. M. Suppo, “EU Starts Five-year Countdown for the IVD Directive," IVD Technogy, January, 1999 (Text available on the internet at: http://www.devicelink.com/ivdt/archive/99/01/006.html)

5. "Council Directive 98/79/EC of the European Parliament and of the Council of 27 October 1998 on In Vitro Diagnostic Medical Devices," Official Journal of the European Communities L331, December 1998. (Text available on the Internet at http://www.edma-ivd.be).

6. Trahey, N. M., editor, NIST Standard Reference Materials Catalog 199899 edition, NIST Spec. Publ. 260, January 1998 (Text available on the internet at http//www.nist.gov).

7. U.S. Industry and Trade Outlook '99, U.S. Department of Commerce/International Trade Administration, McGraw-Hill Companies, 1999.

8. "Cholesterol Measurement - Test Accuracy and Factors that Influence Cholesterol Levels," General Accounting Office Report GAO/PEMD-95-8, December 1994.

9. "Mutual Recognition of National Measurement Standards and of Calibration and Measurement Certificates Issued by National Metrology Institutes," Edited by the BIPM, Paris, France, September 1999. 


\section{HRATCH G. SEMERJIAN}

\section{National Institute of Standards and Technology Chemical Science and Technology Laboratory}

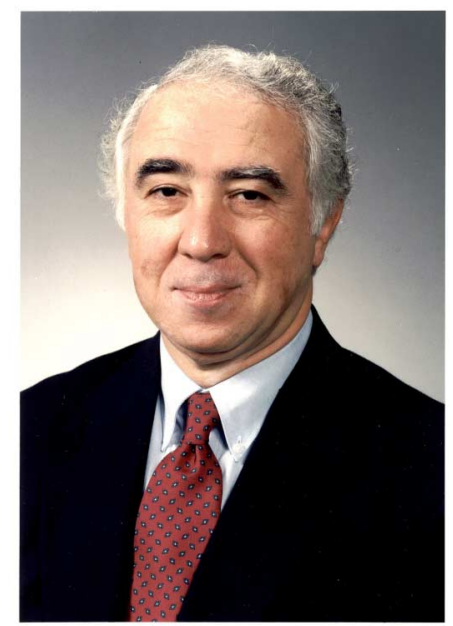

Dr. Semerjian, Ph.D., has been the Director of the Chemical Science and Technology Laboratory (CSTL), one of seven Laboratories in NIST, since 1992. He is responsible for chemical engineering, biotechnology, analytical chemistry, process technology, and physical chemistry research activities at NIST. The mission of CSTL is to promote the U.S. economy by providing technical leadership for the Nation's measurement and standards infrastructure, and assuring the availability of essential data and measurement capabilities. CSTL provides these infrastructural capabilities for U.S. industry, government agencies, and the scientific community, to enhance U.S. industry's productivity and competitiveness, assure equity in trade, and improve public health, safety and environmental quality. CSTL has a total staff of about 450, and an annual budget of about $\$ 60 \mathrm{M}$. Dr. Semerjian received his B.Sc. with honors in Mechanical Engineering from Robert College (Turkey) in 1966, and his M.Sc. (1968) and Ph.D. (1972) in Engineering from Brown University. He served as a Lecturer and PostDoctoral Research Fellow in the Chemistry Department at the University of Toronto. He then joined the research staff of Pratt \& Whitney Aircraft Division of United Technologies Corp. in East Hartford, CT. In 1977, Dr. Semerjian joined the National Bureau of Standards (now the National Institute of Standards and Technology). His research has focused on combustion processes, pollutant formation, and the application of optical techniques to chemically reacting systems. Dr. Semerjian has authored over 100 publications. He is an active member of AIChE, ACS, ASME, AIAA, AAAS, the Combustion Institute, and the Council for Chemical Research. He has also served on advisory and review boards for the Department of Energy, National Science Foundation, Environmental Protection Agency, National Oceanic and Atmospheric Administration and the Department of Defense. Awards he has received include AM\&F Corp. Fellowship at Robert College, the Fullbright Fellowship, C.B. Keen Fellowship at Brown, the U.S. Department of Commerce Meritorious Federal Service (Silver Medal) Award in 1984, and the U.S. Department of Commerce Distinguished Achievement in Federal Service (Gold Medal) Award in 1995; he was also selected as the DoC "Federal Engineer of the Year" in 1991. In 1996, he was elected a Fellow of the American Society of Mechanical Engineers. In 1997, he received the Brown Engineering Alumni Medal. Dr. Semerjian served as Acting Deputy Director, NIST, January through October, 1997. In 2000, Dr. Semerjian was elected a member of the National Academy of Engineering. 


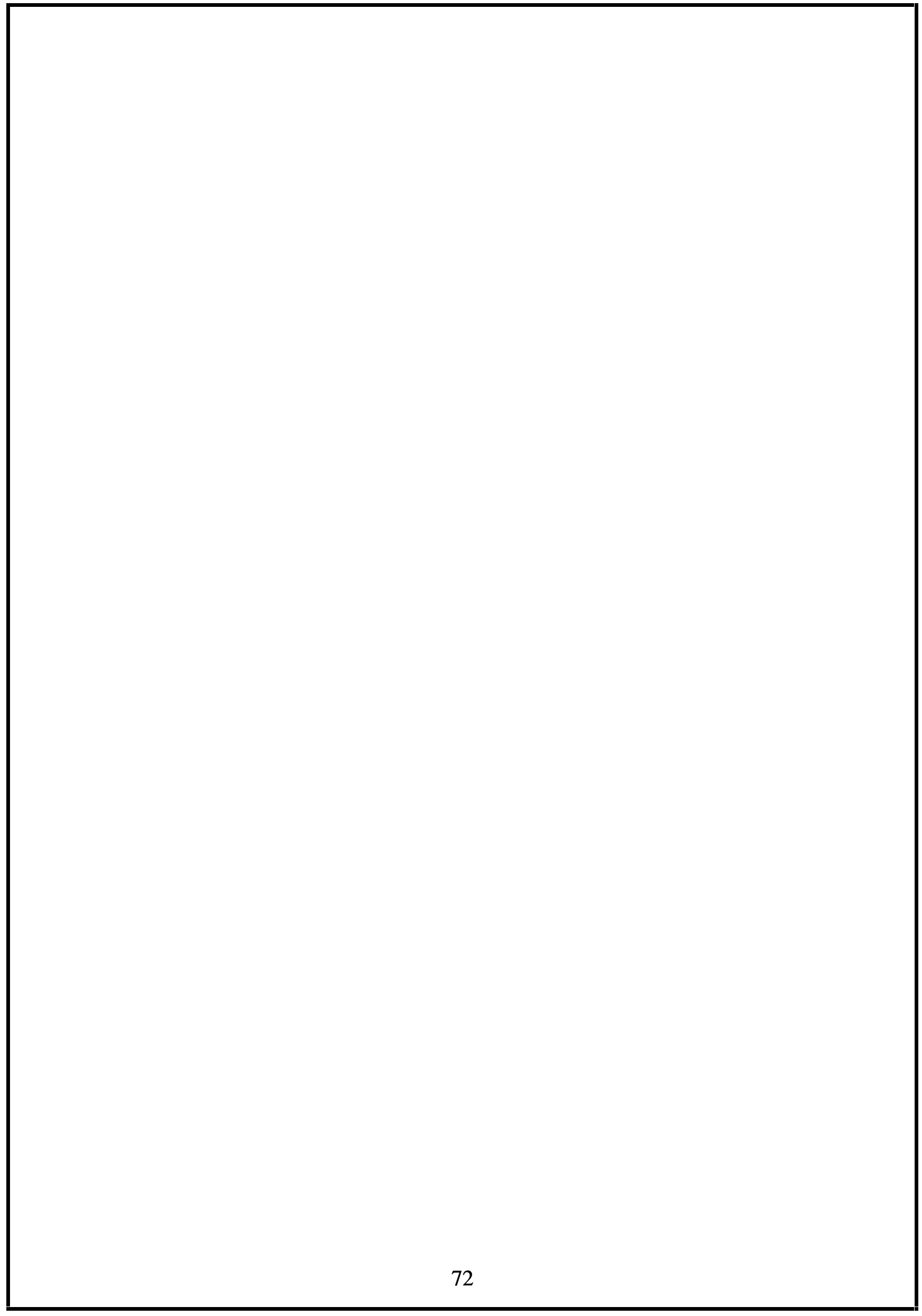


Mutual Recognition of Measurements: How Do They Impact International Trade?

Visuals Presented By

Hratch G. Semerjian

National Institute of Standards and Technology 

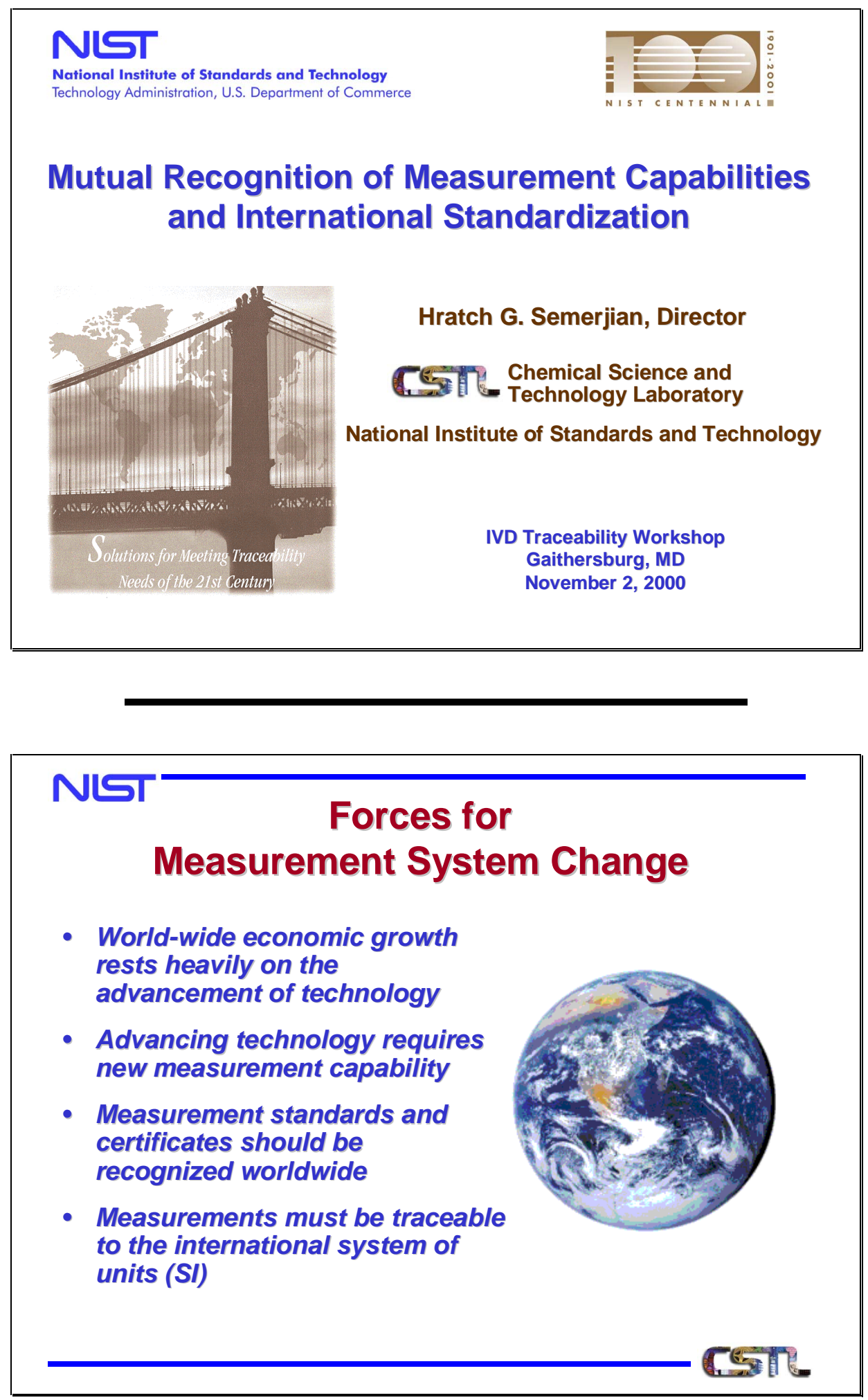


\section{Traceability vs Comparability}

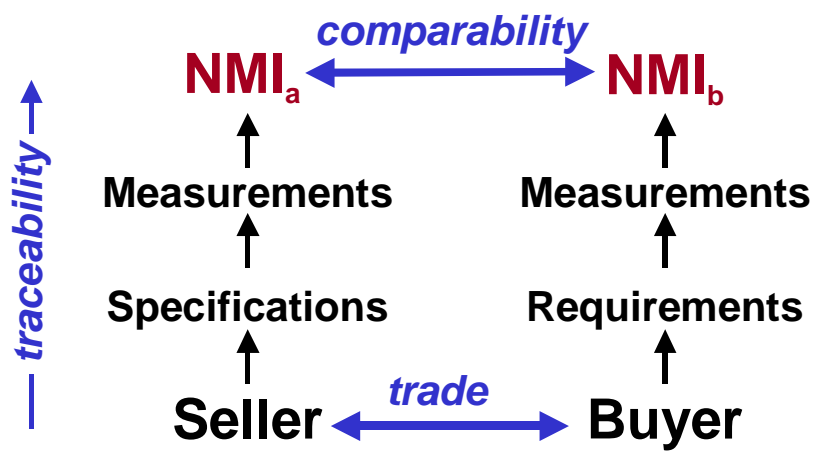

NMI: National Measurement Institute

\section{NGT}

Impact of NIST Measurement and Standards Programs

ADVANCING TECHNOLOGY...

- Is vital for commerce and international trade

- Accounts for $~ 50 \%$ of U.S. economic growth

- Drives demand for new measurements and standards

- Requires that NIST maintain state-of-the art scientific facilities

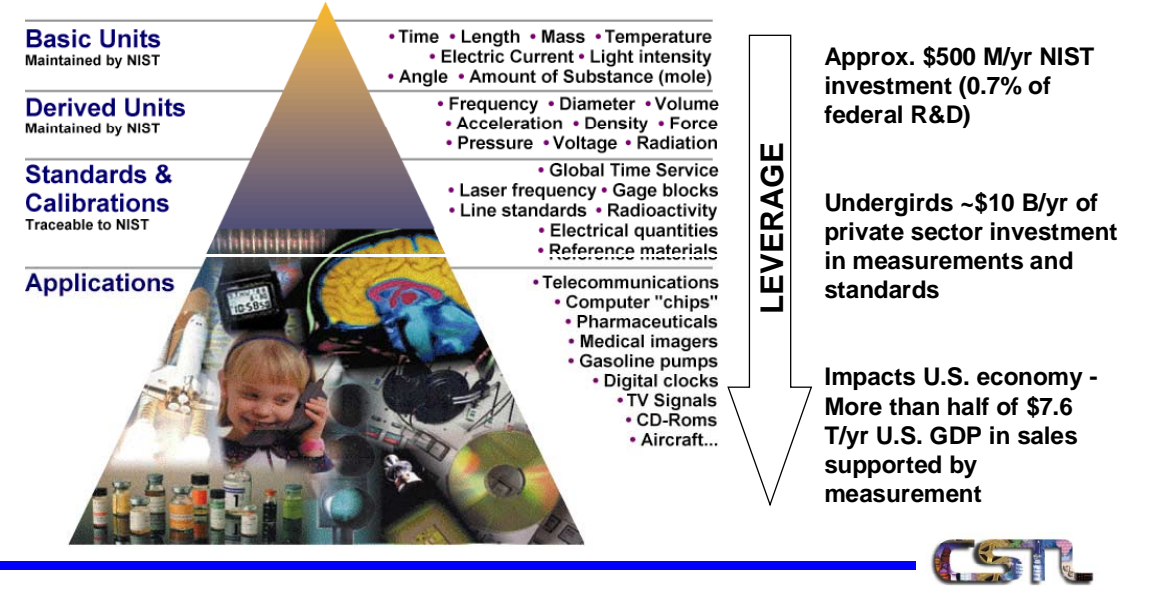



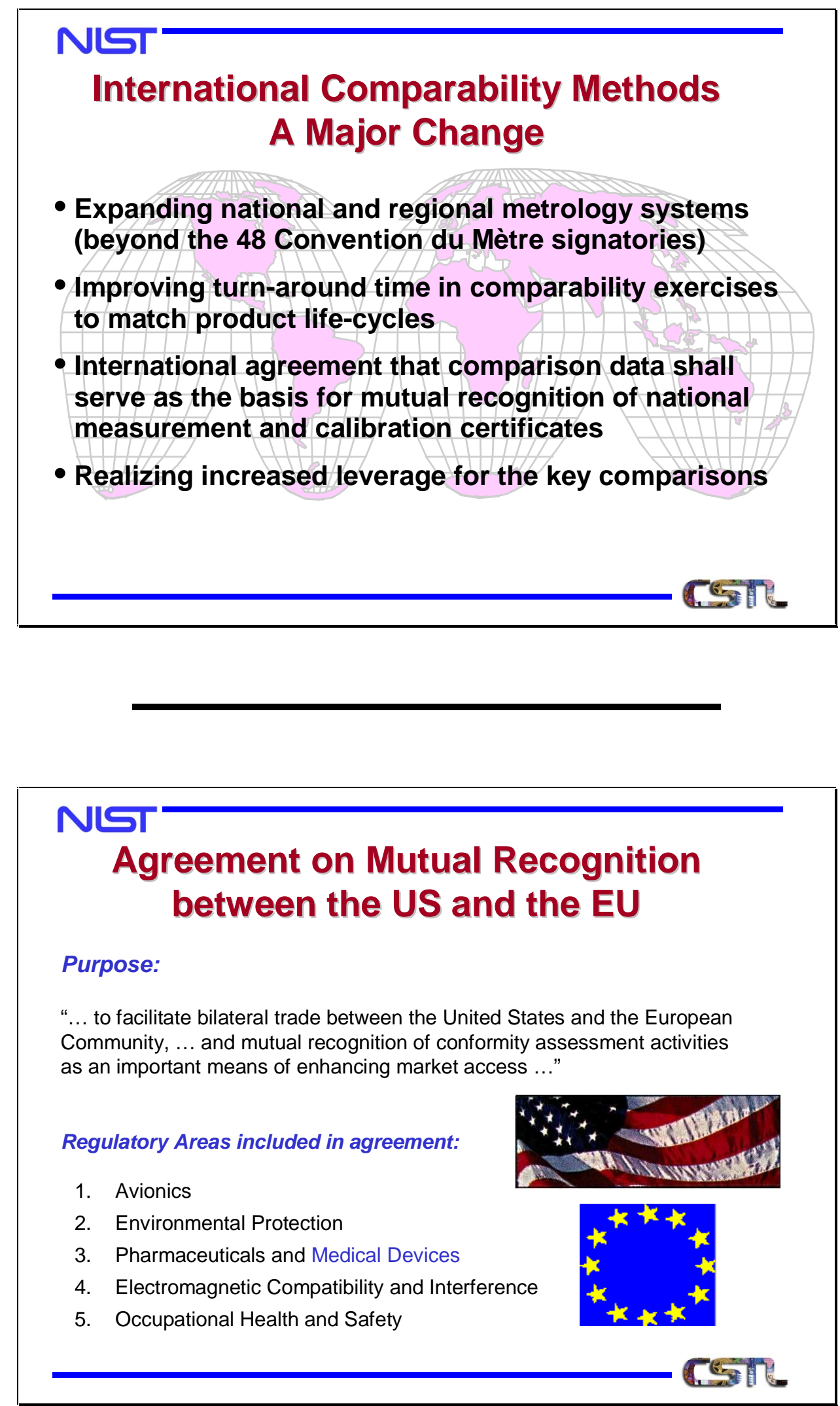


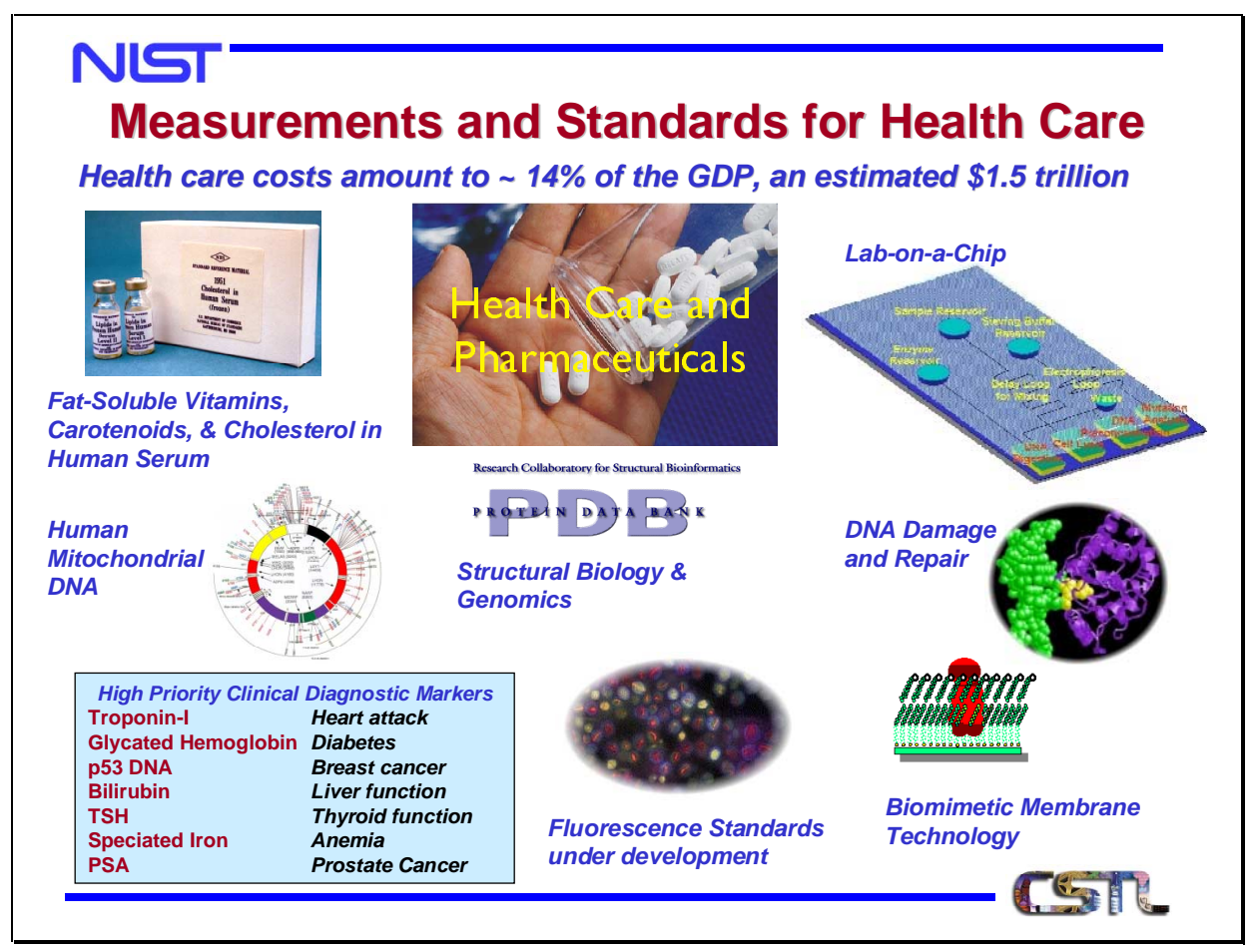

\section{NLاك}

\section{Health Care Markers: EU IVD Directive}

Magnitude and Scope:

- U.S. Spends \$1.5 trillion on Health Care (14\% of GDP)

- $\sim 13 \%$ of this amount is associated with measurements $(\$ 140 \mathrm{~B})$

- Non-diagnostic measurements cost $\sim \$ 39 B$

- Improvement in accuracy of one marker alone (cholesterol) is estimated to have saved \$100M per year

New Driver: EU IVD Directive to go into effect 2003

- Worldwide in vitro diagnostic device market is $\sim 20 \mathrm{~B}$;

- $>60 \%$ of European market is supplied by U.S. and Japan

Stated Purpose of Directive

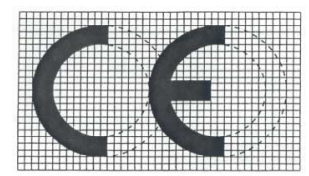

- Eliminate trade barriers within Europe by ensuring access to the entire EU market with one single product approval (CE Mark)

Essential Requirements

- IVD Calibrators and/or control materials must be traceable to "standards of a higher order"

- nationally/internationally recognized certified reference materials

Implementation

- First IVD product with CE Mark may be placed from June 2000 onwards

- All new IVD products must have mark by December 2003

- Existing IVD products may be sold without the CE mark until December 2005 


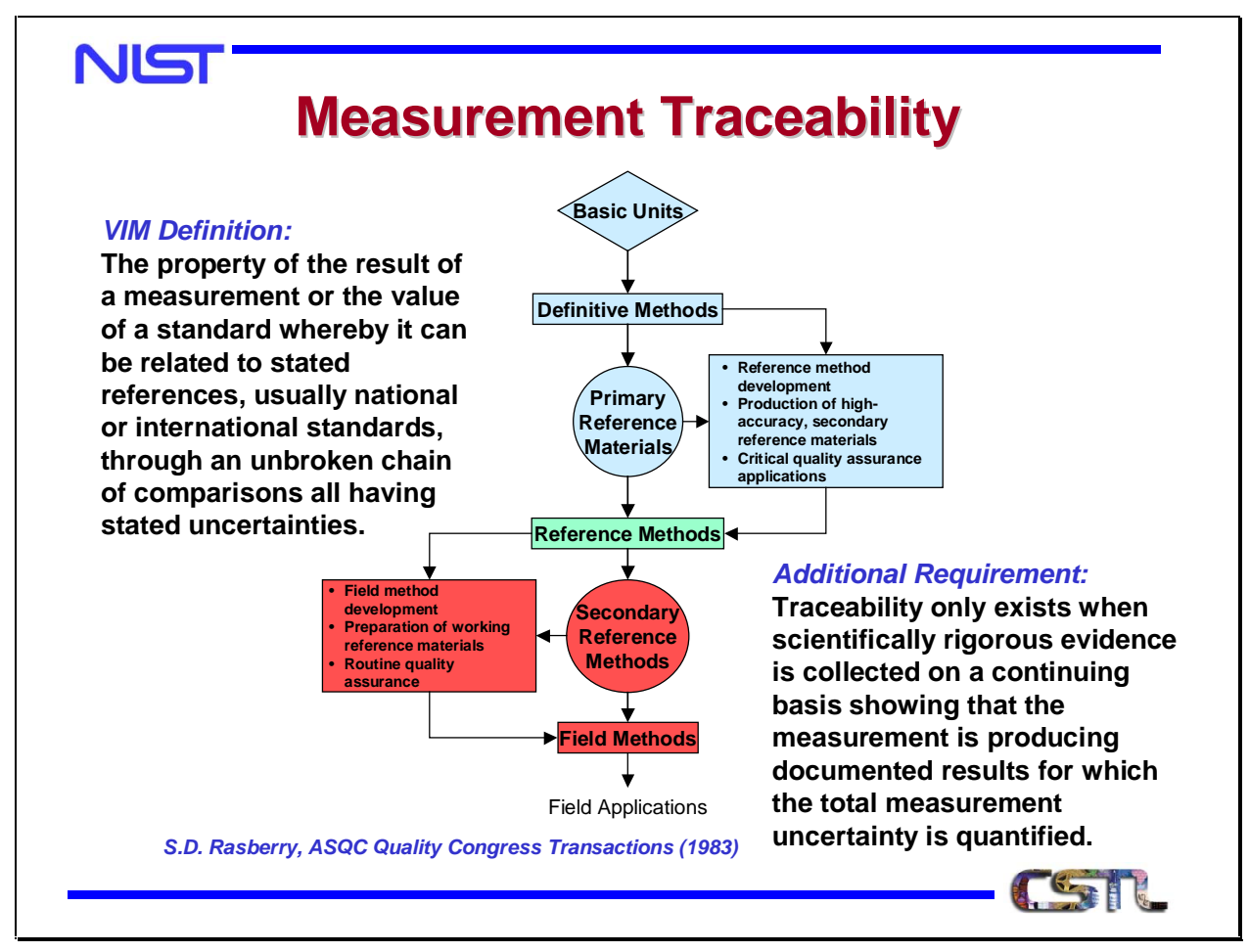

NIST

\section{A National Traceability System}

- Tools for the dissemination of measurement standards

- reference materials

- reference data

- calibrations

- special arrangements for greater leverage

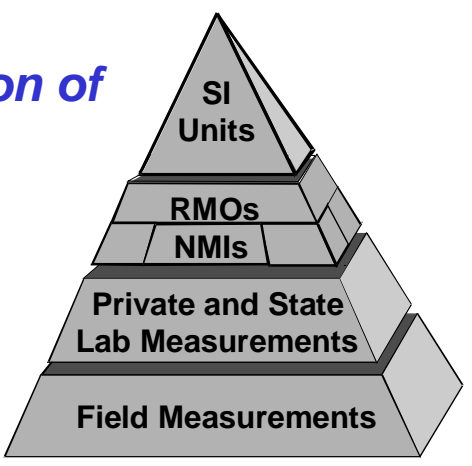




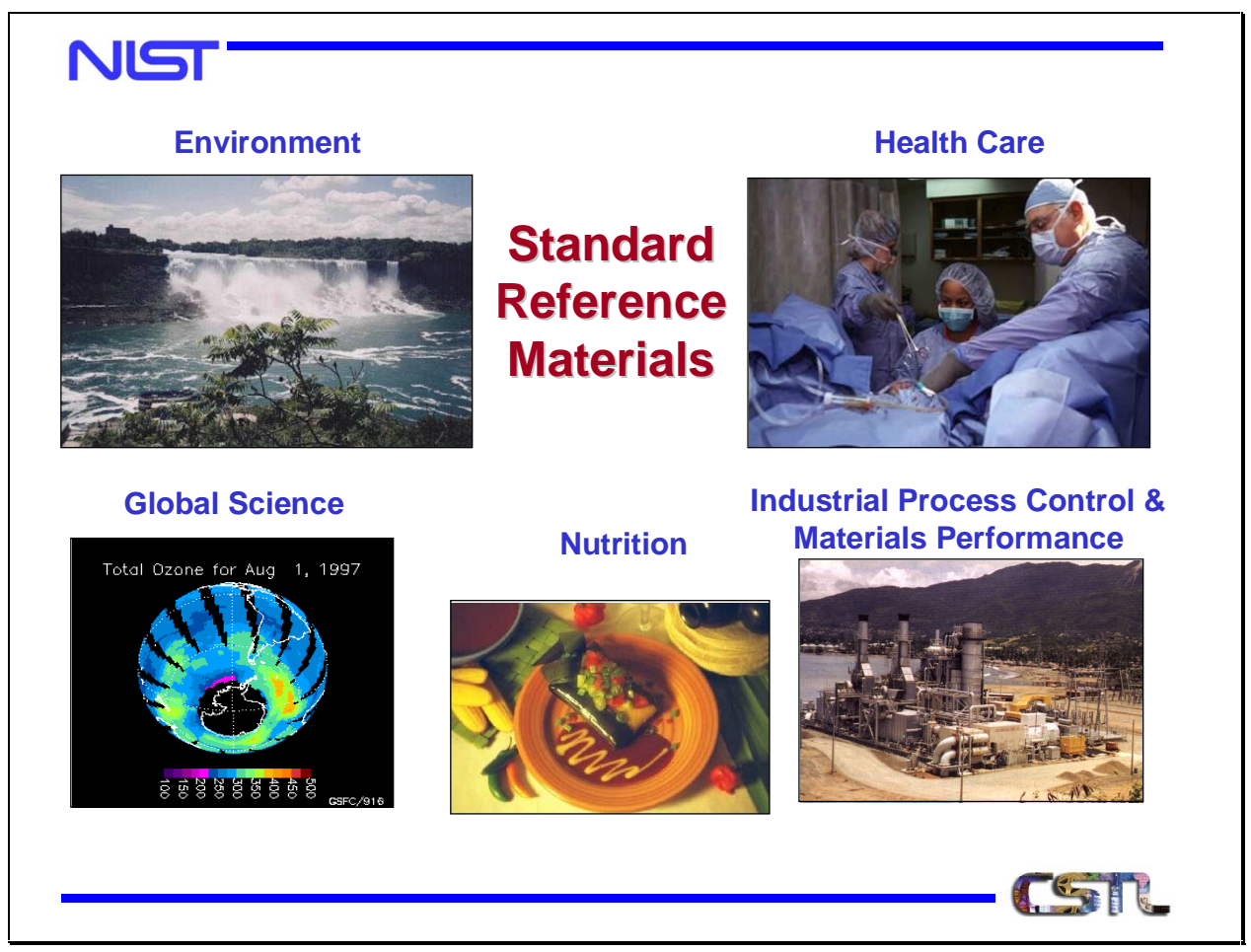

NIST

\section{CIPM Mutual Recognition Arrangement}

\section{Objectives:}

- Establish the degree of equivalence of national measurement standards maintained by NMls

- Provide for the mutual recognition of calibration and measurement certificates issued by NMIs

- Provide a secure technical foundation for wider agreements related to international trade, commerce and regulatory affairs 
NIكT

\section{CIPM Mutual Recognition Arrangement}

\section{Process:}

- International comparisons of measurements, to be known as key comparisons

- Supplementary international comparisons of measurements

- Quality systems and demonstration of competence by NMIs

\section{Outcome:}

- Statements of the measurement capabilities of each NMI in a database publicly available on the Web

NLT

\section{CIPM Key and Supplementary} Comparisons

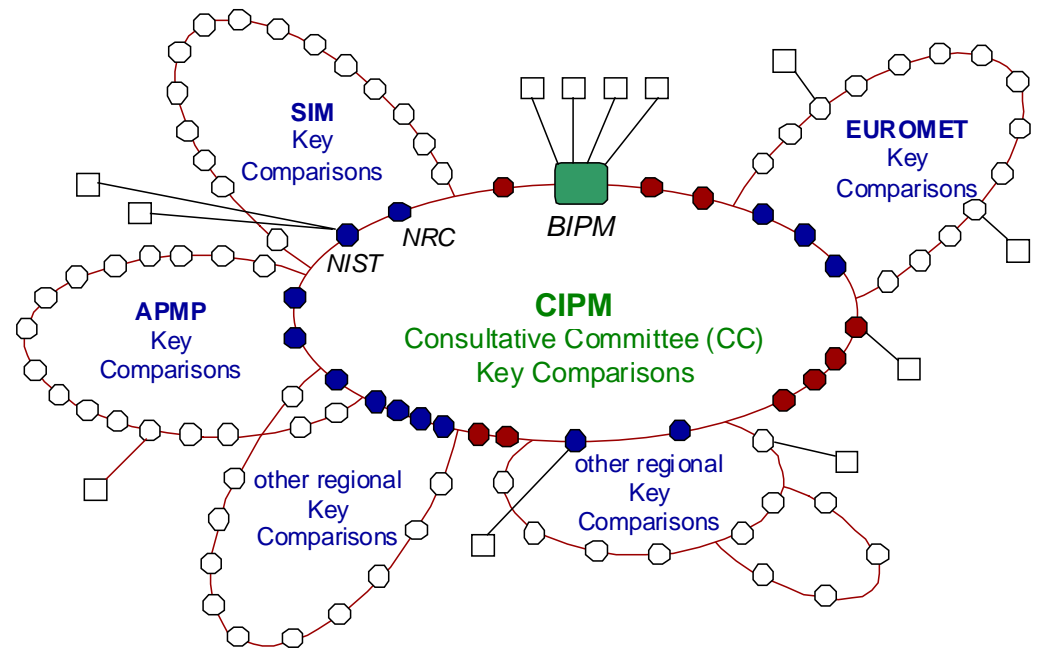




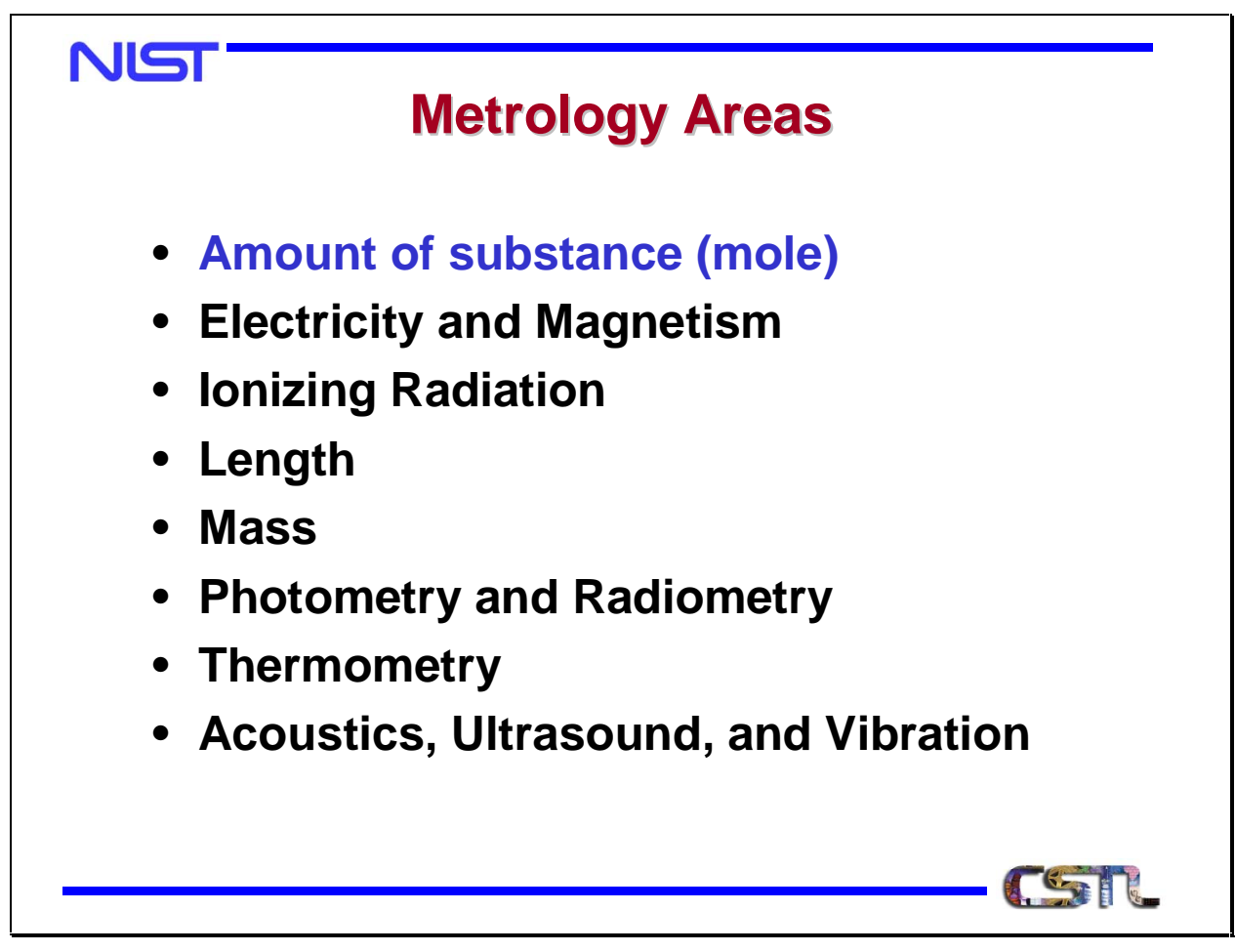

- Amount of substance (mole)

- Electricity and Magnetism

- lonizing Radiation

- Length

- Mass

Photometry and Radiometry

Acoustics, Ultrasound, and Vibration

\section{NLT}

\section{Principal MRA Components}

Appendix B

Comparisons

- Metrology areas

- Protocol specifications

- Participants

- Measuring conditions

- Results and uncertainties
Appendix C

Capabilities

- Measurement or calibration area

- Applicable range

- Uncertainties attainable

- Means of traceability to the SI

Recorded in International Comparisons Database developed by NIST and maintained by BIPM 

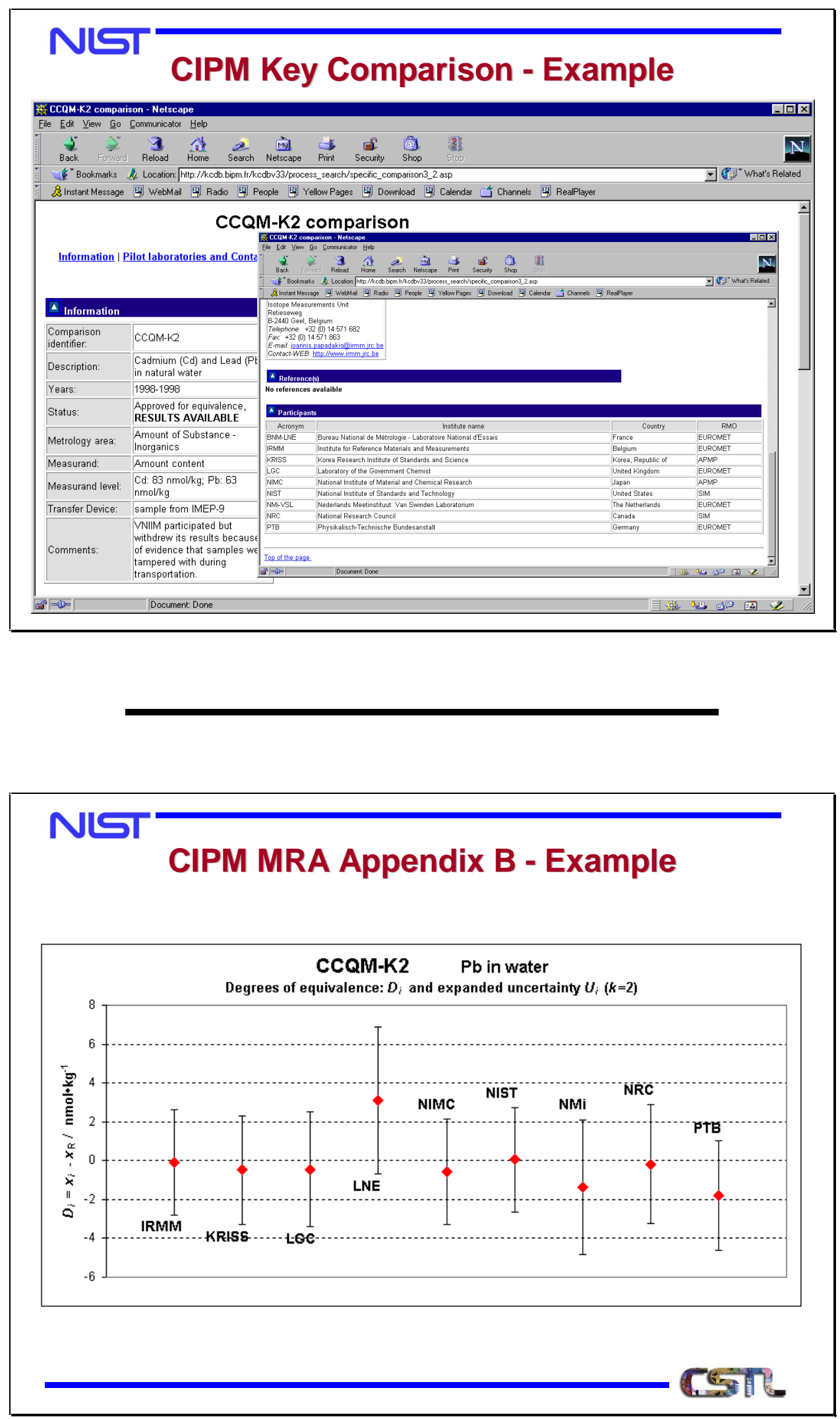

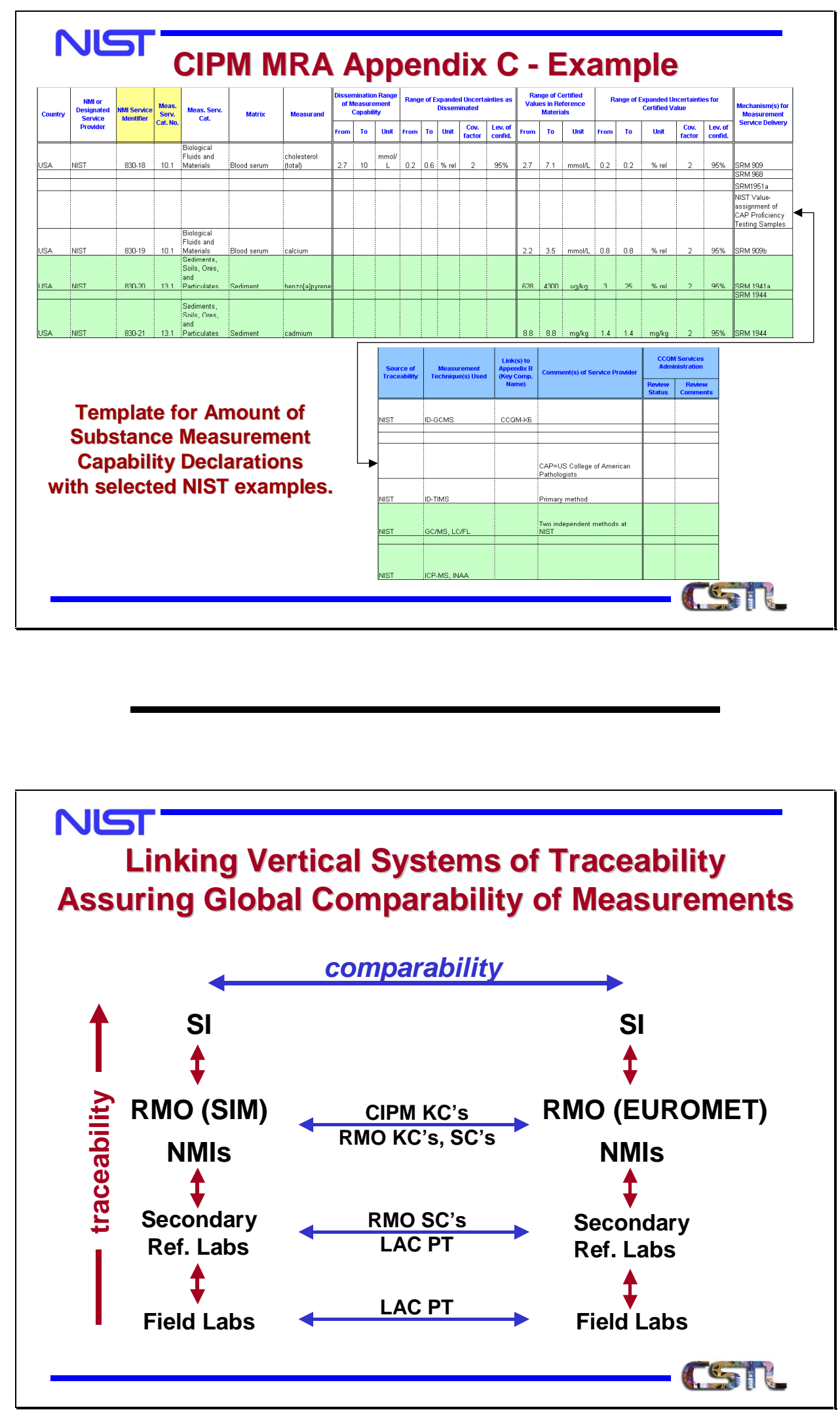
NLI

\section{Summary}

- Health Care has great impact on the economy and everyday life

- US-EU MRA provides framework for promoting international trade

- CIPM MRA facilitates the mutual recognition of measurements performed in other countries

- EU IVD Directive identifies an area where current standards are inadequate

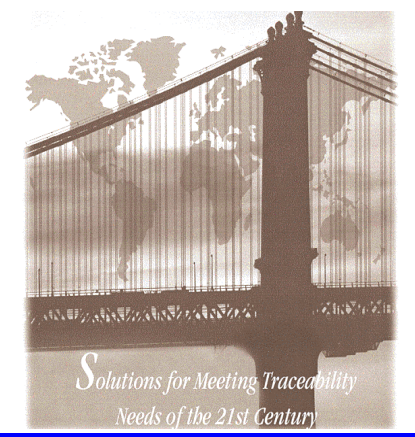

A cooperative international effort is required to meet the challenges of providing necessary measurement standards 


\section{Traceability of Calibration for IVD Industry: Process for Credentialing Reference Materials, Reference Methods and Reference Laboratories in Europe Lothar Siekmann University of Bonn • Institut für Klinische Biochemie}

The concept of measurement traceability has been established in general chemical metrology and is now also being introduced to the field of clinical chemical analyses. Traceability provides probably the most important strategy to achieve standardisation in laboratory medicine aimed at comparable measurement results regardless of the method, the measurement procedure (test kit) and of the laboratory where analyses are carried out.

According to the 'Vocabulary in Metrology (VIM)' and the 'Guide to the Expression of Uncertainty in Metrology (GUM)' measurement traceability is defined as:

property of the result of a measurement or the value of a standard whereby it can be related to stated references, usually national or international standards, through an unbroken chain of comparisons all having stated uncertainties

Traceability of a value attributed to a routine sample, a calibrator or a control material is established by a series of comparative measurements using measurement procedures and reference materials in a chain of increasing hierarchical order as shown in Fig.1. Since each link in the traceability chain contributes to the uncertainty of the result it is advisable to omit as many steps as possible. In terms of metrology it would be ideal to omit all in between steps of the traceability chain and to measure the routine sample directly by use of a primary reference procedure; this of course is not feasible.

The complete traceability chain as presented here is valid only for those measurable quantities that can have a value expressed in SI units. When primary or secondary calibrators are not available, the traceability chain for many measurands in laboratory medicine ends at a lower level, e.g. at the manufacturer's selected measurement procedure. In a case where a manufacturer detects a new diagnostic marker and defines the measurable quantity by establishing a measurement procedure for this marker, the maunufacturer's measurement procedure will form the top of the traceability chain. Nevertheless even in this simple situation the principles of the traceabilty concept are applicable.

An inevitable precondition for establishing traceable results to calibrators and control materials is the specificity of the measurement procedures applied. Results of measurement cannot be traceable when the procedure applied partially detects components that are not consistent with the definition of the measurand.

Traceability is not really a new fundamental concept in our science. Many years before traceability has been mentioned in general chemical metrology, reference meas- 
urement procedures and reference materials have been established in clinical chemistry. The early developments in this field in the U.S., particular by the NIST colleagues Cohen, Ellerbe, Hertz, Mandel, Schaffer, Sniegoski, Welch, White and others [1-12] as well as by individual scientists such as Cali [13], Tietz [14-16], Eckfeld [17], and Doumas [18-19] as well as the relevant NCCLS standards [20-23] probably had an important influence on the development of the concept of traceablility in general chemical metrology. Some basic experimental work for the development of reference measurement procedures and reference materials had already been undertaken in Europe, e.g. by Björkhem [24-26], Büttner [27-29], Külpmann [30- 31] Schumann [32-33], Siekmann [34-43], Stöckl [44-46], Thienpont [47-53], and others.

FIGURE 1. Calibration Hierarchy and Traceability

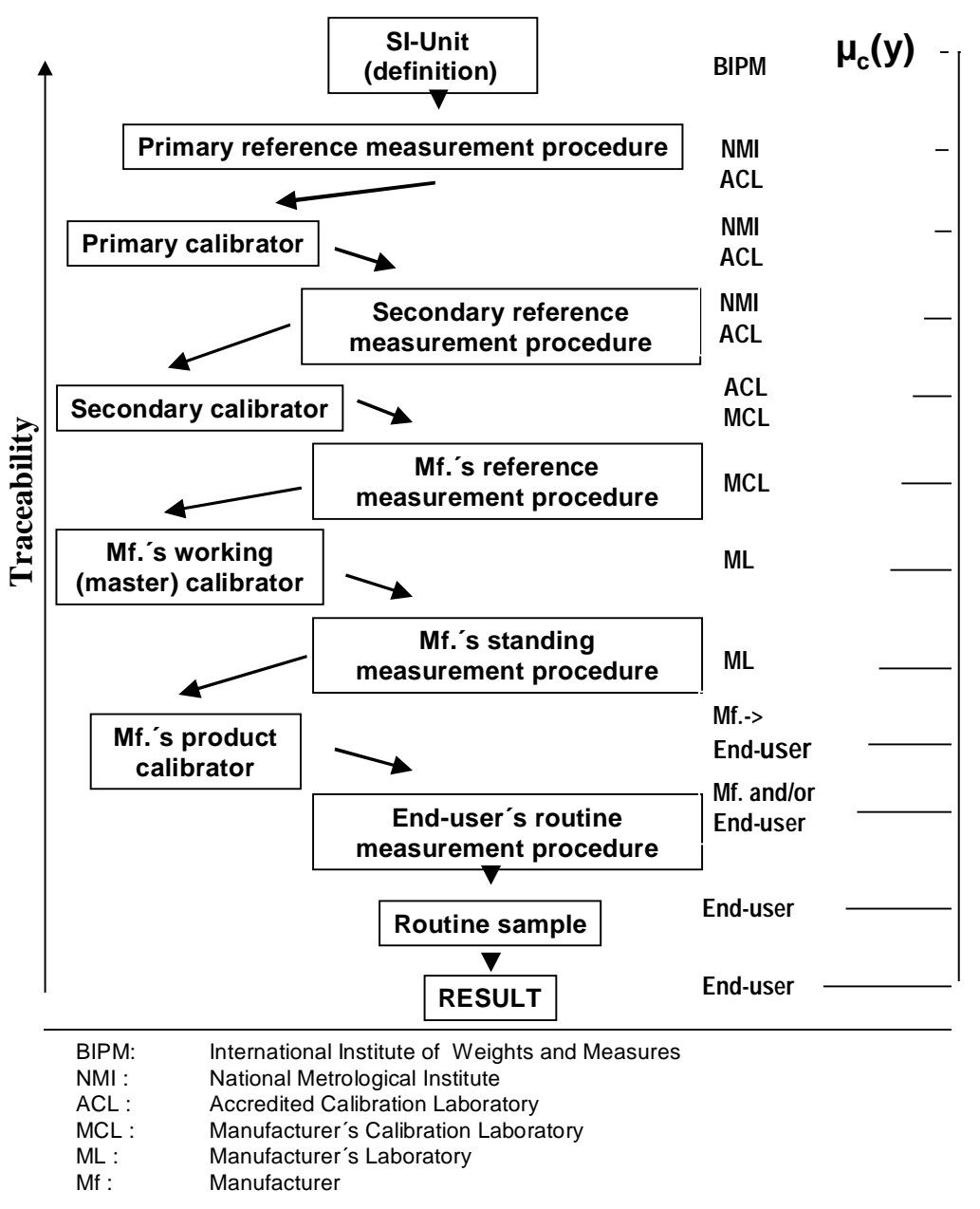

In 1970, a long time before the concept of traceability became popular, the technique of isotope dilution mass spectrometry had been developed for the first time in a clinical chemical reference laboratory and was applied as a reference procedure for the measurement of estrogens in human body fluids [35]. Ever since that time, this technique 
provides one of the most powerful tools for establishing reference method values for many substrates and metabolites in calibrators, controls and reference materials.

In view of the globalisation of economics, science and, hopefully, also social developments, the process of credentialing, the traceability concept and its implementation and acceptance, is not only a regional (European) or national task, but it is a global task. Traceability is a task that concerns all members of our scientific community involved in the field of clinical chemical analyses.

Such as:

- Legislative bodies, when issuing regulations or directives concerning measurements in laboratory medicine, e.g. FDA, CEC.

- $\quad$ The national metrology institutes, e.g. NIST, which are responsible for dissemination of the SI units (Système International d'Unités) and for establishing correctness of measurement according to national legislation.

- International organisations issuing reference materials, e.g. NIST, IRMM, WHO.

- International and national standardisation organisations, e.g. ISO, CEN, NCCLS.

- International and national scientific societies, e.g. IFCC.

- External quality assessment organisations, e..g. CAP, Reference Institut of Bioanalysis (DGKC).

- Reference laboratories.

- Diagnostic kit manufacturers.

- Clinical chemical laboratories when applying commercial or home-made diagnostic tests, and

- Physicians determining diagnosis and therapy on the basis of laboratory results.

Credentialing traceability in clinical chemistry implies the demonstration of applicability and usefulness of the concept as a basis of credibility. In practice, reference systems are to be established consisting of reference measurement procedures, reference materials and reference laboratories, which should preferably be accredited and organised within a network.

The introduction of such reference systems has been proposed in clinical chemistry for about 30 years. However, neither the international scientific community nor any national or international body have addressed the question of which agency or authority should be responsible for formal authorization of these reference systems; including reference materials, reference procedures and reference laboratories. 
For reference materials it may not be too difficult to solve the problem. Materials, which fulfil the requirement for higher metrological order standards, are now provided by the NIST and the IRMM. The WHO, although usually of lower metrological order, issues additional useful materials. Authorization of these materials is a question of mutual acceptance, which, in view of the "Implementing Arrangement for Cooperation in the Fields of Metrology and Measurement Standards" signed by the directors of the NIST and the EU`s Research Directorate, has almost been achieved.

For the authorization of reference procedures and reference laboratories the situation is somewhat more complicated and the question of how the concept of traceablity should be implemented arises. There is no simple answer and probably there is no general rule that can be applied to all situations. The new International Standards dealing with 'traceability' (prEN ISO 17511) and the 'requirements for reference laboratories' (ISO/DIS 15195) developed by CEN TC140 and ISO TC212, can at least give some guidance for the credentialing process for reference laboratories and reference procedures. In fact, the strategy for establishing reference systems depends on the nature of the analyte.

For low molecular substances - electrolytes, organic substrates and metabolites like cholesterol, creatinine or steroid hormones - as well as for many drugs, the only meaningful way is to aspire to results that are traceable to the SI units. For these measurands, where traceability to SI units is achievable, national metrology institutes as legal custodians of units and measurements hold the highest authority (Fig 2). The metrology institutes are connected globally with each other by so-called CCQM (Consultative Committee on Amount of Substance) key comparisons, where they demonstrate, by the means of ring trials, their ability to perform measurements of the highest available metrological level, usually by the use of so-called primary methods. Such key comparisons were carried out for cholesterol, creatinine and glucose. A group of European metrology institutes has now started a global initiative to address the problem of traceability in clinical chemical measurements in collaboration with the NIST and the metrology institutes in Australia and Japan.

In addition to the national metrology institutes there exist a number of highly specialised reference laboratories most of which are situated in university hospitals and at manufacturer's sites. Usually these laboratories have developed their own reference procedures. Some of them have long-standing experience and perform measurements at a high metrological level.

According to the ISO standard on reference laboratories in laboratory medicine their competence may be approved by the national metrology institutes, e.g. by accreditation according to ISO 17025. The German metrology institute (PTB) has so far accredited two reference laboratories; the first one is the 
reference laboratories; the first one is the author's laboratory which mainly serves the proficiency testing organisation 'Reference Institute of Bioanalysis' of the German Society of Clinical Chemistry (DGKC) and also establishes target values for calibrators and control materials of commercial diagnostic kits; the second laboratory is that of an industrial diagnostic kit manufacturer; further accreditations will follow in due course.

The competence of reference laboratories concerning the environmental, personnel and management performance may be approved by accreditation; this still leaves a question that sometimes appears in letters to the editors of scientific journals, e.g. Clinical Chemistry. The question is: "When is a 'proposed' reference method a reference method?". The answer here is that the competence of a reference laboratory should not only be evaluated according to its management quality laid down in a quality manual and monitored by regular inspections of the laboratory, but also on the basis of the documented reference procedures and - most importantly - on the results of parallel comparative measurements. In this way the accrediting body also approves the measurement procedures and their performance. Consequently, accreditation is not valid for all measurements of the laboratory but only for particular measurands for which agreement of results with those of the laboratory of the accrediting body and thereby traceability to the SI has been demonstrated in comparative measurements.

Therefore, accreditation of a reference laboratory for a particular measurable quantity also contains an approval of the measurement procedure that includes the measurement principle, e.g. IDMS as a 'primary method' for the measurement of cholesterol, the complete standard operating procedure and the uncertainty of results. This may serve as an answer to the question "When is a 'proposed' reference method a reference method?"

In Europe the concept of traceability was promoted during the last 15 years by the organisers of external quality assessment schemes (EQAS). In the German proficiency testing system in particular the use of reference measurement procedures for several measurands is prescribed by legislation since 1988. As a result the "Reference Institute for Bioanalysis" has established reference measurement procedures for electrolytes, metabolites and substrates, hormones and drugs (Fig. 3). Reference methods for 13 of these 20 analytes have been developed using the analytical principle of the so-called 'primary

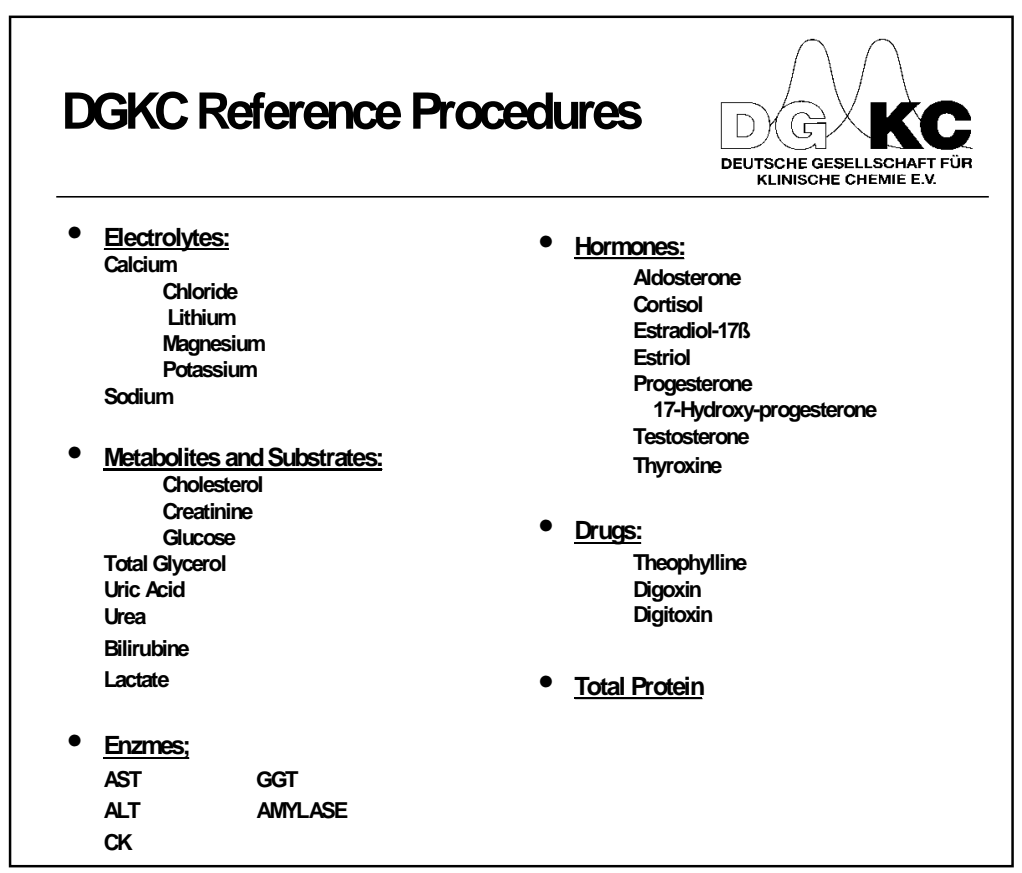

Figure 3. Reference Procedures Established by the Reference Institute of Bioanalysis (DGKC) 
method' isotope dilution mass spectrometry in the reference laboratories of the DGKC. These include creatinine, urea, cholesterol, total glycerol, uric acid and glucose, as well as the steroid hormones and thyroxine. The reference methods are now applied regularly for the setting up of target values in the control samples of internal and external quality assessment as well as for certifying matrix reference materials of the IRMM.

We currently share the system with partners in Portugal, the Czech Republic and occasionally in Denmark by exchanging samples for external quality assessment with reference procedure target values.

How the introduction of the concept of traceability improved the performance of diagnostic tests since 1988 may be of some interest. A look at the list of routine method target values for creatinine, uric acid, total cholesterol and total glycerol in the control material of one manufacturer issued before 1988, clearly shows that a large scatter of up to $30 \%$ existed, depending on which method or test kit was used (Fig 4). This situation was particularly untenable considering the fact that only one value for creatinine concentration in serum can be the "true" one. Obviously any progress towards improving the comparability of analytical results from different laboratories is hindered as long as methods with a known or even unknown bias are accepted.

\begin{tabular}{lr} 
CREATININE & $\mu \mathrm{mol} / \mathrm{I}$ \\
\hline$-\mathrm{-anymatic/}$ PAP & 151 \\
Enzymatic UV system & 161 \\
Jaffe without deproteinisation (Merck) & 168 \\
Jaffe after deproteinisation (Boehr.) & 177 \\
Jaffe without deproteinisation (Boehr.) & 189
\end{tabular}

\begin{tabular}{lr} 
URIC ACID & $\mu \mathrm{mol} / \mathrm{I}$ \\
\hline Fully enzymatic (Boehr./Merck) & 457 \\
UV - system (Boehr.) & 476 \\
UV - system (Merck) & 539 \\
Phosphotungstic acid (Goed.) & 583
\end{tabular}

\begin{tabular}{lr} 
CHOLESTEROL & $\mathrm{mmol} / \mathrm{I}$ \\
\hline CHOD - lodide & 4.02 \\
CHOD - PAP & 4.30 \\
CHOD - Katalase & 4.61 \\
Peridochrom & 4.69 \\
Liebermann-Burchard & 5.49
\end{tabular}

\begin{tabular}{lc} 
TRIGLYCERIDES & $\mathrm{mmol} / \mathrm{I}$ \\
\hline$-\mathbf{y u}^{-}$ & 1.15 \\
\hline Fully enzymatic (Behr.) & 1.34 \\
Fully enzymatic (Merck) & 1.30 \\
Eully enzymatic (Roche) & 1.36
\end{tabular}

Figure 4. Procedure Dependent Target Values in a Commercial Control Serum

This unsatisfactory situation also became visible in external quality assessment. For example, two different samples were distributed in a routine ring trial of the DGKC for cholesterol to about 1300 laboratories and the results were then displayed in a YOUDEN diagram (Fig. 5). Each dot in this diagram represents the two results from one laboratory, whereby the result for sample A can be read from the abscissa and that for the sample B from the ordinate. A laboratory with its dot just in the middle of the screen is in full agreement with the target value, which here is the reference method value certified by isotope dilution mass spectrometry. 


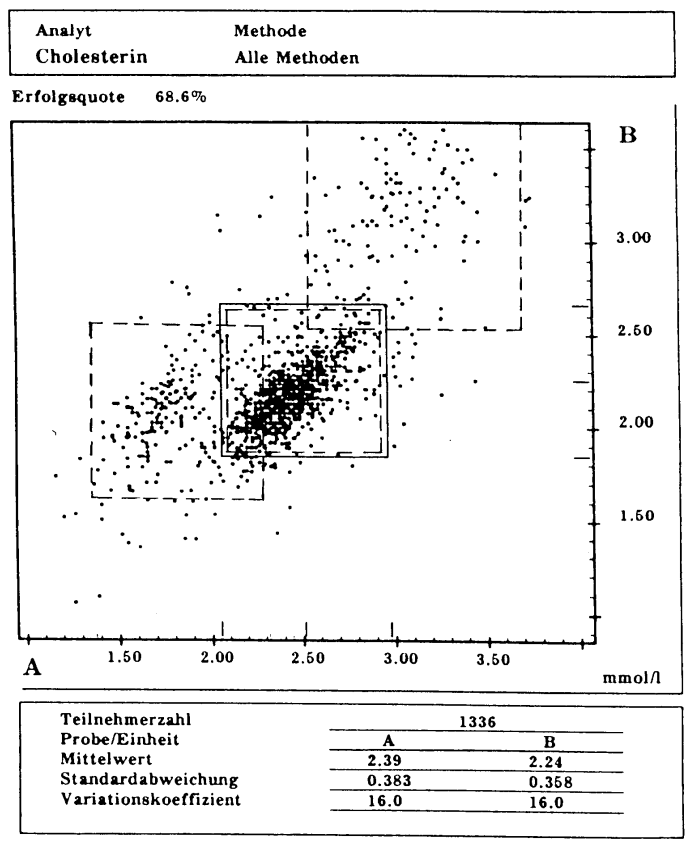

FIGURE 5. YOUDEN Diagram of a Collaborative Survey for Cholesterol (1987)

The three broken-line squares show the methoddependent evaluation limits for the Liebermann-Burchard method (upper-right), for the CHOD-PAP methods (middle) and the CHOD-Iodide method (lower-left). The solidline square in the middle shows the acceptance limits on the basis of the IDMS reference method value as target.
Participants' results from this survey for cholesterol in 1987 clearly show that three different groups of data have been reported according to three different methods of cholesterol determination. The participants with relatively high cholesterol results had used the Liebermann-Burchard procedure, which was still in use in 1987. The group with low cholesterol values had applied the cholesterol oxidase iodide method, and the data of laboratories using the CHOD/PAP method are situated in the middle of the screen. Until 1988 participants results were evaluated by comparison with the means of their peer group according to the different methodological principles used. Differences of up to $50 \%$ between the peer group target values could be observed for cholesterol measurements. In view of the fact

that there can be only one true cholesterol concentration value in a serum, this situation was clearly untenable.

After introducing the reference procedure values for cholesterol, based on IDMS measurements, the different peer group target values have now been replaced by reference method values, which in our case are represented as the exact middle of the screen. The corresponding limits of acceptance are shown as the solid square. As a consequence, methods with inherent systematic errors like the Liebermann-Burchard method and the CHOD-iodide method, disappeared from the market. Today only methods which are within the limits of acceptance with the reference method values established by isotope dilution mass spectrometry exist.

In fact, 12 years ago there was an unacceptably wide scatter of method-dependent target values for many clinical chemical parameters. In order to improve accuracy in clinical chemistry it was absolutely essential to replace these method-dependent target values with reference method values. 
The measurement of hormone concentrations in human body fluids has proved to be a valuable diagnostic tool in the field of clinical endocrinology. Thyroxine and the various steroids are the most commonly determined hormones and are usually measured by radio immunoassay (RIA) or by enzyme immuno assay (EIA) with a fairly high degree of sensitivity. However, a manufacturer's list of aldosterone-, cortisol-, progesterone- and oestradiol-17ß target concentrations in a commercial serum pool indicates that given the same sample and using immunoassay, assigned values varied considerably from one test kit to another (Fig 6). For cortisol and aldosterone the range of results was between $100 \%$ and $200 \%$; for progesterone and oestradiol-17ß determinations the results differed by a factor of 7 . This was probably due to the different qualities of the antibodies and reagents used in the various commercial test kits. What could a consensus value mean in such a context? A target value based on a consensus mean or median was of little use in judging test kits that gave such variable results.

\begin{tabular}{|c|c|c|c|c|}
\hline & $\begin{array}{l}\text { Aldosterone } \\
\text { pmol/1 }\end{array}$ & $\begin{array}{r}\text { Cortisol } \\
\mathrm{nmo} / 1\end{array}$ & $\begin{array}{c}\text { Progesterone } \\
\mathrm{nmo} / 1\end{array}$ & $\begin{array}{c}\text { Estradiol } \\
\mathrm{pmol} / 1\end{array}$ \\
\hline ABBOTT & 121.9 & & & \\
\hline AMERSHAM & 113.1 & & & \\
\hline BAXTER DADE DIR & & 104.8 & 2.16 & 396.4 \\
\hline BAXTER DADE AG ER & & & & 244.1 \\
\hline BAXTER DADE AD EXT & & & 196.0 & \\
\hline BECTON DICINSON & & 88.0 & & \\
\hline BIOCLONE & & & 1.91 & \\
\hline BIOMERIEUX & & & 2.54 & 539.6 \\
\hline BIOTEX PREMIX & 99.4 & 70.6 & 3.72 & 759.9 \\
\hline CAMBRID 'GE MEDICAL & 120.8 & & 0.86 & \\
\hline CIBA CORNING & & 110.3 & & \\
\hline CLINICAL ASSAYS & & 99.3 & & \\
\hline CYBERFLUOR FIAGEN & 88.2 & & & \\
\hline DIANOSTIC PRODUCTS $\quad 207.7$ & 113.1 & 3.12 & 119.3 & \\
\hline DUPONT RIANEN & & 135.1 & & \\
\hline EURODIAGNOSTICS & & 115.8 & & \\
\hline FARMOS DIAGN. & & 99.3 & 4.67 & 394.9 \\
\hline IMMUNCHEM COV. COAT & & 110.3 & 5.41 & 348.7 \\
\hline LEECO & & 113.1 & 2.99 & 144.2 \\
\hline MALLINCKRODT & & 88.3 & & \\
\hline NML RIA & & 96.6 & & \\
\hline NMS PHARMACEUTICALS & & & 3.18 & 205.5 \\
\hline PANTEX IMMUNO DIRECT & & & & 143.1 \\
\hline PANTEX IMMUNO & & 118.6 & 4.13 & 190.8 \\
\hline PANTEX IMMUNOCOAT & 132.4 & & 7.00 & 154.9 \\
\hline PHARMACIA DELFIA & & 99.9 & & 790.0 \\
\hline RSL & 169.2 & & 4.77 & 117.4 \\
\hline SCLAVO LISO PHASE & & 277.4 & 126.9 & 3.82 \\
\hline SERONO & & 112.0 & & \\
\hline SIBAR ELISA & & 121.3 & 1.27 & \\
\hline SORIN & 165.9 & 68.9 & 2.86 & 139.5 \\
\hline SYVA EMIT & 137.9 & & & \\
\hline TECHLAND RIA & & & 4.77 & \\
\hline VITEK SYSTEMS & & 110.0 & & \\
\hline
\end{tabular}

FIGURE. 6 Target Concentrations for Steroids Hormones in a Commercial Control Serum

Using method-dependent assigned values for external quality control means having many different target values for the same analyte in the same control serum is a very impractical and, from a theoretical point of view, very unsatisfactory procedure since 
they generate many different results for a substance of known molecular weight and with a defined number of molecules.

It, therefore, seemed imperative to establish a methodology that would provide the basis for the development of reference methods. As a result, the target values for the collaborative surveys of the DGKC for steroid hormones have been determined by reference methods since 1977 and more recently also for thyroxine.

Only recently we had to reply to a complaint of a manufacturer who suspected that the bad performance of his customers in our proficiency system surveys for progesterone was due to commutability problems of the quality control materials used in our ring trials. The unsatisfactory performance of the test became visible as an increasing bias of the test kit results at lower progesterone lower concentrations (Fig 7, sample B).

FIGURE 7. YOUDEN Diagram (left) and Test-Kit Specific Evaluation (right) of a Collaborative Survey for Progesterone

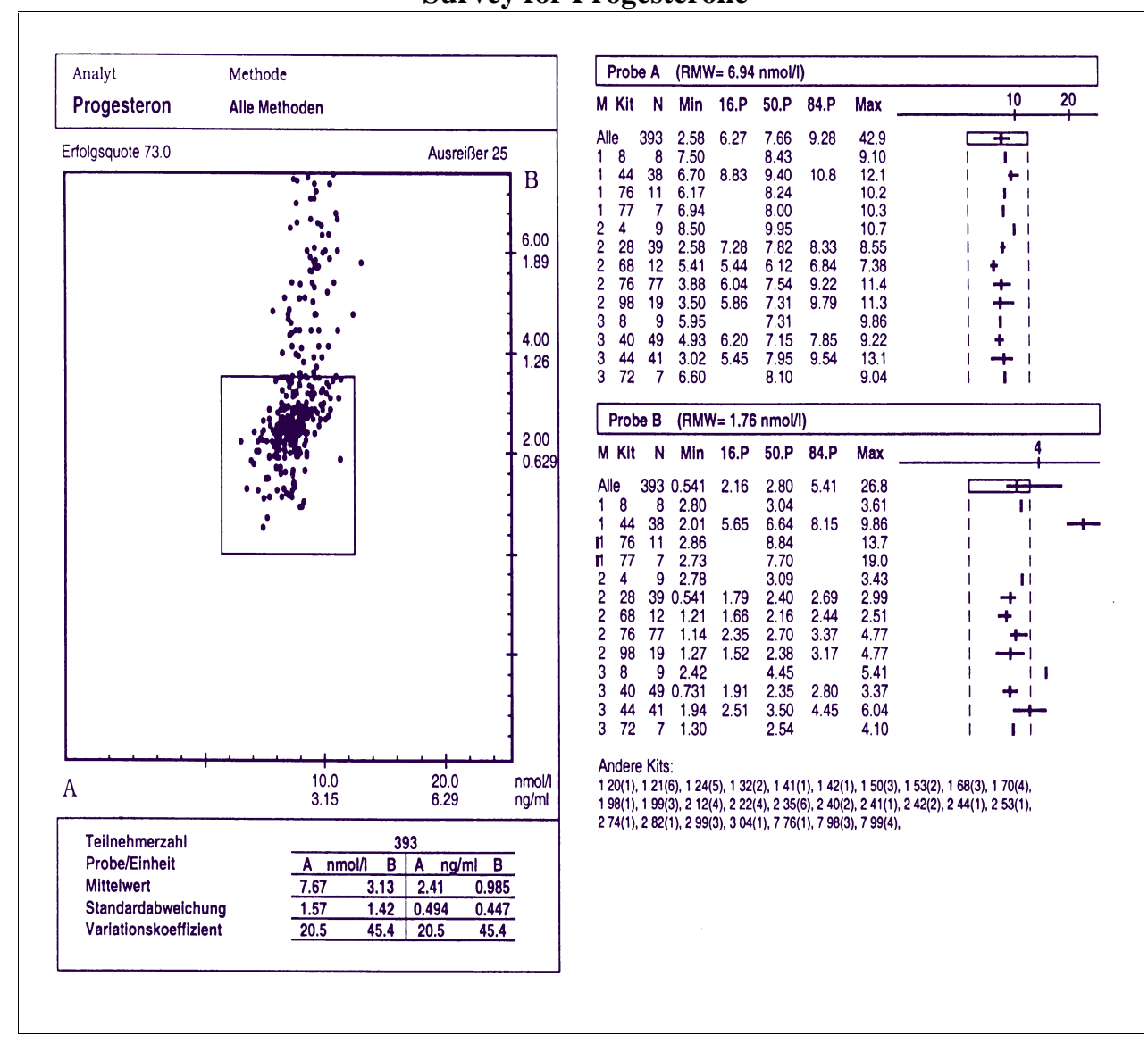

In order to validate the commutability of our control materials it was necessary to perform split sample measurements with patient samples using the test kit in parallel to the IDMS reference procedure for progesterone. At the first glance a good correlation between the test kit and the IDMS reference procedure could be observed (Fig. 8a).

However the difference plot of the same data revealed for both the patient sera and the ring trial results a considerable bias in relation to the reference procedure at low 
progesterone concentrations (Fig 8b). The reason for the bad performance of the test was obviously a lack of specificity rather than a lack of commutability of the control materials. At even lower progesterone concentrations the bias increased up to $1000 \%$. It should be noted that the kit manufacturer, unfortunately, did not issue any lower limit of determination for his measurement procedure.
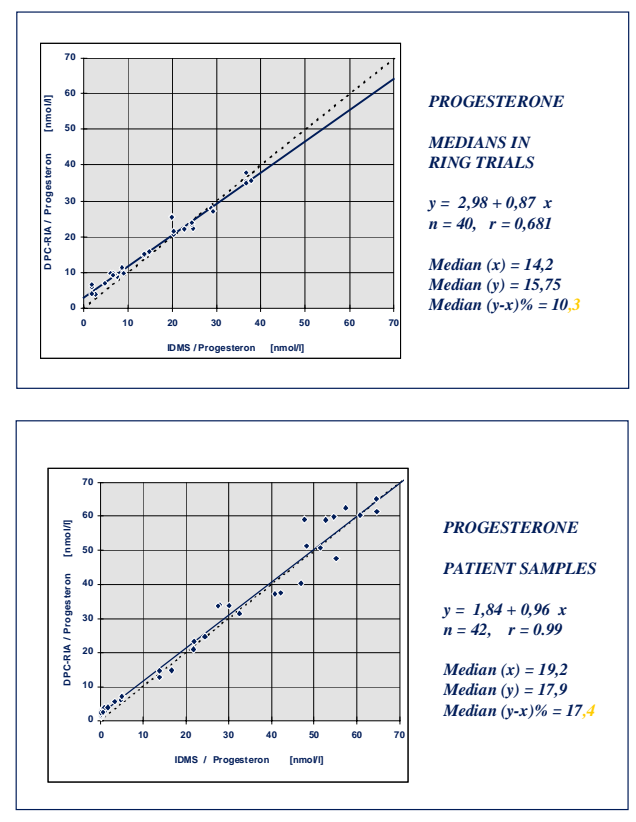

Figure 8a. Method Comparison for Progesterone

Test-Kit D - IDMS - Regression Analysis
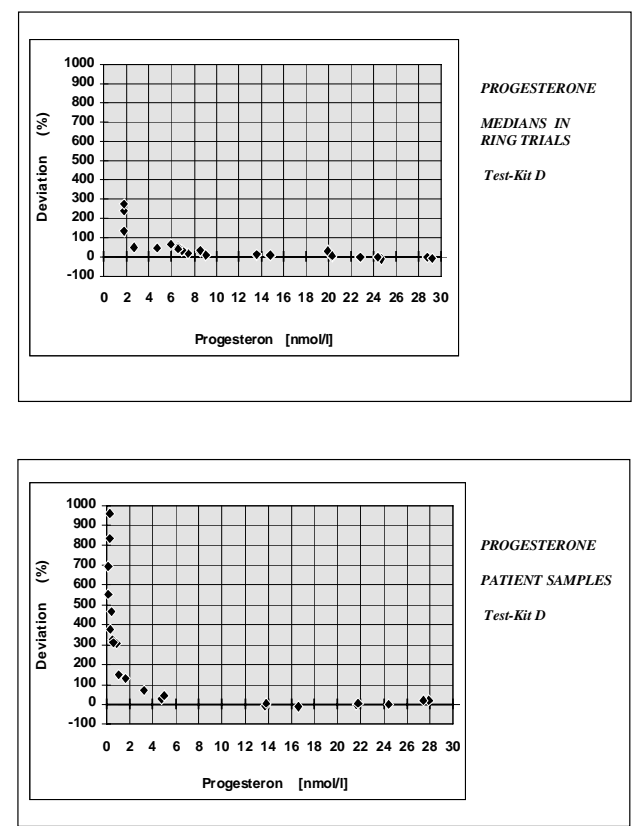

Figure 8b. Method Comparison for Progesterone

Test-Kit D - IDMS - Plot of Difference

During the early years of external quality control, the accuracy of unconjugated estriol in serum proved to be astonishingly high. This changed dramatically to-

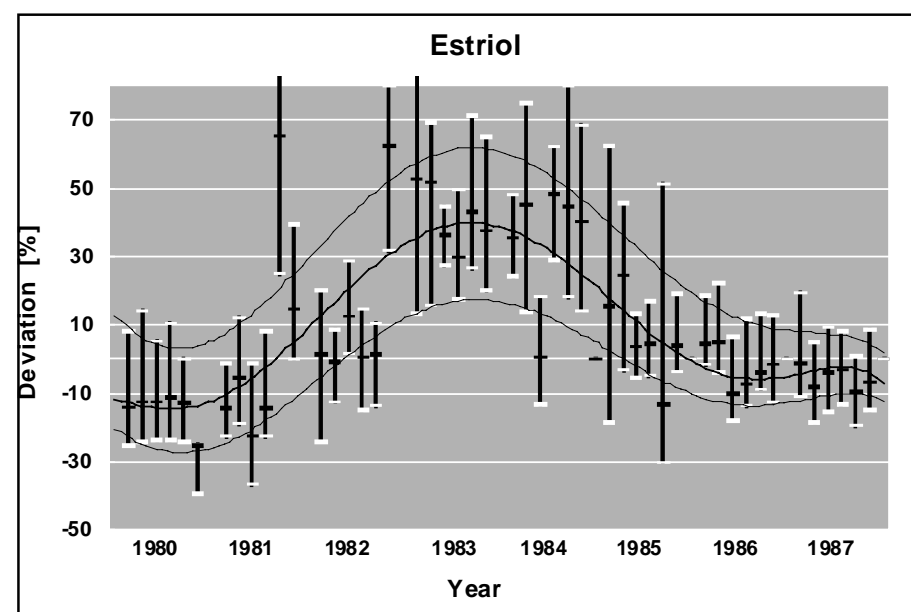
wards the end of 1981 (Fig. 9). Especially when the control samples contained conjugated estriol, the ranges of participants' results were significantly higher than the mass spectrometric target values.

Figure 9.

Results of Collaborative Surveys for Estriol Columns Indicate the Range Between the $16^{\text {th }}$ and $84^{\text {th }}$ Percentile of Distribution of Each Survey 
As it turned out, just at that time a kit manufacturer, who dominated the estriol-determination market in Germany, started using a new antibody. This obviously gave rise to cross-reactions with the conjugated steroid. Meanwhile a small group of participants who used their own laboratory methods to determine estriol continued to produce results that accorded with mass spectrometric values. It was possible to convince the manufacturer that this state of affairs required correction and, mainly due to this, results have improved greatly since 1985 . However, it must be assumed that in the time span from 1981 to 1984 test kits did not only fail to measure estriol in control samples but also in patient samples. Estriol determinations are mainly used to monitor fetal well-being in the last months of pregnancy. Since not only estriol but also estriol conjugates are elevated in this period, we suspect that nonconjugated estriol was probably overestimated due to the test kit's lack of specificity not only in control samples, but also in patient samples.

For non-SI traceable quantities the strategy for introducing traceability has to be different. This concerns a large number of analytes for which no defined molecular structure can be assigned, such as for many enzymes, proteo-hormones, tumour markers, cardiac markers. Before it is possible to establish reference systems (reference procedures, materials and laboratories) the measurand under consideration must first be defined. Whenever possible, a global consensus on the definition of the measurand should be achieved. Consequently, definition of the measurand and establishment of reference systems is the objective of several working groups and committees of the Scientific Division of IFCC.

In many instances a selected and agreed reference measurement procedure forms the basis of the definition of the measurand and thereby represents the top of the hierarchical traceability chain. This is particularly true for establishing reference systems for the catalytic concentrations of enzyme activities. In 1999 members of the IFCC working group and some enzyme reference laboratories decided to establish new $37^{\circ}$ measurement procedures as 'IFCC reference methods' on the basis of the existing $30^{\circ}$ IFCC methods and to certify enzyme reference materials for ALT, GGT, $\mathrm{CK}$ and LD in collaboration with the IRMM. The project was conducted in three steps:

1. Discussion and decision on primary procedures for the measurement of catalytic activities as 'IFCC reference methods'. Some experimental work was necessary for this in order to achieve optimised conditions for the measurement protocol. The members of the working group agreed upon standard operating procedures (SOP). The standard operation procedures include control and reporting of traceability of all individual steps of the procedures, e.g. for mass, volume, temperature, photometric wavelength and absorbance measurements.

2. The performance of laboratories applying the standard operating procedure was demonstrated in feasibility studies by analysing several commercial control materials. Depending on the enzyme, 10 - 12 laboratories from hospitals and diagnostic kit manufacturers were involved in the project. 
3. In the certification campaign the participating laboratories were asked to carry out the measurements on at least three different occasions. A material from the feasibility study was included in the analytical series for internal quality assessment.

As shown in Fig. 10a and 10b the certification campaign for four different BCR enzyme reference materials (ALAT, GGT, CK and LD) demonstrates that:

1. The $95 \%$ confidence interval of the laboratory results is less than $2.5 \%$ for all enzymes under investigation, which shows the excellent metrological performance of the participating laboratories from the Far East (Japan) to the Far West (California).

2. The standard operating procedures, which were developed in the course of the study, can be used as a reference points for the definition of the measurands as the top of the traceability chain. The procedures will now be published as IFCC reference methods.

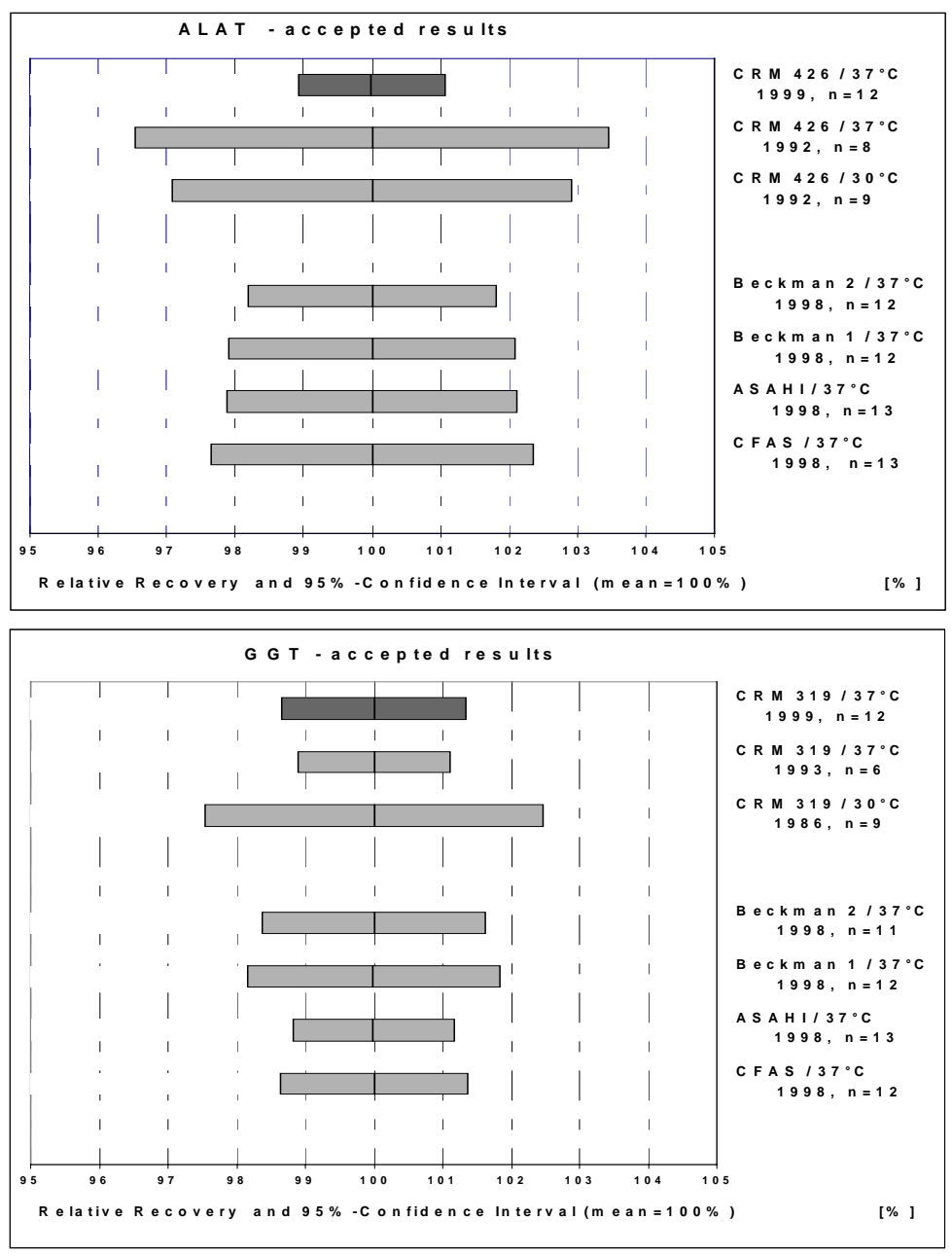

FigurE 10a. Results of the Certification of BCR Enzyme Reference Materials for ALAT and GGT. The bar graphs show the $95 \%$-confidence intervals obtained in the certification experiment in 1999 (top) in comparison to those of former certification campaigns using different methods (temperatures). The lower bar graphs show the $95 \%$-confidence intervals obtained from a feasibility study in 1998 before the certification campaign using different commercial calibrator preparations 

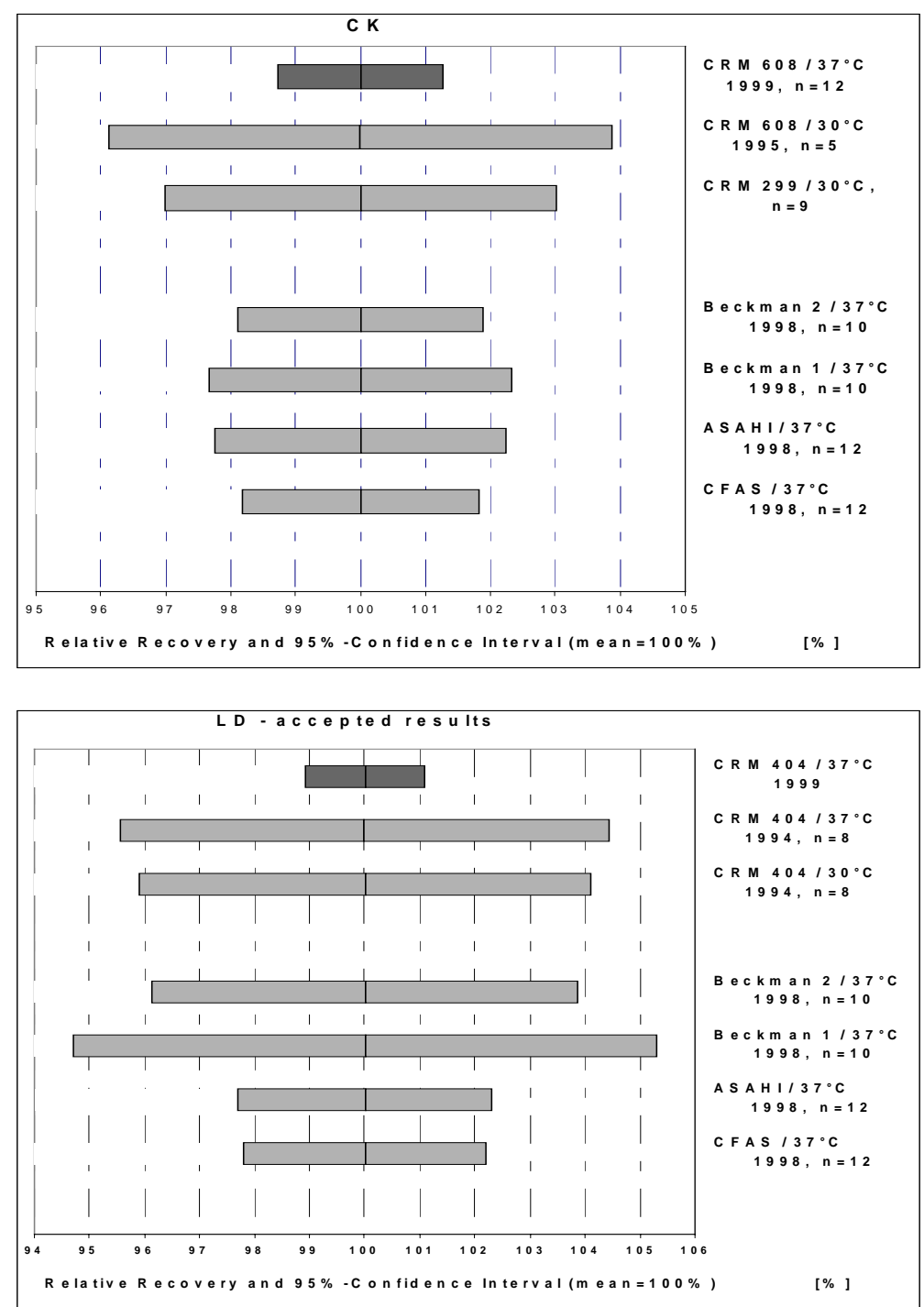

FIGURE 10b. Results of the Certification of BCR Enzyme Reference Materials for CK and LD. The bar graphs show the $95 \%$-confidence intervals obtained in the certification experiment in 1999 (top) in comparison to those of former certification campaigns using different methods (temperatures). The lower bar graphs show the $95 \%$-confidence intervals obtained from a feasibility study in 1998 before the certification campaign using different commercial calibrator preparations.

So far reference systems for the measurement of catalytic activity concentrations for four different enzymes have been successfully established and can now be used for assigning traceable values to calibrators and control materials.

The IFCC enzyme project, which has been conducted together with the IRMM, could be regarded as a model for the development of reference systems in other fields of interest. 
In summary it can be stated that for SI- traceable measurands the credentialing process has to some extent been successful, although the full implementation of the traceability concept on a global basis still requires considerable efforts.

For non-SI-traceable quantities the predominant objective must be an agreement on the definition of these quantities before reference systems comprising reference procedures, materials and laboratories can be established.

\section{References}

1. Cali JP, Mandel J, Moore L, Young DS. A referee method for the determination of calcium in serum. National Bureau of Standards Special Publication 260-36, 1972.

2. Velapoldi RA, Paule RC, Schaffer R, Mandel J, Murphy TJ, Gramlich JW. A reference method for the determination of chloride in serum. National Bureau of Standards Special Publication 260-67, 1979.

3. Velapoldi RA, Paule RC, Schaffer R, Mandel J, Machlan LA, Gramlich JW. A reference method for the determination of potassium in serum. National Bureau of Standards Special Publication 260-63, 1979.

4. Velapoldi RA, Paule RC, Schaffer R, Mandel J, Moody RJ. A reference method for the determination of sodium in serum. National Bureau of Standards Special Publication 260-60, 1978.

5. Velapoldi RA, Paule RC, Schaffer R, Mandel J, Machlan LA, Garner EL, et al.. A reference method for the determination of lithium in serum. National Bureau of Standards Special Publication 260-67, 1979

6. Cohen A., Hertz HS, Mandel J, Paule RC, Schaffer R, Sniegoski LT, et al. Total serum cholesterol by isotope dilution/mass spectrometry: a candidate definitive method. Clin. Chem. 1980; 26: 854-860.

7. Welch MJ, Cohen A, Hertz HS, Ng KJ, Schaffer R, Van der Lijn P, et al. Determination of serum creatinine by isotope dilution mass spectrometry as candidate definitive method. Anala. Chem. 1986; 58: 1681-1685.

8. Schaffer R, Mandel J, Sun J, Cohen A, Hertz HS, Neese JW. Standard reference materials: evaluation by an ID/MS method of the AACC reference method for serum glucose. National Bureau of Standards Special Publication 260-80, 1982.

9. White V E, Welch J, Sun T, Sniegosky LT, Schaffer R, Hertz HS, et al. The accurate determination of serum glucose by isotope dilution mass spectrometry - two methods. Biomed. Mass Spectrom. 1982; 9:395-405.

10. Ellerbe P, Sniegosky LT, Welch MJ. Isotope dilution mass spectrometry as a candidate definitive method for determining total glycerides and triglycerides in serum. Clin. Chem. 1995; 41:397-404. 
11. Ellerbe P, Cohen A, Welch M, White V E. Determination of serum uric acid by isotope dilution mass spectrometry as a new candidate definitive method. Anal. Chem. 1990; 62:2173-2177.

12. Welch MJ, Cohen A, Hertz HS, Ruegg FC, Schaffer R, Sniegisky LT, et al. Determination of serum urea by isotope dilution mass spectrometry as a candidate definitive method. Anal. Chem. 1984; 56:713-719.

13. Cali JP, Rationale for reference methods in clinical chemistry. Pure Appl. Chem. 1976; 45:63-68.

14. Tietz NW. A model for a comprehensive measurement system in clinical chemistry. Clin. Chem. 1979; 25:833-839.

15. Tietz NW, Rodgerson DO, Laessig RH. Are clinical laboratory proficiency tests as good as they can be? Clin. Chem. 1992; 38: 473-475.

16. Tietz NW, Accuracy in clinical chemistry - does anybody care? Clin. Chem. 1994; 40: 859-861.

17. Eckfeldt JH, Lewis LA, Belcher JD, Singh J, Frantz Jr ID. Determination of serum cholesterol by isotope dilution mass spectrometry with a bench top capillary gas chromatograph/mass spectrometer: comparison with the national reference system's definitive and reference methods. Clin. Chem. 1991; 37: 1161-1165.

18. Doumas BT, Kwok-Cheung PP, Perry BW, Jendrzejczak B, McComb RB, Schaffer $\mathrm{R}$, et al. Candidate reference method for determination of total bilirubin in serum: development and validation. Clin. Chem. 1985; 31: 1779-1789.

19. Doumas BT, Bayse DD, Carter RJ, Peters jr. T, Schaffer R. A candidate reference method for the determination of total protein in serum. I Development and validation. Clin Chem. 1981; 27: 1651-1654.

20. National Committee for Clinical Laboratory Standards. Development of definitive methods for the national reference system for the clinical laboratory; approved guideline. Villanova (PA): NCCLS; 1991 NCCLS Publication NRSCL1-A.

21. National Committee for Clinical Laboratory Standards. Development of reference methods for the national reference system for the clinical laboratory; approved guideline. Villanova (PA): NCCLS; 1991 NCCLS Publication NRSCL2-A.

22. National Committee for Clinical Laboratory Standards. Development of certified reference materials for the national reference system for the clinical laboratory; approved guideline. Villanova (PA): NCCLS; 1991 NCCLS Publication NRSCL3-A.

23. National Committee for Clinical Laboratory Standards. Source book of reference methods, reference materials, and related information for the clinical laboratory; proposed guideline. Villanova (PA): NCCLS; 1994 NCCLS Publication NRSCL 12-P.

24. Björkhem I, Blomstrand R, Lantto O, Svensson L, Öhman G. Towards absolute methods in clinical chemistry: application of mass fragmentography to high-accuracy analyses. Clin. Chem. 1976; 22: 1789-1801.

25. Björkhem I, Blomstrand R, Eriksson S. Use of isotope dilution-mass spectrometry for accuracy control of different routine methods used in clinical chemistry. Scand. J. Clin. Lab. Invest. 1980; 40: 529-534. 
26. Björkhem I, Bergman A, Falk O, Kallner A, Lantto O, Svensson L, et al. Accuracy of some routine methods used in clinical chemistry as judged by isotope dilution-mass spectrometry. Clin. Chem. 1981; 27: 733-735.

27. Büttner J. Reference methods as a basis for accurate measuring systems. Eur. J. Clin. Chem. Clin. Biochem 1991; 29: 223-235.

28. Büttner J. Reference materials and reference methods in laboratory medicine: a challenge to international cooperation. Eur. J. Clin. Chem. Clin. Biochem. 1994 38: 1401.

29. Büttner J. The need for accuracy in laboratory medicine. Eur. J. Clin. Chem. Clin. Biochem 1995; 33: 981-988.

30. Külpmann WR. Grundprinzioien von Referenzmethoden am Beispiel der Elektrolytbestimmungen. Fresenius Z. Anal. Chem. 1986; 324: 205-207.

31. Külpmann WR, Lagemann J, Sander R, Maibaum P. A comparison of reference method values for sodium, potassium and chloride with method dependent assigned values. J. Clin. Chem. Clin. Biochem. 1985; 23: 865-874.

32. Schumann G, Büttner J. A candidate reference method for creatinine with HPLC. Fresenius Z. Anal. Chem. 1986; 321: 279-280.

33. Schumann G, Büttner J. Determination of glucose in reference materials by isotope dilution - mass spectrometry. Fresenius Z. Anal. Chem. 1988; 330: 424-425.

34. Siekmann L. Reference methods. IN: Lawson AM, editor. Mass Spectrometry. Berlin, New York: Walter de Gruyter, 1989: 647-681.

35. Siekmann L, Hoppen HO, Breuer H. Zur gas-chromatographischmassenspektrometrischen Bestimmung von Steroidhormonen in Körperflüssigkeiten unter Verwendung eines Multiple Ion Detectors (Fragmentographie). Fresenius Z. Anal. Chem. 1970; 252: 294-298.

36. Siekmann L, Spiegelhalder B, Breuer H. Bestimmung von Aldosteron im Plasma des Menschen unter Verwendung der Kombination Gaschromatographie Massenspektrometrie. Fresenius Z. Anal. Chem. 1972; 261: 377.

37. Siekmann L. Determination of steroid hormones by the use of isotope-dilution mass spectrometry: a definitive method in clinical chemistry. J. Steroid. Biochem. 1979; 11: 117-123.

38. Siekmann L, Breuer H. Determination of cortisol in human plasma by isotope dilution - mass spectrometry. J. Clin. Chem. Clin. Biochem. 1982; 35: 532-536.

39. Siekmann L. Determination of estradiol-17ß in human plasma by isotope dilutionmass spectrometry. J. Clin. Chem. Clin. Biochem. 1984; 22: 551-557.

40. Siekmann L. Determination of uric acid in serum by isotope dilution-mass spectrometry. J. Clin. Chem. Clin. Biochem. 1985; 23: 129-135.

41. Siekmann L. Determination of creatinine in human serum by isotope dilution-mass spectrometry. J. Clin. Chem. Clin. Biochem. 1985; 23: 137-144.

42. Siekmann L. Reference methods for total cholesterol and total glycerol. Eur. J. Clin. Chem. Clin. Biochem. 1991; 29: 277-279.

43. Siekmann L. Measurement of thyroxine in human serum by isotope dilution-mass spectrometry. Biomed. Mass Spectrom. 1987; 14: 683-688. 
44. Stöckl D, Reinauer H. Development of criteria for the evaluation of reference method values. Scand. J. Lab. Invest. 1993; 53 Suppl. 212: 16-18.

45. Stöckl D, Reinauer H. Candidate reference methods for the determination of target values for cholesterol, creatinine, uric aid, and glucose in external quality assessment and internal accuracy control. I. Method setup. Clin. Chem. 1993; 39: 993-1000.

46. Stöckl D, Franzini C, Kratochvila J, Middle J, Ricos C, Siekmann L, Thienpont LM. Analytical specifications of reference methods; compilation and critical discussion. Eur. J. Clin. Chem. Clin. Biochem. 1996; 34: 319-337.

47. Thienpont LM, Stöckl D. Reference methods and reference method values. Klin. Biochem. Metab. 1994; 2: 4-9.

48. Thienpont LM, Franzini C, Kratochvila J. Middle J, Ricos C, Siekmann L, Stöckl D. Analytical quality speciications for reference methods and operating specifications for networks of reference laboratories. Recommendations of the European EQAOrganizers Working Group B. Eur. J. Clin. Chem. Clin. Biochem. 1995; 33: 949-957.

49. Thienpont LM, Van Nuwenborg JE, Stöckl D. Ion chromatography as a potential reference methodology for the determination of total calcium and magnesium in human serum. Anal. Chem. 1994; 66: 2404-2408.

50. Thienpont LM, De Leenheer AP, Stöckl D. Reinauer H. Candidate reference methods for the determination of target values for cholesterol, creatinine, uric acid and glucose in external quality assessment and internal accuracy control. II Method transfer. Clin. Chem. 1993; 39: 1001-1006.

51. Thienpont LM, Van Landuyt K, Stöckl D, De Leenheer AP. Candidate reference method for determining serum creatinine based on isocratic high performance liquid chromatography: validation with isotope dilution - gas chromatography/mass spectrometry and application for accuracy assessment of routine test-kits. Clin. Chem. 1995; 41: 995-1003.

52. Thienpont LM, Verhaeghe PG, Van Brussel KA, De Leenheer AP. Estradiol-17ß quantified in serum by isotope dilution-gas chromatography-mass spectrometry: reversed phase C18 high performance liquid chromatography compared with immunoaffinity chromatography for sample pretreatment. Clin. Chem. 1988; 34: 2066-2069.

53. Thienpont LM, De Brabandere VI, Stöckl D, De Leenheer AP. Development of a new method for the determination of thyroxine in serum based on isotope dilution-gas chromatography mass spectrometry. Biol. Mass Spectrom. 1994; 23: 475-482 


\section{LOTHAR SIEKMANN}

\section{University of Bonn • Institut für Klinische Biochemie}

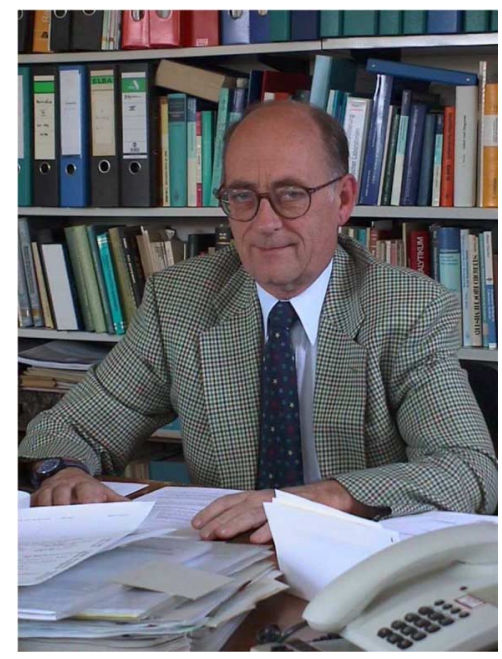

Lothar Siekmann, Ph.D., is a Professor of Clinical Chemistry at the University of Bonn, Germany. He is the Director of the Department for Clinical Chemical Analysis and Technology as well as the Director of the Reference Institute of Bioanalysis of the German Society of Clinical Chemistry. His current research includes development of isotope dilution mass spectrometry and the development of reference measurement systems in internal and external quality assessment. Dr. Siekmann has received the Gabor Szasz Prize of the German Society for Clinical Chemistry for "Development and Application of Reference Methods in Clinical Chemistry". He is a member numerous of standards organizations and committees, including being the Chairman of the Commission of Standardization of the German Society of Clinical Chemistry, Convener of an S, M\&T Project (BCR) of the European Union for the Certification of Reference Materials for Creatinine in Serum, Coordinator of an IFCC/IRMM Project for Establishing Reference Systems for Measurement of Catalytic Concentrations of Enzyme, an Expert in Working Groups on Steroid Hormones of the European Union, and a member of working groups of CEN TC 140 and ISO TC 212. 
Traceability of Calibration for IVD Industry: Process for Credentialing Reference Materials, Reference Methods and Reference Laboratories in Europe

Visuals Presented By

\section{Lothar Siekmann}

University of Bonn •Institut fur Klinische Biochemie 


\section{Traceability in Laboratory Medicine:}

Process for Credentialing Reference Materials,

Reference Methods and Reference Laboratories

\section{Lothar Siekmann}

Institut für Klinische Biochemie, Universität Bonn

Reference Institute of Bioanalysis, DGKC

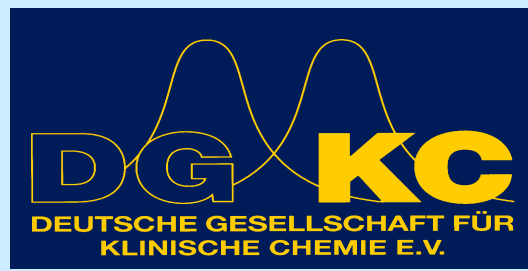

\section{Measurement Traceability}

property of the result of a measurement or the value of a standard whereby it can be related to stated references, usually national or international standards, through an unbroken chain of comparisons all having stated uncertainties 


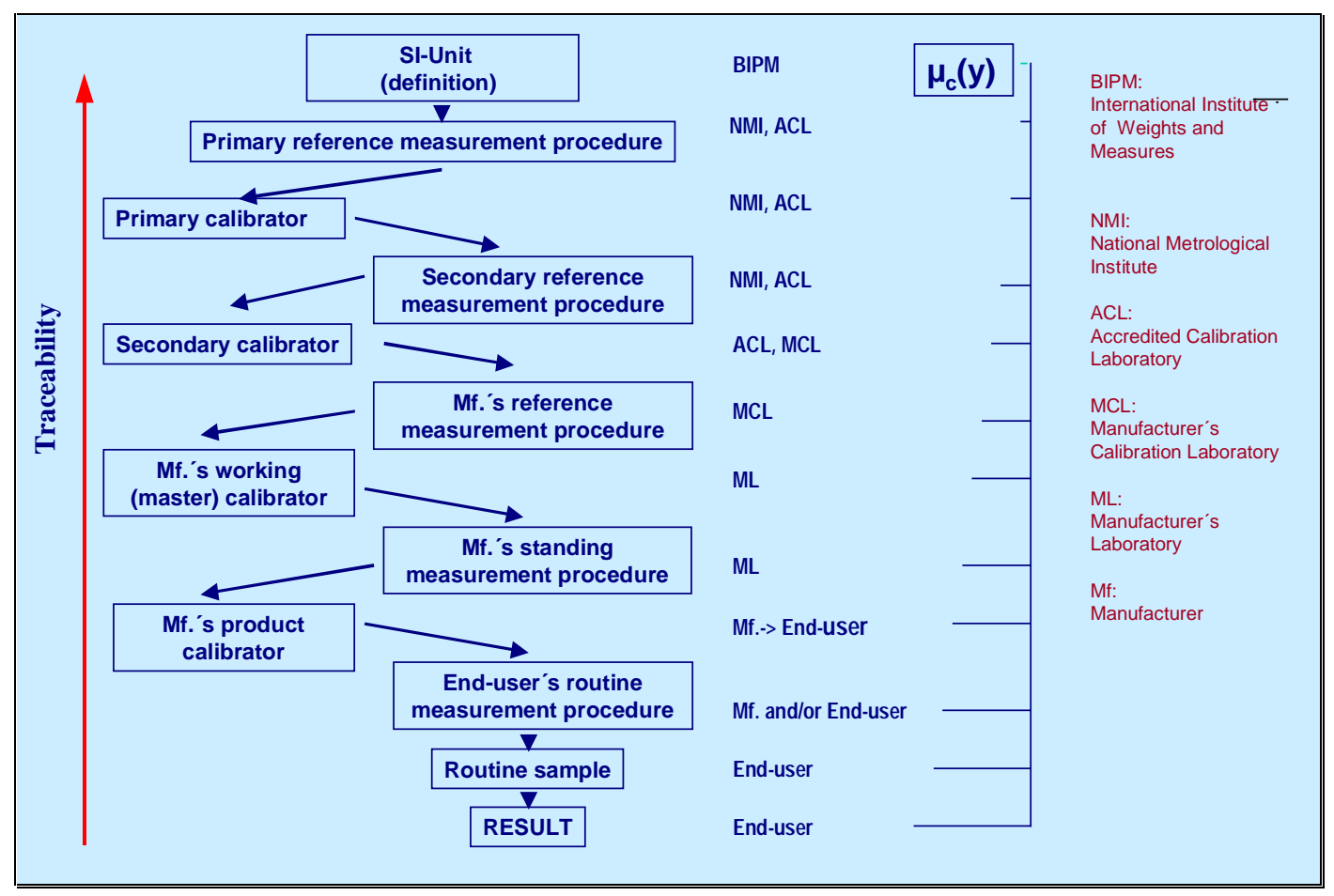

Zur gas-chromatographisch-massenspektrometrischen Bestimmung von Steroidhormonen in Körperflüssigkeiten unter Verwendung eines Multiple Ion Detectors (Fragmentographie)

L. SIERMANN*, H.-O. HOP'EN und H. BREUER

Lnatitut firr Klinische Bise:hemnie der Universititt Bonn

Fingegange: am 29. Mai 1971

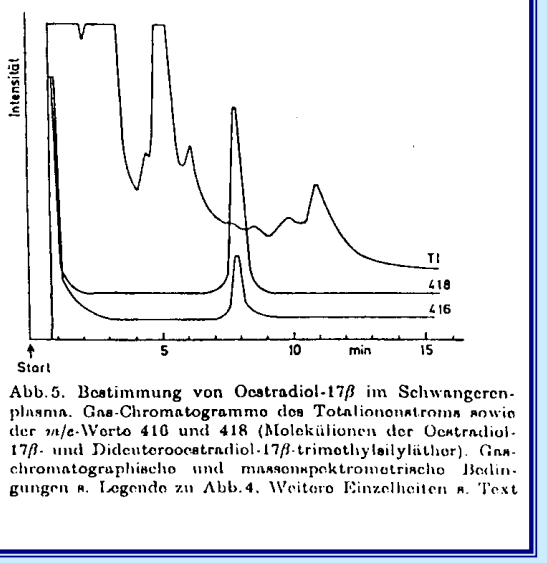


Organisations and Individuals involved in the Implementation of the Concept of Traceability

- Legislative Bodies

- National Metrology Institutes

- International Institutions issuing Reference Materials (WHO, IRMM, NIST)

- International and National Standardisation Organisations (ISO, CEN, NCCLS, DIN)

- International and National Scientific Societies (IFCC)

- External Quality Assessment Organisations

- Reference Laboratories

- Diagnostic Kit Manufacturers

- Clinical Chemical Laboratories

- Physicians

\section{Reference Systems in Clinical Chemistry}

- Reference Materials

- Reference Measurement Procedures

- Reference Laboratories 


\section{Suppliers of Reference Materials}

- National Institute of Standards and Technology (NIST), USA

- Institute for Reference Methods and Materials (IRMM), EU

- World Health Organisation (WHO), Geneva

- National Institute of Biological Standards (NIBSC), U.K.

- National Metrology Institutes

Hierarchy of Laboratories

\begin{tabular}{|c|}
\hline \hline National Metrology Institutes \\
\hline$\downarrow$ \\
\hline $\begin{array}{c}\text { Accredited Reference Laboratories } \\
\text { (universities, hospitals, manufacturers) }\end{array}$ \\
\hline$\downarrow$ \\
\hline $\begin{array}{c}\text { Manufacturer's Standardisation } \\
\text { Laboratories }\end{array}$ \\
$\downarrow$ \\
\hline Routine Laboratories \\
\hline
\end{tabular}




\section{Approval of \\ Reference Laboratories and Methods}

by:

- establishing and monitoring of a quality management system,

- regular inspections of the reference laboratory,

- regular comparisons using split sample measurements at the reference laboratory and the national metrology institute.

\section{DGKC Reference Procedures}

- Electrolytes:

Calcium

Chloride

Lithium

Magnesium

Potassium

Sodium

- Metabolites and Substrates:

Cholesterol

Creatinine

Glucose

Total Glycero

Uric Acid

Urea

Bilirubine

Lactate

- Enzmes;

AST

ALT

CK
- Hormones:

Aldosterone

Cortisol

Estradiol-17B

Estriol

Progesterone

17-Hydroxy-progesterone

Testosterone

Thyroxine

- Drugs:

Theophylline

Digoxin

Digitoxin

- Total Protein 


\section{TARGET CONCENTRATIONS IN A CONTROL SERUM}

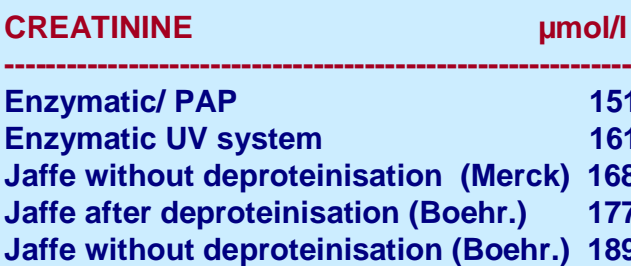

\begin{tabular}{lr} 
CHOLESTEROL & $\mathrm{mmol} / \mathrm{l}$ \\
\hline CHOD - lodide & 4.02 \\
CHOD - PAP & 4.30 \\
CHOD - Katalase & 4.61 \\
Peridochrom & 4.69 \\
Liebermann-Burchard & 5.49
\end{tabular}

URIC ACID $\quad \mu \mathrm{mol} /$

Fully enzymatic (Boehr./Merck) $\quad 457$

UV - system (Boehr.) 476

UV - system (Merck) 539

Phosphotungstic acid (Goed.) 583

TRIGLYCERIDES

$\mathrm{mmol} / \mathrm{l}$

Fully enzymatic (Behr.)

1.15

Fully enzymatic (Merck)

1.34

Fully enzymatic (Roche)

1.30

Enzymatic (Boehr.)

1.36

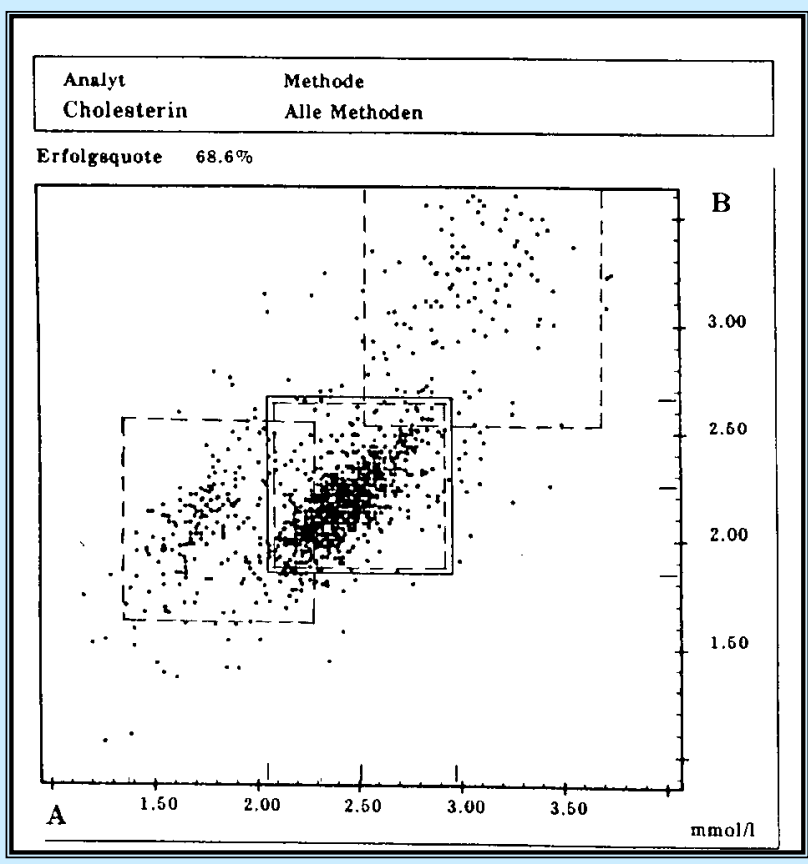




\begin{tabular}{|c|c|c|c|c|}
\hline \multicolumn{5}{|c|}{ Target Concentrations for Steroid Hormones in a Commercial Control Serum } \\
\hline & $\begin{array}{c}\text { Aldosterone } \\
\mathrm{pmol} / \mathrm{l}\end{array}$ & $\begin{array}{c}\text { Cortisol } \\
\text { nmo/l }\end{array}$ & $\begin{array}{l}\text { Progesterone } \\
\text { nmo/l }\end{array}$ & $\begin{array}{l}\text { Estradiol } \\
\mathrm{pmol} / 1\end{array}$ \\
\hline ABBOTT & 121.9 & & \multirow{4}{*}{2.16} & \\
\hline AMERSHAM & 113.1 & \multirow{4}{*}{104.8} & & \\
\hline BAXTER DADE DIR & & & & 396.4 \\
\hline $\begin{array}{l}\text { BAXTER DADE AG ER } \\
\text { BAXTER DADE AD EXT }\end{array}$ & & & & $\begin{array}{l}244.1 \\
196.0\end{array}$ \\
\hline $\begin{array}{l}\text { BIOMERIEUX } \\
\text { lil }\end{array}$ & & & 2.54 & 539.6 \\
\hline BIOTEX PREMIX & 99.4 & \multirow[t]{2}{*}{70.6} & \multirow{4}{*}{$\begin{array}{l}3.72 \\
0.86\end{array}$} & 759.9 \\
\hline CAMBRID'GE MEDICAL & 120.8 & & & \\
\hline CIBA CORNING & & \multirow{2}{*}{$\begin{array}{r}110.3 \\
99.3\end{array}$} & & \\
\hline CLINICAL ASSAYS & & & & \\
\hline CYBERFLUOR FIAGEN & \multirow[t]{2}{*}{88.2} & & \multirow[b]{2}{*}{3.12} & \multirow[b]{2}{*}{119.3} \\
\hline $\begin{array}{l}\text { DIAGNOSTIC PRODUCTS } \\
\text { EURODIAGNOSTICS }\end{array}$ & & $\begin{array}{l}113.1 \\
115.8\end{array}$ & & \\
\hline FARMOS DIAGN. & & 99.3 & 4.67 & 394.9 \\
\hline IMMUNCHEM COV. COAT & & 110.3 & 5.41 & 348.7 \\
\hline LEECO & & \multirow[t]{2}{*}{113.1} & \multirow[t]{2}{*}{2.99} & 144.2 \\
\hline MALLINCKRODT & & & & 96.6 \\
\hline NMS PHARMACEUTICALS & & \multirow[b]{2}{*}{118.6} & \multirow{2}{*}{$\begin{array}{l}3.18 \\
4.13\end{array}$} & 205.5 \\
\hline PANTEX IMMUNO & & & & 190.8 \\
\hline PANTEX IMMUNOCOAT & 132.4 & \multirow{3}{*}{99.9} & \multirow[t]{2}{*}{7.00} & 154.9 \\
\hline PHARMACIA DELFIA & & & & 790.0 \\
\hline RSL & \multirow[t]{3}{*}{169.2} & & \multirow{3}{*}{3.82} & 117.4 \\
\hline SCLAVO LISO PHASE & & \multirow{2}{*}{$\begin{array}{l}277.4 \\
112.0\end{array}$} & & \\
\hline SERONO & & & & \\
\hline SIBAR ELISA & & $\begin{array}{l}112.0 \\
121.3\end{array}$ & \multirow{2}{*}{$\begin{array}{l}1.27 \\
2.86\end{array}$} & \multirow{4}{*}{139.5} \\
\hline SORIN & 165.9 & \multirow[t]{2}{*}{68.9} & & \\
\hline SYVA EMIT & 137.9 & & \multirow{3}{*}{4.77} & \\
\hline TECHLAND RIA & & \multirow[b]{2}{*}{110.0} & & \\
\hline VITEK SYSTEMS & & & & \\
\hline
\end{tabular}

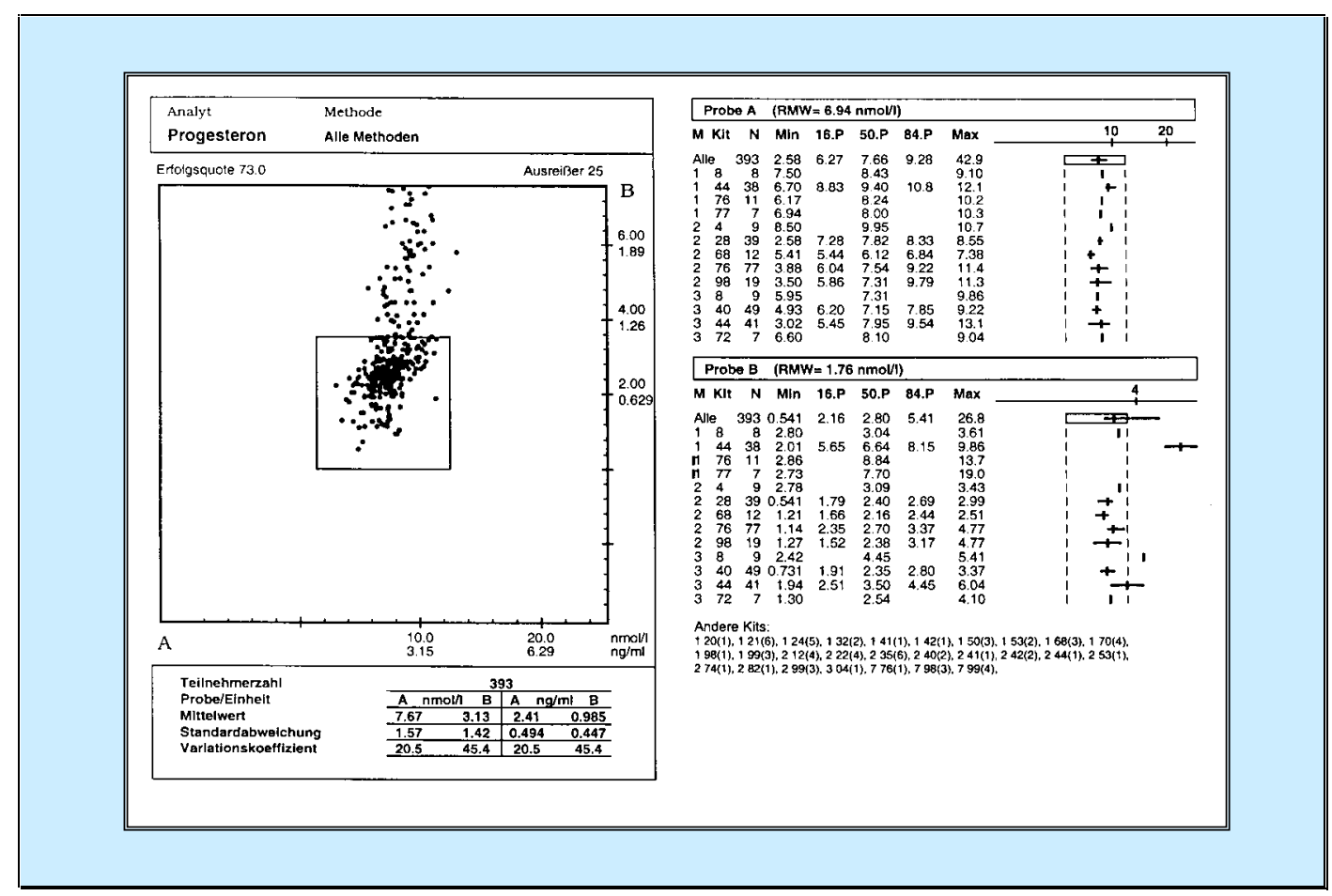



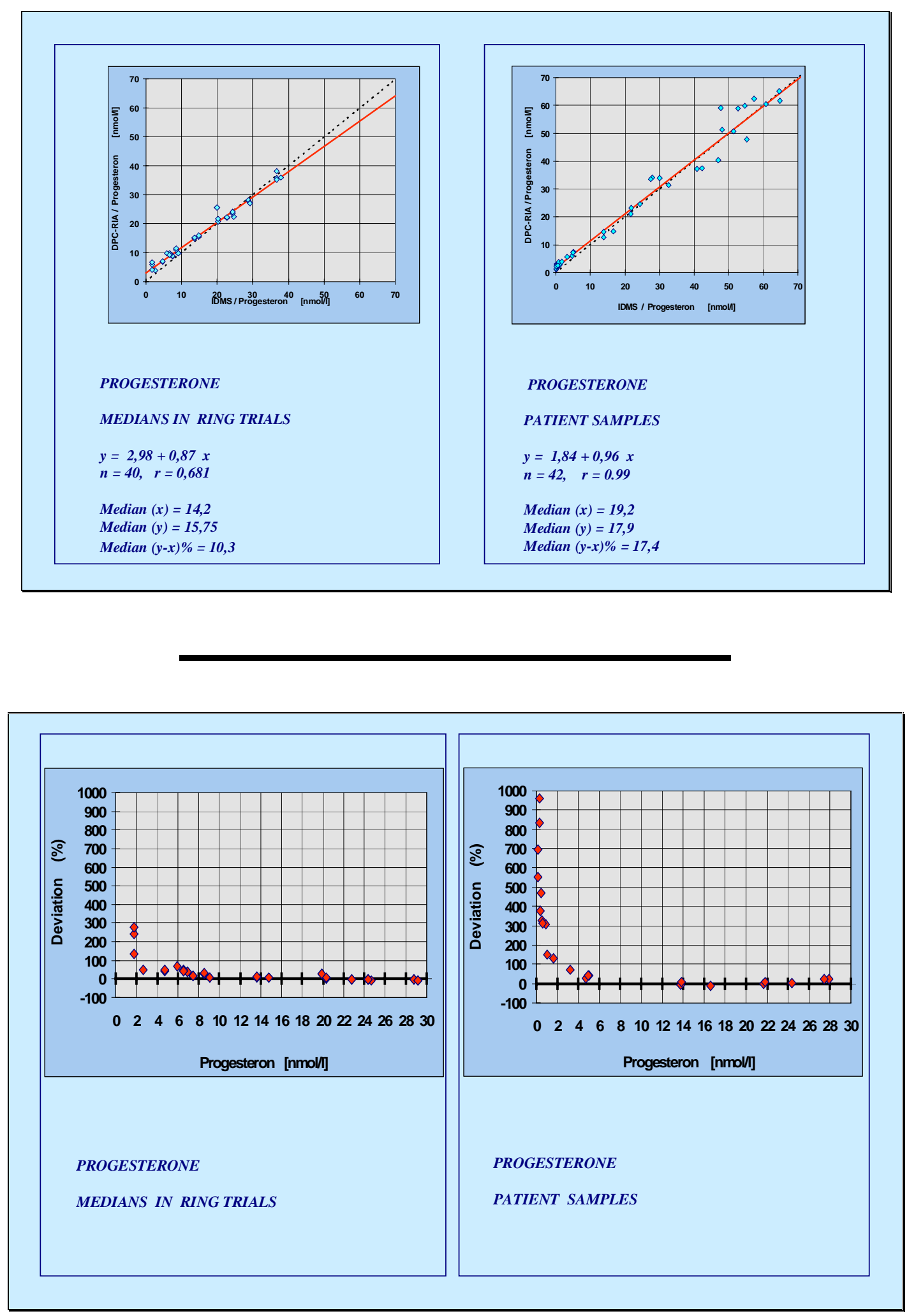


\section{Strategy to establish Reference Systems for non-SI Traceable Quantities}

- Definition of the analytes

- Development of a Reference Procedure

- Development of a Reference Material

\section{Establishing a Reference System for the Measurement of the}

\section{Catalytic Concentration of Enzymes}

- Decision on a primary reference measurement procedure (IFCC working group)

- Establishing of the reference procedure within a network of reference laboratories according to stringent metrological principles

- Selection of commutable reference material and certification by network laboratories 

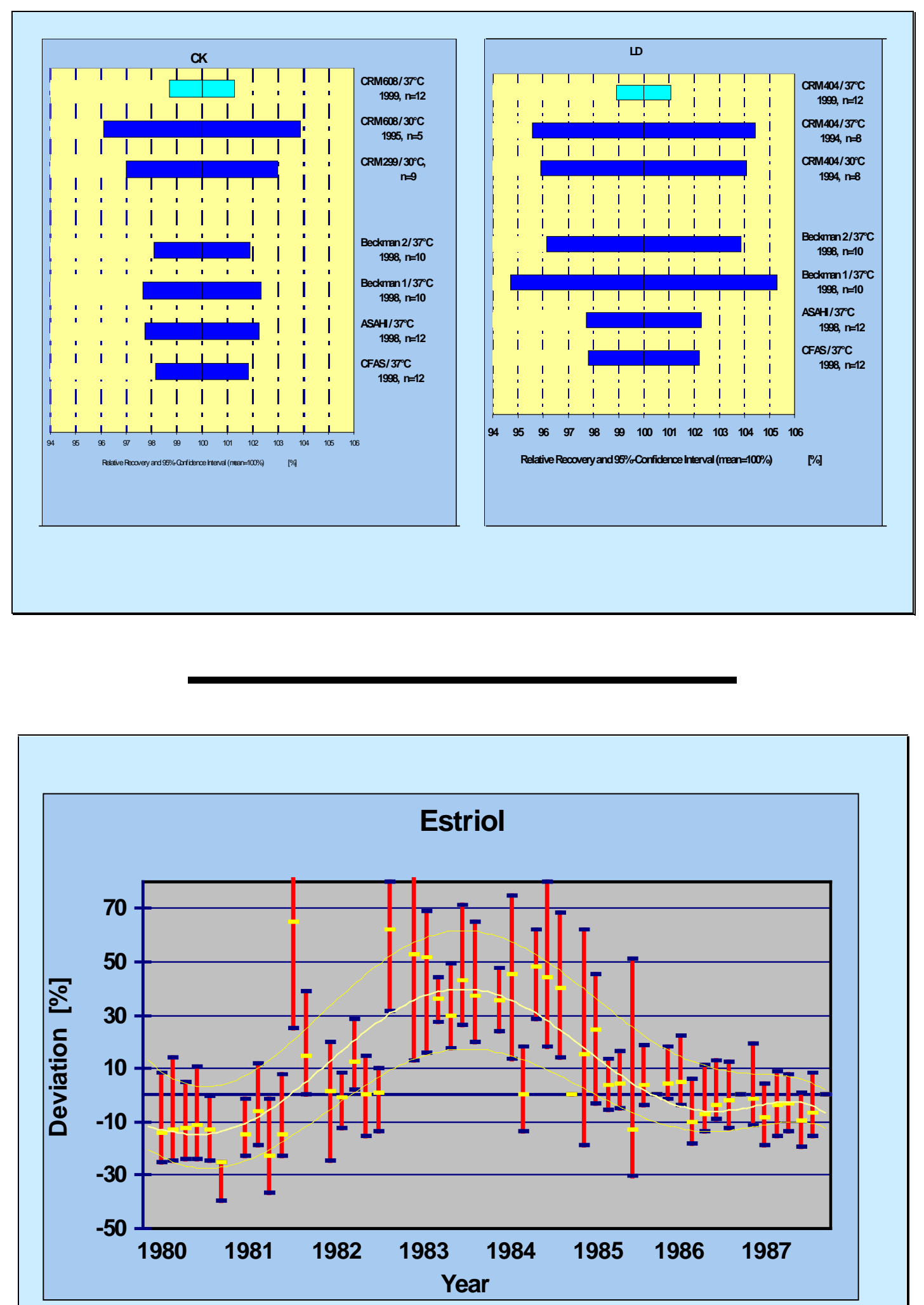


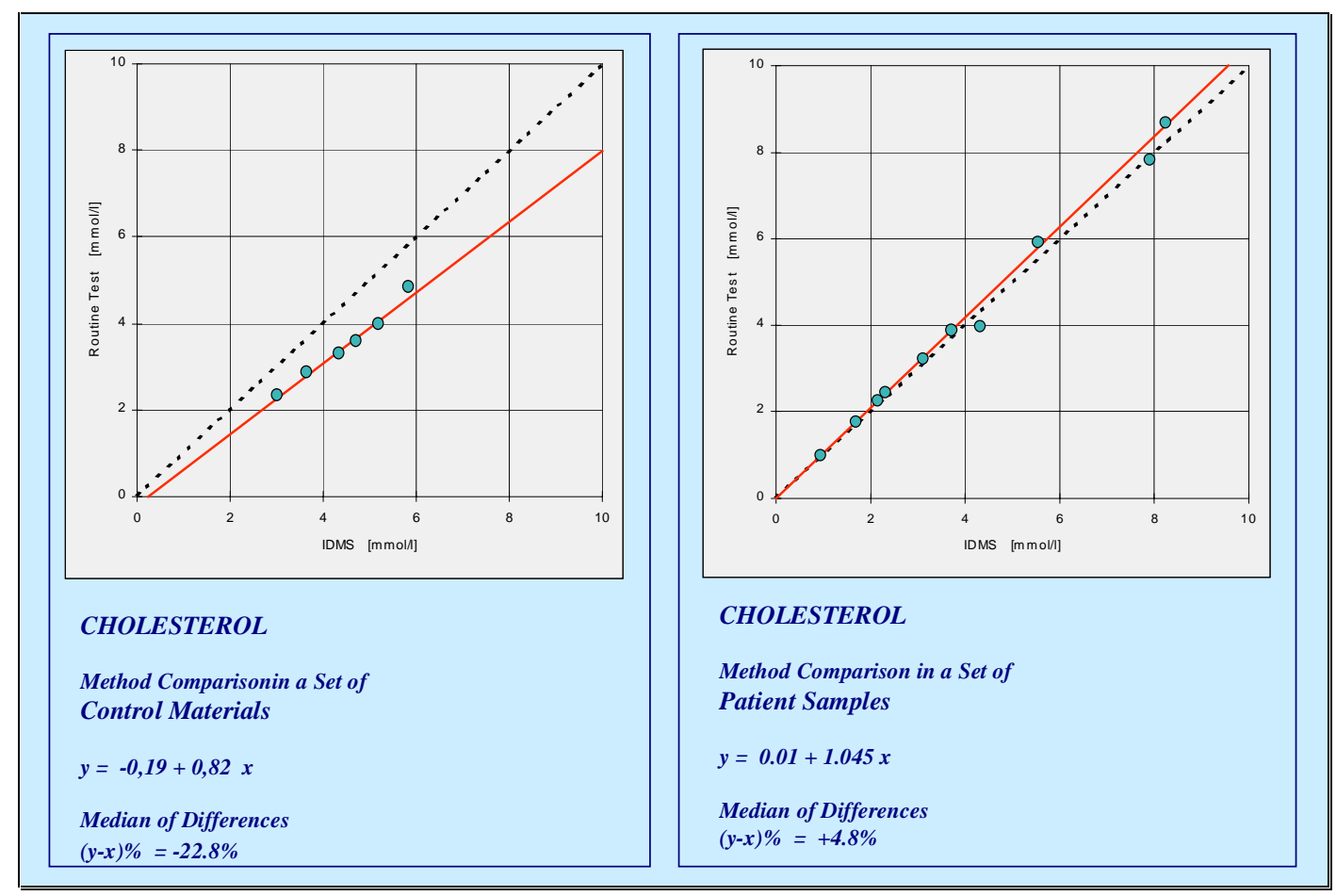




\section{European Reference Materials Activities in the Clinical Field H. Schimmel, M. Baptist, N. Kristiansen, T. Linsinger, P. Taylor, J. Pauwels \\ European Commission, Joint Research Centre Institute for Reference Materials and Measurements, Belgium}

Activities on the production of certified reference materials (CRMs) have been ongoing for more than 20 years, starting in the BCR programme, which evolved into the current M\&T programme, of DG Research. Several projects focusing on the production of clinical CRMs are continuing. Presently 34 BCR - CRMs related to clinical chemistry, or more generally to medical applications, are available (see Table 1). Since 1995, the Institute for Reference Materials and Measurements (IRMM) of the Joint Research Centre of the European Commission has taken over the responsibility for the distribution, monitoring and replacement of exhausted batches of all BCR CRMs.

\section{Table 1 : List of BCR Certified Clinical Reference Materials}

\section{PROTEINS}

BCR-148 : lyophilised thromboplastin Bovine

BCR-149S : lyophilised rabbit thromboplastin

BCR-393 : lyophilised Apo A I from human serum

BCR-394 : lyophilised Apo A II from human serum

BCR-486 : purified alphafoetoprotein

BCR-457 : human Thyroglobulin

BCR-470 : 15 serum proteins

BCR-405 (RM) : glycated haemoglobin in human haemolysate

BCR-522 : haemiglobincyanide in bovine blood lysate

BCR-613 : prostate specific antigen in reconstituted material

\section{ENZYMES}

BCR-299 : creatine kinase BB partially purified, from human placenta

BCR-319: $\gamma$-glutamyltransferase partially purified, from pig kidney

BCR-371 : alkaline phosphatase partially purified, from pig kidney

BCR-404 : human lactate dehydrogenase isoenzyme

BCR-410 : prostatic acid phosphatase highly purified, from human prostate

BCR-426 : alanine aminotransferase partially purified, from pig heart

BCR-476 : pancreatic $\alpha$-amylase, from human pancreas

BCR-608 : creatine kinase CK-MB from human heart 


\section{HORMONES}

BCR-192 and BCR-193 : cortisol in human serum (2 levels)

BCR-347 : progesterone in human serum

BCR-576, BCR-577, BCR-578 : 17 $\beta$-estradiol in human serum (3 levels)

\section{ELECTROLYTES and METABOLYTES}

BCR-194, BCR-195, BCR-196 : lead and cadmium in lyophilised bovine blood

BCR-304 : calcium, magnesium and lithium in lyophilised human serum

BCR-573, BCR-574, BCR-575 : creatinine in human serum

\section{BLOOD CELL SIZE REFERENCE MATERIAL}

BCR-165, BCR-166, BCR-167 : latex spheres $(2,4.8$ and 9.6 of certified size $\mu \mathrm{m})$

The European "in vitro diagnostic and medical device Directive" (98/79/EC), that requires manufacturers of in-vitro diagnostics (IVD) who wish to sell their products in the EU demonstrate that they are "traceable to reference systems (i.e. reference materials and/or reference methods) of a higher order", will generate an enormous need of internationally recognized certified reference materials (CRMs). On October 30 ${ }^{\text {th }}, 1996$, anticipating this need, the IRMM entered into a Co-operation Agreement with the International Federation of Clinical Chemistry (IFCC) with the aim of producing joint IRMM/IFCC certified reference materials. These reference materials are to be produced in close collaboration with IFCC working groups and committees involving experts in clinical chemistry, reference laboratories and IVD industry.

In the past, different approaches have been followed for the certification of reference materials. The two extreme approaches have been applied: one focused mainly on measurands where SI traceability could be achieved, and the other, based on consensus, mainly involved complex measurands where the biological activity was important and SI traceablity could not be achieved. Both approaches suffer from drawbacks, if all aspects relevant in clinical chemistry, especially for complex analytes, are not taken into consideration. By following purely the metrological approach for many clinical analytes, no CRMs were produced, because SI traceability could not be achieved or was too costly. Consequently, for most of the clinically relevant parameters there are no reference materials available. In addition, the certified quantity may not or not fully have represented the clinically relevant form, and the materials may not have been appropriate for direct use with IVDs because of the problems with commutability. Commutability is defined as the ability of a material to show interassay properties comparable to those of human sera; i.e., fresh patient samples and a commutable reference material show the same behaviour when submitted to an IVD routine test system. Commutability problems are very common for complex proteins where the matrix and the sample treatment influences the behaviour of the measurand and antibodies usually used in IVD tests. It is evident that commutability should be investigated and interpreted with care in order to ensure a maximum standardisation of these effects. The scheme for the assessment of commutability should allow a clear separation of true matrix and sample treatment effects due to lack of specificity. In case of lack of specificity, the potential standardization effect will be reduced due to the varying composition of patient samples. However, the acceptance and use of reference materials depends on applicability and commutability. Only com- 
mutable materials can be used to directly control the various steps in the production of IVDs, including the direct calibration of calibrators and performance checks of IVD's. Therefore, commutable CRMs are critical for assuring an unbroken traceability chain.

The second approach, based on consensus only, was predominantly aiming at normalization of various methods to a common reference material. The disadvantage of such systems is that they are arbitrary and difficult to reproduce. Often the characterization was done without having a clear concept, using poorly understood methods and without assessing the calibration of the methods used for the characterization. If several of such CRMs exist for the same analyte, discrepancies between methods can arise if normalized to different CRMs. Therefore, it is felt that a more structured and coordinated approach is required on an international level involving all relevant parties. This networking approach will also help to better identify the needs and specification requirements for new reference materials.

Currently, joint IRMM - IFCC certification projects attempt to implement a widely recognized reference system including methods and CRMs that are based on metrological principles. With reasonable efforts, and current state of the art technical possibilities, the clinically relevant form of an analyte should be identified as enabling traceability to the SI system. The properties, quality and foreseen use of CRMs are defined at an early stage, and the production of commutable reference materials is envisaged. International cooperation will help to evaluate all relevant aspects of CRM production and will certainly increase the acceptance of the CRMs. IRMM is also supporting international networks on traceability according to IVD directive involving all relevant parties such as expert or reference laboratories, IFCC, IVD and CRM producers, accreditation bodies and metrology institutes.

The definition of priorities for projects aiming at the production of reference materials is based on a several criteria. There should be a balance between production efforts, clinical relevance and level of uncertainty to be achieved. The clinical relevance and level of uncertainty to be achieved is determined by the significance of the parameter in the diagnosis of diseases. The reference ranges of healthy patients, the daily variation, and the expected improvement of measurement quality, need to be taken into consideration. The anticipated need for a reference material and the number of analyses needed, as well as the impact on individuals and public health care; (e.g. disease prevention) and the assessment of cost / benefit ratio, are also important criteria.

So far, 4 IRMM/IFCC CRMs for enzyme activity and an IRMM/IFCC reference panel for cortisol have been certified (see Table 2).

\section{Table 2 : List of IRMM/IFCC Certified Clinical Reference Materials}

IRMM/IFCC-451 : cortisol reference panel of fresh frozen human sera

IRMM/IFCC-452 : gamma-glutamyltransferase partially purified, from pig kidney

IRMM/IFCC-453 : human lactate dehydrogenase isoenzyme 1

IRMM/IFCC-454 : alanine aminotransferase partially purified, from pig heart

IRMM/IFCC-455 : creatine kinase CK-MB from human heart 
The enzyme CRMs IRMM/IFCC-452, 453, 454 and 455 have been certified for catalytic activity in cooperation with the IFCC committee on enzyme activity according to newly developed SOPs at $37^{\circ} \mathrm{C}$. These SOPs have been proposed to become IFCC reference methods. Compared to the former IFCC reference methods, crucial parameters in the procedure have been optimized for the new temperature, or have been clearly defined (examples see Table 3). The reproducibility of results has been improved using the new SOPs that could be demonstrated by an approximately $50 \%$ reduction of the $95 \%$ confidence interval calculated from the means of means of the data sets from the laboratories having carried out the characterization measurements. There was also a move to get conditions that are closer to the routine procedures as applied in clinical laboratories. The uncertainty of the certified value was calculated according to the GUM and includes possible heterogeneity and instability contributions. The collection of data by the IFCC committee for definition of preliminary reference ranges is ongoing and publication of the new IFCC reference method is envisaged. Certification has been carried out in collaboration with various Universities, hospitals and IVD producers, including partners from the USA and Japan.

Table 3: Examples for Changes in SOPs for Measurement of Catalytic Activity:

$\begin{array}{lll}\text { GGT } & 37^{\circ} \mathrm{C} & 37^{\circ} \mathrm{C}(\text { old }) \\ \text { pH } & 7.7 & 7.9 \\ \text { Incubation time } & 180 \mathrm{~s} & \text { not specified } \\ \begin{array}{l}\text { Delay time } \\ \text { Measurement interval : }\end{array} & 60 \mathrm{~s} & \text { not specified } \\ & 180 \mathrm{~s} & 300 \mathrm{~s} \\ & & \\ \mathrm{LD} & 37^{\circ} \mathrm{C} & 30{ }^{\circ} \mathrm{C}(\mathrm{old}) \\ \mathrm{pH} & & \\ \text { Incubation time } & 9.4 & 7.2 \\ \text { Delay time : } & 180 \mathrm{~s} & 60-300 \mathrm{~s} \\ \text { Measurement interval : } & 90 \mathrm{~s} & 90 \mathrm{~s} \\ & 180 \mathrm{~s} & 60 \mathrm{~s}\end{array}$

The serum panel for cortisol has been certified in cooperation with the IFCC working group on steroid hormones. A panel consisting 34 individual sera (80 - 770 $\mathrm{nmol} / \mathrm{l})$ has been characterized using a primary method (GC-IDMS) in two independent reference laboratories. Uncertainties were calculated according to GUM. The panel is intended to be used by IVD producers directly to calibrate their test kits. The approach using a panel with samples having individual characteristics and concentrations will enable the IVD producers to properly assess a calibration curve and the specificity of the test system they are producing. The final goal is to establish more reliable reference interval ranges, and to improve the inter-assay comparability. Further certification projects are being conducted. 
Three other CRMs for enzyme catalytic activity will be certified. For two of them (aspartate aminotransferase (ASAT) and $\alpha$-Amylase) the SOPs have been developed for certification at $37^{\circ} \mathrm{C}$. The characterization measurements for $\alpha$-Amylase have been completed and a certification report and certificate are currently being drafted. For ASAT and alkaline phosphatase (ALP) new batches of materials need to be produced, and a commutability study for candidate ASAT reference materials is ongoing.

Work is also underway, in cooperation with the IFCC working group, on cardiac markers to produce a myoglobin CRM. Five candidate CRMs have been collected and the commutability is currently being assessed. A rigorous protocol has been applied to determine linearity and parallelism of various test systems when using the candidate CRMs. In parallel to the commutability study, we intend to develop a reference method for quantification. The project is being carried out in cooperation with IVD industry from US, France, Germany, UK and Finland.

Other ongoing CRM projects are for the replacement of a high level progestorone CRM in human serum (freeze dried), and the certification of total proteins in human serum. An important area that the IRMM has entered recently is the production of CRMs for genetic markers.

Further, a variety of projects are currently being discussed that include pure calibrants and a panel for glycated haemoglobin (Hb1Ac), purified human chorion gonadotropin (hCG) and its isoforms, osteocalcin (osteoporosis marker), steroid hormone (progestorone, testosterone, $17 \beta$-estradiol) serum panels similar to the cortisol serum panel mentioned above and pure progesterone, testosterone, $17 \beta$-estradiol and aldosterone as primary calibrants in cooperation with the German metrology institute PTB, Brauschweig.

In addition to providing CRMs, IRMM is also providing other means for improving the quality of measurements in the clinical field by organizing IMEP exercises (proficiency testing rounds that are based on traceable reference values). IMEP-17 focuses on trace and minor constituents in human serum. Candidate measurands are $\mathrm{Na}, \mathrm{Cl}, \mathrm{Ca}, \mathrm{Cu}$, $\mathrm{Mg}, \mathrm{Li}, \mathrm{K}, \mathrm{Se}, \mathrm{Zn}, \mathrm{Fe}$, glucose, cholesterol, urea, total thyroxine (T4), uric acid and creatinine. The test material will be unmodified/modified human serum. The traceable reference values will be obtained preferably by primary methods of measurements by experienced reference measurement institutes. The exercise will be executed in 2001-2002. 


\section{HEINZ SCHIMMEL}

\section{European Commission, Joint Research Centre Institute for Reference Materials and Measurements Belgium}

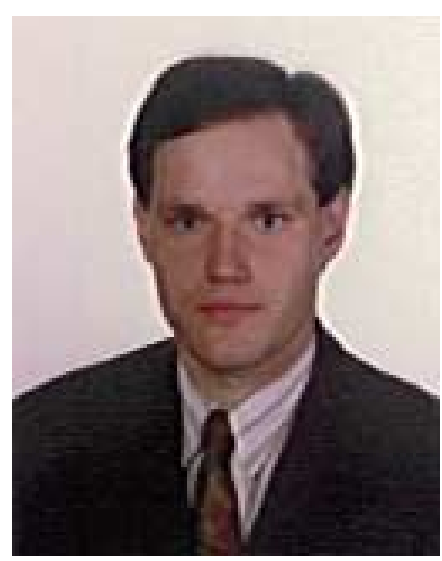

Heinz G. Schimmel, Ph.D., is a Scientific Officer at IRMM. He received his Ph.D. at the University of Ulm in 1992 for his work on the analysis and quantitation of polychlorinated dibenzodioxins and dibenzofurans. He then worked at the Community Bureau of Reference (BCR) of the Commission of the European Communities, providing technical assistance in certification projects on trace organics in environmental matrices. In 1993, Dr. Schimmel took over the management of organic trace analysis projects at the University of Ulm. He assumed his current position at IRMM in 1995 and is in charge of the management of recertification projects, certification projects, and validation studies in the field of environmental and food chemistry, clinical chemistry microbiology and biotechnology. He is head of production control for the analysis of organic and metalorganic compounds. He is also responsible for setting up a new sterile production facility, and a molecular biological and biochemical production control laboratory. Since 1996, he has been involved in the development of clinical reference materials in cooperation with IFCC. 
European Reference Materials Activities in the Clinical Field

Visuals Presented By

Heinz Schimmel

Institute for Reference Materials and Measurements

Belgium 

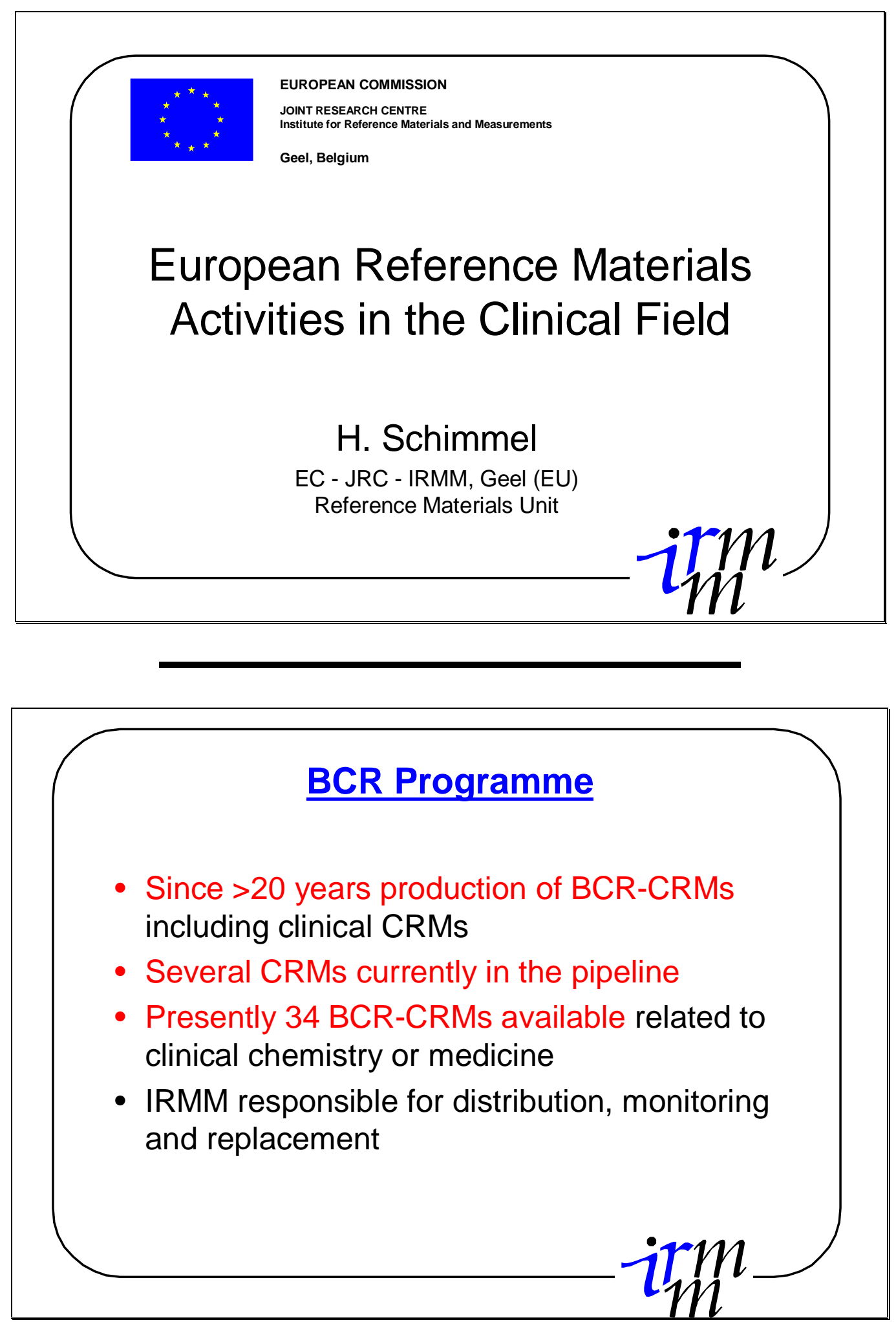


\section{Existing BCR CRMs}

- PROTEINS

(e.g. thromboplastin, Apo A I, Apo A II, serum proteins, PSA)

- ENZYME ACTIVITY

(e.g. CK-BB, CK-MB, ALP, LD, ALAT, $\alpha$-amylase)

- HORMONES (cortisol, progesterone, 17 $\beta$-estradiol)

- ELECTROLYTES and METABOLYTES (Pb, Cd, Ca, Mg, Li, creatinine)

- BLOOD CELL SIZE (latex spheres)

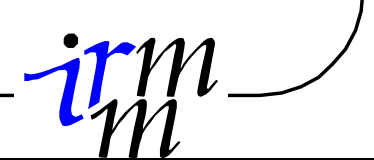

\section{Approaches for CRM production}

- Metrological approach (SI tracability)

- For many clinical analytes no CRMs were produced because SI traceability could not be achieved or was too costly

- The certified quantity may not or not fully represent the clinically relevant form

- The materials may not be appropriate for direct use with IVDs (problems with commutability)

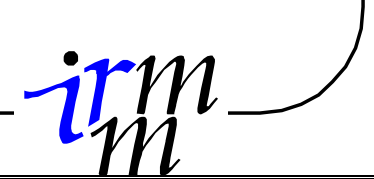




\section{Commutability}

- Ability of a material to show interassay properties comparable to those of human sera

- Not to mixed up with lack of specificity

- Acceptance and use of reference materials depends on applicability and commutability

- Only commutable materials can be used for direct calibration of calibrators and performance check of IVD's and therefore have large importance to ensure unbroken traceability chain
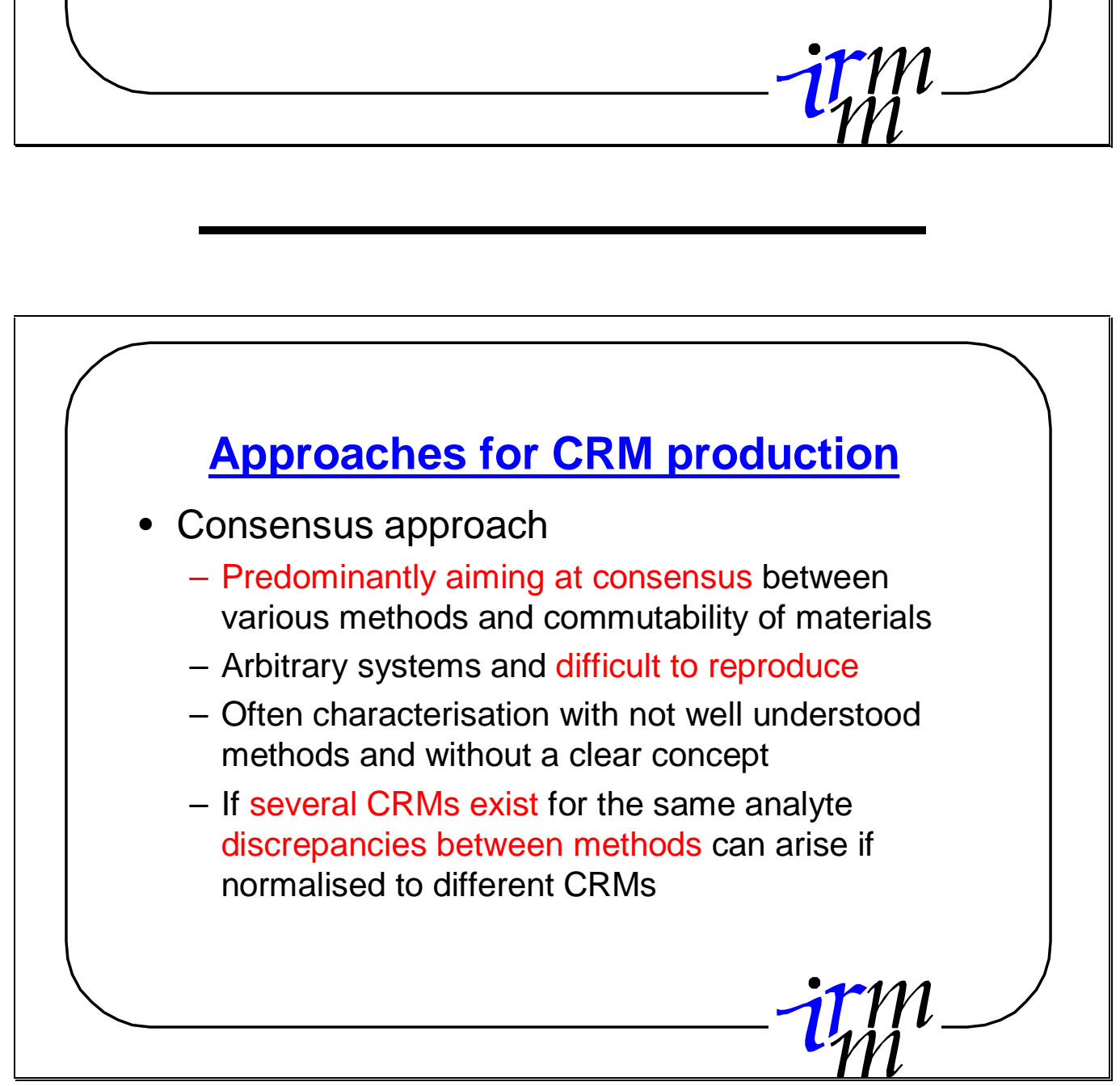


\section{IVD-harmonisation}

- More structured and co-ordinated approach is required involving experts in clinical chemistry, IVD and CRM producers

- Support to networks of relevant institutions on an international level

- Since 1996 direct co-operation with IFCC (International Federation of Clinical Chemistry, $>70$ clinical chemical societies and $>40$ industrial members)
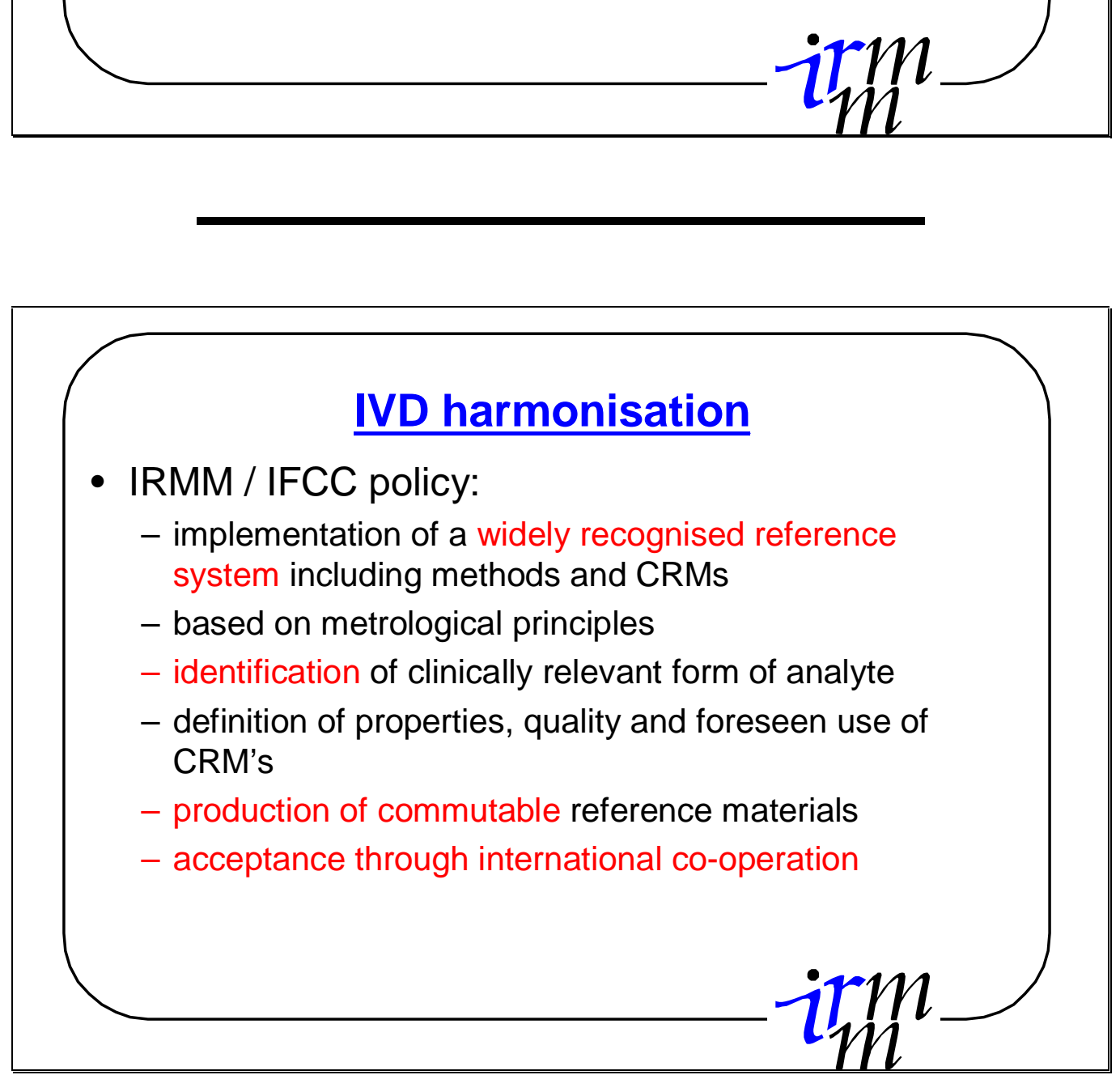


\section{Certification of enzyme CRM's}

in co-operation with the IFCC committee on enzymes

- Certification of enzyme CRM's at $37^{\circ} \mathrm{C}$

- Development of optimised SOP's at $37^{\circ} \mathrm{C}$ based on official IFCC methods at $30^{\circ} \mathrm{C}$

- Certification of 4 enzymes completed and certified according to GUM

- GGT ( $\gamma$-glutamyltransferase)

- ALAT (alanine aminotransferase)

- CK-MB (creatine kinase from human heart)

- LD (lactate hydrogenase)

\section{Certification of enzyme CRM's}

in co-operation with the IFCC committee on enzymes

Changes in SOPs:

GGT

$\mathrm{pH}$

Incubation time

Delay time

Measurement interval :

LD

$\mathrm{pH}$

Incubation time

Delay time :

Measurement interval : $37^{\circ} \mathrm{C}$

7.7

$180 \mathrm{~s}$

$60 \mathrm{~s}$

$180 \mathrm{~s}$

$37^{\circ} \mathrm{C}$

9.4

$180 \mathrm{~s}$

$90 \mathrm{~s}$

$180 \mathrm{~s}$ $37^{\circ} \mathrm{C}$ (old)

7.9

not specified

not specified

$300 \mathrm{~s}$

$30^{\circ} \mathrm{C}$ (old)

7.2

60-300s

$90 \mathrm{~s}$

$60 \mathrm{~s}$ 


\section{Certification of enzyme CRM's}

in co-operation with the IFCC committee on enzymes

- CRM's available for sale

- Collection of data for re-definition of reference ranges by IFCC ongoing

- Publication as official IFCC reference method envisaged

- Collaboration of IFCC / IRMM with

- various Universities and hospitals (including Japan)

- IVD producers (including USA and Japan)

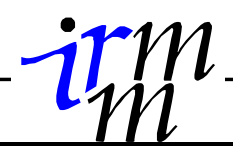

\section{Certification of enzyme CRM's}

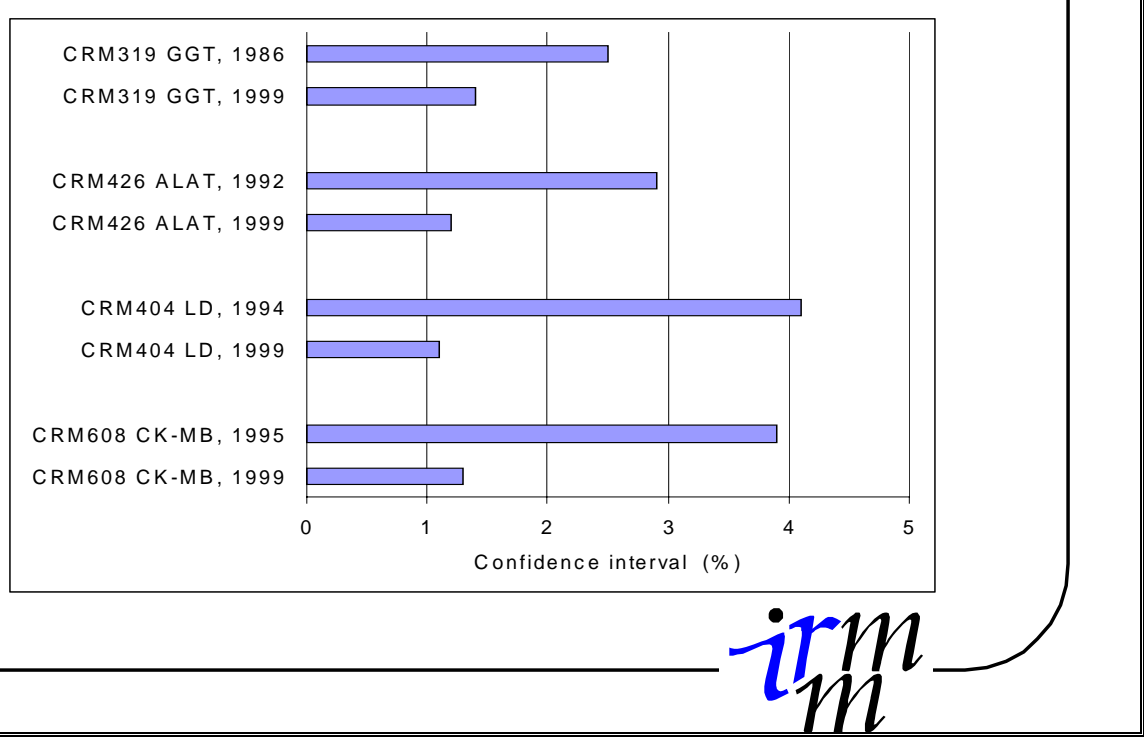



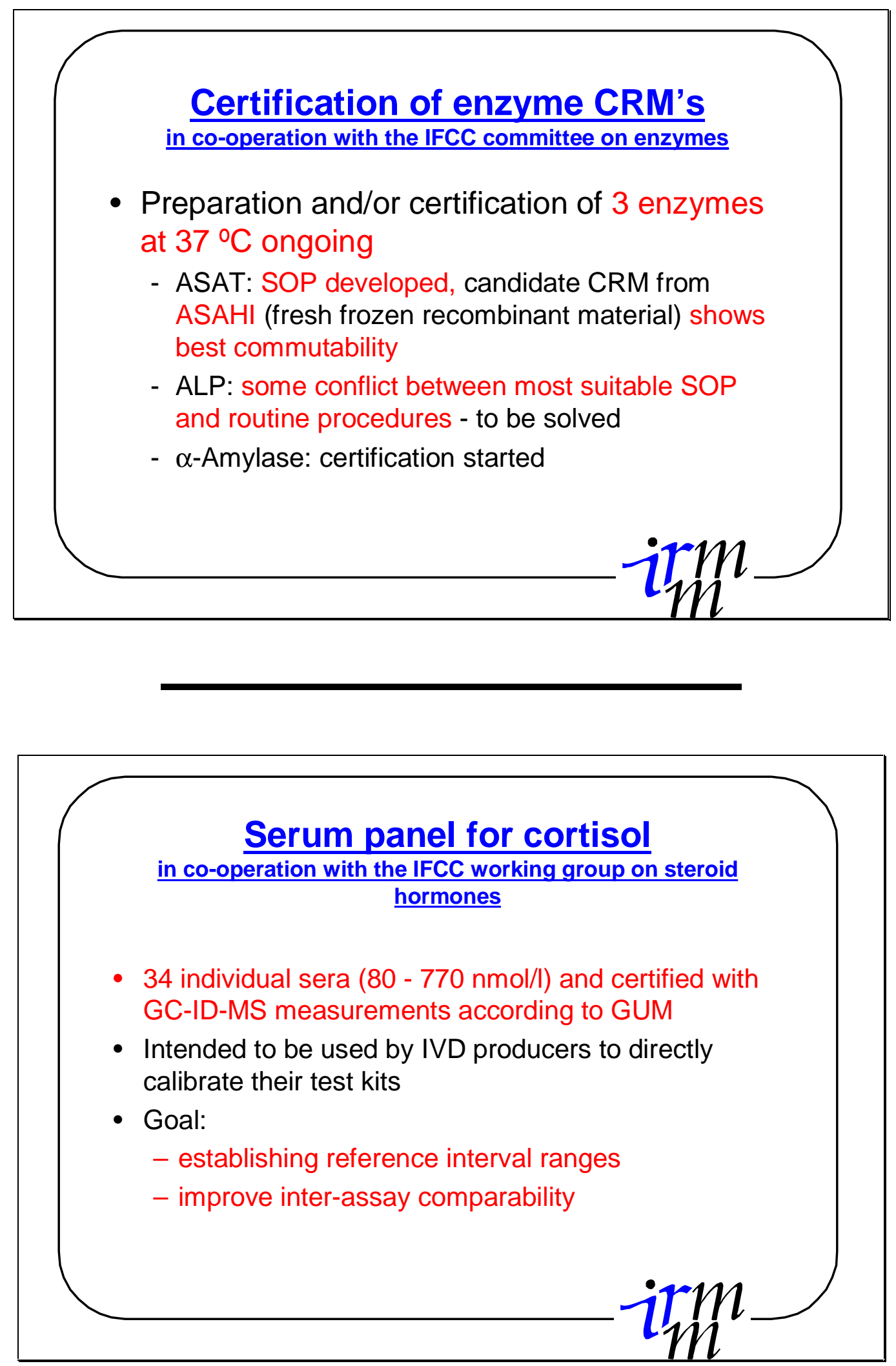

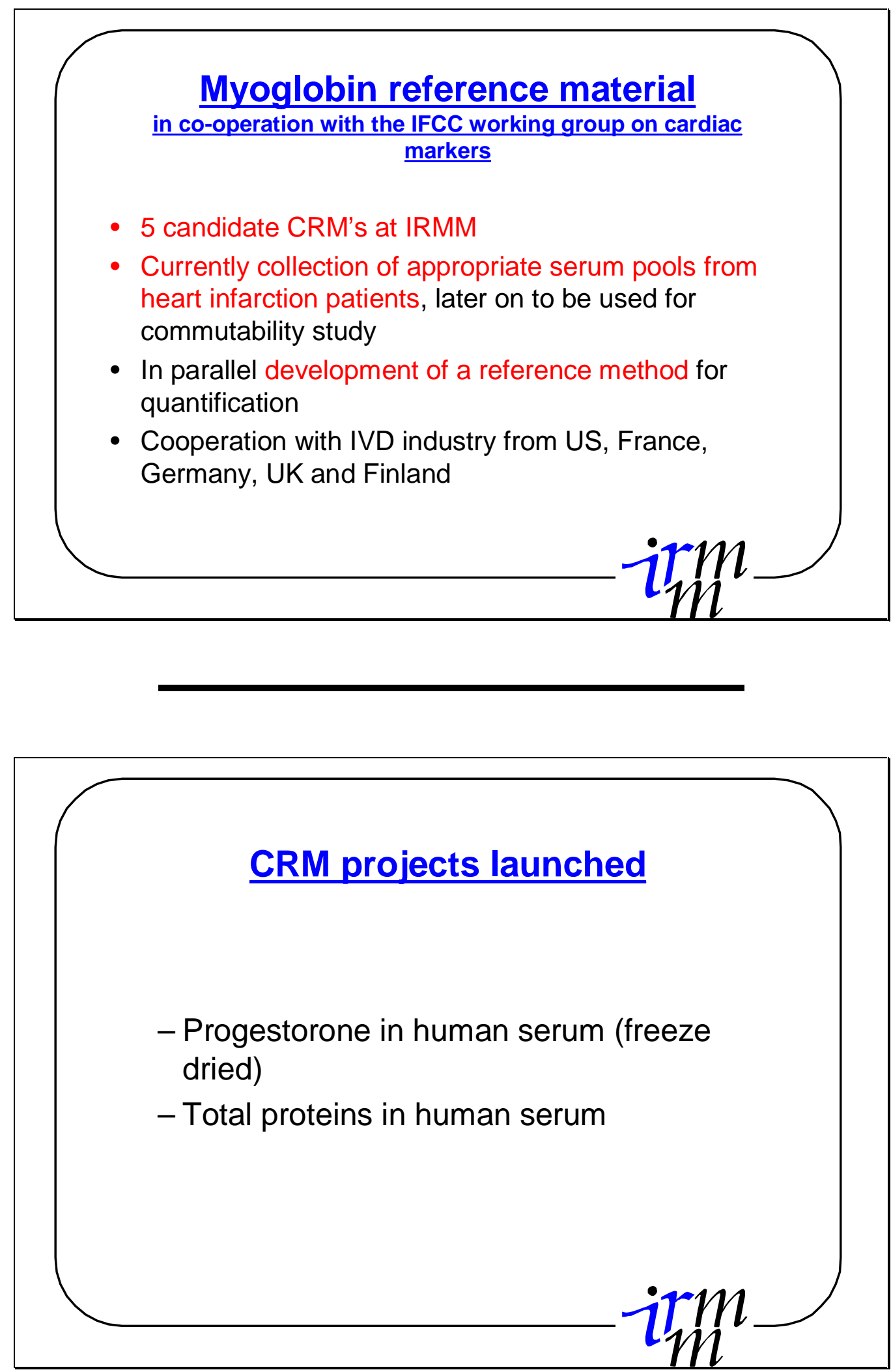


\section{CRM projects under discussion}

- Panel for glycated haemoglobin ( $\mathrm{Hb} 1 \mathrm{Ac})$

- Purified hCG and its isoforms

- Certification of pure progesterone, testosterone, $17 \beta$-estradiol and aldosterone as primary calibrants in co-operation with PTB

- Progestorone, testosterone and/or 17 $\beta$-estradiol serum panels

- Osteocalcin

- Genetic marker CRMs
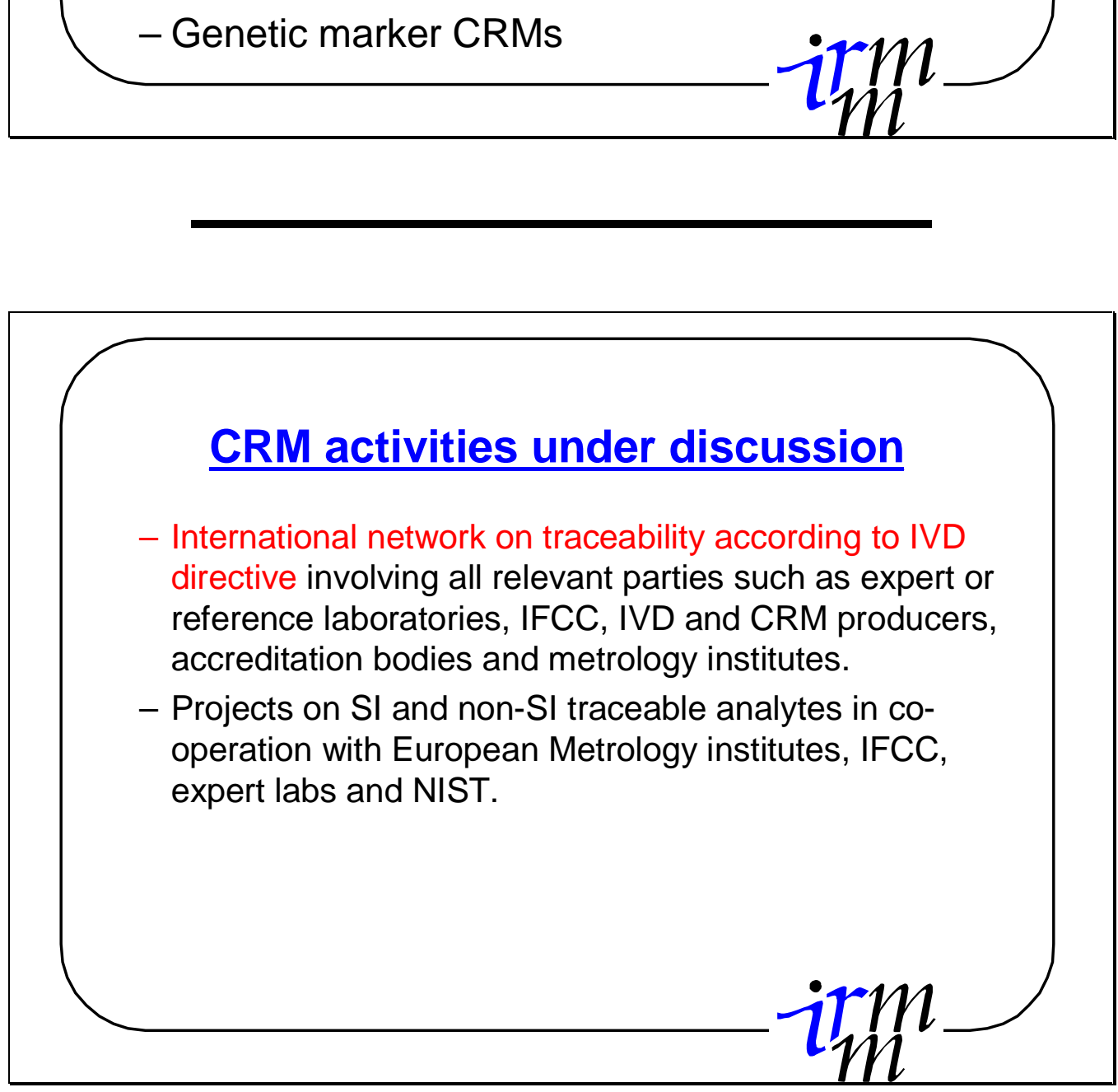

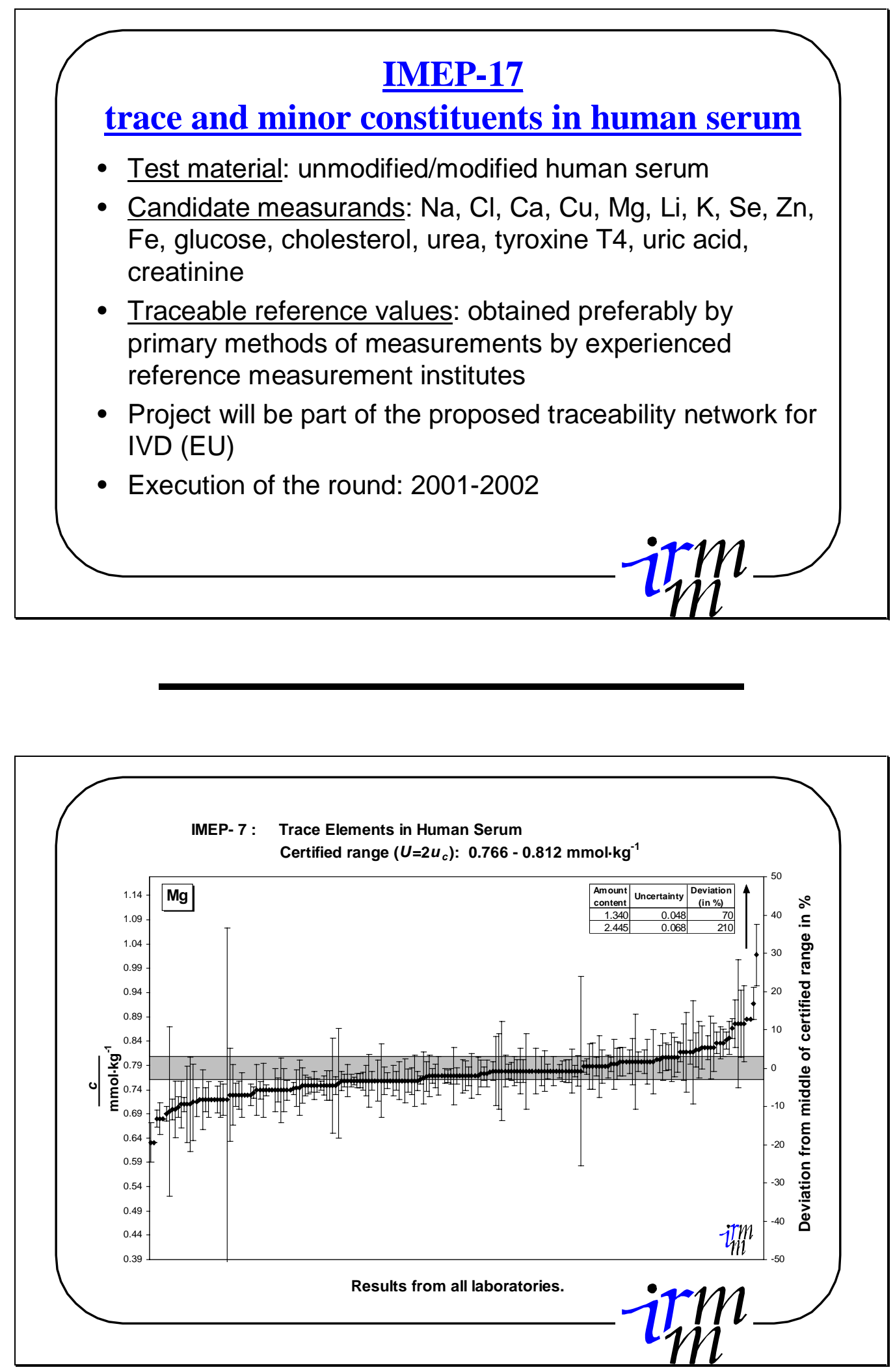


\section{Defining priorities}

- Balance between production efforts, clinical relevance and level of uncertainty

- clinical relevance and level of uncertainty to be achieved determined by

- significance of the parameter in diagnosis

- reference ranges of healthy patients and daily variation

- expected improvement of measurement quality

- number of analyses carried out and impact on individuals and public health care (e.g. disease prevention) i.e. assessment of cost / benefit ratio

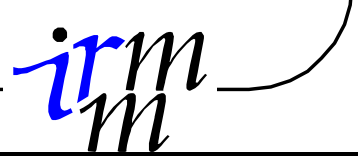




\section{The IVD Directive Requirement for Calibrator Traceability: Impact on IVD Manufacturers \\ Neil Greenberg \\ Ortho-Clinical Diagnostics a Johnson \& Johnson Company}

The new ISO/CEN written standard on traceability of calibrators for in vitro diagnostic test systems is currently being circulated for comment as a committee draft (ISO/CD $17511^{1}$ ) prior to a formal vote. As noted previously in the Regulations and Standards column of the July-August, 2000, issue of IVD Technology ${ }^{2}$, this new standard was developed to support one of the 'essential requirements' of the European Union's In Vitro Diagnostics Medical Devices Directive (Directive 98/79/EC ${ }^{3}$ ). The key text of the Directive states that... "the traceability of values assigned to calibrators and/or control materials must be assured through available reference measurement procedures and/or available reference materials of a higher order." Now that the Directive transition period is officially underway, and CE marking is mandatory for IVD products exported to Europe by December 2003, global manufacturers are beginning to define strategies to achieve compliance, and are recognizing that the word "traceability" is not merely a synonym for the word "accuracy."

Measurement traceability, as defined under the Directive, means that a calibrator's assigned value is systematically derived from higher order reference materials or reference methods, through an unbroken and well-characterized chain of comparisons. This chain of comparisons comprises a known hierarchy of reference methods and reference materials, all with known uncertainties. Trueness (i.e. closeness of agreement between the average value obtained from a large series of test results and an accepted reference value ${ }^{4}$ ) is transferred from an international reference system, through secondary or intermediate reference materials and methods to product calibrators, which are used to standardize routine measurements on human samples.

With recognition of the state of the art in clinical laboratory methods, methodological differences in test results are generally well known and accepted by physicians, laboratory practitioners, independent quality assurance organizations, regulators, and the IVD industry. Many national and regional proficiency (EQAS) programs account for such known differences by employing peer grouping in grading laboratory performance. In contrast, European advocates for 'true' clinical laboratory measurements have succeeded in encoding the objective of measurement truth into European Union law, with the approval of the Directive.

Implications of the metrological traceability requirement for IVD test systems calibrators are far reaching. It is expected that some IVD manufacturers may need to alter not only the amount of documentation they maintain, but also in some cases their procedures and protocols for establishing the calibration of assays they are currently selling in global markets. It is also expected that the calibrator traceability requirement will re-energize a currently static environment for investment in development of 
reference methods and materials. As new reference tools eventually emerge, manufacturers will ultimately need to revise procedures and adjust calibration of their commercial kits and systems over the long term, to remain competitive.

\section{ISO/CD 17511 - Scope \& Specific Requirements}

ISO/CD 17511 was co-developed by a collaboration among working groups of scientists in ISO Technical Committee 212 (Clinical Laboratory Testing and in vitro Diagnostic Test Systems) and CEN Technical Committee 140 (in vitro Diagnostic Medical Devices). In addition to ISO/CD 17511, the same working groups also codeveloped a companion vertical standard, ISO/CD $18153^{5}$, Metrological traceability for catalytic concentration of enzymes in calibrators and control materials.

The membership of the working groups represented a broad range of constituencies, including laboratory medicine professional societies, national metrology institutes, and IVD industry trade associations. Both ISO/CD 17511 and ISO/CD 18153 apply to product calibrators, for reagent kits or systems, and also apply to some "trueness" control materials (i.e., controls intended to evaluate closeness of agreement of the test method with accepted reference values.) The primary objective of both of these ISO standards and the Directive is to ensure trueness of test results based on available internationally recognized reference materials or procedures, when using a routine (lower metrological order) test method. ISO/CD 17511 specifies how to ensure traceability, and highlights the documentation required to be in the manufacturer's technical files in support of the assignment of particular calibration values to a product calibrator. The values assigned to a calibrator must be backed up with a complete, unbroken series of linked steps, starting with the highest order available reference methods or materials, and working stepwise down the metrological hierarchy to product calibrators. As stated in ISO/CD 17511, section 4.1.7, “...the responsibility of the manufacturer for describing the traceability chain ...start(s) at the value of the manufacturer's product calibrator and end(s) at the metrologically highest reference used by the manufacturer."

\section{ISO/CD 17511 - Items Excluded from Scope}

Manufacturers need to be aware that certain IVD products within the general device categories of calibrators or quality control materials are outside the scope of ISO/CD 17511.

\section{Items in this group include:}

- Commercial quality control samples sold without assigned values, and used only for assessing precision within a laboratory (precision control materials)

- Quality control materials intended for intralaboratory quality control purposes, and supplied with intervals of suggested acceptable values that were obtained by interlaboratory consensus, and are limited for use with only certain methods. 
In addition, certain approaches to value assigning IVD calibration materials are considered to be non-traceable under the rules of ISO/CD 17511.

\section{Examples include:}

- Calibrators with values assigned based on "horizontal" or predicate method correlation studies, using results for split patient samples tested with two different measurement procedures operating at the same metrological level.

- Calibrations derived from correlation between two analytes with different physico-chemical characteristics. Example: Total CO2 in whole blood, calibrated against a serum bicarbonate reference procedure.

\section{Key Issues and Concerns: The State of the Art in Calibrator Traceability}

\section{Definition of the Analyte.}

With IVD measurement devices, a detailed definition of the analyte is an often overlooked, but nevertheless important aspect of an analytical reference system. When the target analyte is actually a class or group of substances, rather than a single, welldefined chemical species, tracing measurements to metrologically higher order calibrators or measurement procedures may be quite challenging. Often, very different forms of a particular class of substances may be important in different pathological conditions. An example is human chorionic gonadotropin (HCG), which exists in a variety of different molecular forms in serum, some of which are predominant in women during pregnancy, and others that predominate in certain tumors ${ }^{6}$.

\section{Two General Analyte Classes Defined in ISO/CD 175.}

To differentiate those substances that are chemically well defined and present in a narrowly definable range of molecular species from those substances that are typically found as a range of heterogeneous sub-species, ISO/CD 17511 defines Type A (physicochemically well-defined) and Type B (heterogeneous mixture) analytes. In principle, the Type A substances are expected to be traceable to metrologically higher-order reference materials and methods, often going back up the hierarchical chain to the fundamental unit of measure, as defined according to the System Internationale (SI meter convention). Due to the complexities of analyte definition and molecular structures, the Type B substances are not expected to be traceable to SI units. Because of their metrological complexity, members of the Type B analyte group present the greatest challenges to IVD manufacturers from the standpoint of the task of defining the metrological traceability of the commercially available measurement systems and test kits. 


\section{Some Typical Situations Where Manufacturers May Encounter Difficulty in Defining}

Metrological Traceability, Due to Analyte Complexity and Heterogeneity:

- Analyte chemico-physical complexity is the norm, not the exception: there are perhaps up to 1000 different analytes routinely measured in clinical laboratories, less than $10 \%$ of which are chemically well-defined (Type A). The Type A substances may be more than $80 \%$ of the total volume of procedures actually performed annually, but in terms of the available test menu, the vast majority of analytes are actually Type B. Examples of some of the items from the extensive menu of type B analytes typically offered include tumour markers, coagulation parameters, endocrine hormones, immune factors, virology markers, and bacteriology markers. Many of these substances are complex proteins, which may be present in blood and tissues as a group of various molecular species.

- Immunoassay methods commonly used in routine measurement systems for many complex (Type B) analytes often demonstate antibody selectivity for specific antigens and/or epitopes. This may be due to underlying physical attributes of the analytical antibodies, owing to their being produced in a biological system that consists of a purified antigen/stimulus containing only a limited portion of the spectrum of possible epitopes of the antigen existing in nature.

- The so-called Type A analytes can also display chemico-physical heterogeneity. Seemingly 'simple' analytes such as serum calcium are present in human serum in a chemically broad array of molecular forms, including ionized species, inorganic and organic salts, water-complexed, and protein-bound fractions. Other analytes such as the most commonly measured serum electrolytes, sodium and potassium, can be quantified as either ion concentrations or ion activities, depending on the measurement technology chosen. According to strict analytical chemistry interpretation, these different chemical entities are considered to be different 'quantities,' and therefore, their calibrations must follow along separate pathways of traceability.

\section{Is the Global IVD Reference Systems Infrastructure Sufficiently Mature to Support Calibration Traceability?}

There are a number of gaps in the present system of reference materials and methods for IVDs. In the US, the National Reference System for the Clinical Laboratory (NRSCL), a steering committee operating within NCCLS and originally created for the purpose of credentialing a US IVD reference systems infrastructure, credentialed reference systems for approximately a dozen clinical chemistry analytes over a period of activity spanning approximately 10 years. However, NRSCL is not currently actively involved in credentialing additional reference systems.

In the meantime, a number of other organizations have participated in this arena. As a result, a variety of clinical laboratory reference methods and materials (but few full reference systems) have been certified by groups such as the US National Institute of Standards and Technology (NIST), the (US) Centers for Disease Control and Prevention (CDC), the European Union - Institute for Reference Materials and Methods (IRMM), the World Health Organization (WHO), the International Federation of Clinical Chemistry 
and Laboratory Medicine (IFCC), the Japanese Committee for Clinical Chemistry (JCCLS), the German Society for Clinical Chemistry (DGKC), and the Scandinavian Society for Clinical Chemistry. Across this highly faceted array of organizations, there are some program redundancies, including different reference materials and reference methods for certain analytes. Patient results will significantly differ, in several of these cases, depending on which reference material or reference method is chosen for standardization of a particular procedure.

The current situation is therefore one of having parallel and sometimes redundant standards programs around the world. The participants include both regional and international organizations (e.g. European Union/IRMM, NIST, IFCC, WHO, DS, etc.), many of which are currently, in the wake of the IVD Directive, in the process of initiating new projects to develop calibration standards, without the benefit of formal and comprehensive global intra-organizational coordination. All of the groups have slightly different constituencies, different funding sources, and different (and sometimes even competing) objectives.

\section{Frequently Encountered Deficiencies with Some Existing Reference Materials and Reference Methods.}

1. Absence of a complete definition of the analyte intended to be calibrated. Reference methods and/or materials are often specifically targeted to support measurement of an analyte in a particular body fluid (e.g., potassium in serum, creatinine in serum, amylase in serum). While this is helpful, in fact the clinical laboratory field may be interested in application of routine methods to a broader array of body fluids (e.g. urine, cerebrospinal fluid, pancreatic fluid, whole blood, etc.), and reference materials and methods simply do not exist to support the other applications.

2. Non-suitability of available reference materials for their intended purpose. Many analytes of clinical interest demonstrate chemical instability. Some examples are bilirubin, cardiac troponin and cardiac CK-MB. As a result, highly pure preparations of these analytes prepared for use as reference materials typically demonstrate similar instability. While this problem presents a great technical challenge for the developers of reference materials, the absence of stable preparations of reference materials to support calibration processes in a manufacturing environment renders such materials relatively useless for routine applications.

3. High uncertainty of assigned values for certain reference materials. Of the reference materials that are currently available, some have assigned values that are defined using a statistical consensus approach, involving multiple measurements on a range of diverse analytical methods that are currently commercially available. Since there are no prior calibration standards for these existing methods, their calibrations may be arbitrary. The consensus methodology enables all currently marketed devices to meet calibration requirements, since the statistical range of uncertainty in the face of often highly dispersed data, typically accommodates differences between the methods included in these studies. This approach, while expedient and seemingly 'fair', does not assist in progressing toward the goal over time of achieving 'true' values. This approach also favours the presently available range of 'true' values observed with existing methods, and 
could force the calibration of newer technologies and devices against a status quo that may be scientifically flawed.

4. Non-commutability of reference materials. Commutability is an attribute that describes the ability of a reference material to emulate actual patient samples in its performance characteristics, over a broad range of analytical methods and systems. A number of reference materials contain chemical 'surrogates' of the analyte of interest, not the actual natural compounds, making them useful only in higher order chemical measurement systems (e.g. gas chromatography/ mass spectrometry, high-performance liquid chromatography, atomic absorption spectroscopy, etc.) Instances of incompatibility of many such reference materials with routine field methods, render these reference materials significantly less useful, forcing manufacturers to add additional steps and layers of complexity to their internal product calibration processes, and contributing to increasing uncertainty in the final assigned values for product calibrators.

5. Lack of portability of reference methods. Some reference methods are analytically complex, requiring expensive, rare and sophisticated instruments, as well as highly trained technologists to operate these systems. As a result, there are very few or even a complete absence of laboratories in most regions of the world capable of performing laboratory medicine reference measurements at an attractive cost, in a way that ensures access to all interested parties, especially smaller device manufacturers and clinical labs.

6. Recognition and status of existing reference methods and materials. Currently, there are some instances where certain IVD reference systems, methods or materials have international recognition. However, there are also many cases where recognition and status of a particular standard is only at the national or regional level. At the international level, there is presently no universally recognized authority charged with ensuring harmony among the regional and national bodies involved in accreditation of IVD measurement standards. Further, there is no established internationally accepted umbrella organization or process to accredit either existing or new reference methods, materials or even the reference laboratories that are performing some of the metrologically "higher level" measurements. Indeed, some of the organizations participating in this arena may have not yet established their scientific credibility at a level sufficient to command recognition or status with their peer organizations. Rapid resolution of these issues is clearly necessary in order to define the path forward, in the direction of progress.

\section{Additional IVD Industry Concerns}

\section{ISO/CD 17511 Requires That the Uncertainty of Assigned Values of Calibrators be} Made Available Upon Request.

A fundamental element of the concept of measurement traceability is that each link in the chain of measurement must have known uncertainty. Since the ultimate goal is 'accurate' measurement (i.e. deviations from 'truth' no greater than acceptable levels), knowledge of the uncertainty at each step in the traceability chain is important, in order to 
identify opportunities for improvement ${ }^{7}$. However, clinical laboratory personnel and IVD manufacturers have little experience with the concept of uncertainty of assigned values for reference materials, as it pertains to the calibrators provided for use with routine measurement procedures. This demands that manufacturers proceed through essentially uncharted waters. From the IVD manufacturers' perspective, this is a completely new and unknown requirement, for which no current models exist in the medical device industry. Traditionally, uncertainty calculations have been the concern only of higher order metrology institutes. Only in recent years, as part of the quality movement, has the measurement uncertainty concept begun to filter down to calibration laboratories in the industrial setting.

The primary resource for manufacturers and others interested in the 'how to' of determining and expressing measurement uncertainty is an ISO document known as the GUM (Guide to the Expression of Uncertainty in Measurement ${ }^{8}$ ). Due to the highly complex statistical language and academic discussion presented in the GUM, nonmetrologist readers frequently find the document to be cumbersome, and are left with a number of unknowns in terms of how to actually calculate uncertainty. There are no readily available interpretations that are applicable to IVD manufacturers, and as a result it is expected that different manufacturers will develop a variety of interpretations, leading to lack of uniformity in the estimates reported.

Aside from the concerns about how manufacturers will actually establish their uncertainty estimates, the value of the information about uncertainty of assigned values in calibrators to routine clinical laboratory customers is questionable. This is especially in the case of many typical clinical measurement systems where overall measurement variation is only a very small part of the total variation of the analyte, in the context of the much greater relative magnitude of the biological fluctuations of the analyte within a given individual ${ }^{9}$.

\section{Declaration of New or Improved International Reference Materials and Reference} Methods - Some Manufacturers May Need to Change Calibrations for Certain Analytes

Development of new or improved reference systems is not a specific requirement of the IVD Directive. The Directive states that routine methods need to be traceable to "... available reference measurement procedures and/or available reference materials of a higher order." Similarly, ISO/CD 17511 allows for a wide range of scenarios, include situations where there is no recognized higher order method or material available to trace back to. Under these circumstances, the highest order reference point available to a given IVD manufacturer may be a measurement procedure or reference material that is uniquely defined, controlled, and maintained by each manufacturer of the various commercial assay systems for a given analyte. When new reference methods or materials are developed and internationally accredited for such analytes, certain manufacturers will inevitably have to make changes in their processes in order to adjust their products to the new reference systems. All manufacturers are willing to step up to implementation of changes necessitated by customer needs, and which will ultimately be beneficial in bringing about improved health care. Hopefully, the changes required are important ones, representing needs articulated by a consensus of clinicians and laboratory professionals, since all changes are associated with disruption and cost. 


\section{Some of the short term costs for manufacturers include:}

1. Labeling changes and inventory obsolescence,

2. Customer and EQAS program advisory notices,

3. Filings with regulatory authorities,

4. Manufacturing process changes,

5. Training of customers and intracompany personnel,

6. Reference interval updates requiring additional communications and training for clinicians.

Given these costs, it is especially important that the initiative and the leadership for change be customer-focused, originating from a clear expression of need for improvement on the part of the end-users, not the manufacturers.

ISO/CD 17511, in contrast with the Directive, takes a slightly stronger position in terms of commitment to the cause of continuous improvement in reference methods and materials. As the standard states, "It is the aim of metrology in laboratory medicine to improve traceability...by providing the missing reference measurement procedures and reference materials, based on international consensus." Because of this commitment, it is expected that many national and international standards organizations, scientific, professional, and industry groups, will interpret the Directive's intent, arriving at an interpretation which says that the Directive demands investment in upgrades to the international reference system for the clinical lab. What is most important, as new reference materials or reference method projects inevitably get underway in the name of the Directive, is that adequate scientific support from industry be sought after when staffing the working groups. This will help to ensure that a reasonable balance is achieved between commercial interests, pragmatic realities of manufacturing materials and process limitations, and academic clinical and metrological interests.

\section{Potential for Mis-Use of the Directive Requirement for Calibration Traceability.}

One of the fears occasionally voiced by manufacturers is that once the Directive is fully in force throughout the European Union, country level authorities may be inclined to overinterpret and mis-use the expectation of calibration traceability, to prematurely declare that specific types of IVD products (e.g. test kits for serum troponin) available within their own borders, must be traceable to a particular reference method or material, which is recognized or preferred only within that particular country. Such actions could create protectionism and artificial trade barriers, perhaps favoring the marketing of products manufactured only by companies located within certain countries. Such an approach could also be used to limit exploitation of new or currently existing technology 
options, due to limitations in the capabilities and/or availability of recognized reference methods and materials.

\section{Examples of leading edge IVD technical development where there} currently exists the potential for barriers to new technologies include:

- Restriction of immunoassay methods to specific epitopes or gene products that are currently known markers for a particular disease

- Selection of defined substrates as standards for enzymes, e.g., serum amylase

- Selection of reference methods or materials, which rely on products (equipment or materials) that must be licensed from a single vendor, due to patented technology.

\section{Do IVD Manufacturers Want or Need More Reference Materials And Methods?}

Despite short-term concerns, international calibration standards (reference systems) do in fact help IVD manufacturers by providing well-defined market needs and customer requirements, clear and universal definition of goals, and tools for objective assessment of product attributes. Table 1 provides a more detailed list of a few of the global trade-offs that should be considered by all the participating organizations (manufacturers and distributors of IVD products, industry associations, user groups, customer advocacy and professional groups, government and regulatory bodies, etc.) proposing to undertake development of new calibration standards (reference systems) for IVDs. It is clearly a matter of cost vs. benefit, and the decision to move forward demands careful analysis on a case-by-case basis.

\section{TABLE 1. Trade-Offs in Decision to Invest in New Reference Systems for IVDs}

\section{BENEFITS}

- Product Quality

- Interchangeability of data between products

- Competitiveness - level playing field for competition

- Defined quality needs

- Lower long-term costs

- Clearer pathway to market access

- Transferable technology 


\section{COSTS}

- Availability of appropriate qualified people to participate in standards work

- Risk of investing in standards that, upon completion, are not accepted by all stakeholders, especially customers

- Lengthy cycle time to achieve deliverables

- Costs of transition (both within manufacturing companies \& for customers) to make changes to comply with new standards

- Less variety; fewer alternatives for customers

- Barriers to innovation

- Barriers to market entry

\section{Does Industry Support Initiatives for New IVD Calibration and Reference Systems?}

Public interest requires good quality and safe health care products. It is easy to demonstrate that improved standardization contributes to furthering these goals, and all IVD manufacturing companies share this interest. Reference materials and reference methods for calibration are an important underlying element, contributing to quality and safety through the assurance of interchangeability of information across time and space.

Given these shared goals, it is inevitable that new reference systems projects will emerge, and new reference materials and methods will ultimately be defined, which will impact the definition of the state of the art. Industry must play a role in this process, using its collective wisdom achieved through years of real-world experience, to ensure technically sound and practical solutions to the challenges encountered in development projects undertaken in the name of the desire for better standards.

\section{What are the Priorities of IVD Manufacturers for Development of New Calibration Standards?}

A short-term objective of many manufacturers is to ensure that adequate tools are in place to support priorities established under the IVD Directive. The IVD Directive has designated certain IVDs, included within a list known as Annex II, as ones which require special certification by independent, notified bodies, as a prerequisite to granting and maintaining of the CE mark. Thus, even in the absence of internationally recognized calibration standards, manufacturers are likely to have much less latitude in calibrating these tests. Evaluation and certification of these products will be performed against new de facto standards known as Common Technical Specifications, created especially to support conformance assessment of these products, for CE marking under the Directive. To ensure fairness and equity in these assessments, common reference materials are needed both by the manufacturers and the notified bodies to assist in assessing 
performance, and in controlling analytical sensitivity at medical decision limits or cutoff values.

Some specific analytes urgently needing better international standards and included in the Directive, Annex II, are infectious disease markers used for blood screening and diagnostics for hepatitis, such as HBsAg, anti-HCV, anti HBs; markers for immuno-deficiency viral infections, including anti-HTLV and anti-HIV; and blood grouping reagents used to ensure donor unit compatibility in markers for Anti D, Anti A, Anti B.

\section{Recommendations}

What factors are necessary to get to the future state? To begin, laboratory medicine, scientific, and professional organizations need to provide leadership and guidance relative to what standards are needed. In doing so, project priorities should be defined with an appreciation that resources are limited, while taking into account factors such as (1) the public health significance and disease course, (2) expectations of the degree of improvement anticipated in overall clinical effectiveness of a given test if a new standard is developed, (3) time and cost estimated to reach a desirable endpoint, and (4) the overall likelihood of success.

High priority should be given to establishing a defined, international, customerfocused, and consensus-based process for setting priorities and contracting projects. This process should be led by the major laboratory professional associations (e.g. IFCC, AACC, CAP, WASP and others) and should proceed in an atmosphere of open and public dialogue, employing decision tools that emphasize quantification and metrics, and is inclusive of all key stakeholders (i.e., profession, government, industry, lay public). One possible solution is formation of an 'IRSCL' ('I' standing for international), following the NCCLS-NRSCL model. This body must be international in scope and responsibility, and could be staffed under contract with an organization such as NCCLS, to provide infrastructure needs.

Sufficient project funding (grants or contracts) must be provided, and will need to include government sources (e.g. EU Commission, US Department of Commerce, etc.), professional societies (e.g. IFCC, AACC, CAP, WASP), as well as industry groups.

Projects must be closely managed, with accountability for deliverables and schedule. Project teams must be staffed with appropriate clinical and scientific experts, coming equally from the professions and industry.

\section{Conclusions}

Although the EU IVD Directive does not specifically require it, reference materials and reference methods development projects will be initiated in the name of the Directive. New calibration standards are likely to increase short term costs for IVD manufacturers, but this is a minor consideration if there is a clear need for improved standards and an expectation of improved quality of health care as an outcome, as expressed by a consensus of customers and professional associations. Professional and 
customer advocacy groups should take the lead role in advocating for new calibration standards, especially in defining where improved standards are needed. Whenever IVD calibration and reference systems standards projects are undertaken, whether professional, government, or public health groups sponsor them, inclusion of IVD industry scientists and experts on the technical team is an absolute prerequisite for project success. Creative strategies are needed to ensure adequate project funding, and should involve a combination of contributions from public, professional and industry sources.

\section{References}

1. In Vitro Diagnostic Medical Devices-Measurement of Quantities in Samples of Biological Origin-Metrological Traceability of Values Assigned to Calibrators and Control Materials, ISO/CD 17511 (Geneva: International Organization for Standardization, February, 2000).

2. Powers, Donald M., Regulations and Standards - Traceability of assay calibrators: The EU's IVD Directive raises the bar, IVD Technology, July-August, 2000.

3. Council Directive 98/79/EC of the European Parliament and of the Council of 27 October 1998 on In Vitro Diagnostic Medical Devices, Official Journal of the European Union L331 (December 12, 1998).

4. International Standard ISO 3534-1 Statistics - Vocabulary and symbols, Part 1: Probability and general statistical terms, International Organization for Standardization, first edition, 1993-06-01, Geneva, Switzerland.

5. In Vitro Diagnostic Medical Devices-Measurement of Quantities in Samples of Biological Origin-Metrological Traceability of Values for Catalytic Concentration of Enzymes Assigned to Calibrators and Control Materials, ISO/CD 18153 (Geneva: International Organization for Standardization, February, 2000).

6. Galina Kovalevskaya, Steven Birken, Tatsu Kakuma, John Schlatterer and John F. O'Connor, Evaluation of Nicked Human Chorionic Gonadotropin Content in Clinical Specimens by a Specific Immunometric Assay, Clinical Chemistry, 1999; 45: 68-77.

7. Charles D. Ehrlich and Stanley D. Rasberry, Metrological Timelines in Traceability, J. Res. Natl. Inst. Stand. Technol. 103, 93-105 (1998).

8. International Organization for Standardization, Guide to the Expression of Uncertainty in Measurement, Geneva, Switzerland (1993) (corrected and reprinted 1995).

9. Fuentes-Arderiu, X. Clin Chem 2000;46:1437-1438, Uncertainty of Measurement in Clinical Laboratory Sciences. 


\section{NEIL GREENBERG \\ Ortho-Clinical Diagnostics a Johnson \& Johnson \\ Company}

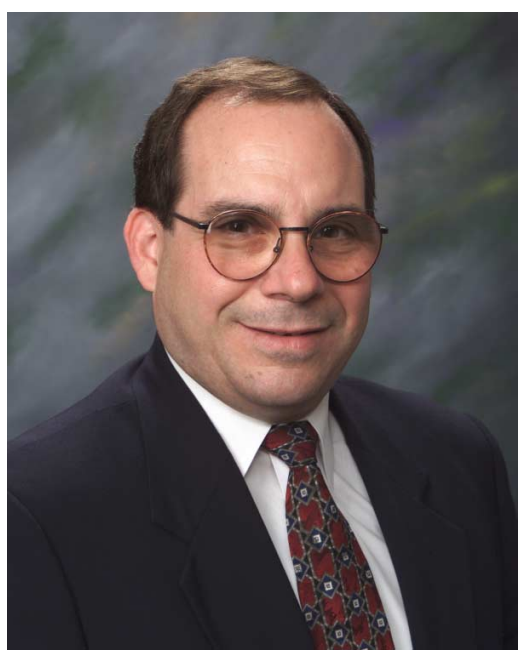

Neil Greenberg, Ph.D., DABCC, is a Project Manager, Regulatory Affairs, OrthoClinical Diagnostics, a Johnson \& Johnson Company. He received his doctorate in Physiological Chemistry and Clinical Chemistry from The Ohio State University, Columbus, Ohio, and served as a Post Doctoral Fellow in Clinical Chemistry at Hahnemann University, Philadelphia. He joined Eastman Kodak in 1978, and has held various managerial roles in manufacturing and quality assurance for the Ortho-Clinical Diagnostics Vitros (formerly Ektachem) line of in vitro diagnostic products. As Manager of Kodak's Reference Laboratory, he became actively involved in standardization of measurement procedures and reference materials for laboratory medicine in the early 1980's, and continues to work actively with NCCLS and the American Association for Clinical Chemistry on various aspects of measurement standardization for in vitro diagnostics. He is a member of Working Group 2, ISO/TC 212, which is developing international standards for reference systems in laboratory medicine. Dr. Greenberg also is the liaison for ISO/TC 212 Working Group 2. 
The IVD Directive Requirement for Calibrator Traceability: Impact on IVD Manufacturers

Visuals Presented By

Neil Greenberg

Ortho-Clinical Diagnostics a Johnson and Johnson Company 


\section{The IVD Directive Requirement for Calibrator Traceability: Impact on IVD Manufacturers}

Ortho-Clinical Diagnostics, a Johnson \& Johnson Company Presented at

Workshop on Measurement Traceability for Clinical Laboratory Testing and In Vitro Diagnostic Test Systems Gaithersburg, MD, November 2, 2000

\section{Traceability: Impact on Manufacturers}

Overview of ISO/CD 17511

Scope \& Specific Requirements

Exclusions

Limitations

Key Issues and Problem Areas

IVD Industry Concerns

Recommendations

Conclusions 


\section{Traceability: Impact on Manufacturers}

ISO/CD 17511 - Scope \& Specific Requirements

Primary Objective: Ensure TRUENESS of test results based on available internationally recognized reference materials or procedures, when using a routine (lower metrological order) test method Specifies documentation requirements for assigning calibration values in a calibrator (reference material) Requires a complete, UNBROKEN series of linked steps from highest order available reference methods or materials, stepwise "downward" to product calibrators

\section{Traceability: Impact on Manufacturers}

ISO/CD 17511 - Scope \& Specific Requirements

- Applies to calibrators and some control materials sold for use with IVDs

- Specifies how to ensure traceability of values assigned to calibrators and control materials that establish or verify TRUENESS of a measurement method.

- 4.1.7 "The responsibility of the manufacturer for describing the traceability chain ...start(s) at the value of the manufacturer's product calibrator and end(s) at the metrologically highest reference used by the manufacturer." 


\section{Traceability: Impact on Manufacturers}

\section{ISO/CD 17511 - Items Excluded from Scope}

Control materials without assigned values, used only for assessing precision (precision control materials)

Control materials intended for intralaboratory quality control purposes \& supplied with intervals of suggested acceptable values (obtained by interlaboratory consensus, for particular methods).

Correlation between results of two measurement procedures at the same metrological level.

"Horizontal" or predicate correlation studies do not provide traceability

\section{Traceability: Impact on Manufacturers}

\section{ISO/CD 17511 - Items Excluded from Scope}

Calibrations derived from correlation between two procedures at different metrological levels, for analytes with different physico-chemical characteristics.

e.g.: Total $\mathrm{CO}_{2}$ in whole blood, calibrated against a serum bicarbonate reference procedure

Properties defined with 'nominal' scales, when no magnitude is involved (e.g. blood group). 


\section{Traceability: Impact on Manufacturers}

\section{$\underline{\text { ISO/CD } 17511 \text { - Items Excluded from Scope }}$}

- Traceability of routine patient results to a calibrator

- Relationship of routine patient results to medical discrimination limits

- Traceability of patient result may be influenced by method specificity

- Non-specificity (random interaction between method and patient samples) may have little or no impact on assigned values of calibrators

\section{Traceability: Impact on Manufacturers}

\section{Key Issues \& Problems}

- Chemico-Physical Nature of Measured Quantities

- Of $\sim 1000$ analytes, less than 100 are well-defined chemical entities

- Analyte heterogeneity

- 'Total Protein', enzymes, hormones, etc.

- Bound vs. free analyte forms

- Immunologic method variability

- Selectivity for specific epitopes 


\section{Traceability: Impact on Manufacturers}

Key Issues \& Problems

\section{- Reference Systems}

- Gaps in reference materials and methods

- Parallel/redundant programs

- Regional \& international organizations (e.g. NIST, IFCC, IRMM, WHO, DS) are initiating programs

- Lack of coordination among programs

- Few laboratories capable of performing reference measurements at reasonable cost

\section{Traceability: Impact on Manufacturers}

Key Issues \& Problems

- Reference Systems

- Deficiencies in existing reference materials and reference methods

- Non-commutability of many reference materials

- Large uncertainties in assigned values of certain reference materials (e.g CK-MB)

- Lack of portability of many reference methods

- No recognized authority to assure harmony or accredit (new or existing) reference methods, materials or labs at international level 


\section{Traceability: Impact on Manufacturers}

Key Issues \& Problems

- Uncertainty of Assigned Values

- Information must be available on request

- Complexity, assumptions, and unknowns in calculating combined total uncertainty

- Directly measurable/observed (type A) uncertainties

- A priori uncertainties (type B)

- Value to customers in routine applications is questionable

\section{Traceability: Impact on Manufacturers}

\section{IVD Industry Concerns}

Development of new or improved reference materials

NOT required by the IVD Directive ...

“...available reference measurement procedures and/or available reference materials of a higher order."

ISO/CD 17511...

"It is the aim of metrology in laboratory medicine to improve traceability...by providing the missing reference measurement procedures and reference materials, based on international consensus." 


\section{Traceability: Impact on Manufacturers \\ IVD Industry Concerns}

- Traceability can be mis-used to limit new or existing technology options, due to limitations in capability and/or availability of recognized reference methods \&/or materials.

- Restricting immunoassay methods to specific epitopes or gene products

- Defined substrates for enzymes, e.g., serum amylase

- Limiting reference (methods or materials) to licensed or patented technology

- Limiting development of in vivo techniques

\section{Traceability: Impact on Manufacturers}

\section{IVD Industry Concerns}

- International recognition status of existing reference methods and materials

- international vs. regional recognition of methods and materials - How to resolve?

- recognition and scientific status of organizations responsible for existing and/or future standards

- Documentation of available reference procedures and materials

- Is documentation compliant with ISO/DIS 15193 \& 15194? 


\section{Traceability: Impact on Manufacturers}

\section{IVD Industry Concerns}

- Available reference materials suitable for intended purpose?

- Analyte clearly defined \& stable?

- e.g. troponin

- Matrix options

- e.g. whole blood vs. serum

- Low uncertainty of assigned values?

- Reference lab accreditation

- compliant with ISO/CD 15195?

- Internal company labs vs. outsourcing?

\section{Traceability: Impact on Manufacturers}

\section{Do IVD Manufacturers want more IVD} reference materials $\&$ reference methods?

- Standards help IVD companies by providing...

- Well-defined market needs

- Clear, universal definition of goals

- Objective assessment of product attributes 


\section{Traceability: Impact on Manufacturers}

\section{Standards Trade-Offs}

\section{- BENEFITS}

- Quality

- Interchangeability

- Competitiveness

- Defined quality needs

- Lower long-term costs?

- Clearer pathway to market access

- Transferable technology

\section{- COSTS}

- Cost of working on standards projects

- Lengthy cycle time to deliverables

- Transition to comply with new standards

- Initially higher costs?

- Less variety; fewer alternatives for customers

- Barriers to innovation

- Barriers to market entry

\section{Traceability: Impact on Manufacturers}

Does Industry support new IVD standards?

- Public interest requires good quality \& safe health care products

- Reference materials \& reference methods are an important underlying element

- Calibration standards will inevitably be defined

- Standards will impact the 'state of the art'

- Industry must play a role to ensure technically sound and practical standards 


\section{Traceability: Impact on Manufacturers}

High Priorities for (some) Manufacturers

- IVD Directive ANNEX II Products -

- Draft EU 'Common Technical Specifications' (CTSs) call for development of critical biological standards (reference materials)

- Common reference materials needed by manufacturers and notified bodies for assessing performance; controlling analytical sensitivity at medical decision limits (cutoff values)

\section{Traceability: Impact on Manufacturers} High Priorities for (some) Manufacturers

-IVD Directive Priorities:

- International standards (IS) for Infectious Disease markers, for blood screening and diagnostics

- Hepatitis - HBsAg, anti HCV, anti HBs

- anti HTLV \& anti HIV

- International blood grouping reagent standards

- Anti D, Anti A, Anti B 


\section{Traceability: Impact on Manufacturers}

\section{Global Cooperation in Developing Standards}

Reference Reagents for Prostate-specific Antigen (PSA): Establishment of the First International Standards for Free PSA and PSA (90:10) Brian Rafferty, ${ }^{1 *}$ Peter Rigsey, ${ }^{2}$ Matthew Rose, ${ }^{1}$ Thomas Stamey, ${ }^{3}$ and Rose GaINEs Das ${ }^{2}$

\section{Traceability: Impact on Manufacturers}

\section{Recommendations}

- Scientific \& Professional Organizations should provide guidance re: what standards are needed

- Define project priorities with recognition that resources are limited, taking into account...

- Public health significance and disease course

- Anticipated improvement in overall clinical effectiveness of tests, if a standard is developed

- Time/cost needed to reach a desirable endpoint

- Likelihood of success 


\section{Traceability: Impact on Manufacturers}

\section{Recommendations}

- Establish a defined, INTERNATIONAL consensus-based process for setting priorities and contracting projects

- Open, public dialogue \& decision process

- All key stakeholders must have a seat (i.e., profession, government, industry, lay public)

- Consider formation of an "IRSCL", following the NCCLS-NRSCL model

- Must be International in scope \& responsibility

- NCCLS could hold the secretariat role

\section{Traceability: Impact on Manufacturers Recommendations}

- Sufficient project funding (grants or contracts) must be provided

- Government sources (e.g. EU Commission, US Dept of Commerce, etc.)

- Professional societies (e.g. IFCC, AACC, CAP, WASP)

- May be supplemented with company grants

- Projects must be closely managed, with accountability for deliverables and schedule

- Project teams must be staffed with appropriate clinical and scientific experts, from the profession and industry 


\section{Traceability: Impact on Manufacturers}

\section{Conclusions}

- Although the Directive does not specifically require it, reference materials and reference methods development projects will be initiated in the name of the Directive

- New standards are likely to increase short term costs

- Manufacturers are NOT likely to take a lead position in advocating for new standards

- especially which standards

\section{Traceability: Impact on Manufacturers}

\section{Conclusions}

- Whenever IVD standards projects are undertaken (via professional, government and/or public health group sponsorship), inclusion of manufacturing scientists \& experts is absolutely required for project success

- Significant project funding from the public sector is necessary 


\section{History of Reference Systems for Clinical Measurements John H. Eckfeldt \\ University of Minnesota \\ Fairview-University Medical Center}

I must confess at the outset that my "history" will emphasize the U.S. activities, particularly the role the College of American Pathologists (CAP) played, because developments in this region are what I know best and have the majority of published reports in English. Nevertheless, I am certain that similar concerns by the medical and laboratory communities worldwide were being expressed and parallel efforts in other parts of the world are progressing. I am sure other speakers at this workshop will mention many of these developments from other parts of the world in their presentations.

Efforts to improve traceability of clinical laboratory measurements in the U.S. date back approximately to the middle of the twentieth century. One of the most seminal reports that described these early efforts was that of a study describing a comparison of clinical laboratory test results performed in 1946 by the Committee on Laboratories of the Medical Society of the State of Pennsylvania (Belk \& Sunderman, 1947). At that time only about a dozen analytes were routinely measured in hospital laboratories. In their study, interlaboratory variability of hemoglobin, glucose, sodium, total protein, albumin, calcium, and urea results were examined. Samples were distributed to 59 laboratories in Pennsylvania and surrounding states that had expressed willingness to participate. Data was collated from two different mailings approximately a month apart. Figures 1 and 2, which are reproduced from this early report, show the results for glucose and hemoglobin. In both cases, a number of the reported results were completely discarded, because
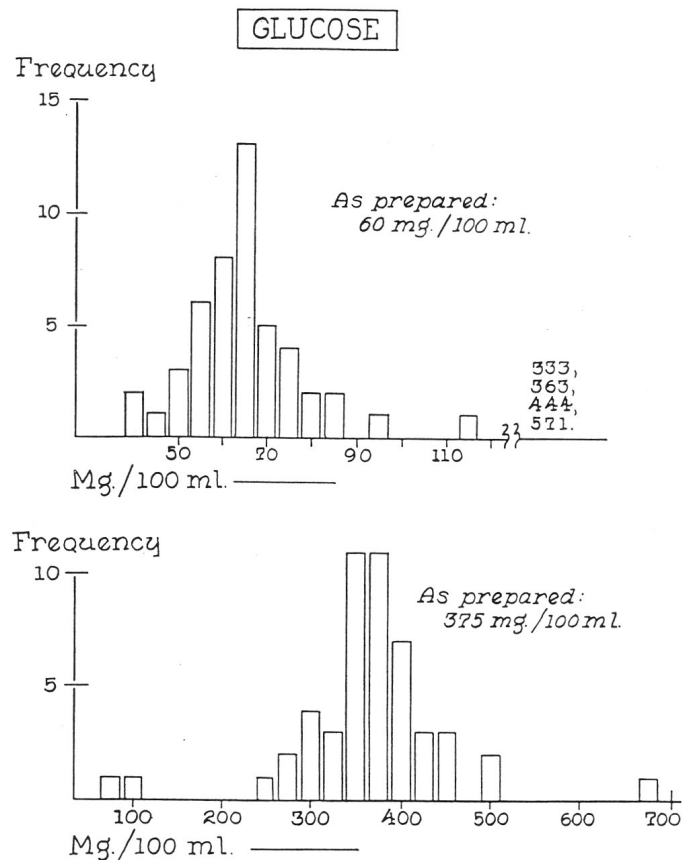

Figure 1. Frequency of results for an aqueous glucose control material from clinical laboratories in Pennsylvania and surrounding states in calendar year 1946. (Reproduced from Belk and Sunderman 1947 by permission). they were so totally out of the range that they would not fit on the graph as presented. In their discussion, Drs. Belk and Sunderman concluded, based on the opinions and information expressed by the participating laboratory staff and pathologists, that poorly or inadequately trained technicians, poor equipment, and a variety of miscellaneous factors 

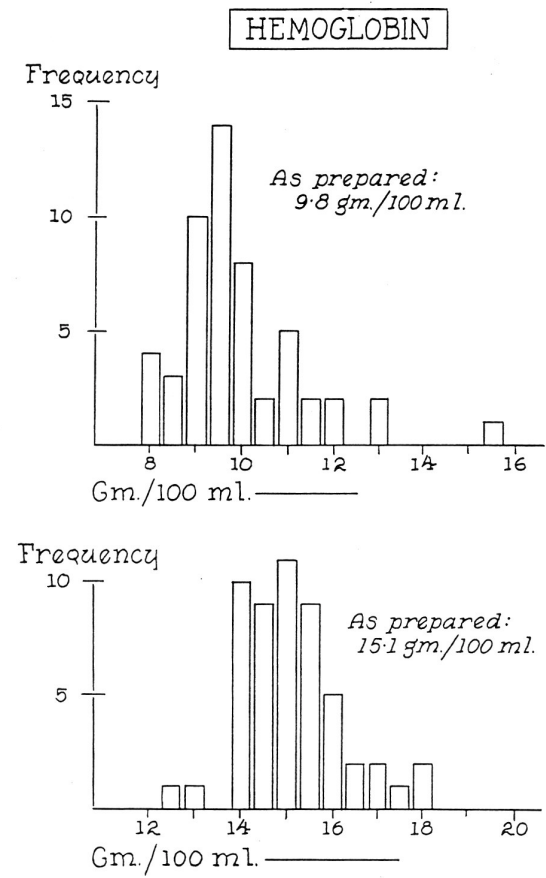

Figure 2. Frequency of results for a whole-blood hemoglobin control material from clinical laboratories in Pennsylvania and surrounding states in calendar year 1946 ( Reproduced from Belk and Sunderman 1947 by permission). contributed to the very high degree of variability between laboratories. The authors concluded that in many cases the clinical laboratories' results were so discordant from the true value that it was difficult to see how any reasonable clinical decisions taking into account laboratory information could be made!

Dr. Sunderman became a founding member of the CAP, guiding many of the CAP's early efforts toward investigation and improvement of inter-laboratory variability of clinical laboratory test results. Interestingly, now at age 100, Dr. Sunderman just contributed a brief report in the August 2000 issue of CAP Today on his personal experiences with today's U.S. medical care system comparing it to personal experiences approximately eight decades ago. Dr. Sunderman and other CAP founders began the CAP Survey's Program in 1949. The CAP Survey's Program was designed, and still is, a proficiency testing (PT) program, often called external quality assessment system (EQAS) in other parts of the world, whose major goal is to improve interlaboratory comparability of medically important analytes. Over the more than 50 years since its inception, the CAP Surveys Program has developed into the largest PT program in the world. In the calendar year 2000, it will include over 25,000 participating laboratories, testing over 250,000 different samples for over 500 different analytes.

Shortly after the beginning of the Survey Program, the CAP Standard Solutions program began in 1951. This program was designed to provide reference materials to clinical laboratories that could be used for calibration of their clinical instruments. In many ways this program marked the beginning of the development of a clinical laboratory "reference system" for the U.S. Because of continued inter-laboratory comparability problems with hemoglobin measurement, and the extreme importance of this analyte for clinical decision-making, the CAP opened a Clinical Standards Laboratory at the Cleveland Clinic, in Cleveland, $\mathrm{OH}$ in 1961. This laboratory provided reference measurements and the materials for the cyanmethemoglobin determination for clinical laboratories and for manufacturers of devices and reagents for hemoglobin measurement through the late 1970's at the Cleveland Clinic. In 1970, this laboratory was moved to the United States National Bureau of Standards (NBS), as the National Institutes of Standards and Technology (NIST) was then called, under a Cooperative Research and Development Agreement between NBS and CAP. Prior to this laboratory's move to the NBS, reference 
methods for billirubin and cholesterol, two other clinically important analytes, had been added to its repertoire. At its peak in the mid-1990's, under this agreement, three fully funded CAP employed Roger K. Gilbert Research Fellows worked side-by-side with NIST staff in the Gaithersburg, MD facilities providing high-level reference method analyses of NIST and CAP reference materials.

At the same time that the CAP's Clinical Standards Laboratory and Surveys Programs were evolving, another very important program sponsored by the college was being developed. In 1962, the CAP's Inspection and Accreditation program began conducting on-site inspections of clinical laboratories and issuing accreditation certificates to those laboratories that met the written standards designated to insure quality of the work they have provided. At approximately the same time, another historically and very important development in clinical laboratory reference systems was developing. Leaders of the CAP and the American Association for Clinical Chemistry (AACC), as well as other clinical laboratory-related organizations recognized the need for a very broad-based organization. These discussions culminated in the formation of National Committee on Clinical Laboratory Standards (NCCLS) as a non-profit corporation in 1968. A critically important aspect of this new organization was the recognition that improvement to clinical laboratory quality required a strong partnership between the professional, governmental, and IVD manufacturing sectors. NCCLS has as a major tenet that consensus building and a balance of industry, government, and professional representatives at all levels of the organization are needed for development of highest quality and workable clinical laboratory standards.

The various programs of these many different organizations continued to evolve through the late 1960's and 1970's. Clinical laboratory efforts on reference methods and materials specifically culminated in 1977 with a conference sponsored by CDC, FDA, and NBS, the published proceedings of which give an excellent overview of early clinical laboratory standardization activities (Boutwell 1978). This conference led to the (U.S.) National Reference System for Clinical Chemistry (NRSCC). The NRSCC was broadened two years later to cover analytes outside of traditional clinical chemistry and was renamed the National Reference System for Clinical Laboratory (NRSCL). Over the ensuing twenty-three years, numerous other efforts and organizations became involved in improving inter-laboratory comparability of reference methods and materials. As will be presented by other speakers, in Europe the clinical laboratory reference system movement culminated with the European Union's IVD Directive in 1998. This directive made traceability of clinical laboratory results to higher-level reference methods mandatory for in vitro diagnostic devices in European Community countries, and is largely the reason for our meeting here today in Gaithersburg.

Besides the NRSCL, which includes only about a dozen clinical chemistry analytes, there are numerous other "reference systems" worldwide. Some are limited to providing a pooled reference material (e.g., World Health Organization reference preparations), while others focus on both reference measurement procedures and reference materials. No doubt, most highly developed reference systems are for cholesterol. It has at its highest level ("definitive" in NRSCL terminology) an isotope dilution-mass spectrometry 
method, at its mid-level ("reference method" in NRSCL terminology) the Abell-Kendall extraction-based, colorimetric method, a NIST-supplied pure cholesterol "certified reference material" (CRM) and a variety of NIST-supplied serum or processed plasma-based "standard reference materials" (SRM). An adaptation of this cholesterol reference system using panels of patient specimens will be described later. Rather than trying to develop an exhaustive list all of the clinical laboratory reference systems, and no doubt missing many which individuals in the audience think are important, I thought it might be more useful to try to offer some of my personal observations of what factors I think have led to significant improvements in inter-laboratory comparability over my 23 years as a hospital-based clinical pathologist, directing progressively larger, and more diverse clinical laboratories.

I would start by saying that I believe that one of the key factors for improving clinical laboratory test accuracy, which is often not formally recognized as part of a clinical laboratory test "reference system," is a uniformly accepted way to measure interlaboratory variability and to make the finding publicly available for scrutiny by the laboratory community. Without a way to measure the current state-of-the-art and its improvement over time, nobody can really know how good or bad things are and whether they are getting better or worse. PT/EQAS programs are generally thought to fill this role. Certainly Sunderman and Belk's contributions stated a massive effort to improve

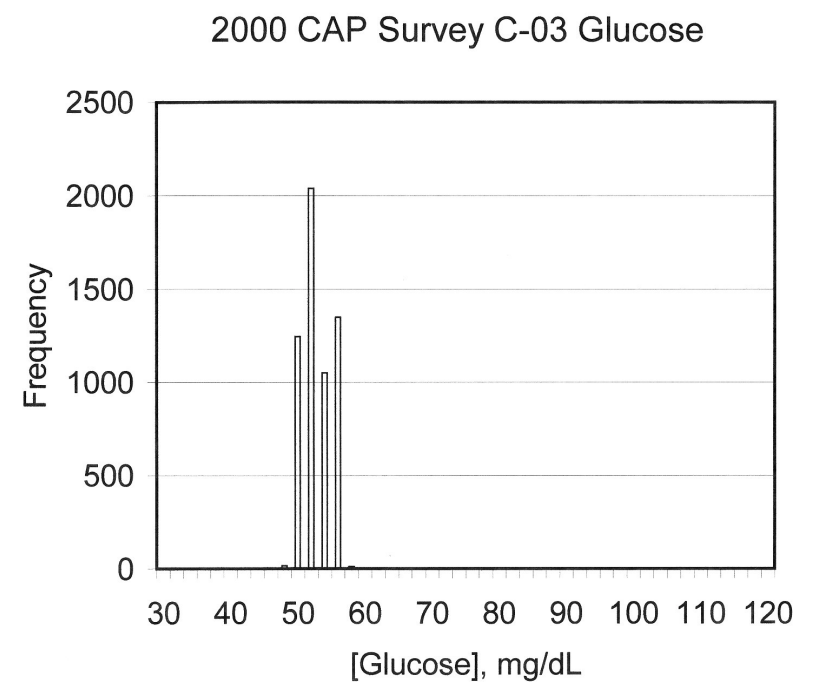

Figure 3. Frequency glucose results from a liquid whole blood control material from clinical laboratories participating in the CAP C-03 Survey in calendar year 2000 . laboratory test result accuracy in the US. Only with publicly available data that is accepted as a reliable representation of comparability of actual patient clinical test results, are clinical laboratories and manufacturers pressured to improve test accuracy. The CAP Surveys Program and other similar programs worldwide certainly yield considerable data on the performance of clinical laboratories. I believe this sort of data has been extremely instrumental in driving the immense improvement seen since Sunderman and Belk's original 1947 report. For example, Figure 3 shows the performance of laboratories reporting glucose results and Figure 4, hemoglobin results in the CAP General Chemistry Survey within the past year. These results obviously represent huge improvements over the findings of Belk and Sunderman. However, there is a subtle weakness of the CAP programs and most other PT/EQAS programs, which relates to the character of the PT/EQAS samples themselves. What are typically distributed are samples processed or preserved in some way (e.g., lyophilized 
serum, gluteraldehyde fixed red cell, etc.). This stabilizing process can lead to specimens that behave somewhat differently on clinical analyzers (but much less commonly in reference methods) than patient materials.

These problems have been termed "commutability" or "matrix effect" problems. The analyte most carefully studied for commutability/matrix effect problems is cholesterol. Figure 5 shows an example of comparing results of both fresh clinical serum specimens and CAP lyophilized Survey PT/EQAS samples as measured by a widely used, clinical laboratory method and the Abell-Kendall high-level reference method (Eckfeldt and Copeland 1993). If one looked only at the PT/EQAS samples, clinical method versus reference method results, one would come

Clinical Method vs. Comparative Method Showing Linear Regression Line and 95\% Confidence Limits

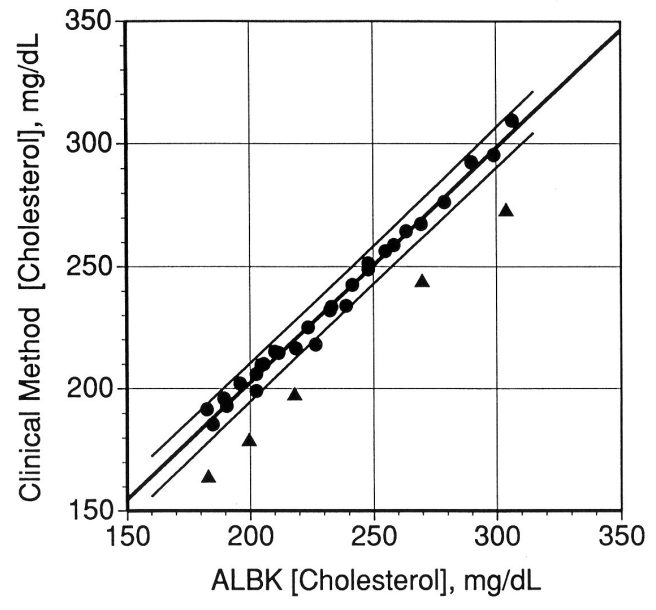

Figure 5. X,Y-plots of cholesterol results by a clinical method and the Abell-Kendall reference method for a set of patient specimens $(\bullet)$ and for five 1991 lyophilized processed human plasma CAP General Chemistry Survey specimens $(\stackrel{\bullet}{\wedge})$.
1999 CAP HE-03 Survey

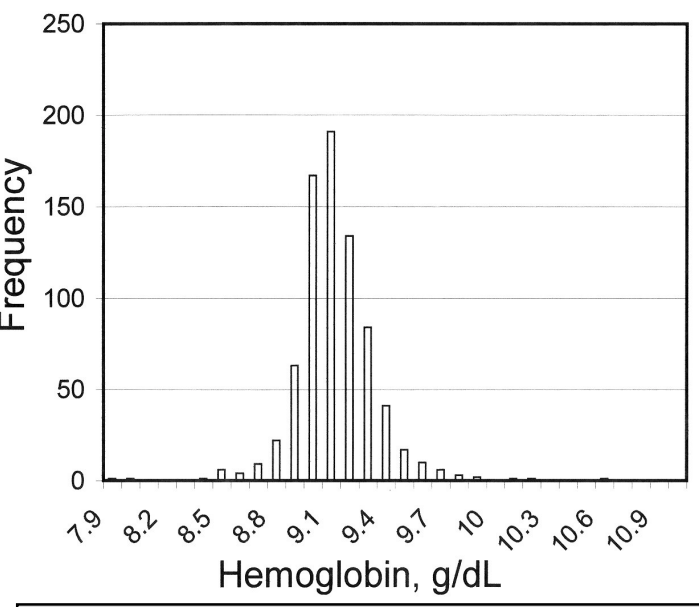

Figure 4. Frequency hemoglobin results from a liquid whole blood control material from clinical laboratories participating in the CAP HE-03 Survey in calendar year 1999. to the conclusion that the clinical laboratory method's results were $10 \%$ to $15 \%$ below the true cholesterol concentration in the samples. However, looking at the fresh patient results one can see that the clinical method shows a very high degree of accuracy. There have been examples where manufacturers have adjusted their IVD devices and reagents to make PT/EQAS samples' results comparable to reference method target values, but in doing so unwittingly making their results on patient samples less accurate. This situation leads to a potential mistrust of PT/EQAS results as being representative of the state-of-the-art for clinical test result accuracy. Commutability issues have created some tension between providers of PT/EQAS programs and the materials they use and IVD manufacturers whose clinical 
laboratory methods are being evaluated by such programs. The IVD manufacturers tend to claim that that "the problem" lies with "inferior" PT materials that do not accurately mimic clinical specimens, while those producing PT/EQAS materials claim it is a lack of "robust" clinical reagent and instrument systems. In reality, the "problem" belongs to the laboratory community in general. Certainly the early efforts started by Belk and Sunderman and their followers have led to major improvements. However, I believe that for many analytes, commutability/matrix effect issues have been a serious impediment to further improvements in inter-laboratory variability for many analytes for approximately the past decade. Commutability concerns raise doubts as to the reliability of the PT/EQAS data as being reflective to inter-laboratory comparability of patient sample results. Other speakers will discuss this topic in more detail.

Inter-laboratory comparability continued to improve during the 1970's and 1980's, but concurrently, commutability/matrix effect issues became a more and more serious concern. Several innovative approaches for reference systems developed that circumvented the commutability/matrix effect problem of more typical PT/EQAS materials. The earliest and most successful programs within North America, and probably worldwide, has been the U.S. National Heart, Lung, and Blood Institute (NHLBI)/Centers for Disease Control and Prevention (CDC) Lipid Standardization Program. Shortly after the landmark NIH-funded Coronary Primary Prevention Trial results were published showing that reducing serum cholesterol reduced coronary heart d6isease risk, major efforts

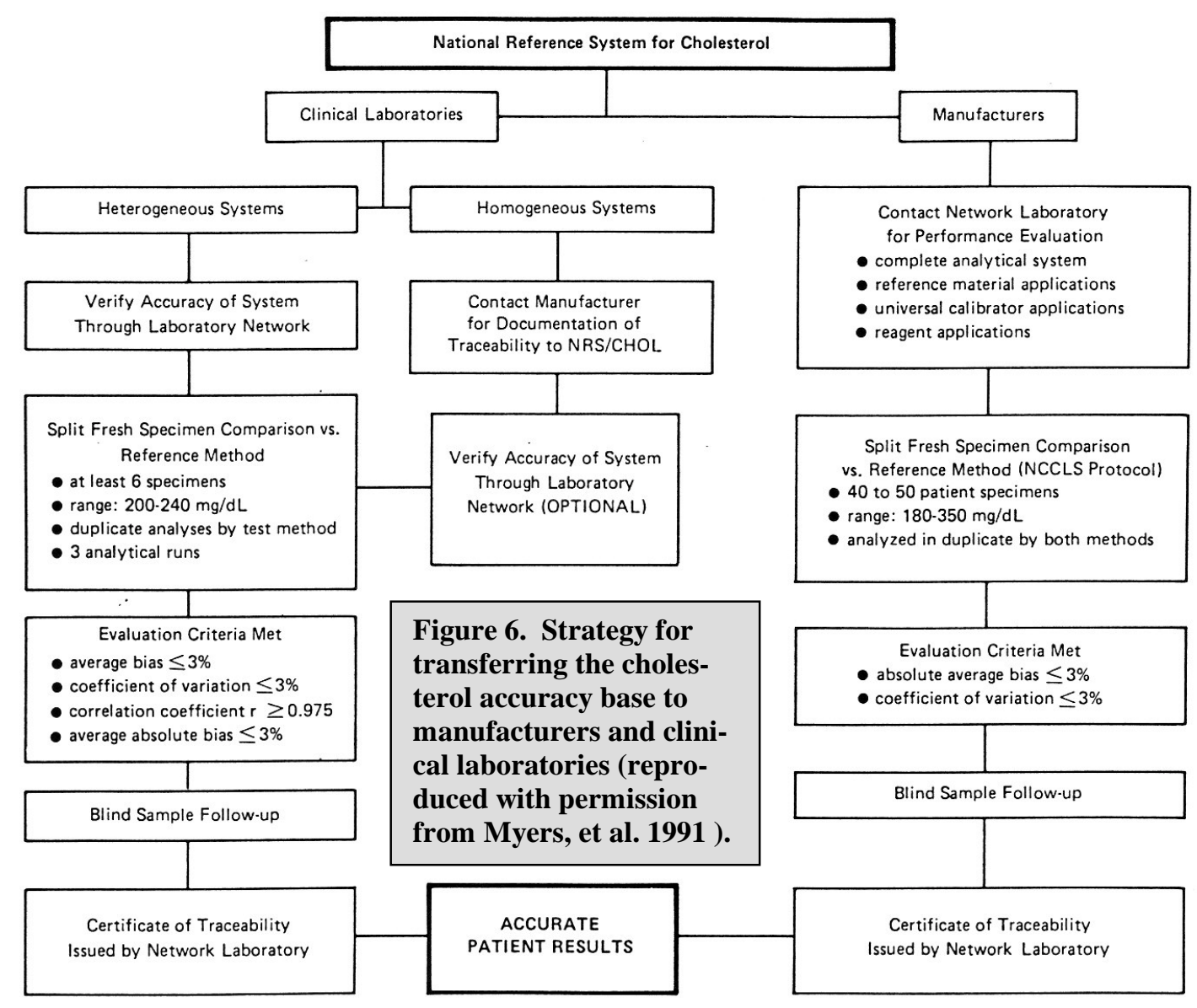


were begun to improve clinical laboratory cholesterol test accuracy. The National Cholesterol Education Program (NCEP) was formed as a cooperative NHLBI/CDC venture. An integral part of the NCEP program was the NCEP Laboratory Standardization Program (NCEP LSP). I think one of the key factors that lead to the immense success of the NCEP was that early on it garnered strong support from both the clinical and medical associations such as the American Heart Association and American Medical Association. Physicians were asked by these professional medical organizations to inquire whether the clinical laboratory that they used had NCEP Lipid Standardization (NCEP LSP) certification for lipid testing (Myers et al. 1991).

Early on, the NCEP LSP recognized the limitations of using lyophilized reference materials for calibration and PT/EQAS purposes. Two different formats of the program were designed. One distributed frozen serum pools that were prepared at CDC and shipped to laboratories, primarily those involved in NIH funded research projects on a periodic basis. These pools were frozen quickly, never lyophilized, and stored at $-70{ }^{\circ} \mathrm{C}$. As a result, they tended to behave much more like fresh clinical specimens in the field methods than the typically lyophilized reference materials for lipids that were available in the early 1960's. Abell-Kendall cholesterol, as well as high-level reference method HDLcholesterol and triglyceride values, served as the top of the traceability chain for the NCEP LSP. In a slightly different format offered to any manufacturer of cholesterolmeasuring IVDs and to clinical laboratories, fresh patient sample analysis by CDCcertified Network Laboratories became available to document the accuracy of the clinical test results. The major shortcoming of the program, particularly for clinical laboratories, was the significant expense involved. As a result, although most major manufacturers of cholesterol-measuring reagents and instruments became NCEP certified, only a very small percentage of clinical laboratories ever went through the process. A parallel initiative begun in the mid-1990 to improve the traceability of lipid values in clinical laboratories in a more cost-effective way was begun by NCCLS, CAP, and NIST. The project's aim was to develop a reliable method for producing frozen serum reference materials for lipids that was free of commutability/matrix problems. A summary of this project was recently published (Myers et al., 1999).

A more recent example of another analyte standardization effort that was largely motivated by clinical professional society demands is the use of glycohemoglobin. Shortly after the publication of the NIH-sponsored Diabetes Control and Complications Trial (DCCT) study results in 1993, hemoglobin A1c or glycohemoglobin became a pivotal clinical laboratory test predicting the risk of diabetic complications and managing diabetic care of individual patients. The clinical diabetes societies, principally the American Diabetes Association (ADA), began proposing numerical glycohemoglobin target values for diabetic care, without regard for the method-specific reference ranges being used by different clinical laboratories performing the test. Suddenly, interlaboratory comparability of test results became a major issue. In 1994, AACC chartered a subcommittee of its Standards Committee to develop a standardization program to assure that clinical laboratories would report glycohemoglobin values equivalent to the DCCT method. This subcommittee evolved eventually into the National Glycohemoglobin Standardization Program (NGSP). In the mid-1990's, NCCLS began developing a 
guideline document for this process, which is now nearing finalization. Not only was the ADA and AMA giving strong support for reporting NGSP-certified (i.e., DCCTequivalent) results, but also the CAP became involved through its Glycohemoglobin Survey, which began in the early 1990's. However, this initial Survey (GH Survey) used lyophilized samples. Fortunately, it was quickly realized that these materials suffered from commutability/matrix effect problems quite analogous to the cholesterol lyophilized PT/EQAS materials. In 1996, to circumvent the commutability/matrix effect problems, the CAP initiated a new fresh whole blood proficiency testing program (GH2 Survey), where blood was collected from diabetics and shipped in a liquid form to clinical laboratories so that it could be tested within a few days of collection. This GH2 Survey allowed for direct comparison of various methods that were used in clinical laboratories. By the year 2000, glycohemoglobin results reported in the GH2 Survey have seen a greater than 2 -fold reduction in the variability of the method-specific glycohemoglobin results. This improvement was possible only through close cooperation of the manufacturers of IVDs for glycohemoglobin and the NGSP. I believe that this program's great success resulted not only from the previously mentioned important ability to measure and publicly display specific methods results on samples that very closely mimic clinical specimens, but there was another very important factor. What it had was large, very influential clinical organizations (i.e., the ADA and AMA) that formally and strongly endorsed the need for comparable patient results, regardless what IVD manufacturer instruments and reagents were being used.

Another example of a program aimed at improving inter-laboratory comparability, with what I personally view as having had much more limited success is the reference system for prostate-specific antigen (PSA). The use of PSA in monitoring and more recently diagnosing prostate cancer goes back about 15 years. However, many reports including the CAP Survey data and others, had shown inter-assay variability of as much as two-fold for some samples in the clinically important 4 to $15 \mathrm{ng} / \mathrm{mL}$ range. In the mid1990 's there was even a recommendation that the change of PSA, over 2 to 4 years, should be used for diagnosis of prostate cancer. This recommendation made interlaboratory variability even more problematic. In an era where patients often change health care plans and laboratories every few years, a large change in PSA might be due totally to analytical difference in the methods used by two labs, or vice versa, and they may mask clinically important changes. Several PSA standardizations conferences were held and recommendation for a calibrator with a fixed ratio of free and bound PSA were made. The proposal to use free and bound mixtures of PSA in the reference materials were based on the fact that some assays were much more sensitive to free PSA than to alpha-1-antichymotrypsin bound PSA. The problem was magnified by the fact that many of the PT/EQAS materials used semen added to normal human plasma leading to a very atypical bound-to-free ratio giving widely discordant results using different clinical methods that were not representative of what was seen when clinical specimens were analyzed. This well-known problem of PT/EQAS materials not being representative of results on clinical specimens, severely hampered progress in improving the inter-method variability. In the September 2000 issue of Clinical Chemistry, the "first international standards" for PSA are described (Rafferty et al., 2000). However, the serious limitations of even these reference materials to reduce inter-method variability without manufactur- 
ers significantly changing their method design, was pointed out in an accompanying editorial (Chan and Sokoll, 2000). How this attempt to improve inter-laboratory comparability of a complex protein that is present in different forms in clinical samples succeeds is yet to be seen. I would emphasize that I give the PSA standardization example not to cast any aspersions on those involved in these efforts, but to highlight the extreme complexity of trying to develop traceability of an analyte that is present in different molecular forms in patient materials. Standardization of cardiac troponin methods offers very similar challenges.

One could go on describing other clinical laboratory standardization efforts, but I believe the examples already presented are reasonably representative of the problems faced. It might be instructive to speculate why some clinical laboratory analyte traceability programs have been reasonably successful, and others less so. My observations are that key factors leading to improvement in inter-laboratory variability include:

1) Results of a widely publicized clinical research study comes to the conclusion that clinical action should be based on uniform cut-points of some laboratory test and this leads to strong pressure from large clinical organizations,

2) A high-level reference method and/or material must exist or must be developed,

3) There must be a way to easily and reliably disseminate this accuracy base provided by the reference method or material,

4) There must be a way to reliably evaluate and publicly display inter-method and interlaboratory comparability results.

Examples of key factor \#1 are the CPPT study results and NCEP clinical cut-points as well as massive "know your number" (i.e., serum total cholesterol) campaign by the NCEP, the DCCT and ADA glycohemoglobin cut-points for good, marginal, and poor control of diabetics. The fact that several studies showing utility of serum PSA testing have not led to comparable improvement in inter-method variability of PSA results, suggests that key factor \#1 while perhaps necessary, is really not sufficient. Traceability schemes typically have at the top of the hierarchy a "high-level" reference material. Even when there is a high-level reference method or a material as listed in key factor \#2 at the top of the chain, the major limitation in practical distribution of this accuracy base to the field is the lack of commutable reference materials (key factor \#3). The NGSP glycohemoglobin and NHLBI/CDC Lipid Standardization Program circumvented this problem by direct analysis of patient samples as part of the system. Efforts such as the NCCLS/CAP/NIST frozen serum reference material for cholesterol is an example of efforts to address key factor \#3. Finally with respect to key factor \#4, unless we know how good or bad inter-method and inter-laboratory variability are, little progress is typically made. Here is where PT/EQAS programs that use commutable reference materials are needed. 
I believe that prior to starting the development or improvement of a reference system, looking at the scattergram comparisons of field method results versus high-level reference method results, using a number of clinical samples from normal healthy subjects as well as samples from patients with the diseases the test designed to manage or diagnose, is extremely useful. If all the clinical field methods show very high degrees of correlation (i.e., " $r$ " approaching 1.0 and $\mathrm{S}_{\mathrm{y} . \mathrm{x}}$ not much larger than the intra-assay SD of the methods), the problem is relatively straightforward. Recalibration with some or all of the field methods with a commutable calibration material should be relatively easy. However, if the correlation is not good (i.e., $r<<1.0$ and $\mathrm{S}_{\mathrm{y} . \mathrm{x}}$ much larger than the intra-assay $\mathrm{SD}$ of the method) the problem is much more complicated. Recalibration may improve the traceability on average, but it will not correct the inter-method comparability of many of an individual patient specimen. While recalibration may give the impression that traceability has improved, for that individual patient whose result is still two-fold different with one clinical method compared to another, there is still a very serious problem when his doctor tries to develop a rational diagnosis or treatment program. This situation is still fundamentally where PSA and many other protein/peptide hormones as well as several chemically well-define hormone assays (e.g., cortisol) stand. These analytes, unfortunately, are those that are most problematic for the clinical laboratories and the clinicians that use them. Frankly, clinical practitioners largely lost interest in interlaboratory variability of sodium and cholesterol more than a decade ago.

I believe it is worth examining the reasons for poor correlation between clinical field method results versus reference method results more carefully. When results are not highly correlated, there are basically two possible causes. For analytes that are well defined chemically, it is likely that the field method has significant analytical interferences from substances found in varying concentrations in normal clinical samples. These interfering substances are often not found in reference materials, especially those prepared by adding purified preparations of the analyte of interest to normal pooled samples. An example of this situation would be cortisol and other steroid hormones. For many nonextraction or non-chromatographic assays, interfering steroids with similar chemical structures can be found at reasonably high concentrations in samples from patients, particularly in those with the very disorders that clinicians are trying to diagnose. Calibrators with increasing amounts of cortisol prepared by adding pure cortisol to pooled normal serum, or even worse an aqueous-based calibration solution, hardly mimic clinical samples. Nevertheless, the problem with chemically well-defined analytes is solvable, although it may require major changes in the fundamental design of the clinical methods to make them analytically more specific.

The second general cause that leads to poor correlation between the field and reference methods ((i.e., $r<<1.0$ and $S_{y . x}$ much larger than the intra-assay SD of the method) is that the analyte itself not well defined or is present in multiple molecular forms that are heterogeneously distributed. Examples of this situation are PSA, troponin, and chorionic gonadotropin. All of these proteins exist in multiple molecular forms in patient samples. Different immunoassays recognizing different epitopes present on the various molecular forms. Thereby, the different field methods yield different quantitative results depending on what forms are present and in what relative amounts they are present. In such a situa- 
tion, it is not at all clear, at least to me, what the approach should be for improving interlaboratory comparability. First, it seems that there must be some agreement on what is the clinically most appropriate "analyte" to measure through some national or international consensus process. However, the logistical difficulties and economic incentives become real barriers to progress. Various manufacturers often contend that their method is clinically superior to others, because it gives different answers. Only with relatively large and expensive clinical outcome studies can the question of superiority or inferiority of various assay designs be made. In my experience, such studies are extremely difficult to design with adequate statistical power and in an unbiased manner. Unfortunately, some of the most problematic analytes tend to fall into this category that is very difficult to solve.

In summary, establishing an on-going documentation of the traceability of field methods to reference methods is both difficult and expensive. Clinical studies must document a clinical benefit of treating a uniform numerical laboratory result or diagnosis or management of a disease process. There must be a widely perceived clinical payback for the reduction of inter-method and inter-laboratory variability for the clinical laboratory and manufacturing community to make it a priority. There must be close cooperation of the users, producers, and regulators of laboratory testing for the effort to be a success. Finally, a poorly defined or heterogeneous clinical analyte creates a special problem. Successful inter-laboratory standardization for analytes such as PSA and troponin will take new and innovative approaches to successfully improve inter-laboratory comparability. Hopefully, this workshop will provide a sound foundation and workable ideas leading to major progress in these efforts. 


\section{References}

1. Belk WP, Sunderman W. A survey of the accuracy of chemical analyses in clinical laboratories. Am J Clin Pathol 1947;17:854-61.

2. Boutwell JH, Editor. A national understanding for the development of reference materials and methods in clinical chemistry: proceedings of a conference. Washington, DC: American Association for Clinical Chemistry, 1978.

3. Chan DW, Sokoll LJ. WHO first international standards for prostate-specific antigen: the beginning of the end for assay discrepancies? Clin Chem 2000;46:1291-2 Editorial.

4. Eckfeldt JH, Copeland KR. Accuracy verification and identification of matrix effects: the College of American Pathologists' Protocol. Arch Pathol Lab Med 1993;117:381-6.

5. Myers GL, Eckfeldt JH, Greenberg N, Levine JB, Miller WG, Wiebe DA. Preparation and validation of commutable frozen human serum pools as secondary reference materials for cholesterol measurement procedures; Approved Guideline. NCCLS Document C37-A. Wayne, PA: NCCLS, 1999.

6. Myers GL, Henderson LO, Cooper GR, Hassemer DJ. Standardization of lipid and lipoprotein methods In: Rifai N, Warnick GR, Eds. Methods for clinical laboratory measurement of lipid and lipoprotein risk factors. Washington, DC: AACC Press, 1991:101-25.

7. Rafferty B, Rigsby P, Rose M, Stamey T, Das RG. Reference reagents for prostatespecific antigen (PSA): establishment of the first international standards for free PSA and PSA (90:10). Clin Chem 2000:46:1310-7. 


\section{JOHN H. ECKFELDT}

\section{University of Minnesota Medical School Department of Laboratory Medicine and Pathology}

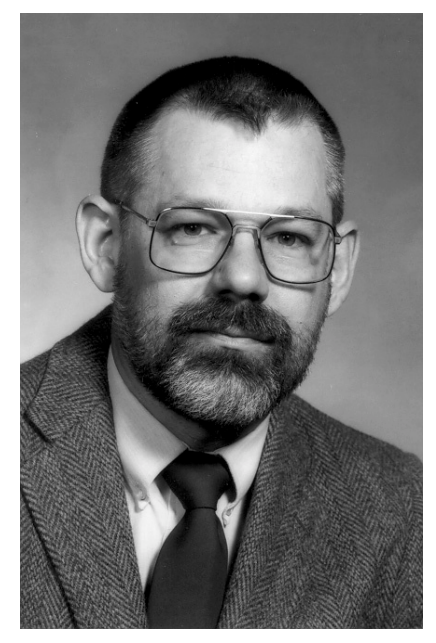

John H. Eckfeldt, M.D., Ph.D., is currently a Professor in the Department of Laboratory Medicine and Pathology in the University of Minnesota's Medical School having served on the University's faculty since 1978. His clinical responsibilities include the medical direction of the Clinical Laboratories at the Fairview University Medical Center in Minneapolis that is the tertiary care hospital for the Fairview Health System, a seven hospital and thirty clinic health care system which provides care for approximately one-half million individuals in Minnesota. He holds a masters degree in physical chemistry from Cornell University and an M.D. and Ph.D. in biophysics from the University of Pennsylvania. His primary research interests include risk assessment for cardiovascular disease, hypertension, and iron overload disorders using traditional blood and body fluid analyses as well as genetic testing. He has a long-term interest in proficiency testing and clinical laboratory method standardization. In the past he has served as a member of the College of American Pathologists' Chemistry and Therapeutic Drug Monitoring and Endocrinology Resource Committees. Presently, he is a member of the College's Biochemical and Molecular Genetics Resource Committee and chairs their Standards Committee. He has also served on the American Association for Clinical Chemistry's Standards Committee since the early 1990's. Since 1995, he has also served on Working Group 2 on reference methods and materials of ISO Technical Committee 212. 


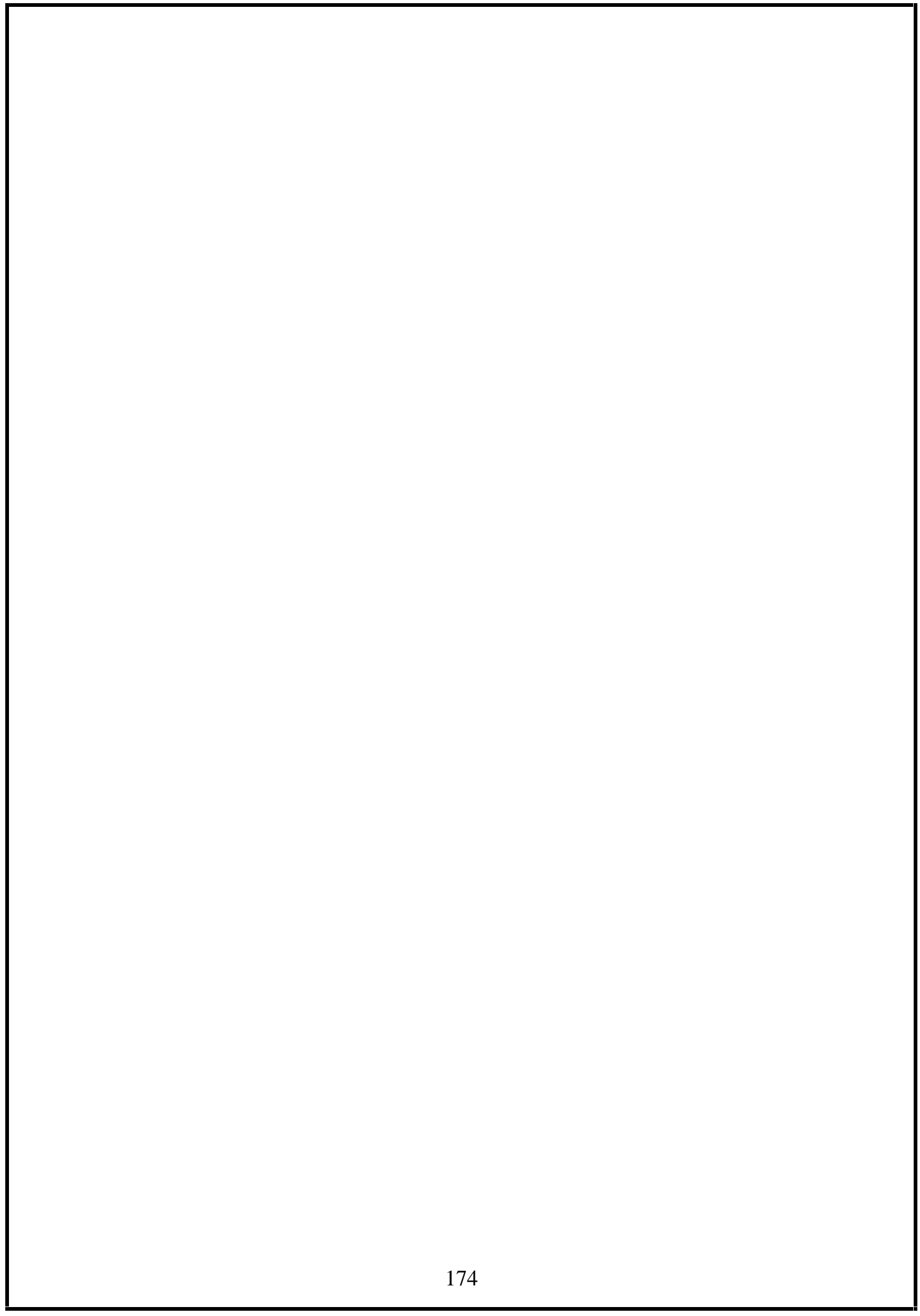


History of Reference Systems for Clinical Measurements

Visuals Presented By

John H. Eckfeldt

Department of Laboratory Medicine and Pathology

University of Minnesota

Clinical Pathology, Fairview-University Medical Center 


\section{History of Reference Systems for Clinical Measurements}

\section{John H. Eckfeldt, MD, PhD}

Medical Director, Clinical Pathology, Fairview-University Medical Center

Professor, Department of Laboratory Medicine and Pathology

University of Minnesota

\section{Topics To Be Addressed}

- Historical view of traceability efforts with an emphasis on US and CAP experiences,

- Role of PT for assessing traceability/trueness and its shortcomings,

- Two examples of working systems within the US to establish traceability of important clinical analytes (NHLBI/CDC Cholesterol Reference Method Laboratory Network and National Glycohemoglobin Standardization Program),

- Example of program with very limited success (PSA)

- Characteristics of "successful" programs to establish traceabilty. 


\section{Milestones in US Inter-laboratory Comparability Improvement}

1947 Belk \& Sunderman's study of clinical laboratory chemistry analytes in Pennsylvania and surronding states,

1949 CAP Surveys Program begin,

1951 CAP "Standards Solutions” begin,

1961 CAP Clinical Standards Laboratory instituted

for cyanmethemoglobin,

1977 CDC/FDA/NBS conference on reference

methods and material --> NRSCC and NRSCL

1998 EC IVD Directive issued

\section{Eras of Traceability}

1940's and 1950's discovering how bad things are, 1950's and early 1960's better reference methods for clinical analytes begin to be developed, 1970's and 1980's develop wider variety and availability of reference methods and materials for clinical analytes, 1990's better understanding of matrix effect/ noncommutability problems--more commutable reference materials are developed for several analytes,

2000's develop better inter-laboratory traceability for clinically important analytes??? 

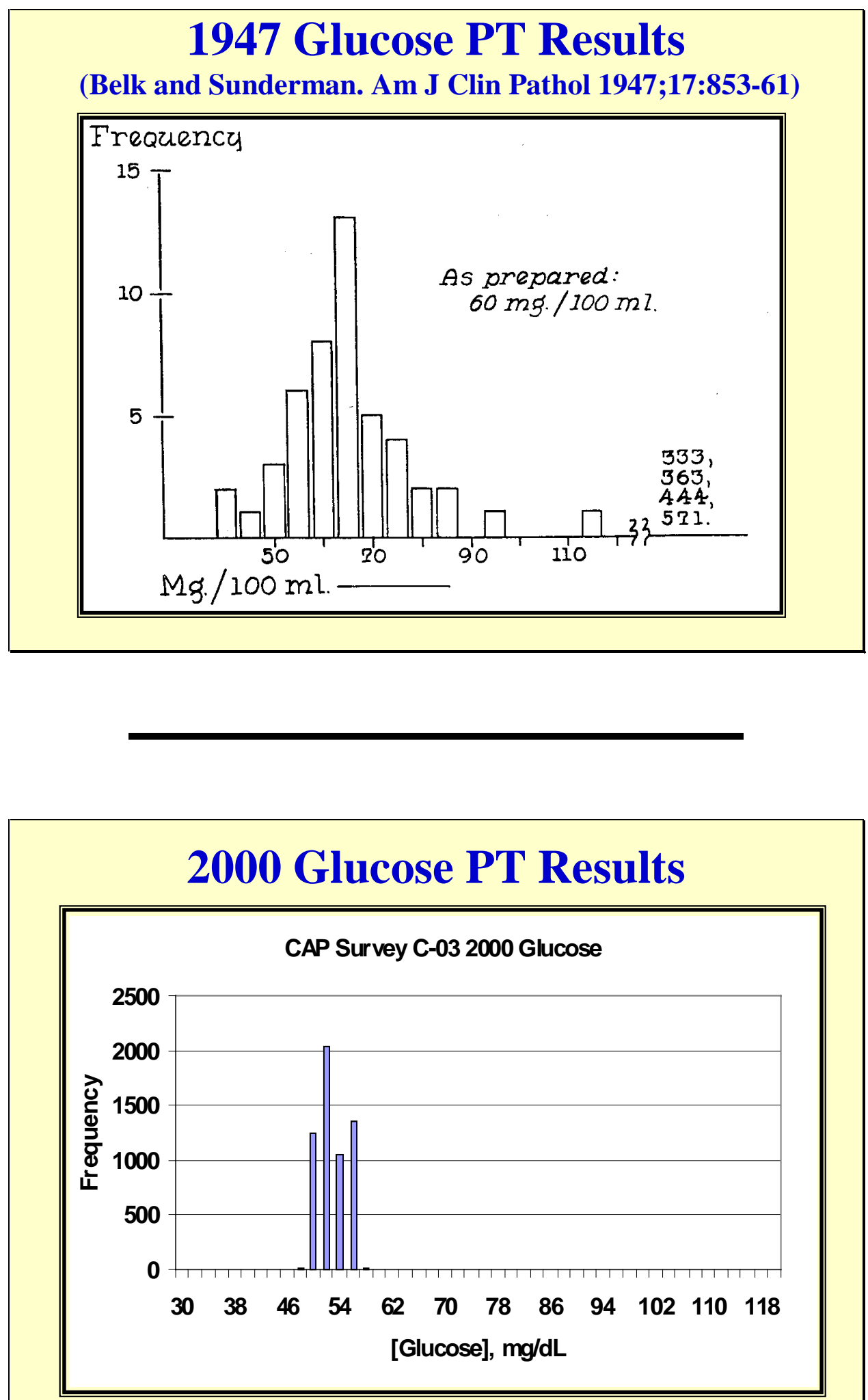


\section{Hemoglobin Proficiency Testing Results}

(Belk and Sunderman. Am J Clin Pathol 1947;17:853-61)

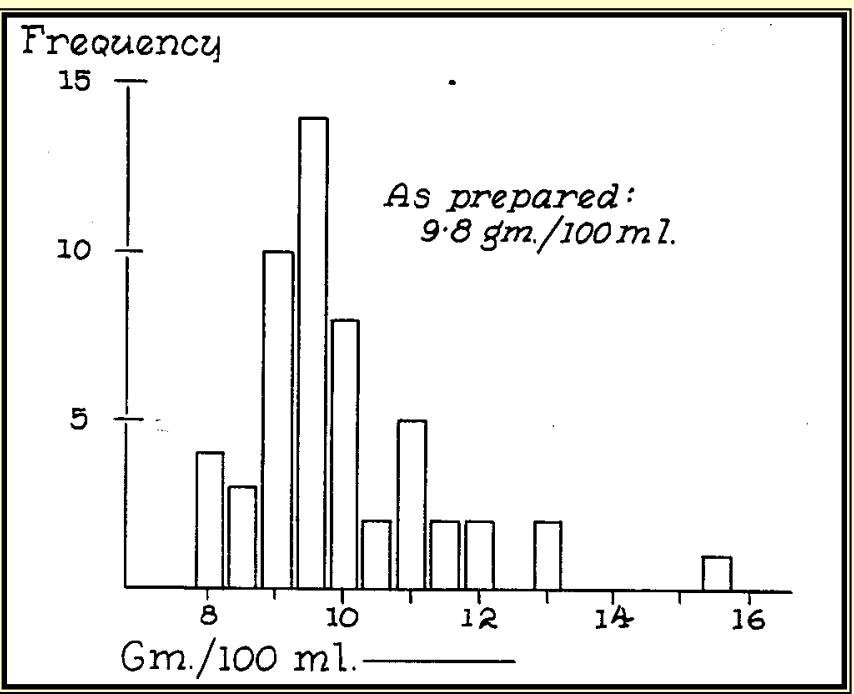

\section{Hemoglobin PT Results}

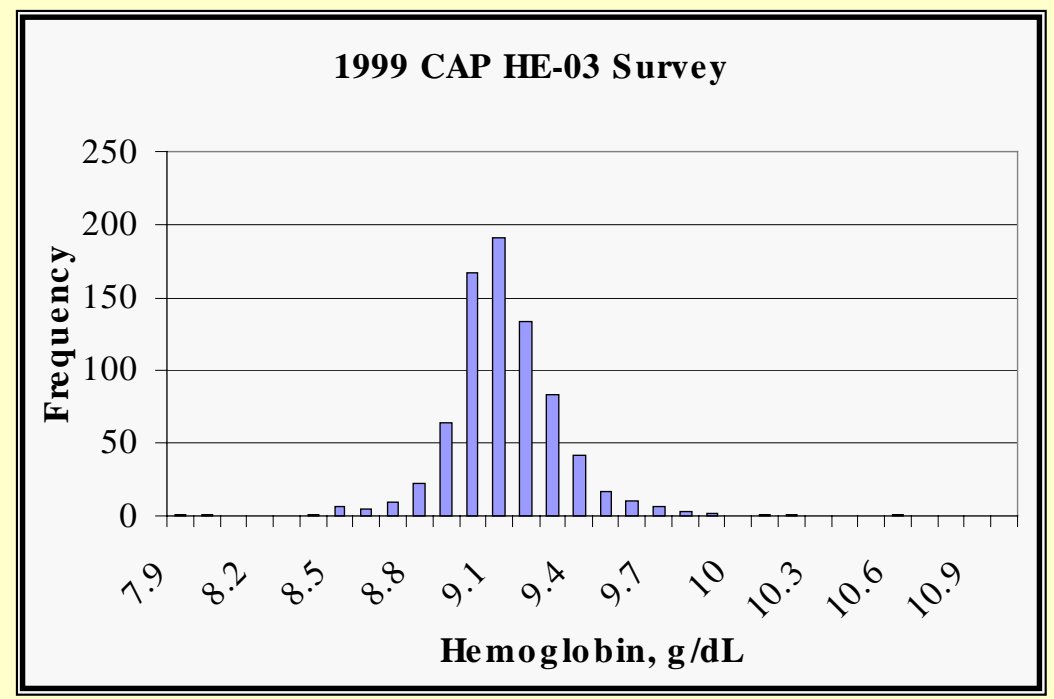




\section{CAP Surveys PT/EQAS Program}

- Evolved from Belk and Sunderman's 59 labs with 24 samples testing performance for seven analytes distributed in 1947 to $>25,000$ labs with $>250,000$ samples testing $>500$ analytes in 2000

- Liquid, lyophilized, frozen serum, plasma, whole blood, urine, CSF samples sent from 2 to 4 times/yr

- Strengths:

- Largest clinical laboratory performance data base in the world

- Largest number of analytes of any PT program

- Weaknesses:

- Large dependence on "peer group" comparisons

\section{Limitations of Lyophilized Reference Materials as "Trueness Controls" and Calibrators}

- Non-commutable "trueness controls" can lead to erroneous conclusions as to accuracy of a method in a PT/EQAS situation,

- Non-commutable calibration materials can lead to erroneous patient values if the high-level reference method value is used to calibrate a clinical instrument. 


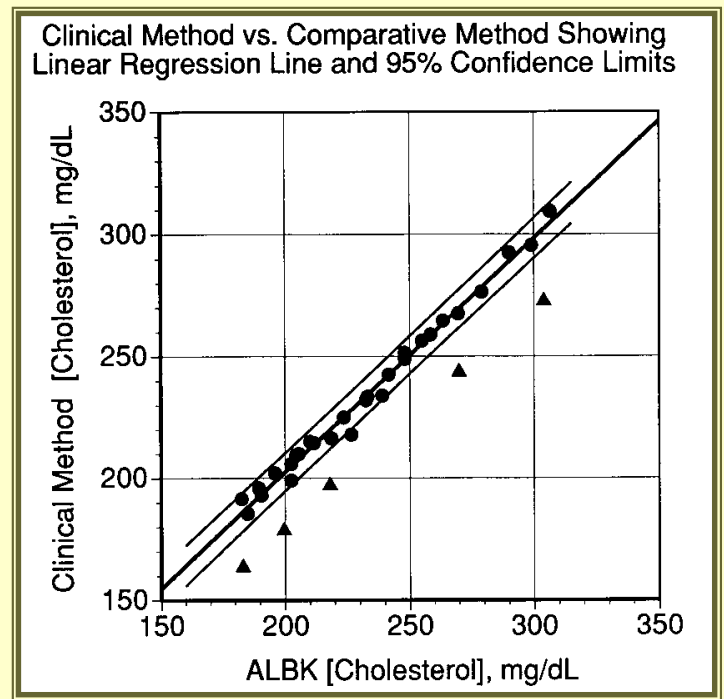

Eckfeldt and Copeland. Arch Pathol Lab Med 1993;117:381-6.

\section{Example \#1: NHLBI/CDC Lipid Standardization Program}

- Cholesterol Coronary Primary Prevention Trial and shortly thereafter numerous other research studies publish results that reducing serum cholesterol reduces CHD risk,

- NCEP is formed with CDC/NHLBI Laboratory Standardization Program formed as an integral part,

- NCEP gets strong support from American Heart Association, AMA, etc.

- Shortcomings of lyophilized reference materials recognized early as calibrator and PT samples, 


\section{Example \#1: NHLBI/CDC Lipid Standardization Program (Continued)}

- Frozen serum pools and fresh patient sample comparisons used for accuracy transfer,

- Manufacturers and clinical laboratory certification based on sound statistical principles,

- Shortcoming: expensive and time consuming for clinical lab participation,

- Led to development of a NCCLS/CAP/NIST frozenserum reference material for lipids (cholesterol, triglyceride, HDL-cholesterol, LDL-cholesterol) to assist IVD manufacturers calibrate their methods better.

\section{Example \#2: National Glycohemoglobin}

\section{Standardization Program}

- In 1993, DCCT and later other research group publish results that reducing average blood glucose reduces risk of diabetic complications,

- In 1994, AACC Subcommittee of Standards Committee begins to develop a standardization program for clinical laboratories to enable them to report "DCCT-equivalent"

- AACC Subcommittee evolves into the National Glycohemoglobin Standardization Program (NGSP) and NCCLS draft guideline document, 


\section{Example \#2: National Glycohemoglobin}

Standardization Program (Continued)

- American Diabetes Association and AMA give strong support of reporting NGSP-certified (i.e., DCCT-equivalent) results,

- In 1997, CAP begins fresh whole blood PT program (GH2 Survey) which directly shows comparability of field methods to reference methods,

- By 2000, there is a more than two-fold reduction in inter-method variability of commercial glycohemoglobin results in the CAP Surveys.

\section{Example \#3: PSA Standardiztion}

\section{Program}

- In the early 1990's, several studies document the usefulness of PSA in tracking prostate cancer treatment and to some degree diagnosis in combination with digital rectal examination,

- Inter-assay variability is $>\mathbf{2}$-fold for some samples in the 4 to $10 \mathrm{ng} / \mathrm{mL}$ range,

- In the mid-1990's, change in PSA over 2 to 4 years proposed as more indicative of prostate cancer than a single value,

- In the mid-1990's, the first and second "Stanford Conferences" recommend "90:10 calibrator," 


\section{Example \#3: PSA Standardiztion Program (Continued)}

- At the urging of the American Cancer Society, in 1993, CAP develops a frozen serum-based reference material,

- In the late 1990's free/bound PSA ratios are proposed as giving additional diagnostic information,

- In 2000, there has been little change in inter-assay variability of the commercially available total PSA assays.

\section{Why Are Some Traceability Programs} Successful and Others Not?

- Clinical research study results recommend clinical action based on uniform cut-points nationally or internationally,

- Strong clinical consensus on treatment or diagnosis based on these uniform cut-points was developed,

- A well accepted "higher level" reference method exist or are developed to which the clinical study can be traced,

- Reference materials are commutable across the reference and field methods OR traceability schemes using clinical samples are devised and used, 


\section{Why Are Some Traceability Programs Successful and Others Not? (Continued)}

- Although different field methods may be biased compared to reference method, they are reasonably well correlated to it (i.e., $r \sim 1.0$ and Sy.x is the same order of magnitude as analytical SD of the methods),

- "Analyte" that is clinically important is reasonably well defined

\section{Outcome "A" When Comparing Field} Method to Reference Method Results

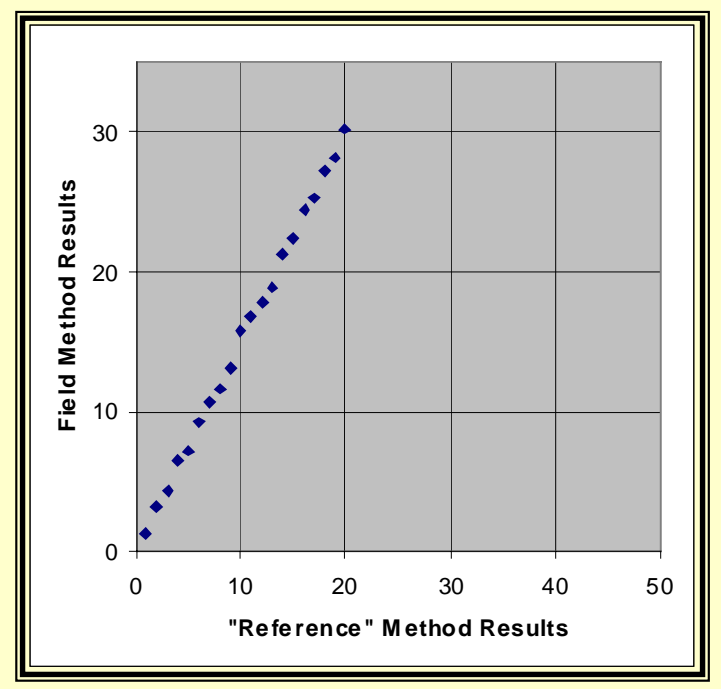




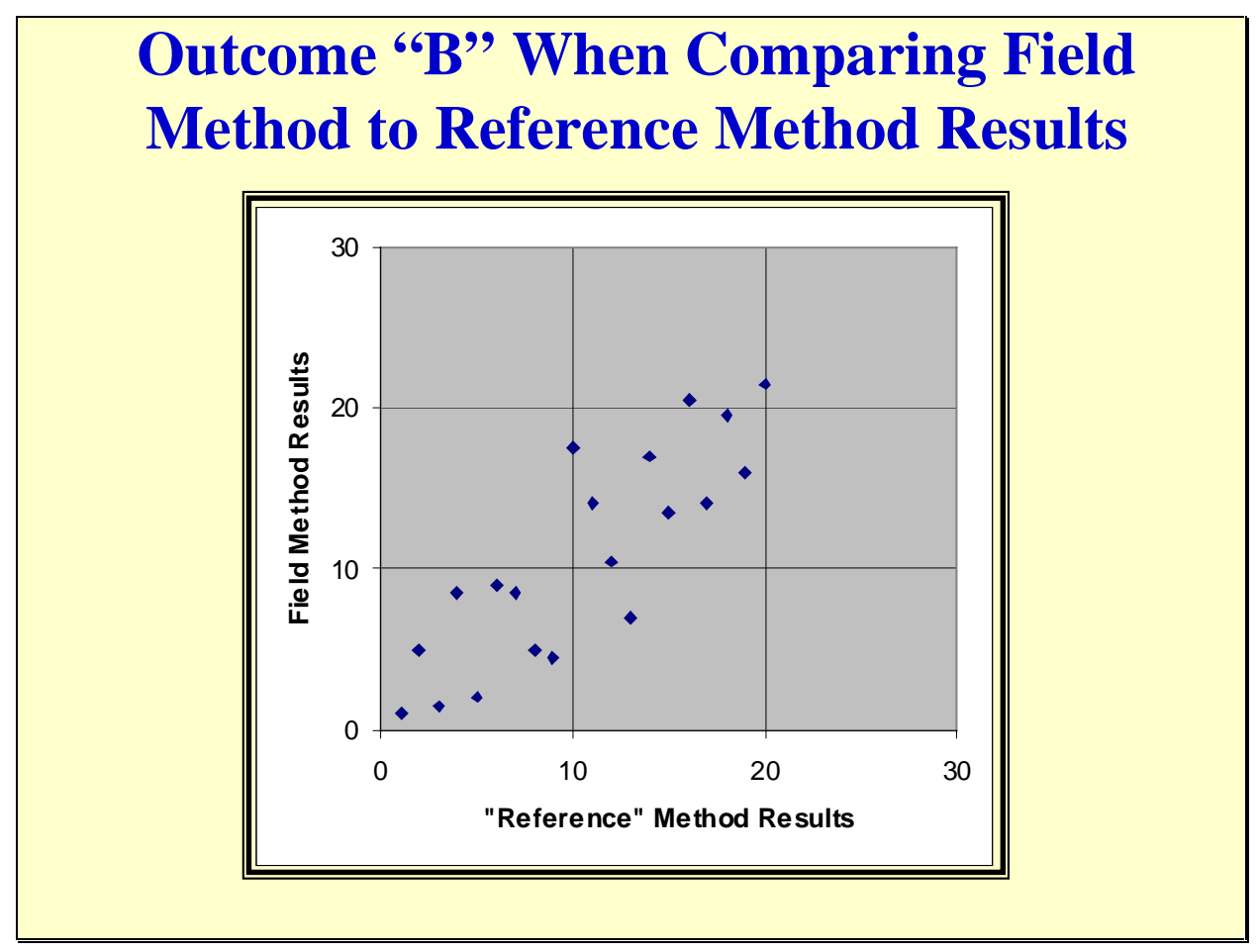

\section{Outcomes When Comparing Field Method to Reference Method Results}

- Outcome A: Results are highly correlated (i.e., r close to one, $S$ y.x approximates analytical CV's of methods)

- Outcome B: Results are not highly correlated (i.e., $r$ much less than one, $S$ y.x much larger than analytical CV's of methods)

- Outcome B1: Analyte is well defined, but field method has significant analytical interferences from substances normally found in typical clinical samples (e.g., cortisol)

- Outcome B2: Analyte itself is not well defined or heterogeneous (e.g., troponin, PSA, HCG, etc.) 


\section{Solutions to Traceability Problems}

- Outcome A: Relatively easy to solve by recalibration,

- Outcome B1 (well defined analytes): Major measurement procedure redesign needed to minimize impact of "influence quantities"--for example, change to more selective primary antibody); change to more selective analytical methods (e.g., chromatography, mass spectrometry, etc.).

\section{Solutions to Traceability Problems (Continued)}

- Outcome B2 (poorly defined analytes):

- First there needs to be agreement on what is clinically most appropriate "analyte" to measure through national/international consensus process,

- Potentially major redesign of some or all field methods--e.g., PSA, HCG, troponin, etc. 


\section{Summary}

- Establishing and on-going documentation of traceability in field methods is both difficult and expensive,

- Therefore, there must be clinical pay-back for this to become a clinical laboratory priority,

- Clinical studies must document clinical benefit of treating a numerical lab result,

- With poorly defined or heterogeneous clinical analytes even clinical studies suggesting treating a lab number are not in and of themselves sufficient to lead to successful inter-laboratory standardization (e.g., PSA). 


\section{NIST Measurement Methods and Standards Reference Materials for Health Status Markers: Current Program and Future Challenges \\ Willie E. May \\ National Institute of Standards and Technology}

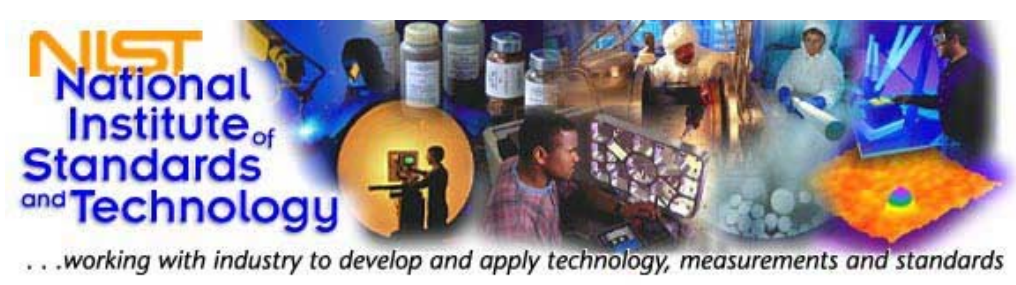

\section{About NIST}

NIST is an agency of the U.S. Department of Commerce's Technology Administration. NIST's seven discipline-based Measurement and Standards Laboratories work at all stages of the pipeline from advancing basic science and pioneering new measurement methods to the development of standard test methods, materials, and data to ensure the quality of commercial products. The mission of the NIST Laboratories is to promote the U.S. economy and public welfare by providing technical leadership for the Nation's measurements and standards infrastructure, and assure the availability of essential reference data and measurement capabilities.

\section{Why NIST and Healthcare?}

\section{Problem Magnitude and Scope:}

- U.S. Spends \$1.5 trillion on Health Care (14\% of GDP) (per year)

$-\sim 13 \%$ of this amount is associated with measurement $(\$ 140 \mathrm{~B})$

- Non-diagnostic measurements cost $\sim$ \$39

- Improvement in accuracy of one marker alone (cholesterol) is estimated to have saved $\$ 100 \mathrm{M}$ per year

New Driver: EU IVD Directive to go into effect 2003

- Worldwide in vitro diagnostic device market is $\sim \$ 20 \mathrm{~B}$;

- $>60 \%$ of European market is supplied by U.S. based companies
A 1999 study by the National Academy of Sciences Institute of Medicine estimates that up to 98,000 people die annually in the United States due to medical errors. While many medical errors are not due to inaccurate measurements, improved measurement accuracy could save lives as well as time and money. In addition, International standards organizations are beginning to require traceability of the accuracy of medical measurements to national standards. For example, since the European Union now requires that certain diagnostic medical devices be "traceable to standards of a higher order", we must develop internationally accepted reference methods and certified reference materials to meet this requirement. NIST's mission is to provide those standards. 


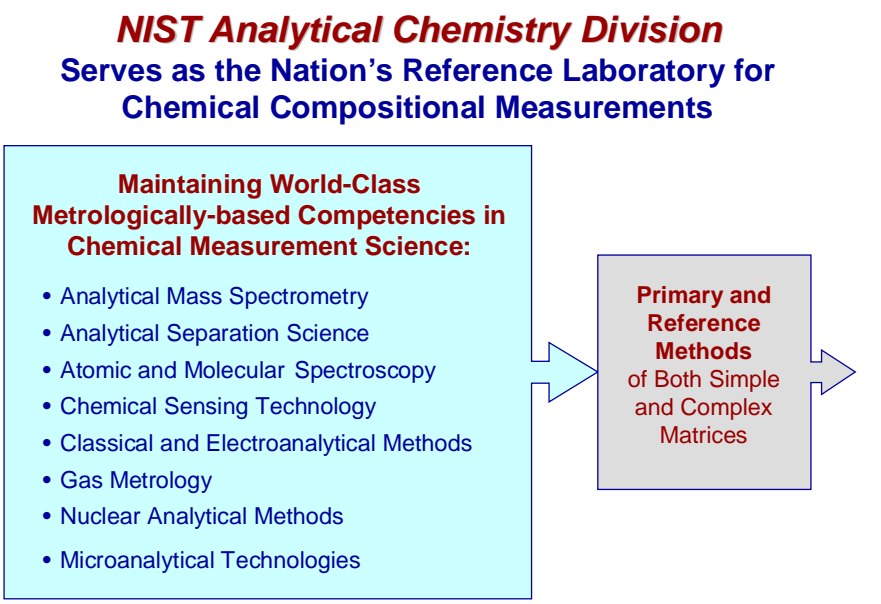

The Analytical Chemistry Division is one of five divisions in NIST's Chemical Science and Technology Laboratory. Core Competencies are maintained in a wide range of analytical technology areas. The skills and knowledge gained from research in these areas support the development of the internationally recognized reference methods and reference materials that NIST provides to facilitate "traceable" measurements in a

wide range of application areas from healthcare and food authenticity, nutritional value and contamination to chemical monitoring and open path sensing of stack emissions.

NIST provides about 840 certified reference materials that support chemical measurement processes; those certified for chemical composition and used for instrument and/or method calibration, and those not certified for chemical composition per se, but extensively used to support chemical measurements and processes. There are also natural samples/matrices with both macroscopic and often trace constituents value assigned for use in validating the accuracy of chemical meas-

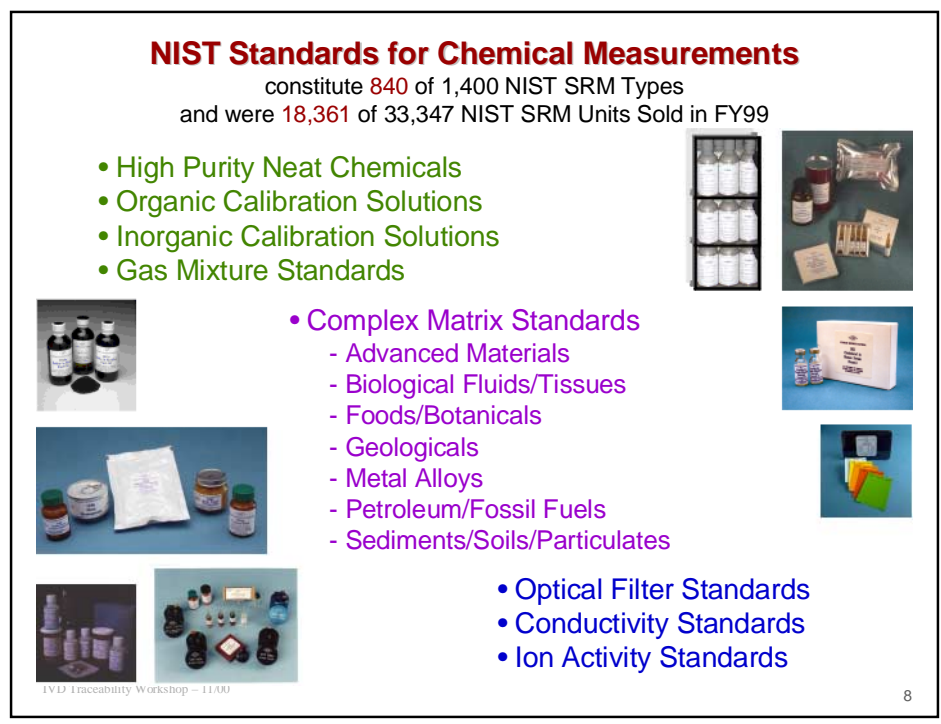
urement methods and processes.

NIST's Role in Health Care Measurements

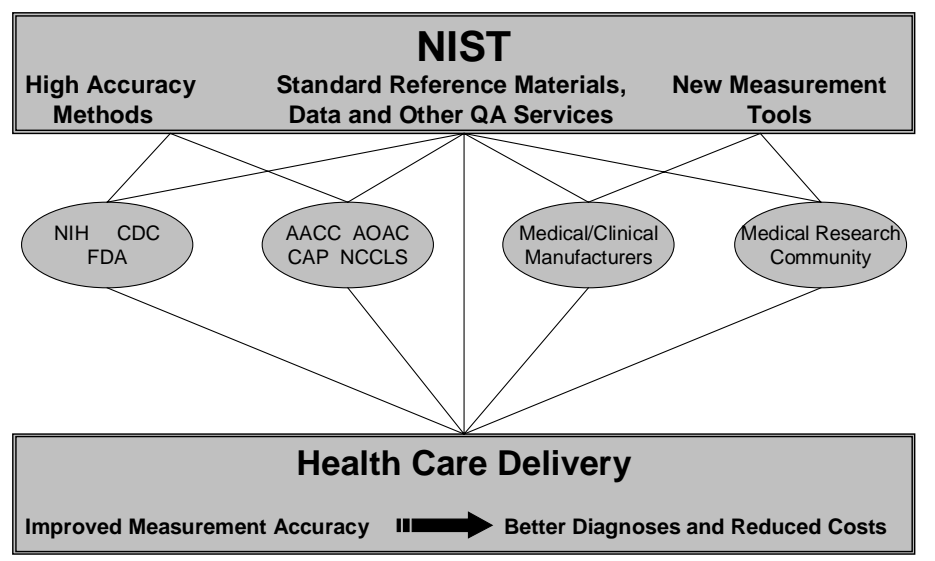

NIST/CSTL works closely with the American Association for Clinical Chemistry (AACC), the Centers for Disease Control and Prevention (CDC), NCCLS, and other organizations interested in health-related standards to help prioritize our standards activities and the development of SRMs. NIST's Chemical Science 
and Technology Laboratory serves as the US Reference Laboratory for heath related chemical measurements providing high accuracy measurement methods and Standard Reference Materials. CSTL is also involved in measurement QA programs and international comparison exercises with other National Metrology Institutes.

\section{History of Clinical Standards Program \\ - Pure Materials \& Artifacts (29)}

- 1914

- 1945

- 1967

- 1968

- 1970's
SRM 41, Dextrose

SRM 1861 \& 186 II, phosphates for $\mathrm{pH}$

SRM 911, Cholesterol

SRM 912, Urea

SRM 913, Uric Acid

SRM 914, Creatinine

SRM 915, Calcium Carbonate

21 SRMs with NIH support

URL: HTTP://ts.nist.gov/srm

E Mail: SRMINFO@nist.gov
NIST has provided a variety of Pure and Artifact Standard Reference Materials (SRMs) for the clinical community for nearly 90 years. First released in 1914, SRM 41c is still available as is SRM $915 \mathrm{a}$, and many of the standards produced with $\mathrm{NIH}$ support. At the request of clinical laboratories, the pure materials have been supplemented with the analyte of interest in serum, for more reliable quality control and calibration of clinical methods.
Since the 1980s, NIST has developed and refined "Definitive Methods" for several health status markers to support the national reference system for clinical measurements: NIST definitive methods for the health status indicators have been used to valueassign SRMs and highpriority serum pools used to serve as the anchor point for CDC developed reference methods and by the College American Pathologists for proficiency testing of more than 20,000 U.S. clinical laboratories.
National Reference System for Clinical Chemistry - Hierarchy of Methods for Cholesterol

- Definitive (NIST)

- Highest accuracy and precision

- Thoroughly tested for bias

- Generally not within the capability of clinical laboratories

- Used for primary RMs and validation of reference methods

- Reference (CDC Network)

- Carefully tested vs definitive method; high accuracy and precision

- Within capability of most clinical labs, but too time consuming

- For secondary RMs and validation of field methods

- Field (Clinical Labs)

- Routine clinical use

- Sufficient accuracy and precision for reliable diagnosis

- Simple, rugged and cost-effective 


\section{Cholesterol Reference System}

Cholesterol Reference System - the CDC Network: Improved accuracy facilitated by this program has led to better diagnosis, treatment and reduced health-care costs.

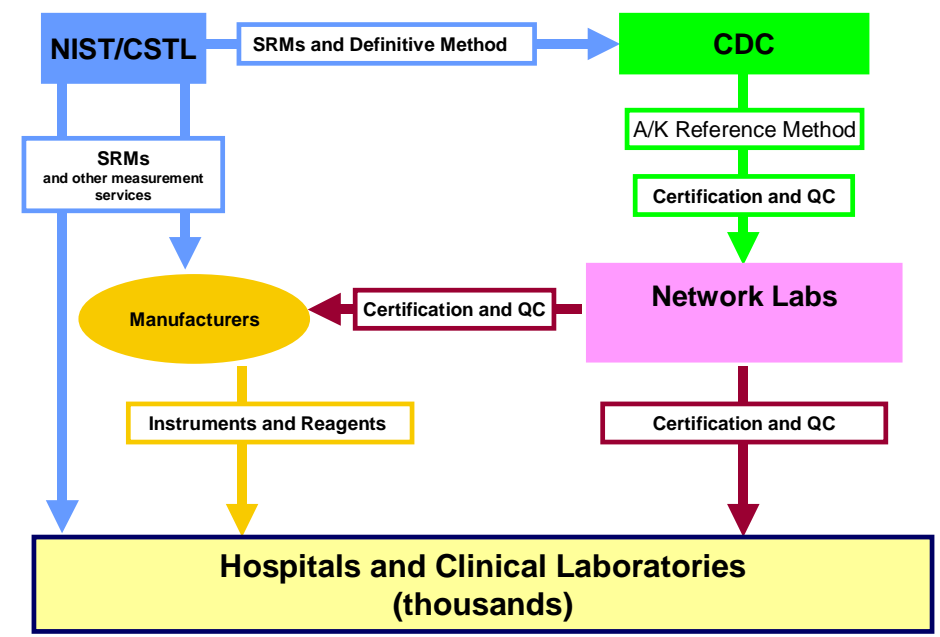

\begin{tabular}{|lll|}
\hline \multicolumn{3}{|c|}{ Definitive } \\
MNethods for Clinical Analytes \\
ANALTE & METHOD & CONDITION \\
\hline Calcium & ID/MS & Cancer, Blood Clotting \\
Chloride & ID/MS, Coulometry & Kidney Function \\
Cholesterol & ID/MS & Heart Disease \\
Creatinine & ID/MS & Kidney Function \\
Glucose & ID/MS & Diabetes \\
Lithium & ID/MS & Antipsychotic treatment \\
Magnesium & ID/MS & Heart Disease \\
Potassium & ID/MS & Electrolyte Balance \\
Sodium & Gravimetry, ICP/MS & Electrolyte Balance \\
Triglycerides & ID/MS & Heart Disease \\
Urea & ID/MS & Kidney Function \\
Uric Acid & ID/MS & Gout \\
\hline
\end{tabular}

NIST maintains and refines definitive methods for the twelve health status markers shown in the box to the left. Classical primary methods are utilized as well as isotope dilution mass spectrometry (IDMS).

Over the last three decades, NIST, in cooperation with the College of American Pathologists (CAP) has developed a series of highly accurate and precise methods, isotope dilution mass spectrometry (IDMS) for a number of clinically important serum constituents, including cholesterol (see figure above). These IDMS methods are recognized by the international clinical laboratory community as "definitive"

The illustration is a schematic representation of ID/MS methodology for organic constituents in a complex matrix. It includes the addition of the isotopically-labeled analog of the species to be measured for use as an internal standard, followed by wet chemical procedures for isolation of the analyte and isotopically-labeled analog from the matrix prior 
to separation of the analyte species (and coeluting isotope diluent) in time from other species co-extracted from the matrix via gas chromatography (GC), before introduction into the mass spectrometer for determining the relative amounts of analyte and isotope diluent in the sample. Since the absolute of amount of isotope diluent is known, the concentration of the analyte can be calculated.

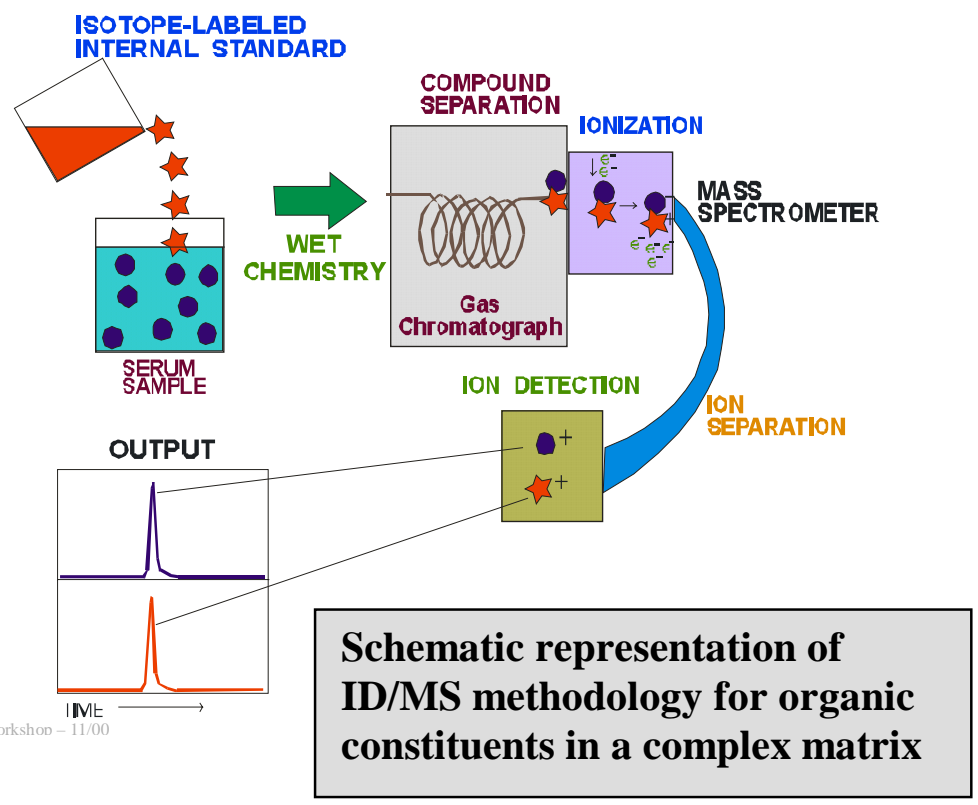
This general approach is used for all small molecule organic species, however the wet chemical procedures used for assuring that the isotope diluent fully equilibrates with the matrix, for isolation of the analyte from the matrix, and for conversion of the analyte to a stable, volatile derivative for GC, must be developed specifically for each individual health status marker.

Typical precisions of the ID-GC/MS definitive methods are shown in the diagram

\section{PRECISION OF ID-GC/MS}
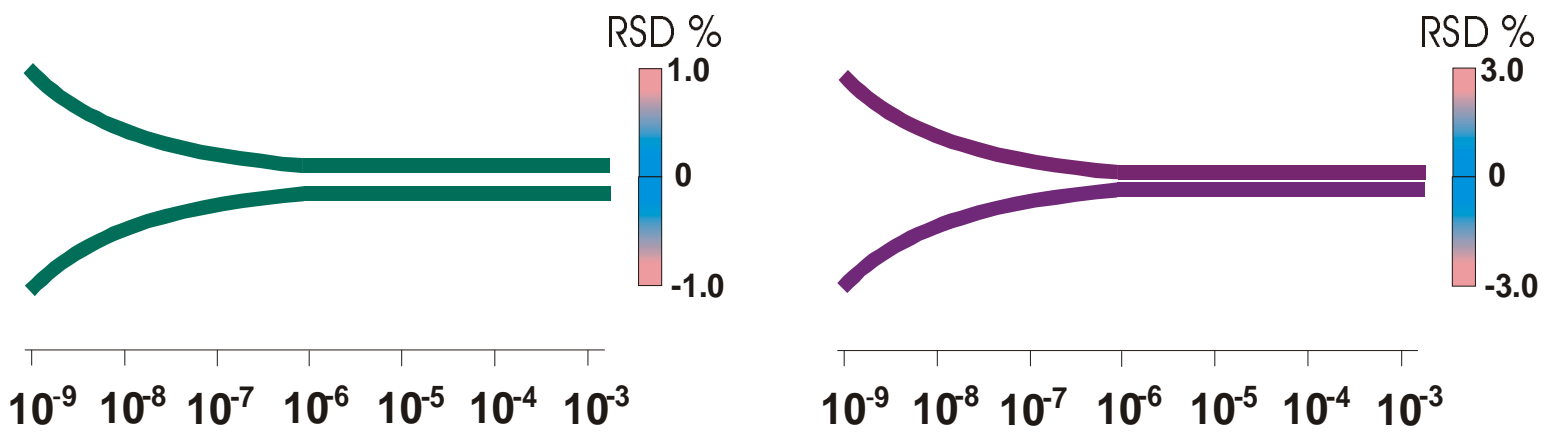

\section{Mass Fraction}

Intensity Ratio Measurements

Measured Mass Fractions of Independently Prepared Samples 


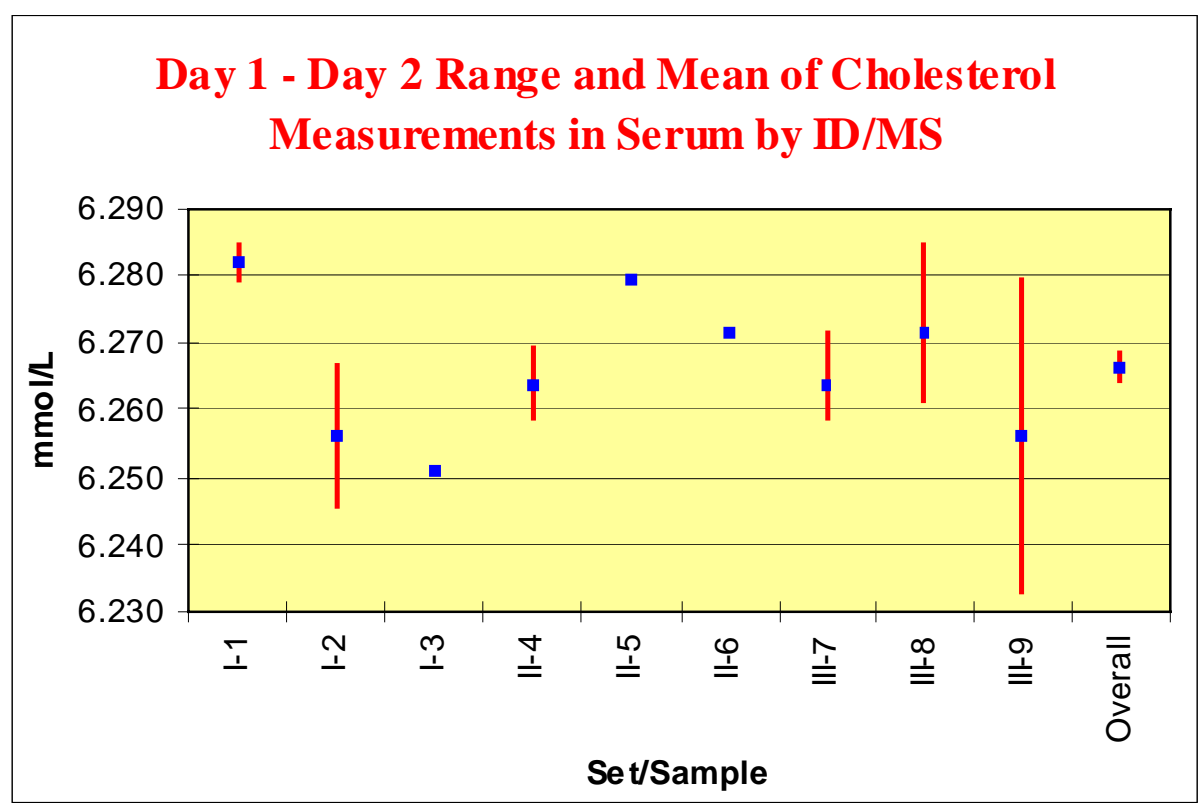

An example of the repeatability of the ID/MS procedure for cholesterol over a period of time is shown in the graph to the left.

A history of serum matrix SRMs available from 1979 to the present is listed in the figure. SRMs provide an accuracy base for the clinical laboratory system.

The following are currently available through NIST's Standard Reference Material Program:

SRMs 900, 909b, 956a, 965, 968c, and 966; in addition to SRMs 1951a, and 1952a.

\section{History of Clinical Standards Program}

- Selected Serum Matrix Materials

- 1979 SRM 900, Antiepilepsy Drug Level Assay Standard

- 1980 SRM 909, Human Serum

- 1982 SRM 1599, Anticonvulsant Drug Level Assay Standard

- 1988 SRM 1951, Cholesterol in Human Serum (Frozen) SRM 1952, Cholesterol in Human Serum (Freeze Dried)

- 1989 SRM 968, Fat Soluble Vitamins in Human Serum

- 1991 SRM 956, Electrolytes in Blood

- 1996 SRM 956a, Electrolytes in Frozen Human Serum

- 1996 SRM 965, Glucose in Frozen Human Serum (3 levels)

- 1997 SRM 1951a, Lipids in Frozen Human Serum (2 levels)

- 2000 SRM 966, Toxic Metals in Blood SRM 2070a, Toxic Elements in Human Urine

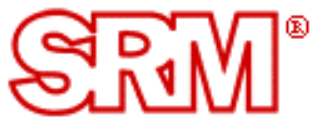

Catalog Home

About | Users Guide | Browse | Ordering Download | Search | Site Index | Contact 


\section{Cholesterol in Blood Experiment - Impact of Inaccurate Measurements}

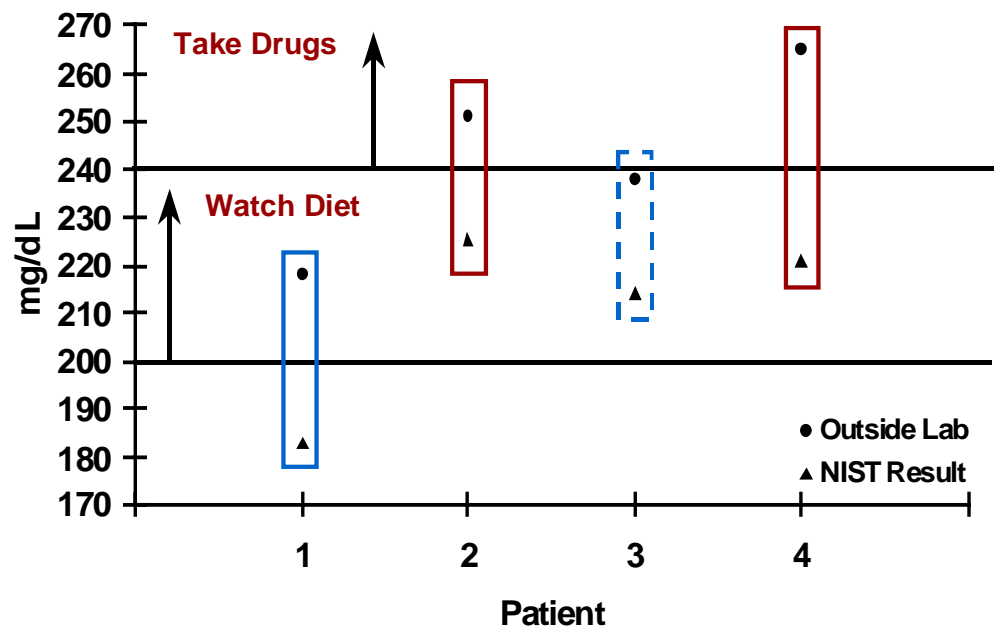

To the left is an application in which calibration control would be of benefit. Notice that values determined by the clinical laboratory on four patient samples are all biased by a similar amount versus the values obtained by NIST using the definitive method.

In three out of the four cases, the results provided by the clinical laboratory would have led to incorrect assessment of heart disease risk based on the guidelines of the National Cholesterol Education Panel and perhaps, unnecessary medical costs.

In the area of cholesterol measurements alone, it has been estimated that measurement uncertainty was on the order of $\pm 18 \%$ relative in 1969, before any reference materials were available. The first pure crystalline cholesterol (SRM 911) was introduced in 1967. Using the definitive method, serum cholesterol SRMs were developed in 1981 (SRM 909) and again in 1988

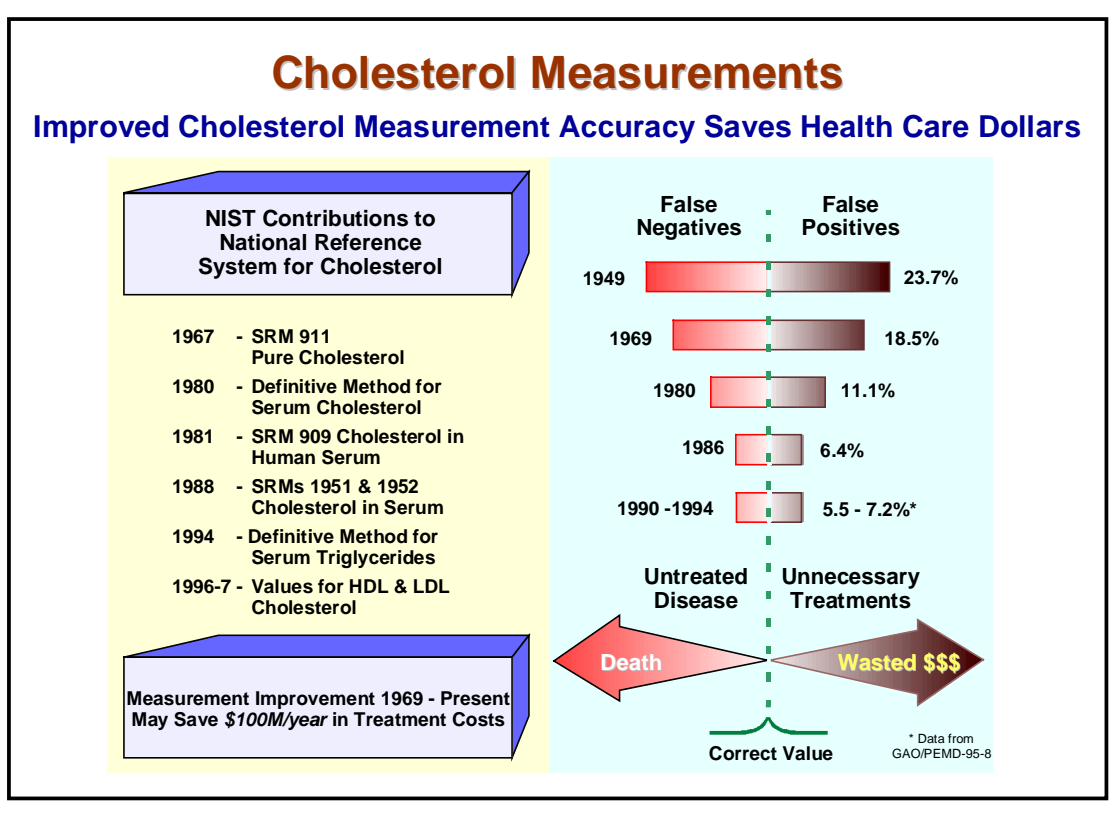
(SRMs 1951 and 1952). These SRMs have contributed to a steady decrease in the number of false positives and negatives resulting from clinical laboratory results for cholesterol in blood to between $\pm 5.5 \%$ to $7.2 \%$, relative. 
NIST conducts privately contracted, formal economic of various programs to assess the impact of its work. The latest impact study quantifies a portion of the economic benefits associated with the Cholesterol Standard reference materials beginning in 1986. The economic consequences of NIST's Cholesterol Standards Program are experienced at several levels of the supply chain from
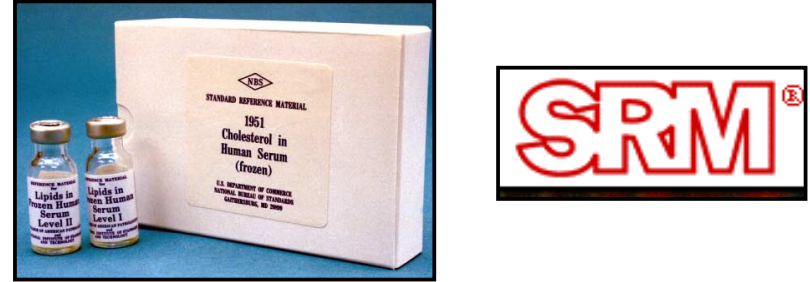

Title: SRMs for Cholesterol Measurements

Products: NIST SRMs 911, 909, 1951 and 1952

(Clinical Standards)

Results (3 levels of supply chain):

$\begin{array}{ll}\text { Benefit-Cost Ratio } & 4.5 \\ \text { Social Rate of Return } & 154 \% \\ \text { Net Present Value } & \$ 3.5 \mathrm{M}\end{array}$

manufacturers, to network laboratories, to clinical laboratories that ultimately deliver medical services to the consumer. The benefits to industry resulting from the NIST investment have changed over the more than three decades of NIST involvement. However, this analysis timeframe was limited to 1986-1999 covering only part of the program's life cycle, thus biasing the measured impacts downward. The results indicate that NIST has played an important economic role in support of a national effort to monitor, measure, and control cholesterol levels, thereby contributing to the reduced level of heart disease. This economic impact study estimates a benefit-to-cost ratio of 4.5, and a social rate of return of $154 \%$. The Net Present Value was calculated to be more than \$3.6 million. However, this study fails to capture the impact of incorrect clinical measurements on patient care, which have been estimated to be approximately $\$ 100 \mathrm{M}$ per year.

The NIST Micronutrients Measurement Assurance Program supports laboratories

\section{NIST Provides:}

- Tools for Comparability Assessment

- Interlaboratory Comparison Exercises

- SRMs and Control Materials

- Performance Database

- Relevant Research in Measurement Science

- Workshops, Site Visits, Tutorials, and Consultations

Program Result:

- Improved accuracy of laboratory measurements resulting in increased interlaboratory comparability that measure selected fatand water-soluble vitamins and carotenoids in human serum and plasma. Participants are from more than 60 laboratories worldwide. These include participants from Academia, Government and Industry (62\% domestic and $38 \%$ foreign).

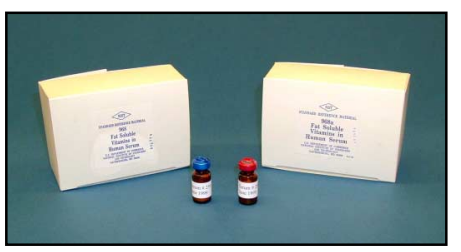




\section{International Activities \\ Key Comparisons in Chemical Metrology}

Formal international cctivities in chemical measurements provide a metrological infrastructure to facilitate trade. National Metrology Institutes (NIST in the U.S.) provide a national traceability structure. However, to facilitate international trade, NMIs participate in Key Comparisions to establish comparability of measurements. This infrastructure can link field measurements among nations via traceabil-

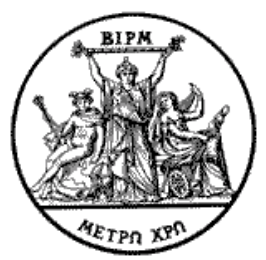
ity to their home NMI. NIST/CSTL has taken a leadership role in the International Committee of Weights and Measures (CIPM) Consultative Committee for the Amount of

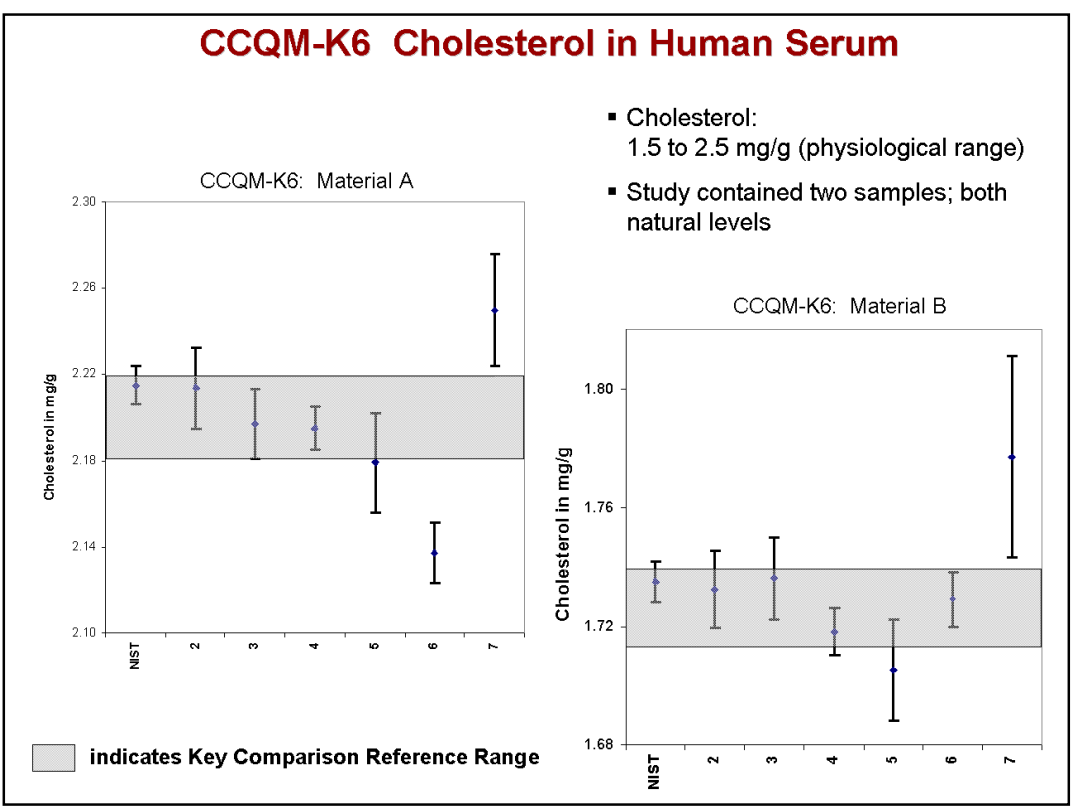

Substance (CCQM) and has leadership roles in many of the Key Comparisons.

CSTL recently lead a Key Comparison on "Cholesterol in Human Serum". The NMIs that agreed to participate were sent samples from two frozen human serum pools. The results of this Key Comparison study demonstrated that the participating NMIs could successfully measure serum cholesterol for normal and elevated levels, using ID/MS-based methods, with interlaboratory expanded uncertainties of less than $1 \%$. However, every serum analyte of interest as a health marker provides a unique set of challenges. To provide a more comprehensive measure of the capabilities of NMIs for measuring well-defined serum analytes, the CCQM is conducting pilot studies for the determinations of serum glucose and creatinine that, if successful, will be followed by Key Comparisons.

CSTL supports the IVD Industry by continuing to maintain and refine definitive methods for the health status markers discussed previously. In addition, CSTL researchers are also working to standardize a new and emerging generation of health status markers that show great promise from the clinical diagnostic perspective.

\section{CARDIAC TROPONIN I (cTnl)}

- Protein found in Heart Muscle

- Released into the Blood following Myocardial Infarction

- Useful Clinical Marker for Myocardial Infarction

- Occurs in Blood as Free cTnl and as Complex with Troponins C \& $T$ Results vary widely among Different Immunoassays 
Analytes had been identified as important prior to the EU IVD directive - and preliminary work was already underway to develop standards. However, these efforts must be accelerated in order to meet future industry needs in light of the IVD Directive. These analytes offer new and more difficult analytical challenges for standardization. Many of the new markers are proteins, peptides, or other large biomolecules, usually present at very low concentrations. Because of the vast market for tests for

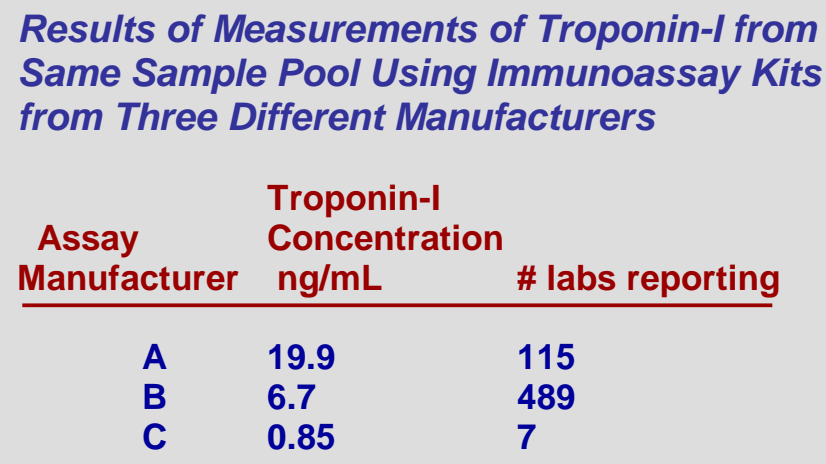
Same Sample Pool Using Immunoassay Kits from Three Different Manufacturers

\begin{tabular}{|c|c|c|}
\hline $\begin{array}{l}\text { Assay } \\
\text { Manufacturer }\end{array}$ & $\begin{array}{l}\text { Troponin-I } \\
\text { Concentration } \\
\mathrm{ng} / \mathrm{mL}\end{array}$ & \# labs reporting \\
\hline $\begin{array}{l}\text { A } \\
B \\
C\end{array}$ & $\begin{array}{l}19.9 \\
6.7 \\
0.85\end{array}$ & $\begin{array}{l}115 \\
489 \\
7\end{array}$ \\
\hline
\end{tabular}

From G. S. Bodor, Denver Health and Hospitals personal communication 1997

these new markers, many different approaches have been developed and these approaches often provide different answers.

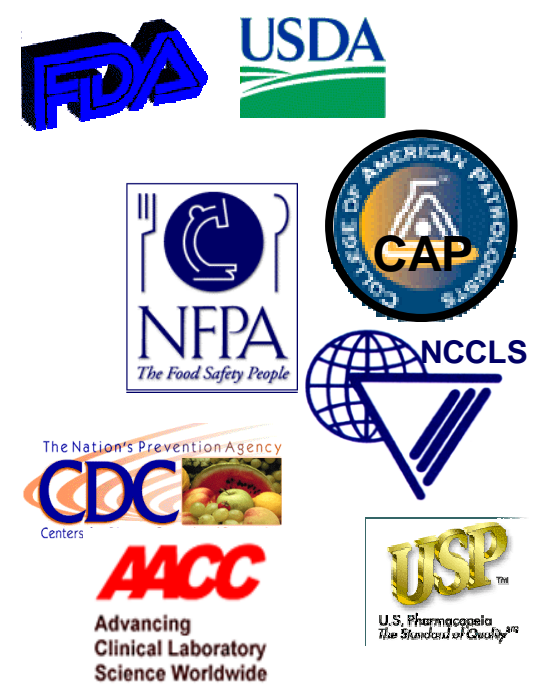

NIST maintains its links to the large clinical measurement community through interactions with a variety of healthcare-related organizations. For example, based on input from the American Association for Clinical Chemistry, the National Committee for Clinical Laboratory Standards, the College of American Pathologists and clinical instrument manufacturers, a number of other health markers have been identified that are medically very important (such as markers for anemia and hemochromatosis, nutritional health status, risk of heart attack, prostate cancer, breast cancer, thyroid function, and neural tube defects) but for which there is high variability among clinical laboratory results.

The international metrological and clinical communities have jointly identified analytes that require standardization. They grouped as follows: "A list"consists of approximately 100 well-defined chemical species, potentially traceable to SI units; "B list" are less well defined, potentially not traceable to SI units, and number $>500$ (for example: method dependent analytes such as liver enzymes) 


\section{Establish Reference Systems for New Clinical Markers - Highest Priorities}

Troponin-I
Gylcated Hemo.
Homocysteine
P $_{53}$ DNA
TSH
Speciated Iron
Human Serum Alb.
PSA
Cadmium \& Mercury
Cortisol
Thyroxine
Folates

\author{
Myocardial Infarction \\ Diabetes Status \\ Risk of Heart Disease \\ Breast Cancer \\ Thyroid Function \\ Hemochromatosis, Anemia \\ Renal Failure \\ Prostate Cancer \\ Toxic Metal Poisoning \\ Endocrine Function \\ Thyroid Function \\ Neural Tube Defects
}

Over the past three years, we have tripled our investment in the healthcare measurements area and are currently focused on the development of reference methods and materials for the health status markers shown in the box below. Some information regarding the clinical application of one of the markers, glycated hemoglobin,

follows. Details of the research for the remaining markers can be found in the extended abstracts from the Poster Session (pages 267 -281).

\section{Diabetes Status}

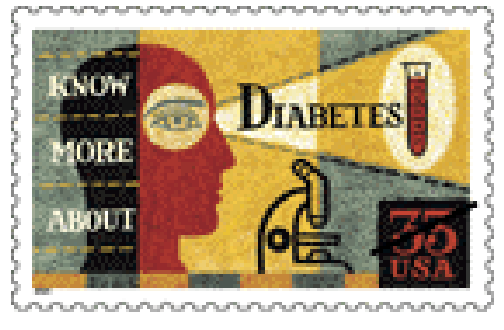

Glucose accumulates in the blood of those with type II diabetes who fail to produce insulin since the cells in their bodies are "insulin resistant" and do not respond properly to the hormone. Left untreated, diabetes can result in cardiovascular disease, kidney disease, eye diseases, and nervous-system maladies. In addition to the daily monitoring of blood glucose levels, the measurement of glycated hemoglobin plays an important role in the diagnosis and treatment of diabetes. When blood glucose levels rise above normal, the glucose can react with hemoglobin in

NIST has participated in inlaboratory comparison studies of IFCC reference materials and has provided critiques for improvements. the blood, forming glycated hemoglobin. Since the lifetime of hemoglobin in the bloodstream is approximately 3 months, the measurement of glycated hemoglobin provides a record of the levels of blood glucose over the course of 3 months, thus providing physicians with information on the efficacy of diabetes treatment over a longer period than can the daily blood glucose measurements. While the medical utility of glycated hemoglobin measurement has been clearly demonstrated, there have been considerable problems with its clinical application due to method-to-method measurement variability. It is clear that there is a strong need measurement standardization. The International Federation for Clinical Chemistry (IFCC) has developed two reference methods for the determination of $\mathrm{HbA}_{1 \mathrm{c}}$, one specific form of glycated hemoglobin, in blood hemolysates. One method uses liquid chromatography coupled with mass spectrometry (LC/MS) and the other used capillary electrophoresis (CE) for $\mathrm{HbA}_{1 \mathrm{c}}$ determination. Both methods have demonstrated high precision (average inter-laboratory coefficient of variance $\leq 2 \%$ ) and excellent agreement with each other. These reference meth- 
ods have been implemented in a network of laboratories worldwide. Currently the IFCC reference methods are being used to value-assign an international $\mathrm{HbA}_{1 \mathrm{c}}$ reference material.

\section{Future Directions}

Expand existing clinical standards program to encompass all major diagnostic markers [perhaps through more effective networking]

- Expand food standards program to address GMO, adulteration and other issues of US concern

- Establish program for credentialing commercial instrument calibrators

- Work with the U.S. College of American Pathologists and appropriate EU counterparts to establish trans-Atlantic Clinical Laboratory Chemical Measurements Proficiency Demonstration Program

\section{Preliminary Plan of Action}

- NIST would develop and maintain reference methods and SRMs for up to $40 \%$ of the A list analytes*

- NMIs in the EU, Japan and Australia would also develop methods and CRMs for the remainder of analytes

- Mechanisms for mutual recognition of reference methods and assigned values for CRMs are being discussed [two models] 


\section{WILLIE E. MAY}

\section{National Institute of Science and Technology}

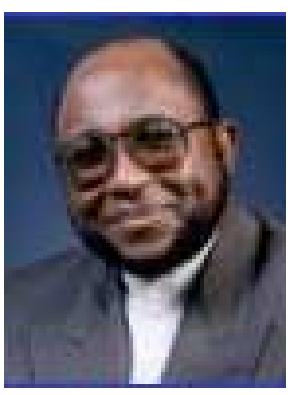

Willie E. May, Ph.D., is Chief, Analytical Chemistry Division, Chemical Science and Technology Laboratory, National Institute of Standards and Technology. He received his B.S. degree in chemistry from Knoxville College, Knoxville, TN in 1968 and his Ph.D. in analytical chemistry from the University of Maryland at College Park, MD in 1977. He joined NIST, then the National Bureau of Standards, in 1971. He became Group Leader for Liquid Chromatography, Organic Analytical Research Division in 1976, and became Chief of the Division in 1983. In 1994, Dr. May became Chief of the Analytical Chemistry (a combination of the Organic Analytical and Inorganic Analytical Chemistry Divisions). As Chief of the NIST Analytical Division, Dr. May is responsible for policy development, planning, priority setting, fiscal allocation, staffing, and management of the operations of the Division's 100 scientists and support staff. The Division has an annual budget of approximately \$15M. Dr. May has several leadership responsibilities in addition to those at NIST. Currently he is Chair, Organic Analysis Working Group, Consultative Committee on the Quantity of Material, International Committee for Weights and Measures; Chair, Technical Committee, North American Metrology Cooperation; and Chair, Chemical Metrology Working Group, Interamerican System for Metrology. In the past he has served as President and Executive Board Member for the International Society for Polycyclic Aromatic Compounds; Chair, Physical Sciences Panel, Ford Foundation Predoctoral Fellowship Evaluation Board; Chair, Chemistry Panel, Texas Higher Education Coordinating Board; Chair, Chemical Metrology Subpanel, Institute for National Measurement Standards, Canadian National Research Council; and Chair, Committee on Minority Affairs, American Chemical Society.

Dr. May's personal research activities were focused in the area of trace organic analytical chemistry, with special emphasis on the development of liquid chromatographic methods for the determination of individual organic species in complex mixtures (i.e., extracts of environmental, food, and clinical samples) and the development of liquid chromatographic methods for the determination of physico-chemical properties such as aqueous solubilities, octanol/water partition coefficients, and vapor pressures of organic compounds. This work is described in more than 100 peer-reviewed publications. He has won numerous awards, including the U.S. Department of Commerce Superior Federal Service (Bronze Medal) Award in 1981, the U.S. Department of Commerce Meritorious Federal Service (Silver Medal) Award in 1985, the Arthur S. Flemming Award for Outstanding Federal Service, in 1987, the Percy Julian Award, NOBCChE, in 1992, the Presidential Rank of Meritorious Executive in Federal Service in 1992 and the U.S. Department of Commerce Distinguished Achievement in Federal Service (Gold Medal) Award in 1992. 


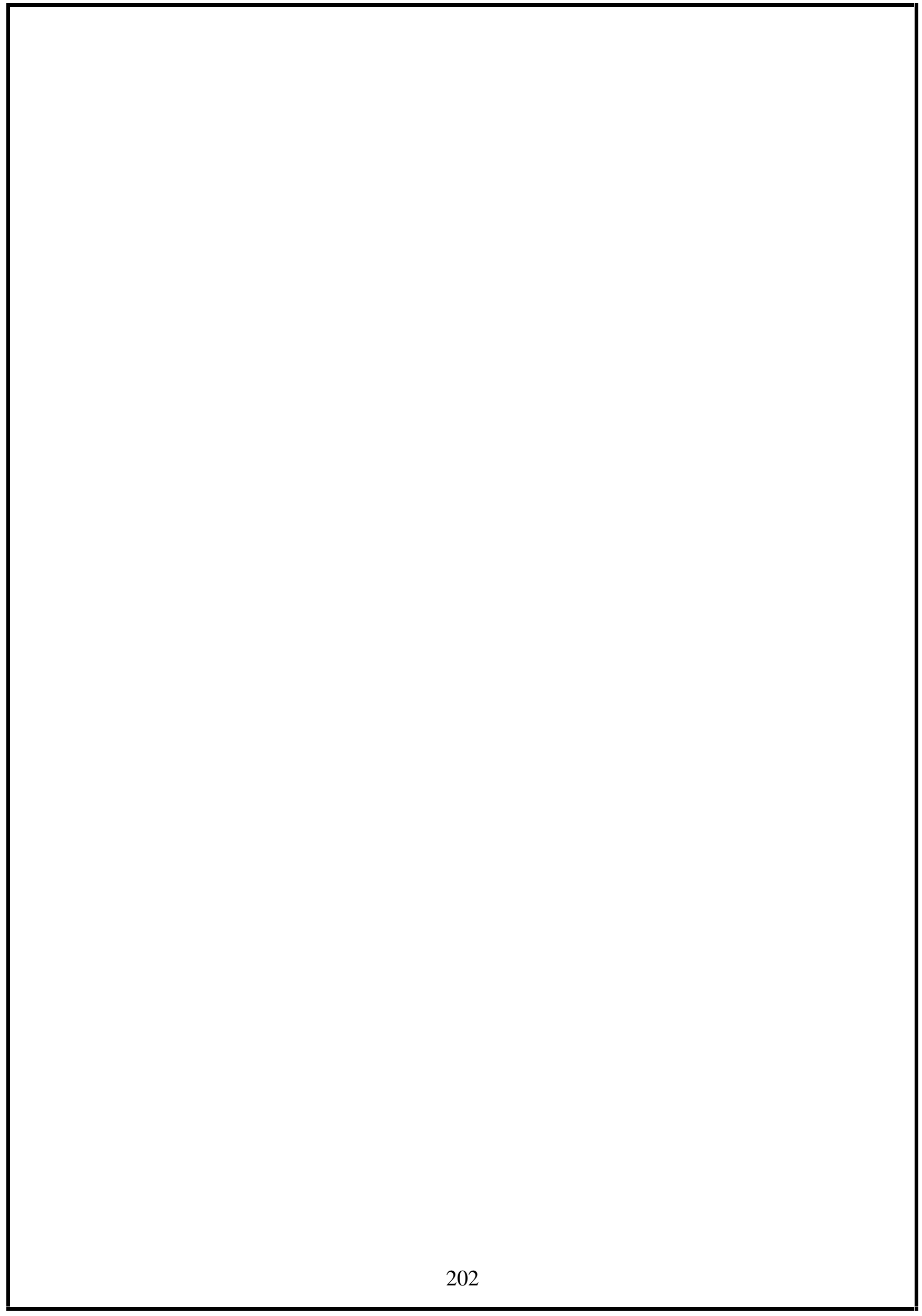


NIST Measurement Methods and Standards Reference Materials for Health Status Markers:

Current Programs and Future Challenges

Visuals Presented By

Willie E. May

National Institute of Standards and Technology 
Development of Reference Methods and Reference Materials in the U.S.

Willie E. May

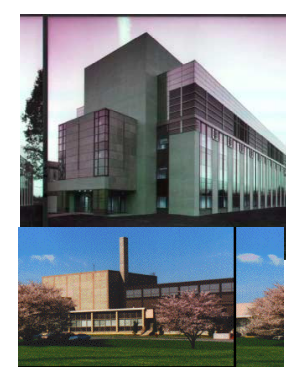

Analytical Chemistry Division

Chemical Science and Technology Laboratory National Institute of Standards and Technology Gaithersburg, Maryland 20899 USA

\begin{tabular}{|ll|}
\hline $\begin{array}{l}\text { For further information: } \\
\text { Mail: }\end{array}$ & Analytical Chemistry Division, MS 8390 \\
& 100 Bureau Dr.,Gaithersburg, MD 20899-8390 USA \\
E-mail: & Willie.May@NIST.gov \\
Phone: & Voice +301-975-3108 \\
& FAX +301-926-8671 \\
Websites: & ACD http://www.cstl.nist.gov/nist839/ \\
& NIST http://www.nist.gov/
\end{tabular}

\section{NLT}

\section{NIST Measurement Methods and Standards for Health Care Markers}

- Mission of NIST Laboratories

- NIST Program in Chemical Measurements and Standards

- Why Health-Care?

- Examples of Measurement Problems and Impact of NIST Activities

- New Drivers

- Protein-based markers

- EU in vitro Diagnostic Devices Directive

- NIST Response to New Drivers 

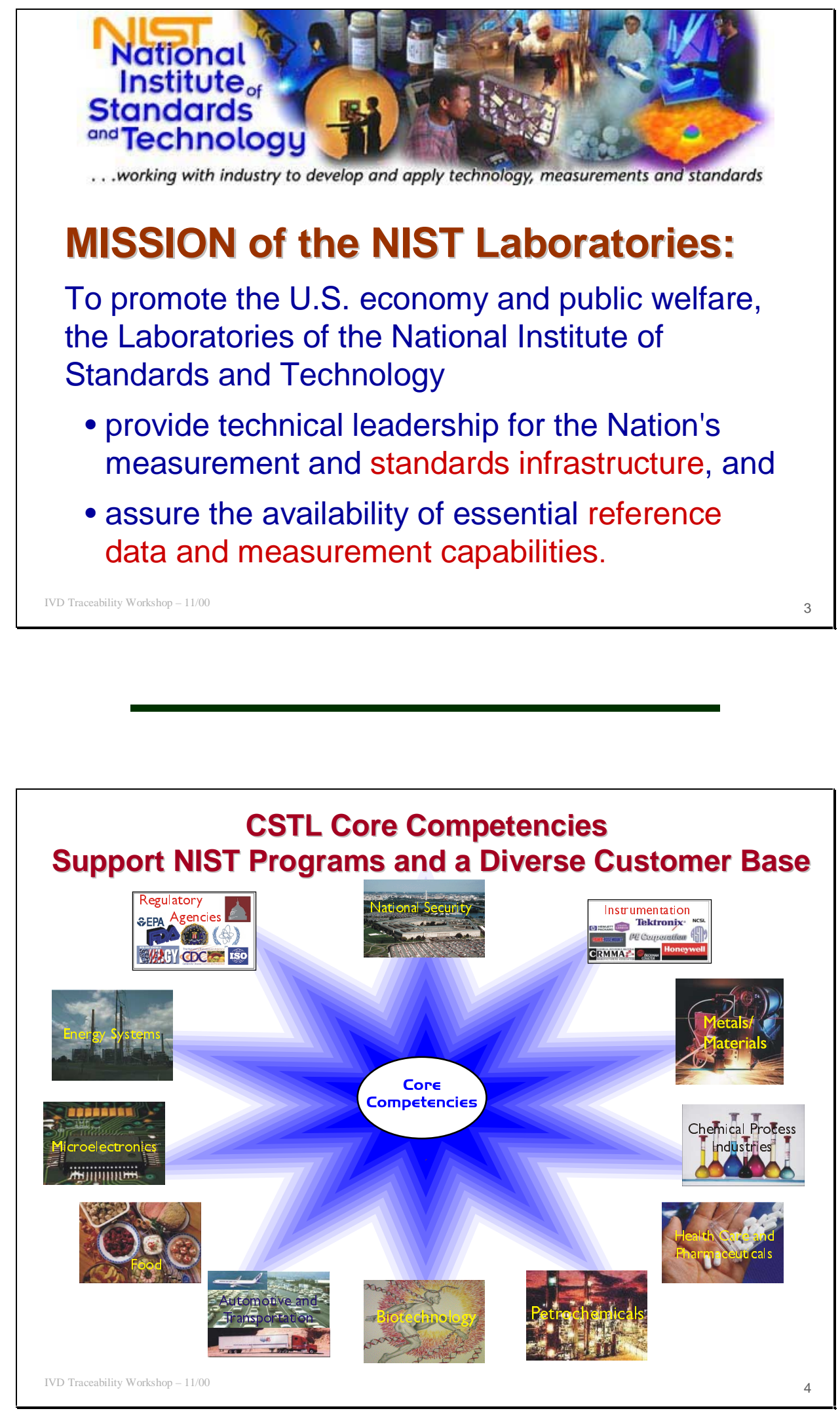


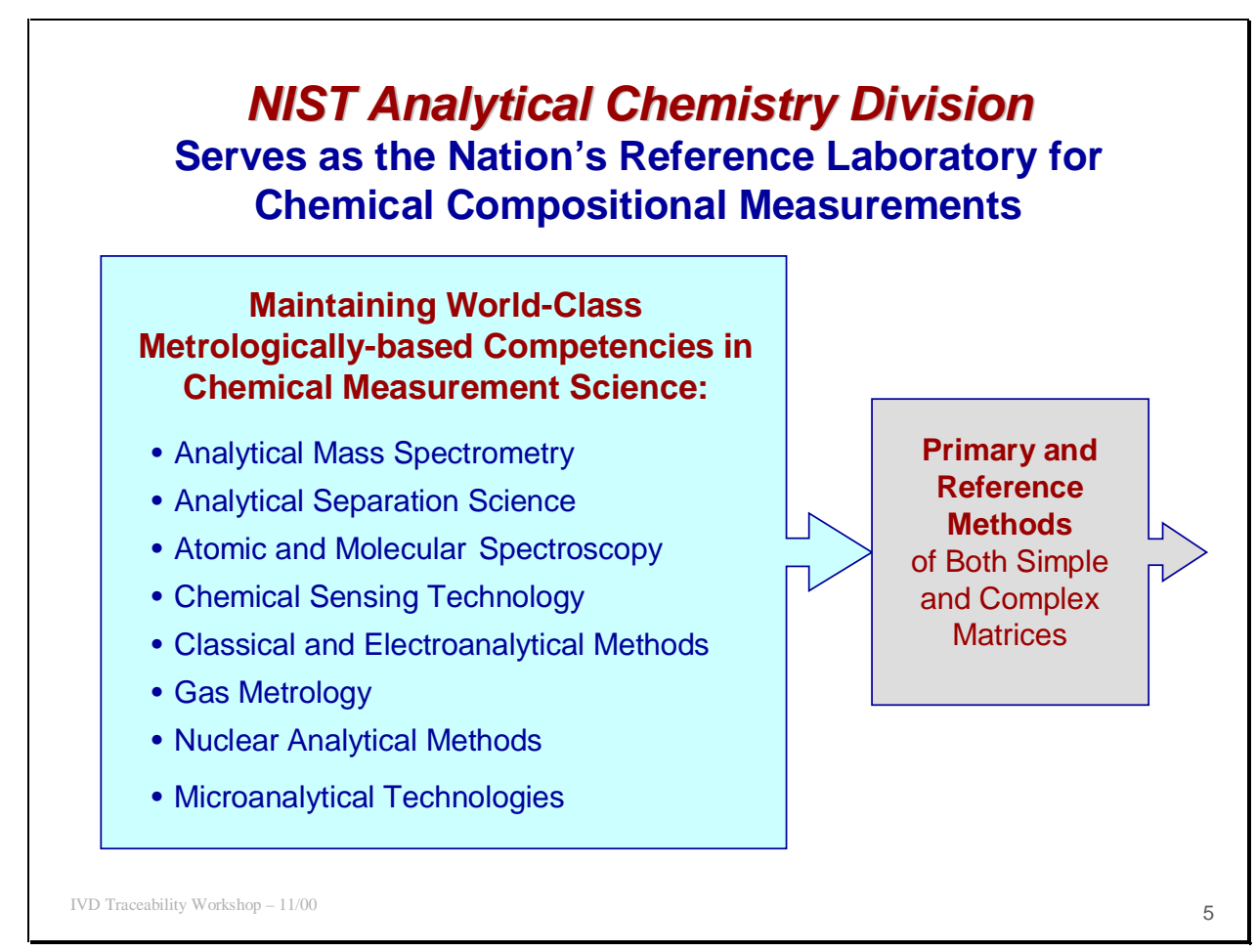

\section{Tools for Achieving National Traceability and International Comparability of Chemical Measurements}

- Standard Reference Materials and Standard Reference Databases

- NIST Traceable Reference Materials

- Measurement Proficiency Assessment for Selected U.S. Environmental, Health, and Industrial Studies

- Strategic International Comparisons
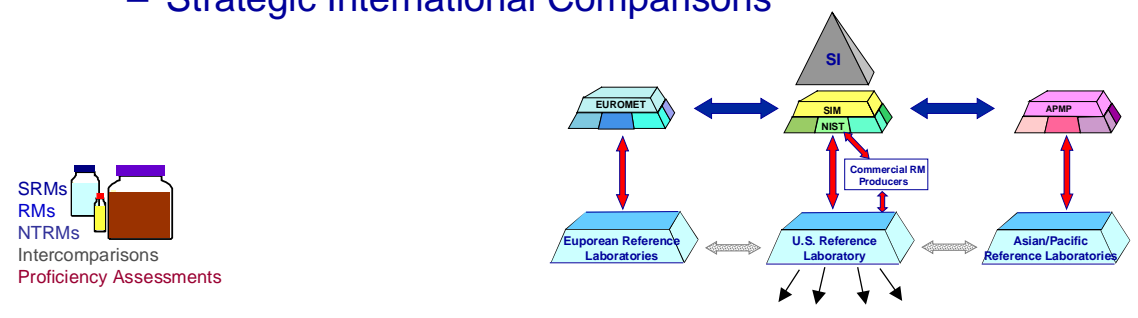


\section{Why NIST and Healthcare?}

Problem Magnitude and Scope:

- U.S. Spends \$1.5 trillion on Health Care (14\% of GDP) (per year) $-\sim 13 \%$ of this amount is associated with measurement (\$140B)

- Non-diagnostic measurements cost $\sim$ \$39B

- Improvement in accuracy of one marker alone (cholesterol) is estimated to have saved $\$ 100 \mathrm{M}$ per year

New Driver: EU IVD Directive to go into effect 2003

- Worldwide in vitro diagnostic device market is $\sim 20 \mathrm{~B}$;

- $>60 \%$ of European market is supplied by U.S. based companies

\section{NIST's Role in Health Care Measurements}

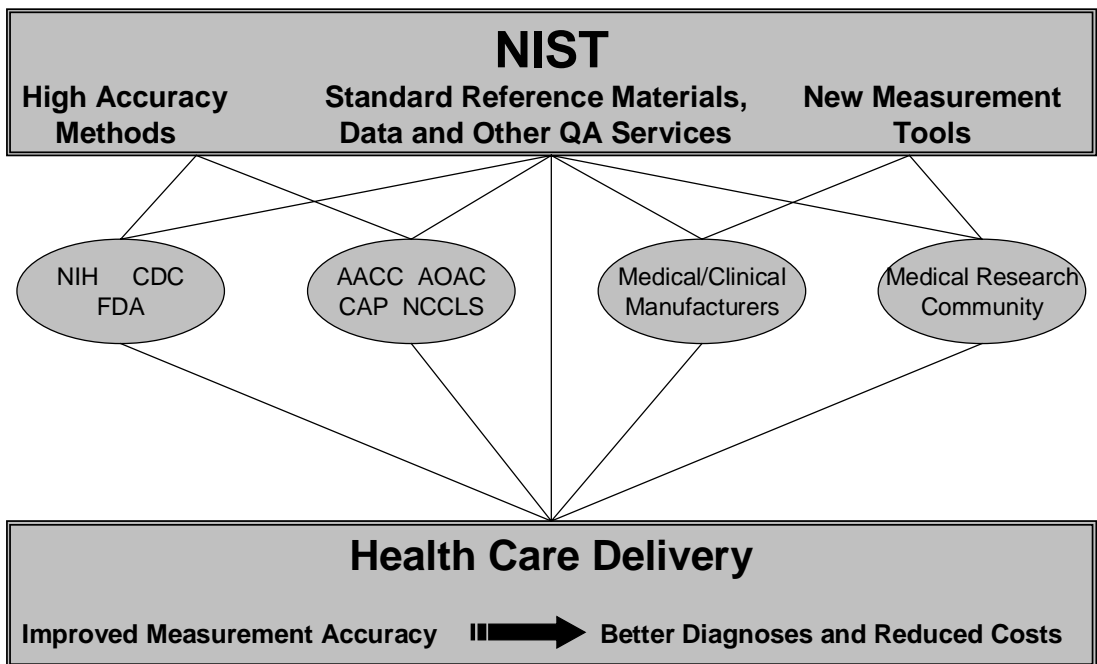




\section{Program Objective and Activities}

To Serve as the US Reference Laboratory for Health-Related Chemical Measurements

- high accuracy measurement methods

- Standard Reference Materials

- pure primary standards

- matrix-based materials

- instrument calibration materials \& artifacts

- interactive measurement quality assessment/demonstration activities

- international comparison exercises with other National Metrology Institutes (NMIs)

\section{History of Clinical Standards Program}

- Pure Materials \& Artifacts (29)

- 1914 SRM 41, Dextrose

- 1945 SRM 1861 \& 186 II, phosphates for pH

- 1967 SRM 911, Cholesterol

- 1968 SRM 912, Urea

SRM 913, Uric Acid

SRM 914, Creatinine

SRM 915, Calcium Carbonate

- 1970's 21 SRMs with NIH support 


\section{National Reference System for Clinical Chemistry - Hierarchy of Methods for Cholesterol}

- Definitive (NIST)

- Highest accuracy and precision

- Thoroughly tested for bias

- Generally not within the capability of clinical laboratories

- Used for primary RMs and validation of reference methods

- Reference (CDC Network)

- Carefully tested vs definitive method; high accuracy and precision

- Within capability of most clinical labs, but too time consuming

- For secondary RMs and validation of field methods

- Field (Clinical Labs)

- Routine clinical use

- Sufficient accuracy and precision for reliable diagnosis

- Simple, rugged and cost-effective

\section{Cholesterol Reference System}

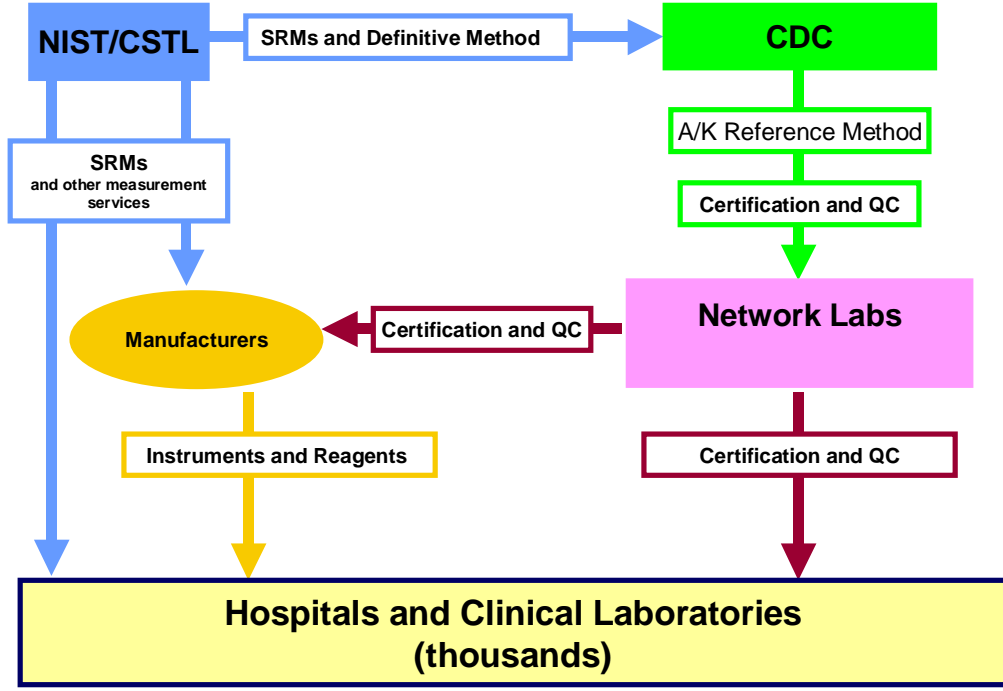




\section{Definitive Methods for Clinical Analytes}

\begin{tabular}{|c|c|c|}
\hline ANAL YTE & METHOD & CONDITION \\
\hline$\overline{\text { Calcium }}$ & $\overline{I D / M S}$ & $\overline{\text { Cancer, Blood Clotting }}$ \\
\hline Chloride & ID/MS, Coulometry & Kidney Function \\
\hline Cholesterol & ID/MS & Heart Disease \\
\hline Creatinine & ID/MS & Kidney Function \\
\hline Glucose & ID/MS & Diabetes \\
\hline Lithium & ID/MS & Antipsychotic treatment \\
\hline Magnesium & ID/MS & Heart Disease \\
\hline Potassium & ID/MS & Electrolyte Balance \\
\hline Sodium & Gravimetry, ICP/MS & Electrolyte Balance \\
\hline Triglycerides & ID/MS & Heart Disease \\
\hline Urea & ID/MS & Kidney Function \\
\hline Uric Acid & ID/MS & Gout \\
\hline
\end{tabular}

\section{Organic Definitive Methods}

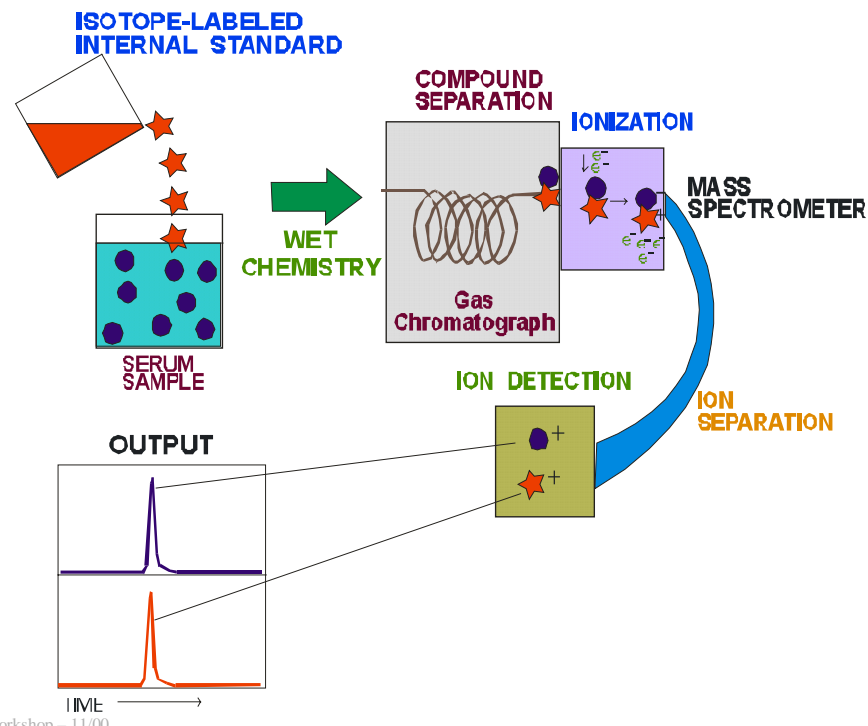




\section{PRECISION OF ID-GC/MS}

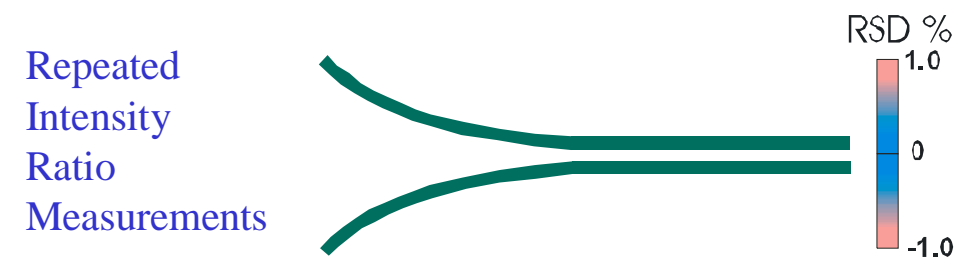

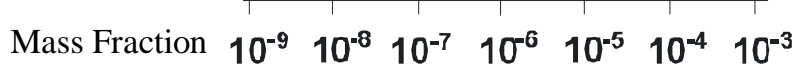

Measured

Mass Fractions

of Independently

Prepared Samples
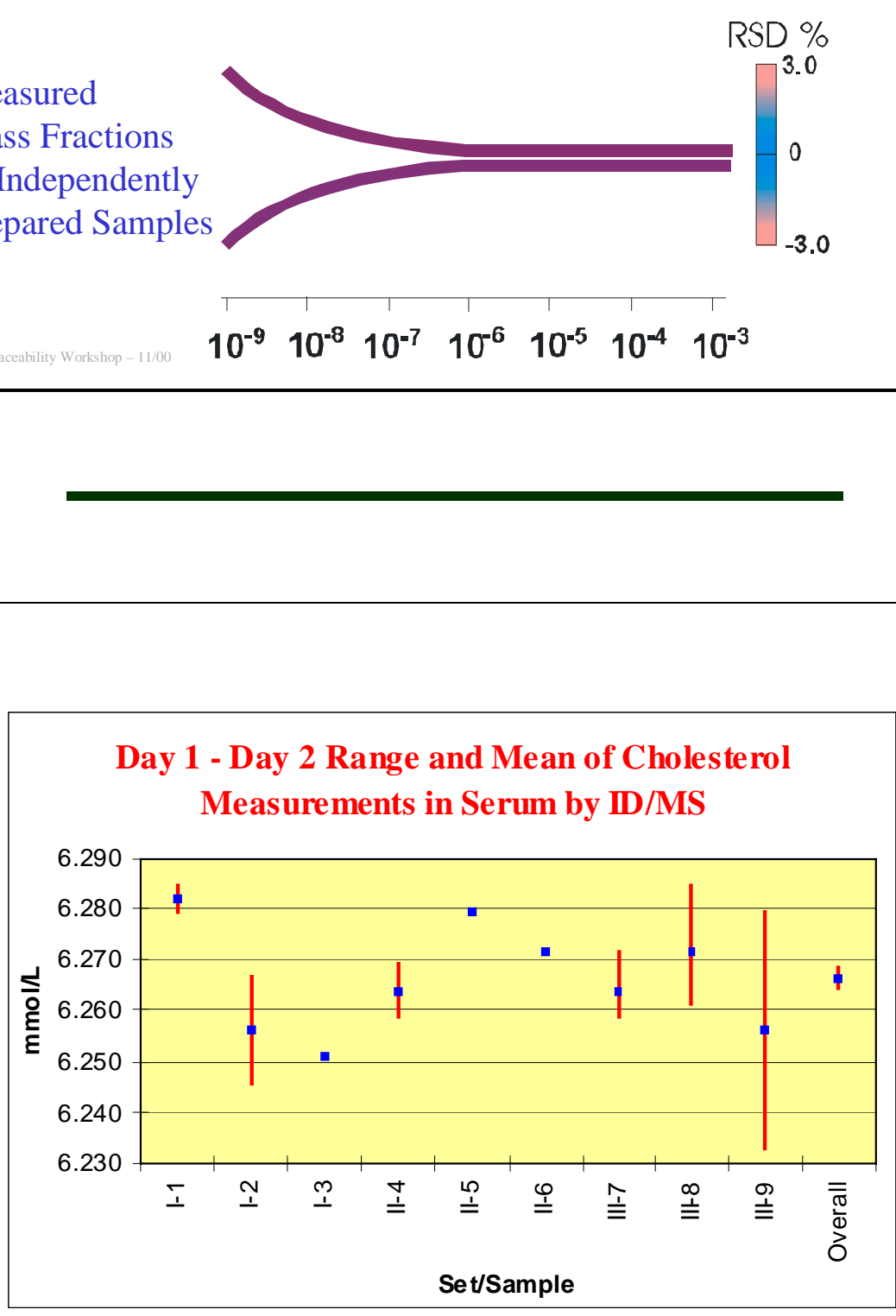
History of Clinical Standards Program

- Selected Serum Matrix Materials

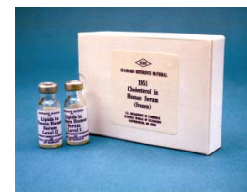

- 1979 SRM 900, Antiepilepsy Drug Level Assay Standard

- 1980 SRM 909, Human Serum

- 1982 SRM 1599, Anticonvulsant Drug Level Assay Standard

- 1988 SRM 1951, Cholesterol in Human Serum (Frozen) SRM 1952, Cholesterol in Human Serum (Freeze Dried)

- 1989 SRM 968, Fat Soluble Vitamins in Human Serum

- 1991

- 1996

- 1996 SRM 956, Electrolytes in Blood

SRM 956a, Electrolytes in Frozen Human Serum

- 1997

- 2000 SRM 965, Glucose in Frozen Human Serum (3 levels) SRM 1951a, Lipids in Frozen Human Serum (2 levels) SRM 966, Toxic Metals in Blood SRM 2070a, Toxic Elements in Human Urine

\section{CSTL Economic Impact Studies}

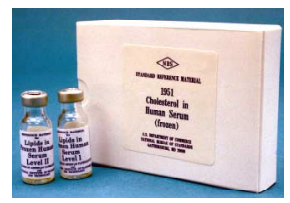

Title: SRMs for Cholesterol Measurements Products: NIST SRMs 911, 909, 1951 and 1952 (Clinical Standards)

Results(3 levels of supply chain): Benefit-Cost Ratio $\quad 4.5$ Social Rate of Return $\quad 154 \%$ GDV Net Present Value

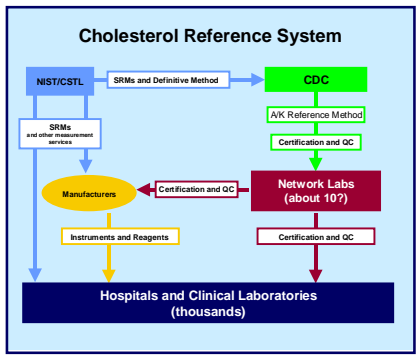

Title: SRMs for DNA Profiling Products: NIST SRMs 2390, 2391

Contract Awarded: July, 1998 expands existing NIJ Study

Comments:

- Web-based survey for data collection

- Representative data collected from state and local forensic laboratories
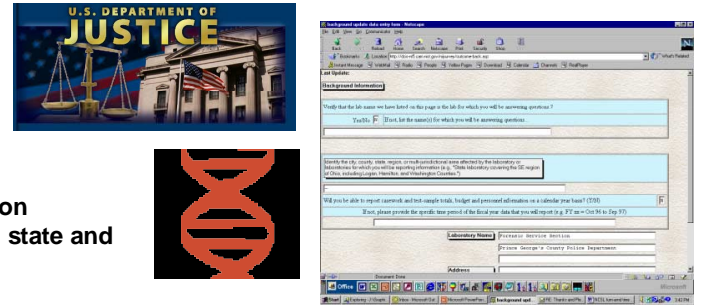


\section{Cholesterol Measurements}

Improved Cholesterol Measurement Accuracy Saves Health Care Dollars
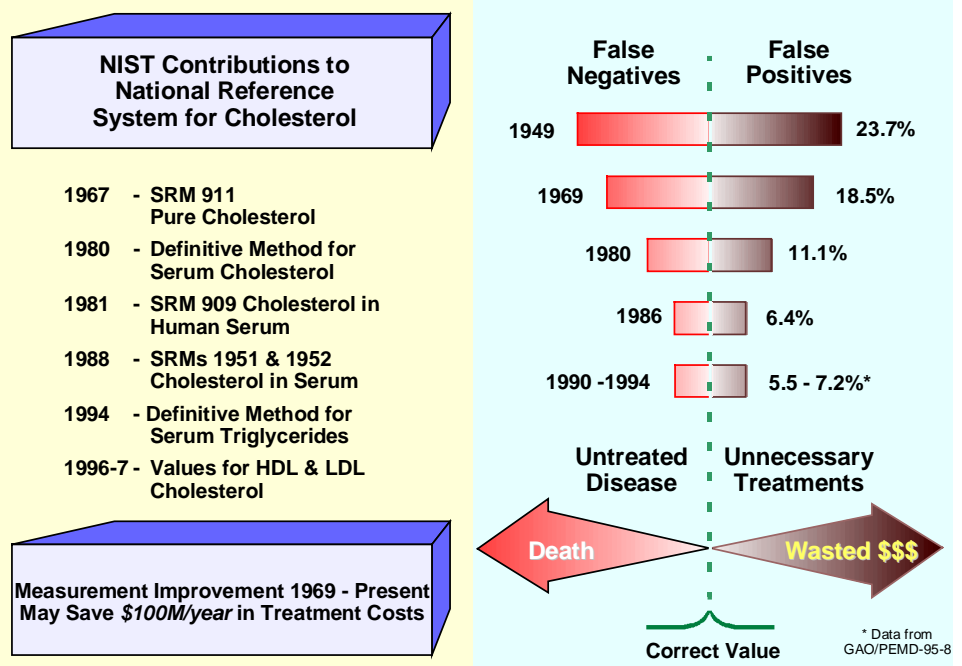

\section{Cholesterol in Blood Experiment - Impact of Inaccurate Measurements}

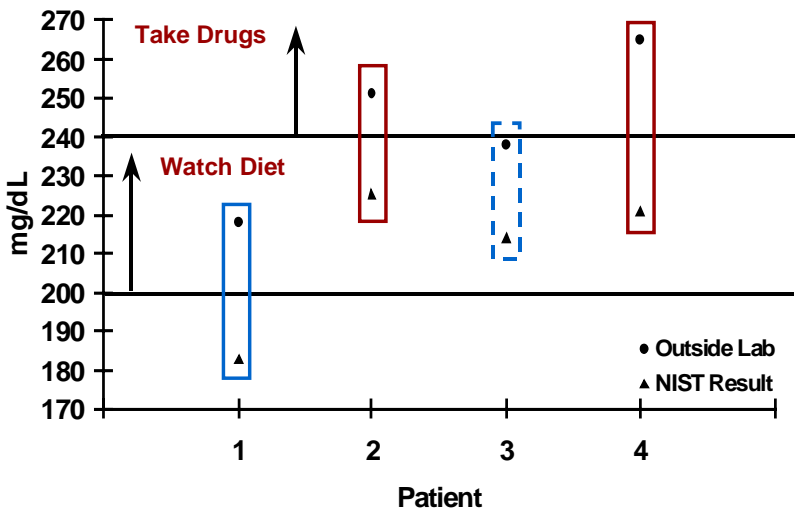




\section{The NIST Micronutrients Measurement Quality Assurance Program}

- to support laboratories that measure selected fat- and water-soluble vitamins and carotenoids in human serum and plasma

\section{NIST Provides:}

?Tools for Comparability Assessment - Interlaboratory Comparison Exercises -SRMs and Control Materials

-Performance Database

?Relevant Research in Measurement Science Workshops

?Site Visits, Tutorials, and Consultations

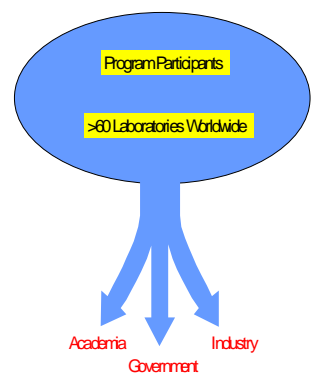

Program Result:

Improved accuracy of laboratory measurements resulting in increased interlaboratory comparability
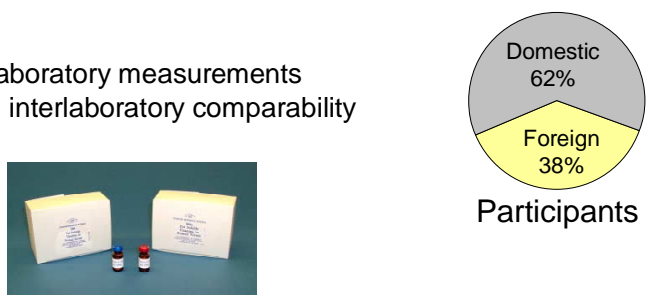

Participants

(1)

Quotes from letters ACD has received regarding the impact of the NIST Micronutrients Measurement Quality Assurance Program:

"We have been participating in the Micronutrients Measurement Quality Assurance Program for four years, and we think it is the best quality program for vitamins in the world. Moreover, the calculations are performed with the highest scientific and statistical level. I do not know any other program which proposes quality assurance for carotenoids."

Dr. H. Faure

Laboratoire de Biochimie C

La Tronche, France

"Currently, it (NIST Micronutrients Measurement QA Program) is the only QA program available for the fat-soluble vitamins and carotenoids. As a CAP/CLIA accredited laboratory, we are required to participate in external $Q A$ programs when available. We have used the data for this $Q A$ program at all of our accreditation inspections. A QA program from NIST carries a certain reputation that could be a benefit to any laboratory taking part in the program."

I. Osberg

The Children's Hospital

University of Colorado Health Sciences Center 
Quotes from letters ACD has received regarding the impact of the

NIST Micronutrients Measurement Quality Assurance Program: (continued)

"Obviously the Program benefits us by providing the independent assessment of our ability to measure antioxidant nutrients. In addition, the fact that we are registered in the scheme and can show independent documentary evidence of our analytical rating helps in our applications for funding to various bodies including the Ministry of Agriculture Fisheries and Food. I would also point out that as sub-optimum intakes of antioxidant nutrients are increasingly implicated in many diseases including coronary heart disease and cancer, there is a need for a QA scheme to monitor the accuracy of results of the growing number of epidemiological and clinical trials undertaken in this areas. As far as I am aware, no other scheme such as the [NIST] Micronutrients Measurement Quality Assurance scheme exists, certainly not in Europe."

Dr. G. Duthie

Rowett Research Institute

Scotland, UK

"The addition of vitamin A to my list of analyses would have been essentially impossible without SRM 968 and the assistance of the capable staff at NIST. The truth was then (and still is) that there are no other programs, no other reference materials out there for those of us who measure these analytes...the availability of the Micronutrients Program (SRM and round robin samples) has enabled me to provide service to patients"..

P. Radmacher

Department of Pediatrics

University of Louisville

\section{CCQM Key Comparison Areas:}

\section{Health}

- clinical diagnostic markers

(cholesterol/heart disease, diabetes/glucose,

creatinine/kidney function, trace hormones)

- electrolytes ( $\mathrm{Na}, \mathrm{K}, \mathrm{Ca})$

- Pb in blood

- Anabolic steroids in urine

\section{Food}

- pesticide residues

- antibiotics in meat

- growth hormones in meat

- vitamins and minerals

- drinking water (EPA List)

\section{Environment}

- air (EPA HAPs List)

- soil/sediments

- biological tissues

- waste water (EPA List)

\section{Advanced Materials}

- semiconductors

- metal alloys

- polymers and plastics

\section{Forensics}

- drugs of abuse

- explosive residues

- breathalyzer (ethanol-in-air)

- DNA profiling

\section{Commodities}

- emissions trading $\left(\mathrm{SO}_{2}\right.$ in stack emissions)

- sulfur in fossil fuels

- natural gas

- sucrose

- cement (Ca, Si, Al, S, Ti, Na, Mg)

- source of origin/adulteration

\section{Pharmaceuticals}

\section{Biotechnology}



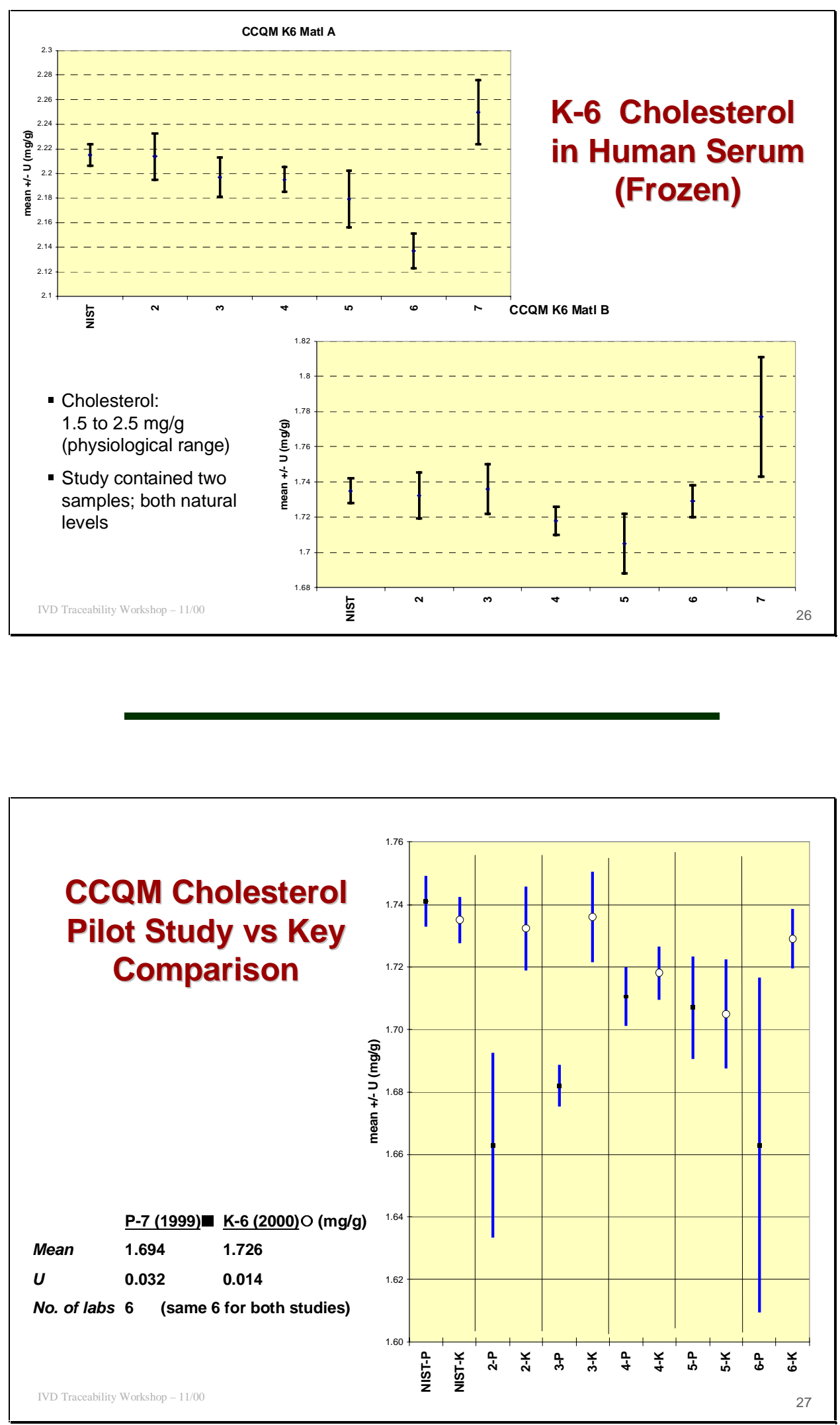


\section{Health Care Measurements:}

\section{... supporting the national reference system and the IVDD industry}

\begin{tabular}{ll} 
Methods for 12 Health Status Markers \\
Metain and Refine Definitive \\
Calcium & Cancer, Blood Clotting \\
Chloride & Kidney Function \\
Cholesterol & Heart Disease \\
Creatinine & Kidney Function \\
Glucose & Diabetes \\
Lithium & Antipsychotic Treatment \\
Magnesium & Heart Disease \\
Potassium & Electrolyte Balance \\
Sodium & Electrolyte Balance \\
Triglycerides & Heart Disease \\
Urea & Kidney Function \\
Uric Acid & Gout \\
\hline
\end{tabular}

Characteristics of current markers:

- Relatively small well-defined molecular or elemental species

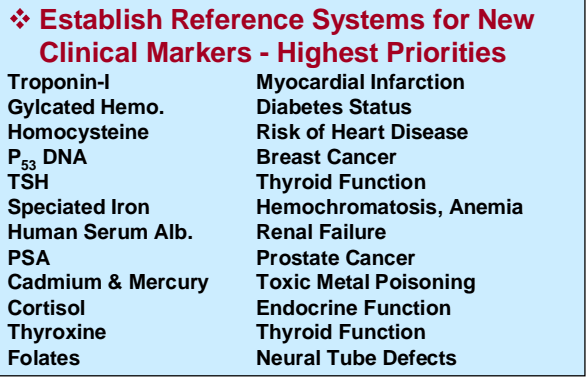

Measurement Challenges for new markers:

- High molecular mass proteins (>20,000 daltons)

- Heterogeneity of the protein

- Separation of different forms of the proteins

- Serum matrix complex; analyte level low

- Stability of analytes

- Standardization necessary before medical

diagnostic benefit can be realized

\section{Assessing Needs and Setting Priorities}

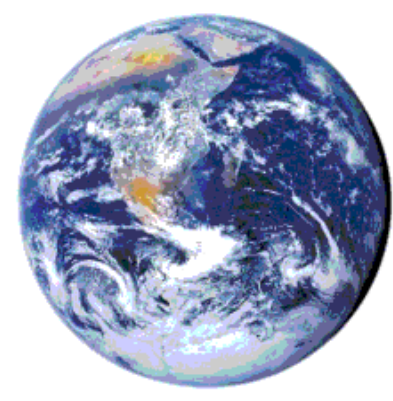




\section{Heart Disease}

Heart disease is the number one cause of death in the United States and accounts for $1 / 3$ of all deaths

Myocardial infarction (AMI) is responsible for $30 \%$ of these deaths

Approximately 6 million people visit ERs annually for chest pain

Approximately 3 million are admitted for possible AMI

Of these, 2 million are not diagnosed as having AMI

[FALSE POSITIVE $\Rightarrow$ UNNECESSARY MEDICAL COSTS!!!]

Of those not admitted , 2-8 \% actually had an AMI

[FALSE NEGATIVE $\Rightarrow$ DELAYED TREATMENT $\Rightarrow$ DEATH??]

\section{CARDIAC TROPONIN I (cTnl)}

v Protein found in Heart Muscle

$\checkmark$ Released into the Blood following Myocardial Infarction

$\checkmark \quad$ Useful Clinical Marker for Myocardial Infarction

- Occurs in Blood as Free cTnl and as Complex with Troponins C \& $T$

$\checkmark \quad$ Results vary widely among Different Immunoassays 


\section{Example of Variability of Results Among Currently Used Clinical Immunoassays}

Results of Measurements of Troponin-I from Same Sample Pool Using Immunoassay Kits from Three Different Manufacturers

\begin{tabular}{ccc}
$\begin{array}{c}\text { Assay } \\
\text { Manufacturer }\end{array}$ & $\begin{array}{c}\text { Troponin-I } \\
\text { Concentration } \\
\mathrm{ng} / \mathrm{mL}\end{array}$ & \# labs reporting \\
\hline A & 19.9 & 115 \\
B & 6.7 & 489 \\
C & 0.85 & 27
\end{tabular}

From G. S. Bodor, Denver Health and Hospitals -- personal communication 1997

"Failure to use effective treatments . . . for acute myocardial infarction for all patients who could benefit from these interventions may lead to as many as 18,000 preventable deaths each year in the United States."

- JAMA, 280, 1000, September 16, 1998.

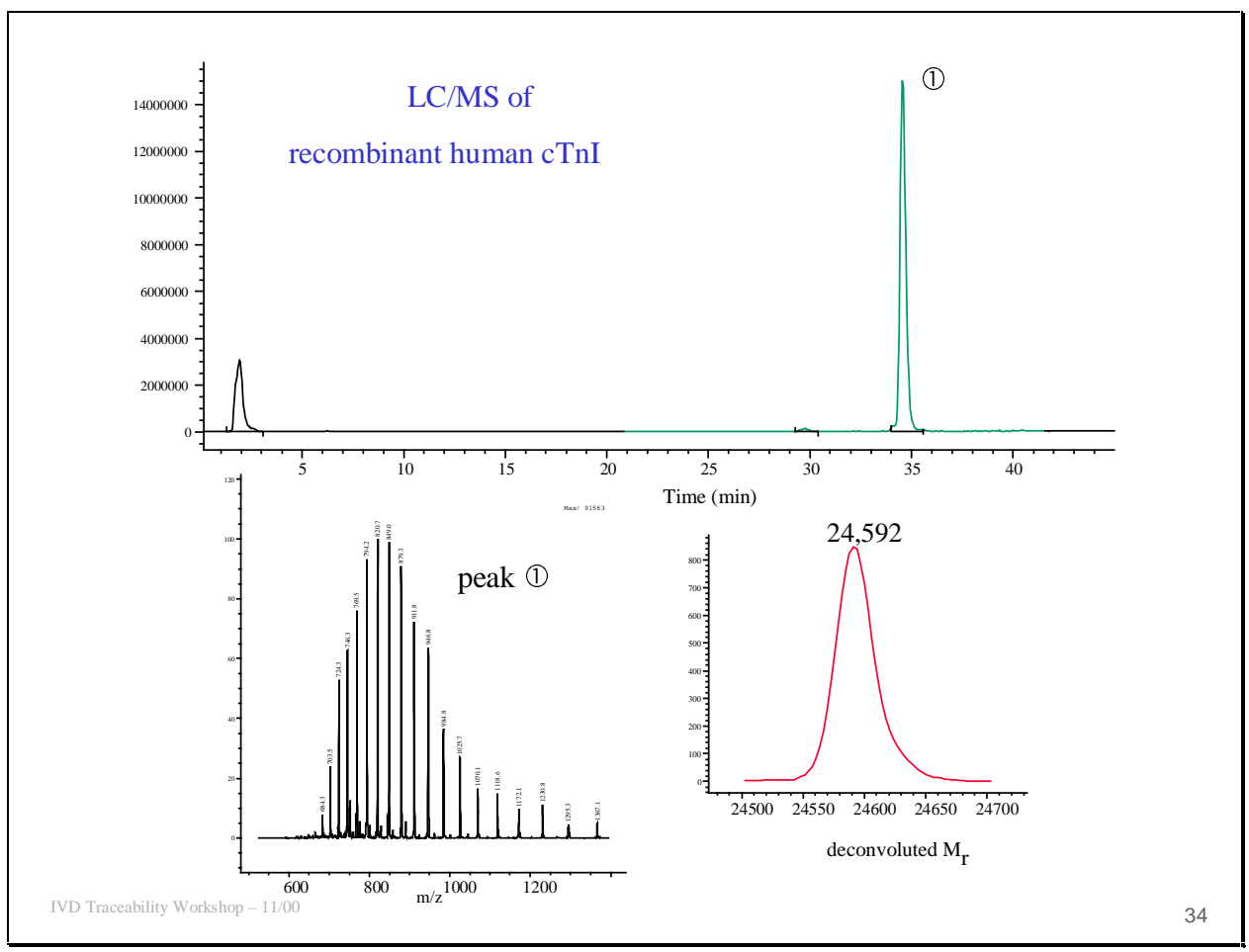




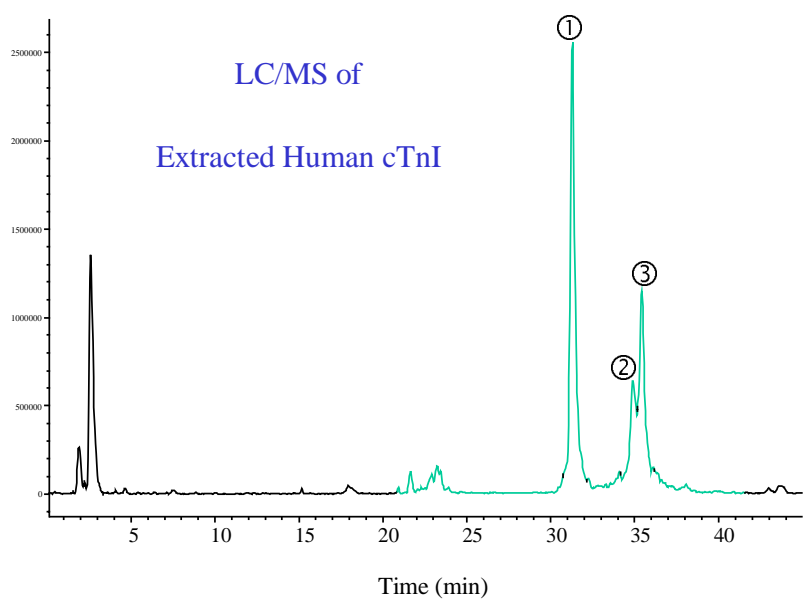

\section{NIST Program Vision:}

-Expand existing clinical standards program to encompass all major diagnostic markers [perhaps through more effective networking]

-Expand food standards program to address GMO, adulteration and other issues of US concern

-Establish program for credentialing commercial instrument calibrators

-Work with the U.S. College of American Pathologists and appropriate EU counterparts to establish trans-Atlantic Clinical Laboratory Chemical Measurements Proficiency Demonstration Program 


\section{Preliminary Plan of Action}

- NIST would develop and maintain reference methods and SRMs for up to $40 \%$ of the A list analytes

- NMls in the EU, Japan and Australia would also develop methods and CRMs for the remainder of analytes

- Mechanisms for mutual recognition of reference methods and assigned values for CRMs are being discussed [two models]

\section{Assessing Needs and Setting Priorities for Health Status Markers: How do we Proceed??}

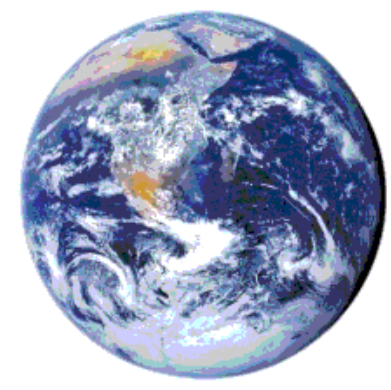




\section{Acknowledgement:}

Health-Care Markers Team

it David Bunk

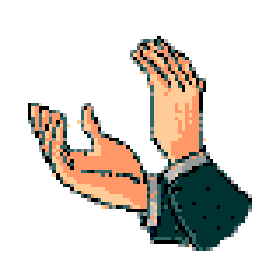

is Joe Dalluge

is Yolanda Davidson

is Polly Ellerbe

is Steve Long

is Bryant Nelson

it Chad Nelson

is Curtis Phinney

is Lorna Sniegoski

is Susan Taia

is Bob Vocke

it Michael Welch 


\section{Importance of Commutable Reference Materials and Patient Test Distributions for Assay Calibration George G. Klee \\ Department of Laboratory Medicine and Pathology \\ Mayo Clinic and Mayo Foundation}

\section{Introduction}

The development of integrated health care systems and consensus agreements of practice guidelines has increased the requirements of uniformity and standardization for clinical laboratory measurements. Small charges in analytic set-points produce major charges in the number of patients having test values which cross critical decision thresholds. Most practice guidelines and many clinicians assume that all test methods produce equivalent test values and therefore provisions for laboratory differences seldom are included in practice decisions. Therefore, it is important for laboratorians, industries producing laboratory diagnostic reagents, and laboratory regulatory agencies, to develop systems for minimizing these differences in laboratory test values. This paper illustrates the effects of analytical bias on the changes in the number of patients crossing key decision thresholds, and shows that certified reference materials by themselves are not sufficient to assure uniform laboratory practice. A novel protocol is proposed for calibration adjustment that utilizes a combination of reference analytic methods, commutable control materials and statistical processing of the distributions of test measurements from welldefined patient groups to minimize the variation of reported test values.

\section{Effect of Analytic Bias on Medical Decisions}

There are multiple sources of errors in laboratory measurements, but one helpful way of classifying these errors is to divide them into random and systematic errors. Random errors, as the term implies, occur sporadically and can be evaluated in terms of the test imprecision (standard deviation (SD) or coefficient of variation (CV)). In clinical decisions, assay imprecision combines with patient biologic variability to produce a total variability that is larger than either of these two components. Therefore, analytical imprecision only significantly increases the total variability when it is large relative to the biologic variation. This relationship had formed the basis for laboratory precision performance goals (1-5). If the analytical imprecision (SD) is less than one-quarter of the biological variation, the total variation (SD) is only about $3 \%$ larger than the biological variation. On the other hand, systematic errors directly effect all clinical decisions without the buffering effect of biological variance. The effect of these bias errors can be evaluated by examining shifts in the distribution of test measurements. Figure one illustrates this concept using the Gaussian distribution. Although most laboratory test distributions are not Gaussian, the general effects of analytic shifts for most laboratory test distributions is similar to the effects on Gaussian distributions (6). In the figure, the clinical decision threshold is set at +2.0 SD. When the laboratory test is operating with the cor- 
Figure 1: Effect of Analytic Bias on Decisions Illustration of effect of analytic bias on the percentage of a Gaussian distribution that crosses the +2 SD decision threshold. Reproduced and modified with permission of Clinical Chemistry (1993:39:1514-1518)

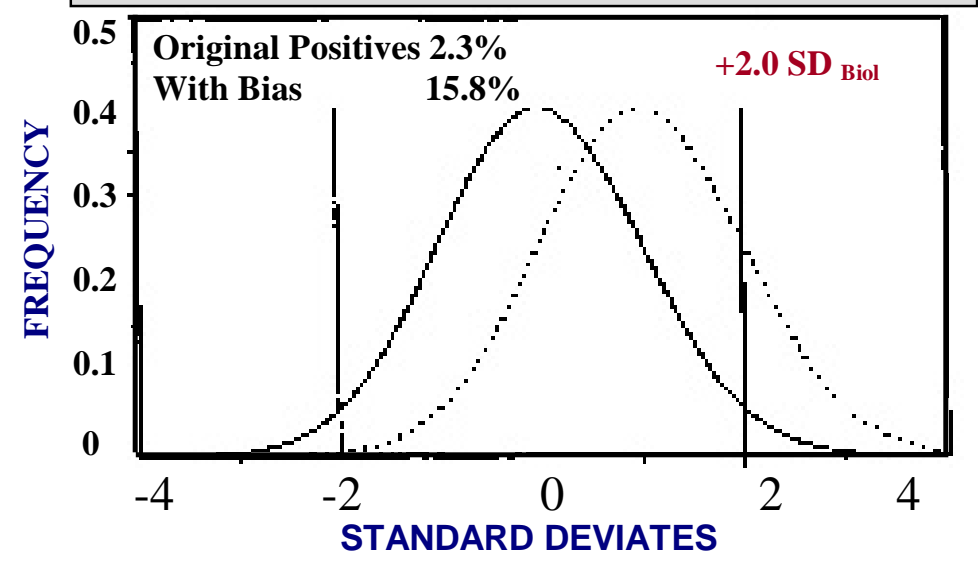

correct set-point, about $2.3 \%$ of the patients without the disease in question have values which exceed this threshold (falsepositives). However, if the analytic test is biased 1.0 SD high (as illustrated by the dotted line) then $15.8 \%$ of the patients without disease have test values which cross this threshold. This causes an almost seven-fold increase in false-positives. Obviously, most clinical decisions are not based solely on laboratory test values, but for some key analytes especially those used for screening, laboratory tests are important factors for medical decisions and analytical bias can cause major problems $\mathbf{( 7 , 8 )}$. Since many of these medical decisions involve expensive second and third level clinical investigations and/or the prescribing of expensive drugs, small differences in laboratory test calibrations can have major effects on downstream medical costs.

Table-one illustrates the effect of analytical bias on the number of patients having cholesterol or thyrotropin (TSH) test measurements which exceed the $200 \mathrm{mg} / \mathrm{dL}$ or 5.0 $\mathrm{mIU} / \mathrm{L}$ decision thresholds. An assay for cholesterol that is biased $1 \%$ high results in a $3.0 \%$ increase in the number of patients having "high" cholesterol values (using actual test distributions rather than Gaussian

Table 1

Effect of Analytic Bias on Clinical Decisions

\begin{tabular}{|c|c|c|c|}
\hline Analyte & alytic Bias & $\underline{\text { Patients }}$ & Multiplier \\
\hline $\begin{array}{l}\text { Cholesterol } \\
@ 200 \mathrm{mg} / \mathrm{dL}\end{array}$ & $\begin{array}{r}+1 \% \\
+3 \% \\
+10 \%\end{array}$ & $\begin{array}{l}+3.0 \% \\
+\mathbf{8 . 8 \%} \\
+\mathbf{2} 1.8 \%\end{array}$ & $\begin{array}{c}3.0 X \\
2.9 X \\
2.8 X\end{array}$ \\
\hline $\begin{array}{l}\text { TSH } \\
@ 5.0 \text { mIU/L }\end{array}$ & $\begin{array}{r}+6 \% \\
+10 \% \\
+20 \%\end{array}$ & $\begin{array}{l}+\mathbf{1 0 . 8 \%} \\
+21.7 \% \\
+\mathbf{5 0 . 8 \%}\end{array}$ & $\begin{array}{l}1.8 X \\
2.2 X \\
2.5 X\end{array}$ \\
\hline
\end{tabular}
models). Similarly, a $3 \%$ bias causes a $8.8 \%$ increase, and a $10 \%$ bias causes a $27.8 \%$ increase. These numbers are based on the average distribution of cholesterol test values for out-patients seen at the Mayo Clinic (7). Overall, the change in the percentage of patients crossing this $200 \mathrm{mg} / \mathrm{dL}$ threshold increases about three fold greater than the percentage change in the analytical shift due to the multiplier effect of the distribution curves. Similar effects are found at other decision levels for cholesterol, such as 240 
$\mathrm{mg} / \mathrm{dL}$, and for other tests. Table one illustrates that the multiplier is about 2 to 2.5 fold for comparing the effect that changes in analytical bias for the TSH assay have on the changes in the percentage of patients with TSH values greater than $5 \mathrm{mIU} / \mathrm{L}$. Again, similar effects are found at other decision levels.

\section{Analytic Differences Across Assays Utilizing the Same Standard Reference Material}

Most assays for human chorionic gonadotropin (hCG) currently are standardized to the WHO $3^{\text {rd }}$ International Reference Preparation (WHO IRP 75/537); however even with the use of this common standard, major differences are found between test values measured with different assays (9). Table 2 illustrates the differences across theses assays found with a recent College of American Pathologists (CAP) Survey (10).

\begin{tabular}{|c|c|c|c|}
\hline \multicolumn{4}{|c|}{$\begin{array}{c}\text { Human Chorionic Gonadotropin (hCG, mIU/L) } \\
\text { (Who Third IRP) } \\
\text { College of American Pathologist K-A Survey } 2000\end{array}$} \\
\hline Method & $\underline{K-02}$ & $\underline{K-05}$ & $\underline{K-01}$ \\
\hline Abbott Architect & 7.36 & 176.84 & 841.36 \\
\hline Abbott Axsym & 9.87 & 229.28 & 969.64 \\
\hline Beckman Access & 13.54 & 158.20 & 667.14 \\
\hline Biomerieux Vidas/Mini & - & 247.08 & 1213.04 \\
\hline Chiron ACS:180 & 11.02 & 241.22 & 946.92 \\
\hline Chiron ACS:Centaur & 11.21 & 240.76 & 923.22 \\
\hline Dade Dimension HM & 0.76 & 238.88 & 1093.49 \\
\hline DPC Immulite & 9.12 & 253.78 & 1060.02 \\
\hline DPC Immulite 2000 & 12.14 & 270.82 & 1269.82 \\
\hline Roche/BMC Elecsys 10/2010 & 1.56 & 212.73 & 968.82 \\
\hline Technicon Immuno-1 & 5.97 & 197.01 & 916.01 \\
\hline Tosoh AIA-Pack BHCG & 5.42 & 230.02 & 1094.46 \\
\hline Vitros ECI & 6.04 & 222.79 & 949.76 \\
\hline ALL METHODS & 9.37 & 224.22 & 953.53 \\
\hline
\end{tabular}

This survey utilizes intact hCG spiked into human serum. Matrix effects of the survey material may cause part of these differences, but major between-assay differences also are seen with patient samples. Even within the same manufacturer, different assays often do not give harmonious results. Even larger differences have been seen when proficiency specimens which include both intact and free beta subunits of hCG were evaluated $(\mathbf{1 1}, \mathbf{1 2})$. Several factors may contribute to these differences in hCG assays:

1) Differences in the immunoreactivity of the antibodies used in the assays for specific epitopes on the hCG molecule which may be differentially expressed in the standard compared to the control or patient specimens. 
2) Cross-reactivity and/or inhibition with alternate molecular forms or metabolic fragments of the hCG molecule which may be present in the standards, controls and/or specimens tested.

3) Matrix differences between the standards and the specimens tested.

4) Differences in the glycosylation and (or protein nicking) between the standard and the specimens.

5) Lack of true traceability of the assay to the reference standard. (The first generation of the assay may have been directly standardized and subsequent generations may be only indirectly linked by comparison studies)

Biochemical, antigenic and metabolic differences in complex glycoproteins such as hCG make standardization and harmonization difficult; however, even simpler molecules like triiodothyronine (T3), which have homogenous crystalizable standards, have major analytical differences among assays. Table 3 illustrates the differences found in T3 measurements across methods for the CAP K-A, 2000 survey. Again, major differences are found even between assays from the same manufacturer. It is assumed that these differences are related to matrix issues and/or differences in standardization protocols and/or large tolerances in manufacturing specifications.

\begin{tabular}{|lccc|}
\hline \multicolumn{4}{|c|}{ Table 3} \\
Triiodothyronine $(T 3, n g / d L)$ \\
College of American Pathologist $K$-A Survey 2000 \\
Method & $\underline{K-02}$ & $\underline{K-05}$ & $\underline{K-01}$ \\
Abbott Architect & 115.9 & 288.5 & 490.6 \\
Abbott Axsym & 108.0 & 259.1 & 519.3 \\
Beckman Access & 131.4 & 309.1 & 660.2 \\
Chiron ACS:180 & 107.2 & 260.8 & 700.2 \\
Chiron ACS:Centaur & 124.5 & 286.7 & $\mathbf{7 3 3 . 0}$ \\
DPC Immulite & 74.5 & 218.8 & 458.7 \\
DPC Immulite 2000 & 67.9 & 199.1 & 418.6 \\
Roche/BMC Elecsys 10/2010 & 168.6 & 438.5 & - \\
Technicon Immuno-1 & 89.8 & 243.2 & 497.6 \\
Tosoh AIA-Pack BHCG & 101.6 & 294.0 & 655.5 \\
Vitros ECI & 131.6 & 301.2 & 658.1 \\
ALL METHODS & 109.0 & 268.1 & 574.0 \\
\hline
\end{tabular}

Significant assay differences also have been found between reagent lots and across time within the same assay system, especially when key components are changed. Even when no components in the reagents are changed, significant lot-to-lot variations frequently occur do to large tolerance specifications in reagent manufacturing and large tolerances in the assignment of calibration set-points for production instruments. 


\section{Proposal for improving Assay Calibration Adjustment}

This proposal for assay calibration adjustment has five basic parts: 1) certified reference materials, 2) reference measurement systems capable of measuring both the reference material, controls and human specimens, 3) commutable control specimens which have values traceable to reference analytical systems, 4) human specimens from patients with well characterized clinical states, and 5) real-time data processing systems for processing both the control specimen data and the patient test values. A combination of statistical rules for monitoring the traceable control results and mathematical algorithms for tracking the patient test value distributions are used to adjust assay calibration to assure uniform test performance.

The term "commutable" is defined by Webster as "capable of being exchanged or interchanged" (13). The phrase "commutable control specimens" refers to control specimens that perform identically to patient specimens in both the reference system and the production assay system. "Commutability" means that measurements of these control specimens on the production system that recover the same set-points that were assigned to the controls by the reference system, will assure that the production system also will recover the same population set-point as the reference system for a group of patient specimens. A potential protocol for testing this definition of commutability of a control would be to measure the control 10 times on both the reference assay and the production assay. Then compare the difference in the averages of the control values on the two systems with the average difference found by testing 10 patient samples on both systems. If the average of the controls can be used to harmonize the patient values, then the control is commutable. When these commutability requirements are met, statistical factors can be developed to provide real-time calibration adjustments, which can be used to correct for inaccuracies in production measurement systems, caused by manufacturing variations and/or inaccuracies in assay calibration assignment.

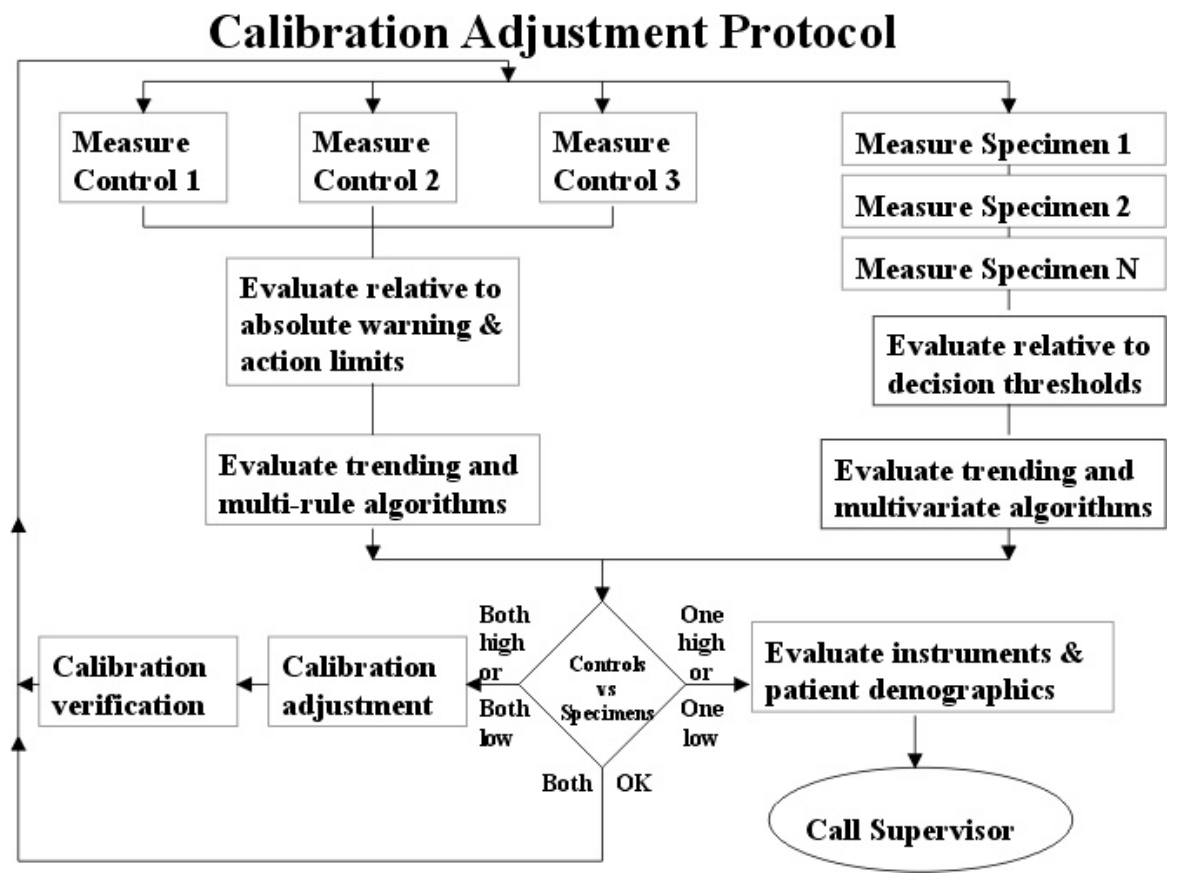

Figure two outlines a protocol for calibration adjustment using a combination of three commutable control sera and the statistical distributions of patient test values. The control test values are evaluated relative to absolute warning and action limits assigned to the controls using es- 
tablished reference methods. Multi-rule tracking algorithms help to evaluate these changes (14). Patient test values are normalized for demographic and clinical differences, then evaluated relative to clinical decision thresholds. Again, statistical algorithms help to track trending differences (14-16). If both systems indicate that the same analytical bias shift has occurred, the assay calibration is adjusted statistically to bring it back to the prior level. After adjustment, the calibration is verified by replicate (5 to 10) measurements of reference calibrators. If the two tracking systems (controls versus patient specimens) differ, then the controls and the patient demographics, as well as preanalytical factors and instrument performance are evaluated.

For large homogeneous groups of patients, central distribution parameters such as the median, $25^{\text {th }}$ and $75^{\text {th }}$ percentiles are remarkably constant. When analytical systems are tightly controlled the variation of these parameters is less than the usual reagent manufacturers tolerance specification for many key analytes. Consequently, tighter reagent control could translate directly into smaller short-term variation in key analytical parameters used in clinical practice. Some laboratory tests have large variations in patient test value distributions due to demographic and pathophysiologic differences. When these differences are well characterized, mathematical corrections can be used to make the distributions of patient test values more homogeneous. Examples of these corrections are: factors to correct for gender differences in hemoglobin concentrations; age adjustment for some hormone measurements; and renal function corrections for compounds cleared by the kidney. Further normalization can occur by sub-setting test distributions into separate tracking groups according to ordering location and/or time of the request. Examples of this type of normalization is to subset patients from pediatric and/or oncology areas, or to exclude patients from intensive care units or burn units, or to exclude weekend or late-night specimens in the calibration adjustment factor calculations.

\section{Conclusions}

Lack of laboratory test harmonization can cause major problems in patient care and assay calibration with standard reference preparations does not assure harmonization of patient test results. There are multiple reasons for this lack of harmonization, but nonhomogeneity of analytes and matrix effects could be major factors. A combination of statistical rules using commutable control material with traceable reference values and mathematical algorithms using distributions of normalized patient test values could be used for calibration adjustment. A preliminary protocol for this form of calibration adjustment is proposed.

\section{Disclaimer}

All conclusions and interpretations in this article with respect to the College of American Pathologists' data base are those of the author and not those of the College. 


\section{References}

1. Analytical Goals in Clinical Chemistry. College of American Pathologists' Conference, Aspen, CO, 1976, F.R.Elevitch, ed., College of American Pathologists, Northfield, IL 60093-2750.

2. Fraser CG, Petersen PH, Ricos C, Haeckel R: Proposed quality specifications for the imprecision and inaccuaracy of analyitical systems for clinical chemistry. Eur $\mathbf{J}$ Clin Chem Biochem. 30;311-317, 1992.

3. Fraser CG, Petersen PH: Desirable performance standards for imprecision and bias in alternate sites. Arch Pathol Lab Med. 119:909-913, 1995.

4. Fraser CG, Petersen PH, Libeer JC, Ricos C: Proposals for setting generally applicable quality goals solely based on biology. Ann Clin Biochem 34:8-12, 1997.

5. Fraser CG: General strategies to set quality specifications for reliability performance characteristics. Scand J Clin Lab Invest. 1999;59:487-490.

6. Klee GG: Tolerance limits for short-term analytical bias and analytical imprecision derived from clinical assay specificity. Clin Chem 39(7):1514-1518, 1993.

7. Klee GG, Schryver PG, Kisabeth RM. Analytic bias specifications based on the analysis of effects on performance of medical guidelines. Scand J Clin Lab Invest 59:509-512, 1999.

8. Petersen PH, de Verdier CH, Groth T, Fraser CG, Blaabjerg O, Horder M: The influence of analytical bias on diagnostic misclassification. Clin Chim Acta 260:189206, 1997.

9. Cole LA: Immunoassay of human chorionic gonadotropin, its free subunits, and metabolites. Clin Chem 43(12):2233-2243, 1997.

10. 2000. K/KN-A Ligand (general). Participant Summary Report. 2000, College of Surveys American Pathologists, Northfield, IL.

11. Klee GG: Serum HCG Assays: Wide across-method differences. College of American Pathologist. Therapeutic Drug Monitoring/Endo Resource Committee, March 1994.

12. Klee GG: Human Chorionic Gonadotropin. Mayo Clin Proc 69:391-392, 1994.

13. Woolf HB, ed. Websters New Collegiate Dictionary: Springfield, Massachusetts: G. \& C. Merian Company, 1979:229.

14. Westgard JO, Klee GG. In: Burtis CA, Ashwood ER, eds. Tietz Textbook of Clinical Chemistry $3^{\text {rd }}$ Edition. WB Saunders. Philadelphia. 1999:384-418

15. Klee GG: A conceptual model for establishing tolerance limits for analytic bias and imprecision based on variations in population test distributions. Clin Chim Acta 260:175-188, 1997

16. Smith FA, Kroft SH: Optimal procedures for detecting analytic bias using patient samples. Am J Clin Pathol 108:254-268, 1997. 


\section{GEORGE G. KLEE}

\section{Mayo Clinic and Mayo Foundation}

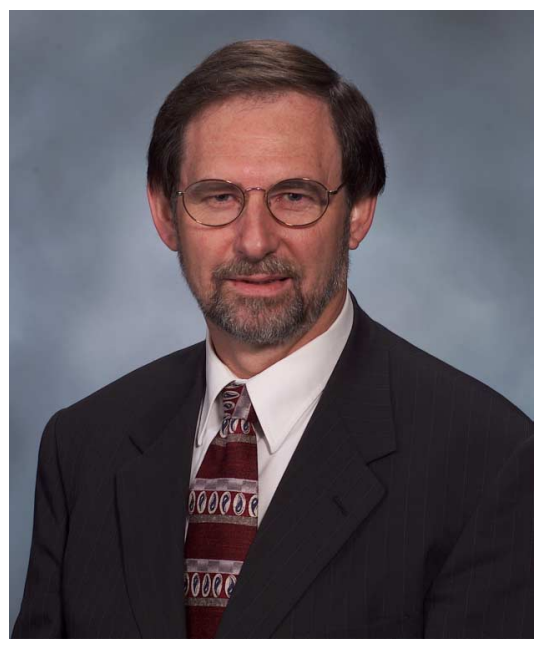

George G. Klee, M.D., Ph.D., is a clinical pathologist with over 20 years experience working with clinicians to provide quality laboratory support. He is a medical consultant at the Mayo Clinic with appointments in both the Department of Laboratory Medicine and Pathology and in the Department of Health Sciences Research. He holds the title of Professor in the Mayo medical School and is on graduate faculty of both Mayo Graduate School and The University of Minnesota. He has published over 100 papers related to endocrine testing, quality assurance, and health care outcomes. He is an active member of numerous societies including CAP, AACC, ACLPS, ATA, CLMA, and the AMA. 
Importance of Commutable Reference Materials and Patient Test Distributions for Assay Calibration

\author{
Visuals Presented By \\ George G. Klee
}

Department of Laboratory Medicine and Pathology

Mayo Clinic and Mayo Foundation 
Importance of Commutable Human Specimens and

Test Value Distributions for Assay Calibration Adjustment

George G. Klee, M.D., Ph.D.

Mayo Clinic

Rochester, Minnesota, USA

\section{Clinical Necessity for Standardization \& Harmonization}

- Differences and changes in analytic setpoints cause major discrepancies in clinical decisions.

- Practice guidelines with standardized action limits require better standardization of laboratory tests.

- Most clinicians assume all test methods are equivalent. 


\section{Two Analytic Control Issues}

- Precision (scatter)

- Accuracy (bias)

\section{Tolerance Limits for Precision}

- Analytic precision is clinically filtered by biologic variation

- If analytic SD $<1 / 4$ biologic SD, total SD only increases by $3 \%$ 


\section{Tolerance Limits for Analytic Bias}

- Bias directly affects test values

- Small analytic changes can produce major shifts in frequency distributions of clinical test values

\section{Effect of Analytic Bias on Decisions}

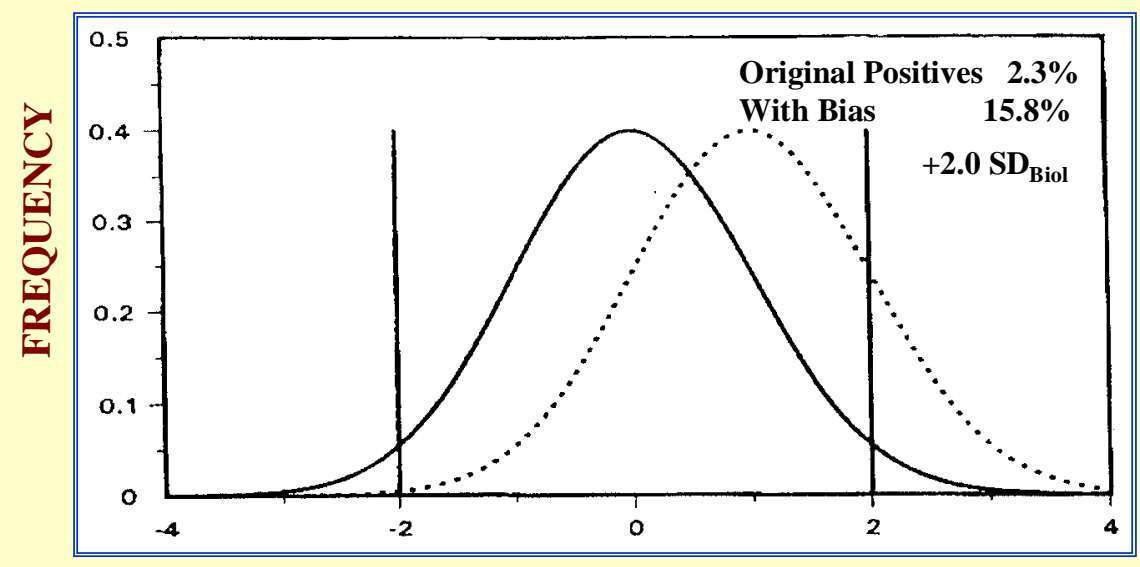

STANDARD DEVIATES 


\section{Frequency Distribution of Cholesterol Values}

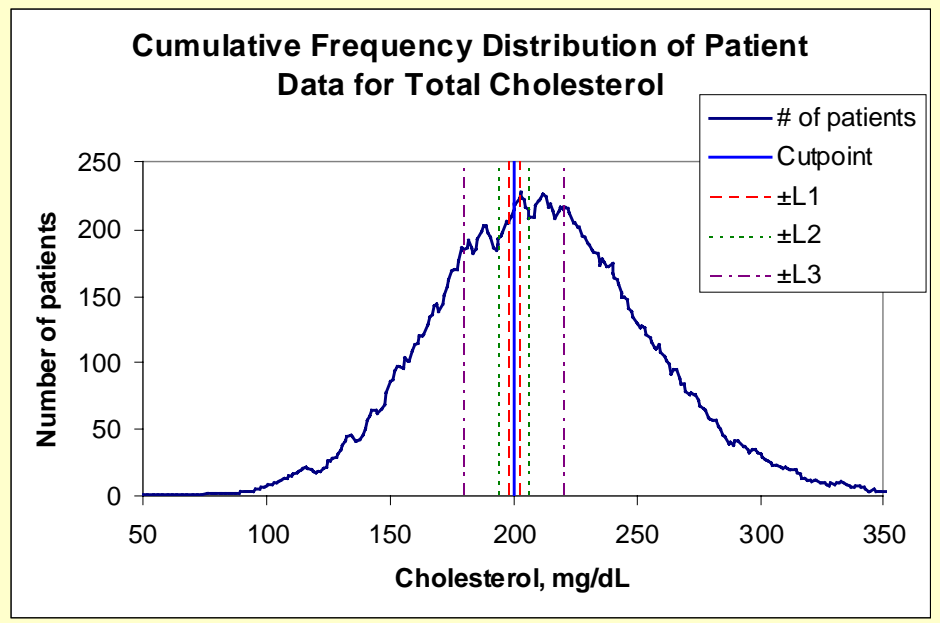

\section{Frequency Distribution of TSH Values}

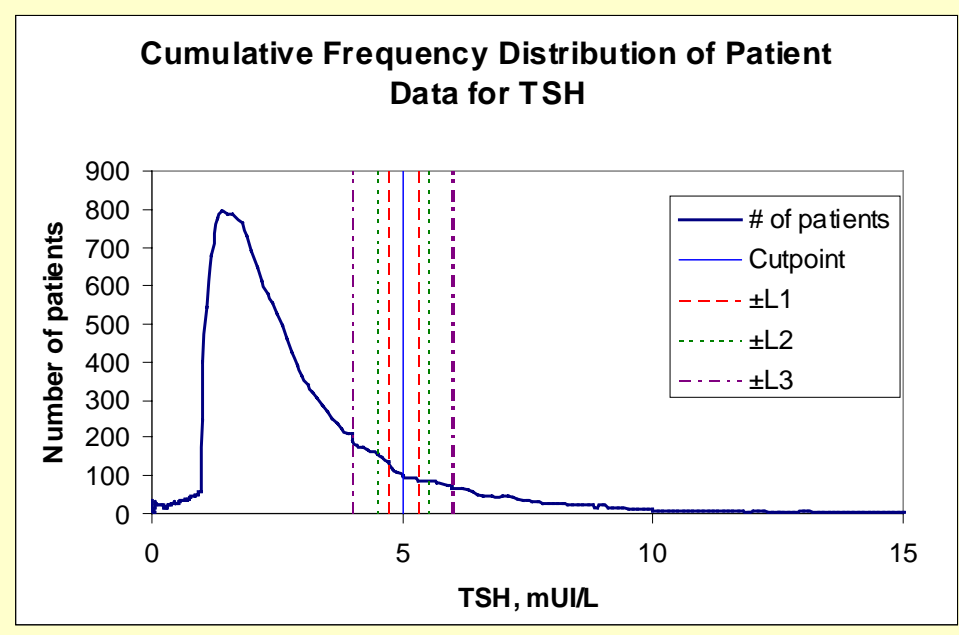


Effect of Analytic Bias on Clinical Decisions

\begin{tabular}{|c|c|c|c|}
\hline Analyte & Analytic Bias & Patients & Multiplier \\
\hline Cholesterol & $+1 \%$ & $+3.0 \%$ & $3.0 x$ \\
\hline \multirow[t]{2}{*}{$@ 200$ mg/dL } & $+3 \%$ & $+8.8 \%$ & $2.9 x$ \\
\hline & $+10 \%$ & $+21.8 \%$ & $2.8 x$ \\
\hline TSH & $+6 \%$ & $+10.8 \%$ & $1.8 x$ \\
\hline \multirow[t]{2}{*}{ @5.0 mlU/L } & $+10 \%$ & $+21.7 \%$ & $2.2 x$ \\
\hline & $+20 \%$ & $+50.8 \%$ & $2.5 x$ \\
\hline
\end{tabular}

\section{Problems with Current Laboratory Standardization}

- Lack of consistency between analytic methods even within the same diagnostic reagent companies

- Standardization with the same reference material does not assure consistency of patient results across methods

- Reagent and manufacturing processes can not economically maintain adequate consistency of analytic set-points. 


\section{Two Examples of Tests With Standards That Still Have Wide Method Differences}

- Human Chorionic Gonadotropin (hCG)

- Triiodothyronine (T3)

\section{Human Chorionic Gonadotropin (hCG) (Who Third IRP)}

College of American Pathologist K-A Survey 2000

Method

Abbott Architect

Abbott Axsym

Beckman Access

Biomerieux Vidas/Mini

Chiron ACS: 180

Chiron ACS:Centaur

Dade Dimension HM

$\begin{array}{ccc}\frac{K-02}{7.36} & \frac{K-05}{176.84} & \underline{K-01} \\ 9.87 & 229.28 & 969.64 \\ 13.54 & 158.20 & 667.14 \\ - & 247.08 & 1213.04 \\ 11.02 & 241.22 & 946.92 \\ 11.21 & 240.76 & 923.22 \\ 0.76 & 238.88 & 1093.49\end{array}$




\section{Human Chorionic Gonadotropin (hCG) (Who Third IRP) continued}

College of American Pathologist K-A Survey 2000

Method

DPC Immulite

DPC Immulite 2000

Roche/BMC Elecsys 10/2010

Technicon Immuno-1

Tosoh AIA-Pack BHCG

Vitros ECl

ALL METHODS

$\begin{array}{rcr}\frac{\mathrm{K}-02}{9.12} & \underline{\mathrm{K}-05} & \underline{\mathrm{K}-01} \\ 12.14 & 270.82 & 1060.02 \\ 1.56 & 212.73 & 969.82 \\ 5.97 & 197.01 & 916.01 \\ 5.42 & 230.02 & 1094.46 \\ 6.04 & 222.79 & 949.76 \\ 9.37 & 224.22 & 953.53\end{array}$

\section{Triiodothyronine (T3)}

College of American Pathologist K-A Survey 2000

Method

Abbott Architect

Abbott Axsym

Beckman Access

Chiron ACS: 180

Chiron ACS:Centaur

$\begin{array}{lll}\frac{K-02}{115.9} & \frac{K-05}{288.5} & \frac{K-01}{490.6} \\ 108.0 & 259.1 & 519.3 \\ 131.4 & 309.1 & 660.2 \\ 107.2 & 260.8 & 700.2 \\ 124.5 & 286.7 & 733.0\end{array}$




\section{Triiodothyronine (T3) \\ continued}

College of American Pathologist K-A Survey 2000

Method

DPC Immulite

$\frac{K-02}{74.5}$

$\underline{K-05}$

K-01

DPC Immulite 2000

67.9

218.8

458.7

Roche/BMC Elecsys 10/2010

Technicon Immuno-1

Tosoh AIA-Pack BHCG

89.8

199.1

418.6

168.6

438.5

Vitros ECI

101.6

243.2

497.6

ALL METHODS

131.6

294.0

655.5

109.0

301.2

658.1

268.1

574.9

\section{Proposal for Improving Standardization}

- Develop robust reference methods standardized with certified reference materials.

- Develop commutable control materials for comparison of routine methods with reference methods.

- Develop mathematical algorithms for tracking patient test value distributions.

- Use the combination of the traceable results and the patient test distribution to adjust assay calibrations to homogeneity. 


\section{Tandem Mass Spectrometry}

- Can have a dual role as a standard reference method and a production assay system

- Faster, cheaper and higher quality

- Can simultaneously measure multiple forms of an analyte to allow better determination of physiologically active forms which could potentially lead to better diagnoses and treatments

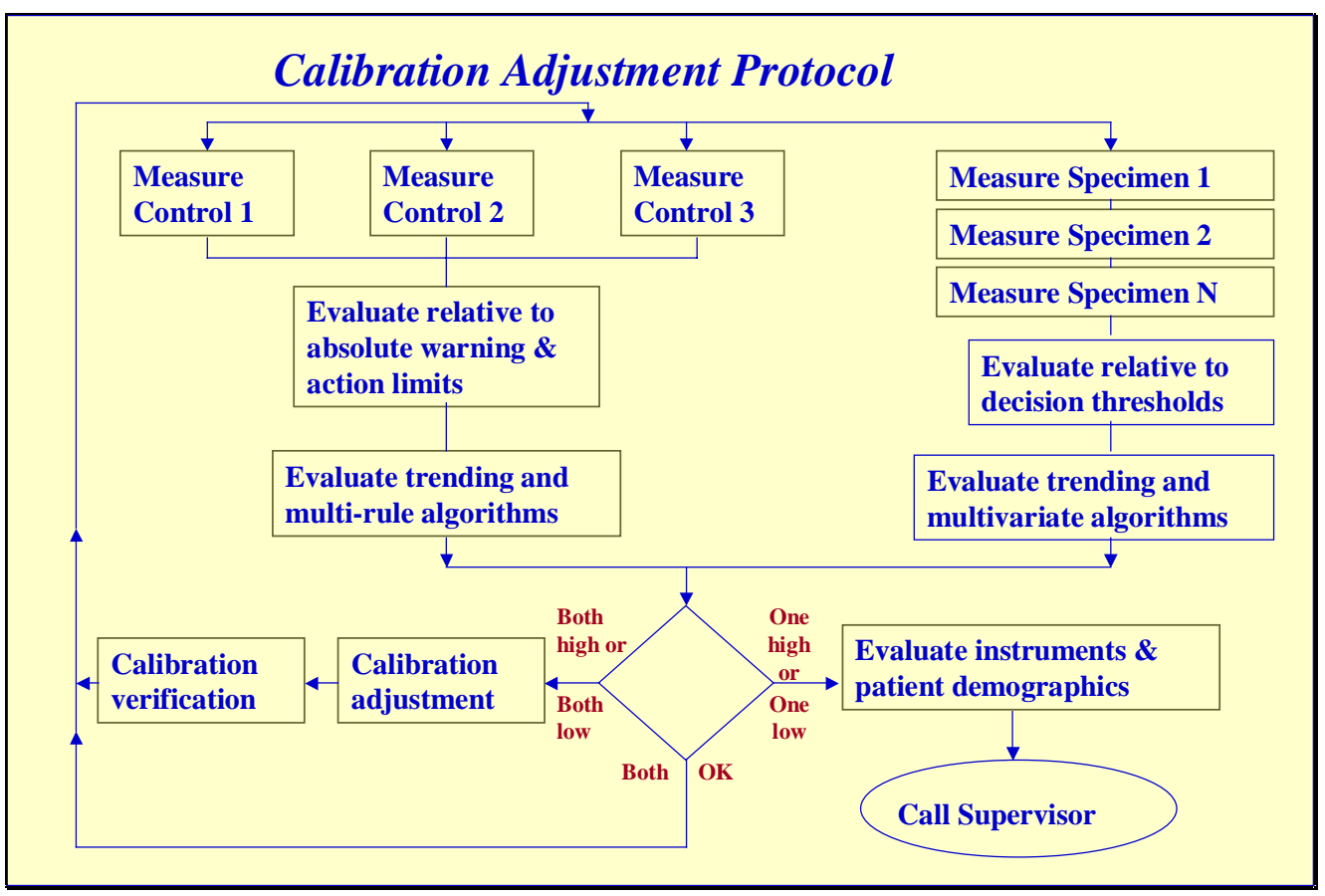




\section{Definition of Commutable}

Webster's definition for Commutable.

- "Capable of being exchanged or interchanged" Specific definition for Commutable Controls.

- "Controls that perform the same as patient samples"

- "Recovery of traceable values with controls assures recovery of traceable values with patient samples"

\section{Constancy of Normalized Distributions of Patient Values}

- For large homogenous groups of patients the median and key percentiles of test values are remarkably stable.

- For smaller non-homogenous groups mathematical corrections for demographic and pathophysiologic differences produces greater stability. 


\section{Mathematical Corrections for Demographics Differences}

- Age normalization (Inverse of Regression)

- Gender correction

- Corrections for Race and Ethnic groups

- Geographic correction

\section{Mathematical Corrections for Pathophysiology Differences}

- Normalize by creatinine

- Elimination of values from patients in

- End Stage Renal Failure/Dialysis

- End Stage Hepatic Failure

- ICU/CCU

- Subset Ambulatory versus Hospitalized Patients. 


\section{Illustration of Stability of Patient Test Distributions, Influenced Mainly by Analytic Changes}

- Collected calcium test values 20 days during a time when laboratory QC was acceptable.

- Median, 25th, 75th percentiles for each day (>1000 pts/day) tracked over time.

- Shift in these percentiles by about $0.1 \mathrm{mg} / \mathrm{dL}$ occurred after about 10 days.

- Shift in patient distributions corresponded to shift of time-averaged QC data.

\section{Patient Calcium Values at the 50th Percentile}

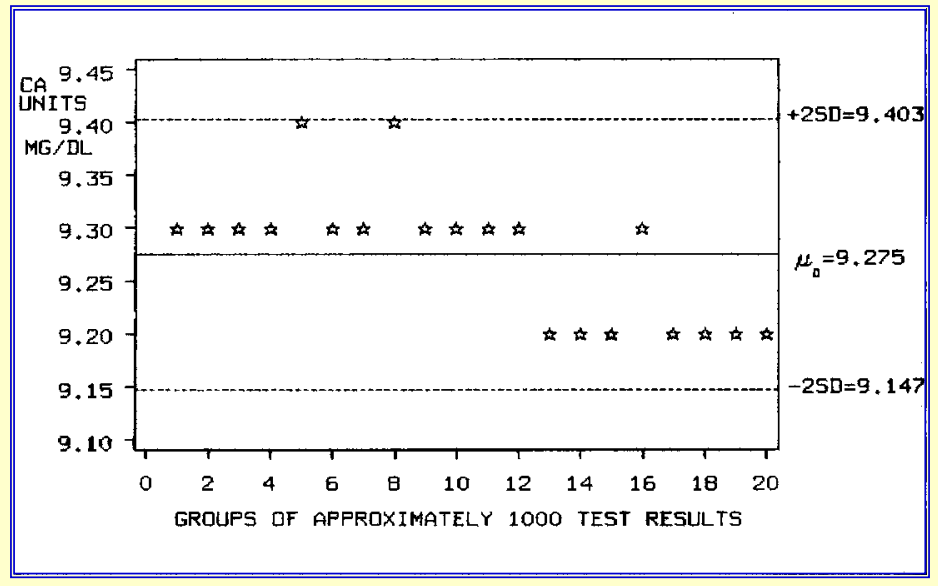




\section{Patient Calcium Values at the 25th Percentile}

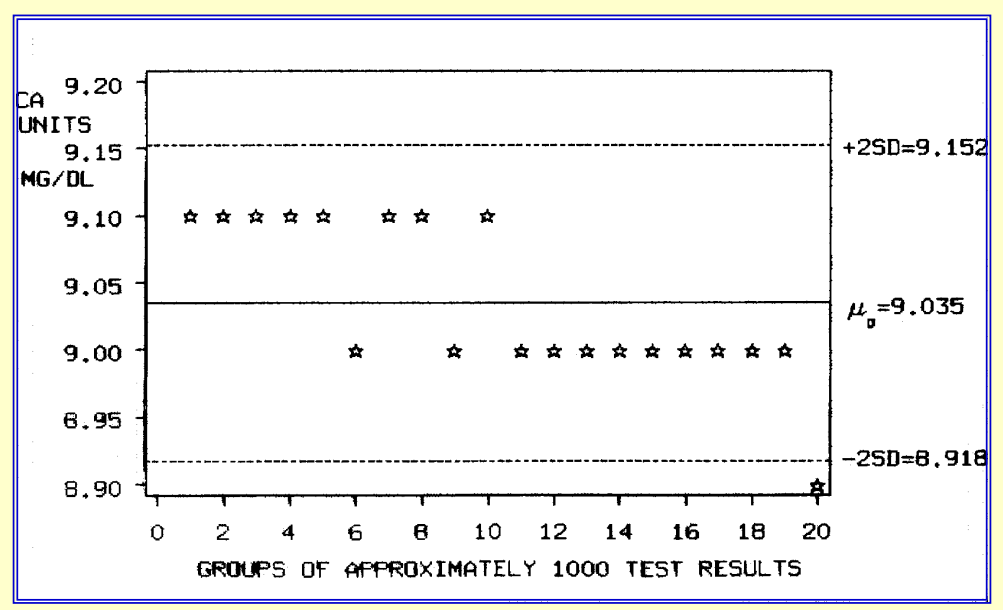

\section{Calcium Pool - CHEM 1}

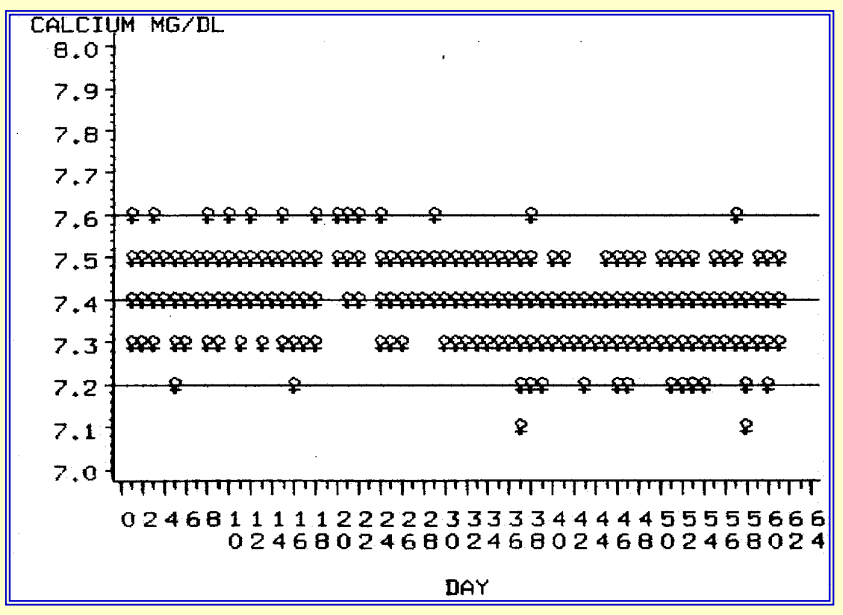




\section{Calcium Pool - CHEM 3}

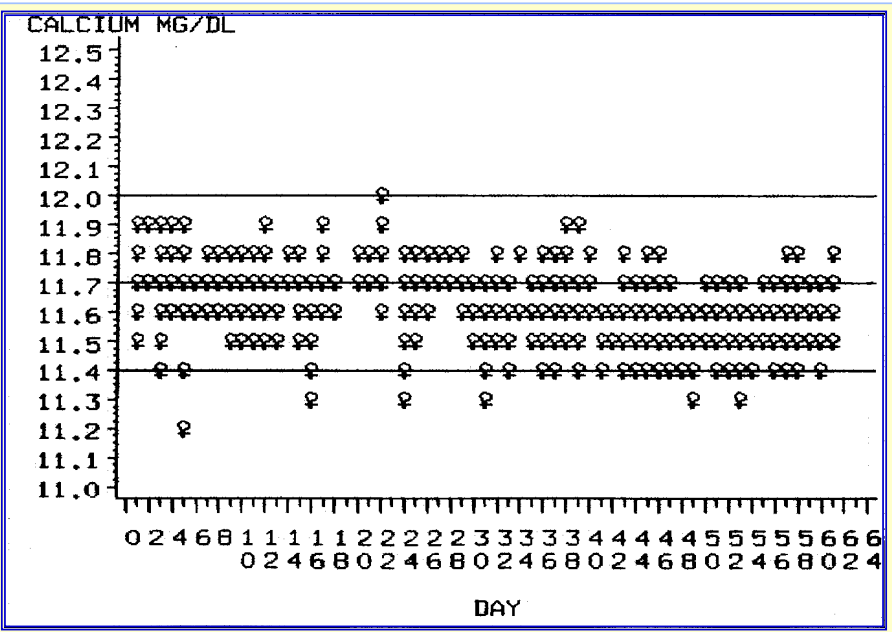

\section{Mean Analysis of Calcium Pool CHEM 1}

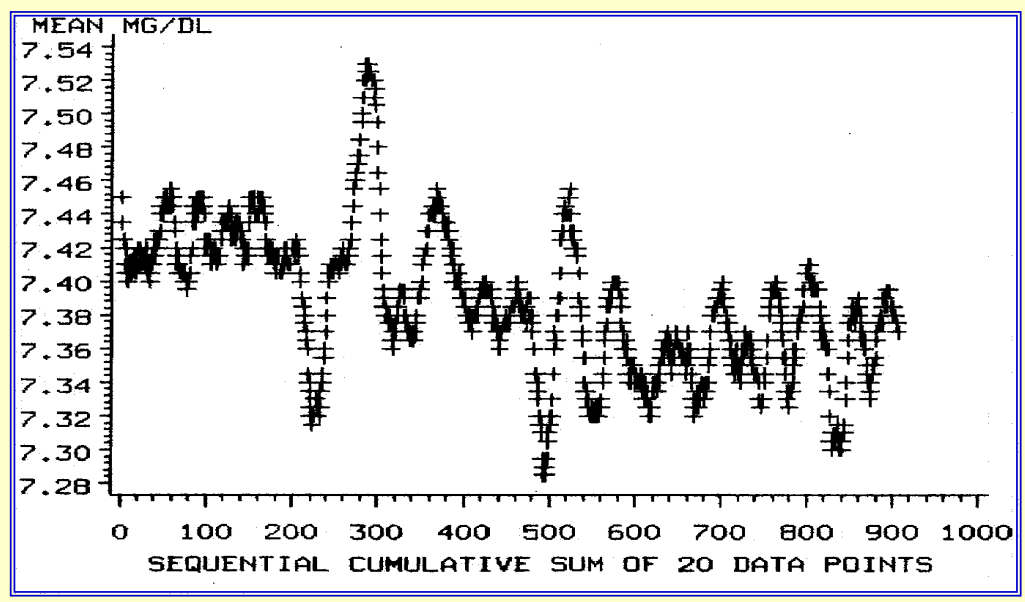




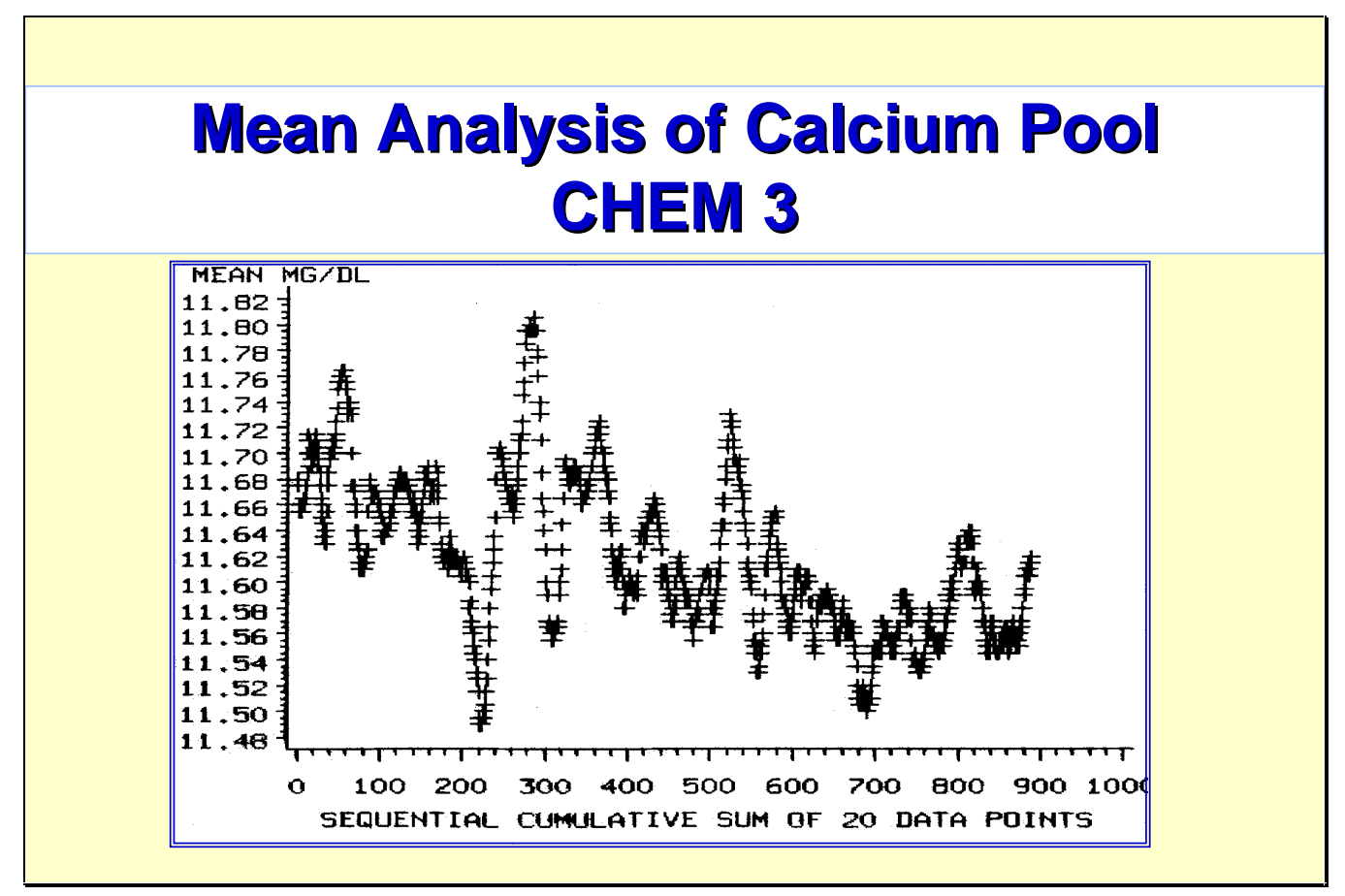

\section{Conclusions}

- Lack of laboratory standardization can cause major problems in patient care.

- Assay calibration with standard reference preparations does not assure harmonization of patient test results.

- A combination of commutable control material with traceable reference values and mathematical algorithms using adjusted patient test values could be used for calibration adjustment. 


\section{Reference Systems Models from Other Industries Joan Walsh Cassedy \\ Executive Director of The American Council of Independent Laboratories}

Measurement traceability is applicable to a variety of industry segments. These segments have defined reference systems models that may be adapted to other business activities, such as clinical laboratory operations.

The ACIL membership performs calibration and testing services that require the implementation of measurement traceability. The ACIL membership includes industry sectors such as civil engineering, environmental sciences, microbiology testing, food testing, mineral analysis, structural evaluation and conformity assessment. These industries have adopted measurement traceability as a result of international quality system requirements, the need for comparable data and as a basis for sound scientific practices.

The foundation for the traceability of measurements is fundamental whenever a test result or measurement is performed. Measurement traceability during calibration activities ensures the comparability of data among manufacturers, regulators and users of the test results. Calibrations performed without traceability result in questionable, noncomparable and unsubstantiated results. Sound scientific practice is assured by selecting a laboratory that is competent. Laboratory competency is defined by international quality system standards such as ISO/IEC Guide 25 and ISO/IEC 17025.

The extent and degree of traceability necessary during calibration is defined by each industry. No two industries require the same degree of traceablity for similar activities. Every industry segment develops standards that define the requirements for traceability of the measurement to a national or international reference standard.

Laboratories adopting conformity assessment standards are implementing the ISO/IEC Guide 25 and its recent replacement ISO/IEC 17025. These standards require operations that trace the measurement of test items (samples) to the standard used for making the measurement. These ISO standards also define the elements required for a laboratory to be uniformly defined as competent.

The international definitions for traceability and calibration define traceability as a property and calibration as a set of operations. These terms go hand in hand to identify the measurement system.

Traceability -"property of the result of a measurement or the value of a standard whereby it can be related to stated references, usually national or international standards, through an unbroken chain of comparisons, all having stated uncertainties".

Calibration - "set of operations that establish, under specified conditions, the relationship between values of quantities indicated by a measuring instrument or measuring system, or values represented by a material measure or a reference materials, and the corresponding values realized by standards". 
During the calibration activity, the laboratory must establish values realized by standards. The source and identification of these standards provide the basis for the measurement. If traceability of the measurement is achieved, the documentation from the laboratory provides the chain of comparisons to national or international standards. It must be noted that the chain must include stated uncertainties. In many cases the standards used for the calibration do not include the uncertainty of the measurement or metrological specification. This results in measurements that do not meet the definition of traceability.

In many industry sectors measurements are sometimes assumed to be traceable. Many industries requiring comparability of measurements made by testing laboratories identify uniformity of measurements through conformity assessment requirements (also termed standards). These requirements define the specific activities for the product or program requiring testing. In order for the conformity assessment requirements to be completed in a consistent manner many of these requirements specify that laboratories perform work within quality system models defined for laboratories. This requirement helps to ensure that the testing is performed in a consistent manner and by a laboratory that can demonstrate it is competent to perform such testing.

The quality system models found in the ISO/IEC Guide 25 and its recent replacement, ISO/IEC 17025, are cited and recognized by ACIL member laboratories. These quality system models define the quality system elements required by any testing and calibration laboratory. They are applicable to any facility or body that calibrates or tests. These models provide specific requirements for the laboratory to document the measurement calibration and verification activities.

ISO/IEC Guide 25 requires laboratories to calibrate or verify equipment used in testing or calibration. The laboratory defines the extent of the calibration and verification. The extent of the activities including the documentation of the calibration or verification is based on client requirements, data used for meeting product specification or the standards cited by the client as required for testing or calibration. The requirement for the client to specify the calibration or verification needed is specifically cited in ISO/IEC 17025.

Calibration of equipment is often performed using standard reference materials (SRM) from a nationally or internationally recognized body. These bodies produce standards that may be certified as a reference material since they are traceable to International System of Units (SI) (Système international d'unités) of the primary standard. These bodies perform their testing in conformance to the ISO/IEC Guide 25 or ISO/IEC 17025 requirements. The report or Certificate of Analysis of the reference standard must include all the information from cited requirements for calibration laboratories as the Certificate of Analysis provides the details of the certified reference materials (CRM).

In the United States, calibration standards are often traced to the National Institute of Technology (NIST). NIST provides standard reference materials for a variety of measurement activities. These SRMs provide a stated uncertainty and the laboratory uses this material as a national or reference standard for calibrating test equipment. Whenever NIST reference materials are not available, the laboratory often explores the use of stan- 
dard reference materials from other internationally recognized bodies that are comparable to NIST.

If these materials are not available from international bodies, then the laboratory must review the testing requirements with the client to determine the process to ensure traceability to a defined specification. The calibration certificates indicate the traceability to national standards with either the uncertainty of the material used for calibration, or provide the statement of compliance with an identified metrological specification

In all of these cases, the laboratory must maintain the certificate of analysis or other documentation. This documentation provides the information required for traceability of the measurement to a recognized standard. The documentation must include the statement of uncertainty and provide an unbroken chain of documents that show the basis for the final reported result.

Whenever materials for traceability are not available from the national or international bodies, ISO/IEC Guide 25 requires that proficiency testing be conducted. This is sometimes referred to as round robin testing. This round robin testing provides the data users with information on the comparability of the data between laboratories.

Laboratories must ensure that reference standards are used for calibration only. Reference standards used by laboratories for routine testing may find that the standard deteriorates, is destroyed or altered during routine testing use. The laboratory must evaluate the use of the reference standards to ensure that it does not mishandle the standard. This mishandling may invalidate the standard or its performance as a reference standard.

The laboratory must ensure that a program for calibration and verification of the reference standards exists. Testing may become suspect if a reference standard that was calibrated 10 years ago is not recalibrated or verified as to its continuing suitability for the testing or calibration being performed. The reference standard certification body (i.e.; NIST) or the accredited calibration laboratory sometimes provides information on the handling of the material and defines when the reference standard requires calibration or verification. This information is presented on the certificate of analysis presented with the reference standard. It is important to note here that the reference standard or the container in which the standard resides should be clearly linked to the certificate of analysis. This is often identified through a unique serial number or identifier.

In many testing activities, the laboratory verifies its testing by using one or more of the national, reference or working standards. The primary standard is the standard used by the national certification body (NIST) to compare the national standard to the working standard. In many cases the laboratory routinely uses a working standard that is one that may or may not be traceable to a national standard certification body. This working standard(s) is verified by analyzing the reference standard to ensure that the measurement is comparable to the national standard, which is demonstrated in Figure 1. 


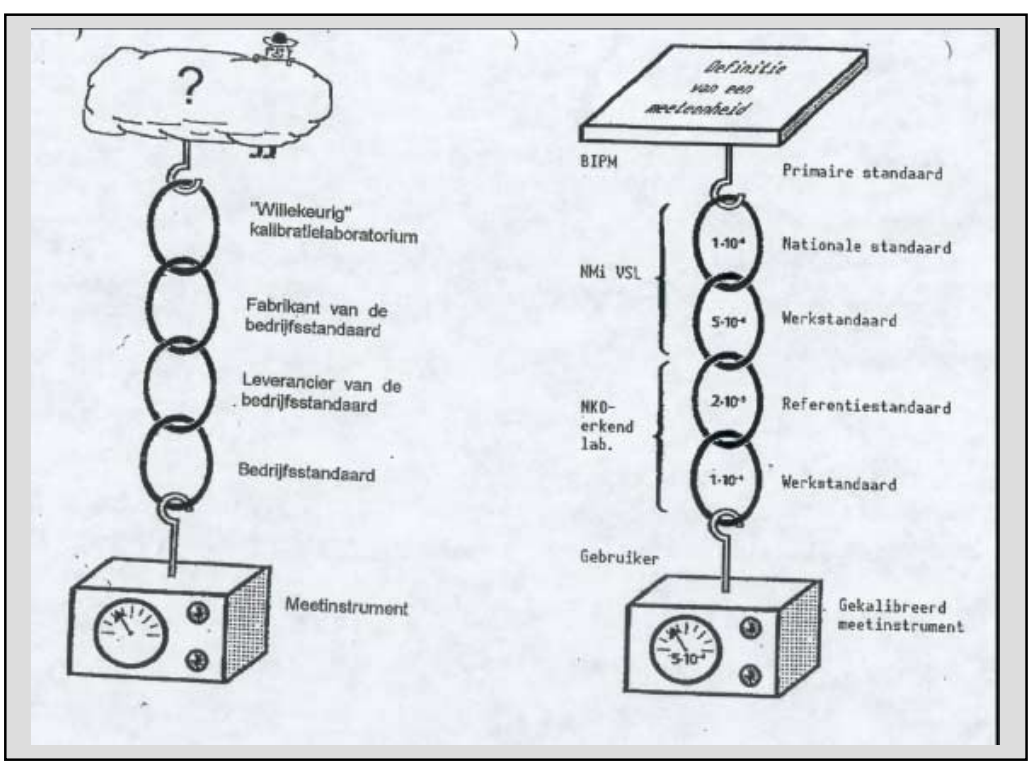

Figure 1: Left shows a testing operation without traceability. Right identifies a testing operation with all the appropriate traceability items identified.
ISO/IEC Guide 25 has always required the traceability of measurements made by an accredited laboratory to national or international standards. In some industry sectors this has not always been possible due to the lack of reference materials available from national or international certification bodies. In lieu of these reference materials all accreditation bodies require the use of proficiency testing or round robin testing to ensure the comparability of data.

With the recent updating of ISO/IEC Guide 25 by ISO/IEC 17025, laboratories performing calibrations are specifically required to document and have procedures that ensure traceability by defining reporting and operational requirements.

ISO/IEC 17025 requires the laboratory to have procedures for selecting, using, calibrating, checking, controlling and maintaining measurement standards, reference materials used as measurement standards, and measuring and test equipment used to perform tests and calibrations. This includes equipment used for ancillary measurements such as environmental conditions, when these conditions effect the result of the measurement.

ISO/IEC 17025 defines separate criteria for calibration and testing laboratories. A laboratory providing calibration services must design and operate its program to ensure traceable measurements to the International System of Units. This is done by the calibration laboratory through an unbroken chain of calibrations or comparisons to the relevant primary standards of the SI units of measurement.

The international system of units is based at the present on the following seven base units. These are length (meter), mass (kilogram), time (second), electric current (ampere), thermodynamic temperature (kelvin), amount of substance (mole) and luminous intensity (candela). The unbroken chain of calibrations or comparisons may be achieved in several steps carried out by different laboratories that can demonstrate traceability. 
The following is an example of traceability to mass using a spring scale. Figure 1 presents a pictorial presentation of the use of primary, national or reference and working standards. As an example, a load cell calibrates the spring scale to an accuracy of $1 \%$ with an uncertainty of $\pm 0.25 \%$. The load cell is certified by dead weights with an uncertainty of $\pm 0.0625 \%$. The dead weights are a reference standard with a certificate from an ISO/IEC 17025 accredited laboratory that includes the dead weight value and the uncertainty.

The load cell is a working standard that has a certificate from an accredited secondary laboratory that indicates the value and uncertainty for the load cell. The secondary laboratory determines the uncertainty of the load cell using the reference standard of the dead weights. The testing laboratory uses the load cell to evaluate the spring scale on a quarterly basis to verify the measurements. The uncertainty of the load cell is greater than the reference standard of the dead weights.

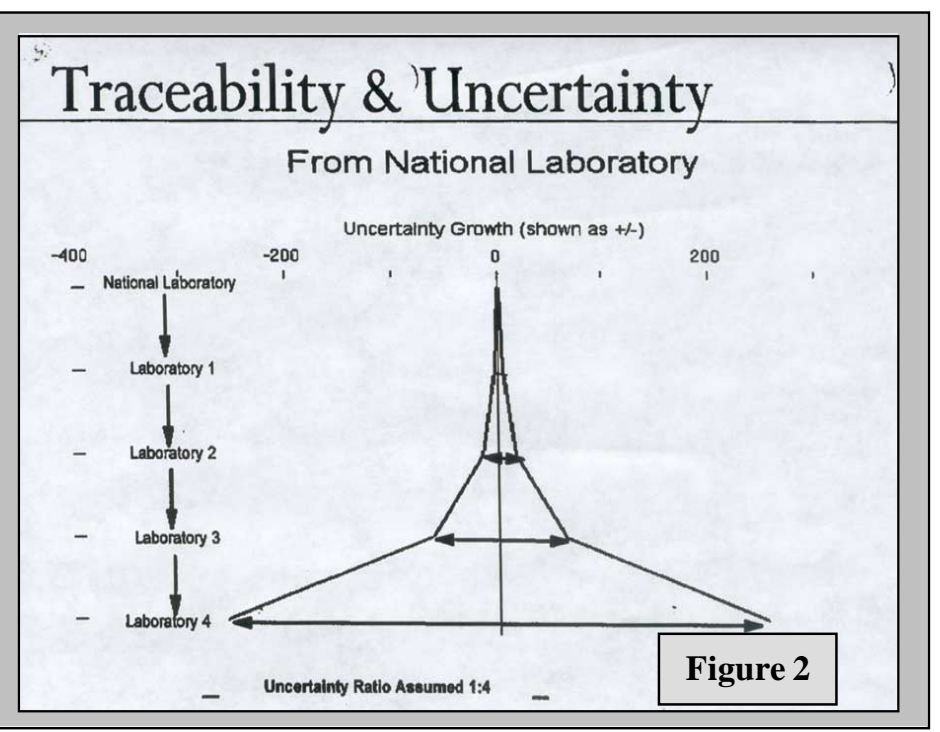

As presented in Figure 2, the uncertainty is greater when the testing is performed using the working standards. Metrologists and laboratories often use the standard of $4: 1$ to perform measurements. This $4: 1$ ratio attempts to minimize the uncertainty associated with any measurement. The laboratory must attempt to control the measurement in order to maintain this uncertainty ratio. As illustrated in Figure 2 the uncertainty grows very rapidly even when this is maintained. Users of testing laboratories accept this uncertainty for costs, ease of performing the measurement and final data requirements or specifications.

The laboratory program for using the spring scale requires the operators to verify the spring scale every quarter. This requirement may be more or less frequent depending on the scale's stability and reproducibility over time. The record of the measurement identifies the load cell and the reading obtained from the spring scale. The personnel are instructed to notify a supervisor when the load cell measurement is not within the specification for the spring scale and determined by the data user laboratory.

In order to verify the value of the load cell, the laboratory program requires the spring scale calibration by the secondary laboratory every quarter. The secondary laboratory follows its procedure for measuring the load cell results using the dead weights. The secondary laboratory program may require the replicate results to be averaged and calculated for the standard deviation. This information and the uncertainty of the measurement made by the secondary laboratory are compared to the reference standard. 
In addition the quarterly readings from the load cell are compiled. The average and standard deviation are compared to the secondary standard criteria. The records must include the serial number of the reference standard, working standard and all raw and calculated values. These records provide the traceability of the results reported by the laboratory. A process using a calibrated spring scale measures the results. The scale is calibrated using reference standards with a stated uncertainty. The spring scale laboratory determines the uncertainty budget if requested by the client. The records from the primary calibration and secondary verification provide an unbroken chain with stated uncertainties for the measurements performed by the laboratory using the spring scale.

A calibration laboratory must design and operate a process to ensure that the equipment calibrations are traceable to the International System of Units. A calibration laboratory establishes traceability of its own measurement standards and measuring instruments to the SI by means of an unbroken chain of calibrations or comparisons linking them to relevant primary standards of the SI units of measurement. The link to SI units may be achieved by reference to national measurement standards. National measurement standards may be primary standards, which are primary realizations of the SI units or agreed representations of SI units based on fundamental physical constants, or they may be secondary standards, which are standards calibrated by another national metrology institute.

When using external calibration services, traceability of measurement are assured by the use of calibration services from laboratories that can demonstrate competence, measurement capability and traceability. The calibration certificates issued by these laboratories present the measurement results, including the measurement uncertainty and/or a statement of compliance with an identified metrological specification. The term "identified metrological specification" means that it must be clear from the calibration certificate which specification the measurements have been compared with, by including the specification or by giving an unambiguous reference to the specification.

Calibration laboratories meeting the requirements of ISO/IEC 17025 are considered to be competent. A calibration certificate bearing an accreditation body logo from a calibration laboratory accredited to ISO/IEC 17025 or Guide 25, is sufficient evidence of traceability of the calibration data reported. Accredited calibration laboratories maintain their own primary standard or representation of SI units based on fundamental physical constants.

When the terms "international standard" or "national standard" are used in connection with traceability, it is assumed that these standards fulfill the properties of primary standards for the realization of SI units.

There are certain calibrations that currently cannot be made strictly in SI units. In these cases calibration includes traceability to appropriate measurement standards such as: 
- The use of certified reference materials provided by a competent supplier to give a reliable physical or chemical characterization of a material. A competent supplier is often defined as a supplier with registration to an ISO 9000 or equivalent standard. This type of registration provides the purchaser with the assurance that a documented quality system is operating within the supplier organization;

- The use of specified methods and/or consensus standards that are clearly described and agreed to by all parties.

- Participation in a suitable proficiency testing or round robin testing program, where possible.

Testing laboratories must meet the requirements of calibration laboratories unless it has been established that the associated contribution from the calibration contributes little to the total uncertainty of the test result. When this situation arises, the laboratory shall ensure that the equipment used can provide the uncertainty of measurement needed. If calibration is the dominant factor in the testing, the testing laboratory must follow the requirements of a calibration laboratory.

Where traceability of measurements to SI units is not possible and/or not relevant, the same calibration laboratory requirements are required for the testing laboratory.

The laboratory shall have a procedure for the calibration of its reference standards. A body that can provide traceability shall calibrate reference standards. Such reference standards of measurement are used for calibration only and for no other purpose. The laboratory must demonstrate that the reference standard is not affected or invalidated if used for other purposes. Reference standards shall be calibrated before and after any adjustment.

Reference materials shall, where possible, be traceable to SI units of measurement, or to certified reference materials. Internal reference materials shall be checked as far as is technically and economically practicable. Checks needed to maintain confidence in the calibration status of reference or working standards are performed according to defined procedures.

The laboratory shall have procedures for safe handling, transport, storage and use of reference standards and reference materials in order to prevent contamination or deterioration and in order to protect their integrity.

Why is this traceability important to both the users of the equipment and test data and the laboratory?

The importance of traceability is founded not only in the quality system program requirements for international standards, but it is also founded in sound scientific practices. Worldwide comparability of data is based on the use of the same standards for defining the measured result. One prime example is "time." Our time standard worldwide 
allows comparability to a single atomic clock. Everyone in the world uses this international standard when critical time measurements are required. The extent of the use of the international standard for "time" is based on the user requirements. Without this common reference point for "time," both local and international travel, meetings and other activities, would be chaos.

The demonstration of traceability for "time" occurs when documentation identifies and provides the information necessary to trace the recorded time back to this international standard. (i.e., time measurements by the atomic clock)

The assurance that the measurement is traceable allows the user to compare data although the same principle of operation is not used to generate the data. A clock, no matter what size, shape or mechanism, measures time. The extent of agreement is based on the user's need to measure time to a defined reference standard.

For another example, in environmental monitoring, air sampling is performed using a personal air-sampling pump in the field. This pump is a precision rotameter that is calibrated using an electronic bubble meter. The following summarizes the laboratory activities for traceability of the measurement:

- Personal air sampling pump set at $2 \mathrm{~L} / \mathrm{min}$.

- Precision rotameter sets this flow rate in the field

- Measurement uncertainty of the rotameter is maintained by a calibration curve in laboratory using an electronic bubble meter.

The electronic bubble meter is calibrated by an accredited calibration laboratory and is traceable to the ampere or electronic signal generated by the bubble meter. A reference standard material is used and the electronic meter has a certificate of calibration, which indicates the uncertainty of the measurement. Since the electronic meter calibrates the rotameter, the air-sampling rate of the pump is traceable to a reference standard. Through measurement and calculations the uncertainty budget may be determined for the flow rate measurement performed in the field using the rotameter.

Any valid certification of measurement has all the information necessary to trace standards used to NIST or other internationally recognized calibration body. Recertifying calibration equipment is ongoing and not a one time activity. The frequency is determined by manufacturer's recommendation, but the user's company quality assurance program is paramount. Frequency of use is one determinant, as is the difference in measurement uncertainty between the calibration equipment and the standard.

Periodic recertification, traceable to NIST, is the heart of any quality assurance program. Without stringent recertification, product quality suffers, resulting in product unreliability detrimental to public health and safety and the cost of doing business.

Uncertainty measurements are tools for the calibration house and should not be compared between companies. However, because the accredited calibration laboratory 
documents the uncertainty and standards used in the measurement, the traceability of the measurement is known, which allows the comparison of test data based on sound scientific principles while meeting the needs of the data user

\section{References}

1. "International Vocabulary of Basic and General Terms in Metrology" (VIM) - BIPM, IEC, IFCC, ISO, IUPAC, IUPAP, OIML, 1993.

2. "New National Standard for Expression of Uncertainty in Measurement", SI/NCSL Z540-2-1997.

3. NIST, Reference on Constants, Units and Uncertainty at http://www.physics.nist.gov/cuu/Uncertainty/bibliography.html

4. "General Requirements for the Competence of Calibration and Testing Laboratories," ISO/IEC Guide 25, 1990.

5. "General requirements for the competence of testing and calibration laboratories" ISO/IEC 17025:1999, First edition 1999-12-15.

6. "General Methods and Instrumentation", Annual Book of Standard, Volume 14.02, 1996.

7. "General Requirements for Bodies Operating Product Certification Systems", ISO/IEC Guide 65, 1996.

8. "Guide for General Criteria Used for Evaluating Laboratory Competence", ASTM E548-94, Volume 14.02, 1996.

9. "Clause 9: Measurement Traceability and Calibration" J. Horlick, NIST, October 1997.

10. "The Occupational Environment - Its Evaluation and Control" Edited by Salvatore R. DiNardi, Copyright AIHA Press 1997, p. 156, Chapter 8, Principles and Instrumentation for Calibrating Air Sampling Equipment, Peter F. Waldron, MPH, IHIT. 


\section{JOAN WALSH CASSEDY}

\section{American Council of Independent Laboratories}

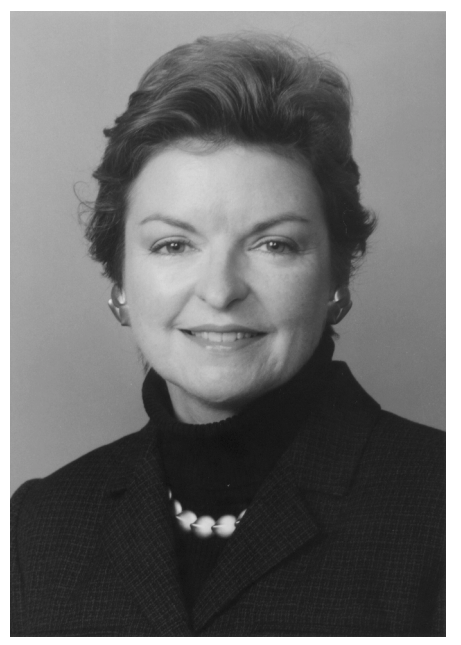

Joan Walsh Cassedy, M.B.A., is the Executive Director of the ACIL, formerly the American Council of Independent Laboratories. She received her M.B.A. from the Wharton School of the University of Pennsylvania in 1982 while serving as Director of Information and Staff Director of Studies at the National Petroleum Council from 1972 to 1984. In 1984, she served as the Chairman and Co-Founder of the International Management Group, Inc. representing clients, including the Society of Toxicology, the International Association for Energy Economics and the Transportation Research Forum, in government and public affairs activities. In 1994, she became the President and Chief Executive of the King Publishing Group and King Communications Group, both award-winning business information services companies. During this time, she was the Associate Publisher of The Energy Daily, Defense Week, New Technology Week, High Performance Computing \& Communications Week, Military Space, BMD Monitor, Navy News \& Undersea Technology, The Brownfields Report and White House Weekly. In addition, she was the Associate Producer of White House Chronicle, a weekly public affairs talk show broadcast on commercial and public radio and television stations. In 1999, she took her present position at ACIL, providing testing, certification and accreditation services to U.S. and international companies and governments in such areas as civil engineering, environment, microbiology, food testing, mineral analysis, conformity assessment, and structural evaluation. 


\section{Reference Systems Models from Other Industries}

Visuals Presented By

Joan Walsh Cassedy

The American Council of Independent Laboratories 


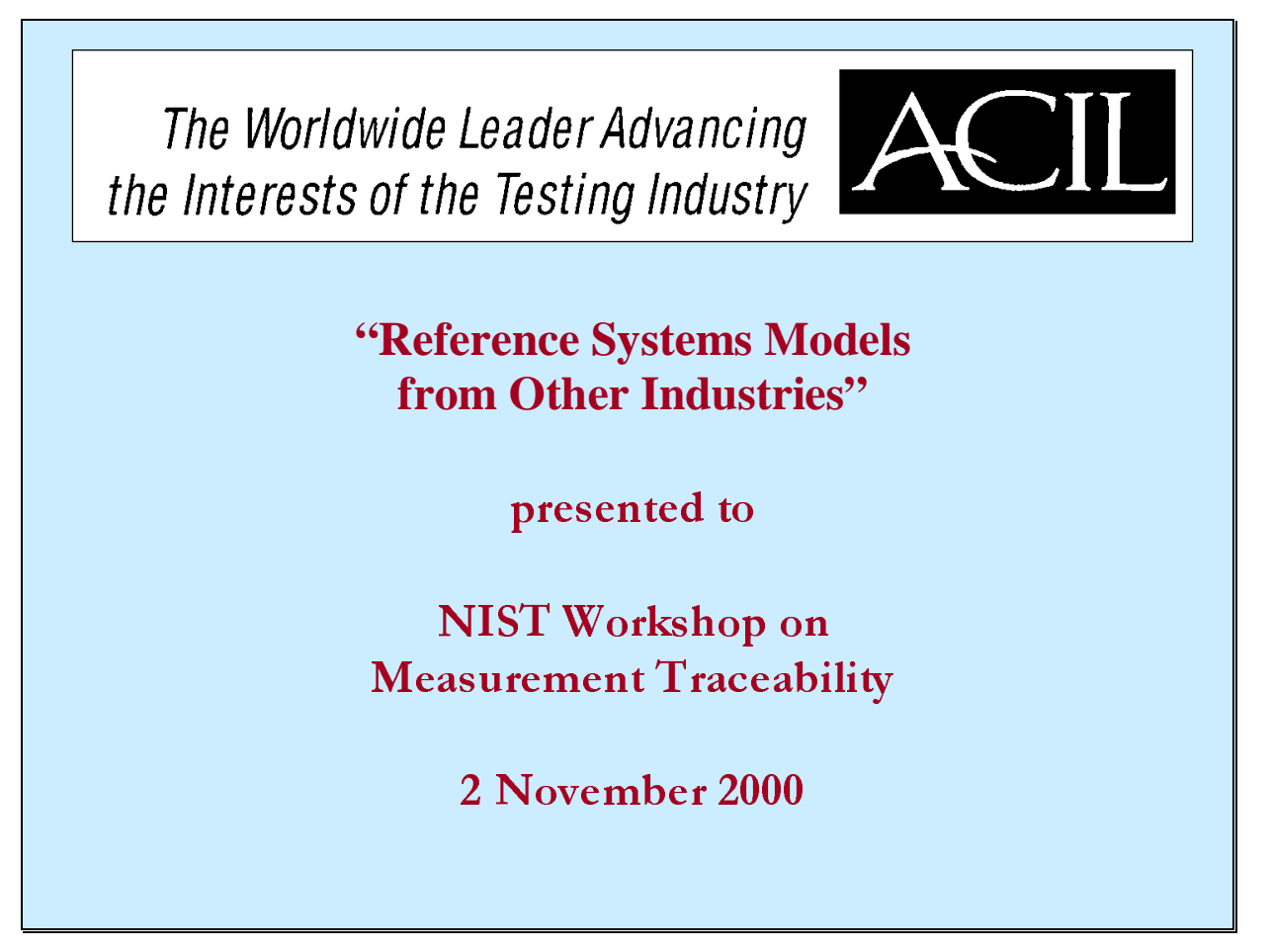

"Reference Systems Models

from Other Industries"

presented to

NIST Workshop on

Measurement Traceability

2 November 2000

\section{Importance of Traceability}

- Foundation of a quality program

- Absence of traceability leads to misleading results--chaos 

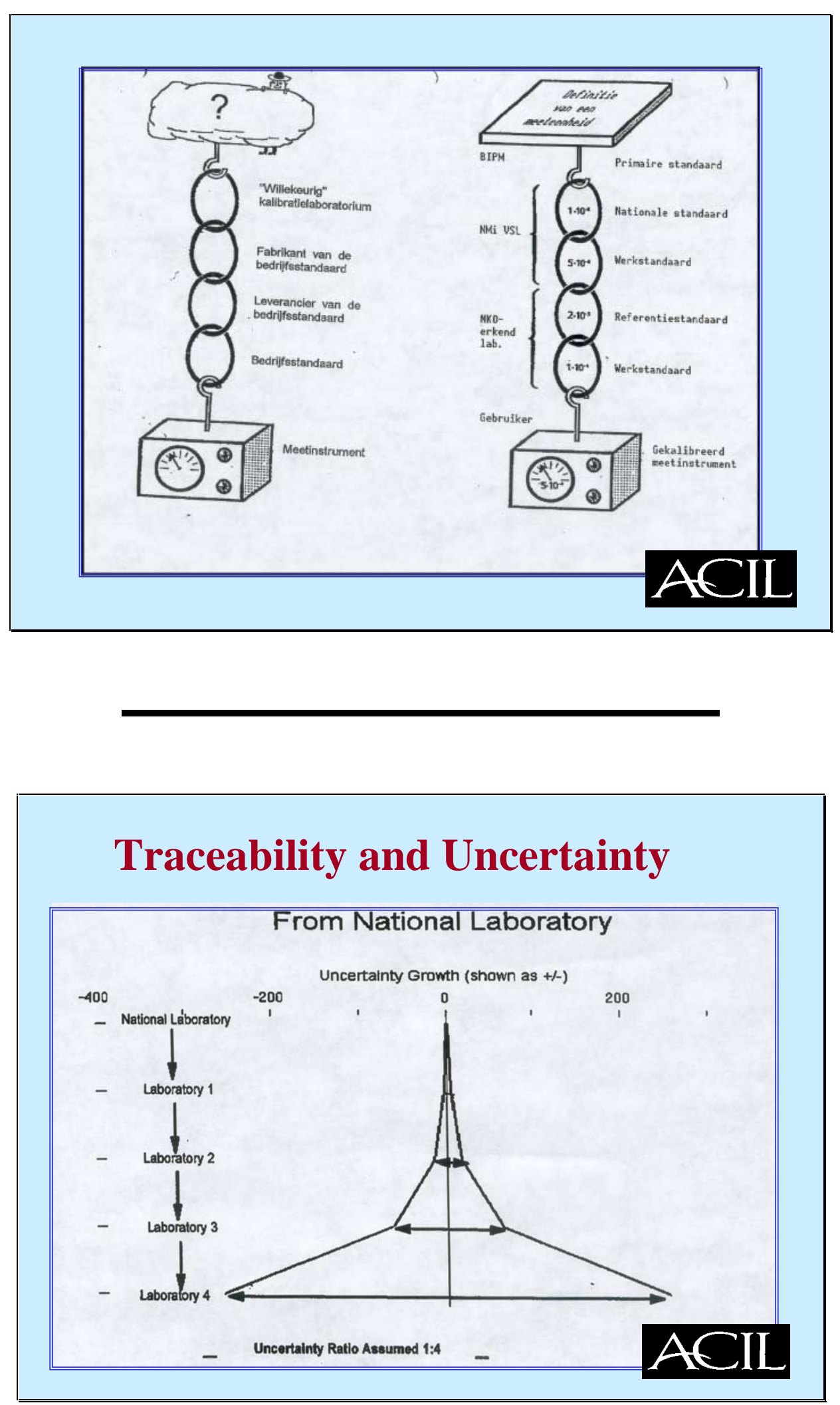
Same principle of operation not

\section{necessary}

- Example: A spring scale with an accuracy of $\pm 1 \%$ could be certified by a load cell with an uncertainty of \pm $0.25 \%$, which in turn could be certified by dead weights with an uncertainty of $\pm 0.0625 \%$

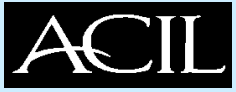

- The load cell is a secondary standard

- The calibrated weights are a primary standard

- Secondary standards are used for convenience, but must be traceable to primary standards. 


\section{Air Sampling Model}

- personal air sampling pump set at 2 L/min.

- precision rotameter sets this flow rate in the field

- measurement uncertainty of the rotameter maintained by a calibration curve in laboratory using an electronic bubble meter

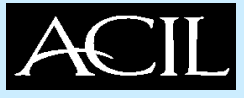

\section{Bottom line}

$\checkmark$ Any valid certification of measurement has all the information necessary to trace standards used to NIST. 


\section{Recertifying calibration equipment}

- Frequency determined by manufacturer's recommendation, but company's quality assurance program is paramount.

- Frequency of use is one determinant, also the difference in measurement uncertainty between the calibration equipment and the standard.

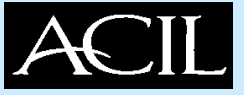

- Periodic recertification, traceable to NIST, is the heart of any quality assurance program.

- Without stringent recertification, product quality suffers, resulting in product unreliability detrimental to public health and safety and the cost of doing business. 


\section{WARNING:}

- Uncertainty measurements are tools for the calibration house and should not be compared between companies.

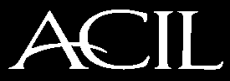

Resources on uncertainty calculations:

- ANSI/NCSL Z540-2-1997, "New National Standard for Expression of Uncertainty in Measurement"

- NIST, Reference on Constants, Units and Uncertainty at http://www.physics.nist.gov/cuu/Uncert ainty/bibliography.html 
For more information about ACIL, please contact:

\section{Joan Walsh Cassedy}

Executive Director

American Council of Independent Laboratories

1629 K Street, NW, Suite 400

Washington, D.C. 20006

Tel: (202) 887-5872 Fax: (202) 887-0021

E-mail: jcassedy@acil.org www.acil.org

\section{Traceability}

- property of the result of a measurement or the value of a standard whereby it can be related to stated references, usually national or international standards, through an unbroken chain of comparisons, all having stated uncertainties. 


\section{Calibration}

- set of operations that establish, under specified conditions, the relationship between values of quantities indicated by a measuring instrument or measuring system, or values represented by a material measure or a reference materials, and the corresponding values realized by standards.

- Source: "International Vocabulary of Basic and General Terms in Metrology" (VIM) - BIPM, IEC, IFCC, ISO, IUPAC, IUPAP, OIML, 1993.

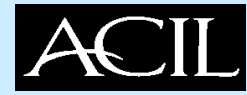

- Ideal minimum accuracy $=4: 1$

- 10:1 not uncommon

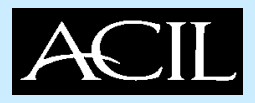




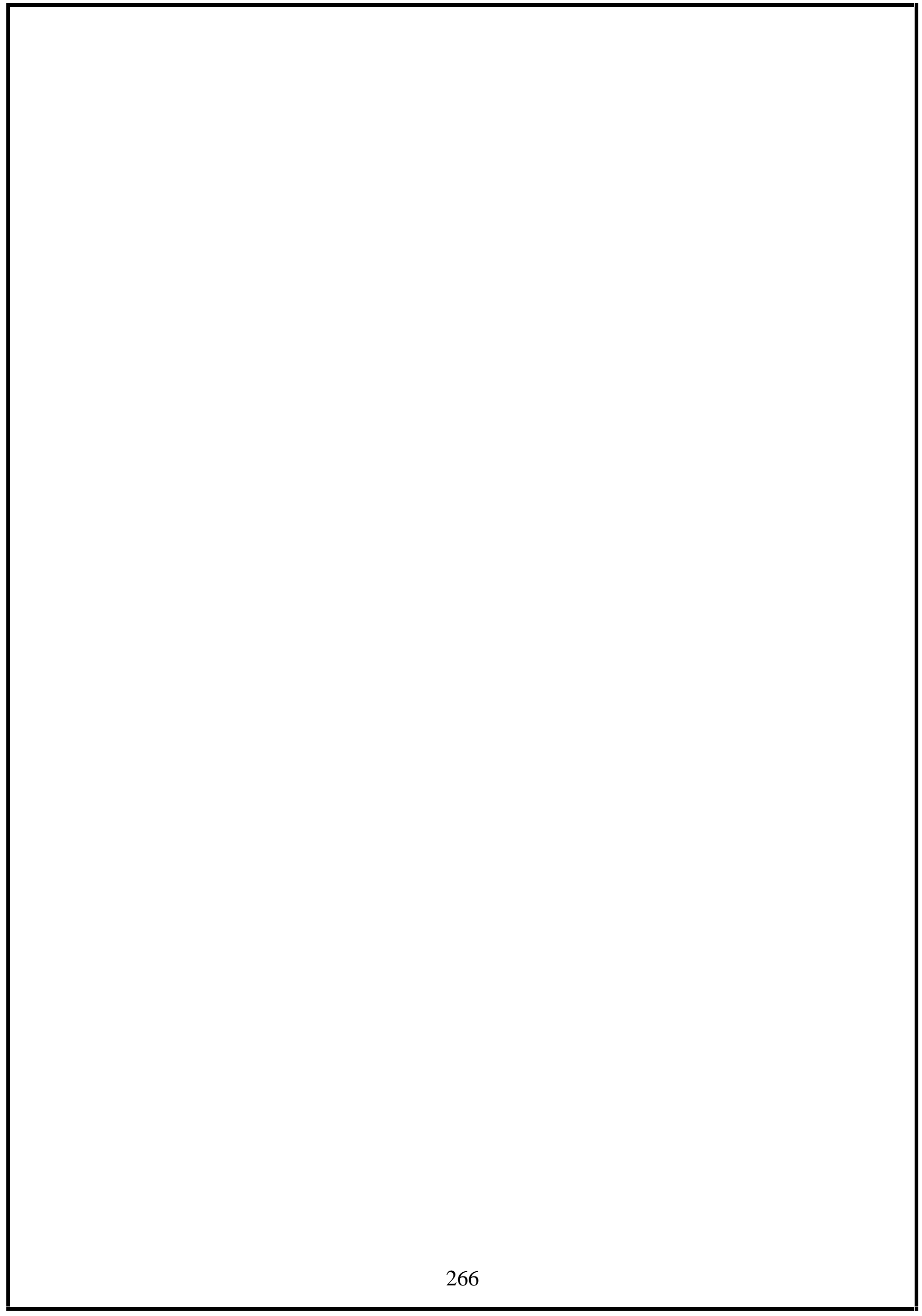




\section{Poster Session \\ Michael J. Welch, Chair \\ National Institute of Standards and Technology, USA \\ Held November 2, 2000}

\section{SRMS TO SUPPORT ACCURACY AND TRACEABILITY IN HEALTH- RELATED MEASUREMENTS \\ Jennifer Colbert and Michael Welch \\ NIST}

Background: Inaccuracy in health-related measurements raises overall health care costs, results in misdiagnoses, and leads to inaccurate conclusions in clinical studies. In addition, lack of certified reference materials (CRMs) hampers traceability, and with the EC IVD directive requiring traceability to recognized national standards, there is an increased need for CRMs for many analytes.

NIST Activities: NIST has a long history of providing reference materials to support accuracy in health-related measurements. Initial efforts in the 1960's focused on providing pure reference materials to serve as primary calibrators. A series of crystalline materials of stated purity and uncertainty were developed for many of the most commonly measured analytes in human blood, such as cholesterol, glucose, uric acid, bilirubin, creatinine, and urea. Most such organic compounds cannot be directly assayed for purity. The determination of the purity of these materials is carried out at NIST and generally involves using a variety of analytical techniques to measure impurities and then to subtract the total mass of these impurities from $100 \%$ to determine the purity.

In the 1970's, NIST began developing very accurate and precise isotope dilution mass spectrometric methods for important serum analytes, both organic and inorganic. These methods are described in another section of this document. These methods were then applied to the determination of these analytes in human serum-based SRMs. SRM 909 Human Serum and its successors, 909a and 909b, are lyophilized serum materials with certified concentrations of approximately 12 analytes. Other lyophilized serum materials were developed specifically for serum lipids (SRM 1952) and vitamins and carotenoids (SRM 968).

Because some routine methods demonstrate strong matrix effect biases between lyophilized serum and fresh serum, recent SRM activity has focused on providing frozen human serum, stored at $-80^{\circ} \mathrm{C}$. Extensive studies using a large variety of routine methods have shown little or no matrix effect problems with frozen serum. SRMs using frozen human serum matrices include SRM 956, certified for many electrolytes, SRM 965, certified for glucose, and SRM 1951a, with certified and reference values for cholesterol, HDL- and LDL-cholesterol and triglycerides.

Further information about NIST SRMs can be found at:

http://ts.nist.gov/ts/htdocs/230/232/232.htm 


\section{ID/GC/MS METHODS FOR HEALTH-STATUS MARKERS Michael Welch, NIST}

\section{Diagnostic Markers for Diabetes, Cardiovascular Risk, and Other Conditions}

Well-defined organic species in blood have been measured for many decades to determine a variety of conditions. Among the most widely measured substances (and condition) are glucose (diabetes), cholesterol (cardiovascular disease risk), uric acid (gout), urea (kidney function), creatinine (kidney function), and triglycerides (cardiovascular disease risk).

\section{Measurement Challenge:}

Routine clinical measurements have been developed by a large variety of manufacturers, often using different approaches for a given analyte. Results from these different methods may vary considerably; with no clear-cut means of determining which is more accurate, unless reference systems are in place to provide an accuracy base.

\section{NIST Research Activities:}

Over the last twenty years NIST has developed highly accurate and precise isotope dilution/gas chromatography/mass spectrometry-based methods for the six analytes listed above. These methods are recognized as definitive methods by the National Reference System for Clinical Laboratories, and as such, sit atop the measurement hierarchy for providing traceability for routine clinical measurements.

These methods involve careful gravimetric measurement of all critical quantities, addition of a known mass of a stable isotope-labeled analog of the compound of interest (as an internal standard) to a known mass of serum, equilibration of the resulting solution, a series of chemical steps to isolate the compound of interest from the serum matrix, a chemical conversion of the compound to a thermally stable derivative, injection of the derivatized sample into a specially designed gas chromatograph/mass spectrometer system, and careful measurement of the ratio of the ion signals from the native compound and the isotopically labeled internal standard. The ratio is compared with ratios measured for standards with known weight ratios of pure native compound and the isotope-labeled analog to determine the quantity of native analyte in the serum sample.

These methods are not intended for use in routine applications. The primary function of such methods is to certify high-level reference materials that can then be used in evaluating the performance of routine methods and the accuracy of the value assignment of lower order reference materials and controls. To facilitate this transfer of accuracy to manufacturers and users of routine clinical measurement methods, NIST has developed a series of Standard Reference Materials ${ }^{\circledR}$ with concentrations of important analytes, such as those listed above and all of the major electrolytes, in human serum matrices. These materials are described in more detail in an accompanying section.

For a review of the development and use of isotope dilution mass spectrometric methods for clinical analytes, both organic and inorganic, see: Bowers, G.N., Fassett, J. D., and White V, E., Anal. Chem., 65, 475R-479R (1993). 


\section{DEVELOPMENT OF A REFERENCE MATERIAL FOR CARDIAC TROPONIN I \\ David Bunk, Joseph Dalluge, Michael Welch, NIST \\ Robert Christenson (U. of MD), Show Hong Duh (U. of MD)}

\section{Diagnostic Marker for Heart Attack}

Cardiac Troponin I is a diagnostic marker for cardiac injury, including myocardial infarction. This protein, together with the Troponin $\mathrm{T}$ and $\mathrm{C}$ subunits, make up the Troponin CIT complex, which regulates heart muscle contraction. After damage to heart muscle tissue, Troponin I is released into the bloodstream and can be detected within hours and remains at an elevated blood level for days. Compared to other diagnostic markers for myocardial infarction, Troponin I has the advantage of being heart-specific; it is normally found only in heart muscle tissue. Therefore, its presence in serum is always an indicator of heart muscle tissue damage.

\section{Measurement Challenge:}

Cardiac Troponin I is present in serum after myocardial infarction in very low levels $(0.1-50 \mu \mathrm{g} / \mathrm{L})$. A variety of post-translational modifications, including phosphorylation and proteolytic cleavage, make Troponin I very heterogeneous in structure. In heart muscle tissue, Troponin I is complexed to the Troponin $\mathrm{T}$ and $\mathrm{C}$ subunits. When Troponin is released from the damaged heart tissue after a heart attack, it is not known to what degree the I subunit is still complexed to the other subunits. In order to produce an effective reference material, the structure of the protein in the reference material has to reflect the structure of Troponin I found in serum. The challenges are to identify the relevant forms, isolate them from the serum matrix, concentrate them, and measure their concentration.

\section{NIST Research Plan/Activities:}

- Structural characterization of Troponin I standards using LC/MS and LC/MS/MS

- Round-Robin Study of Troponin I standards and their compatibility with commercial clinical immunoassays

- Develop methods for measurement and characterization of Troponin I in serum using LC/MS

- Issue Troponin I reference material (purified preparation)

- Develop serum-based Troponin I reference material

\section{Collaborators:}

American Association for Clinical Chemistry (including affiliations with Bayer Corporation, Beckman Coulter, Abbott Laboratories, Byk-Sangtec Diagnostica, Spectral Diagnostics, Ortho-Clinical Diagnostics, Biosite Diagnostics, Dade Behring, Inc., the University of Maryland, and the University of Miami). 


\section{DETERMINATION OF THYROXINE AND CORTISOL IN SERUM BY ISOTOPE DILUTION LIQUID CHROMATOGRAPHY-MASS SPECTROMETRY WITH ELECTROSPRAY IONIZATION \\ Susan Tai, Lorna Sniegoski, and Michael Welch NIST}

The determination of thyroxine, a thyroid hormone, is widely used for the diagnosis and therapy control of thyroid disorders. A new method based on isotope dilution /liquid chromatography-mass spectrometry using electrospray for ionization (LC/MSESI) has been developed for the determination of total thyroxine in serum. An isotopically labeled compound, thyroxine- $\mathrm{d}_{5}$, was used as an internal standard. Protein precipitation, ethyl acetate extraction and solid-phase extraction were used to prepare samples for LC/MS analyses. LC was performed on a $\mathrm{C}_{18}$ column with an isocratic mobile phase. $(\mathrm{M}+\mathrm{H})^{+}$ions at $\mathrm{m} / \mathrm{z} 778$ and 783 for thyroxine and its labeled internal standard were monitored for positive ions and (M-H) ${ }^{-}$ions at m/z 776 and 781 for negative ions. Samples of frozen serum pools were prepared and measured on three separate sets.

Excellent precision was obtained for three levels of serum samples with relative standard deviations of the mean ranging from $0.1 \%$ to $0.6 \%$. Positive and negative ion measurements agreed within $0.8 \%$. The detection limit is estimated to be $30 \mathrm{pg}$ for positive ions, and $20 \mathrm{pg}$ negative ions. The results of the LC/MS-ESI method and field methods compared well with an average difference of $5 \%$ for all three levels.

Cortisol is a steroid hormone that is a diagnostic marker for endocrine function. ID/GC/MS methods for cortisol have produced results that are about $20 \%$ to $30 \%$ lower than field methods. GC/MS is not a robust method for this molecule as it must be derivatized at several positions, resulting in a large molecule that is difficult to get into the gas phase. Reproducibility of a GC/MS method developed at NIST was poor. LC/MS appears to be a much better alternative. It is polar enough that it should work with polar solvent systems and it should have good sensitivity in electrospray ionization. An approach similar to that used for thyroxine will be investigated. 


\section{CHARACTERIZATION OF THYROID STIMULATING HORMONE AND RELATED PROTEINS USING MASS SPECTROMETRY \\ Maura Donohue, David Bunk, and Joseph Dalluge NIST}

\section{Diagnostic Marker for Thyroid Function}

Human Thyroid Stimulating Hormone (hTSH) is a glycoprotein hormone secreted by the pituitary gland. Its role is in the stimulation of the thyroid gland to produce triiodothyroxine (T3) and thyroxine (T4). Serum TSH levels are used as a diagnostic tool for assessing thyroid function. Thyroid conditions such as hyperthyroidism can arise if the thyroid gland fails to recognize the presence of $\mathrm{TSH}$, and are characterized by increased serum hTSH.

\section{Measurement Challenge}

TSH is structurally heterogeneous and present at very low levels in human serum, and therefore presents a significant measurement challenge. It is measured clinically using immunoassays, which are confounded by differences in antisera binding specificities, lack of internationally accepted standards, and the heterogeneity of TSH. These limitations often lead to significant variability in measurements of this analyte using different FDA-approved immunoassay kits.

\section{NIST Research Plans/Activities}

- Development of analytical chemistry-based methodology for the structural characterization and measurement of hTSH in serum based primarily on directly combined liquid chromatography-mass spectrometry (LC-MS).

- Characterization of a variety of hTSH standards at the molecular level to understand further, the heterogeneity of the analyte, arising predominantly from differential glycosylation of the hormone.

- Determination of a glycoprotein mass map for hTSH, and the nature and extent of site heterogeneity.

- Production of preliminary well-characterized standards for improvement of between-method variations in calibration of immunoassay measurements.

- Determination of specific forms of hTSH in patient samples to understand better the clinically relevant forms of this compound.

- Address the challenge of analyte enrichment in patient samples using nonimmunological methodology including combinatorial production of aptamers with high binding affinities for hTSH.

- Development of a suite of clinically relevant standards.

\section{Collaborators:}

The Mayo Clinic 


\section{CHARACTERIZATION OF PROSTATE-SPECIFIC ANTIGEN USING LIQUID CHROMATOGRAPHY/MASS SPECTROMETRY \\ Chad Nelson, Joseph Dalluge, David Bunk, and Michael Welch NIST}

\section{Diagnostic Marker for Prostate Cancer}

Prostate-specific Antigen (PSA) is a single chain glycoprotein (appproximately 7 $\%$ to $8 \%$ carbohydrate) present in the prostate and seminal fluid. The presence of PSA in the serum is measured as a diagnostic indicator of prostatic cancer. Clinical laboratories currently measure PSA by immunoassays, which are confounded by lack of common standards, and the heterogeneity of the antisera and antigen being measured.

\section{Measurement Challenge}

Measurement of PSA using analytical methods is a significant challenge due to the structural heterogeneity of PSA in serum, it's low concentration in this complex matrix $(<10 \mathrm{ng} / \mathrm{mL})$, and lack of knowledge regarding what compound is actually being measured in clinical PSA tests.

\section{NIST Research Plans/Activities}

- Characterization of a variety of PSA standards at the molecular level to understand further the heterogeneity of the analyte, arising predominantly from differential glycosylation of the protein.

- Production of preliminary well-characterized standards for improvement of between-method variations in calibration of immunoassay measurements.

- Determination of specific forms of PSA in patient samples to understand better the clinically relevant forms of this compound.

- Address the challenge of analyte enrichment in patient samples using nonimmunological methodology including combinatorial production of aptamers with high binding affinities for PSA.

- Development of LC/MS and LC/MS/MS methods for the structural characterization and measurement of PSA in serum.

- Development of a suite of clinically relevant standards.

\section{Collaborators:}

The Mayo Clinic

The Johns Hopkins University 


\section{EVALUATION OF LIQUID CHROMATOGRAPHY/MASS SPECTROMETRY FOR THE DETERMINATION OF HOMOCYSTEINE IN HUMAN PLASMA \\ Joseph Dalluge, Bryant Nelson, Lorna Sniegoski, and Sam Margolis NIST}

\section{Diagnostic Marker for Risk of Heart Disease}

Total homocysteine (tHcy) has emerged as an important independent risk factor for cardiovascular disease, as an indicator of nutritional cofactor deficiency (folate and cobalamin), and as a contributing factor in the pathogenesis of neural tube defects.

\section{Measurement Challenge}

tHcy is measured clinically using a variety of methods including immunoassays and LC-based methods requiring extensive derivatization procedures and frequent analysis of quality control samples. Because clinical laboratories use a variety of different methods for its measurement, interlaboratory comparisons of tHcy measurements are poor.

\section{NIST Research Plan/Activities}

- Develop collaborative effort between NIST and Mayo Clinic to develop $\mathrm{LC} / \mathrm{MS} / \mathrm{MS}$ reference method for the measurement of tHcy toward the production of standards that will be used to calibrate and validate this and other methods currently used in the clinical community.

- Development of the method will include the addition of a chromatographic separation prior to MS/MS measurement of tHcy to improve the selectivity and precision of the measurements without significantly compromising analysis time.

- Investigate development of GC/MS methodology for measurement of tHcy in plasma/urine as a second method for confirmation.

\section{Collaborators:}

The Mayo Clinic 


\title{
8. PRE-COLUMN EXTRACTION AND LIQUID CHROMATOGRAPHY/ ELECTROSPRAY-IONIZATION MASS SPECTROMETRY FOR THE QUANTITATION OF FOLIC ACID DERIVATIVES IN HUMAN PLASMA
}

\author{
Bryant Nelson, Joseph Dalluge, Sam Margolis, Dawit Bezebeh, and Lane Sander \\ NIST
}

Diagnostic Marker for Neural Tube Defects: Folic acid is a water-soluble B vitamin that plays a significant role in the development of the central nervous system during the early weeks of gestation. A deficiency of folic acid during this time period prevents the neural tube from forming correctly, which leads to spina bifida, anencephaly and other serious fetal malformations. Folic acid supplementation reduces the risk of recurrent neural tube defects (NTDs) and recently, the Food and Drug Administration (FDA) issued a regulation "requiring that all enriched grain products be fortified with folic acid to reduce the risk of neural tube defects in newborns."

Folic acid is also directly involved in reducing the levels of homocysteine in the body. High levels of homocysteine have been linked to the increased risk of cardiovascular disease, cerebrovascular disease, nutritional cofactor deficiency and neural tube abnormalities. Folic acid supplementation reduces the level of homocysteine in the body through the transfer of a methyl group from 5-methyl tetrahydrofolate (a folic acid vitamer) to homocysteine to form the amino acid methionine.

Measurement Challenge: The ability to determine folic acid status accurately in segments of the population at risk for NTD births is a significant challenge. Determination of folic acids (folates) in biological samples is extremely difficult due to the existence of numerous folic acid metabolites (folic acid exists in as many as eight different biologically important forms), their extreme instability (folic acids are easily degraded by air, light, temperature and $\mathrm{pH}$ and they frequently interconvert among forms) and their low levels in plasma and serum ( $\leq 10 \mathrm{ng} / \mathrm{ml}$ for total folates).

\section{NIST Research Plans/Activities}

- Develop liquid chromatographic separation methods combined with electrochemical (coulometric), fluorescence and electrospray ionization mass spectrometric detection for the determination of the five most biologically important folic acid vitamers in plasma and serum.

- Step 1 - Develop the separation and detection methods (completed).

- Step 2 - Stabilize the folates (on-going).

- Step 3 - Quantitate the folates in spiked plasma/serum and in control plasma/ serum samples (on-going).

- Apply the developed LC/EC, LC/Fluor and LC/MS methods to the determination of folic acid vitamers in food matrices.

\section{Collaborators:}

The current collaborators are the Centers for Disease Control and Prevention (CDC). 


\section{METHOD DEVELOPMENT FOR THE DETERMINATION OF GLYCATED HEMOGLOBIN (HbA1c) \\ David Bunk and Joseph Dalluge, NIST}

\section{Diagnostic Marker for Control of Diabetes}

Glycated hemoglobin is a diagnostic marker for diabetes and the effectiveness of diabetes care. When the blood glucose levels of diabetics rise in an uncontrolled fashion, some of the glucose binds to blood proteins. This binding is known as glycation and affects many blood proteins including hemoglobin. Because the lifetime of hemoglobin in the blood is several months, monitoring the amount of glycated hemoglobin gives an indication of how well blood glucose levels have remained controlled over the long term. Long term control of blood glucose levels is important in order to alleviate the complications that are associated with diabetes.

\section{Measurement Challenge:}

The challenges are to modify and improve existing methods involving LC/MS and CE to more accurately measure the degree of glycation of hemoglobin and to develop a reference material that will help to harmonize results among the more than forty glycated hemoglobin assays that are used in clinical labs.

\section{NIST Research Plan/Activities:}

- Participate in the evaluation of the two reference methods developed by the International Federation for Clinical Chemistry (IFCC) which use LC/MS and LC/CE

- Modify methods as needed to achieve accuracy and precision goals

- $\quad$ Prepare and evaluate glycated hemoglobin reference material

\section{Collaborators:}

International Federation for Clinical Chemistry (including participation by the Center for Disease Control and Prevention). 


\section{METHOD DEVELOPMENT FOR THE DETERMINATION OF ALBUMIN IN HUMAN SERUM \\ David Bunk, NIST}

\section{Diagnostic Marker for Nutritional Health Status}

Human serum albumin is a diagnostic marker for nutritional health status (in serum), and kidney functions (in urine). Patients who suffer from severe wasting, such as those with cancer or advanced HIV, generally have low serum albumin concentrations from malnutrition or poor adsorption of nutrients. When albumin is found in urine, it generally means that there is kidney damage, as filtration of the blood by healthy kidneys excludes serum proteins from urine.

\section{Measurement Challenge:}

The challenge of serum albumin measurement is dealing with the extreme structural heterogeneity of this protein. The structural heterogeneity combined with the high molecular mass of albumin (approximately $67 \mathrm{kDa}$ ) makes it difficult to find an internal standard for accurate quantitation. In contrast to other protein markers, albumin is present at high levels in serum.

\section{NIST Research Plan/Activities:}

- Evaluate purified albumin preparation for use as reference materials

- Develop analytical methods for serum-based quantification of albumin by LC/MS

\section{Collaborators:}

University of Wisconsin Medical College

\section{METHOD DEVELOPMENT FOR THE DETERMINATION OF SPECIATED IRON IN SERUM \\ David Bunk and Stephen Long NIST}

\section{Diagnostic Marker for Anemia and Hemochromatosis}

In the anemic patient, a low level of iron in the bloodstream results in reduced blood oxygen levels as oxygen is bound to the iron in hemoglobin. In hemochromatosis, the body absorbs too much iron from food; this iron can accumulate in tissues such as the liver, resulting in damage over the long term. Both anemia and hemochromatosis and other iron-related diseases result in an altered level of serum iron concentration, however, the distribution of iron among the various iron-binding and iron storage proteins in serum provides more clinical information than just the traditional measurement of total serum iron. 


\section{Measurement Challenge:}

Total serum iron, as currently measured in metrology laboratories using isotope dilution mass spectrometry, is generally higher than that measured in clinical laboratories using spectroscopic techniques. The cause of this measurement bias is unknown, although it has been speculated that heme contamination in serum is the cause. The iron bound to heme is not detected by spectroscopic techniques but does contribute to total serum iron values obtained my mass spectrometry.

The two most prevalent iron-carrying proteins in serum are transferrin and ferritin. In the case of transferrin, the clinical quantity of interest is the degree of transferrin iron saturation, i.e., the percentage of transferrin iron-binding sites that are occupied by iron. The measurement of transferrin saturation requires both the measurement of the amount of iron bound to transferrin and the amount of transferrin in serum. Additionally, transferrin measurement is hindered by the structural heterogeneity of this protein due to glycosylation. But the characterization of transferrin glycoforms has an addition benefit as increased glycoform heterogeneity of transferrin has been linked to chronic alcoholism as well as a genetic disease called carbohydrate deficient glycoprotein syndrome.

Ferritin measurement presents much more of a challenge. Ferritin is present in very low levels in serum $(10 \mu \mathrm{g} / \mathrm{L}$ to $20 \mu \mathrm{g} / \mathrm{L})$ and is an extremely large protein. Ferritin is made up of 24 subunits and has a molecular mass of approximately $450 \mathrm{kDa}$. Up to 25 ferritin isoforms are thought to exist, composed of various combinations of the two primary subunits. Each ferritin molecule is capable of storing up to 4500 iron atoms.

\section{NIST Research Plan/Activities}

- Discover the source of bias in total serum iron measurements when using mass spectrometry (ICP-MS) as compared to the spectroscopic methods used by clinical laboratories.

- Characterize the heterogeneity of transferrin standards

- Develop analytical methods to quantify transferrin in serum by LC/MS

- Develop analytical methods to quantify the iron bound to transferrin by LC/ICP-MS

- Prepare a serum-based reference material for transferrin iron saturation.

- Investigate glycoform heterogeneity in transferrin as a potential reference material for diagnosis of chronic alcoholism

- Characterize serum ferritin standards and access the degree of structural heterogeneity present.

- Develop analytical methods for isolating and quantifying ferritin from serum using LC/MS

- Prepare serum-based ferritin reference material

\section{Collaborators:}

The College of American Pathologists

The University of Minnesota 


\title{
12. METHOD DEVELOPMENT FOR THE DETERMINATION OF BILIRUBIN IN SERUM
}

\author{
Yolanda Davidson, Lorna Sniegoski, and Michael Welch \\ NIST
}

\section{Diagnostic marker for liver function}

Bilirubin is a tetrapyrrole produced as a metabolic product that is processed by the liver and excreted into the bile. Elevated levels of bilirubin are an indication of impaired liver function, such as is caused by hepatitis. In newborns, jaundice is often observed from build up of blood levels of bilirubin, caused by hemolysis of erythrocytes exceeding the rate at which the liver can conjugate bilirubin and excrete it into the bile. Conversely, a recent study reported that the antioxidant properties of bilirubin are beneficial in reducing heart disease.

\section{Measurement challenge}

Bilirubin is a large, thermally labile molecule that is subject to degradation in light. There are at least three known isomers and it exists in blood in both conjugated and non-conjugated forms. Gas chromatography/mass spectrometry, the methodology of choice for determination of many small organic molecules, has not been feasible because no thermally stable derivatives have been found. The currently accepted reference method is a spectrophotometric procedure, Doumas, et.al., Clin. Chem., 31, 1779-89 (1985).

\section{NIST activities}

Initial NIST efforts were focused on development of a crystalline bilirubin material of known purity. This led to the development of SRM 916 and its successor 916a, certified for purity and isomeric composition. Reference values are provided for the molar extinction coefficients measured under certain conditions. The certification of this material is described in detail in Cohen, et. al., Fresenius J. Anal. Chem., 338, 426-29, (1990).

Recent activities have focused on implementing the spectrophotometric reference method at NIST so that it can be used to value assign bilirubin concentrations in serumbased SRMs. Plans are to organize a small round-robin of laboratories that have experience with this method to perform measurements on SRMs with appropriate levels.

The new technologies now available in liquid chromatography/mass spectrometry instrumentation appear to make it possible to measure serum bilirubin levels with unsurpassed specificity and accuracy. NIST will be investigating this approach as an independent means of determining bilirubin. If the two methods provide results that agree, the confidence in the accuracy of the result will be very high.

\section{Collaborators:}

University of Wisconsin Medical College 


\section{CERTIFICATION OF Pb AND Cd IN SRM 966, TOXIC METALS IN BLOOD, BY ISOTOPE DILUTION ICP-MS AND RADIOCHEMICAL NEUTRON ACTIVATION

\author{
Stephen E. Long, Michael S. Rearick, Robert D. Vocke and Elizabeth A. Mackey
} \\ NIST}

Background: A need was identified in the late 1990's for a whole blood reference material that contained normal as well as elevated levels of selected toxic heavy metals, specifically $\mathrm{Cd}$, inorganic $\mathrm{Hg}$, methyl $\mathrm{Hg}(\mathrm{MeHg}$ ) and endogenous $\mathrm{Pb}$. This need originated from continued interest in workplace and public health monitoring for $\mathrm{Cd}$ and $\mathrm{MeHg}$ in blood and the large number of participants in proficiency testing programs. In addition, new OSHA workplace monitoring standards now require measurement of blood $\mathrm{Cd}$. Measurement methods have been developed at NIST for the certification of $\mathrm{Pb}$ and Cd in SRM 966, a two level bovine whole blood SRM. These are based on isotope dilution inductively coupled plasma - mass spectrometry (ID-ICP-MS), a primary method, and for $\mathrm{Cd}$ only, radiochemical neutron activation analysis (RNAA).

The base material for SRM 966 was prepared at a USDA licensed facility under the direction of the Centers for Disease Control and Prevention (CDC). Cows were dosed with lead nitrate and then bled in units of $500 \mathrm{~mL}$. Multiple pools of blood were prepared. One pool was spiked with $\mathrm{MeHg}$ (as iodide), inorganic $\mathrm{Hg}$ and inorganic $\mathrm{Cd}$. At each concentration level, a minimum of 2000 plastic containers were filled with at least $2 \mathrm{~mL}$ of blood each and frozen at $-20^{\circ} \mathrm{C}$. The vials were subsequently shipped to NIST for value assignment.

Processing and Measurement: For ICP-MS, approximately $2 \mathrm{~g}$ of blood were removed from each vial and spiked with a calibrated mixture of ${ }^{206} \mathrm{~Pb}$ and ${ }^{111} \mathrm{Cd}$ separated isotopes. The samples were digested with $\mathrm{HNO}_{3} / \mathrm{HClO}_{4}$ and analyzed by quadrupole ICP-MS.

For RNAA measurement of cadmium, each vial was freeze-dried at $-20{ }^{\circ} \mathrm{C}$ and a pressure of 20 mtorr, for five days. The dry powder was transferred to linear polyethylene bags, and heat-sealed. Samples were placed in a polyethylene rabbit, irradiated for 8 hours at a neutron flux of $3 \times 10^{13} \mathrm{n} \cdot \mathrm{cm}^{-2} \cdot \mathrm{s}^{-1}$ and allowed to decay for 48 hours to permit the level of ${ }^{24} \mathrm{Na}$ to decrease. Samples were then digested with $\mathrm{HNO}_{3} / \mathrm{HClO}_{4}$. After digestion was complete, zinc-diethyl-dithiocarbamate (DDC) in chloroform was added and the $\mathrm{Cd}$ back extracted from the organic DDC fraction into $\mathrm{HCl}$. The $\mathrm{Cd}$ fraction was then transferred to a polyethylene bottle for $\gamma$-ray spectrometry.

\begin{tabular}{|lcccc|}
\hline & & $\begin{array}{c}\text { SRM 966, Toxic Metals in Blood } \\
\text { (Concentrations of Pb and Cd, } \boldsymbol{\mu g} / \mathbf{d L})\end{array}$ \\
& Element & \multicolumn{2}{c|}{ Certified Value } & Information Value \\
Level 1 & $\mathrm{Pb}$ & 1.56 & $\mu \mathrm{g} / \mathrm{dL} \pm 0.05$ & \\
& $\mathrm{Cd}$ & & & $<0.4 \mu \mathrm{g} / \mathrm{L}$ \\
Level 2 & $\mathrm{Pb}$ & 25.27 & $\mu \mathrm{g} / \mathrm{dL} \pm 0.22$ & \\
& $\mathrm{Cd}$ & 5.22 & $\mu \mathrm{g} / \mathrm{dL} \pm 0.16$ & \\
& & & \\
\hline
\end{tabular}


Certificate Values: The certified values for $\mathrm{Cd}$ were determined by combining data from ID-ICP-MS and RNAA following the ISO GUM procedure. The expanded uncertainty is a $95 \%$ confidence interval and reflects the combined effects of measurement uncertainty, variability in concentrations between vials, blanks and any systematic differences between the two techniques.

The certified values for $\mathrm{Pb}$ were determined by the primary method, ID-ICP-MS. The expanded uncertainties are $95 \%$ confidence intervals and reflect the combined effects of measurement uncertainty, variability in concentrations between vials, and blanks.

\section{NIST STANDARD REFERENCE MATERIALS FOR HUMAN DNA ANALYSIS}

\section{Barbara Levin, Haiyan Cheng, Margaret Kline, Jan Redman, Dennis Reeder, Kristy Richie, Catherine O'Connell, and Lois Tully NIST}

The National Institute of Standards and Technology (NIST) has developed three Standard Reference Materials (SRM 2390, SRM 2391 and SRM 2392) and is working on two more SRMs to provide quality assurance in the analysis of human DNA. SRMs 2390 and 2391- the DNA Profiling SRM and the Polymerase Chain Reaction (PCR)-based DNA Profiling standard - are intended for use in forensic and paternity identifications, for instructional law enforcement, or non-clinical research purposes. SRM 2392 is for standardization and quality control when sequencing the entire or any segment of human mitochondrial DNA (mtDNA) for forensic identification, disease diagnosis or mutation detection. It can also be used for quality control when performing PCR and sequencing any DNA. SRM 2393, under development, is for p53 mutation detection and consists of 12 plasmid clones (one wild-type and 11 with single point mutations in exons 5-9). Since the p53 gene is the most commonly mutated human cancer gene, this SRM will be useful to clinical laboratories examining tumors and laboratories involved in mutation detection. A heteroplasmic mtDNA SRM (SRM 2394 also under development) consists of mixtures of various concentrations of two mtDNA templates differing in one base pair. This SRM is designed for medical, forensic and toxicological scientists who wish to determine the limit of detection of low frequency mutations, polymorphisms or heteroplasmic DNA sites.

This work was funded in part by the U.S. National Institute of Justice through the NIST Office of Law Enforcement Standards and the NIST Standard Reference Materials Program. 


\section{THE MICRONUTRIENTS MEASUREMENT QUALITY ASSURANCE PROGRAM}

\section{Jeanice Brown Thomas, NIST}

The Micronutrients Measurement Quality Assurance (QA) Program was organized to support measurement technology for selected fat- and water- soluble vitamins and carotenoids in human serum and plasma. This program was initiated in 1984 as part of investigations supported by the National Cancer Institute (NCI) Division of Cancer Prevention and Control to study the possible role of these analytes in reducing the risk of developing certain types of cancers and diseases. The program initially supported a core of approximately 10-NCI grantee laboratories and has since expanded to include more than 60 laboratories worldwide. Today, the program is $100 \%$ fee-supported and is "the only QA program available internationally for the fat-soluble vitamins and carotenoids."

One of the services that the QA program provides laboratories is that of measurement comparability assessment. NIST provides laboratories with the tools for comparability assessment through use of interlaboratory comparison studies, Standard Reference Materials (SRMs) and control materials, and methods development and validation. Serum-based samples with assigned values for the target analytes and performanceevaluation standards are distributed by NIST to laboratories for analysis. NIST staff provides the laboratories with technical feedback concerning their performance and suggestions for methods development and refinement. The results from the comparison studies are used to establish a laboratory performance database, which is used to help laboratories to improve their measurement comparability and to obtain reliable data needed to make accurate clinical and health-care decisions.

As a result of the QA program, the accuracy of laboratory measurements resulting in increased interlaboratory comparability for retinol, $\forall$-tocopherol, and $\exists$-carotene has improved substantially over time. The average estimated coefficient of variation for retinol and $\forall$-tocopherol has been approximately $5 \%$, and about $10 \%$ for $\exists$-carotene, for the past five years.

Future plans for FY 2001 include the continuation of the round robin exercises for selected vitamins in serum, including the measurement of coenzyme $\mathrm{Q}_{10}$ and vitamin $\mathrm{K}_{1}$. As an effort to continue the program beyond FY 2001, a two-phase plan is currently underway to expand the program. Beginning November 2000, an external QA web page will be available. Phase I will allow customers to enroll into the program via the internet. They will also be able to register on-line for the upcoming QA workshop, which will be held as a symposium at the Experimental Biology conference on April 4, 2001 in Orlando, Florida. Phase II (pending resources) will provide electronic data entry and feedback with security capabilities.

\section{Publications/Outputs:}

Brown Thomas et al. "Preparation and Value Assignment of Standard Reference Material 968c: Fat-Soluble Vitamins, Carotenoids, and Cholesterol in Human Serum," accepted by Clin. Chim. Acta, September 2000. 
Duewer, D.L., Kline, M.C., Sharpless, K.E., and Brown Thomas, J., "NIST Micronutrients Measurement Quality Assurance Program: Characterizing Individual Participant Measurement Performance Over Time," Anal. Chem.,72, 15, 3611-3619 (2000).

Duewer, D.L., Kline, M.C., Sharpless, K.E., and Brown Thomas, J., "NIST Micronutrients Measurement Quality Assurance Program: Characterizing the Measurement Community's Performance Over Time," Anal. Chem., 72, 17, 4163-4170 (2000).

\section{ANABOLIC STEROID CRMS FOR IN VITRO ANALYSIS Steven Westwood and Bernard King Australian Government Analytical Laboratory, Australia}

The Pure Substance Reference Materials team at NARL has produced a collection of anabolic steroid metabolites, conjugates and deuterates. The initial motivation for their production was for use as traceable Reference Materials in sports drug analyses, but they have the potential for use in general clinical diagnostic, veterinary residue, toxicology and forensic testing applications. Key compounds, particularly those used to provide quantitative estimations of steroid concentrations in vivo, are available as full Certified Reference Materials. ${ }^{1}$ Over one hundred steroids will be produced and characterised when the project is completed.

We have developed a flexible but rigorous approach to establishing the key properties of identity and chemical purity for these materials that in principal confers traceability to the SI units the mole and kilogram, respectively. ${ }^{2}$

Chemical identity is established by combining information from the synthetic route used to prepare the material, comparison with established literature data and firstprinciples examination of the spectroscopic properties of the material using modern instrumental techniques. High-field ${ }^{1} \mathrm{H}$ and ${ }^{13} \mathrm{C}$ NMR is especially valuable in this regard, particularly for establishing with acceptable confidence what can be quite subtle questions regarding the three-dimensional configuration of a molecule.

Chemical purity is assessed by summation of impurity analysis and subtraction from $100 \%$ to give the purity estimate for the compound with an accompanying uncertainty budget. Analysis techniques utilised include GC, HPLC, differential scanning calorimetry, thermogravimetric analysis, ${ }^{1} \mathrm{H}$ NMR, elemental microanalysis and Karl-Fischer titrimetry. Isotopic purity is also determined in the case of labeled compounds. Key components of the quality system recommended in ISO Guide $34^{3}$ for reference material producers have been implemented and we have also recently achieved accreditation to ILAC G12:2000 for the production of pure substance organic Reference Materials and Certified Reference Materials in the areas of anabolic steroids, illicit drugs and agrochemicals. 


\section{References:}

1. "The Role of Reference Materials" (ISO Publication 2000-01/1000)

2. Bernard King and Steven Westwood; "GC-FID as a Primary Method for Establishing the Purity of Organic CRMs Used for Drugs in Sport Analysis". Accreditation and Quality Assurance, submitted.

3. ISO Guide 34:2000 "Quality system requirements for reference materials producers"

\section{OBSERVATIONS ON WHO CONSULTATION MEETING ON INTERNATIONAL BIOLOGICAL STANDARDS FOR IN-VITRO DIOAGNOSTIC PROCEDURES (SEPTEMBER 14-15, 2000; GENEVA) \\ Rudolf M. Lequin \\ Diagnostics Consultancy, The Netherlands}

\section{Attended By:}

International Professional Societies: IFCC, ISTH, ICSH, WASP, IABS, IUPAC, IUIS

Regulatory Bodies: FDA (CBER / CDRH); EU IVD Taskforce

Notified Bodies: Japanese Regulatory Agency

WHO Collaborative Centers: NIBSC, London; CLB, Amsterdam; CBER, Washington IVD Industry: EDMA; HIMA (AdvaMed)

The report of this meeting will be presented to the WHO Expert Committee on Biological Standardization (ECBS) meeting to be held first week of November 2000. The ECBS has to approve the recommendations!

\section{Terminology}

Biological substance For control of vaccines or therapeutics

Analyte For in-vitro diagnostic/monitoring procedures

Note: the analyte may or may not have a defined biological activity 


\section{Traceability}

1. Clearly define the analyte in relation to medical needs

2. TYPE A analytes

SI traceable

Defined Compounds

Reference Measurement
TYPE B analytes

Not SI traceable, but arbitrary units e.g.

WHO IUs

Poorly defined analytes, especially

in biological fluids

International Conventional Reference

Systems (RMS) *

Measurement Systems (ICRMS )*

consisting of

Reference Measurement

Procedure ( RMP )

Reference Material ( RM )

For many in place

* Independent of routine measurement procedures

\section{consisting of} Material ( ICRM)
International Conventional Reference

Procedure ( ICRMP)

International Conventional Reference

For virtually all, not in place

3. A network of reference measurement laboratories is needed to perform the measurements employing the RMSs or ICRMSs

4. For Type B analytes, value assignment is proposed as follows:

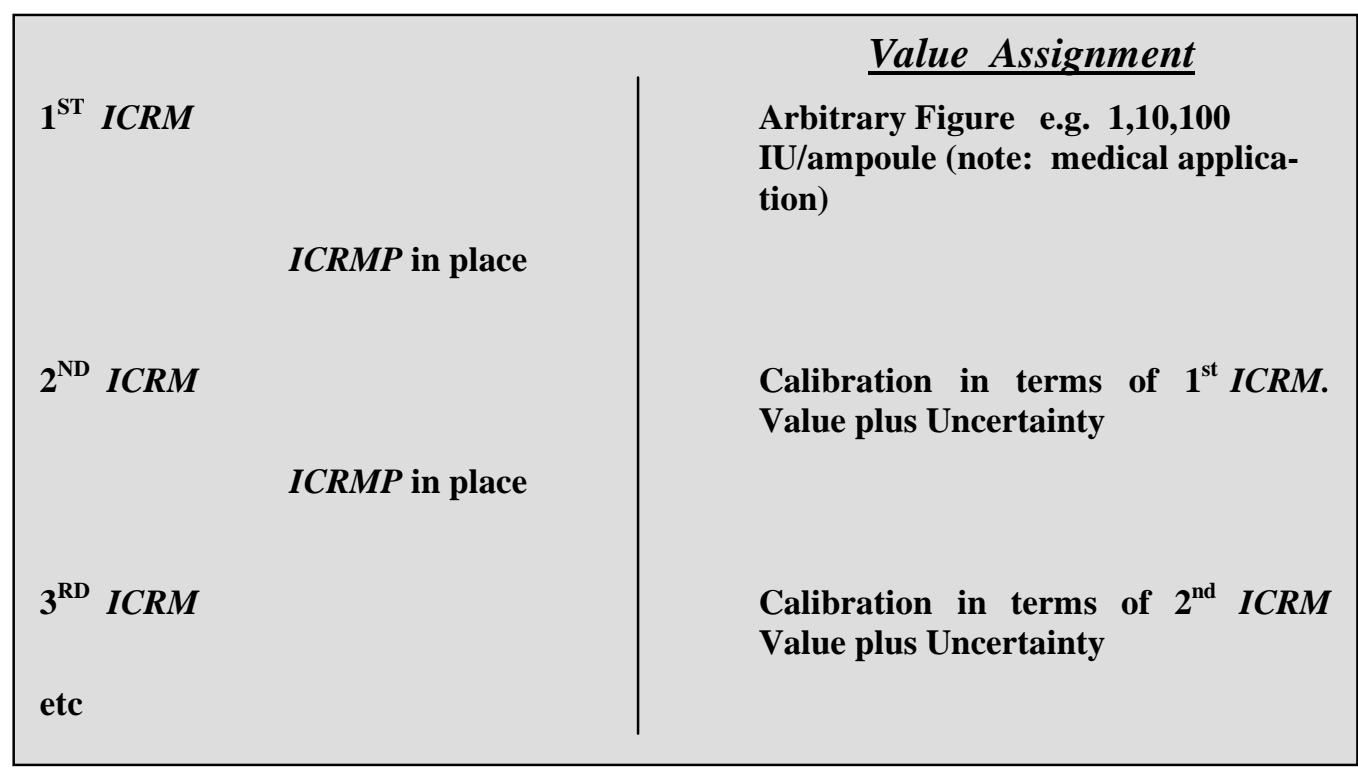


5. For the purpose of labeling, the following is proposed:

$1^{\mathrm{ST}}, 2^{\mathrm{ND}}, 3^{\mathrm{RD}}$ etc., WHO (?) International Conventional Reference Material (ICRM) of analyte "X", for in-vitro diagnostic procedures: "Y" IU per ampoule.

\title{
18. THEMATIC NETWORK PROJECT PROPOSAL ON TRACEABILITY ISSUES RELATED TO THE EU DIRECTIVE 98/79/EC IN VITRO DIAGNOSTIC MEDICAL DEVICES
}

\author{
Annarita Baldan, Ed de Leer \\ Nederlands Meetinstituut, The Netherlands
}

\section{Background}

This Thematic Network project proposal is under submission to the European Commission and it is the response to the dedicated call for proposals that appeared in April 2000, under the specific programme Competitive and Sustainable Growth of the $5^{\text {th }}$ Framework Programme.

This Network proposal is based on the traceability requirements reported in Annex I A.3 of the EU directive 98/79/EC In Vitro Diagnostic Medical Devices:

"The traceability of values assigned to calibrators and/or control material must be assured through available reference measurement procedures and/or available reference materials of a higher order".

It aims at the creation of an international network to promote the understanding and knowledge transfer of concepts such as traceability, comparability and measurement uncertainty in the field of In Vitro Diagnostics.

Relevance of a Network on IVDs

- to give support to the essential requirements introduced by the EU directive 98/79/EC, which will come into force in the year 2003

- $\quad$ to provide the proper infrastructure (network) and tools (deliverables) for building up an IVD traceability chain and for transferring knowledge about metrological issues (dissemination)

- to contribute to the improvement of the quality, accuracy and comparability of IVD medical devices' measurement results

- to set up an international co-operation between a variety of organisations with different backgrounds that are dealing with IVDs 


\section{World-wide spirit of the Network}

The strength of this Network is its international character. In order to create a traceability network in the IVD area, and to establish new policies and trends that will have world-wide effect, it is necessary to cooperate on a global scale.

The Network project will appoint an international advisory board, the IIGTH, "International Initiative on Global Traceability in Healthcare". This board, composed of IVD experts from all continents, has the task of supervising the Network, and to setting the main guidelines for the creation of a global consensus.

Furthermore, the Network's broad composition will provide complete coverage of the IVD community:

- IVD Manufacturers

- Professional Scientific organisations

- Certified Reference Materials producers (Europe and United States)

- National Metrology institutes (Europe and United States)

- Reference Measurement Laboratories

- Accreditation bodies (ILAC)

- Standardization institutes (ISO,)

- IVD experts

\section{Objectives and Deliverables}

The Thematic Network is structured in work packages, which will focus on:

$>$ Understanding, and knowledge transfer of metrological issues in the IVD area, realised by the preparation of a booklet on IVD quantities traceable and not traceable to SI, by the creation of a network web site and by making available on internet an IVD reference systems data-base;

$>$ investigation of the needs in IVD for new certified reference materials (CRMs) and new reference measurement procedures, realised by the preparation of guides;

$>$ global collaboration to harmonise measurement procedures and to mutually recognise reference materials, for the reduction of trade barriers (especially Europe and United States);

$>$ identification of measurement areas that need strengthening, and support the organisation of interlaboratory comparison exercises. 


\section{DEVELOPMENT OF REFERENCE SYSTEMS OF MEASUREMENT FOR THE IMPLEMENTATION OF THE IVD DIRECTIVE FOR CHEMICALLY DEFINED MEASURANDS}

\section{A European Project Proposal \\ Detlef Schiel \\ Physikalisch-Technische Bundesanstalt, Braunschweig, Germany}

\section{Motivation and Objectives:}

To provide the metrological basis for the implementation of the IVD-Directive by means of :

$>$ reliable reference measurement procedures and reference materials for some measurands for which traceability to the SI units can be established

$>$ improvement of the accuracy and comparability of routine measurements in clinical chemistry for an enhancement of the quality of heath care through:

- reliable diagnoses and therapies,

- strengthening of the confidence in clinical measurements

- avoiding superfluous repeat measurements that will aid in the reduction of health care costs

On the basis of the Mutual Recognition Arrangement of the national metrological institutes (NMI) to contribute to the preservation of the competitiveness of the EU reference material manufacturers.

\section{Measurands:}

b) Digitoxine, Digoxine Cardiac insufficiency, therapeutic drug monitoring

c) Testosterone, Estriol Endocrine disorders

d) $\mathrm{PH} \quad$ Acid-base disorders

e) Potassium Electrolyte balance disorders

\section{R\&D Work:}

$>$ develop reference measurement procedures (primary methods) linked up to the SI system

- Isotopic dilution mass spectrometry (except for $\mathrm{pH}$ )

- Characterization of pure materials of the measurands $(a, b)$

- Development of separation methods as GC or IC (a, b, d)

$>$ international comparison measurements to validate the procedures

$>$ a study to develop the ability to certify matrix-based higher order reference materials 


\section{Participants:}

The work is divided among eight participants of five EU countries and two collaborating partners from Australia and USA. The consortium is composed of NMI's, universities, reference institutes for clinical chemistry and other RM producers and manufacturers of diagnostic devices. The partners are highly experienced and recognized in the field of chemical analysis in clinical chemistry.

\section{An EU Proposal Rudolf M. Lequin Diagnostics Consultancy, The Netherlands}

20. IN-VITRO DIAGNOSTIC MEASUREMENT in LABORATORY MEDICINE

In a joint effort with IFCC and IRMM a proposal has been developed on In-vitro Diagnostic procedures in diagnosis and monitoring of Thyroid Disease entitled:

\section{"Feasibility to Develop Reference Measurement Systems for Thyrotropin} (TSH) and for Free Thyroxine (FT4), and Validation of Reference Measurement Systems (procedure and material) for Thyroxine (T4) and Triiodothyronine (T3) in Human Serum"

Main Deliverables of this project:

1. Preparation, purification and characterisation of a subgroup of serum TSH glycoforms (possibility to develop an International Conventional Reference Material)

2. A possible solution to an international agreement of the procedure to separate free from bound T4 in human serum without disturbance of the equilibrium. This is the first and crucial step to a future reference measurement system for FT4 in human serum, independent of routine measurement procedures for FT4.

3. Validation of published primary reference measurement procedure (RMP) for total T4 in human serum, and establishment of a primary reference material for T4.

4. Validation of published primary reference measurement procedure (RMP) for total T3 in human serum, and establishment of a primary reference material or T3.

Note 1: the results of \# 3 and \# 4 should be endorsed by an international advisory group

Note 2 : the results of \# 2 and \# 1 will be communicated to an international advisory group who then may recommend to proceed with development of a reference measurement system and an international conventional reference measurement system, respectively. 


\section{BREAKOUT SESSIONS: Reports and Discussion Held November 3, 2000}

\section{Breakout Session One}

Neil Greenberg, Ph.D., D.A.B.C.C.

Priorities for National and International Investments in New or Improved Reference Systems in Support of Clinical Laboratory Measurement

\section{Breakout Session Two}

\section{Don Powers, Ph.D.}

Reference Materials and Reference Measurement Procedures to Support

Traceability Requirements of the IVD Directive

\section{Breakout Session Three}

\section{Greg Miller, Ph.D.}

Impact of Method-Material Matrix Interactions on Calibration Traceability

Protocols for Successful Harmonization of Patient Results

\section{Breakout Session Four}

\section{William F. Koch, Ph.D., F.A.C.B.}

Development of International Consensus on the Credentialing Process for Reference Systems

\section{Breakout Session Five}

Gary L. Myers, Ph.D., F.A.C.B.

Creating and Sustaining Reference Method Laboratory Networks 


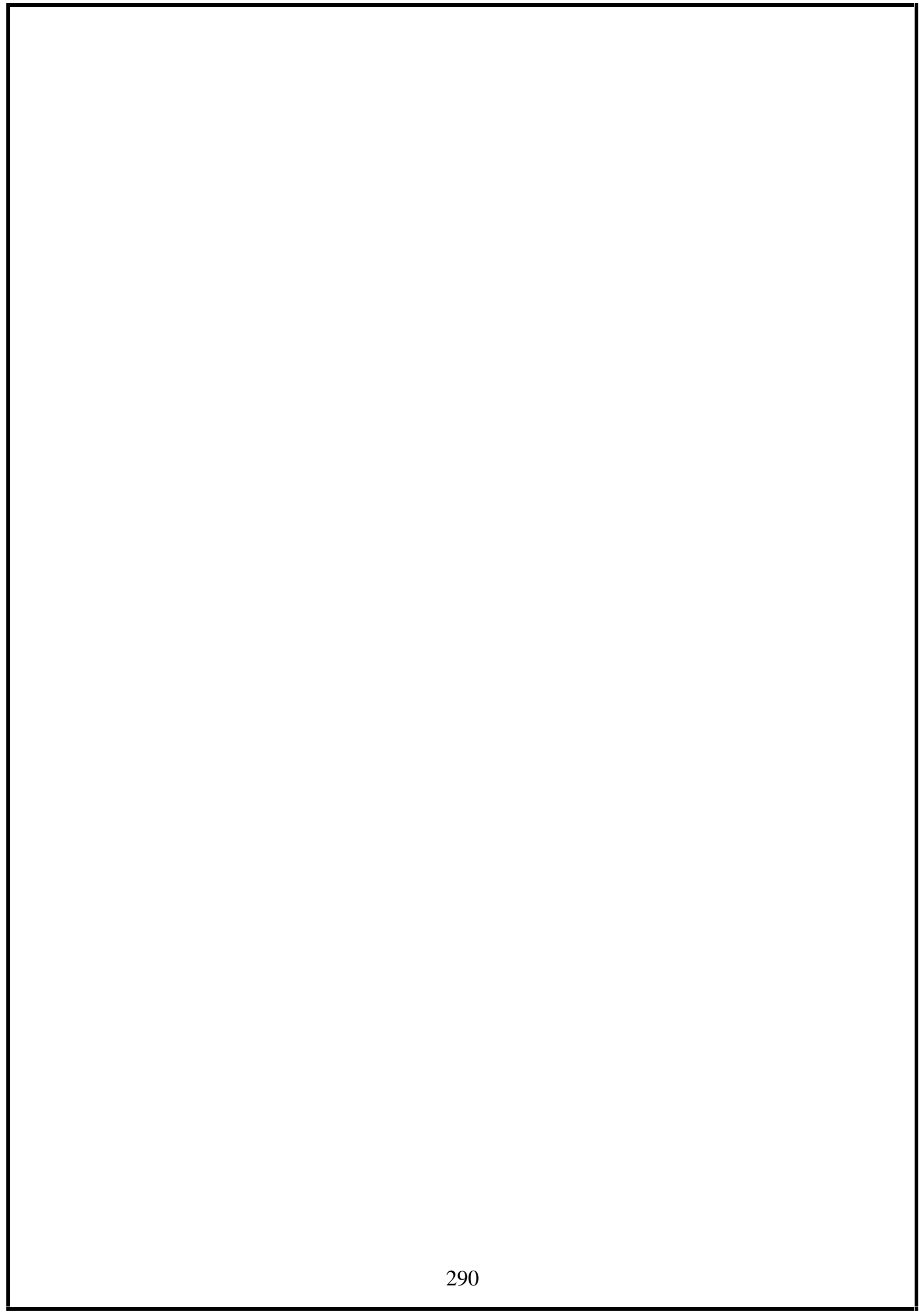




\section{Breakout Session Reports: Summaries and Discussion \\ Willie E. May, Moderator \\ Edited for Clarity by Ellyn S. Beary \\ National Institute of Standards and Technology}

The Discussion came to order at 1:15 p.m. at NIST in the Green Auditorium, Building 101, 100

Bureau Drive, Gaithersburg, MD

Willie May, Ph.D., presiding.
DR. MAY: Now comes the difficult part of the meeting where we have to try to synthesize and assimilate all of the hard work of this morning. As you know, we are going to do this by having reports from the five breakout groups.

During this morning's session, I walked through and spent some time with most of the breakout groups. In reviewing the subject matter, and listening to the discussions, I think there are some common themes. So, what I propose is that the groups report back in the following order -- group one, group four, group five, group two, and group three.
... in some other housekeeping rules, this entire session is being transcribed. I hope no one has a problem with that. The purpose is to help us to put together the final report of the meeting. Transcribed by Neal Gross and Co, Inc.

Groups one, four and five were focused on reference systems, and priority setting. Groups two and three were focused on reference materials, reference methods, and they are tools to be used as a part of the reference systems. So I think it may be easier to collate the feedback from the separate groups if we collect the information in that order. So, if no one objects, that's how we'll proceed.

We will begin with the reports from each of the working group leaders, restricting that report to no more than 25 minutes per group. We will allow questions for clarification only. I think that we can have a more coherent discussion after all the reports are given. During the discussion period, we ask that you use the microphones on either side of the room: please state your name, your affiliation, so that we can maintain an accurate

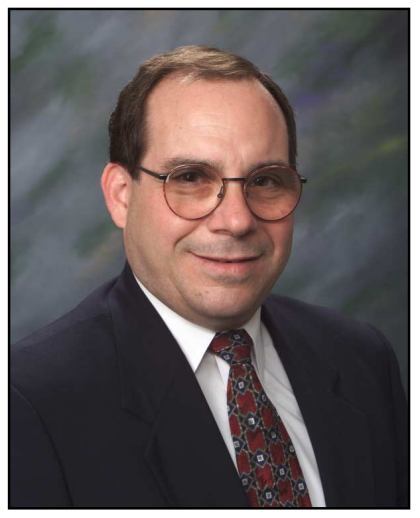
record. Before we proceed, are there any questions? If not, I'll introduce the first speaker, Neil Greenberg.

Report from Breakout Session One: Priorities for National and International Investments in New or Improved Reference Systems in Support of Clinical Laboratory Measurement, Neil Greenberg, convener.

Neil Greenberg, Ph.D., DABCC, is a Project Manager, Regulatory Affairs, Ortho-Clinical Diagnostics, a Johnson \& 
Johnson Company. He received his doctorate in Physiological Chemistry and Clinical Chemistry from The Ohio State University, Columbus, Ohio, and served as a Post Doctoral Fellow in Clinical Chemistry at Hahnemann University, Philadelphia. $\mathrm{He}$ joined Eastman Kodak in 1978, and has held various managerial roles in manufacturing and quality assurance for the Ortho-Clinical Diagnostics Vitros (formerly Ektachem) line of in vitro diagnostic products. As Manager of Kodak's Reference Laboratory, he became actively involved in standardization of measurement procedures and reference materials for laboratory medicine in the early 1980's, and continues to work actively with NCCLS and the American Association for Clinical Chemistry on various aspects of measurement standardization for in vitro diagnostics. He is a member of Working Group 2, ISO/TC 212, which is developing international standards for reference systems in laboratory medicine. Dr. Greenberg also is the liaison for ISO/TC 212 Working Group 2.

\section{Breakout Session One}

Editor's Note: Prior to the Meeting Dr. Greenberg provided the following details about his discussion group.

Title: Priorities for National and International Investments in New or Improved Reference Systems in Support of Clinical Laboratory Measurement Objective: To identify and rank the factors that are necessary for determining priorities for development of new reference systems.

Summary of Topics/Discussion Points:

- Who determines when there are measurement problems with IVDs? Who are the stakeholders? (e.g., government/regulators, professional organizations and/or proficiency providers, manufacturers, laboratory personnel, physicians, or patients?) How should we balance these perspectives?

- What metrics are useful in assessment of process capability (PCI) for clinical laboratory measurements? What are the right measurement specifications (e.g., CLIA performance criteria), and how does the clinical laboratory industry perform (generically) relative to an overall objective of "design for six-sigma"?

- What needs have been identified by industry? This topic will include more detailed review and discussion of findings from AdvaMed's (formerly HIMA) industry needs/gap analysis (just getting underway).

- What factors should be considered in a "performance scorecard" approach for evaluation of current suitability of available measurement systems for each analyte in laboratory medicine? (e.g., volume of testing performed, criticality/clinical significance factors, interlaboratory proficiency, etc.)

DR. GREENBERG: There were about 20 of us in this breakout session, with an excellent cross section of people from industry professionals, the people from standards institutes, and regulatory bodies. We had a very healthy discussion. We decided to break out our discussion in terms of what it is that we needed and then further deal with some of the whos and the hows. So, to start off with, I'd like to just highlight some of the key points. 


\begin{tabular}{|ll|}
\hline$\underline{\text { Participant }}$ & Affiliation \\
Dave Armbruster & Abbott Labs \\
David Bunk & NIST/CSTL \\
Mary Burritt & Mayo Clinic \\
Jennifer Colbert & NIST/SRMP \\
Sylvia Crush-Stanton & Boston Biomedica, Inc. \\
Yolanda Davidson & NIST/CSTL \\
Ed de Leer & NMi, Delft \\
Eleanor Ling & Beckman Coulter \\
John Eckfeldt & CAP \\
Glenn Ehlers & Ortho-Clinical \\
Adolfas Gaigalas & NIST/CSTL \\
Patricia Garrett & Boston Biomedica, Inc. \\
Farrah Kamalian & Bio-Rad Labs \\
Rudy Lequin & Diagnostics Consultancy \\
Bryant Nelson & NIST/CSTL \\
Max Robinowitz & FDA \\
John Thompson & Dade Behring, Inc. \\
Willard Tuthill & UL, Inc. \\
Emil Voelkert & Roche Diagnotics \\
\end{tabular}

First of all there was a consensus among the participants that we needed global reference systems, and these reference systems needed to harmonize the clinically reported results. We also felt it was important to minimize redundancies in development and reference systems. In addition, we want to assure that barriers to new technology are not created. So, these reference systems certainly need to encourage new technologies.

\footnotetext{
$\left(\frac{1}{2}\right.$

\section{NIST Workshop on IVD Calibration} Traceability - Breakout Session I

"Strawman" position/viewpoint...

To be successful at a global level, the establishment of a clearly defined, credible IVD reference systems project selection and prioritization process is required. The process...

- should be an open and public one, that actively seeks out and welcomes input from all the relevant stakeholders.

- should engage industry to participate in project prioritization.

- should use consensus as a decision-making tool

- should be data-driven, and systematically examine a series of key questions and factors prior to recommending and/or undertaking a given reference systems development program.

- would engage active support and participation from the laboratory medicine community/industry in the selected standards development programs.

The (clinical laboratory medicine) professional sector should play the most significant role in setting the priorities.
}

\section{The figure to the left is a summary of the features of a globally recognized reference system, and an identification of who should be engaged in priority setting activities.}

Setting Priorities: Key points are as follows:

We felt it was very important that the numeric results in diagnosis and or management of disease states in terms of the degree of overlap between diseased and non-diseased populations needs to be taken into account as a factor in determining whether or not there was any improvement that could be had by improved standards and improved reference systems. 


\section{NIST Workshop on IVD Calibration Traceability - Breakout Session I}

\section{Strawman Position/viewpoint (continued)...}

- The project prioritization process should (1) recognize that there are limited resources available to do the work, and (2) take into account decision factors such as...

- technical feasibilty and likelihood of success

- impact (both positive or negative?) on the clinical utility of the measurement, if a new or improved standard were to be made available

- public health significance of disease states toward which the given measurement is targeted.

- costs

- estimated time needed for the project to deliver the desired output (e.g., materials, methods, or both)
We talked a great deal about their project prioritization strategy, and this is really, I think, the heart of the discussion, and the primary intent of this particular breakout session.

Another important factor was the clinical outcome for the patients as a result of incorrect diagnosis or patient management decisions. If indeed there was going to be some change in clinical outcomes envisioned as a result of improved reference systems, that factor had to be part of the equation. In addition, demographic factors like disease prevalence and the volume of the tests and the number of labs performing the tests should be considered. For example, if there are only a small number of laboratories that are conducting a specific test, then the need for standardization might not be as great.

Then we considered success factors such as the "treatability" of the disease, especially in the case of broadly used screening tests. If the disease "treatability" is high, then of course standardization efforts would be encouraged.

In addition, current inter-method variability must be taken into account as a determining factor. For that, we felt that it was important to leverage experience from manufacturers. That coupled with data from some of the PT (Proficiency Testing) and EQAS (European Quality Assessment Scheme) type organizations could be extremely valuable in terms of helping us to differentiate the tests that demonstrated larger amounts of method-to-method variability.
One of the manufacturers present was from a quality control product manufacturer, and they felt that their data from method to method variability would be extremely valuable. 


\section{NIST Workshop on IVD Calibration Traceability - Breakout Session I}

Some background questions \& points of discussion...

- Defining the stakeholders in the process,e.g....

- government/regulators, professional organizations and/or proficiency providers, manufacturers, laboratory personnel, physicians, or patients?

- How should we balance or weight the stakeholder perspectives?

- What IVD reference system needs have been identified by industry? professional associations? government authorities?
Identifying stakeholders and reference systems needs:

There is an immediate need to inventory and communicate information about current reference systems. There seems to be a lack of that information available to the general public. I know that this is one of the projects that has been proposed under the IIGTH consortium in Europe. We have a couple of representatives here.

As we make the transition to new reference systems as we inevitably will, we will need to develop new standards.

The following priority lists were excerpted from the draft report on

Reference Materials and Systems Priorities identified at WHO

Consultation on International Biological Standards for in vitro

Diagnostic Procedures.

September $14-15,2000$, Geneva

\section{International Federation of Clinical Chemistry and Laboratory Medicine}

HCG Isoforms, Lp(a), Myoglobin \& Troponins, HbA1c, PSA, Homocysteine, ATIII, Osteocalcin TSH, T4/T3, FV Leiden DNA, Alkaline Phosphatase, CRP, S-Transf.

\section{EU Notified Bodies}

HBsAG reference panel, HCV Ag, Anti-HCV genotype reference panel, Anti-HIV subtype reference panel, HCV-RNA, HBV-DNA, HIV-RNA (geno-, subtypes)

\section{U.S. Food \& Drug}

Administration's (FDA) Center for

Biologics Evaluation and Research

HCV, HbsAG

Anti-HBs, HIV-1, HIV-2, HIV subtype

Group M, HIV subtype Group O, HTLV

EU Notified Bodies are organizations designated by the national governments of member states as being competent to make independent judgments about whether or not a product complies with the protection requirements laid down by each $\mathrm{CE}$ marking directive. 


\section{WHO Priority lists, continued}

\section{FDA's Center for Devices and Radiological Health (CDRH)}

CDRH concurs with statements and suggestions made by the International Council for the Standardization of Hematology (ICSH) and other Hematology groups about a need for standards in general hematology, hemostasis, thrombosis and for clotting factor assays. Included in this would be d-dimers and Leiden Factor V. Development of reference materials are encouraged for any of the tumor markers being used clinically such as CA 15-3 and CA 125. International standards are also needed for FISH assays and immunohistochemical assays for molecular markers such as HER2/neu and other molecular oncology markers. Standards for in vitro allergy testing are needed, and international reference materials for $\operatorname{Lp}(\mathrm{a})$, bone markers, telopeptides and anti cardiolipins.

\section{National Institute of Health, Japan}

Development of viral safety markers applied to the virological safety testing of blood, and blood products.

Training: In addition, there is an important need to develop tools for training and increasing the awareness. That training has to occur in a number of areas. The company's manufacturers, of course, need to train their people, but the clinical laboratories, the customers, and the clinicians also need to be trained. We felt that some of those tools could be developed by organizations such as the professional societies, IFCC (International Federation of Clinical Chemistry and Laboratory Medicine), AACC (American Association for Clinical Chemistry), and so on. They can do that in collaboration with some of the manufacturers groups such as EDMA (European Diagnostic test Manufacturers Association) and AdvaMed (Advanced Medical Technology Association). And in fact, that could build on some information, which is already being developed within EDMA to provide guidance specifically for manufacturers.

Global Consortiums: Now, a bit more about the whos and the hows. We talked a lot about global consortiums. In fact, we had some very interesting inputs from some of the people in the room about other models in some of the other industries, such as the plasma industry, where manufacturers consortia have gotten together.

Those consortia have happened essentially through the manufacturing associations for those particular industries. They have been instrumental in terms of helping those particular industries develop standards.

A couple of examples of this type of consortium would be the IIGTH, which I have already mentioned, that has formed in Europe to implement the ISO 17511 standard (In vitro Diagnostic Medical Devices - Measurement of quantities in samples of biological
We felt that it was important to offer a proposal here that we establish a global consortium of IVD manufacturers, professional societies, NMIs, and regulatory bodies. 
The function of the consortium would be to agree on projects, channel funding, also they would apply for funding to various sources such as government agencies, or even private or commercial sources, or nongovernmental sources. origin - Metrological traceability of values assigned to calibrators and control materials). There is another consortium, already existing, named SOGAT (WHO International WG on the Standardization of Gene Amplification Tests) that is concerned with the infectious disease marker devices. They seem to be having a great deal of success with this model.

Funding sources need to be explored and pursued by this proposed consortium (or could be

multiple consortia - depending on the focus). In fact, we felt that perhaps we ought to leave the infectious disease marker activity to the existing organization, SOGAT. Perhaps we could pursue expansion of the IIGTH model for full implementation of some of the other so called type A analytes, and type B analytes and the infectious disease markers could be left into a special class.

The funding sources that these consortia could pursue would certainly be the industry type funding pools that could be brought together through industry associations such as AdvaMed. Also some

\section{NIST Workshop on IVD Calibration Traceability - Breakout Session I}

Some Additional Concepts and discussion points...

- In determining where there is need for improved standardization, which metrics may be useful in assessment of process capability (PCI) for clinical laboratory measurements?

- Can EQAS data be used to define the present state?

- What are the right measurement specifications (e.g., US CLIA performance criteria,German Federal Medical System requirements, etc.)?

- What international and/or national organization(s) should...

- Participate in or be represented in the priorization process?

- Manage the project selection/prioritization process?

- How should IVD reference systems projects be funded? tax dollar sources, of course, because IIGTH is already applying to the European Commission for support.

Government organizations that have a long history of this type of funding are: U.S. Department of Commerce, the National Institutes of Health, and Centers for Disease Control and Prevention. Active nongovernment foundations include: the American Diabetes Association, American Heart Association, American Cancer Society. This is all sort of American in flavor, but these were examples that we could think of off the tops of our heads, and we know that similar organizations exist in Europe and other parts of the world.

We need to consider approaching those organizations in the interest of their mission to support programs in various aspects of health care, to consider funding some of these programs for reference systems. 
Advocacy: We felt that professional advocacy is another important piece of the overall program. We felt that this advocacy - even some leadership - could be solicited from organizations like the American Medical Association and other medical societies around the world. We could have those groups highlight the importance of standards to assure quality in health care. The smaller professional groups such as CAP (College of American Pathologists), and AACC, and IFCC, and so forth could follow along and support statements that were developed in some of the more powerful organizations such as AMA.

The industry must articulate to Congress and the Department of Commerce how important the standards activities are to our success ...

Industry advocacy for the investment in standards is extremely important, particularly with respect to facilitating funding through organizations or government agencies like NIST.

We also look to trade organizations such as AdvaMed and EDMA to develop statements and advocacy plans.

Process Proposed by this Discussion Group:

- Define a future state: Where would we like to be (in terms of process, organization \& infrastructure for setting priorities) 3 years from now?

- Define gaps: Where are we now vs. where do we want to be in the future state?

- Recommend ways to close gaps and get to future state.

These conclude the recommendations from Breakout Session One.

\section{Breakout Session Four}

\section{Report from Breakout Session on the Development of International Consensus on the Credentialing Process for Reference Systems, William F. Koch, convener.}

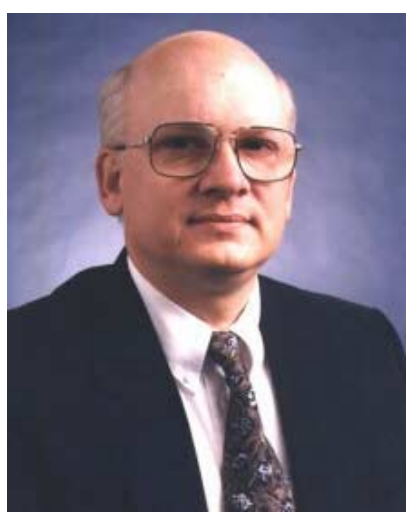

Dr. William F. Koch is the Deputy Director of the Chemical Science and Technology Laboratory at the National Institute of Standards and Technology. He began his career at NIST as a research chemist in the Center for Analytical Chemistry at NBS, where he expanded his research interests to include $\mathrm{pH}$, electrolytic conductivity and ion chromatography. He has had direct involvement in the development and certification of over 70 Standard Reference Materials, and has authored over 70 scientific publications.

Dr. Koch has been an elected member of the Board of Directors of the National Committee for Clinical Laboratory Standards (NCCLS) since 1990, and is currently the 
Immediate Past President of this organization. He is on the Governing Board of the Council for Chemical Research and chair of the CCR Science Education and Human Resources Committee. He was recently elected as a Fellow in the National Academy of Clinical Biochemistry. He is an active member of the Directors of Industrial Research Analytical Group, the American Chemical Society, the Society for Electroanalytical Research, and the American Association for Clinical Chemistry.

\section{Breakout Session Four}

Editor's Note: Prior to the Meeting Dr. Koch provided the following details about his discussion group.

Title: Development of international consensus on the credentialing process for reference systems.

Objective: To develop an international consensus on the credentialing process for reference systems.

Discussion Points:

Is there a need for an international reference system for clinical laboratory testing (IRSCL)?

Who are the main players and how would an IRSCL be funded?

Who should maintain the IRSCL?

Are Mutual Recognition Agreements among nations necessary?

Does a process for accreditation or conformity assessment need to be established?

DR KOCH: Group four was charged with developing an international consensus on the credentialing process for reference systems.

\begin{tabular}{|c|c|}
\hline Participant & Affiliation \\
\hline Mara Caler & Beckman Coulter \\
\hline Kim Carneiro & Danish Inst. of Metrology \\
\hline Donna Chapman & Bio-Rad \\
\hline Ellen Chen & FDA \\
\hline Nancy Dubrowny & Becton Dickinson \\
\hline Rene Dybkaer & H:S Frederiksberg Hospital \\
\hline Bernard King & LGC \\
\hline Alfred Hartman & CAP \\
\hline Jean-Michel Lacroix & Visible Genetics, Inc \\
\hline Barbara Levin & NIST \\
\hline Stanley Lo & Med. College of Wisconsin \\
\hline Richard Miller & Dade Behring \\
\hline Ronald Ng & Abbott Labs \\
\hline Mauro Panteghini & Azienda Ospeoaliera \\
\hline Richard Ross & Beckman Coulter \\
\hline Lothar Siekmann & University of Bonn \\
\hline Ken Slickers & Roche \\
\hline Lorna Sniegoski & NIST \\
\hline Beth Ann Wise & NCCLS \\
\hline
\end{tabular}

There was some hang up on the use of the words "credentialing process". Apparently that does not translate well for our European friends, and so they would like to strike the word "credentialing", and call it an "approval process for reference systems".
We quickly agreed that international consensus was necessary and that the reference systems were necessary. 
An International Reference System, composed of:

- Reference Materials

- Reference Methods

- Mechanism for Demonstrating Competence and Equivalence for making Reference measurements

We spent some time deciding or trying to figure out whether we were talking about reference methods, reference materials, reference laboratories, and we went down several paths. But I think we ultimately came back to the agreement that a reference system was going to be needed, and that it was comprised of lots of components. We focused on those components that were considered essential elements of a reference system. So, here are our recommendations. One, is we need an international reference system that's composed of reference materials, reference methods, and a mechanism - key word mechanism - for demonstrating the competence and equivalence for making reference measurements.

This reference system is realized through Reference Laboratories

- Demonstrated Traceability to International References compatible with Meter Convention

- Demonstrated competence through Accreditation compatible with ILAC)

- Compensation

- Incentives

- Regulation
This reference system is then realized through reference laboratories. So, the reference laboratories become a way of realizing our reference system, since these reference laboratories would have demonstrated traceability to the international references that were

compatible with the Meter Convention, realizing that not all analytes are going to be directly applicable to the convention.

However, all the things that we want to do for the reference lab have to be at least compatible with the Meter Convention. The reference labs must demonstrate competence through accreditation and we would charge that it be compatible with ILAC. Reference labs will need adequate compensation in order to carry this off.

In addition to compensation incentives, they may need other incentives, which were left undefined. I think the reference labs and the manufacturers will know what these incentives are. We have to realize

A bit of history...

\section{Convention du Metré}

As far back as 1875 when the Convention $d u$ Metré was signed in Paris, the necessity for international collaboration and agreement on metrological issues was recognized. The United States, while not a world power at the time was one of the seventeen original signatory nations. The Convention remains the basis of all international agreement on units of measurement. Currently, there are forty-nine Member States, including all the major industrialized countries.

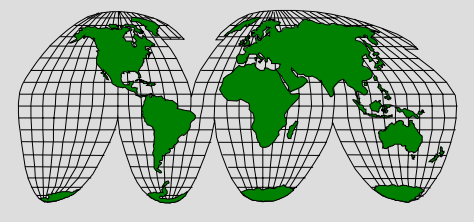


that regulation is also a factor that the reference labs have to take into account. We would like IFCC to be the main conductor, if you will, of this orchestra. IFCC could use a consensus process (perhaps the NCCLS process) to achieve consensus on a reference system. Remember that the reference system consists of the materials, the methods, and the mechanisms to ensure equivalency. Other stakeholders would necessarily involve WHO, CCQM, ILAC, and probably a host of other organizations. Unlike breakout session one that advocated creating a whole new organization, we spent some time thinking that we shouldn't

Who is going to pull this international reference system off?

Perhaps IFCC using consensus process (eg. NCCLS-like) in cooperation with WHO, CCQM, ILAC have a whole new organization because industry was already stressed with the number of memberships they must maintain in existing organizations.

\section{Funded by Members of: - AdvaMed \\ - MDMA \\ - EDMA \\ - AACC \\ - Governments}

So, we wanted to use existing structures, and IFCC seemed to be the most viable of those organizations. But we agree on who's going to pay for this. (Laughter) And that would be AdvaMed, MDMA, EDMA, AACC, and governments. We didn't go into detail of which government agencies were going to do it. They would have to be all the governments, not just the U.S. The European governments would have participate.

The next step in our recommendation would be to engage IFCC. Professor Siekmann and Dr. Panteghini agreed to talk with the leadership of IFCC as soon as they return to Europe to try to get this dialogue started, at least to find out if IFCC is willing to take this on. IFCC would then be in the driver seat. They would have to consult the stakeholders, pull together a meeting to develop a formal proposal in order flesh out the reference system.

Next Steps:

- Engage IFCC

- Consult stakeholders

- Formal proposal (6 months)

- Acquire funding

With that proposal, we could go to funding agencies and say, "Here is our proposal. How about up fronting some money for it?"

"Son of Traceability" Meeting to discuss progress (one year)
Our group proposes to have a second traceability meeting in about a year to discuss the progress in this endeavor.

This concludes the report from Breakout Session Four. Questions? 
PARTICIPANT: Why can AACC can be a funding organization, and not CAP, for example?

DR. KOCH: We can expand this list. If IFCC takes the lead, and puts the proposal together, the stakeholders will decide where they think they can get money. We'll go to as many mortgage companies as we can and let them all pay for it.

DR. LEQUIN: Lequin, the Netherlands.

Bill, you mentioned international reference systems. I, and many people now regard that we have international reference systems for the type A, well-defined compounds and where you have a reference material and method. However, for the type B compounds, which were discussed at length at a WHO meeting about six weeks ago, we came to the conclusion on the basis of the standard ISO 17511, to call the related reference system an international conventional reference system. As I said, this may be playing with words, but I think we should start to make this distinction right from the beginning. In that case you call the material an international conventional reference material.

DR. KOCH: So noted. We will take that into consideration.

DR. DOUMAS: If we can hold other specific questions until later.

\section{Breakout Session Five}

\section{Report from Breakout Session on Creating and Sustaining Reference Methods Laboratory Networks, Gary L. Myers, convener.}

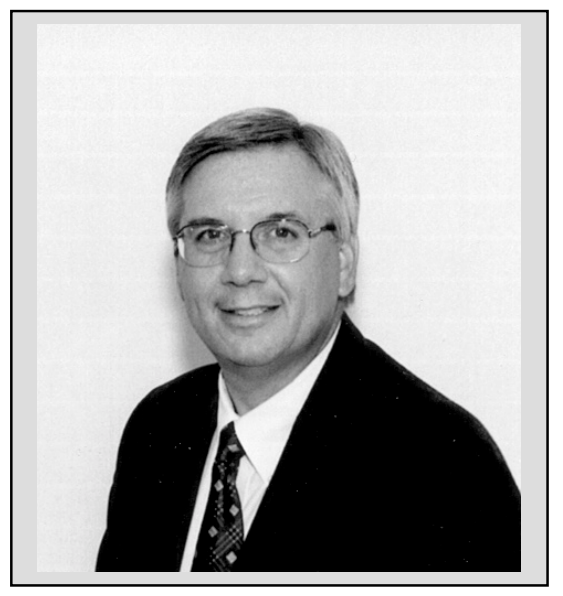

Gary L. Myers, Ph.D. is the Chief, Special Activities Branch, Division of Laboratory Sciences at the Centers for Disease Control and Prevention (CDC). Dr. Myers is responsible for $\mathrm{CDC}^{\prime} \mathrm{s}$ activities in laboratory standardization and methodology research to assure the comparability and accuracy of blood lipid and lipoprotein measurements performed in support of coronary heart disease research worldwide. In this capacity he also serves as the Scientific Director of the World Health Organization Collaborating Center for Reference and Research in Blood Lipids established at CDC. He represents the CDC on various national and international committees dealing with laboratory measurement issues. Currently he serves as Vice-chair of the NCCLS Area Committee on Clinical Chemistry and Toxicology. He is a consultant for lipid and lipoprotein measurement issues to the College of American Pathologists' Chemistry Resource Committee. He is a member of the ISO/TC 212/Working Group 2 on reference systems. Dr. Myers also serves on the Steering Committee for the National Glycohemoglobin 
Standardization Program and is a member of the International Federation of Clinical Chemistry's Working Group on HbA1c Standardization. Dr. Myers has received numerous awards for his efforts in laboratory standardization and laboratory improvement. In 1991 Dr. Myers received the Public Health Service's Special Recognition Award for his leadership in improving the measurement of cholesterol in the nation's laboratories.

Breakout Session Five
Editor's Note: Prior to the Meeting Dr. Myers provided the following details
about his discussion group.
Title: Creating and sustaining reference method laboratory networks.
Objective: To develop criteria necessary for the implementation and
maintenance of reference laboratory networks
Discussion points:
- When is a network needed?
- How many labs does a network make?
- Resources needed to establish and maintain a network.
- How much does a network really cost to operate?
- Function of network in validating traceability.
- Administration of network: How will this be accomplished?
- How will manufacturers use the reference laboratory networks?

DR. MYERS: Group five was charged with discussing "creating and sustaining reference lab networks". We had a good cross section of representatives. We actually had representatives from some of the networks that are already currently in operation.

We also had representatives from manufacturers, and from NMIs (such as NIST and IRMM). We had some very good discussions on some key points concerning reference lab networks.

Funding was obviously a key component across all of the Breakout Sessions.

\begin{tabular}{|ll|}
\hline$\underline{\text { Participant }}$ & Affiliation \\
Ellyn Beary & NIST \\
Patrick Caines & Ortho-Clinical \\
Joan Cassedy & ACIL \\
Stanley Cernosek & Beckman Coulter \\
Basil Doumas & Med. College of Wisconsin \\
Mark Manak & BBI Biotech \\
Mary Kimberly & CDC \\
Masakazu Nakamura & Osaka Med. Center \\
Paula Radmacher & Univ. of Louisville \\
Curt Rohlfing & Univ. of Missouri \\
Heinz Schimmel & IRMM \\
David Secombe & Canadian External Q/A \\
Michael Welch & NIST \\
Ruth Wu-Wong & Abbott Labs \\
\hline
\end{tabular}

I think you will see that as I go through just a few of the recommendations that we have, there is some overlap with some of the other groups that you have already heard from, particularly in the area of funding. 
We recommend that a needs assessment be performed, and we recommended that this be done by AdvaMed. We think that this should be done immediately before any more resources are committed in any areas.
One thing that we discussed was that we needed to determine where reference lab networks are necessary. For this we need to have a needs assessment performed of manufacturers to find out what they think their needs are, so we can concentrate resources in those specific areas. We need to know immediately where those resources should be focused.
Another recommendation was that a network would require a minimum of three laboratories. One lab isn't sufficient. Two labs are close, but you need three labs to have that odd man out. If there are discrepancies, you can use the three labs to help validate which labs or which methods or which results are correct.

\section{Where does the network lab fit in the} traceability chain? Does it:

- Value assign manufacturer calibrators

- Provide reference values for PT providers

- Provide leadership in Reference Method improvement
Based on experience we felt that three labs per network would be a minimum requirement.
Then we discussed where the network labs actually fit in to the traceability chain. I think this is very important, because that is based on how the manufacturers and other clients might use these reference laboratories.

Obviously, one area is to value assign manufacturer's calibrators. That fits into the traceability chain. Also, another way to support the manufacturers is to provide reference values on PT samples for PT providers. Again, we are assuming that these are either commutable materials or fresh frozen patient samples. These services will help close the loop for manufacturers to determine whether their value assignment in the traceability chain is being transferred down to the routine laboratory. So, the reference network laboratories can provide input into the overall process by providing assistance to PT providers.

These reference laboratories should provide leadership to the community in reference method improvement. Some of the current reference methods have been in existence for around 15-20 years. Reference laboratories may be able to provide leadership in coming up with improvements in those reference methods.

Our group thought that it is very important to have an oversight body for operations of the network labs to help develop SOPs for the network. I think this fits with what you've heard from the first two working groups as far as having an oversight body for these 
reference systems. One example of this would be to design and develop the minimum requirements for a transfer protocol to help educate manufacturers. If the manufacturers

\section{An oversight body for operations of the network labs would develop SOPs for the network \\ for example: minimum requirements for a transfer protocol}

know the requirements when they go a reference network laboratory or a group of reference network laboratories, they may say, "I want my value assignment done on two days in duplicate."

When looking at the overall situation, a more appropriate protocol may be developed having input from statisticians and so on. So, this is a minimum developed protocol, and we feel there should be an oversight group that could help develop these type of SOPs which the network labs then would follow, and would also transfer that information to the manufacturers.

Finally, the one area that probably we spent as much time as any on, was the development of mechanisms of support for the network labs. Network labs, reference labs are expensive. Networks of reference labs are expensive to operate. No doubt about that. So, we have to have some ways to fund these laboratories. Creating network labs requires a certain amount of developing the infrastructure if it's not already there. So, one suggestion was to approach the Hughes Foundation for funding to create infrastructure.

Apparently, the Hughes Foundation has money that they invest in the medical and public health area. The representative from ACIL said that she would help facilitate this approach to looking at the Hughes Foundation to come up with possible funding to help

It is relatively easy to create and develop networks, the hard part is coming up with the funding to sustain those laboratories over one, five, ten years or more. build the infrastructure for developing reference network laboratories. However funding to get started is only one part of it, the second part is sustaining those laboratories. We thought we could establish an advisory group of the stakeholders for developing mechanisms of sustaining these networks. This might be tied into a workshop that IRMM is going to have in the spring of 2001.

The purpose of this meeting would be to develop business plans. We thought that it was important to develop a business plan, not only by the individual manufacturers, but by the industry in general. AdvaMed might be the group that would facilitate this.

The network labs could also develop a business plan. What does it cost to operate a network laboratory on a sustained basis? To come up with this plan, we felt it would be good to include representatives from: CDC and the NGSP who operate networks; and, NIST who develops reference and definitive methods. Professor Siekmann, would also provide valuable input to this group since he has some experience globally in operating a reference laboratory and reference networks in Europe. Stakeholders have to buy into 
these things. But I think that we have to have a very defined approach to going and getting this money and to deciding how it has to be distributed. These decisions must be based on a very sound business plan.
Again, the overriding area that we discussed is funding. You have to have funding to put these reference systems into place. You have to have funding to sustain these reference labs.

\section{These conclude the recommendations from Breakout Session Five.}

\section{DR. MAY: Clarifications?}

DR. KOCH: My question, Gary, had to do with the number of labs. I'm assuming that what you mean is you need to have three labs for any particular measurand, and those three labs could be totally different for a different measurand.

DR. MYERS: They could be. For each measurand there should be at a minimum of three network labs in a network. Obviously, each network labs could provide reference services for a variety of measurands. It would be most cost effective if one network provides services for many measurands. We should try not to create individual reference lab networks for one or maybe two measurands, but rather try to have as many as possible within that reference lab network.

DR. DOUMAS: Maybe it was one before that there was something where you described the three laboratories and what they were going to do.

DR. MYERS: Where they fit into the traceability chain?

DR. DOUMAS: No - I got the impression that you thought that these laboratories should somehow be accredited, right? Their capability should somehow be accredited, and it was not clear to me which process you were thinking about.

DR. MYERS: The oversight body?

DR. DOUMAS: No, that's another one. It was before that.

DR. MYERS: I think that may have been part of Bill's.

DR. DOUMAS: No, because I was at Bill's session.

(Laughter)

It was part of Bill's, but not in that sense. But anyway, so you didn't treat the mechanism part of it. The mechanism for say an accreditation or something like that.

DR. MYERS: No, we didn't actually talk about the oversight body as far as selecting and establishing network labs. That would be done by an oversight body based on the needs. 
Accreditation of the reference labs would fit in more to the discussion on the reference system that Bill's group had. We just recommended that there be an oversight body to help develop the SOPs under which those network labs would operate, and also the criteria for selecting those reference laboratories. That there needs to be a series of SOPs for operation and selection of those reference labs.

DR. DOUMAS: Do you really mean that that could be a mechanism for selecting the laboratories? It would not be open field, so to speak, where the laboratory could offer its services and ...

DR. MYERS: Oh, certainly. Certainly. I mean, we would not exclude that. But if there is a need and no one volunteers, there still has to be a mechanism possibly to go out and select laboratories to fill that need. It is the old idea of looking for champions when you talk about developing reference methods. Same thing -- looking for laboratories that want to serve as part of a reference lab network. That can go two ways. A lab can volunteer, but if there are no volunteers, then there must be a process of solicitation, trying to find labs that we feel like are adequate to provide the service. Recruitment.

DR. KESSNER: Art Kessner from Beckman Coulter.

I just wanted to understand your mentioning the IRMM meeting in the spring.

And where was that going to be? Are we talking about an American network with American manufacturers meeting overseas to talk about this or do we need to have a domestic site to talk about a network system?

DR. MYERS: No, the purpose there was to have this stakeholders group meet in conjunction with an IRMM workshop and I may have to refer to Dr. Schimmel to give more explanation on that. We discussed that stakeholders could meet at that time to look at a business plan on developing funding mechanisms to sustain the proposed network.

MR. KESSNER: I was just concerned about the logistics of shipping everybody from here over there to talk about it.

DR. MYERS: Well, it wouldn't be shipping everyone. Stakeholders could send a representative.

DR. MAY: It seems that that's an issue, and perhaps we can discuss that a bit later after we've heard all.

DR. LASKY: Fred Lasky, Ortho Clinical.

I had a couple of questions. One is just for clarification. You proposed a whole series of things that a reference laboratory might do, and I presume that is "wish to do", and that it would be up to the laboratory. One of the things that has come up in discussion, and I haven't heard any objections, was that manufacturers laboratories are able to join this network and get certified or accredited or whatever. My guess, based on our own experience, is that the manufacturer's laboratory would not be interested in picking up any additional services because it is working in a production mode. So, I just wanted to 
get clarification that your group agreed with that sort of approach, or if it was discussed at all.

DR. MYERS: Actually, we didn't discuss that particular aspect of it, but that is something that we certainly could consider in our further discussions.

DR. LASKY: And the second was you mentioned that it would take three labs for a viable network, and I do not disagree with your estimate. But I can also envision places where there might be a specialized analyte or an orphan analyte. This was discussed in the working group I was in. Did your group talk about alternatives if indeed there's only one laboratory, or if there are only two laboratories, and you don't hit the magic three? What then?

DR. MYERS: Well, that's where I think you would have to recruit additional laboratories to make a network. I mean, our feeling was one lab does not make a network.

DR. LASKY: Okay. Well, obviously I don't mean to put you on the spot, but I think that's one of the things that perhaps we should discuss afterwards.

DR. MYERS: Yes.

DR. LASKY: This is an important concern, because for instance, if a manufacturer comes up has an analyte that they think is going to change the course of health care in any particular area, they are the prime source for traceability of the highest order from that standpoint. So, we have to consider that also in our discussions.

DR. MAY: This isn't the time to do that, but I know there are differing opinions of what traceability means, and perhaps we can discuss that later when we have the entire group back. Because I think we have to come closer to a unified agreement of what we are talking about when we talk about traceability. 


\section{Breakout Session Two}

\section{Report from Breakout Session Reference System Infrastructure to Support Traceability Requirements, Donald M. Powers, convener.}

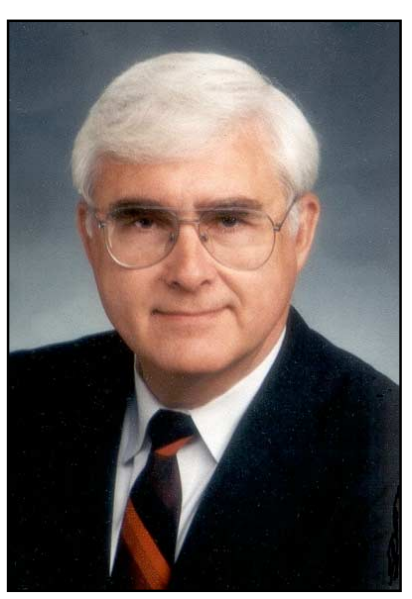

Donald M. Powers, $\mathrm{PhD}$ is President of Powers Consulting Services, providing advisory and training support to the global IVD industry in the areas of regulatory compliance, product licensing and quality management systems, and an affiliate of Quintiles Consulting. Dr Powers served 22 years in the IVD industry, most recently as Director of Regulatory Affairs for Ortho-Clinical Diagnostics, a Johnson and Johnson company, where he had responsibility for worldwide regulatory affairs for the Vitros chemistry and immunochemistry systems.

He previously held various scientific and management positions in Quality Assurance, Customer Technical Support, Clinical Evaluation and Research and Development at Eastman Kodak's Clinical Diagnostics Division. After earning his $\mathrm{PhD}$ in biochemistry from Cornell University, he was a research scientist at the National Institutes of Health in Bethesda, MD and a clinical chemist at Hahnemann University and Hospital in Philadelphia, PA.

Dr. Powers is a member of the US Delegation to ISO/TC212 and Convenor of the Working Group on IVD product standards. He recently completed two terms on the NCCLS Board of Directors and is past chairholder of the Area Committee on Evaluation Protocols. He is an active member of RAPS, ASQ and AACC.

\section{Breakout Session Two}

Editor's Note: Prior to the Meeting Dr. Powers provided the following details about his discussion group.

Title: Reference Materials and Reference Measurement Procedures to Support Traceability Requirements of the IVD Directive.

Objective: To identify practical ways to close important gaps in available reference measurement procedures and materials, and to explore ways to maximize cooperation among existing organizations developing and providing reference systems.

\section{Discussion Points:}

- Does industry need or want additional reference materials and measurement procedures?

-What elements of the network are in place today? Who are the players?

- How can they work together to provide the missing elements?

- When are they needed? When can they be in place? 


\begin{tabular}{|ll|}
\hline Participant & Affiliation \\
Nina Chace & FDA \\
Robert Chi & Taiwan Committee for \\
& Clin. Lab. Standards \\
William Childress & FDA \\
Sae Choo & First Medical \\
Lou Dunka & LifeScan \\
Ronald Elin & CAP \\
Patrick Ford & Ortho-Clinical \\
Theresa Gratiano & BBI Technologies \\
Chandra Jain & Beckman Coulter \\
Andy Jaunzemis & BD Biosciences \\
Carolyn Jones & AdvaMed \\
Patricia Klimley & Bio-Rad Labs \\
Manfred Kratzer & Roche Diagnostics GMBH \\
Fred Lasky & Ortho-Clinical \\
Nate Lawrence & BBI Technologies \\
Ed Levine & Diagnostic Products Corp. \\
Randie Little & U. of Missouri \\
Roy Marcus & Bayer Corp. \\
Laurence Potter & Dade Behring, Inc. \\
Terry Pry & Abbott Labs \\
R. Sridharan & Instrumentation Lab \\
Charles Tonkin & UL International, Ltd. \\
Steven Westwood & Australian Govt. Labs \\
Richard White & Dade Behring \\
Thomas Yager & Visible Genetics, Inc. \\
& \\
\hline
\end{tabular}

DR. POWERS: Our group looked at the reference system infrastructure to support traceability requirements, the reference measurement procedures and reference materials.

As others have mentioned, there is a lot of overlap with the other reports. I think it's all converging very nicely. We took a bit of time in the morning figuring out what the future states should look like. We did some brainstorming, and captured some visions or ideas of what should be elements of the future state.

We kept reminding ourselves that the goal of traceability is to end up clinically relevant reproducible results over time and space. And at times, we found ourselves focusing perhaps a bit too much on the analytical or the metrological requirements. And we kept bringing ourselves back to the ultimate goal.

We felt a global infrastructure needs to be in place yesterday. We would take it by 2003 , but everyone recognized that it's really needed now in order to allow manufacturers to meet the 2003 requirements of the EU Directive.

Global kept coming up everywhere. There is nobody thinking about establishing a parallel structure on this continent to what's already going on in Europe. It needs to be collaborative. A lot of effort needs to be put into making sure that the overlaps are minimized, and whatever we do needs to be cost effective in terms of process and the end results.

\section{Future State}

- Reproducible, clinically relevant results over time and space (goal of traceability)

- Global infrastructure in place by 2003 (but needed earlier)

- Collaborative, not overlapping

- Cost-effective process and results 
We need reference materials available for at least the medically important analytes. So, the prioritization is going to be extremely important. The process needs to be robust.

\section{Future State}

- Reference materials available for medically important analytes

- Robust process, easily integrated into industry development processes

- Reasonable transition to traceable values

- Accuracy-based proficiency testing
I'm talking now about

the overall global transferability process.

It needs to be easily integrated into what the industry does today. It could be extremely disruptive if manufacturers have to go back and redo processes for the literally dozens or hundreds of analytes. There needs to be a

reasonable transition into the world of traceable values. It's not going to be an easy thing to do for some manufacturers. Also the impact goes beyond manufacturers. It goes to the clinical lab and ultimately the physicians.

They're going to see changes. If the laboratories change, the physicians are going to see change. Those are not going to be welcome. Nobody we've heard has been talking about what kind of transition is allowed. Two thousand and three seems to be a drop-dead time that may not be reasonable.

In addition, we need accuracy-based proficiency testing as a way to verify that the traceability is working as implemented. Along with that goes things like commutable survey materials and so on. So, this is not going to be easy. But we feel it's something that's important.

We ended up with a number of recommendations, which were consolidated, like I think group one. We were favoring the international consortium that was discussed yesterday as the foundation for whatever is done. It said "form", but we really mean "expand" what's already in place. The industry is represented at least in Europe. The IFCC is representing the professions. A number of metrology institutes, including NIST, are represented. So, why not just build on what already exists?

Stakeholders: We felt like IFCC should be a player, but no one suggested that they would actually be the lead or coordinate the whole thing. We also felt medical professionals should be involved in this, because prioritization of the
Form/expand International

Consortium within 6 months to include all major stakeholders in the major geographic areas (eg, medical and laboratory professional organizations, industry trade associations, reimbursement agencies) 
analytes to be worked on is very important. Also reimbursement agencies since cost is going to be a huge factor here. We felt they should play a role. We weren't sure exactly what, but someone is going to pay for this. I don't think the list was long enough to fund everything that needs to be done.

We see this consortium being developed for the purpose of developing global consensus, and then for coordinating the activities. We lumped most of our recommendations into this.

\section{Summary of Recommendations}

This global consortium will be formed for the purpose of developing global consensus and coordinating the following:

- Prioritize analytes based on medical needs

- Coordinate development of reference materials/methods

- Develop interpretive guidelines for manufacturers, NMIs, clinical laboratories and medical practitioners to assist implementation of traceability requirements
They need to prioritize the analytes based on medical needs. We did not attempt to specify who would do this. So, I think it's understood that this would be IFCC and the medical professional groups working together.

We were reluctant to specify individual organizations here because we felt that was the purpose of the consortium.

The seeds of the consortium

are already there. Let's expand it, and let them decide among themselves who is in the best position to do these things.

Obviously the development of new reference materials and methods needs to be very well coordinated to avoid the redundancies. We didn't really dwell on that.

One thing that kept coming up is the need for interpretive guidelines. Manufacturers certainly need them. The traceability standards are not exactly something you can sit down with and figure out immediately what to do. Someone needs to provide guidelines, and we know that EDMA is producing a set of guidelines. It seemed obvious to us that the trade associations would probably get this assignment. But again, the consortium ought to decide who should do that.

For NMIs, there needs to be guidelines and procedures in place that they would follow. Perhaps they already exist. We didn't have that knowledge in the room. Also clinical laboratories and practitioners are going to need some guidelines on how to cope with the changes they're going to see.

We talked a bit about clinical traceability and where that fit in. We decided it was probably outside the scope. But I think it's important to recognize that there are analytes that have very well established values in the clinical community, and they are not going to want to change. 
There are clinical trials that have been done over many years, epidemiological studies and so on. So, the laboratories are going to figure out how to deal with this, because they will be obligated to conform to the analytical traceability requirements.

Summary of Recommendations, continued

- Establish and maintain an information network to inform users of available reference materials, methods and laboratories.

- Develop and oversee processes for credentialing reference materials/methods and accrediting reference laboratories.

- Establish and maintain an information network to inform users of available reference materials, methods and laboratories.
Again, we wanted to make sure that as we go through all of this, the clinical and medical communities are very well represented, because they are going to bear the brunt of a lot of these changes.

This consortium also would be charged with establishing and maintaining an information network. This was a pretty important element that kept coming up again and again.

We need a data bank of what materials, methods, reference laboratories are available.

We would see the consortium establishing its own web site. They can identify somebody who can actually run it for them. There are a lot of possibilities there. And then all the stakeholders would establish links to this web site.
... we could use a web site as a way to capture what is already available and what needs to be done.

Developing and overseeing processes for approving reference materials and methods, and accrediting reference laboratories need to be established. We knew those were being covered by other groups, and we didn't dwell on them.

We need to make sure there is a global distribution process in place so that reference materials are available everywhere in the world to whoever needs them. There are

Summary of Recommendations, continued

- Oversee a global distribution process for reference materials.

- Verify effectiveness of processes and impact on clinical outcomes.

- Establish procedures for arbitration of differences.

- Follow up with additional conferences to monitor progress and educate stakeholders in 6-month timeframe. systems already in place that could be used, and there are probably new ones that need to be invented. In the end, somebody needs to verify the effectiveness of what we are doing, the processes. Also, the impact. Did all this traceability activity make an impact on the clinical use of these tests?

It is going to cost a lot of money, and it needs to be 
justified in terms of the impact it has on medicine. We also brought up the possibility of differences. How would they be arbitrated if there were disagreements between a manufacturer and another party.

There ought to be some way that these type of differences can be acknowledged and arbitrated. We think the consortium ought to be able to deal with these things if it's made up of the right organizations.

We also felt that a follow up conference to this conference is essential to monitor progress. There should be a series, but we were looking more toward the six-month time frame, and having at least some people that are here meeting again to see how things are going.

\section{This concludes the report from Breakout Session Two.}

DR. MAY: Questions? Clarifications?

In reference to your second from the last slide: I remember that it said that the consortia should oversee the distribution of reference materials. Can you explain what that means?

DR. POWERS: Yes. Again, we don't see this consortium actually doing things, but making sure they have the right organizations involved so that everything gets done. We would see them overseeing the distribution processes in place today, making sure they're effective, and recognizing gaps and creating new ones.

DR. WEINMEYER: Hsiao-Mei Weinmeyer, from the University of Missouri, Columbia. I think that your working group came out very clearly that the involvement of the medical profession in decision making is a very, very important step in this whole process of establishing of traceability, and that the clinical outcome is also a very important.

DR. POWERS: Exactly right. That is the way we felt. 


\section{Breakout Session Three}

\section{Report from Breakout Session on the Impact of Method-Material Matrix Interactions on Calibration Traceability Protocols for Successful Harmonization of Patient Results, Greg Miller, convener.}

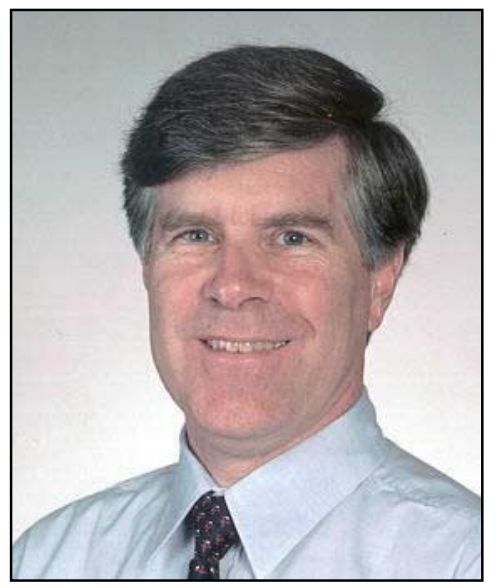

Greg Miller, Ph.D. is a Professor in the Pathology Department at Virginia Commonwealth University where he has been on the faculty since 1977. He serves as Director of Pathology Information Systems, Co-Director of Clinical Chemistry and Director of Pathology Quality Management for the Medical College of Virginia Hospitals of the Virginia Commonwealth University Health Systems Authority. He received a Ph.D. degree in Biochemistry from the University of Arizona in 1973; did post-doctoral training in Clinical Chemistry at the Ohio State University; and became a Diplomat of the American Board of Clinical Chemistry in 1976. Current professional activities include Chair of the NCCLS Area Committee on Clinical Chemistry and Toxicology and Consultant to the College of American Pathologists Chemistry Resource Committee. Past positions include Chair of the AACC Standards Committee and Chair of the AACC Lipids and Lipoproteins Division. Research and publications have been in the areas of instrument and method performance, quality control, proficiency testing, laboratory standardization, and fiber optic immunosensors.

\section{Breakout Session Three}

Editor's Note: Prior to the Meeting Dr. Miller provided the following details about his discussion group.

Title: Impact of method-material matrix interactions on calibration traceability protocols for successful harmonization of patient results.

Objective: To develop a statement of limitations in the commutability of reference materials and the appropriate use of such reference materials for traceability and harmonization of results.

\section{Discussion Points:}

- Reference material matrix alterations, non-native analyte components and commutability with patient specimens

- Role of authentic patient specimens in traceability validation

- Commutable reference materials: how to validate performance; role in traceability documentation

- Non-commutable materials: role in method calibration; how to value assign

- Requirements for commutability between reference materials and patient specimens, and between method calibrators and patient specimens

- Impact of matrix modified reference materials on traceability for new technology applications

- Impact of method non-specificity on traceability validation 


\begin{tabular}{|ll|}
\hline Participant & Affiliation \\
Caryl Antalis & Roche \\
Sabine Arends & Roche, GmbH \\
Edward Ashwood & CAP \\
Steven Binder & Bio-Rad \\
D. Bozimowski & Abbott Labs \\
Joe Bugler & Abbott Labs \\
F. Ceeriotti & H.S. Raffaele \\
Cynthia Coady & Diagnostic Chemicals, Ltd. \\
Sherb Edmondson & Abbott Labs \\
F-Javier Gella & Biosystems \\
Monica Giguere & BD Biosciences \\
Isabelle Giuliani & Bio-Rad \\
Omer Guzel & Biruni Laboratories \\
Tuija Halonen & PE Life Sciences \\
Art Kessner & Beckman Coulter \\
George Klee & Mayo Clinic \\
Peter Maxim & FDA \\
Joseph Murnane & UL, Inc. \\
Chad Nelson & NIST \\
Donald Parker & Bayer Corp. \\
Barbara Pizza & Genzyme Corp. \\
Nancy Ring & Diametrics Medical, Inc. \\
R. San George & Inverness Med.Tech. \\
Nick Smit & i-STAT Canada \\
H-M Wiedmeyer & U. of Missouri-Columbia \\
& \\
\hline
\end{tabular}

DR. MILLER: Session three took on the topic of "method, material and matrix interactions for noncommutability, and its effect on traceability protocols".

\section{We really focused on the reference materials themselves, some of their limitations, and had some specific recommendations to how to deal with these problems.}

The entire focus of the group was on reference materials that have assigned values. We didn't consider other situations. One key recommendation was that it is important to validate the commutability of the material for all the methods for which it is intended or designed to be used.

There is a document from NCCLS EP14, is a matrix evaluation protocol that gives some statistical tools, selection of patients, and so on, which can be used to accomplish this.

We spent most of our time dealing with what happens if the material is found not to be commutable. There are a number of specific recommendations around this area. First of all, that a patient specimen panel can be used to transfer accuracy to working calibrators.

There are a couple of If a material is not commutable patient specimen panel can be used to transfer accuracy to working calibrators

- Method specific target values can be assigned to a reference material using an algorithm that achieves commutability for a patient panel

- Method specific target values can be assigned to a transfer material

- Method specific target values may be reagent lot specific

aspects of using a patient specimen panel. The first is that method specific target values can be assigned to reference materials using an appropriate algorithm that allows that material to become commutable for a panel of patient specimens. 
In addition, method specific target values can be assigned to a transfer material, which may be some type of intermediate calibration product or other reference material used typically by a manufacturer for an internal process to maintain the transferability process.

It is important to recognize that when method specific target values are assigned to a reference material, they may be specific to a particular reagent lot. Additional work needs to be done to demonstrate that the correction factors or the target values can be used on subsequent lots in the manufacturing process.

\section{Reference material may be incompatible with} the method used in a clinical setting

- This condition can look like noncommutability

$>$ For example: epitopes in immunoassay; lead isotopes by mass spectrometry

- In this case, that reference material should not be used
Second point is that a reference material may be incompatible with the method. By incompatible, we mean that it may not actually measure the analyte that we think it's measuring. A typical example here would be the different epitopes in immunoassay assay.

We also discussed an example of different lead isotopes ratios and their geographic distribution. It may be a similar situation in a non-immunoassay example. This can look like a noncommutability condition when you do a typical commutability validation where you compare the reference material against a panel of patient specimens.

\section{... it is important to investigate why a non-commutability issue exists before jumping to the conclusion that a method specific target value will in fact deal with the situation appropriately.}

\section{Guidelines should be developed for assigning method-specific target values to reference materials}

A group such as the NCCLS might be an appropriate place to undertake the important task of developing guidelines for assigning method specific target values to reference materials. In addition to specific guideline development, such a project might include tools, statistical procedures, etc. to do these processes.

Patient panels then take on a very important role in the transferability process. We also felt it would be important that guidelines be developed on how you select patient panels for a variety of purposes.

Some of the key attributes that we felt should be included in this approach would be that the patient panel represent typical measurand conditions and not have unusual molecular 
forms. So, for purposes of traceability and calibration, the patient panel needs to represent the type of analytes that are typically encountered in an average mix of patients.

The problem of a particular method not reacting correctly with epitopes, is considered a method problem. Or if in some type of pathology, a very unusual variant of a molecule might be present, that's a

Patient Panels; guidelines should be developed

- Represent typical measurand conditions

- Not have unusual molecular forms for traceability use measurand content

- For commutability: near values of the reference material

- For transferability: cover measurement range

- Collection and storage issues

- Availability

- Demographics

method specificity issue that doesn't need to enter into the situation in which you are trying to establish accuracy or calibration traceability.

... patient panels can be used in different ways, and it's important that, as this guideline is developed and clarified, these situations are distinguished from each other to avoid confusion.
For commutability evaluation, the patient panel needs to have values that are near the values of the reference material. But for a transferability purpose, the patient panel needs to cover the measurement range.

Other points that must be addressed are: pre-analytical collection storage issues; the availability of the panels to people who need them; and, demographics. Different patient demographics and ethnic backgrounds can, in fact, influence analyte content and method specificity. These are just some of the considerations that we think are important to consider before moving forward.

In addition, patient pools can be used to simulate individual patient specimens if they have the appropriate attributes. This obviously represents a material, then becomes a reference material of sorts. They can, in fact, substitute for patient panels under the right circumstances.

\section{Patient Pools}

- Intended to simulate individual patient specimens

- NCCLS C37 document provides a procedure for preparing and validating pool commutability

- Stability - reevaluate on a regular basis
The NCCLS C37

document, which addresses the development of patient pools for cholesterol and lipid measurements, does have protocols on how to develop a fresh frozen material, and particularly

contains a protocol how to validate that that pooled material is in fact commutable among all the methods for its intended use. We recommend that this be a starting place for the 
validation process. Again, stability of patient pools, just as stability of any other type of reference material, is quite important and needs to be reevaluated regularly.

\section{Harmonization is important clinically in absence of reference method}

- Include reference materials in harmonization process

- Validate reference material commutability in process

- Consensus value can be assigned without a reference method
We felt that harmonization was another very important goal, independent of reference methods development or traceability issues. However, we felt that it was important when harmonization exercises are performed, that reference materials be included. Even if they are only candidate reference materials, and harmonization process is based on patient specimens for any given analyte or measurand, it would be useful to make sure reference materials are included in the process. In that case, when it's all over, we may have a validated reference material that can then be value assigned by an appropriate mechanism, either by a reference method or a type of consensus process of available methods. This would utilize resources

It is also important to harmonize the measurement units in the process of doing harmonization exercises. in the most efficient manner.

\section{Reference Material versus Proficiency Testing (PT; EQAS) material}

- Commutability for PT is desirable but difficult and not mandatory

- Do not use PT as a reference material

- Do not harmonize to PT results

- Education is needed
Another very important point we discussed was that reference materials and proficiency testing materials are not the same thing. This is an important distinction. It is desirable for PT materials to be commutable. In a perfect world, they would be commutable, and they could in

fact play a key role in evaluating the success of accuracy transfer right into the field methods. In practice, this is difficult to achieve and is not in fact mandatory for a successful proficiency testing program. However, the critical recommendation is that PT materials cannot be used as reference materials, and we must not harmonize methods to PT results. Due to the frequency of non-commutability problems with these materials, you can easily make a mistake in using them. We felt that it was important for education on this point to be made available through whatever appropriate mechanisms are out there, because people's experience was that there are laboratories that rely on PT results as a way to adjust calibration or make changes, and that would be inappropriate. 


\section{Patient Population}

- Stable patient population can provide traceable calibration for some analytes (e.g. RBC mean cell volume)

- Based on clinical validation exercise
There are situations where a stable patient population can in fact provide traceable calibration for very select analytes. One example is for red blood cells the mean cell volume of a large population is very stable and can in fact be used as a calibration point. However, this approach must be based on clinical validation exercises.

Also, the role of reference materials in new technology development is important. As new technology to measure analytes or measurands is developed, it's important to validate the commutability of reference materials for that particular

New technology development

- Reference Material - validate commutability

- Encourage use of multi-analyte material

- For new analyte consider an existing material

- Must requalify for new analyte methodology.

We also suggest that wherever possible, multi-analyte materials be used because it is quite expensive to have a different reference material for each measurand in our laboratories.

...when new analytes are developed, and the technology to measure new analytes is developed, it is important to consider using an existing material if possible.
There are probably many examples where existing materials contain the analyte for which we're measuring a new test. Somebody just needs to go look for it and see if in fact that material can be used rather than developing an entirely new one.

Finally, in the process of reference method development, we recommend that in all cases, reference materials be developed as a parallel process with the reference method so that we don't get into a situation where we have a method, but suddenly no reference material that can be used to ... reference materials should be developed as a parallel process with the reference method ... transfer that to other methods.

This concludes the reports from the five Breakout Session Conveners. 
DR. MAY: We will have a coffee break in a few minutes, but I wanted you to know that we will re-convene afterwards to discuss the proposals and recommendations. We need that input from you because, this evening, a group of us will be sitting down together trying to synthesize these recommendations and make them more coherent. I'm sure there are a number of issues that need discussion further, and get even further clarification.

I would like to share with you a few things that I jotted down. I'll give you something to talk about when you go out for the coffee break.

In listening to this discussion, I thought it was important to differentiate between a certified reference material - that is, one that is going to be produced in very large batches for a distribution world wide, and a manufacturer calibrator that will be developed primarily to support one type of instrumentation.

I see some heads shaking. Well, Some Points to Consider

- Difference between CRMs and manufacturer calibrators

- Role of NMIs and role of Reference Labs

- Who should provide CRMs?

- What do we mean by:

$>$ Traceability

$>$ Linkages to stated reference

- Identify lead players for action items that's my perspective.

It wasn't clear to me in the discussions that there was a clear distinction between the role of national metrology institutes and the role of reference laboratories. Or can they be interchangeable? Perhaps they can be. But I think we need to discuss that.

Who provides CRMs? Are we talking about providing CRMs through the reference laboratories? Providing them through NMIs? Providing them through whomever wants to provide them? I think that's important. We have a lot of confusion out there as to what a certified reference material means. This is based on what I was getting from the discussion, I think we can have further clarification.

What do we mean by traceability? In my view, certainly this is a complicated area, we're talking about linkages to some stated reference. I'm not going to sit in an ivory tower and say it has to be the SI, but I think it has to be an internationally accepted stated reference. Then there has to be some agreement on what we mean by linkages to that stated reference. When we say an unbroken chain of intercomparisons, what are we talking about? There needs to be some consensus on that.

In much of the discussions, the appropriate lead organization was not indicated. Certainly we can't identify all of the lead players because not everyone in the universe is here. But I think we need to be careful to talk about the types of organizations, if not specifically who would be appropriate to take the lead in some of these areas. 
Bill (Koch), any housekeeping? I think the coffee is ready and it is now slightly past 2:30. We should come back at 3:00 for discussion and wrap-up.
(Whereupon, the foregoing matter went off the record at 2:33 p.m. and went back on the record at 3:05 p.m.)

DR. MAY: The next order of business, from my perspective anyway, is to have some discussion on where do we go from here in terms of getting together again. There have been some recommendations that we get together in roughly six months. There have

\section{When should we get together again?} been others that say a year. I heard some people say two years. I think that's probably too far out. So what's your pleasure? Should we look to try to get together someplace say six to nine months from now, or should we wait and think about this -- say the fall of 2001? What's appropriate? I really think we need to hear from the manufacturers here.

\section{PARTICIPANT: Six months.}

DR. MAY: I think the manufacturers see that 2003 is coming in a hurry. (Laughter)

PARTICIPANT: I actually think there is an important point to consider. If you want to get funding, you need to get into manufacturer's budget cycles, and you want to make sure you do that as early as possible so six months would be advisable.

DR. MAY: Let me clarify one thing. Certainly from the NIST perspective, we don't expect to have our activities funded based on manufacturer contribution, because realistically, they will not be able to fund all the things that we need to do. Certainly having some funds from a consortium of the manufacturers certainly shows good faith, and that you believe that this is an important issue.

Six months is sort of on the table.

Where should the meeting be? There are some people who feel that perhaps looking at the needs of the U.S. based manufacturers, we need to have a meeting here. There have been other recommendations that perhaps we should alternate this between the U.S. and Europe with the thought that if this series continues, maybe it should move around the world.

What is our pleasure?

PARTICIPANT: Gaithersburg is centrally located. It is not too expensive for Europeans, or people from the West Coast. 
DR. MAY: That is the first time I've ever heard that Gaithersburg was the center of the world.

(Laughter)

When I came here about 30 years ago, I had this T-shirt, "Where in the heck is Gaithersburg, Maryland?"

(Laughter)

MR. LEQUIN: I think we should alternate between U.S. and Europe.

DR. MAY: He's giving me instructions. It's always you, Rudy.

(Laughter)

Rudy knows I like him. That's why I can pick on him.

DR. DOUMAS: May I suggest that we have an alternating schedule. I think what we have done yesterday and today was a marvelous cooperation between people in the United States and Europe. I suggest strongly that we would have the next session in Europe so we can receive and we can welcome our American friends there. The next meeting, as you say, might be even at another place in the world. Just to let everybody have the feeling that we work indeed globally. Thank you.

DR. GREENBERG: Yes, I just heard a suggestion that we have the next ISO TC212 meeting I believe it's in Ireland in June. So, that conceivably is a venue that might be appropriate. We could have it a day or two before or a day or two after. So, let's think about that.

DR. MAY: Yes, I guess there are really two competing forces here. One is the realization that this truly is a global issue. This is not a U.S. issue alone, so it makes sense to think about moving the meeting around. However, the other consideration is purely financial. I think in order to get the right people to attend, the costs have to be reasonable. Everybody has lots of meetings to go to, and we want them to choose the next meeting or the next gathering of

There are two competing forces for deciding a meeting place:

- Global nature of the issue on the one hand, and

- Finances on the other this group as one of the meetings they choose to attend.

Having it in conjunction with the meeting that Neil mentioned, does that meet the need? Is that a meeting that most of you are going to attend anyway?

PARTICIPANT: I think I want to second that because IFCC meeting follows the TC212 meeting. So, if you want to get IFCC involvement, have it in between. If people want to go to the IFCC meeting, that might work. 
DR. MAY: Now, who would be willing to organize it? Any volunteers?

The dates of the meetings are the 6th through the 8th of June. We are talking about Dublin as the location. I guess once we get together, we can talk about whether or not we need to expand the Steering Committee to accommodate this. So we have decided that the follow on meeting will be about seven months from now, and it will be in Dublin. I guess that takes care of that issue.

Just one other general comment in listening to some of the discussions during the coffee break, I think there are a number of people who see producing these reference materials (because the need is great) as a viable business enterprise. I'll be quite frank with you.

We've been doing this for a long time, and you do not make a profit at it. To do it correctly, it's going to cost you a lot more than you're going to be able to charge customers. Now, you don't have to take my word for it ... Making reference materials in a metrologically sound way costs a lot of money, and you need to be subsidized by someone.

DR. DOUMAS: I just would like to confirm your statement as the second largest reference material producer. You will never earn the money you are investing in making reference materials if you do it in the proper way.

DR. MAY: Now we would like to have some further discussions on the reports from the working group chairs. Are there any burning questions that you have of any of the presenters, and that will sort of trigger the order of the discussion. Or, I can begin drafting people.

DR. SEMERJIAN: I don't remember now which group it was, but there was a recommendation for a needs assessment. I think that this is very important, especially since funding must be generated. Also if various companies need to be convinced

... there was a recommendation for "needs assessment" ... I think that this is a very important activity. that this is going to work, some attempt must to be made to quantify what the need is, what the impact will be. I think that's a very important activity. I'm not quite sure who would do it or how it would be done. It's not an easy task since it's such a diffuse activity, very broad based. I think that this is something that we should pay special attention to make sure that it gets done early enough to have an impact.

DR. MAY: I'll just second what Dr. Semerjian just said, and I want to mention something about quantifying the impact of the work we do. I think we shared with some of you that we have recently done some impact studies (one was for the cholesterol standards program), and we found also that it is probably best to think about commercial impact in two parts, if you will. That is: 1 . What is the impact on commerce of not doing certain things; and 2. What would be the benefit of doing certain things on health care decision-making based on clinical results. 
DR. MYERS: I just have three clarifications that came up during the discussion at coffee break. One was before -

DR. MAY: For the recorder, that's Dr. Gary Myers from CDC.

DR. MYERS: I'm sorry. You don't recognize me?

You made a comment before we broke earlier about the difference between the NMIs and the reference labs. One thing we discussed in our breakout session and that is the national metrology institutes are not set up on a cycle to provide real time reference services. They are to anchor these reference systems. The reference methods that we're talking about and the reference lab networks are the ones that can interact with the manufacturers to provide real time reference services as needed when they're needed. We don't see the organizations like NIST and IRMM and so on as being able to provide those types of reference services. That's what

One difference could be that NMIs anchor reference systems while

Reference Labs provide real time reference services our group defined as one of the differences.

To clarify a comment from Dr. Myer's report.

A question was raised during the break concerning the comment I made that one area in which the reference network labs could interact in the traceability cycle was to provide value assignment to manufacturer's calibrators. That is not something that the reference labs are going to go out and seek. They are providing those services to the manufacturers as the manufacturers need those services. Rudy (Lequin) asked during the break if there will be quality assurance within the reference lab networks to make sure they're providing the highest quality possible. Yes, we talked about that in great detail. We saw that as being one of the operations of the oversight body that develops the SOPs for the

... laboratory networks would have QA procedures in place... reference lab networks. One of their functions would be to provide QC or PT for the network labs themselves, which is in addition to what they would normally provide as reference services for the manufacturers. This is to make sure internally the reference lab networks are providing the best analytical services possible.

DR MAY: I'd like to ask one question, though. I think we need to be careful and not be too prescriptive, and not say certainly there are two different roles that an NMI has and that a reference laboratory has. I don't think you mean to say that an NMI might not choose to wear both hats. For example, I think PTB is wearing both hats in Germany, and they are actually

NMIs "Wearing Two Hats" ... until these other networks get formed, it might be most feasible in the short term to have some NMIs fulfill the role of "reference lab" if they choose to do so. 
providing some credentialing (I'll use that word we shouldn't use) for some of the calibrators ...

DR. MYERS: No, my earlier comment was not an exclusion statement. During our discussion of reference labs and reference lab networks, that was just one of the ways we differentiated between NMIs and the secondary reference labs.

DR. MAY: Sure.

DR. MYERS: Looking at more of the real time type services that are needed.

DR. MAY: I think I understood that, but I was just trying to clarify it so that everyone understood what we were talking about.

MR. LASKY: Fred Lasky, Ortho Clinical Diagnostics.

There are a couple of points that I discussed with Don Powers during the break that came up during our breakout session that I just want to emphasize and be sure it gets on the record.

One was the issue about guidelines and guidances. And I think that is going to be a key function to give an example, to illustrate why it's so important. I think this standard on traceability is extremely detailed and frankly, very difficult standard to understand. It is not for the casual reader by any stretch of the imagination. In our experience, we passed this draft document over to our biochemists in development and they came back and said, "Well, it's nice of you to pass it on, but I didn't understand how we're going to deal with these things like uncertainty." And it's not a trivial exercise. So, that was just as an example to emphasize why we feel that guidelines are so important. Particularly for new terms and words for us to deal with as we're rubbing elbows with our friends, the metrologists.

DR. MAY: Let's provide a response to that. Help, Bernard. I think you are planning to do that as a part of the consortium that we're putting together, that actually will provide some explanatory notes, if you will, regarding traceability in clinical measurements.

\section{DR KING:}

Bernard King, LGC, UK.

I'm happy to respond to that. I think there are some developments and some new terminologies such as how do you actually achieve traceability, what is this thing called measurement uncertainty, which is relatively new to some people. There is a conference going on in another part of Washington at the moment - it is the Laboratory of Accreditation people who are meeting. One of the two big topics on their agenda is this issue of 
traceability and measurement uncertainty. They have just published a new Laboratory of Accreditation Standards 17025, which requires the use of traceability and measurement uncertainty in a fairly firm way for the first time. There have been many discussions on this topic, for five years it's been in development at least, and you can see its origins further back than that. There seems to be a recognition that perhaps this is not such a fearful and enormous task as we first thought if we go about it in a pragmatic and sensible fit for purpose

In the last year, I've seen quite a substantial move from the sort of denial and fear - "we can't cope with these things" - to recognizing that perhaps it does make sense. way. I'm not downhearted about these things, and I suspect in a year or two, we will not see them as being such big hurdles and big obstacles as perhaps some see them now.

... people all around the world in all sectors are asking themselves this question: How do we establish traceability, how do we determine measurement uncertainty, and how do we do it in a cost effective, affordable, manner to fit the purpose without going way overboard on it.
To help that along, and to respond to the question you asked, is that in the European project that we hope will be funded, we see one component of that as being able to produce some user friendly guides on how to achieve these things, and how to understand and interpret them for both industry, the people who go around doing accreditation, and the people in the laboratories who have to deal with them on a routine basis. I think this task is doable.
DR. MAY: Sure. I think that there are other documents that might be useful as well. I think it is helpful to realize that traceability is not an end unto itself. But it's actually a pathway or tool to be used in improving the comparability of measurements. That's really what we're after. To make measurements more comparable, and certainly by using this concept of traceability, we believe that that will provide that end.

DR. LASKY: Thanks. I'm feeling better already. (Laughter)

DR. MAY: Do you promise?

DR. LASKY: I work in regulatory affairs. I know that what we need to do is already specified; now I'm really concerned about how we do it. I'm encouraged, but I really want to emphasize that a year is probably the maximum that we could probably tolerate 
in order to meet the 2003 deadline. However, that's beside the point. That's just an editorial. I know people understand the urgency.

The other comment that I wanted to make (this also came up in our session) was this idea of reference laboratories. Just to bring to light an idea that had surfaced was something similar to what NCCLS used to have with their reference system for clinical laboratories. Essentially, it was a proposal to reintroduce that concept as an international reference system for the clinical laboratories. From my own perspective, I think that that was a good start. It dealt mostly with papers and methods that are certainly invaluable. I don't want to diminish that.

I don't think we should lose sight of all the good work that has gone on before. But we also need real laboratories to take those proposed reference methods and actually work through them and demonstrate that in fact they do come up to the standard -- the standard for being a reference method.

DR. MAY: Are you as a manufacturer willing to provide resources to compensate laboratories to do that? I think there are a lot of people that would be more than willing to do that work.

PARTICIPANT: We discussed that extensively today. I want to answer a question that you raised before first. Yes, industry addressed their desire to have reference laboratories. However, the question is how those reference laboratories will be financed, and how they will survive. I don't think they can depend on grants, and I don't think they can depend on some kind of retainer. They need volume of work to survive. My question is: "Is industry willing to support it?"

In terms of starting up new reference lab, we should think very, very carefully about finances. It's the volume of work that keeps reference labs in business. We must keep the people busy and guarantee employment.

There must be a long-term commitment to the laboratory, because a laboratory cannot start and invest a lot of money and train people, and then after six months, "Sorry, we cannot send you anything anymore."

DR. MAY: Other concerns?

DR. SEMERJIAN: I think this meeting in nine months in Dublin is fine, but I also sense another urgency: The 2003 deadline will be here before we know it. As a result, I think we need to do some parallel processing. Just because the meeting is in nine months does not mean that nothing can be done in between.

I think the prioritization process has to happen. It may not be the final priorities or it may not be

A prioritization process must happen now ... I think there is an urgency here, and we cannot wait for each little step to fall in place. 
the final word, but I think we must attempt to make some decisions, develop some consensus on what are the most important analytes. We cannot afford to wait for the completion of one step to do the next.

Even if there is some overlap or maybe some redundancy, I think we need to get going on the needs assessment and prioritization, et cetera, even though it may be more efficient in the long run to wait for the other step. I don't think we can afford it time-wise. I would like to know if I'm wrong about that.

DR. MAY: I think what Dr. Semerjian is saying, and this is from the U.S. perspective, that maybe we need to think about having a meeting to get our house in order prior to the next meeting of this group. And I think there has been some discussion bubbling up in that regard.

DR. POWERS: Don Powers.

I think you're right. I think we need to move forward with a lot of urgency. But it just occurred to me that we ought to keep in mind that the directive, which is imposing the 2003 deadline, only requires that traceability be made to available reference materials. Now may not be the right time to go map out all the other analytes that need reference materials, but those that exist, we ought to be using those to figure out how to put a process in place that can accommodate the manufacturers, including the reference laboratories. I would defer spending time trying to identify all the other analytes that don't yet have reference materials.

DR. MAY: I would like to bring up an issue. There are no reference materials right now, as I understand it, for troponin, and glycated hemoglobin, however, those are tests are already being used by physicians. We know that there are measurement problems. If we just stop our efforts on that, all of a sudden, come 2003, 2005, or whenever, we've got this big gap. So, again, I think you almost have to do some parallel processing.

For the purpose of the directive, manufacturers need to identify those materials that are available today that they can show traceability to and focus on those.
I think we need to think about how we can use the existing tools to put together reference systems, but I think part of our activities have to be looking forward, also developing those next set of tools to be used to put together reference systems for analytes that are becoming more and more clinically relevant. 
can show traceability to and focus on those. Those driven by medical needs ought to continue anyway, but on a different timeline.

DR. MAY: I really think that exactly what you said probably should be the focus of a meeting a few months from now with the manufacturers that are present here. That should probably be one of the points of emphasis for that meeting.

DR. COLLINS: Sure.

DR. BINDER: Steven Binder from Bio-Rad.

I would like to agree with the previous speaker that when we talk about prioritization, we are trying to meet several different needs here: regulatory and medical. The medical need really has two parts. One is the need to produce better numbers, which are useful to the patients. I agree with you completely, that is something we should all focus on. But another medical necessity, is this idea of eliminating repeat testing by making methods that have the appearance of being more reliable to physicians, and that's a very, very large goal. I feel that if we focus on that goal of trying to give physicians a higher level of confidence, we may well end up with a two-tiered system, where we have a very small number of analytes that have this glow of certification around them and a large other number of analytes that do not have that glow of certification around them. I think that this will create even more confusion for the physician. I think we really need to prioritize regulatory needs first, urgent medical decisions like cholesterol levels second, and improving the physician view of our industry as more of a moderate, or long-term, and much more challenging goal.

DR. MAY: Should something like that be the focus of a meeting in the very near future prior to the meeting in Dublin? I am hearing a few people say yes, but I don't know that this is a majority.

DR. MILLER: Greg Miller from Virginia Commonwealth University.

It seems to me that the parallel processing is the way to succeed here. What we need to do is drive this process by clinical requirements, not necessarily what we know how to measure. It seems to me that somebody has got to take responsibility to get it done. So, some organization needs to be assigned a task. It seems to me if a formal request is made of the College of American Pathologists, who basically has lab directors that run the pathology labs in this country, they could be asked for a priority list of analytes that are causing problems from a medical interpretation, or a medical use point of view. We could get that list together in a relatively short order. I think the College would be happy to oblige, and they seem a logical group to ask. But someone has to do that. Someone has to be assigned
We have to find a path forward with specific assignments for specific people or organizations to do. 
the job of doing that. If we just talk about how nice it would be to do it, we'll be talking about it again in June, and it won't have happened yet. A second point that I would like to make is, during the last two days, I have not heard anybody ask for somebody to specify what is the highest metrological level that exists for a list of analytes. It seems to me, if we had a list of what is considered the highest metrological order, whether it be a material or a method, then manufacturers would know exactly what they have to trace to, and they could just get on with doing it. I don't know how to achieve that. I don't know what that is, but I think if that could be done, it would be quite helpful.

DR. MILLER: Rick Miller from Dade Behring.

Just to key on Greg's statements and a couple of other things I have heard now, and something that Art Kessner touched on earlier. Should we be, in the short-term, developing a resource web site or resource list to help us understand where we are with some of these things, and help us determine

Should we develop a website and perhaps start putting together what resources are already available? what milestones we have to make, and even perhaps start putting together some of the already existing standards in such a way that they are more identifiable for each of us?

DR. MAY: Okay. I'm writing these things down.

DR. KESSNER: Art Kessner from Beckman Coulter.

... in the reality of today, we should ask:

1. what do we have?

2. what do we need?, and

3. how do we make the information available?

It sounds like we're developing an incredibly complex list of questions and a very short list of answers. I think there is a need not only for manufacturers to understand the process but we need to have a process in order to understand it. Right now I think we have a lot of pieces. So, I would think that the next meeting in the short-term that you just mentioned should really focus on, like you say, putting your house in order and understanding or sketching out what is the process and what are the pieces that need to be fulfilled now, not in some prioritized future. We need an action plan so by the time we get to next June we at least understand what it is we are trying to accomplish in some areas, and which areas are more important than others. It is incredibly complex, and there are a lot of priorities to be set, but right now the priority seems to be to know what to do tomorrow when I go to work. I'd like to see a very focused type of a meeting to understand what the American situation is relative to the global situation.

DR GUZEL: Omer Guzel, World Trade, from Istanbul.

I think most of us were thinking before we attended this meeting that these items were all discussed, and most of the problems might be solved. Suddenly we realize that at the end of these two days meetings there is a lot to be done. I second Dr. Semerjian's proposal that we should not lose time. There must be an official governing body to keep things 
moving. Perhaps the organizing committee of this meeting can continue until the next meeting and it can be delivered later to some other institutions or some other people willing to do it. It should be done in organized way. At least to coordinate the information gathered from here .... Thank you.

DR. KING: I would just like to make a suggestion. I was sitting there trying to think of who has the information that the manufacturers need about which reference materials are available, that is, reference materials of a higher order. It strikes me that NIST probably has that information. You certainly know which ones that you offer. Through the MRAs you should

Would it be reasonable for perhaps NIST to establish a page on its web site that would list these reference materials. know what others are available in Europe and so on.

DR. MAY: We had actually put down to develop a web site. I had asked Dr. Koch here if we would be willing to do this? We would be willing to perhaps take a shot at it for a while and then maybe pass that off to someone else after we get the information there. I have to give credit to the other folks. The European Consortium has already put together a lot of that information, and I am fairly sure that they would be willing to work with us. So, we would get that information together in partnership with them.

DR. KING: Okay. All we need is a date.

DR. MAY: Before our next meeting. It really shouldn't take that long. I think we have all the information at hand to do that.

PARTICIPANT: It seems that most manufacturers, if not all, do not understand what exactly the traceability document is saying. I have a suggestion, at least for this country, to translate it for lay people:

maybe have a one-week workshop, or maybe ask AACC to put it on the web. But translate it, tell an

We need to translate the "traceability document" for lay people. example of an analyte for which we have a reference method and a reference material, and then go down the line and tell them this is how it is done, how one thing is linked to the other, graphically, in very simple words.

DR. MAY: Okay. Just stay there a second.

Bernard, you don't have to go to the microphone, but I assume that something like this is what you had in mind; is that not true?

DR. KING: That is exactly what we had in mind.

DR. MAY: There are plans to do that. Bernard has planned to do this as one of his activities, and we at NIST have already agreed to work with him on that project. This will 
be a document that essentially translates the traceability requirement section of the IVD directive.

PARTICIPANT: Well, then what timeline are you thinking of there?

DR. MAY: Bernard, what timeline did you have in mind for that?

DR. KING: Well we're to seek funding from the European Union to help bring together the players that will contribute to this.

DR. MAY: How much of an effort do you need? I'm sure you don't mind where you get the resources from, so how much will it cost?

DR. KING: I've not gotten to that.

DR. MAY: Roughly.

DR. KING: It is not a single document. It is a series of documents about a series of related issues.

DR. MAY: If it is not proprietary, what you were expecting to get from the EU in order to do this?

DR. KING: What we were envisioning is that the project would take place in two years. We plan to find documents that already exist and material that already exists, and then provide some user-friendly resources.

DR. MAY: Essentially, what you would need would be some resources to support travel and a bit of time for certain key people.

DR. KING: Certainly. We'll be bringing together the people who need to have an input, and then also some more people who will sit down and put the material together.

DR. MAY: That seems to be something that is truly needed, so if you can put together a document, let's say, what it would take to have this done in draft form by the Dublin meeting. Is that impossible? For this time next year?

DR. KING: I'll think about it. It is easy for me to say, yes, oh, yes. The reality is can we have it done at the appropriate cost?

DR. MAY: I guess the statement from Basil (Doumas) here would be a translation for lay people not the "higher order". ... Basil is shaking his head. Is it important, Basil, for lay people, that is for patients, to understand it or is it only important to people in the clinical laboratory and the manufacturers? 
DR. DOUMAS: It needs to be in plain English, simple with examples for all people in the industry who are assigned this to be able to understand it.

(Laughter)

DR. MAY: Now we have our marching orders.

DR. POWERS: Willie, may I make a related comment?

I was going to ask if Emil is still here. If so, I'll let him answer my question. I understood that EDMA was working on a guidance document. I am kind of concerned about creating a document for lay people. I think we need something for manufacturers written in the language manufacturers understand. I would see the trade associations as being the logical owners of that specific document. I think we have enough people who have been working on the traceability standards who could get together and knock it off in a week.

DR. MAY: Okay.

DR. POWERS: Or two.

DR. MAY: Is that true, Emil?

DR. VOELKERT: I'm Emil Voelkert from EDMA.

In fact, I would like to pour some wine into the water. I think we have more than the discussion really shows. First of all, I think we do have lists of reference materials, NIST for one, and there are several others. Secondly, I am also aware that there have been descriptions and publications of reference methods. The only thing I can say right now is that if you look back at the German quality regulation system (I don't know whether it's updated) it lists, I think, about 20 different reference methods. So, you might take a look at that.

Concerning these traceability documents that we are working on, we have tried in a small working group in EDMA, to highlight the positions which we think from the industry side are relevant when transposing the standard into actual life. From my point of view, I think it is understandable.

But I come to another problem I perceive. I am not sure whether we really mean the same thing when we talk about reference methods and reference materials. I perceive from some of the discussions there are still

So, when we talk across the Atlantic, so to say, we have to be aware that there may be differences in semantics. different meanings around the world. This is something we have to address.

I think, also from my point of view, that it would be useful to have another meeting in between so that we can work out the subtle differences. I will be willing to make the 
EDMA document available to all manufacturers that are interested. It will be available through the EDMA sites, and I will also distribute it to the AdvaMed. I think that it will also be available to other interested parties.

If you are asking for the timeline, actually, the document is in the last stages of development. I anticipate that it will be available by end of this year at the latest. However, we would welcome any documents that would provide other perspectives, particular from the point of view of the manufacturers, because that is mainly whom we are working for.

DR. MAY: All right, Dr. Voelkert, is that document drafted so that perhaps some of the manufacturers could review it to ask for clarification, if you will, or to make sure that things are stated clearly enough that they can understand the intention of the directives?

DR. VOELKERT: This is a very difficult question. It depends on the level of understanding of the particular person who reads it. From my point of view, if somebody has dealt with the IVD directive and has a rough idea about traceability, it helps understanding. I think it is up also to this global initiative really to come up with additional tools and educational material really to translate this idea of traceability. So, I would not put the expectations too high, but from my point of view and from my perspective, it is a useful document.

DR. MAY: Let's take this position: We should take a look at the EDMA document. If by some chance it does not meet your needs, then maybe we can take that as the basis of a document, and then modify it so that sufficiently meets your needs.

DR. SMIT: Nick Smit with i-STAT, Canada.

Nine months seems awfully far away, as far as I'm concerned at i-STAT. I have a couple of suggestions that could be an outcome of this workshop. A question: Is there going to be something that resembles a project plan that in a draft form or as an outline, or an interim progress report that so that in nine months when we get together - everybody here has an expectation, and you have your expectation - but when we get together in nine months, we don't disconnect at the other side of the world in Dublin?

DR. MAY: Well, project plan? I am not sure that we have defined a project. What we will provide for you would be the proceedings of this meeting. Regarding the next meeting, I think we've already agreed that we need to have a meeting before the nine months, and the earlier meeting would be one here in the United States. I don't know whether Carolyn Jones is still here or not, but I jotted on my notes that perhaps we would ask AdvaMed to convene that meeting.

We will provide:

- a copy of the presentations that were made yesterday, and

- an editorial version of the discussions that we've had this afternoon.

Then we would discuss many of the issues that we have been discussing this afternoon to 
prepare ourselves to the international meeting in Dublin. I think it's at that meeting that we can sort of clarify some of the issues that are bubbling up here.

DR. SMIT: All right. Just in terms of letting you know what we will be doing - I think that a lot of the industry (including us), will be drafting their own documents and their own process and procedures on how to implement this.

DR. MAY: Sure.

DR. SMIT: I know it's a chicken and egg kind of situation, who is waiting for who to give who direction. However, i-STAT is going to move ahead, and we're going to figure it out. Whether the reference laboratories are in place or not in place, we need to have some kind of documented resolution to traceability. Nine months just seems far away.

DR. MAY: Again, that's certainly something that you have to do. I think you have realized that certainly from a NIST perspective we are here to provide the tools for you, but, obviously, you have to do the work to develop what is required to get your products into the European Union. We are here to try to facilitate that process, and we will commit to do that.

Let me share with you what I have heard here, and we can see if this sort of covers most of the holes we had. Certainly, we had talked about the necessity of perhaps having $\mathbf{a}$ meeting sooner than nine months, and we will talk to some people, perhaps AdvaMed about convening that meeting to address some pressing concerns, primarily of the IVD manufacturers.

Plan of Action:

- Meeting of US IVD manufacturers before next international meeting

- CAP provide priority list of analytes

- EU's "A list" and "B list" analytes

- Web dissemination of information

We talked about requesting that CAP provide a list of high priority analytes based on results that they have from their proficiency testing trials.

We talked about the necessity of communicating information, perhaps having someone volunteer at NIST to look at putting together a web site that would list the tools that are in already in place, both the reference methods, and the reference materials. Perhaps on that, we could pull from the IVD directive the listing, as it stands now, of the "A list" analytes and the "B list" analytes so that you have as much information as we have, basically. We would work with the European Union on putting this information together.

DR. CARNEIRO: This is Kim Carneiro from DFM, Denmark.

I would certainly be happy to do that, because exactly if the European project gets funded, one of my tasks to put up the web site and the information. 
DR. MAY: Okay.

DR. CARNEIRO: But let me be very clear about ...

DR. MAY: NIST would just link to it then.

DR. CARNEIRO: The project that we have -- that you have taken very kindly on board is a proposal that has not even been reviewed yet. So, we will be into the new year before that can start. Then, of course, we will be going full steam. But it would be wrong for anybody to rush, and I think that's partly why Bernard is rather reluctant to publish anything within the next few weeks. We really are not ready yet. We do have a project plan, and it has been nicely received here, but it will take us into the new year before it gets started.

DR. MAY: Sure.

DR. CARNEIRO: In that respect, I think it's very timely to meet in Dublin in June, because there will be some progress, and we can continue the dialogue on a much more specific level than we have been able to do here. We've had very good dialogue on a general level. We all need to go back and think a bit about it. Let's do this well, and still think about timeliness.

DR. MAY: Can we do this? I guess what I am hearing from the manufacturers, from what they say and by reading their faces, is they need this maybe a bit sooner ... That's why I carefully said that maybe NIST would take a crack at this now and maybe pass it off to someone later. I wonder we could work together to try to get something out there now so that, basically, we can provide as much information to the manufacturers as we have now. Then as your (Dr. Carneiro's) project moves forward, you could take that over and perhaps put a lot more resources than we would put into this initial effort to just put the information there without a lot of bells and whistles.

DR. CARNEIRO: I'll be very happy to discuss that and do our utmost. As soon as the project gets going, I think we can easily arrange that everybody in this room is kept electronically informed about what goes on. I think that is the most efficient way of doing it.

DR. MAY: Sure.

DR. CARNEIRO: I was certainly saying to have a similar meeting to this in June next year would be fine, because some work can be done along the lines that we've been proposing. Having another meeting in between is fine. We need to keep this going. We may arrange a similar meeting in Europe to keep closer to the European-based industry. Then we'll meet in June and have a good next round. A meeting before then I think would be too hastily done. 
DR. MAY: Great.

DR. ECKFELDT: John Eckfeldt, CAP in University of Minnesota.

I actually know AACC Standards Committee put together a compilation of reference methods and materials that was and I think it's still on the web site somewhere at AACC. That would be a starting place. It is not that comprehensive, but it is one place and is already on a web site, and worth looking at. As I remember, there was a brief description of the method, literature references, materials, who you got them from, and who the contact people were. There was a format that was already devised. Jean Rhame was involved in putting that together for AACC. It is just some place to start, we could look at it and maybe use it as a template or something. I know some of it is out of date.

Another place that might be able to provide some information is CAP. When we looked at our Standards and Reference Materials Program, we looked at what other materials or methods were available internationally, particularly materials, just to see if there were things that we were going to discontinue that were not available somewhere else. There was virtually no analyte for which we provide the material that you couldn't get somewhere else. I think we have that on a database somewhere. I could probably share that with you on the material side.

DR. MAY: We'd certainly appreciate that.

Continuing to go down the list that I had, translate the IVD traceability document was one of the things that was discussed. It appears that EDMA is well on the way to doing that, and I think what we agreed to do was to take a look at what they provided and go from there. If that meets our needs, the problem is

Plan of Action, continued: "Translate" IVD traceability document - it appears that EDMA is well on the way to doing that ...

solved. If we need further translation, then we can take that aboard and have it defined who "we" is. Then finally, I think we've agreed that this international group would meet next in Dublin in June. Any other questions, comments, concerns? Not putting you on the spot, Rene, but I know at our coffee break you had some issues on your mind. Do you feel comfortable sharing them with us? I know you aren't shy.

DR. DYBKAER: Rene Dybkaer, H:S Frederiksberg Hospital, Denmark.

First of all, I should like to say that it should be quite clear that although it is nice to be hasty with something that must be done, you shouldn't be too hasty. The facts are that the European Directive will come into force at the end of 2003, and it does not include a demand that everything that the manufacturers provide will suddenly have to be traceable to the SI. As was said a little while ago, the directive says available reference materials and procedures. So, that means that if nothing is available, you can go on with what you have until something comes along. That's very important issue. The other thing I 
would say is that just as Dr. May is a great admirer of Rudy Lequin, so am I. But that does not mean that I always agree with him.

\section{(Laughter)}

DR. MAY: Me either.

MR. DYBKAER: And earlier on he made a plea that we should look differently at the so-called A\&B situations. The "A list" are analytes for which there was obviously existing a possibility of traceability through SI, and the "B list" is the group where this

... we should start at the

bottom and then go as far

up the traceability chain as

possible ... traceability does not exist. I would submit that it is more important to say we should always use the same procedure in principle, and that is we should identify the problem at the bottom in the routine laboratory, and then we should say how we can obtain traceability for the results we deliver. Then we go up the existing chain as far as we can. If we can go to SI, fine. If not, then, as Rudy was saying, we will have to rely on some international conventional reference material or reference procedure. It is all traceability. We just go as far up the chain as is technically possible. I think that was most of what I wanted to submit. Thank you.

DR. MAY: I think what he is saying is that metrology is essentially metrology, and it is our responsibility to make the system as metrologically sound as we possibly can and not say that we're going to look at some measurement problems in a sound metrological basis and the others, well,

It is our responsibility to make the system as metrologically sound as possible. just forget about metrology. We really should be striving, if you will, to convert all of the "B list" analytes to "A list" if we can. Obviously, we know that that's not possible, but that should be our quest.

\section{... thank you to Basil Doumas and Bill Koch for the vision ...}

Well, it's getting late, and some people have to leave. Let me say that perhaps a year or so ago, not even that, Basil and Bill decided that we were going to have this meeting, and many of us were cynical. We weren't sure if we could pull it together so quickly, whether anyone would come other than the folks that work here and the Steering Committee. We've been pleasantly surprised.

I think that we all agree that this certainly is an important issue that we need to discuss. And we thank all of you for taking time out of your very busy schedules to come here and share your thoughts on these issues with us.
... thanks to our many participants for your insights ... 
So, on behalf of the Steering Committee, the NIST staff

... thanks to all who worked to make this workshop a success. that worked on this, and in particular Lyn Beary, Donna Sirk, Jeanice Brown-Thomas, Amy Grafmuller, Mike Welch, who worked behind the scenes to make this a success, and certainly on behalf of Bill Koch and Hratch Semerjian, and myself, we really thank you for your efforts. We are sure that this won't be the last time we'll be seeing you and talking to you about these issues. Thank you very much.

(Applause)

Whereupon, the Workshop was concluded at 4:00 p.m. 


\section{Workshop on Measurement Traceability for Clinical Laboratory Testing and In Vitro Diagnostic Test Systems \\ A Report on the Workshop \\ William F. Koch \\ National Institute of Standards and Technology}

In early November of the year 2000, the footings for a new bridge were laid in Gaithersburg, Maryland, just outside Washington, DC. Representatives of government, the IVD industry, and the medical professions from 15 nations representing four continents gathered at the National Institute of Standards and Technology to participate in the "Workshop on Measurement Traceability for Clinical Laboratory Testing and in vitro Diagnostic Test Systems". Their goal: to develop recommendations regarding the needs for measurement traceability for health status markers to (1) address IVD industry needs for compliance with international standards (e.g., EU IVD Directive) and (2) improve comparability of clinical measurement data to facilitate better decision making by medical professionals.

As these 135 scientific experts and stakeholders from around the globe (25\% were from outside the United States) entered the NIST "Green Auditorium" for the start of this intense two-day workshop, their eyes were drawn to the huge screen displaying the icon for the meeting - a bridge superimposed on a map of the world. This bridge symbolized three fundamental concepts related to the workshop. First, a bridge is a means to get from one place to another, often across a formidable gap. The first day of the Workshop was filled with 10 talks delivered by experts providing the background and current status, thereby anchoring one end of the bridge. The second day was spent in breakout sessions and general discussions intended to plan the size and direction of the path forward. Secondly, just as a bridge is a critical part of the infrastructure for the transportation system that facilitates free and open commerce, so too is traceability a vital piece of the measurement infrastructure for the global healthcare system that supports equitable and open trade, as well as assuring improved comparability and reliability of clinical testing. And finally, the bridge on this Workshop's logo spans the waters of the world. Healthcare is now more than ever a global issue and a global commodity, demanding global solutions.

Before recounting the details and conclusions of the meeting, it is essential to understand the driving force behind the need for this workshop. Traceability to internationally recognized and accepted standards is an important component in assuring the accuracy and comparability of clinical laboratory measurements. In addition, the global marketplace is presenting new demands for measurement traceability. NIST has a long history of providing certified measurement standards in several fields including the clinical laboratory disciplines, and is continuing its efforts to develop new reference methods and materials for important health-status markers to meet on-going and future needs for traceability. NCCLS is a globally recognized, voluntary consensus standards-developing organization that enhances the value of medical testing within the healthcare community 
through the development and dissemination of standards, guidelines, and best practices. NCCLS has the Secretariat responsibility for the ISO Technical Committee 212 (ISO/TC 212) on Clinical Laboratory Testing and In Vitro Diagnostic Test Systems, and is the home of the National Reference System for the Clinical Laboratory (NRSCL), a collection of broadly understood reference systems intended to improve the comparability of test results, consistent with the needs of medical practice. Recently, an important opportunity has emerged that applies new pressure to the quest for traceability and the demand for reference systems. Prompted by the European Union's In Vitro Diagnostics Directive (IVDD), the European Committee for Standardization's Technical Committee 140 (CEN/TC 140), in vitro diagnostic systems, began drafting a standard on metrological traceability. By working closely together, CEN/TC 140 and ISO/TC 212 will develop identical European and ISO standards on this topic. Full implementation of the IVD Directive is expected by December 2003 and will require that calibration of all IVD assays be traceable to available reference materials or methods of higher metrological order. (See Don Powers Article in IVD Tech, July, 2000, http://www.devicelink. com/ivdt/archive/00/07/003.html).

During the first day of lectures, the attendees learned directly from the leading authorities in Europe about the European IVD Directive. Dr. Kim Carniero, Danish Institute of Fundamental Metrology and representing the European Commission, presented a succinct summary of the Directive, its basis, purpose, implementation, and implications. Dr. Emil Voelkert, Roche Diagnostics GmbH and chair of CEN/TC140, described the standards activities within CEN and ISO, focusing on CEN/TC 140 and ISO/TC212, in support of the IVD Directive and with the goal of harmonized standards within Europe. Professor Lothar Siekmann, University of Bonn and representing the IFCC, discussed the concept of traceability as applied to the field of clinical chemical analysis, remarking that traceability provides probably the most important strategy to achieve standardization in laboratory medicine. He went on to illustrate the process for credentialing reference laboratories, methods and materials in Germany. Rounding out the European viewpoint was Dr. Heinz Schimmel, Institute for Reference Materials and Measurements (IRMM), who informed the audience of the EU's current and planned clinical reference material development activities.

To segue back to the United States, Dr. Hratch Semerjian, National Institute of Standards and Technology, related the important and increasing role that national metrology institutes (NMI), such as NIST, PTB and DFM, have in establishing mutual recognition of measurements and tests between nations. In October 1999, the NMI Directors of the 38 member states of the Meter Convention signed "the mutual recognition arrangement (MRA) on national measurement standards and calibration and measurement certificates issued by national metrology institutes." In addition, the US and EU have entered into an MRA to facilitate bilateral trade between the US and European Community realizing that mutual recognition of conformity assessment activities is an important means of enhancing market access. Continuing with efforts at NIST, Dr. Willie May, NIST, explained how NIST serves as the primary US reference laboratory for healthrelated chemical measurements through: the development of high-accuracy measurement methods; the development, certification and distribution of Standard Reference Materials; interactive measurement quality assessment activities; and international comparison exercises with other NMIs. Highlighting a subtle but significant change in his title, Dr. Neil 
Greenberg, Ortho-Clinical Diagnostics, presented the perspective held by the global (originally the US) IVD industry of the Directive's requirement for calibrator traceability. He made a special note that the IVD Directive does not specifically call for the development of new or improved reference materials, but only that available reference materials and measurement procedures of higher metrological order be used for traceability purposes. Nevertheless, ISO/CD 17511 states that it is the aim of metrology in laboratory medicine to improve traceability by providing the missing reference measurement procedures and materials, based on international consensus. As a result, reference material and methods development projects will be initiated in the name of the Directive. Whereas IVD companies value standards because they help define market needs and provide a clear universal definition of goals, as well as an objective assessment of product attributes, it is unlikely that they will take a lead position in advocating for new standards at this time. However, the inclusion of manufacturing scientists and experts in IVD standards projects led by professional, government and public health groups is absolutely necessary for project success.

Adhering to the philosophy that one should know where one has been in order to better know where to go next, Dr. John Eckfeldt, Fairview-University Medical Center and representing the College of American Pathologists, presented a historical review or reference systems for clinical measurements. Evolving from the Belk and Sunderman's study in 1947 involving 59 clinical laboratories, using 24 samples, and testing for 7 analytes, the CAP PT/EQUAS Program today encompasses over 25,000 laboratories with over a quarter of a million samples and testing for over 500 analytes. He cited several examples to illustrate the role of performance testing for assessing traceability, noting that some programs were more successful than others, often due to limitations of the analyte being tested (e.g., non-commutability, poorly defined or heterogeneous analyte). He concluded that establishing and maintaining the documentation for traceability in field methods is both difficult and expensive. Dr. George Klee, Mayo Clinic, expanded on the need for commutability, noting that reference materials, even when available and used in conjunction with established reference methods, do not necessarily assure harmonization of test values on patient samples. He concluded that a combination of commutable control materials (based on panels of commutable human specimens) with traceable reference values and mathematical algorithms using adjusted patient test values could be used for calibration adjustment. This approach will complement the role of reference materials in improving patient care. And finally, Ms. Joan Walsh Cassedy, ACIL, presented models of reference systems used by other industries, emphasizing that measurement traceability forms the foundation of a quality program, and that absence of traceability leads to chaos.

Armed with this information, the participants reconvened the next morning in five separate breakout sessions led by: Neil Greenberg (Priorities for National and International Investments in New or Improved Reference Systems in Support of Clinical Laboratory Measurement); Don Powers (Reference Materials and Reference Measurement Procedures to Support Traceability Requirements of the IVD Directive); Greg Miller (Impact of Method-Material Matrix Interactions on Calibration Traceability Protocols for Successful Harmonization of Patient Results); William F. Koch (Development of International Consensus on the Credentialing Process for Reference Systems); and Gary L. Myers (Creating and Sustaining Reference Method Laboratory Networks). Four hours of spirited discussions and consensus-building within each group resulted in five reports that 
were presented to the re-assembled audience in the afternoon. Although each group brought forward unique perspectives and recommendations based on the topical area, there was general agreement on some critical attributes of the traceability bridge. The need for global reference systems composed of reference methods, reference materials and a mechanism for demonstrating competence and equivalence was of paramount importance. Internationally recognized and accepted reference laboratories should implement these reference systems, using a networked approach. In order to meet the immediate requirements of the IVD Directive, a catalog of the available reference methods and reference materials must be communicated to the IVD manufacturers. There was concurrence that when properly implemented, traceability is a value-added exercise that will improve patient care, testing accuracy, reliability and availability, market access, and, in the long run, reduce costs. However, it was emphasized that efforts undertaken must be designed to minimize redundancy and barriers, encourage new technologies and facilitate global collaborations.

And now that we know where to build our bridge and have some idea of what the bridge will look like, what are the next steps? The closing session of the workshop achieved concurrence on the following actions:

- Develop a web-enabled database of currently available reference methods and internationally recognized CRMs. NIST will undertake this activity while realizing that the EU has submitted a proposal to be reviewed in several months for a similar exercise. If the EU proposal is funded, NIST will coordinate with the EU.

- Organize an internationally accepted "oversight" group that is industry-led (but composed of government experts and medical professionals as well as industry experts) whose task will be to:

$>$ Set priorities for new reference methods and materials based on medical importance, need, and commutability.

$>$ Identify sources and secure funding for developing reference methods and materials.

$>$ Develop the process for international consensus on reference systems

- Develop interpretive guidelines on traceability requirements.

- Develop a global process for approving reference methods and for distributing certified reference materials (CRMs).

- Identify alternative approaches for those analytes for which SI traceable reference methods and/or materials are impossible, at this time.

- Convene a follow-up meeting in Dublin, preceding the ISO/TC212 Meeting in June 2001. Progress in the above bulleted action items will be reviewed and further design and construction of the bridge will proceed.

It is clear that the Traceability Bridge is far from complete. This Workshop was only the beginning, but we should be encouraged by the broad representation, both geo- 
graphic and sector, and by the level of effort, enthusiasm and commitment exhibited at this groundbreaking in Gaithersburg. The waters under the bridge need not be troubled if international consensus on measurement traceability can be achieved.

\section{WILLIAM F. KOCH}

\section{National Institute of Standards and Technology}

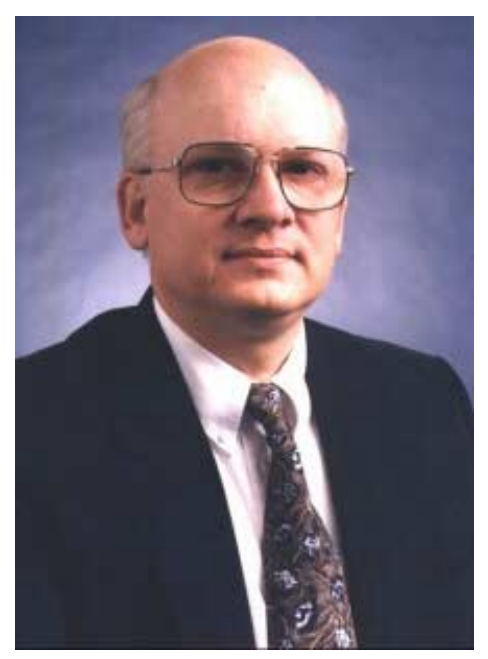

Dr. William F. Koch is the Deputy Director of the Chemical Science and Technology Laboratory at the National Institute of Standards and Technology. He began his career at NIST as a research chemist in the Center for Analytical Chemistry at NBS, where he expanded his research interests to include $\mathrm{pH}$, electrolytic conductivity and ion chromatography. He has had direct involvement in the development and certification of over 70 Standard Reference Materials, and has authored over 70 scientific publications.

Dr. Koch has been an elected member of the Board of Directors of the National Committee for Clinical Laboratory Standards (NCCLS) since 1990, and is currently the Immediate Past President of this organization. He is on the Governing Board of the Council for Chemical Research and chair of the CCR Science Education and Human Resources Committee. He was recently elected as a Fellow in the National Academy of Clinical Biochemistry. He is an active member of the Directors of Industrial Research-Analytical Group, the American Chemical Society, the Society for Electroanalytical Research, and the American Association for Clinical Chemistry. 


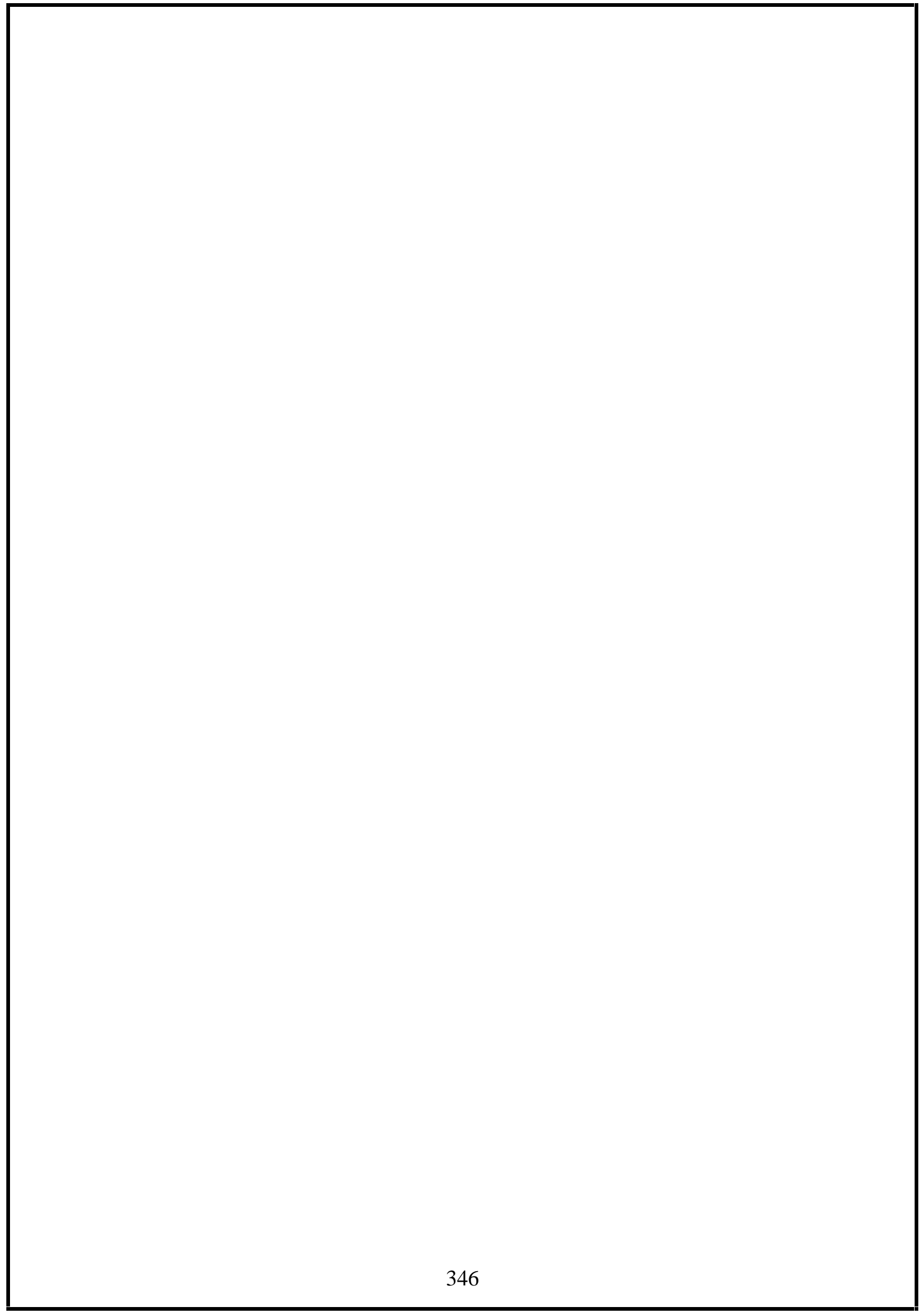




\section{Workshop on Measurement Traceability for Clinical Laboratory Testing and In-Vitro Diagnostic Test Systems \\ November 2-3, 2000}

\section{Participants List}

Ahnell, Joseph

BD Biosciences

7 Loveton Circle, MC 622

P.O. Box 999

Sparks, MD 21152-0999

USA

410/316-4078

410/316-4046

Joseph_E_Ahnell@ms.bd.com

Antalis, Caryl

Roche Diagnostics

9115 Hague Rd.

Indianapolis, IN 46250

USA

317/576-3120

317/576-3085

caryl.antalis@ roche.com

Arends, Sabine

Roche Diagnostics GmbH

Bahnhofstrasse 9-15

Tutzing

Germany

82327

49/81 58224349

49/8158224152

sabine.arends@ roche.com

Armbruster, David

Abbott Laboratories

1920 Hurd Drive, MS 1-9

Irivng, TX 75038

USA

972/518-6016

972/753-3295

david.armbruster@abbott.com
Ashwood, Edward

College of American Pathologists

ARUP Laboratories

University of Utah

500 Chipeta Way

Salt Lake City, UT 84108

USA

801/583-2787

$801 / 584-5207$

ashwood@med.utah.edu

Beary, Ellyn

NIST

100 Bureau Drive, MS 8300

Gaithersburg, MD 20899-8300

USA

301/975-8307

301/975-3845

ellyn.beary@nist.gov

Binder, Steven

Bio-Rad Laboratories

4000 Alfred Nobel Dr.

Hercules, CA 94708

USA

510/724-7000

steve_binder@bio-rad.com

Blessum, Cynthia

Beckman Coulter, Inc.

200 S. Kraemer Blvd.

Brea, CA 92822

USA

714/993-8980

714/961-3759

crblessum@beckman.com 
Bozimowski, Dennis

Abbott Laboratories

1920 Hurd Dr.

Irving, TX 75038

USA

972/518-7546

972/753-3233

dennis.bozimowski@add.ssw.abbott.com

Bugler, Joe

Abbott Laboratories

MediSense Products

Unit 14/15 Eyston Way

Abingdon, Oxon

OX14 1TR

United Kindom

44/1/235-542-310

44/1/235-555-705

joe.bugler@abott.com

Bunk, David

NIST

100 Bureau Drive, MS 8392

Gaithersburg, MD 20899-8392

USA

301/975-5071

301/977-0685

david.bunk@nist.gov

Burritt, Mary

Mayo Clinic

2001 st St., SW

Rochester, MN 55905

USA

507/284-3954

507/266-4176

mburritt@mayo.edu

Caines, Patrick

Ortho-Clinical Diagnostics

100 Indigo Creek Dr.

MC 24149 B 642

Rochester, NY 14626

USA

716/453-5089

716/453-5170

pcaines@ocdus.jnj.com
Caler, Mara

Beckman Coulter, Inc.

P.O. Box 269006

San Diego, CA 92196

USA

$858 / 621-4583$

$858 / 621-4752$

mcalerchapman@beckman.com

Canouse, Patricia

Abbott Laboratories

5440 Patrick Henry Dr.

Santa Clara, CA 95054

USA

408/567-3377

408/567-3568

patricia.canouse@add.ssw.abbott.com

Carneiro, Kim

Danish Institute of Fundamental Metrology

Building 307, Anker Engelunds Vej 1

DK-2800 Lyngby

Denmark

Tel: +4545255867

Fax: +45 45931137

kc@dfm.dtu.dk

Cassedy, Joan Walsh

ACIL

1629 K St., NW - Suite 400

Washington, DC 20006

USA

202/887-5872

jcassedy@acil.org

Ceriotti, Ferruccio

H.S. Raffaele

Via Olgettina 60

Milan 20132

Italy

$39 / 226432282$

$39 / 226432640$

ceriotti.ferruccio@hsr.it 
Cernosek, Stanley

Beckman Coulter, Inc.

200 S. Kraemer Blvd.

Brea, CA 92822

USA

714/993-8805

714/961-3740

sfcernosek@beckman.com

Chace, Nina

FDA

2098 Gaither Road

Rockville, MD 20850

USA

301-594-1293

Chapman, Donna

Bio-Rad Laboratories

9500 Jeronimo Rd.

Irvine, CA 92618

USA

949/598-1280

949/598-1555

donna_chapman@bio-rad.com

Chen, Ellen

Food and Drug Administration (FDA)

12725 Twinbrook Pkwy.

FDA/CDRH/DMMS, Rm.22

Rockville, MD 20852

USA

301/827-4716

301/827-4877

exc@cdrh.fda.gov

Chi, Robert

Taiwan Committee for Clinical Laboratory

Standards

Department Clinical Path

Tri-Service General Hospital

Taipei

Taiwan

$886 / 2-3653825$

$886 / 2-23655582$

chin422@ndmctsgh.edu.tw
Childress, William

Food and Drug Administration

109 Holton St.

Winchester, MA 01890

USA

781/729-5700

781/729-3593

wchildre@ora.fda.gov

Choo, Sae

First Medical

530 Logue Ave.

Mountain View, CA 94043

USA

650/903-5974

650/903-9040

saechoo@firstmedical.com

Clark, Douglas

Dade Behring, Inc.

P.O. Box 6101, MS 707

Building 700

Newark, DE 19714-6101

USA

302/631-7545

302/6316998

clarksdp@dadebehring.com

Coady, Cynthia

Diagnostic Chemicals Ltd.

16 McCarville St

Charlottetown, PE

C1A 2 A6

Canada

902/566-1396

902/566-2498

lwoold@dclchem.com

Cockerham, Kenneth

Bio-Tech Imaging, Inc.

5711 Industry Lane

Unit 31

Frederick, MD 21704

USA

301/695-9900

301/695-0030

krcham@aol.com 
Colbert, Jennifer

NIST

100 Bureau Drive, MS 2321

Gaithersburg, MD 20899

USA

301/975-2579

jennifer.colbert@nist.gov

Crush-Stanton, Sylvia

Boston Biomedica, Inc.

375 West St.

West Bridgewater, MA 02379

USA

508/580-1900

508/580-0250

scrush-stanton@bbii.com

Dalluge, Joseph

NIST

100 Bureau Drive, MS 8392

Gaithersburg, MD 20899-8392

USA

301/975-3651

301/977-0685

joseph.dalluge@nist.gov

Davidson, Yolanda

NIST

100 Bureau Drive, MS 8392

Gaithersburg, MD 20899-8392

USA

301/975-3116

301/977-0685

yolanda.davidson@nist.gov

de Leer, Ed W.B.

NMi Van Swinden Laboratorium

Department of Chemistry

PO Box 654

2600 AR Delft

The Netherlands

31/15-269-1712

31/15-261-2971

edeleer@nmi.nl
Doumas, Basil

Medical College of Wisconsin

Plenary Session Chair

2430 Whipple Tree Lane

Brookfield, WI 53045

USA

262/786-1038

262/786-4171

bdoumas@core.com

Dubrowny, Nancy

BD Vacutainer Systems

1 Becton Drive, Mail Code 310

Franklin Lakes, NJ 07417

USA

201/847-4409

Dunka, Lou

LifeScan

1000 Gibraltar Drive

Milpitas, CA 95035

USA

408/942-5762

Dybkaer, Rene

H:S Frederiksberg Hospital

Nordre Fasanvej 57

Department Standards Laboratory Medicine

Frederiksberg, DK-2000

Denmark

$45 / 38163870$

$45 / 38163879$

Eckfeldt, John

Fairview University Medical Center

420 Delaware St., SE

Minneapolis, MN 55455

USA

612/626-3176

615/273-6994

eckfe001@tc.umn.edu

Edmondson, Sherb

Abbott Laboratories

5440 Patrick Henry Dr.

Santa Clara, CA 95054

USA

408/567-3424

408/982-4882

sherb.edmondson@add.ssw.abbott.com 
Ehlers, Glenn

Ortho-Clinical Diagnostics

100 Indigo Creek Dr.

Rochester, NY 14626

USA

716/453-5025

716/453-5188

gehlers@ocdus.jnj.com

Elin, Ronald

College of American Pathologists

University of Louisville

7015 Windham Pkwy.

Prospect, KY 40059

USA

502/852-4464

502/852-1771

rjelin01@gwise.louisville.edu

Files, Nancy

Immunicon Corp.

3401 Masons Mill Road, Suite 100

Huntingdon Valley, PA 19006

USA

215/830-0777

Fish, Terri

U.S. House of Representatives

Science Committee Staff

2319 Rayburn House Bldg.

Washington, DC 20515

USA

202/225-8844

teresa.fish@mail.house.gov

Fitzgerald, Marc

American Chemical Society

1155 16th St., NW

Washington, DC 20036

USA

202/872-6296

202/872-6060

m_fitzgerald@acs.org
Ford, Patrick

Ortho-Clinical Diagnostics

Cardiff Laboratories

Forest Farm Estate

Cardiff CF47YT

South Wales

United Kindom

44/029 20521693

44/029 20521632

pford@ocdgb.jnj.com

Gaigalas, Adolfas

NIST

100 Bureau Drive

Gaithersburg, MD 20899

USA

301/975-2873

301/975-5449

adolfas.gaigalas@nist.gov

Garrett, Patricia

Boston Biomedica, Inc.

47 West Street, Suite 1

Portland, ME 04102

USA

860/225-1900

Gella, F-Javier

Biosystems

Costa Brava 30

Barcelona 08030

Spain

34/9331 10811

Giguere, Monica

BD Biosciences

7 Loveton Circle, MC 614

Sparks, MD 21152

USA

410/316-4287

410/316-4499

mgiguere@bd.com 
Giuliani, Isabelle

Bio-Rad

UMR CNRS 5094

Faculte de pharmacie

15 Avenue Charles P1

Montpellier 34060

France

$33 / 467548607$

33/4 67548610

isabelle.giuliani@ibph.pharma.univ-

montp1.fr

Gratiano, Theresa

BBI Biotech Research Labs., Inc.

217 Perry Pkwy.

Gaithersburg, MD 20877

USA

301/208-8100

301/208-8829

tgratiano@bbii.com

Greenberg, Neil

Ortho-Clinical Diagnostics

100 Indigo Creek Dr.

Rochester, NY 14626

USA

716/453-3768

716/453-3982

ngreenbe@ocus.jnj.com

Guzel, Omer

Biruni Laboratory

Buyukdere Cad Gokfil

Ishani Mecidiyekoy

Istanbul TR80310

Turkey

90/2122174106

90/21221 74106

oguzel@biruni.com.tr

Halonen, Tuija

PerkinElmer Wallac

Customer Support Laboratory

P.O.Box 10

20101 Turku

Finland

$358 / 22678111$

$358 / 22678357$

tuija.halonen@wallac.fi
Hartmann, Alfred

College of American Pathologists

Physicians Laboratory

1000 E. 21 St.

Suite 4100

Sioux Falls, SD 57105

USA

$605 / 322-7190$

605/322-7222

al.hartmann@mckennan.org

Hawes, Katherine

Immunicon Corp.

3401 Masons Mill Road, Suite 100

Huntingdon Valley, PA 19006

USA

215/830-0777

Hughes, John

LifeScan, Inc.

1000 Gibraltar Dr

Milpitas, CA 95035

USA

408/956-4064

408/942-5906

jhughes@lfsus.jnj.com

Jain, Chandra

Beckman Coulter, Inc.

200 South Kraemer Blvd.

Brea, CA 92822

USA

714/961-4116

714/961-3740

cpjain@beckman.com

Jaunzemis, Andris

Becton Dickinson

7 Loveton Circle, MS 622

Sparks, MD 21152

USA

410/316-4860

Jones, Carolyn

AdvaMed

200 G Street NW, Suite 400

Washington, D.C. 20005-3814

USA

cjones@advamed.org 
Kahn, Stephen

Loyola University Medical Center

Rm. 0122 Clinical Lab

2160 S. $1^{\text {st }}$ Ave.

Maywood, IL 60153

USA

708/216-4725

708/216-4146

skahn@lumc.edu

Kamalian, Farrah

Bio-Rad Laboratories

9500 Jeronimo Rd.

Irvine, CA 92618

USA

949/598-1319

949/598-1553

Farrah_kamalian@bio-rad.com

Kessner, Art

Beckman Coulter, Inc.

200 South Kraemer Blvd.

Brea, CA 92822

USA

714/993-8999

714/961-3740

akessner@beckman.com

Kimberly, Mary

Centers for Disease Control and Prevention

4770 Buford Hwy., NE, MS F25

Atlanta, GA 30341

USA

770/488-4683

770/488-4192

mmk1@cdc.gov

King, Bernard

LGC

Queens Rd

Teddington

Middlesex TW11 0LY

United Kindom

44/2089437667

44/2089432767

bmjk@lgc.co.uk
Klee, George

Mayo Clinic

200 1st St., SW

Stabile 104C

Rochester, MN 55905

USA

$507 / 284-8213$

507/266-4341

klee.george@mayo.edu

Klimley, Patricia

Bio-Rad Laboratories

4000 Alfred Nobel Drive

Hercules, CA 94547

USA

510/741-6263

Koch, William

NIST

100 Bureau Drive, MS 8300

Gaithersburg, MD 20899-8300

USA

301/975-8301

301/975-3845

william.koch@nist.gov

Kratzer, Manfred

Roche Diagnostics GmbH

Nonnenwald 2

Penzberg 82372

Germany

49/8856-603032

49/8856-602130

manfred.kratzer@roche.com

Lacroix, Jean-Michel

Visible Genetics, Inc.

700 Bay St.

Suite 1000

Toronto, Ontario

M5G 1 Z6

CANADA

416/813-3240, x4883

416/813-3250

lacroix@visgen.com 
Lasky, Fred

Ortho-Clinical Diagnostics

100 Indigo Creek Dr., MC 00881

Rochester, NY 14626-5101

USA

716/453-3880

716/453-3113

flasky@ocdus.jnj.com

Lawrence, Nate

BBI Biotechnology

217 Perry Pkwy.

Gaithersburg, MD 20877

USA

301/208-8100

301/208-8829

nlawrence@bbii.com

Lequin, Rudolf

Diagnostics Consultancy

P.O. Box 4963

Eindhoven CD 5604

The Netherlands

31/40-290-8620

31/40-290-8621

r.m.lequin@wxs.nl

Levin, Barbara

NIST

100 Bureau Drive, MS 8311

Gaithersburg, MD 20899-8311

USA

301/975-6682

barbara.levin@nist.gov

Levine, Edward

Diagnostic Products Corporation

5700 West $96^{\text {th }}$ Street

Los Angeles, CA 90045-5597

USA

310/645-8200

310/645-9999

elevine@dpconline.com

Ling, Eleanor

Beckman Coulter

1000 Lake Hazeltine

Bloomington, MN 55318

USA

952/368-1208
Little, Randie

University of Missouri-Columbia

One Hospital Dr, M767

Department of Child Health

Columbia, MO 65212

USA

573/882-1257

573/884-4748

LittleR@health.missouri.edu

Lo, Stanley

Medical College of Wisconsin

9000 W. Wisconsin Avenue

Children's Hospital

Milwaukee, WI 53226

USA

414/266-2508

Long, Stephen

NIST

100 Bureau Drive, MS 8391

Gaithersburg, MD 20899-8391

USA

301/975-4140

Stephen.long@nist.gov

Manak, Mark

BBI Biotech

217 Perry Pkwy.

Gaithersburg, MD 20877

USA

301/208-8100

301/208-8829

mmanak@bbii.com

Marcus, Roy

Bayer Corporation

511 Benedict Ave.

Tarrytown, NY 10598

USA

914/524-2687

914/524-3323

roy.marcus.b@bayer.com

Maxim, Peter

FDA/CDRH

2098 Gaither Road

Rockville, MD 20850

USA

301/594-1293 
May, Willie

NIST

100 Bureau Drive, MS 8390

Gaithersburg, MD 20899-8390

USA

301/975-3108

301/926-8671

willie.may@nist.gov

Miller, Greg

Virginia Commonwealth University

Department of Pathology

P.O. Box 980286

Richmond, VA 23219

USA

804/828-0375

804/828-0353

millerg@hsc.vcu.edu

Miller, Richard

Dade Behring, Inc.

P.O. Box 6101, M/S 1

Glasgow Business Community

Newark, DE 19711

USA

302/631-6116

302/631-6122

rrmiller@ix.netcom.com

Murnane, Joseph

Underwriters Laboratories, Inc.

1285 Walt Whitman Rd.

Melville, NY 11747

USA

631/271-6200

631/439-6042

Joseph.P.MurnaneJr@us.ul.com

Myers, Gary

Centers for Disease Control and Prevention

4770 Buford Hwy., NE

Atlanta, GA 30341

USA

$770 / 488-4603$

770/488-4192

glm1@cdc.gov
Nadel, Debbie

AACC

2101 L St., NW

Washington, DC 20037

USA

202/835-8704

202/887-5093

dnadel@aacc.org

Nakamura, Masakazu

Osaka Medical Center for Cancer

3 Nakamichi 1-chome

Higashinari-ku

Osaka 537-8511

Japan

81/6-6972-1181

81/6-6972-7749

xnakamur@iph.pref.osaka.jp

Nelson, Bryant

NIST

100 Bureau Drive, MS 8392

Gaithersburg, MD 20899-8392

USA

301/975-8540

301/977-0685

bryant.nelson@nist.gov

Nelson, Chad

NIST

100 Bureau Drive, MS 8392

Gaithersburg, MD 20899-8392

USA

301/975-3389

301/977-0685

chad.nelson@nist.gov

$\mathrm{Ng}$, Ronald

Abbott Laboratories

4A Crosby Dr.

Bedford, MA 01730

USA

781/276-6212

781/271-1417

ng@medisense.com 
Pankratz, Thomas

Dade Behring, Inc.

Glasgow 707

P.O. Box 6101

Newark, DE 19714

USA

302/631-7608

302/631-7487

pankratj@dadebehring.com

Panteghini, Mauro

Azienda Ospeoaliera

'Spedali Civili'

Rm. 25125, Brescia

Italy

39/030 3995470

39/030 3995365

panteghi@bshosp.unibs.it

Parker, Donald

Bayer Corporation

1884 Miles Ave.

Elkhart, IN 46514

USA

219/264-8962

219/262-7826

don.parker.b@bayer.com

Pizza, Barbara

Genzyme Corporation

One Kendall Square

Bldg. 200, $4^{\text {th }}$ Floor

Cambridge, MA 02139

USA

617/252-7953

617/374-7470

barbara.pizza@genzyme.com

Potter, Laurence

Dade Behring, Inc.

P.O. Box 6101, M/S 514

Newark, DE 19714-6101

USA

302/631-0441

302/631-6299

potterla@dadebehring.com
Powers, Don

Powers Consulting Services

One Wandering Trail

Pittsford, NY 14534

USA

716/381-7575

425/952-1450

powers@frontiernet.net

Pry, Terry

Abbott Laboratories

1920 Hurd Dr.

MS 1-10, D2252, LC-1

Irving, TX 75038

USA

972/518-6046

972/518-7692

terry.pry@add.ssw.abbott.com

Quear, Mike

U.S. House of Representatives

Science Committee Staff

2319 Rayburn House Bldg.

Washington, DC 20515

USA

202/225-8844

mike.quear@mail.house.gov

Radmacher, Paula

University of Louisville

511 South Floyd St.

Rm. 107 MDR Bldg.

Neonatal Nutrition Research Laboratory

Louisville, KY 40292

USA

$502 / 852-5532$

502/852-8826

paula.radmacher@louisville.edu

Ring, Nancy

Diametrics Medical, Inc.

2658 Patton Rd.

Roseville, MN 55113

USA

651/638-1250

651/368-1060

nring@diametrics.com 
Robinowitz, Max

FDA HFZ-440

2098 Gaither Road

Rockville, MD 20850

USA

301/594-1293

Rohlfing, Curt

University of Missouri-Columbia

One Hospital Dr., M772

Columbia, MO 65212

USA

573/884-2385

573/884-4748

rohlfingc@health.missouri.edu

Ross, Richard

Beckman Coulter, Inc.

P.O. Box 8000

Brea, CA 92822-8000

USA

714/961-4912

714/961-4123

RTRoss@Beckman.com

San George, Richard

Inverness Medical Technology, Inc.

Two University Office Park

51 Sawyer Road

Waltham, MA 02453

USA

richardcsg@aol.com

Sander, Lane

NIST

100 Bureau Drive, MS 8392

Gaithersburg, MD 20899-8392

USA

301/975-3117

301/977-0685

lane.sander@nist.gov

Schiel, Detlef

PTB

Bundesallee 100

Braunschweig

Germany

$49 / 5315923220$

49/5315923045

detlef.schiel@ptb.de
Schimmel, Heinz

Institute for Reference Materials and

Measurement (IRMM)

Geel B-2440

Belgium

32/014-571-720

32/014-590-406

schimmel@irmm.jrc.be

Seccombe, David

Canadian External Quality Assessment

Laboratory

307-2083 Alma St.

Vancouver BC

V6R 4N6

Canada

604/222-1355

604/222-1373

dseccombe@ceqal.com

Semerjian, Hratch

NIST

100 Bureau Drive, MS 8300

Gaithersburg, MD 20899-8300

USA

301/975-8300

301/975-3845

hratch.semerjian@nist.gov

Siekmann, Lothar

University of Bonn

Bonn

D-53127

GERMANY

49/228-287-5911

49/228-287-5033

siekmann@mailer.meb.uni-bonn.de

Sieberman, Steven

Bio-Tech Imaging, Inc.

5711 Industry Lane, Unit 31

Frederick, MD 21704

USA

301/695-9900

301/695-0030

silb@biotechimaging.com 
Slickers, Kenneth

Roche Diagnostics Corporation

9115 Hague Road

P.O. Box 50457

Indianapolis, IN 46250-0457

USA

317/576-7196

317/576-4249

ken.slickers@roche.com

Smit, Nick

i-STAT Canada

436 Hazeldean Road

Kanata, Ontario, KsL 1T9

CANADA

613-831-2725, x381

Sniegoski, Lorna

NIST

100 Bureau Drive, MS 8392

Gaithersburg, MD 20899-8392

USA

301/975-3133

301/977-0685

lorna.sniegoski@nist.gov

Sridharan, Ramamurthi

Instrumentation Laboratory

113 Hartwell Ave.

Lexington, MA 02421

USA

781/861-4246

781/861-4452

ssridharan@ilww.com

Tai, Susan

NIST

100 Bureau Drive, MS 8392

Gaithersburg, MD 20899-8392

USA

301/975-3127

301/975-0685

susan.tai@nist.gov
Thomas, Jeanice Brown

NIST

100 Bureau Drive, MS 8392

Gaithersburg, MD 20899-8392

USA

301/975-3120

301/977-0685

jeanice.brownthomas@nist.gov

Thompson, John

Dade Behring

P.O. Box 6101

707

Newark, DE 19714

USA

302/631-7584

302/631-7487

thompsjc@dadebehring.com

Tonkin, Charles

UL International Ltd

Old Portsmouth Rd.

Wonersh House

Guildford GU3 1LR

United Kindom

44/1483 302130

44/1483-302230

tonkinc@ul.com

Trahey-Bale, Nancy

NIST

100 Bureau Drive

Gaithersburg, MD 20899

USA

301/975-2021

nancy.trahey@nist.gov

Tuthill Jr., Willard

Underwriters Laboratories, Inc.

1285 Walt Whitman Rd.

Melville, NY 11747

USA

631/271-6200

631/439-6132

Willard.E.TuthillJr@us.ul.com 
Vocke, Robert

NIST

100 Bureau Drive, MS 8391

Gaithersburg, MD 20899-8391

USA

301/975-4103

robert.vocke@nist.gov

Voelkert, Emil

Roche Diagnostics GmbH

Dept. GH

Sandhofer Str. 116

D-68305 Mannheim

GERMANY

49/621/759-1656

49/621-759-4583

emil.voelkert@roche.com

Wadhera, Ashwani

Health Canada

Main Stats Building, Room 1601

Ottawa, Ontario K1A 0L2

CANADA

613/954-0387

ashwani.wadhera@ha-sc.gc.ca

Wahed, M.A.

Nutritional Biochemistry

ICDDR, B

GPO Box 128

Dhaka-1000

BANGLADESH

Welch, Michael

NIST

100 Bureau Drive, MS 8392

Gaithersburg, MD 20899-8392

USA

301/975-3100

301/977-0685

michael.welch@nist.gov

Westwood, Steven

NARL, AGAL

P.O. Box 385

Pymble NSW 2073

AUSTRALIA

61/2-9449 0111

61/2-9449 1653

steven.westwood@agal.gov.au
White, Richard

Dade Behring Corporation

20400 Mariani Ave.

Cupertino, CA 95014

USA

408/366-3730

408/996-9216

rjwhite@jps.net

Wiedmeyer, Hsiao-Mei

University of Missouri-Columbia

School of Medicine

1 Hospital Drive, Rm. M767

Columbia, MO 65212

USA

573/882-2705

573/884-8823

wiedmeyerh@heath.missouri.edu

Wielgosz, Robert

BIPM

Pavillon De Breteuil

Sevres Cedex F-92312

FRANCE

33/1 45076251

33/1 45342021

rwielgos@bipm.org

Williams, Christopher

Abbott Laboratories

1401 Sheridan Rd.

D-736/M4

North Chicago, IL 60064

USA

847/938-9124

847/937-4634

christopher.williams@ln.ssw.abbott.com

Wise, Beth Ann

NCCLS

940 West Valley Rd.

Suite 1400

Wayne, PA 19087

USA

610/688-0100

bawise@nccls.org 
Wise, Stephen

NIST

100 Bureau Drive, MS 8392

Gaithersburg, MD 20899-8392

USA

301/975-3112

301/977-0685

stephen.wise@nist.gov

Wu-Wong, Ruth

Abbott Laboratories

5440 Patrick Henry Dr.

Santa Clara, CA 95054

USA

408/567-3298

408/588-2912

ruth.r.wuwong@abbott.com

Yager, Thomas

Visible Genetics, Inc.

700 Bay St., Suite 1000

Toronto, Ontario

M5G 1Z6 CANADA

416/813-3240

416/813-3250

tom@visgen.com
Onsite Registrants:

Campbell, Michael

DiTuillo, Robert 